\title{
Criticality Benchmark Guide for Light-Water-Reactor Fuel in Transportation and Storage Packages
}

Prepared by

J. J. Lichtenwalter, S. M. Bowman, M. D. DeHart, C. M. Hopper

Oak Ridge National Laboratory

Prepared for

U.S. Nuclear Regulatory Commission

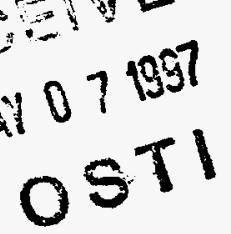




\section{DISCLAMMER}

Portions of this document may be illegible in electronic image products. Images are produced from the best available original document. 
NUREG/CR-6361

ORNL/TM-13211

\section{Criticality Benchmark Guide for Light-Water-Reactor Fuel in Transportation and Storage Packages}

Manuscript Completed: December 1996

Date Published: March 1997

Prepared by

J. J. Lichtenwalter*, S. M. Bowman, M. D. DeHart, C. M. Hopper

Oak Ridge National Laboratory

Managed by Lockheed Martin Energy Research Corp.

Oak Ridge, TN 37831-6370

Prepared for

Spent Fuel Project Office

Office of Nuclear Material Safety and Safeguards

U.S. Nuclear Regulatory Commission

Washington, DC 20555-0001

NRC Job Code B0009

\footnotetext{
* Appointee of U.S. Department of Energy Laboratory Cooperative Postgraduate Research Training Program at Oak Ridge National Laboratory administered by the Oak Ridge Institute for Science and Education.
} 
NUREG/CR-6361 has been reproduced

from the best available copy. 


\begin{abstract}
This report is designed as a guide for performing criticality benchmark calculations for light-water-reactor (LWR) fuel applications. The guide provides documentation of 180 criticality experiments with geometries, materials, and neutron interaction characteristics representative of transportation packages containing LWR fuel or uranium oxide pellets or powder. These experiments should benefit the U.S. Nuclear Regulatory Commission (NRC) staff and licensees in validation of computational methods used in LWR fuel storage and transportation concerns. The experiments are classified by key parameters such as enrichment, water/fuel volume, hydrogen-to-fissile ratio $(\mathrm{H} / \mathrm{X})$, and lattice pitch. Groups of experiments with common features such as separator plates, shielding walls, and soluble boron are also identified. In addition, a sample validation using these experiments and a statistical analysis of the results are provided. Recommendations for selecting suitable experiments and determination of calculational bias and uncertainty are presented as part of this benchmark guide.
\end{abstract}





\section{CONTENTS}

Page

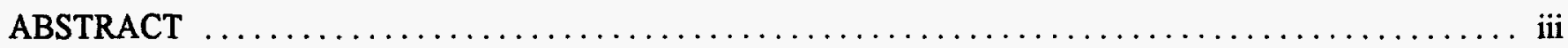

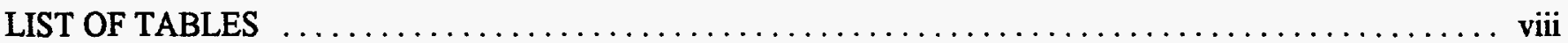

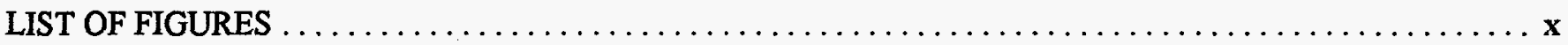

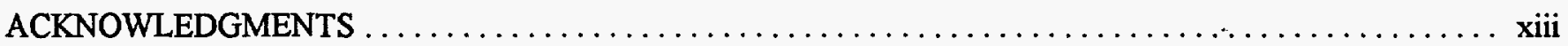

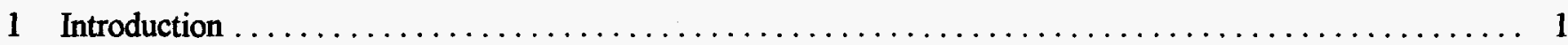

2 Physical Description of Selected Low-Enriched Critical Experiments $\ldots \ldots \ldots \ldots \ldots \ldots \ldots \ldots \ldots \ldots \ldots$

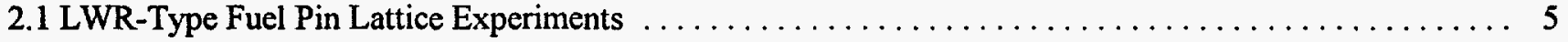

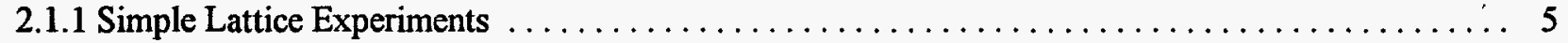

2.1.1.1 Simple Lattice Experiments from ANS Transactions, Vol. $33 \ldots \ldots \ldots \ldots \ldots \ldots \ldots 5$

2.1.1.2 Simple Lattice Experiments from BAW-1484 $\ldots \ldots \ldots \ldots \ldots \ldots \ldots \ldots \ldots \ldots$

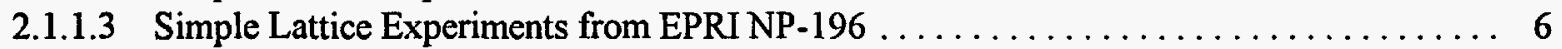

2.1.1.4 Simple Lattice Experiments from NS\&E, Vol. $71 \ldots \ldots \ldots \ldots \ldots \ldots \ldots \ldots \ldots$

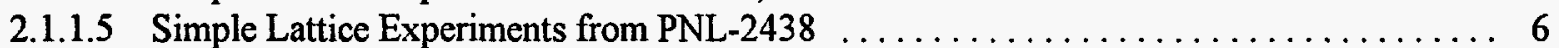

2.1.1.6 Simple Lattice Experiments from PNL-2827 $\ldots \ldots \ldots \ldots \ldots \ldots \ldots \ldots \ldots \ldots \ldots \ldots$

2.1.1.7 Simple Lattice Experiments from PNL-3314 $\ldots \ldots \ldots \ldots \ldots \ldots \ldots \ldots \ldots \ldots \ldots .7$

2.1.1.8 Simple Lattice Experiments from PNL-3926 $\ldots \ldots \ldots \ldots \ldots \ldots \ldots \ldots \ldots \ldots \ldots \ldots$

2.1.1.9 Simple Lattice Experiments from PNL-4267 $\ldots \ldots \ldots \ldots \ldots \ldots \ldots \ldots \ldots \ldots \ldots$

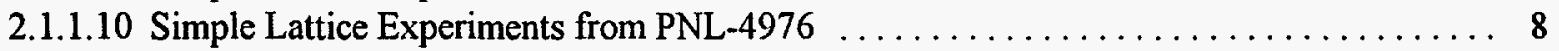

2.1.1.11 Simple Lattice Experiments from WCAP $-3269 \ldots \ldots \ldots \ldots \ldots \ldots \ldots \ldots \ldots \ldots$

2.1.1.12 Simple Lattice Experiments from WCAP $-3385 \ldots \ldots \ldots \ldots \ldots \ldots \ldots \ldots$

2.1.2 Separator Plate Experiments $\ldots \ldots \ldots \ldots \ldots \ldots \ldots \ldots \ldots \ldots \ldots \ldots \ldots \ldots \ldots$

2.1.2.1 Separator Plate Experiments from ANS Transactions, Vol. $33 \ldots \ldots \ldots \ldots \ldots \ldots .9$

2.1.2.2 Separator Plate Experiments from BAW-1484 $\ldots \ldots \ldots \ldots \ldots \ldots \ldots \ldots \ldots$

2.1.2.3 Separator Plate Experiments from BAW $-1645 \ldots \ldots \ldots \ldots \ldots \ldots \ldots \ldots \ldots \ldots \ldots$

2.1.2.4 Separator Plate Experiments from DSN No. $399 / 80 \ldots \ldots \ldots \ldots \ldots \ldots \ldots \ldots \ldots \ldots$

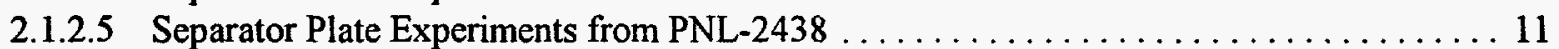

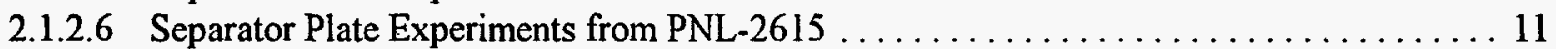

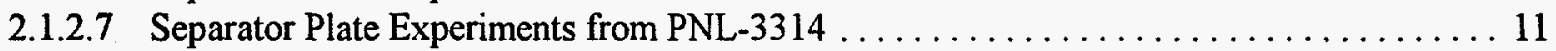

2.1.2.8 Separator Plate Experiments from PNL-6205 and PNL-7176 $\ldots \ldots \ldots \ldots \ldots \ldots \ldots 12$

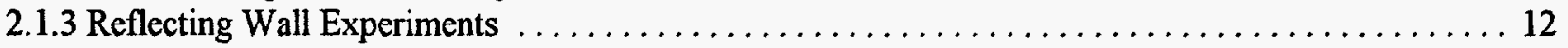

2.1.3.1 Reflecting Wall Experiments from PNL-2827 $\ldots \ldots \ldots \ldots \ldots \ldots \ldots \ldots \ldots \ldots \ldots$

2.1.3.2 Reflecting Wall Experiments from PNL-3602 $\ldots \ldots \ldots \ldots \ldots \ldots \ldots \ldots \ldots \ldots \ldots$

2.1.3.3 Reflecting Wall Experiments from PNL-3926 $\ldots \ldots \ldots \ldots \ldots \ldots \ldots \ldots \ldots \ldots \ldots$

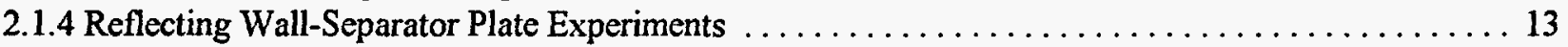

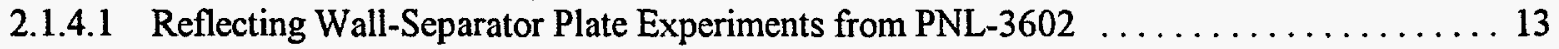

2.1.4.2 Reflecting Wall-Separator Plate Experiments from PATRAM' $80 \ldots \ldots \ldots \ldots \ldots \ldots 14$

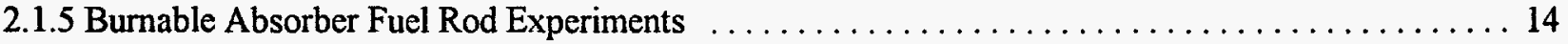

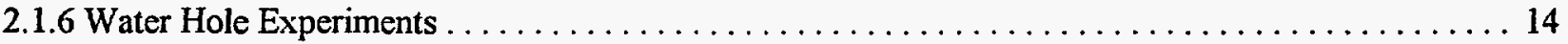

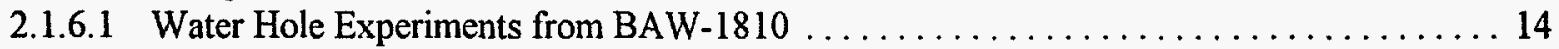


2.1.6.2 Water Hole Experiments from NS\&E, Vol. $71 \ldots \ldots \ldots \ldots \ldots \ldots \ldots \ldots \ldots \ldots \ldots$

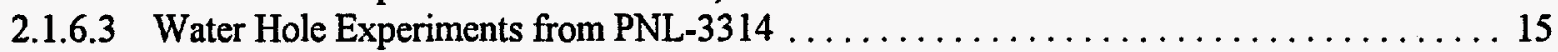

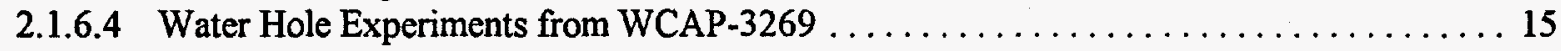

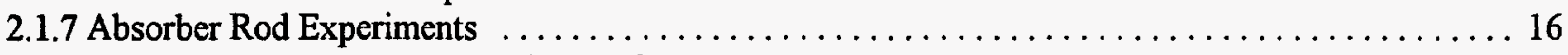

2.1.7.1 Absorber Rod Experiments from BAW-1484 $\ldots \ldots \ldots \ldots \ldots \ldots \ldots \ldots \ldots \ldots \ldots$

2.1.7.2 Absorber Rod Experiments from BAW $-1810 \ldots \ldots \ldots \ldots \ldots \ldots \ldots \ldots \ldots \ldots \ldots$

2.1.7.3 Absorber Rod Experiments from WCAP-3269 $\ldots \ldots \ldots \ldots \ldots \ldots \ldots \ldots \ldots \ldots \ldots 16$

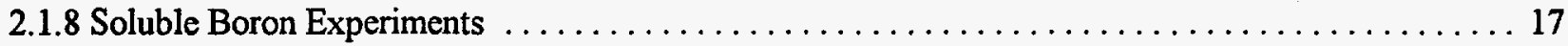

2.1.8.1 Soluble Boron Experiments from BAW-1231 $\ldots \ldots \ldots \ldots \ldots \ldots \ldots \ldots \ldots \ldots \ldots \ldots$

2.1.8.2 Soluble Boron Experiments from BAW-1273 $\ldots \ldots \ldots \ldots \ldots \ldots \ldots \ldots \ldots \ldots \ldots \ldots$

2.1.8.3 Soluble Boron Experiments from BAW-1484 $\ldots \ldots \ldots \ldots \ldots \ldots \ldots \ldots \ldots \ldots \ldots$

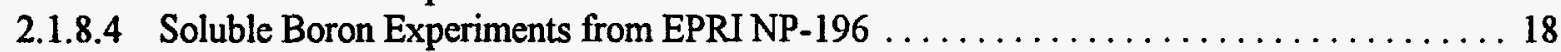

2.1.8.5 Soluble Boron Experiments from PNL-4267 $\ldots \ldots \ldots \ldots \ldots \ldots \ldots \ldots \ldots \ldots \ldots 18$

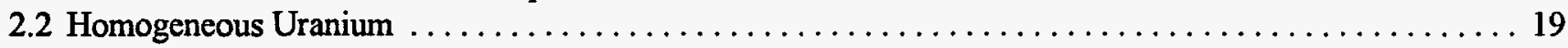

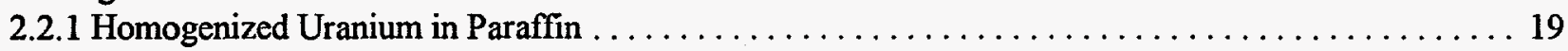

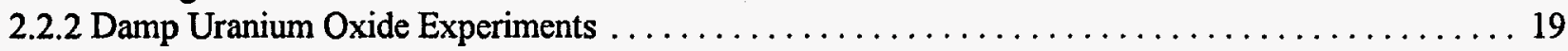

3 Validation of the SCALE 44-Group ENDF/B-V Library with the LWR Critical Experiments . . . . . . . 139

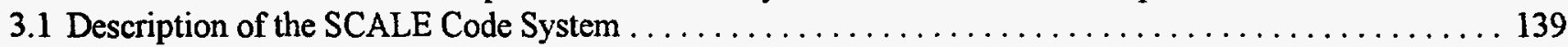

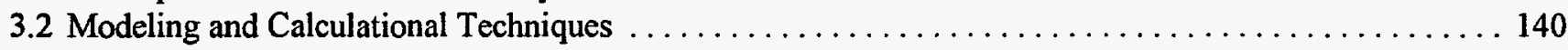

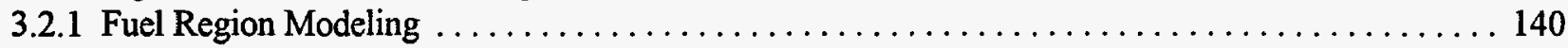

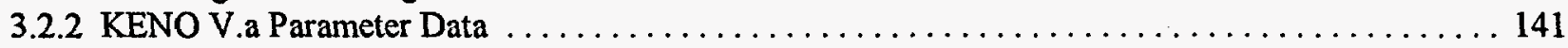

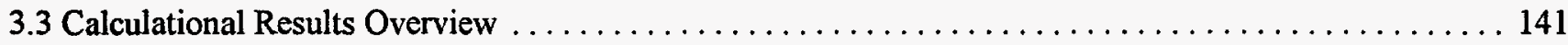

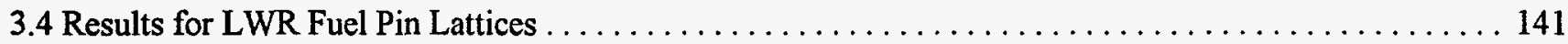

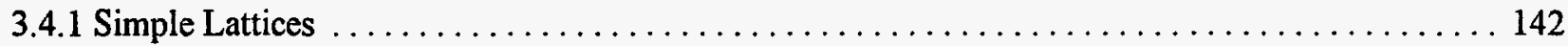

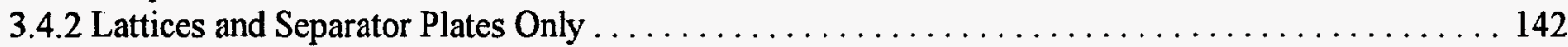

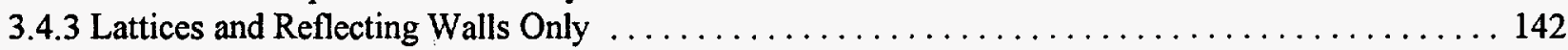

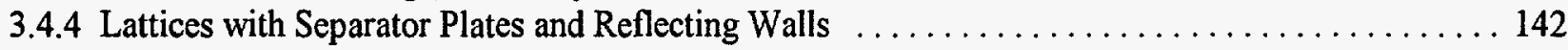

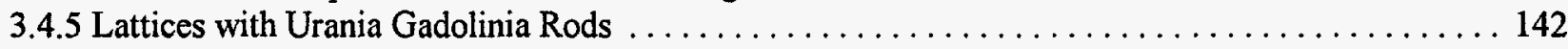

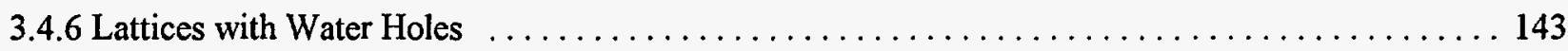

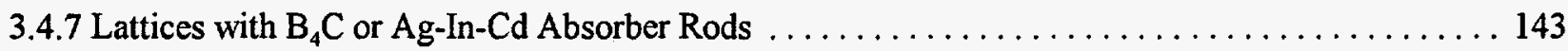

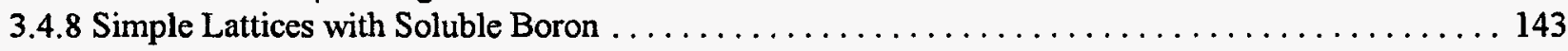

3.5 Results for Homogeneous Uranium Systems $\ldots \ldots \ldots \ldots \ldots \ldots \ldots \ldots \ldots \ldots \ldots \ldots \ldots \ldots \ldots$

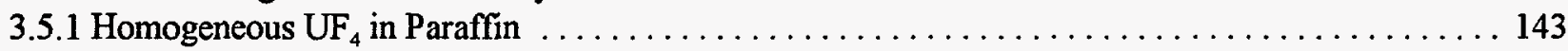

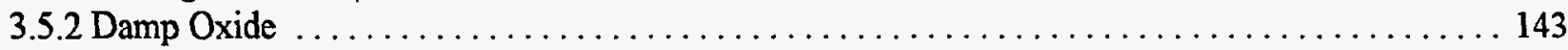

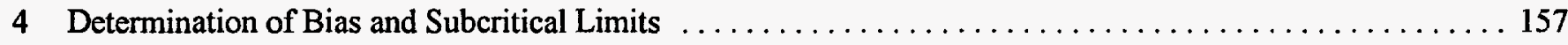

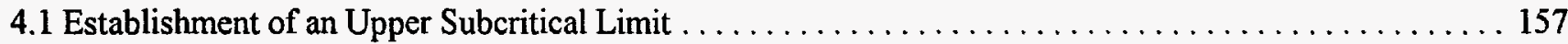

4.1.1 USL Method 1: Confidence Band with Administrative Margin $\ldots \ldots \ldots \ldots \ldots \ldots \ldots \ldots \ldots$

4.1.2 USL Method 2: Single-Sided Uniform Width Closed Interval Approach . . . . . . . . . . . 160

4.1.3 Application of Upper Subcritical Limit Methods $\ldots \ldots \ldots \ldots \ldots \ldots \ldots \ldots \ldots \ldots \ldots \ldots \ldots \ldots \ldots \ldots \ldots$

5 Guidelines for Experiment Selection and Areas of Applicability $\ldots \ldots \ldots \ldots \ldots \ldots \ldots \ldots \ldots \ldots$

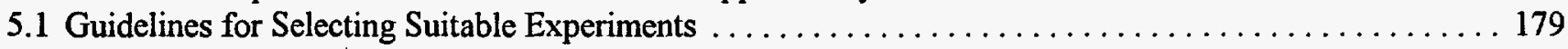

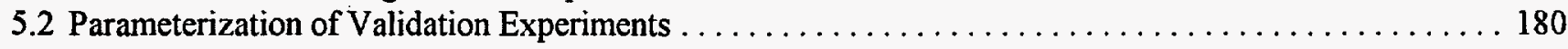

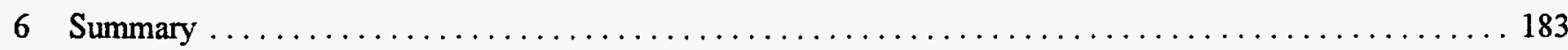

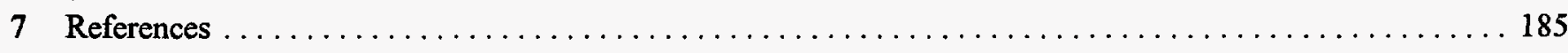

NUREG/CR-6361 vi 
Appendix A. SCALE Input File Listings .

Appendix B. Comparison of 44- and 238-Group Results

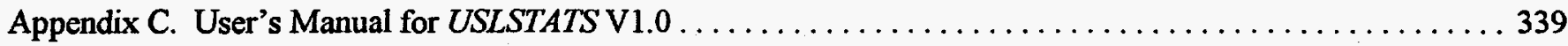

C.1 Introduction

C.2 Background

C.3 Input Description

339

C.4 Output Description

342

C. 5 How to Run USLSTATS

342

C.6 How to Obtain USLSTATS

342 


\section{LIST OF TABLES}

Table

Page

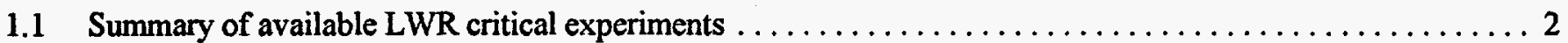

2.1 Case descriptions of $L W R$-type $\mathrm{UO}_{2}$ fuel pin lattice critical benchmark experiments $\ldots \ldots \ldots \ldots \ldots \ldots 20$

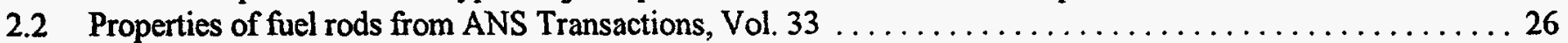

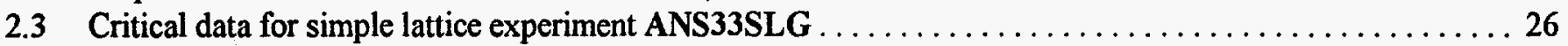

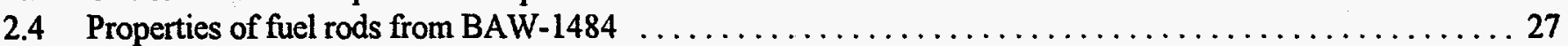

2.5 Critical data for simple lattice experiment BW1484SL from BAW-1484 $\ldots \ldots \ldots \ldots \ldots \ldots \ldots \ldots 27$

2.6 Critical data for simple lattice experiments from EPRI NP $-196 \ldots \ldots \ldots \ldots \ldots \ldots \ldots \ldots \ldots \ldots \ldots \ldots$

2.7 Critical data for simple lattice experiments from NS\&E, Vol. $71 \ldots \ldots \ldots \ldots \ldots \ldots \ldots \ldots \ldots \ldots \ldots .29$

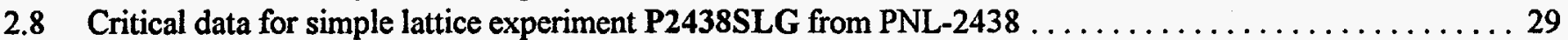

2.9 Critical data for simple lattice experiment P2827SLG fromPNL-2827 $\ldots \ldots \ldots \ldots \ldots \ldots \ldots \ldots \ldots 29$

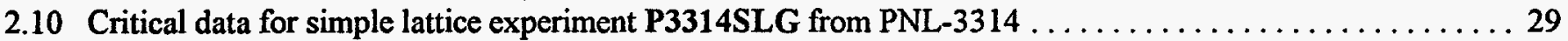

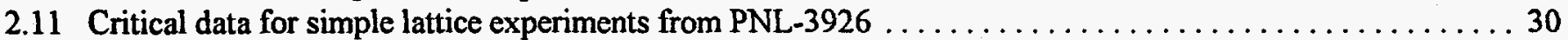

2.12 Critical data for simple lattice experiments from PNL $-4267 \ldots \ldots \ldots \ldots \ldots \ldots \ldots \ldots \ldots \ldots \ldots \ldots \ldots$

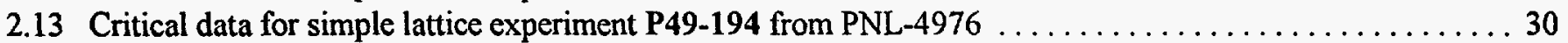

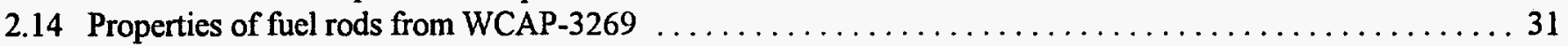

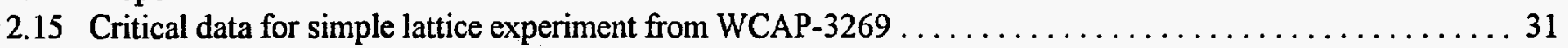

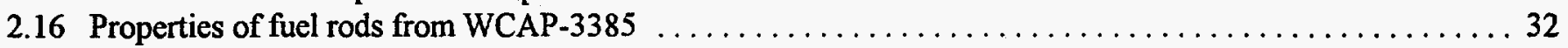

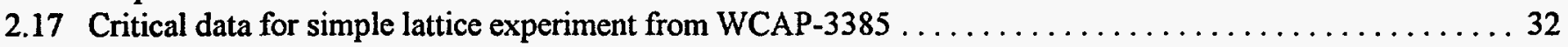

2.18 Critical Data for separator plate experiments from ANS Transactions, Vol. $33 \ldots \ldots \ldots \ldots \ldots \ldots \ldots$

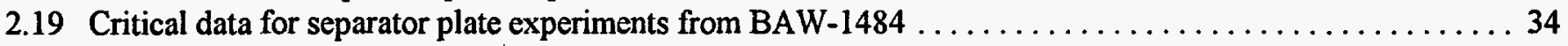

2.20 Critical data for separator plate experiments from BAW $-1645 \ldots \ldots \ldots \ldots \ldots \ldots \ldots \ldots \ldots \ldots \ldots . \ldots \ldots$

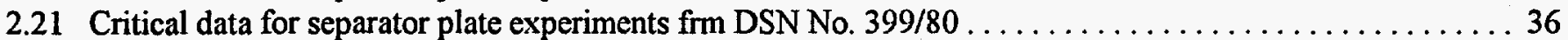

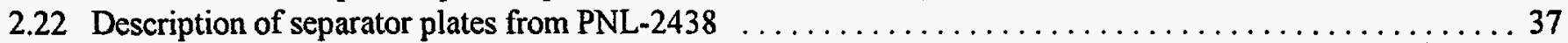

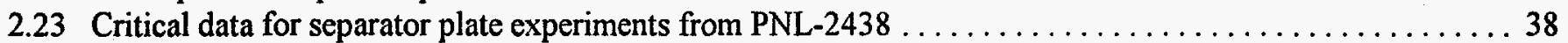

2.24 Critical data for separator plate experiments from PNL-2615 $\ldots \ldots \ldots \ldots \ldots \ldots \ldots \ldots \ldots \ldots \ldots \ldots . \ldots \ldots$

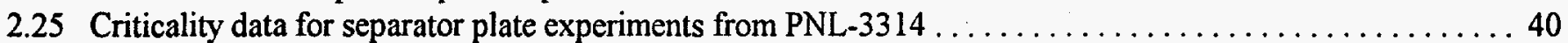

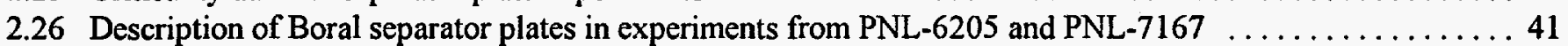

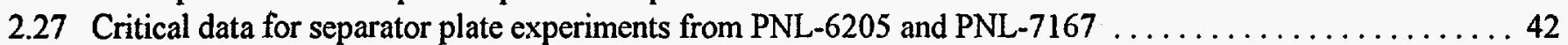

2.28 Modeled assemblies of separator plate experiments from PNL-6205 and PNL-7167 $\ldots \ldots \ldots \ldots \ldots \ldots 43$

2.29 Critical data for reflecting wall experiments from PNL $-2827 \ldots \ldots \ldots \ldots \ldots \ldots \ldots \ldots \ldots \ldots \ldots \ldots$

2.30 Critical data for reflecting wall experiments from PNL $-3602 \ldots \ldots \ldots \ldots \ldots \ldots \ldots \ldots \ldots \ldots \ldots \ldots$

2.31 Critical data for reflecting wall experiments from PNL-3926 $\ldots \ldots \ldots \ldots \ldots \ldots \ldots \ldots \ldots \ldots \ldots \ldots \ldots$

2.32 Critical data for reflecting wall-separator plate experiments from PNL-3602 $\ldots \ldots \ldots \ldots \ldots \ldots \ldots 4$

2.33 Critical data for reflecting wall-separator plate experiments from PATRAM' $80 \ldots \ldots \ldots \ldots \ldots \ldots \ldots 48$

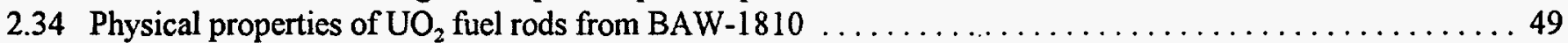

2.35 Physical properties of $\mathrm{UO}_{2} \mathrm{Gd}_{2} \mathrm{O}_{3}$ fuel rods from $\mathrm{BAW}-1810 \ldots \ldots \ldots \ldots \ldots \ldots \ldots \ldots \ldots \ldots \ldots \ldots$

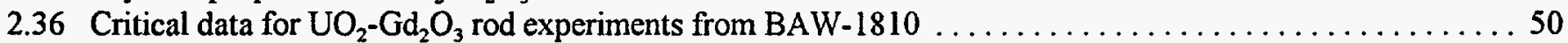

2.37 Critical data for water hole experiments from $\mathrm{BAW}-1810 \ldots \ldots \ldots \ldots \ldots \ldots \ldots \ldots \ldots \ldots \ldots \ldots \ldots \ldots \ldots$

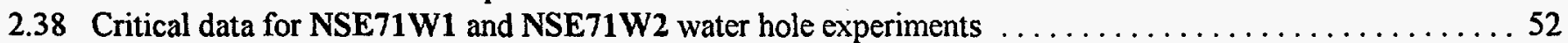

2.39 Critical data for water hole experiments from PNL $-3314 \ldots \ldots \ldots \ldots \ldots \ldots \ldots \ldots \ldots \ldots \ldots \ldots \ldots$

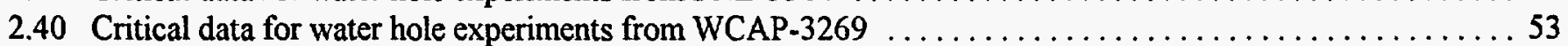

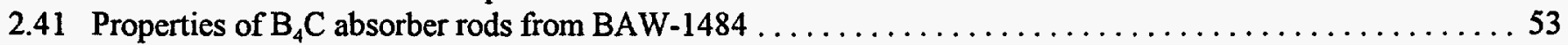

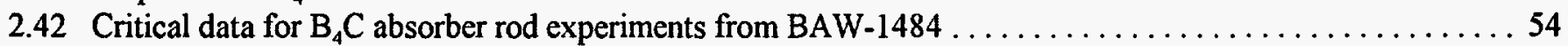

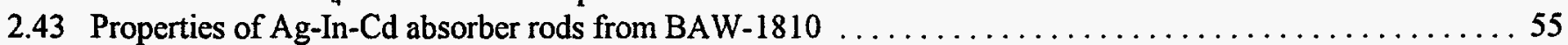

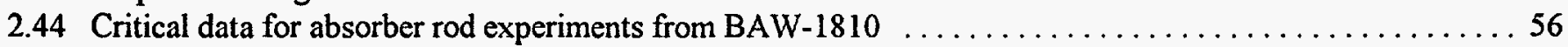

2.45 Critical data for Ag-In-Cd absorber rod experiments from WCAP-3269 $\ldots \ldots \ldots \ldots \ldots \ldots \ldots \ldots$ 
2.46 Critical data for soluble boron experiments from BAW $-1231 \ldots \ldots \ldots \ldots \ldots \ldots \ldots \ldots \ldots \ldots \ldots \ldots$

2.47 Critical data for soluble boron experiment BW1273M $\ldots \ldots \ldots \ldots \ldots \ldots \ldots \ldots \ldots \ldots \ldots \ldots \ldots$

2.48 Critical data for soluble boron experiments from BAW-1484 $\ldots \ldots \ldots \ldots \ldots \ldots \ldots \ldots \ldots \ldots \ldots \ldots$

2.49 Critical data for simple lattice experiment from EPRI NP $-196 \ldots \ldots \ldots \ldots \ldots \ldots \ldots \ldots \ldots \ldots \ldots \ldots$

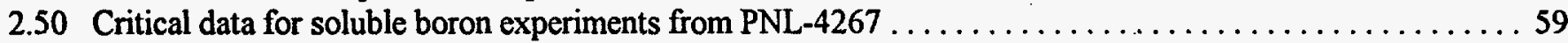

2.51 Atom densities (atoms $/ \mathrm{b}-\mathrm{cm}$ ) of fuel in critical experiments from $\mathrm{Y}-\mathrm{DR}-14 \ldots \ldots \ldots \ldots \ldots \ldots \ldots$

2.52 Criticality data for homogenized uranium experiments from $Y-D R-14 \ldots \ldots \ldots \ldots \ldots \ldots \ldots \ldots \ldots$

2.53 Cuboid fuel can descriptions for CR1071AS, CR1653AS, CR2500S $\ldots \ldots \ldots \ldots \ldots \ldots \ldots \ldots \ldots \ldots 6$

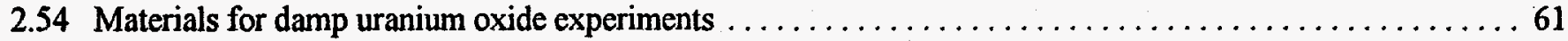

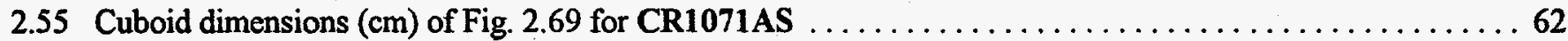

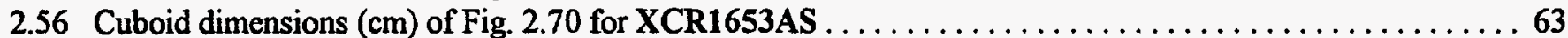

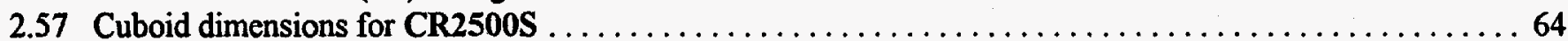

3.1 Broad-group structure of the 44GROUPNDF5 library $\ldots \ldots \ldots \ldots \ldots \ldots \ldots \ldots \ldots \ldots \ldots \ldots \ldots \ldots$

3.2 Explicit fuel rod models for fuel above the moderator $\ldots \ldots \ldots \ldots \ldots \ldots \ldots \ldots \ldots \ldots \ldots \ldots \ldots \ldots$

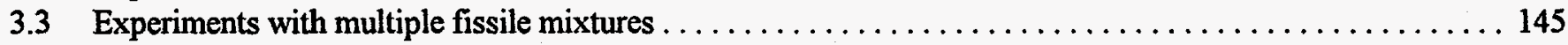

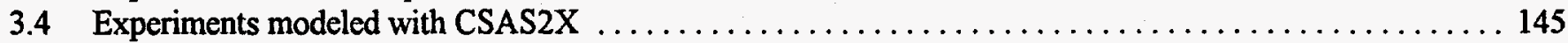

3.5 Calculational results using SCALE 44 -group cross-section library $\ldots \ldots \ldots \ldots \ldots \ldots \ldots \ldots \ldots \ldots \ldots 146$

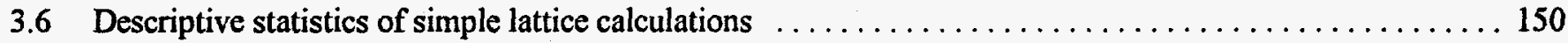

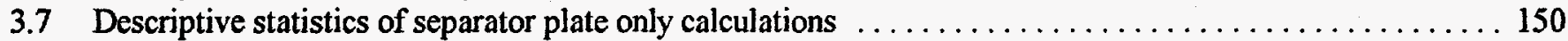

3.8 Descriptive statistics of separator plate-soluble boron calculations $\ldots \ldots \ldots \ldots \ldots \ldots \ldots \ldots \ldots \ldots \ldots \ldots$

3.9 Descriptive statistics of separator plate-void material (flux trap) calculations $\ldots \ldots \ldots \ldots \ldots \ldots \ldots 1$

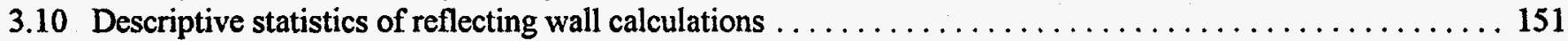

3.11 Descriptive statistics of reflecting wall-separator plate calculations $\ldots \ldots \ldots \ldots \ldots \ldots \ldots \ldots \ldots \ldots 1$

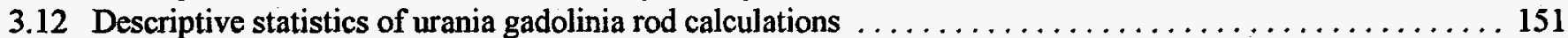

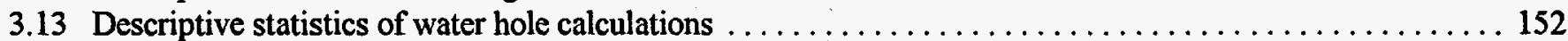

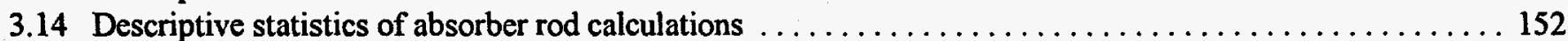

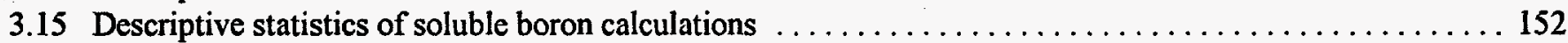

3.16 Calculational results and experimental parameters of homogenized uranium experiments $\ldots \ldots \ldots \ldots 153$

3.17 Descriptive statistics of homogenized uranium calculations $\ldots \ldots \ldots \ldots \ldots \ldots \ldots \ldots \ldots \ldots \ldots \ldots \ldots$

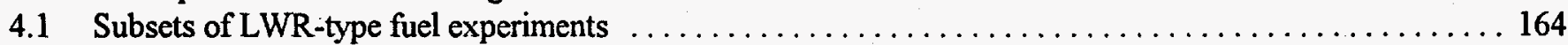

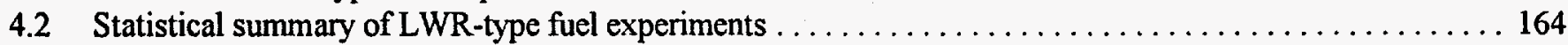

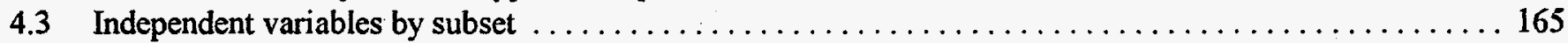

4.4 Regression analyses results for subsets of $L W R$-type fuel experiments $\ldots \ldots \ldots \ldots \ldots \ldots \ldots \ldots \ldots 166$

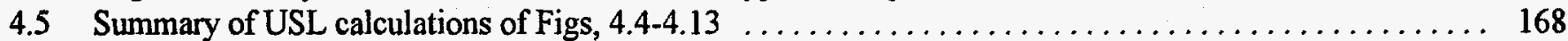

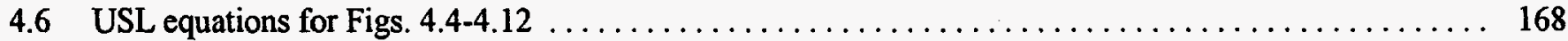

5.1 Characterization variables of $L W R$-type fuel experiments $\ldots \ldots \ldots \ldots \ldots \ldots \ldots \ldots \ldots \ldots \ldots \ldots \ldots$

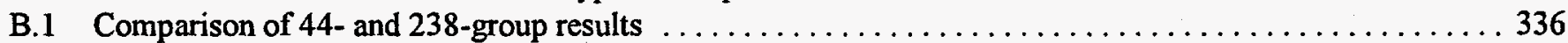




\section{LIST OF FIGURES}

Figure

2.1 Principal dimensions of $4.742 \mathrm{wt} \% \mathrm{UO}_{2}$ fuel rods for ANS Transactions, Vol. 33, and NS\&E, Vol. 71 ,

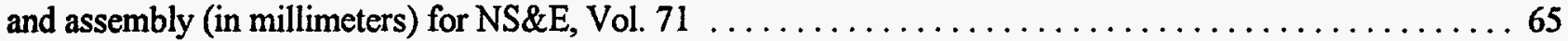

2.2 Planar and half-end view of experiments from ANS Transactions, Vol. $33 \ldots \ldots \ldots \ldots \ldots \ldots \ldots \ldots 6$

2.3 Dimensions of $2.459 \mathrm{wt} \% \mathrm{UO}_{2}$ fuel rods for experiments from $\mathrm{BAW}-1484 \ldots \ldots \ldots \ldots \ldots \ldots \ldots \ldots 6$

2.4 Experiment BW1484SL vertical dimensions $\ldots \ldots \ldots \ldots \ldots \ldots \ldots \ldots \ldots \ldots \ldots \ldots \ldots \ldots \ldots . \ldots \ldots$

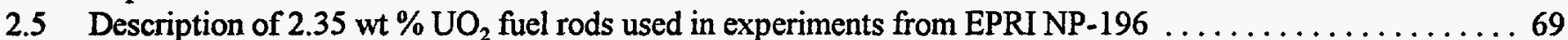

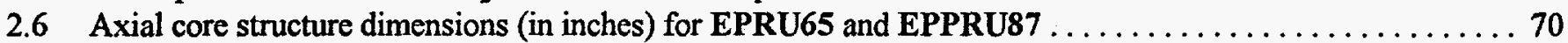

2.7 Axial core structure dimensions (in inches) for EPRU75 $\ldots \ldots \ldots \ldots \ldots \ldots \ldots \ldots \ldots \ldots \ldots \ldots \ldots \ldots$

2.8 Dimensions (in inches) of "eggcrate" lattice grids for EPRU65 and EPRU87 $\ldots \ldots \ldots \ldots \ldots \ldots \ldots \ldots 72$

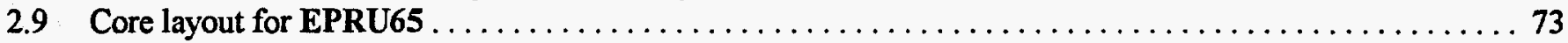

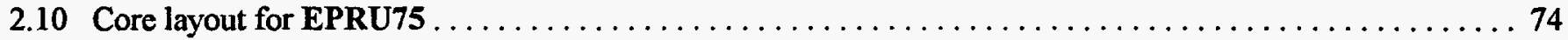

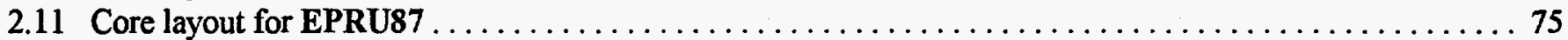

2.12 Core configurations for three hexagonal lattice experiments from NS\&E, Vol. $71 \ldots \ldots \ldots \ldots \ldots \ldots \ldots 76$

2.13 Planar and end views of experiments from PNL $-2438 \ldots \ldots \ldots \ldots \ldots \ldots \ldots \ldots \ldots \ldots \ldots \ldots \ldots$

2.14 Description of $4.31 \mathrm{wt} \% \mathrm{UO}_{2}$ fuel rods used in experiments from PNL-3314 $\ldots \ldots \ldots \ldots \ldots \ldots \ldots \ldots 78$

2.15 Planar and end view of experiments with four-assembly geometry from PNL-3314 $\ldots \ldots \ldots \ldots \ldots \ldots 79$

2.16(a) Fuel assemblies and absorber plate configurations for experiments with $4.31 \mathrm{wt} \%$

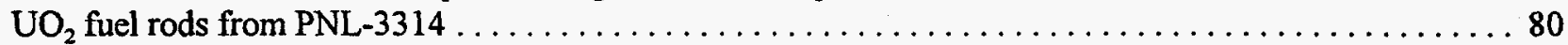

2.16(b) Fuel assemblies and absorber plate configurations for experiments with $2.35 \mathrm{wt} \%$

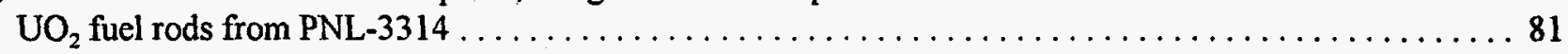

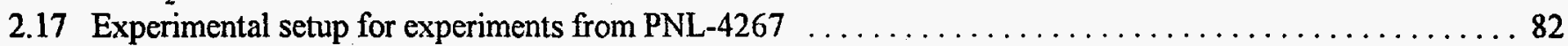

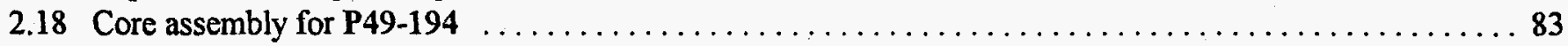

2.19 Experimental assembly elevations for $\mathbf{P} 49-194 \ldots \ldots \ldots \ldots \ldots \ldots \ldots \ldots \ldots \ldots \ldots \ldots \ldots \ldots \ldots$

2.20 Vertical dimensions of experimental assembly for W3269SL1 $\ldots \ldots \ldots \ldots \ldots \ldots \ldots \ldots \ldots \ldots \ldots$

2.21 Vertical dimensions of experimental assembly for W3269SL2 $\ldots \ldots \ldots \ldots \ldots \ldots \ldots \ldots \ldots \ldots \ldots$

2.22 Vertical dimensions of experiments from WCAP $-3385 \ldots \ldots \ldots \ldots \ldots \ldots \ldots \ldots \ldots \ldots \ldots \ldots \ldots$

2.23 Grid plate layouts for experiments from WCAP $-3385 \ldots \ldots \ldots \ldots \ldots \ldots \ldots \ldots \ldots \ldots \ldots \ldots \ldots$

2.24 Vertical dimensions for separator plate experiments from BAW $-1484 \ldots \ldots \ldots \ldots \ldots \ldots \ldots \ldots \ldots . \ldots 89$

2.25 Side sheet locations for separator plate experiments from BAW $-1645 \ldots \ldots \ldots \ldots \ldots \ldots \ldots \ldots \ldots \ldots 9 . \ldots$

2.26 Vertical dimensions of experiments from BAW $-1645 \ldots \ldots \ldots \ldots \ldots \ldots \ldots \ldots \ldots \ldots \ldots \ldots \ldots$

2.27 Intermediate spacing for BW1645T1, BW1645T2, BW1645T3, and BW1645T4 ......... 92

2.28 Cross-sectional view of separator plate experiments from DSN No. $399 / 80 \ldots \ldots \ldots \ldots \ldots \ldots \ldots \ldots$

2.29 Fuel cluster and steel angle iron for DSN399-1 and DSN399-2 $\ldots \ldots \ldots \ldots \ldots \ldots \ldots \ldots \ldots \ldots \ldots$

2.30 Planar and end view of experiments with three-assembly geometry from PNL-3314 . . . . . . . . . 95

2.31 Experimental assembly evaluations for experiments from PNL-6205 and PNL-7176 . . . . . . . . 96

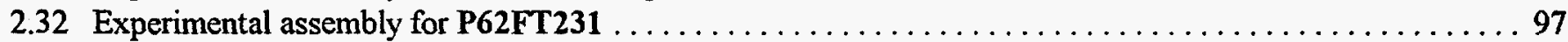

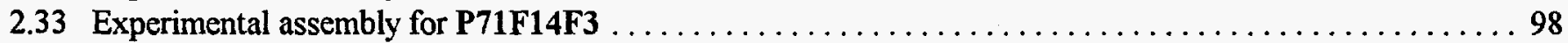

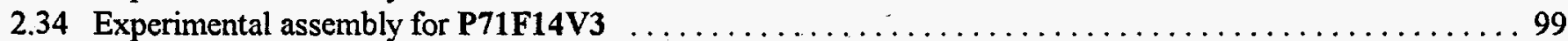

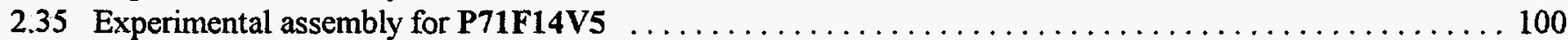

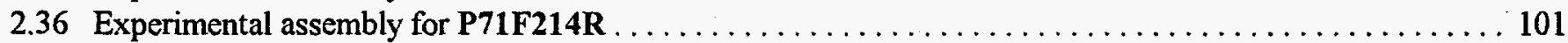

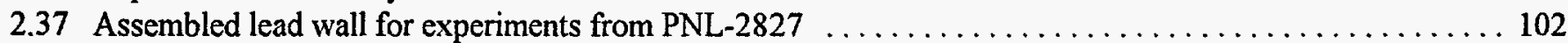

2.38 Assembled depleted uranium wall for experiments from PNL $-2827 \ldots \ldots \ldots \ldots \ldots \ldots \ldots \ldots \ldots \ldots$

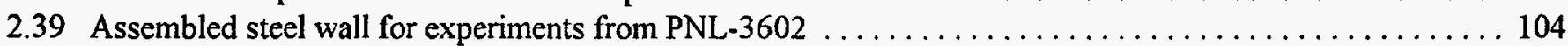

2.40 Quarter-assembly configuration for experiments from PATRAM' $80 \ldots \ldots \ldots \ldots \ldots \ldots \ldots \ldots \ldots \ldots$

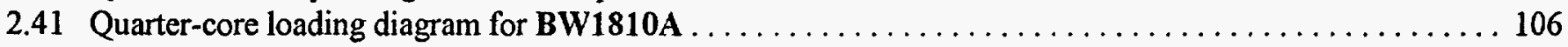




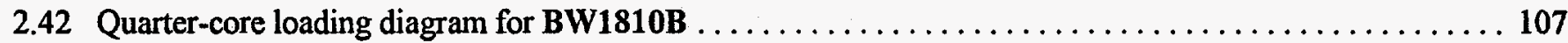

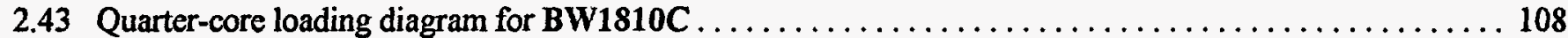

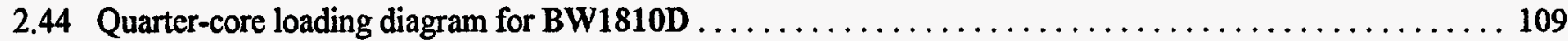

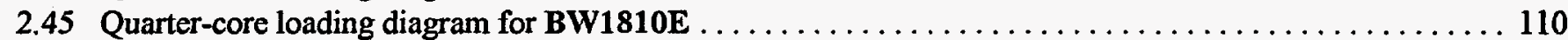

2.46 Quarter-core loading diagram for BW1810F $\ldots \ldots \ldots \ldots \ldots \ldots \ldots \ldots \ldots \ldots \ldots \ldots \ldots \ldots \ldots \ldots$

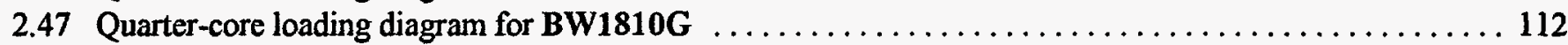

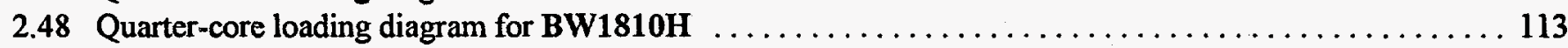

2.49 Cross-sectional view of modeled NSE71W1 and NSE71 W2 assemblies with water holes $\ldots \ldots \ldots \ldots 114$

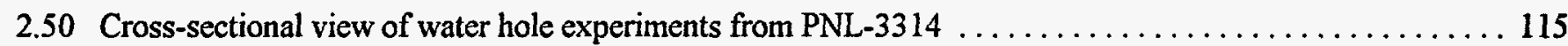

2.51 Cross-sectional view of modeled P3314W1 and P3314W2 assemblies with water holes $\ldots \ldots \ldots \ldots 116$

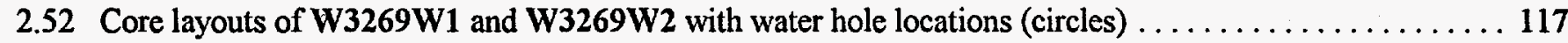

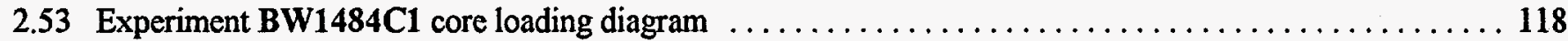

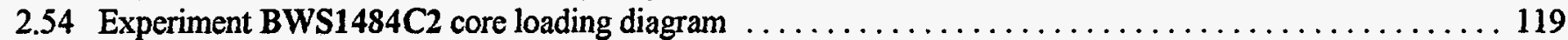

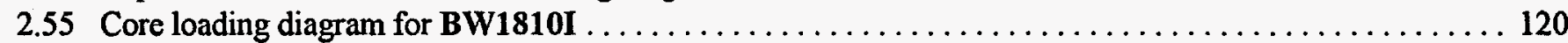

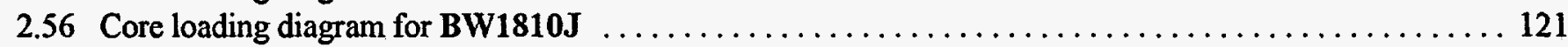

2.57 Vertical cross section of core assembly for experiments W3269B1, W3269B2, and W3269B3 ..... 122

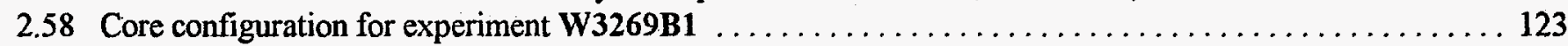

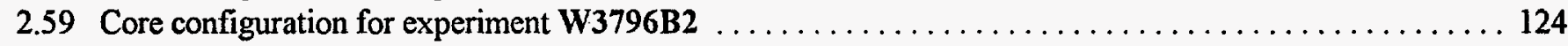

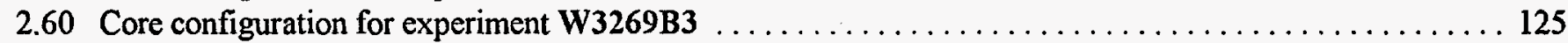

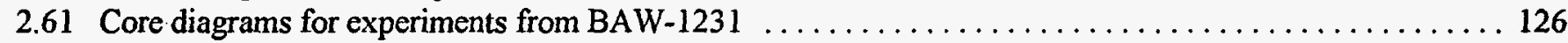

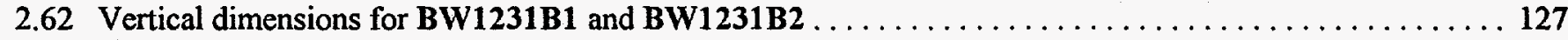

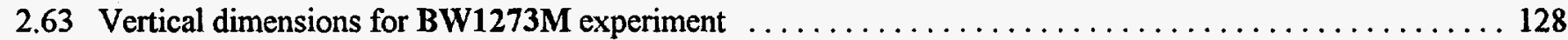

2.64 Cross-sectional view of modeled BW1273M core . . . . . . . . . . . . . . . . . . . . . . . . . . . . . . 129

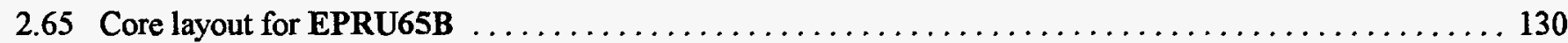

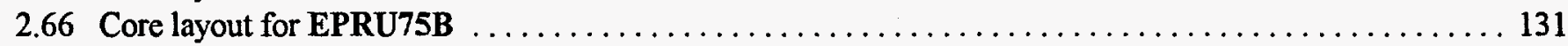

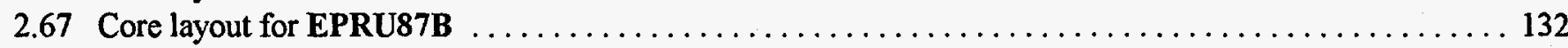

2.68 Isometric drawings of the (a) 42 , (b) 38 , and (c) 30 can configurations of experiment

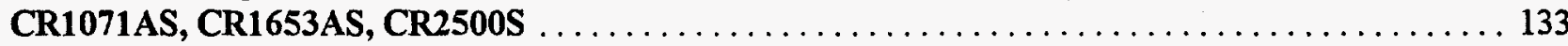

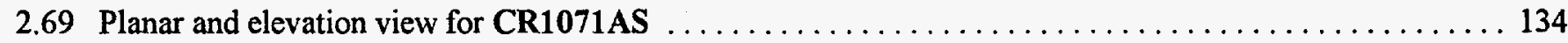

2.70 Planar and elevation view of core boundary (dashed lines) and reflector boundaries (solid lines)

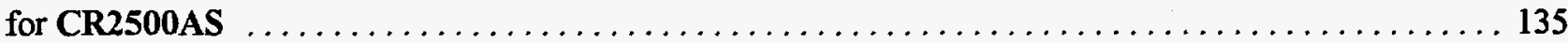

2.71 Dimension (mm) plastic reflector end panel and frame for CR1653AS $\ldots \ldots \ldots \ldots \ldots \ldots \ldots \ldots$

2.72 Three nested cuboids of CR2500S: core, reflector interior, reflector exterior $\ldots \ldots \ldots \ldots \ldots \ldots \ldots \ldots$

3.1 Frequency chart for calculated $k_{\text {eff }}$ of $173 \mathrm{LWR}$ fuel pin lattice experiments $\ldots \ldots \ldots \ldots \ldots \ldots \ldots \ldots$

3.2 Frequency chart for calculated AEF of $173 \mathrm{LWR}$ fuel pin lattice experiments $\ldots \ldots \ldots \ldots \ldots \ldots \ldots$

4.1 Confidence band and additional margin applied to a set of criticality calculations . . . . . . . . ... 169

4.2 USLs for $167 \mathrm{LWR}$-type fuel critical experiments, $k_{\text {eff }}$ vs enrichment $\ldots \ldots \ldots \ldots \ldots \ldots \ldots \ldots \ldots$

4.3 USLs for $167 \mathrm{LWR}$-type fuel critical experiments, $k_{\text {eff }} v s \ln (\mathrm{AEF}) \ldots \ldots \ldots \ldots \ldots \ldots \ldots \ldots \ldots \ldots$

4.4 USLs for 76 separated assemblies with separator plate critical experiments, $k_{\text {eff }}$ vs $\mathrm{H} / \mathrm{X} \ldots \ldots \ldots \ldots 172$

4.5 USLs for 50 reflecting wall experiments, $k_{\mathrm{eff}}$ vs assembly separation $\ldots \ldots \ldots \ldots \ldots \ldots \ldots \ldots \ldots$

4.6 USLs for 36 soluble boron experiments, $k_{\text {eff }}$ vs boron concentration $\ldots \ldots \ldots \ldots \ldots \ldots \ldots \ldots \ldots \ldots$

4.7 USLs for 15 separator plate and reflecting wall experiments, $k_{\text {eff }} v s$ pitch $\ldots \ldots \ldots \ldots \ldots \ldots \ldots \ldots$

4.8 USLs for 10 water hole experiments, $k_{\text {eff }}$ vs enrichment $\ldots \ldots \ldots \ldots \ldots \ldots \ldots \ldots \ldots \ldots \ldots \ldots$

4.9 USLs for 7 borated separator plates and reflecting wall critical experiments, $\mathrm{k}_{\mathrm{eff}}$ vs reflecting

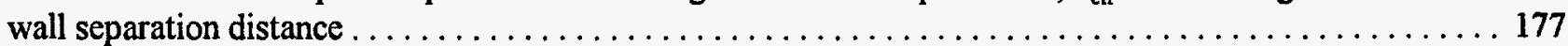





\section{ACKNOWLEDGMENTS}

The authors would like to thank Marissa Bailey, NRC Technical Monitor for this project, who provided valuable guidance for this report. Many ORNL staff contributed their knowledge, experience, and expertise, including $H$. $R$. Dyer, W. C. Jordan, C. V. Parks, L. M. Petrie, and R. M. Westfall. Special appreciation is expressed to C. V. Parks for his leadership and guidance. L. F. Norris also deserves recognition for her outstanding efforts in transforming the drafts into a professional report.

This research was supported in part by an appointment to the U.S. Department of Energy Laboratory Cooperative Postgraduate Research Training Program at the Oak Ridge National Laboratory administered by the Oak Ridge Institute for Science and Education. 


\section{Introduction}

This report is designed as a guide for performing criticality benchmark calculations for light-water-reactor (LWR) fuel applications. The guide provides documentation of 180 criticality experiments with geometries, materials, and neutron interaction characteristics representative of transportation packages containing LWR fuel or uranium oxide pellets or powder. These experiments should benefit the U.S. Nuclear Regulatory Commission (NRC) staff and licensees in validation of computational methods used in LWR fuel storage and transportation concerns. The experiments are classified by key parameters such as enrichment, water-to-fuel volume ratio, hydrogen-to-fissile ratio $(\mathrm{H} / \mathrm{X})$, and lattice pitch. Groups of experiments with common features such as separator plates, reflecting walls, and soluble boron are also identified. In addition, a sample validation using these experiments and a statistical analysis of the results are provided. Recommendations for selecting suitable experiments and determination of calculational bias and uncertainty are presented as part of this benchmark guide.

A literature survey was performed of low-enriched, LWR-type fuel critical experiment reports to select the experiments documented here. Table 1.1 presents a brief summary of the reports surveyed and the types and number of available experiments found. The table also indicates the number of experiments selected for inclusion in this report. This information may be useful to an analyst who wishes to perform a more extensive validation for a particular category of LWR fuel experiments.

Descriptions of the selected critical experiments are presented in Sect. 2 in sufficient detail for independent analysis by any computational tool that is appropriate for performing criticality safety analyses.

Section 3 documents benchmark calculations of these experiments performed with the SCALE code system ${ }^{1}$ using the 44-group ENDF/B-V cross-section library. ${ }^{2}$ Results include neutron multiplication factors $\left(k_{\text {eff }}\right)$, presented individually and combined by experiment type. The SCALE input files used to generate the results of Sect. 3 are listed in Appendix A. Individual results based on the 238-group ENDF/B-V library ${ }^{3}$ are also presented for comparison in Appendix B.

Determinations of calculational biases and subcritical limits are discussed in Sect. 4. Statistical analyses of the SCALE calculational results are performed to parameterize trends. The purpose of these analyses is to determine the correlation of bias in calculated $k_{\text {eff }}$ values to key parameters. The statistical results are then applied in the demonstration of two methods for establishing an upper subcritical limit (USL): (1) a $95 \%$ confidence band for a single future calculation with an administrative subcritical margin and (2) a lower tolerance band based on a $95 \%$ confidence band on $\mathbf{9 9 . 5 \%}$ of all future calculations using the uslstats program documented in Appendix $\mathrm{C}$.

Section 5 discusses the issue of areas of applicability in the validation of computational methods and presents guidance on how to select criticality experiments that are applicable to validate the computational method for the system of concern. Parameters that should be studied to demonstrate areas of applicability are discussed.

A summary of this benchmark guide is presented in Sect. 6 . 
Table 1.1 Summary of available LWR critical experiments

\begin{tabular}{|c|c|c|c|}
\hline Report & $\begin{array}{c}\text { No. of } \\
\text { available } \\
\text { experiments }\end{array}$ & $\begin{array}{l}\text { No. of } \\
\text { selected } \\
\text { experiments }\end{array}$ & Description of criticality experiments \\
\hline $\begin{array}{l}\text { ANS Transactions, Vol. } \\
\text { 33, p.362 } \\
\text { (Ref. 5) }\end{array}$ & 25 & 9 & $\begin{array}{l}4.74 \mathrm{wt} \%{ }^{235} \mathrm{U} \mathrm{UO}_{2} \text { fuel rods in square lattices of } 1.35-\mathrm{cm} \text { pitch; fuel } \\
\text { clusters separated by air, polystyrene, polyethylene, or water, fuel } \\
\text { clusters submersed in aqueous } \mathrm{NaNO}_{3} \text { solution }\end{array}$ \\
\hline BAW-1484 (Ref. 6) & 37 & 10 & $\begin{array}{l}2.46 \mathrm{wt} \%{ }^{235} \mathrm{U} \mathrm{UO}_{2} \text { fuel rods in square lattices of } 1.636-\mathrm{cm} \text { pitch; the } \\
\text { spacing between } 3 \times 3 \text { array of } \mathrm{LWR} \text {-type fuel assemblies is filled with } \\
\text { water and } \mathrm{B}_{4} \mathrm{C} \text { pins, stainless steel sheets, or borated stainless steel } \\
\text { sheets; lattices with borated moderator }\end{array}$ \\
\hline EPRI-NP-196 (Ref. 7) & 6 & 6 & $\begin{array}{l}2.35 \mathrm{wt} \%{ }^{233} \mathrm{U} \mathrm{UO}_{2} \text { fuel rods in square lattices of } 1.562-, 1.905-\text {, and } \\
2.210 \text {-cm pitch; lattices with borated moderator }\end{array}$ \\
\hline $\begin{array}{l}\text { NS\&E, Vol. 71, } \\
\text { p. } 154 \text { (Ref. 8) }\end{array}$ & 26 & 6 & $\begin{array}{l}4.74 \mathrm{wt} \%{ }^{233} \mathrm{U} \mathrm{UO}_{2} \text { fuel rods in square lattices of } 1.26-, 1.60-, 2.10 \text {, } \\
\text { and } 2.52-\mathrm{cm} \text { pitch; triangular and triangular with pseudo-cylindrical } \\
\text { shape lattices of } 1.35-, 1.72-\text {, and } 2.26-\mathrm{cm} \text { pitch; irregular hexagonal } \\
\text { lattices of } 1.35-\mathrm{cm} \text { pitch; lattices with water holes }\end{array}$ \\
\hline PNL-2438 (Ref. 9) & 48 & 6 & $\begin{array}{l}2.35 \mathrm{wt} \%{ }^{235} \mathrm{U} \mathrm{UO}_{2} \text { fuel rods in square lattices of } 2.032 \text {-cm pitch; } \mathrm{Cd} \text {, } \\
\mathrm{Al} \text {, Cu, stainless steel, borated stainless steel, Boral, and Zircaloy } \\
\text { separator plates between assemblies }\end{array}$ \\
\hline PNL-2827 (Ref. 10) & 23 & 9 & $\begin{array}{l}2.35 \text { and } 4.31 \mathrm{wt} \%{ }^{235} \mathrm{U} \mathrm{UO}_{2} \text { fuel rods in square lattices of } 2.032-\text { and } \\
2.540 \text {-cm pitch; reflecting walls of } \mathrm{Pb} \text { or depleted uranium }\end{array}$ \\
\hline PNL-3314 (Ref. 11) & 142 & 27 & $\begin{array}{l}2.35 \text { and } 4.31 \mathrm{wt} \%{ }^{235} \mathrm{U} \mathrm{UO}_{2} \text { fuel rods in square lattices of } 1.684 \text { - and } \\
1.892 \text {-cm pitch; stainless steel, borated stainless steel, } \mathrm{Cd}, \mathrm{Al}, \mathrm{Cu} \text {, } \\
\text { Boral, Boroflex, and Zircaloy separator plates between assemblies; } \\
\text { lattices with water holes and voids }\end{array}$ \\
\hline PNL-3926 (Ref. 12) & 22 & 14 & $\begin{array}{l}2.35 \text { and } 4.31 \mathrm{wt} \%{ }^{235} \mathrm{U} \mathrm{UO}_{2} \text { fuel rods in square lattices of } 1.684-\text { and } \\
1.892-\mathrm{cm} \text { pitch; reflecting walls of } \mathrm{Pb} \text { or depleted uranium }\end{array}$ \\
\hline PNL-4267 (Ref. 13) & 9 & 7 & $\begin{array}{l}4.31 \mathrm{wt} \%{ }^{235} \mathrm{U} \mathrm{UO}_{2} \text { fuel rods in square lattices of } 1.890 \text { - and } 1.715-\mathrm{cm} \\
\text { pitch; lattices with borated moderator }\end{array}$ \\
\hline PNL-4976 (Ref. 14) & 17 & 1 & $\begin{array}{l}4.31 \mathrm{wt} \%(2.35 \mathrm{wt} \%){ }^{235} \mathrm{U} \mathrm{UO}_{2} \text { fuel rods in hexagonal lattices of } \\
2.398-, 1.801-\text {, and } 1.598-\mathrm{cm} \text { pitch; moderator contains Gadolinium }\end{array}$ \\
\hline WCAP-3269 (Ref. 15) & 157 & 9 & $\begin{array}{l}2.7,3.7 \text {, and } 5.7 \mathrm{wt} \%{ }^{235} \mathrm{U} \mathrm{UO}_{2} \text { fuel rods in square lattices of } 1.029-\text {, } \\
1.105-\text {, and } 1.422-\mathrm{cm} \text { pitch; lattices with } \mathrm{Ag}-\mathrm{In}-\mathrm{Cd} \text { absorber rods, water } \\
\text { holes, void tubes }\end{array}$ \\
\hline WCAP-3385 (Ref. 16) & 3 & 2 & $\begin{array}{l}5.74 \mathrm{wt} \%{ }^{235} \mathrm{U} \mathrm{UO}_{2} \text { fuel rods in square lattices of } 1.321-, 1.422-\text {, and } \\
2.012-\mathrm{cm} \text { pitch }\end{array}$ \\
\hline
\end{tabular}


Table 1.1 (continued)

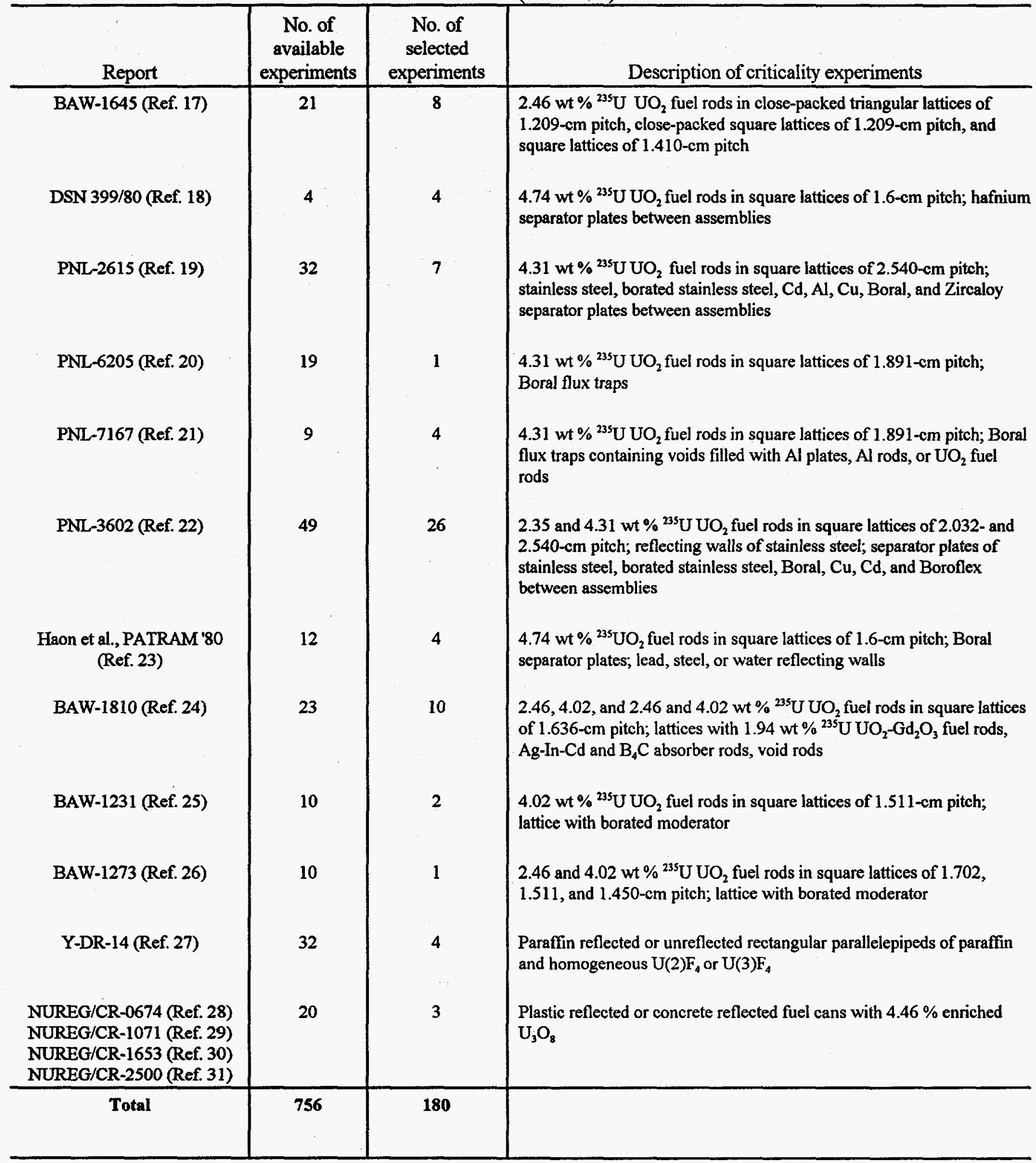





\section{Physical Description of Selected Low-Enriched Critical Experiments}

This section provides descriptions of the materials and physical layouts of 180 low-enriched critical experiments ${ }^{5-28}$ suitable for benchmarking LWR fuel analyses. The experiments were selected based on structural, material, poison, geometry, and spectral similarities to NRC-certified radioactive materials packages. ${ }^{4}$ The 180 experiments can be categorized as 173 LWR-type fuel pin lattice experiments and 7 homogeneous uranium experiments. Brief descriptions of the 180 experiments are presented in Table 2.1. More detailed descriptions and modeling assumptions are found in Sects. 2.1 and 2.2. All experimental data reported are nominal values. The reader may refer to the references for these experiments to obtain data regarding experimental uncertainties. Items that are noted as "not modeled" or "ignored" were generally located near the top or bottom of the experiment where neutron leakage was high. The volume occupied by these items in the experiment were modeled as the surrounding medium, either water or void. General modeling and calculational techniques used in the SCALE-4.3 calculations are discussed in Sect. 3.

\subsection{LWR-Type Fuel Pin Lattice Experiments}

The following sections present descriptions of experiments utilizing $\mathrm{UO}_{2} \mathrm{LWR}$-type fuel in square and hexagonal lattices moderated by light water. The experiments are categorized as simple lattices (2.1.1), separator plates (2.1.2), reflecting walls (2.1.3), reflecting walls and separator plates (2.1.4), burnable absorber fuel rods (2.1.5), water holes (2.1.6), poison rods (2.1.7), and borated moderator (2.1.8).

\subsubsection{Simple Lattice Experiments}

This set of experiments consists of large single lattices (cores) and arrays of assemblies void of poisons, significant reflecting materials, water holes, etc. The experiments represent simple transportation packages with primary criticality control by means of controlling assembly interaction. Modeling of the experiments demonstrates the ability of the calculational techniques to predict the neutron multiplication factor of essentially infinite arrays of rods (cores) or neutronically interacting separated assemblies. The experiments provide baselines for comparisons to more complex problems.

\subsubsection{Simple Lattice Experiments from ANS Transactions, Vol. 33 (Ref. 5)}

ANS33SLG consists of a $2 \times 2$ array of fuel assemblies separated by $5.0 \mathrm{~cm}$ of water. The $4.742 \mathrm{wt} \% \mathrm{UO}_{2}$ fuel rod specifications are given in Table 2.2 and Fig. 2.1. The planar and end views of the experiment are shown in Fig. 2.2; the $0.3-\mathrm{cm}$-thick aluminum wall box was replaced by water in this experiment. Critical data for the experiment are located in Table 2.3 .

Assumptions: The holes in the lower lattice grid plate for accommodating the fuel rods were taken to be $1.0 \mathrm{~cm}$ in diameter from Ref. 23. The aluminum box density, $2.651 \mathrm{~g} / \mathrm{cm}^{3}$, was assumed from Ref. 23.

Model-experiment comparison: The spring of the fuel rods was modeled as void. The end plugs were modeled as $0.94-$ $\mathrm{cm}$-diam aluminum cylinders. The fuel rods above the moderator were modeled by the method explained in Sect. 3.2.1.

\subsubsection{Simple Lattice Experiments from BAW-1484 (Ref. 6)}

BW1484SL consists of a $3 \times 3$ array of fuel assemblies separated by $6.544 \mathrm{~cm}$ of water. Details of the $2.459 \mathrm{wt} \%$ $\mathrm{UO}_{2}$ fuel rod design are outlined in Table 2.4 and Fig. 2.3. The vertical dimensions for the experiment are found in Fig. 2.4. Absent from Fig. 2.4: the 1 in. $(2.54 \mathrm{~cm})$ between the base plate and core tank is mostly water and the core tank is $0.5 \mathrm{in}$. $(1.27 \mathrm{~cm})$ thick. Difficult to distinguish in Fig. 2.4 is that the fuel rod end plugs are located in $0.125 \mathrm{in}$. 
$(0.3175 \mathrm{~cm})$ of water between the bottom grid plate and the base plate. The critical data for this experiment are located in Table 2.5.

Assumptions: The aluminum corner rods are believed to be of fuel rod length. The separation distance between assemblies is believed to have been measured from the cell boundaries of the assemblies. To accommodate fuel rods, square holes with $0.615-\mathrm{cm}$ sides were assumed in the bottom grid plate.

Model-experiment comparison: The $22.9-\mathrm{cm} \times 22.9-\mathrm{cm} \times 2.54-\mathrm{cm}$ aluminum top grid plate was not modeled. The fuel rods and separator plate above the moderator region were not modeled.

\subsubsection{Simple Lattice Experiments from EPRI NP-196 (Ref. 7)}

These experiments consist of large singular arrays of fuel rods. Specifications for the $2.35 \mathrm{wt} \% \mathrm{UO}_{2}$ fuel rods are presented in Fig. 2.5. Figure 2.6 is the axial arrangement of experiments EPRU65 and EPRU87. Figure 2.7 is the axial arrangement of experiment EPRU75. The grid plate holes for EPRU75 are $0.586 \mathrm{in} .(1.488 \mathrm{~cm})$ in diameter. The specifications for the aluminum "eggcrate" grids seen in Fig. 2.6 are given in Fig. 2.8. The core layouts are shown in Figs. 2.9-2.11. The critical data are located in Table 2.6.

Model-experiment comparison: The soluble boron in the moderator was not modeled because of the low concentration. A 182.88-cm-diam, 274.32-cm-tall tank constitutes full-water reflection of the cores. The experiments were modeled with $6 \mathrm{in} .(15.24 \mathrm{~cm})$ of water above the lead shield and $30 \mathrm{~cm}$ of water below the aluminum solid plate.

\subsubsection{Simple Lattice Experiments from NS\&E, Vol. 71 (Ref. 8)}

These experiments consist of large singular arrays of fuel rods in square or hexagonal lattices. Specifications for the $4.742 \mathrm{wt} \% \mathrm{UO}_{2}$ fuel rods are given in Table 2.2 and Fig. 2.1. The principal dimensions of the experiments are also given in Fig. 2.1. The layouts of the three hexagonal lattice cores are presented in Fig. 2.12. The critical data for the experiments are located in Table 2.7.

Assumptions: The holes in the lower grid plate to accommodate the fuel rods were taken to be $1.0 \mathrm{~cm}$ in diameter from Ref. 23.

Model-experiment comparison: The reported upper grid, missing from Fig 2.1, was ignored in the model due to insufficient data. The two grid plates were reportedly attached to the support plate by four fuel rods distanced more than $20 \mathrm{~cm}$ from the fuel. These rods were not modeled. The fuel rods above the moderator were modeled by the method explained in Sect. 3.2.1. The stainless steel support plate was modeled as $71.40 \mathrm{wt} \% \mathrm{Fe}, 19.16 \mathrm{wt} \% \mathrm{Cr}$, and $9.44 \mathrm{wt} \% \mathrm{Ni}$. For NSE71H1, NSE71H2, and NSE71 H3, $0.25 \mathrm{~cm}$ of the bottom end plug was homogenized with the surrounding lower grid plate and water. The remaining $1.55 \mathrm{~cm}$ of the bottom end plug was homogenized with the surrounding water.

\subsubsection{Simple Lattice Experiments from PNL-2438 (Ref. 9)}

P2438SLG consists of a $3 \times 1$ array of fuel assemblies separated by $8.39 \mathrm{~cm}$ of water. Details of the $2.35 \mathrm{wt} \% \mathrm{UO}_{2}$ fuel rods are outlined in Fig. 2.5. Planar and axial views of the experiment can be found in Fig. 2.13; the experiment was minus the poison plates and biological shielding walls shown in Fig 2.13. Critical data for the experiments are located in Table 2.8. 
Model-experiment comparison: The 1.27-cm-thick acrylic grid plates were modeled as water because acrylic plastic has about the same density and neutron moderating characteristics as water. The carbon steel core tank was not modeled because the amount of water on the boundary of the array ensures a maximum increase in $k_{\text {eff }}$

\subsubsection{Simple Lattice Experiments from PNL-2827 (Ref. 10)}

P2827SLG consists of a $3 \times 1$ array of fuel assemblies separated by $8.31 \mathrm{~cm}$ of water. Details of the $2.35 \mathrm{wt} \% \mathrm{UO}_{2}$ fuel rods are outlined in Fig. 2.5. Planar and axial views of the experiment can be found in Fig. 2.13; the experiment was minus the poison plates and biological shielding walls shown in Fig. 2.13. Critical data for the experiments are located in Table 2.9 .

Model-experiment comparison: The 1.27-cm-thick acrylic grid plates were modeled as water because acrylic plastic has about the same density and neutron moderating characteristics as water. The carbon steel core tank was not modeled because the amount of water on the boundary of the array ensures a maximum increase in $k_{\text {eff }}$

\subsubsection{Simple Lattice Experiments from PNL-3314 (Ref. 11)}

P3314SLG consists of a $2 \times 2$ array of fuel assemblies separated by $2.83 \mathrm{~cm}$ and $10.86 \mathrm{~cm}$ of water. Descriptions of the $4.31 \mathrm{wt} \% \mathrm{UO}_{2}$ fuel rods are provided in Fig. 2.14. Planar and axial views of the experiment are shown in Fig. 2.15. Figure $2.16 \mathrm{a}$ (c) illustrates the $2 \times 2$ assembly configuration. Critical data on the experiment are given in Table 2.10.

Model-experiment comparison: The minimum fuel rod pellet column length, $91.44 \mathrm{~cm}$, was modeled. The two polypropylene (neutronic properties similar to water) grid plates were modeled as water. The carbon steel tank was not modeled because the amount of water on the boundary of the array ensures a maximum increase in $k_{\text {eff }}$

\subsubsection{Simple Lattice Experiments from PNL-3926 (Ref. 12)}

The experiments consist of $3 \times 1$ arrays of fuel assemblies separated by 6.59 and $12.79 \mathrm{~cm}$ of water. Details of the 2.35 and $4.31 \mathrm{wt} \% \mathrm{UO}_{2}$ fuel rods are outlined in Figs. 2.5 and 2.14. Planar and axial views of the experiment can be found in Fig. 2.15. Absent in the experiments are the poison plates and biological shielding walls of Fig. 2.15. Critical data for the experiments are located in Table 2.11.

Model-experiment comparison: The minimum fuel rod pellet column length, $91.44 \mathrm{~cm}$, was modeled for the $4.31 \mathrm{wt} \%$ $\mathrm{UO}_{2}$ fuel rods. The 1.27 -cm-thick polypropylene $\left(0.904 \mathrm{~g} / \mathrm{cm}^{3}\right)$ grid plates were modeled as water because polypropylene has about the same density and neutron moderating characteristics as water. The carbon steel core tank was not modeled because the amount of water on the boundary of the array ensures a maximum increase in $k_{\text {eff }}$.

\subsubsection{PNL-4267 Simple Lattice Experiments from PNL-4267 (Ref. 13)}

The experiments consist of single arrays of fuel rods. Specifications for the $4.31 \mathrm{wt} \%$ fuel rod are located in Fig. 2.14. The planar and elevation views of the experiments are illustrated in Fig. 2.17. The Plexiglas box is fully reflected on all four sides by unborated water. Critical data for the experiments are given in Table 2.12.

Model-experiment comparison: The minimum fuel rod pellet column length, $91.44 \mathrm{~cm}$, was modeled.

The PNL-4267 experiments are subcritical configurations. The number of rods required to achieve critical are predicted by the approach-to-critical method. For P4267SL1 and P4267SL2, the nearest whole number of rods 
required for criticality was modeled. The partial rod can be modeled if using CSAS2X. P4267SL1 was modeled with 9 columns of $\mathbf{4 0}$ fuel rods, and 1 far left column of 37 fuel rods arbitrarily placed against the lower Plexiglas box side of Fig. 2.17 (planar view). The array was positioned along its length against the right side of the Plexiglas box with the array boundary next to the Plexiglas box surface. The array was centered along its width in the Plexiglas box. P4267SL2 was modeled with 11 columns of 44 fuel rods, and 1 far left column of 25 fuel rods arbitrarily placed against the lower Plexiglas box side of Fig. 2.17 (planar view). The array was positioned along its length against the right side of the Plexiglas box with the array boundary next to the Plexiglas box surface. The array was centered along its width in the Plexiglas box.

A discrepancy in Fig. 2.17 was noticed. As displayed in the figure, the cell boundaries along the array width are 75.88 $\mathrm{cm}$ apart. Forty and 44 fuel rods placed in lattices of 1.890 - and $1.715-\mathrm{cm}$ pitch give rise to cell boundaries 75.6 and $75.46 \mathrm{~cm}$ apart, not $75.88 \mathrm{~cm}$.

\subsubsection{Simple Lattice Experiments from PNL-4976 (Ref. 14)}

The experiment P49-194 used a large singular array of fuel rods in a hexagonal lattice. Specifications for the $4.31 \mathrm{wt}$ $\% \mathrm{UO}_{2}$ fuel rods are located in Fig. 2.14. The core assembly and experimental assembly elevations are illustrated in Figs 2.18 and 2.19. Missing from Fig. 2.19: The acrylic $\left(1.185 \mathrm{~g} / \mathrm{cm}^{3}\right)$ is composed of $60 \mathrm{wt} \% \mathrm{C}, 32 \mathrm{wt} \% \mathrm{O}$, and 8 wt \% H; the polypropylene $\left(0.905 \mathrm{~g} / \mathrm{cm}^{3}\right)$ lattice plates are $91.44 \mathrm{~cm}$ in diameter. The critical data for P49-194 are given in Table 2.13.

Assumptions: With a core tank diameter of $152 \mathrm{~cm}$, the core can be considered fully reflected by water laterally. The acrylic base plate is believed to have the same diameter as the polypropylene lattice plates.

Model-experiment comparison: The minimum fuel pellet column length, $91.44 \mathrm{~cm}$, was used. The aluminum spacers, fiberglass tank bottom, carbon steel tank, and concrete floor were excluded from the model. The polypropylene lattice plates were modeled as polyethylene using hydrogen in a water thermal kernel.

\subsubsection{Simple Lattice Experiments from WCAP-3269 (Ref. 15)}

W3269SL1 and W3269SL2 use large singular arrays of fuel rods. Descriptions of the 2.72 and $5.7 \mathrm{wt} \% \mathrm{UO}_{2}$ fuel rods are located in Table 2.14. The vertical dimensions of experiments W3269SL1 and W3269SL2 are shown in Figs. 2.20 and 2.21; the guide plates are aluminum. The absorber section of Fig. 2.20 was not part of experiment W3269SL1. Critical data for the experiments are located in Table 2.15.

Assumptions: The moderator height was believed to have been measured from the bottom of the fuel. The guide plate holes to accommodate fuel rods were assumed to be of fuel rod diameter. In Fig. 2.21, all plates were taken to be of assembly width and depth; full-water reflection was assumed on the sides of the experimental assembly; the 2.54-cmthick plate below the bottom guide plate was assumed to be of aluminum. In Fig. 2.20, full-water reflector was assumed at the boundary of the assembly defined by the $60.96-\mathrm{cm}-\mathrm{square}$ guide plates.

Model-experiment comparison: The fuel rods above the moderator were modeled by the method explained in Sect. 3.2.1. In Fig. 2.21 (W3269SL2) the $6.35 \mathrm{~cm}$ above the aluminum base plate was modeled as water only. In Fig. 2.20 (W3269SL1) the top and center guide plates, in air for this experiment, were not modeled; the bottom end plugs in the bottom guide plate were modeled as aluminum; the bottom end plugs in the 0.188 -in. $(0.428-\mathrm{cm})$-thick water slot and the top end plugs were not modeled. 


\subsubsection{Simple Lattice Experiments from WCAP-3385 (Ref. 16)}

W3385SL1 and W3385SL2 consist of large singular arrays of fuel rods. The $5.74 \mathrm{wt} \% \mathrm{UO}_{2}$ rods are described in Table 2.16. Vertical dimensions of the experiments are given in Fig. 2.22. Missing from, or hard to distinguish in, Fig. 2.22 is that: (1) all structures are made of aluminum, (2) the bottom grid plate rests on a 1-in. (2.54-cm)-thick aluminum slab above $2.5 \mathrm{in}$. $(6.35 \mathrm{~cm})$ of water and a 2 -in. $(5.08-\mathrm{cm})$-thick aluminum slab, (3) the fuel pellet column ends are $0.5 \mathrm{in} .(1.27 \mathrm{~cm})$ above the bottom grid plate and $0.4 \mathrm{in}$. $(1.016 \mathrm{~cm})$ below the top grid plate, and (4) the stainless steel support rods are $0.4375 \mathrm{in} .(1.1113 \mathrm{~cm})$ in diameter with 0.625 -in. $(0.476-\mathrm{cm})$-thick aluminum cladding. Figure 2.23 shows the location and size of the fuel rod holes and circulation holes of the grid plates of Fig. 2.22. Critical data for the experiments are located in Table 2.17.

Assumptions: The core tank is $121.92 \mathrm{~cm}$ in diameter constituting full-water reflection. The end plugs are believed to be of cladding material (i.e., 304 stainless steel).

Model-experiment comparison: The support rods were ignored. Homogenized water and aluminum were used to represent the center grid plate and circulation holes. The fuel rods above the moderator were modeled by the method explained in Sect. 3.2.1.

\subsubsection{Separator Plate Experiments}

These experiments have been selected to demonstrate the ability of the calculational techniques to predict the neutron multiplication factor of experiments with neutron absorbing or scattering materials (i.e., separator plates) between fuel assemblies. Separator plates are commonly used in transportation packages to limit neutron interaction and increased reactivity associated with the bringing into close proximity of fuel assemblies.

\subsubsection{Separator Plate Experiments from ANS Transactions, Vol. 33 (Ref. 5)}

The experiments consist of $2 \times 2$ arrays of fuel assemblies separated by aluminum boxes (four sides and a bottom) filled with water, polyethylene powder and balls, and expanded polystyrene. These experiments further test the ability of the calculation method to predict $\mathrm{k}_{\mathrm{eff}}$ for assemblies with various hydrogenous compound moderators. Specifications of the $4.742 \mathrm{wt} \%$ fuel rods are given in Table 2.2 and Fig. 2.1. The planar and end views of the experiment are shown in Fig. 2.2. Critical data for the experiments are located in Table 2.18.

Assumptions: Holes to accommodate the fuel rods in the lower grid plate were taken to be $1.0 \mathrm{~cm}$ in diameter from Ref. 23. The aluminum box density, $2.651 \mathrm{~g} / \mathrm{cm}^{3}$, was assumed from Ref. 23.

Model-experiment comparison: The spring of the fuel rods was modeled as void. The end plugs were modeled as $0.94-$ cm-diam aluminum cylinders. The fuel rods above the moderator were modeled by the method explained in Sect. 3.2.1.

\subsubsection{Separator Plate Experiments from BAW-1484 (Ref. 6)}

The experiments consist of $3 \times 3$ arrays of fuel assemblies separated by borated aluminum plates and water or 304-L stainless steel plates and water. Details of the $2.459 \mathrm{wt} \% \mathrm{UO}_{2}$ fuel rods are outlined in Table 2.4 and Fig. 2.3. The elevations of the experiments can be found in Fig. 2.24. The top grid was not used in the experiments. Missing from, or difficult to distinguish in, Fig. 2.24 is that (1) the fuel pellet column and fuel rod bottom end plugs extend 0.635 and $0.9525 \mathrm{~cm}$ into the aluminum bottom grid, respectively, (2) the aluminum bottom grid and aluminum bottom grid plate are $2.54 \mathrm{~cm}$ thick, (3) and $0.3175 \mathrm{~cm}$ of water exists between the aluminum bottom grid plate and the $5.08-\mathrm{cm}$-thick aluminum base plate. Critical data for the experiments are located in Table 2.19. 
Assumptions: The aluminum corner rods are assumed to be of fuel rod length. The separation distance between assemblies is believed to have been measured from the cell boundaries of the assemblies. Holes, $0.615 \mathrm{~cm}$ on a side, were assumed in the bottom grid plate to accommodate the fuel rods.

Model-experiment comparison: The 22.9-cm $\times 22.9-\mathrm{cm} \times 0.3175-\mathrm{cm}$ aluminum center grid plate was not modeled. The fuel rods and separator plate above the moderator region were not modeled. In the model, the separator plates rest in a channel cut $0.635-\mathrm{cm}$ deep into the bottom grid with the channel thickness equaling the separation distance of assemblies and channel length running the length of the assembly.

\subsubsection{Separator Plate Experiments from BAW-1645 (Ref. 17)}

The experiments consist of $5 \times 5$ arrays of fuel assemblies separated by (presumed) aluminum plates. Details of the $2.459 \mathrm{wt} \% \mathrm{UO}_{2}$ fuel rods are outlined in Table 2.4 and Fig. 2.3. Figure 2.25 is a planar view of the experiments showing the assemblies with mounted aluminum side sheets, or separator plates. Vertical dimensions of the experiments can be found in Fig. 2.26. Missing from, or difficult to distinguish in, Fig. 2.26 is that (1) the end plugs of the fuel rods extend $0.3175 \mathrm{~cm}$ into the $3.2-\mathrm{cm}$-thick aluminum bottom grid plate, (2) the aluminum base plate has dimensions of $122 \mathrm{~cm} \times 122 \mathrm{~cm} \times 8.9 \mathrm{~cm}$, (3) the aluminum base plate is separated from the core tank by $2.86 \mathrm{~cm}$ of water, and (4) the separator plates extend vertically from the base of the bottom grid plate to the top of the top grid plate. Critical data for the experiments are located in Table 2.20 .

Assumptions: The aluminum corner rods are assumed to be of fuel rod length. The separation distance between assemblies is believed to have been measured from the cell boundaries of the assemblies. The core tank is assumed to be $1.0-\mathrm{cm}$ thick and constructed from carbon steel. With a $274-\mathrm{cm}$ inside core tank diameter, the core is assumed to be fully reflected laterally by water.

Model-experiment comparison: The aluminum top and center grid plates were of unknown thickness and were not modeled. The fuel rods and separator plates above the moderator region and the core tank sides were ignored in the model. For experiments BW1645T1, BW1645T1, BW1645T3, BW1645T4, BW1645S1, and BW1645S2, the 3.2$\mathrm{cm}$-thick aluminum bottom grid plate has grooves for coolant flow. The top $0.3175 \mathrm{~cm}$ of the bottom grid plate around the end plugs was modeled as water only, the middle $1.067 \mathrm{~cm}$ of the plate was modeled as a mixture of $25 \%$ aluminum and $75 \%$ water by volume, and the bottom $1.815 \mathrm{~cm}$ was modeled as $100 \%$ aluminum by volume. For B1645SO1 and B1645SO2, the 3.2-cm-thick aluminum bottom grid plate was modeled as water around the 0.3175 $\mathrm{cm}$-long bottom end plugs and $2.882 \mathrm{~cm}$ of aluminum below the bottom end plugs.

\subsubsection{Separator Plate Experiments from DSN No. 399/80 (Ref. 18)}

The experiments consist of $2 \times 2$ arrays of fuel assemblies separated by water and hafnium plates, or single assemblies surrounded by hafnium plates. Specifications for the $4.742 \mathrm{wt} \% \mathrm{UO}_{2}$ fuel rods are outlined in Table 2.2 and Fig. 2.1. The elevations of the experiments are also given in Fig. 2.1. For DSN399-1 and DSN399-2, a 0.4-cm-thick stainless steel assembly support plate (not shown in Fig. 2.1) is situated between the bottom plugs of the fuel rods and the 0.8cm-thick stainless steel support plate. Cross-sectional views of the experiments are provided in Fig. 2.28. Critical data on the experiments are given in Table 2.21 .

Assumptions: The assembly separation distances and plate-to-assembly distances are believed to have been measured from the cell boundaries of the assemblies. The holes in the lower lattice grid plate for accommodating the fuel rods were taken to be $1.0 \mathrm{~cm}$ in diameter from Ref. 23.

Model-experiment comparison: The fuel rods above the moderator were modeled by the method explained in Sect. 3.2.1. The separator plates were modeled as resting on top of the assembly support plate (DSN399-1 and DSN399-2) 
or support plate (DSN399-3 and DSN399-4). The spring of the fuel rods was modeled as void. The upper grid plate of stainless steel, left out of Fig. 2.1, was reported as having an insignificant influence on $\mathrm{k}_{\mathrm{eff}}$; therefore, it was not modeled. The stainless steel support plates were modeled as $71.40 \mathrm{wt} \% \mathrm{Fe}, 19.16 \mathrm{wt} \% \mathrm{Cr}$, and $9.44 \mathrm{wt} \% \mathrm{Ni}$. The steel angle irons, shown in Fig. 2.29 (corner A is positioned at the center of the setup of Fig 2.28a), of DSN399-1 and DSN399-2 were not modeled.

\subsubsection{Separator Plate Experiments from PNL-2438 (Ref. 9)}

The experiments consist of $3 \times 1$ arrays of fuel assemblies separated by aluminum, Boral-A, copper, stainless steel, or Zircaloy-4 plates and water. Table 2.22 describes the separator plates. Details of the $2.35 \mathrm{wt} \% \mathrm{UO}_{2}$ fuel rods are outlined in Fig. 2.5. Planar and axial views of the experiment can be found in Fig. 2.13; the experiments were minus the biological shielding walls shown in Fig. 2.13. Critical data for the experiments are located in Table 2.23.

Model-experiment comparison: The top and middle 1.27-cm-thick acrylic grid plates were modeled as water because acrylic plastic has about the same density and neutron moderating characteristics as water. The carbon steel core tank was not modeled. The separator plates were modeled as resting on top of the bottom 2.54 -cm-thick acrylic grid plate.

\subsubsection{Separator Plate Experiments from PNL-2615 (Ref. 19)}

The experiments consist of $3 \times 1$ arrays of fuel assemblies separated by aluminum, Boral-B, cadmium, copper, stainless steel, and Zircaloy-4 plates and water. Table 2.22 describes the separator plates. Details of the $4.31 \mathrm{wt} \%$ $\mathrm{UO}_{2}$ fuel rods are outlined in Fig. 2.14. Planar and axial views of the experiment can be found in Fig. 2.13; the experiments were minus the biological shielding walls shown in Figs. 2.30 and 2.15. Critical data for the experiments are located in Table 2.24 .

Model-experiment comparison: The minimum fuel rod pellet column length, $91.44 \mathrm{~cm}$, was modeled. The top and middle 1.27-cm-thick acrylic grid plates were modeled as water because acrylic plastic has about the same density and neutron moderating characteristics as water. The carbon steel core tank was not modeled because the amount of water on the boundary of the array ensures a maximum increase in $k_{\text {eff. }}$ The separator plates were modeled as resting on top of the bottom $2.54-\mathrm{cm}$-thick acrylic grid plate.

\subsubsection{Separator Plate Experiments from PNL-3314 (Ref. 11)}

The experiments consist of $2 \times 2$ or $3 \times 1$ arrays of fuel assemblies separated by aluminum, Boral-A, Boral-C, Boroflex, cadmium, copper, copper-cadmium, borated stainless steel, stainless steel, and Zircaloy-4 plates and water. Descriptions of the 2.35 and $4.31 \mathrm{wt} \% \mathrm{UO}_{2}$ fuel rods are provided in Figs. 2.5 and 2.14. Planar and axial views of the $3 \times 1$ and $2 \times 2$ assembly experiments are shown in Figs. $2.30(\mathrm{G}=0)$ and 2.15; the experiments were minus the biological shielding walls shown in Figs. 2.30 and 2.15. Figures 2.16(a) and 2.16(b) illustrate the assembly and fuel rod array configurations of the experiments. Separator plate data are given in Table 2.22 . Critical data on the experiments are given in Table 2.25 .

Model-experiment comparison: The minimum fuel rod pellet column length, $91.44 \mathrm{~cm}$, was modeled for the $4.31 \mathrm{wt} \%$ $\mathrm{UO}_{2}$ fuel rods. The two polypropylene (neutronic properties similar to water) grid plates were modeled as water. The carbon steel tank was not modeled because the amount of water on the boundary of the array ensures a maximum increase in $\mathrm{k}_{\mathrm{efr}}$. The separator plates were modeled as resting on top of the acrylic plate. 


\subsubsection{Separator Plate Experiments from PNL-6205 (Ref. 20) and PNL-7176 (Ref. 21)}

The experiments consist of $2 \times 2$ arrays of fuel assemblies separated by Boral plates and water. The Boral plates, detailed in Table 2.26 , create a flux trap where epithermal neutrons passing through the Boral become thermalized in the water between the Boral plates. Aluminum plates, aluminum rods, and fuel rods were added to the water region of the flux trap in order to investigate the reactivity effect of voiding. The modeling of these experiments tests the ability of the calculation method to predict $\mathrm{k}_{\text {eff }}$ for assemblies with separator plates acting as flux traps and voiding materials. The $4.31 \mathrm{wt} \% \mathrm{UO}_{2}$ fuel rods are detailed in Fig. 2.14; the experimental elevations are provided in Fig. 2.31; and the critical data are located in Table 2.27. The assemblies for experiments P62FT231, P71F14F3, P71F14V3, P71F14V5, and P71F214R are displayed in Figs. 2.32-2.36.

Assumptions: The aluminum rods are assumed to be of fuel rod length. The aluminum voiding plates are assumed to be of Boral plate width and height.

Model-experiment comparison: The minimum fuel rod pellet column length, $91.44 \mathrm{~cm}$, was modeled. The polypropylene lattice plates $\left(0.90 \mathrm{~g} / \mathrm{cm}^{3}\right)$ were modeled as water because of their neutron moderating similarities to water. The separator plates were modeled as resting on top of the base plate.

The PNL-6205 and PNL-7167 experiments are subcritical configurations. The number of rods required to achieve delayed critical is predicted by the approach-to-critical method. Table 2.28 shows the number of rods of the experiment (column 2), shown in Figs. 2.32-2.36, the predicted number of rods needed for delayed critical (column 3), and the number of rods modeled with CSAS25 (column 4). Note the partial rod of column 3 can be modeled if using CSAS2X instead of CSAS25. The extra rods modeled were arbitrary placed in one of the four assemblies. The modeled assembly sizes are listed in Table 2.28.

\subsubsection{Reflecting Wall Experiments}

Large transportation packages have reflectors in the form of biological shielding materials that are part of the packaging. The materials facilitate the return of neutrons leaking from the outer fissile material boundary. Modeling of these experiments demonstrates the ability of the calculational techniques to predict the neutron multiplication factor of water and lead-, steel-, or uranium-reflected assemblies.

\subsubsection{Reflecting Wall Experiments from PNL-2827 (Ref. 10)}

The experiments consist of $3 \times 1$ arrays of fuel assemblies separated by water and reflected by water and two lead or depleted uranium reflecting walls. Details of the 2.35 and 4.31 wt \% $\mathrm{UO}_{2}$ fuel rods are outlined in Figs. 2.5 and 2.14. Planar and axial views of the experiments can be found in Fig. 2.13. Absent in the experiments are the poison plates of Fig. 2.13. Details of the lead and uranium reflecting walls are provided in Figs. 2.37 and 2.38. Critical data for the experiments are located in Table 2.29 .

Model-experiment comparison: The minimum fuel rod pellet column length, $91.44 \mathrm{~cm}$, was modeled for the $4.31 \mathrm{wt} \%$ $\mathrm{UO}_{2}$ fuel rods. The 1.27 -cm-thick acrylic grid plates were modeled as water because acrylic plastic has about the same density and neutron moderating characteristics as water. The carbon steel core tank was not modeled because the amount of water on the boundary of the array ensures a maximum increase in $k_{\text {eff- }}$ 


\subsubsection{Reflecting Wall Experiments from PNL-3602 (Ref. 22)}

The experiments consist of $3 \times 1$ arrays of fuel assemblies separated by water and reflected by water and two steel reflecting walls. Details of the 2.35 and $4.31 \mathrm{wt} \% \mathrm{UO}_{2}$ fuel rods are outlined in Figs. 2.5 and 2.14. Planar and axial views of the experiments can be found in Fig. 2.30. Absent in the experiments are the poison plates of Fig. 2.30. Details of the steel reflecting walls are provided in Fig. 2.39. Critical data for the experiments are located in Table 2.30.

Model-experiment comparison: The minimum fuel rod pellet column length, $91.44 \mathrm{~cm}$, was modeled for the $4.31 \mathrm{wt} \%$ $\mathrm{UO}_{2}$ fuel rods. The $1.27-\mathrm{cm}$-thick acrylic and polypropylene grid plates were modeled as water because these materials have similar densities and neutron moderating characteristics as water. The carbon steel core tank was not modeled because the amount of water on the boundary of the array ensures a maximum increase in $k_{\text {eff }}$

\subsubsection{Reflecting Wall Experiments from PNL-3926 (Ref. 12)}

The experiments consist of $3 \times 1$ arrays of fuel assemblies separated by water and reflected by water and two lead or depleted uranium reflecting walls. Details of the $2.35 \%$ and $4.31 \mathrm{wt} \% \mathrm{UO}_{2}$ fuel rods are outlined in Figs. 2.5 and 2.14. Planar and axial views of the experiments can be found in Fig. 2.15. However, these experiments do not contain poison plates as shown in the figure. Details of the lead and uranium reflecting walls are provided in Figs. 2.37 and 2.38. Critical data for the experiments are located in Table 2.31 .

Model-experiment comparison: The minimum fuel rod pellet column length, $91.44 \mathrm{~cm}$, was modeled for the $4.31 \mathrm{wt} \%$ $\mathrm{UO}_{2}$ fuel rods. The $1.27-\mathrm{cm}$-thick polypropylene $\left(0.904 \mathrm{~g} / \mathrm{cm}^{3}\right)$ grid plates were modeled as water because polypropylene has about the same density and neutron moderating characteristics as water. The carbon steel core tank was not modeled because the amount of water on the boundary of the array is equivalent to an infinite water reflector.

\subsubsection{Reflecting Wall-Separator Plate Experiments}

These experiments have been selected to demonstrate the ability of the calculational techniques to predict the neutron multiplication factor of experiments with neutron absorbing or scattering materials (i.e., separator plates) between fuel assemblies reflected by water and steel. Separator plates are commonly used in transportation packages to limit neutron interaction and increased reactivity associated with the bringing into close proximity of fuel assemblies. In addition, large transportation packages may also have reflectors in the form of biological shielding materials that facilitates the return of neutrons leaking from the outer fissile material boundary.

\subsubsection{Reflecting Wall-Separator Plate Experiments from PNL-3602 (Ref. 22)}

The experiments consist of $3 \times 1$ arrays of fuel assemblies separated by water and Boral, cadmium, copper, borated and unborated stainless steel separator plates and reflected by water and two steel reflecting walls. Details of the 2.35 and $4.31 \mathrm{wt} \% \mathrm{UO}_{2}$ fuel rods are outlined in Figs. 2.5 and 2.14. Planar and axial views of the experiments can be found in Fig $2.30(\mathrm{G}=0)$. Descriptions of the separator plates are provided in Table 2.22. Details of the steel reflecting walls are provided in Fig 2.39. Critical data for the experiments are located in Table 2.32.

Model-experiment comparison: The minimum fuel rod pellet column length, $91.44 \mathrm{~cm}$, was modeled for the 4.31 wt \% $\mathrm{UO}_{2}$ fuel rods. The polypropylene grid plates were modeled as water because polypropylene has similar densities and neutron moderating characteristics as water. The carbon steel core tank was not modeled because the amount of water on the boundary of the array ensures a maximum increase in $k_{e f f}$. The separator plates were modeled resting on top of the bottom $2.54-\mathrm{cm}$-thick acrylic plate. 


\subsubsection{Reflecting Wall-Separator Plate Experiments from PATRAM'80 (Ref. 23)}

The experiments consist of $2 \times 2$ arrays of fuel assemblies separated by water and Boral plates and reflected by water and lead or steel reflecting walls. Details of the $4.742 \mathrm{wt} \%$ fuel rods are outlined in Figs. 2.1 and Table 2.2. The principal dimensions for the experiments are found in Fig. 2.1. A 0.4-cm-thick steel (composition specified on Fig. 2.1) assembly support plate, not shown in Fig. 2.1, is situated between the bottom plugs of the fuel rods and the 0.8$\mathrm{cm}$-thick support plate. The lower grid plate holes for accommodating the fuel rods are $1.0 \mathrm{~cm}$ in diameter. The planarview of a quarter of an experimental assembly is displayed in Fig. 2.40. Critical data for the experiments are located in Table 2.33 .

Assumptions: The four stainless steel corner rods were assumed to be of fuel rod length.

Model-experiment comparison: The upper grid plate was ignored due to lack of information. The spring of the fuel rods was modeled as void. The lower grid plate around the corner rods was ignored. The fuel rods above the moderator were modeled by the method explained in Sect. 3.2.1.

\subsubsection{Burnable Absorber Fuel Rod Experiments ${ }^{24}$}

Modeling these experiments demonstrates the ability of the calculational techniques to predict the neutron multiplication factors of experiments that have $\mathrm{UO}_{2}$ fuel rods fabricated with gadolinia for extended burnup purposes. A set of five experiments were performed with various 2.459 or 2.459 and $4.020 \mathrm{wt} \% \mathrm{UO}_{2}$ and $1.944 \mathrm{wt} \% \mathrm{UO}_{2}$ $\mathrm{Gd}_{2} \mathrm{O}_{3}$ fuel rod configurations. The $2.459 \mathrm{wt} \% \mathrm{UO}_{2}$ rods are detailed in Table 2.4 and Fig. 2.3 , the $4.020 \mathrm{wt} \% \mathrm{UO}_{2}$ rods are detailed in Table 2.34, and the $1.944 \mathrm{wt} \% \mathrm{UO}_{2}-\mathrm{Gd}_{2} \mathrm{O}_{3}$ rods are detailed in Table 2.35. The vertical dimensions are believed to be those presented in Fig. 2.4. In Fig. 2.4 the aluminum core tank is $1.27 \mathrm{~cm}$ thick and $152.4 \mathrm{~cm}$ in diameter. The core configurations for the experiments are located in Figs. 2.41-2.45. Critical data are located in Table 2.36. Note, these experiments contained borated water and water holes.

Assumptions: The vertical dimensions are believed to be those of Fig. 2.4. The 152.4-cm-diam core tank filled with water constitutes full-water reflection of the cores.

Model-experiment comparison: The fuel rods above the moderator were not modeled. The top and bottom grid plates were ignored.

\subsubsection{Water Hole Experiments}

LWR fuel elements have lattice positions, usually in a $5 \times 5$ matrix, filled by control rod and instrumentation clusters. The clusters are sometimes removed when the LWR fuel assemblies are shipped, leaving behind water holes that are richer in thermalized neutrons than the surrounding cells. The modeling of the experiments in this section tests the ability of the calculation techniques to handle cells with different degrees of moderation.

\subsubsection{Water Hole Experiments from BAW-1810 (Ref. 24)}

The experiments consist of 2.459 or 2.459 and $4.020 \mathrm{wt} \% \mathrm{UO}_{2}$ fuel rod configurations with water holes. The 2.459 wt $\% \mathrm{UO}_{2}$ rods are detailed in Table 2.4 and Fig. 2.3 and the $4.020 \mathrm{wt} \% \mathrm{UO}_{2}$ rods are detailed in Table 2.34. The vertical dimensions are believed to be those presented in Fig. 2.4. In Fig. 2.4 the aluminum core tank is $1.27 \mathrm{~cm}$ thick and $152.4 \mathrm{~cm}$ in diameter. The core configurations for the experiments are located in Figs. 2.46-2.48. Critical data are located in Table 2.37. Note, these experiments contained borated water. 
Assumptions: The vertical dimensions are believed to be those of Fig. 2.4. The 152.4-cm-diam core tank filled with water constitutes full-water reflection of the cores.

Model-experiment comparison: The fuel rods above the moderator were not modeled. The top and bottom grid plates were ignored.

\subsubsection{Water Hole Experiments from NS\&E, Vol. 71 (Ref. 8)}

The experiments consist of large singular arrays of $4.742 \mathrm{wt} \% \mathrm{UO}_{2}$ fuel rods with $5 \times 5$ and $8 \times 8$ matrices of water holes. Fuel rod specifications are given in Table 2.2 and Fig. 2.1. The principal dimensions of the experiments are also given in Fig. 2.1. The critical data for the experiments are located in Table 2.38.

Assumptions: The holes in the lower grid plate to accommodate the fuel rods were taken to be $1.0 \mathrm{~cm}$ in diameter from Ref. 23.

Model-experiment comparison: The reported upper grid, missing from Fig. 2.1, was ignored in the model due to insufficient data. The two grid plates were reportedly attached to the support plate by four fuel rods distanced more than $20 \mathrm{~cm}$ from the fuel. These rods were not modeled. The stainless steel support plate was modeled as $71.40 \mathrm{wt} \%$ $\mathrm{Fe}, 19.16 \mathrm{wt} \% \mathrm{Cr}$, and $9.44 \mathrm{wt} \% \mathrm{Ni}$. Figure 2.49 represents the cross-sectional view of the modeled assemblies.

\subsubsection{Water Hole Experiments from PNL-3314 (Ref. 11)}

The experiments consist of single lattices with $5 \times 5$ matrices of water holes. Descriptions of the $2.35 \mathrm{wt} \%$ and 4.31 wt \% $\mathrm{UO}_{2}$ fuel rods are provided in Figs. 2.5 and 2.14. Planar and axial views of the experiment setup are shown in Fig. 2.15. The cross-sectional view of the experimental setup showing water hole positions is presented in Fig. 2.50. Critical data on the experiment are given in Table 2.39 .

Model-experiment comparison: P3314W1 and P3314W2 are subcritical configurations. The number of predicted rods to achieve criticality were obtained by the approach-to-critical method. The number of fuel rods modeled was rounded from the predicted number to the nearest whole number. Note: The partial predicted rod can be modeled if using CSAS2X. The geometric models for P3314W1 and P3314W2 are seen in Fig. 2.51. The partial row of fuel rods was arbitrarily located against the assembly left cell boundary. The two polypropylene (neutronic properties similar to water) grid plates were modeled as water. The carbon steel tank was not modeled because the amount of water on the boundary of the array ensures a maximum increase in $k_{\text {eff }}$.

\subsubsection{Water Hole Experiments from WCAP-3269 (Ref. 15)}

The experiments consist of large single arrays of fuel rods and water holes. Descriptions of the 2.72 and $5.7 \mathrm{wt} \%$ $\mathrm{UO}_{2}$ fuel rods can be found in Table 2.14. The vertical dimensions of experiments W3269W1 and W3269W2 are shown in Figs. 2.20 and 2.21; the guide plates are aluminum. The absorber section of Fig. 2.20 was not part of the experiments. Core layouts showing water hole locations are given in Fig. 2.52. Critical data for the experiments are located in Table 2.40 .

Assumptions: The moderator height was believed to have been measured from the bottom of the fuel. The guide plate holes to accommodate fuel rods were assumed to be of fuel rod diameter. In Fig. 2.21, all plates were taken to be of assembly width and depth; full-water reflection was assumed on the sides of the experimental assembly; the 2.54-cmthick plate below the bottom guide plate was assumed to be of aluminum. In Fig. 2.20, full-water reflector was assumed at the boundary of the assembly defined by the $60.96-\mathrm{cm}$-square guide plates. 
Model-experiment comparison: The fuel rods above the moderator were modeled by the method explained in Sect. 3.2.1. For W3269W2 the $6.35-\mathrm{cm}$ gap above the aluminum base plate was modeled as water only. For W3269W1, the top and center guide plates, in air for this experiment, were not modeled; the bottom end plugs in the bottom guide plate were modeled as aluminum; the bottom end plugs in the $0.188-\mathrm{in} .(0.478-\mathrm{cm})$-thick water slot and the top end plugs were not modeled.

\subsubsection{Absorber Rod Experiments}

Modeling of these experiments tests the ability of the calculational techniques to predict neutron multiplication factors of experiments simulating shipping packages of LWR assemblies with control rod clusters intact.

\subsubsection{Absorber Rod Experiments from BAW-1484 (Ref. 6)}

The experiments consist of a $3 \times 3$ array of fuel assemblies separated by a water gap containing $\mathrm{B}_{4} \mathrm{C}$ absorber rods. Details of the $2.459 \mathrm{wt} \% \mathrm{UO}_{2}$ fuel rod design are outlined in Table 2.4 and Fig. 2.3. Details of the $\mathrm{B}_{4} \mathrm{C}$ rods are outlined in Table 2.41. The vertical dimensions for the experiment are found in Fig. 2.4. Absent from Fig. 2.4: the $1 \mathrm{in} .(2.54 \mathrm{~cm})$ between the base plate and core tank is mostly water and the core tank is $0.5 \mathrm{in} .(1.27 \mathrm{~cm})$ thick. Difficult to distinguish in Fig. 2.4 is that the fuel rod end plugs are located in $0.125 \mathrm{in} .(0.3175 \mathrm{~cm})$ of water between the bottom grid plate and the base plate. Cross-sectional views of experiments BW1484C1 and BW1484C2 are located in Figs. 2.53 and 2.54. The critical data are located in Table 2.42.

Assumptions: The aluminum corner rods are taken to be of fuel rod length. The separation distance between assemblies is believed to have been measured from the cell boundaries of the assemblies. To accommodate the fuel rods, square holes with $0.615-\mathrm{cm}$ sides were assumed in the bottom grid plate.

Model-experiment comparison: The 22.9-cm-wide $\times 22.9$-cm-long $\times 2.54-\mathrm{cm}$-thick aluminum top grid plate was not modeled. The fuel rods and separator plate above the moderator region were not modeled.

\subsubsection{Absorber Rod Experiments from BAW-1810 (Ref. 24)}

The experiments consist of 2.459 or 2.459 and $4.020 \mathrm{wt} \% \mathrm{UO}_{2}$ fuel rod configurations with $\mathrm{Ag}$-In-Cd and $\mathrm{B}_{4} \mathrm{C}$ absorber rods. The $2.459 \mathrm{wt} \% \mathrm{UO}_{2}$ rods are detailed in Table 2.4 and Fig. 2.3. The $4.020 \mathrm{wt} \% \mathrm{UO}_{2}$ rods are detailed in Table 2.34. Properties of the Ag-In-Cd and $\mathrm{B}_{4} \mathrm{C}$ rods are given in Tables 2.43 and 2.41. The vertical dimensions are believed to be those presented in Fig. 2.4. In Fig. 2.4 the aluminum core tank is $1.27 \mathrm{~cm}$ thick and $152.4 \mathrm{~cm}$ in diameter. The core configurations for the experiments are located in Figs. 2.55 and 2.56. Critical data are located in Table 2.44. Note, these experiments have borated water and water holes.

Assumptions: The vertical dimensions are those of Fig. 2.4. The 152.4-cm-diam core tank filled with water constitutes full-water reflection of the cores.

Model-experiment comparison: The fuel and absorber rods above the moderator were not modeled. The top and bottom grid plates were ignored.

\subsubsection{Absorber Rod Experiments from WCAP-3269 (Ref. 15)}

Experiments W3269A and W3269C used large single arrays of 5.7 and $2.72 \mathrm{wt} \% \mathrm{UO}_{2}$ fuel rods and Ag-In-Cd absorber rods. W3269B1, W3269B2, and W3269B3 used large single arrays of $3.7 \mathrm{wt} \% \mathrm{UO}_{2}$ fuel rods and Ag-In-Cd absorber rods. Descriptions of the $2.72,3.7$, and $5.7 \mathrm{wt} \% \mathrm{UO}_{2}$ fuel rods can be found in Table 2.14 . The vertical 
dimensions of experiments W3269A and W3269C are shown in Figs. 2.21 and 2.20. Figure 2.57 displays the vertical dimensions for W3269B1, W3269B2, and W3269B3. All experiments used aluminum guide plates. The core layouts of W3269A and W3269C, with water holes replaced by Ag-In-Cd rods, are given in Fig. 2.52. The core layouts of W3269B1, W3269B2, and W3269B3 are given in Figs. 2.58-2.60. The cruciform in Figs. 2.58-2.60 are control rod fuel followers. Each cruciform has a center fuel rod and four vanes with nine fuel rods on a $0.418-\mathrm{in}$. (1.062-cm) pitch. Critical data for the experiments are located in Table 2.45.

Assumptions: The Ag-In-Cd rods are assumed to be of fuel rod length. The moderator height was believed to have been measured from the bottom of the fuel. The guide plate holes to accommodate fuel rods were assumed to be of fuel rod diameter. For W3269A, W3269B1, W3269B2, and W3269B3 full water reflection was assumed laterally beyond the assembly boundary. For W3269C, full water reflection was assumed outside the assembly boundary defined by the 60.96-cm-square guide plates. In Fig. 2.21 , the $2.54-\mathrm{cm}$-thick plate below the bottom guide plate was assumed to be of aluminum.

Model-experiment comparison: The fuel rods above the moderator were modeled by the method explained in Sect. 3.2.1. For W3269A, the $6.35 \mathrm{~cm}$ above the base plate was modeled as water only. For W3269B1, W3269B2, and W3269B3, no data were available on the fuel rod end plugs so the plugs were modeled as water; the center guide plate, $70.17 \mathrm{~cm}$ above the bottom of the fuel, was not modeled because it was above the moderator level; the eccentricity of the fuel follower was treated as described in Sect. 3.2.1. For W3269C, the top and center guide plates, in air for this experiment, were not modeled; the bottom end plugs in the bottom guide plate were modeled as aluminum; the bottom end plugs in the 0.188 -in. $(0.478-\mathrm{cm})$-thick water slot and top end plugs were not modeled.

\subsubsection{Soluble Boron Experiments}

Boric acid is often used in shipping casks as an additional measure for preventing accidental criticality; boric acid is a strong absorber that can significantly affect the neutronics of a system. The experiments described in this section are used to test the ability of the calculational techniques to predict the neutron multiplication factors of systems with a borated water moderator.

\subsubsection{Soluble Boron Experiments from BAW-1231 (Ref. 25)}

The experiments consist of large arrays of fuel rods moderated by borated water. Properties of the $4.020 \mathrm{wt} \%$ fuel rods are given in Table 2.34. The core layouts and vertical dimensions of the experiments are given in Figs. 2.61 and 2.62. The top and bottom grid plates of Fig. 2.62 are aluminum. Critical data for the experiments are in Table 2.46.

Assumptions: Holes of fuel rod diameter were assumed in the grid plates. The core tank is $274.32 \mathrm{~cm}$ in diameter constituting full-water reflection. The end plugs are believed to be made of aluminum.

Model-experiment comparison: The fuel rods above the moderator were modeled by the method explained in Sect. 3.2.1. The top grid plate, top end plugs, and top end plugs above the top grid plate were modeled as a solid aluminum slab.

\subsubsection{Soluble Boron Experiments from BAW-1273 (Ref. 26)}

BW1273M consists of a large cylindrical array of fuel rods moderated by borated water. Properties of the $2.459 \mathrm{wt} \%$ $\mathrm{UO}_{2}$ fuel rods are given in Table 2.4 and Fig. 2.3. The vertical dimensions of the experiment are given in Fig. 2.63. Missing from Fig. 2.63 are two 0.0625 -in. $(0.1588$-cm)-thick aluminum midplane grid plates with bases 49 and $82 \mathrm{~cm}$ above the reference plane. Critical data for the experiments are in Table 2.47 . 
Assumptions: The 274.32-cm-diam core tank filled with water constitutes full-water reflection of the core. Square holes, $1.27 \mathrm{~cm}$ on a side, were assumed in each grid plate to accommodate the fuel rods.

Model-experiment comparison: The fuel rods above the moderator were modeled by the method explained in Sect 3.2.1. The top grid plate was not modeled. Figure 2.64 shows a cross-sectional view of the modeled core for reference.

\subsubsection{Soluble Boron Experiments from BAW-1484 (Ref. 6)}

The experiments consisted of $3 \times 3$ arrays of fuel assemblies separated by water gaps and moderated with borated water. Details of the $2.459 \mathrm{wt} \% \mathrm{UO}_{2}$ fuel rods are outlined in Table 2.4 and Fig. 2.3. The elevations of experiments BW1484B1 and BW1484B2 can be found in Fig. 2.4. Absent from Fig 2.4: the 1 in. $(2.54 \mathrm{~cm})$ below the base plate is mostly water and the core tank is $0.5 \mathrm{in}$. $(1.27 \mathrm{~cm})$ thick. Difficult to distinguish in Fig. 2.4 is that the fuel rod end plugs are located in $0.125 \mathrm{in} .(0.3175 \mathrm{~cm})$ of water between the bottom grid plate and the base plate. The elevations of experiment BW1484B3 are given in Fig. 2.24. The top grid of Fig. 2.24 was not used in the experiments. Missing from, or difficult to distinguish in, Fig. 2.24 is that: (1) the fuel pellet column and fuel rod bottom end plugs extend 0.635 and $0.9525 \mathrm{~cm}$ into the aluminum bottom grid, respectively, (2) the aluminum bottom grid and aluminum bottom grid plate are $2.54 \mathrm{~cm}$ thick, (3) and $0.3175 \mathrm{~cm}$ of water exists between the aluminum bottom grid plate and the 5.08-cm-thick aluminum base plate. Critical data for the experiments are located in Table 2.48.

Assumptions: The aluminum corner rods are taken to be of fuel rods length. The separation distance between assemblies is believed to have been measured from the cell boundaries of the assemblies. Holes, $0.615 \mathrm{~cm}$ on a side, were assumed in the bottom grid plate to accommodate the fuel rods.

Model-experiment comparison: The fuel rods above the moderator region were not modeled. The 22.9-cm-wide $\times$ 22.9-cm-long $\times 2.54-\mathrm{cm}$-thick aluminum top grid plate were not modeled.

\subsubsection{Soluble Boron Experiments from EPRI NP-196 (Ref. 7)}

These experiments consist of large singular arrays of fuel rods moderated by borated water. Specifications of the 2.35 wt $\% \mathrm{UO}_{2}$ fuel rods are presented in Fig. 2.5. Figure 2.6 shows the axial dimensions of experiments EPRU65B and EPRU87B. Figure 2.7 shows the axial dimensions of experiment EPRU75B. The grid plate holes for EPRU75B are $0.586 \mathrm{in} .(1.488 \mathrm{~cm})$ in diameter. The specifications for the aluminum "eggcrate" grids seen in Fig. 2.6 are given in Fig. 2.8. The core layouts are shown in Figs. 2.65-2.67. The critical data are located in Table 2.49.

Model-experiment comparison: A 182.88-cm-diam, 274.32-cm-tall tank contributes full-water reflection of the cores. The experiments were modeled with $6 \mathrm{in} .(15.24 \mathrm{~cm})$ of water above the lead shield and $30 \mathrm{~cm}$ of water below the aluminum solid plate.

\subsubsection{Soluble Boron Experiments from PNL-4267 (Ref. 13)}

The experiments consist of a single array of fuel rods moderated by borated water. Specifications of the $4.31 \mathrm{wt} \%$ $\mathrm{UO}_{2}$ fuel rods are located in Fig. 2.14. The experimental setup is illustrated in Fig. 2.17. The box is full-water reflected on all four sides with unborated water. Critical data for the experiments are given in Table 2.50. The reader should be aware of the large uncertainty associated with some measured boron concentration values.

Model-experiment comparison: The minimum fuel rod pellet column length, $91.44 \mathrm{~cm}$, was modeled.

The PNL-4267 experiments are subcritical configurations. The number of rods required to achieve critical are predicted by the approach-to-critical method. For P4267SL1 and P4267SL2, the nearest whole number of rods 
required for criticality were modeled. The partial rod can be modeled if using CSAS2X. P4267B1 was modeled with 23 columns of 40 fuel rods, and 1 far-left column of 3 fuel rods. P4267B2 was modeled with 30 columns of 40 fuel rods and 1 far-left column of 37 fuel rods. P4267B3 was modeled with 16 columns of 44 fuel rods and 1 far-left column of 33 fuel rods. P4267B4 was modeled with 20 columns of 44 fuel rods and 1 far-left column of 37 fuel rods. P4267B5 was modeled with 27 columns of 44 fuel rods and 1 far-left column of 4 fuel rods. The far-left columns of the arrays were arbitrarily placed against the lower Plexiglas box side of Fig. 2.17 (planar view). The arrays were positioned along their length against the right side of the Plexiglas box with the boundary next to the Plexiglas box surface. The arrays were centered along their width in the Plexiglas box.

A discrepancy in Fig. 2.17 was noticed. As marked, the cell boundaries along the array width are $75.88 \mathrm{~cm}$ apart. Forty and 44 fuel rods placed in lattices of $1.890-$ and $1.715-\mathrm{cm}$ pitch give rise to cell boundaries 75.6 and $75.46 \mathrm{~cm}$ apart, not $75.88 \mathrm{~cm}$.

\subsection{Homogeneous Uranium}

Modeling this set of experiments tests the ability of the calculational techniques to predict the neutron multiplication factors of homogeneous uranium systems such as shipping containers with low-enriched uranium oxide pellets or powder.

\subsubsection{Homogenized Uranium in Paraffin}

These experiments consisted of paraffin-reflected or unreflected parallelepipeds of homogeneous $U(2) F_{4}$ or $U(3) F_{4}$ and paraffin. The fuel atom densities for each experiment are listed in Table 2.51. Critical data of the experiments are located in Table 2.52.

\subsubsection{Damp Uranium Oxide Experiments ${ }^{28-31}$}

The experiments CR1071AS, CR1653AS, and CR2500S consist of 42, 38, and 30 cans of damp uranium oxide moderated and reflected by Plexiglas. The fuel cans and damp uranium oxide for the three experiments are detailed in Table 2.53. Material compositions of the cans, moderator, and reflector are given in Table 2.54. Isometric drawings of the fuel can configurations are found in Fig. 2.68. Table 2.55 contains CR1071AS core, reflector, faceplate, frame extender, and filler dimensions corresponding to Fig. 2.69. Table 2.56 contains CR1653AS core, reflector, and filler dimensions corresponding to Fig. 2.70. The reflector end panel and frame dimensions of Fig 2.70 are shown in Fig 2.71 for clarification. For CR2500S, Table 2.57 lists the cuboid dimensions corresponding to the three nested cuboids of Fig. 2.72 .

Assumptions: All voids were assumed to be filled with nonfire-retardant Plexiglas in CR2500S except the void to the east of the north and south table cores. For all experiments, nearly $80 \%$ of the reflector is reported to be fire-retardant Plexiglas. The experimenter suggests that the north frame, south frame, and south end panel be modeled as fireretardant Plexiglas and the north end panel, frame extensions, and filler blocks be modeled as nonfire-retardant Plexiglas.

Model-experiment comparison: The fuel can contents (i.e., $\mathrm{U}_{3} \mathrm{O} 8, \mathrm{H}_{2} \mathrm{O}$, and polyethylene) were homogenized over the can cavity. The fuel can, vinyl tape, and mylar tape were homogenized over the fuel can shell volume. 
Table 2.1 Case descriptions of LWR-type UO, fuel pin lattice critical benchmark experiments

\begin{tabular}{|c|c|c|c|c|c|c|}
\hline $\begin{array}{l}\text { Case } \\
\text { No. }\end{array}$ & $\begin{array}{c}\text { Case } \\
\text { designation }\end{array}$ & $\begin{array}{l}\text { Enrich. } \\
\text { (wt \%) }\end{array}$ & $\begin{array}{l}\text { Pitch } \\
(\mathrm{cm})\end{array}$ & $\begin{array}{l}\mathrm{H}_{2} \mathrm{O} / \text { fuel } \\
\text { volume } \\
\end{array}$ & Description & $\begin{array}{c}\text { Reference } \\
\text { No. } \\
\end{array}$ \\
\hline 1 & ANS33AL1 & 4.74 & 1.35 & 2.302 & $\mathrm{H}_{2} \mathrm{O}$ filled $\mathrm{Al}$ cruciform boxes & 5 \\
\hline 2 & ANS33AL2 & 4.74 & 1.35 & 2.302 & $\mathrm{H}_{2} \mathrm{O}$ filled $\mathrm{Al}$ cruciform boxes & 5 \\
\hline 3 & ANS33AL3 & 4.74 & 1.35 & 2.302 & $\mathrm{H}_{2} \mathrm{O}$ filled $\mathrm{Al}$ cruciform boxes & 5 \\
\hline 4 & ANS33EB1 & 4.74 & 1.35 & 2.302 & Polyethylene balls in $\mathrm{Al}$ cruciform boxes & 5 \\
\hline 5 & ANS33EB2 & 4.74 & 1.35 & 2.302 & Polyethylene balls in $\mathrm{Al}$ cruciform boxes & 5 \\
\hline 6 & ANS33EP1 & 4.74 & 1.35 & 2.302 & Polyethylene powder in $\mathrm{Al}$ cruciform boxes & 5 \\
\hline 7 & ANS33EP2 & 4.74 & 1.35 & 2.302 & Polyethylene powder in $\mathrm{Al}$ cruciform boxes & 5 \\
\hline 8 & ANS33SLG & 4.74 & 1.35 & 2.302 & Simple square lattice & 5 \\
\hline 9 & ANS33STY & 4.74 & 1.35 & 2.302 & Expanded polystyrene in Al cruciform box & 5 \\
\hline 10 & $\mathrm{~B} 1645 \mathrm{SO} 1$ & 2.46 & 1.410 & 1.015 & Al separator plates, borated $\mathrm{H}_{2} \mathrm{O}$ & 17 \\
\hline 11 & $\mathrm{~B} 1645 \mathrm{SO} 2$ & 2.46 & 1.410 & 1.015 & Al separator plates, borated $\mathrm{H}_{2} \mathrm{O}$ & 17 \\
\hline 12 & $\mathrm{BW} 1231 \mathrm{B1}$ & 4.02 & 1.511 & 1.139 & Borated $\mathrm{H}_{2} \mathrm{O}$ & 25 \\
\hline 13 & $\mathrm{BW} 1231 \mathrm{~B} 2$ & 4.02 & 1.511 & 1.139 & Borated $\mathrm{H}_{2} \mathrm{O}$ & 25 \\
\hline 14 & $\mathrm{BW} 1273 \mathrm{M}$ & 2.46 & 1.511 & 1.376 & Borated $\mathrm{H}_{2} \mathrm{O}$ & 26 \\
\hline 15 & $\mathrm{BW} 1484 \mathrm{Al}$ & 2.46 & 1.636 & 1.841 & Borated $\mathrm{Al}$ absorber plates, borated $\mathrm{H}_{2} \mathrm{O}$ & 6 \\
\hline 16 & $\mathrm{BW} 1484 \mathrm{~A} 2$ & 2.46 & 1.636 & 1.841 & Borated $\mathrm{Al}$ absorber plates, borated $\mathrm{H}_{2} \mathrm{O}$ & 6 \\
\hline 17 & $\mathrm{BW} 1484 \mathrm{~B} 1$ & 2.46 & 1.636 & 1.841 & Borated $\mathrm{H}_{2} \mathrm{O}$ & 6 \\
\hline 18 & $\mathrm{BW} 1484 \mathrm{~B} 2$ & 2.46 & 1.636 & 1.841 & Borated $\mathrm{H}_{2} \mathrm{O}$ & 6 \\
\hline 19 & BW1484B3 & 2.46 & 1.636 & 1.841 & Borated $\mathrm{H}_{2} \mathrm{O}$ & 6 \\
\hline 20 & $\mathrm{BW} 1484 \mathrm{Cl}$ & 2.46 & 1.636 & 1.841 & $\mathrm{~B}_{4} \mathrm{C}$ rods & 6 \\
\hline 21 & $\mathrm{BW} 1484 \mathrm{C} 2$ & 2.46 & 1.636 & 1.841 & $\mathrm{~B}_{4} \mathrm{C}$ rods & 6 \\
\hline 22 & BW1484S1 & 2.46 & 1.636 & 1.841 & Stainless steel absorber plates, borated $\mathrm{H}_{2} \mathrm{O}$ & 6 \\
\hline 23 & $\mathrm{BW} 1484 \mathrm{~S} 2$ & 2.46 & 1.636 & 1.841 & Stainless steel absorber plates, borated $\mathrm{H}_{2} \mathrm{O}$ & 6 \\
\hline 24 & BW1484SL & 2.46 & 1.636 & 1.841 & Simple square lattice & 6 \\
\hline 25 & $\mathrm{BW} 1645 \mathrm{~S} 1$ & 2.46 & 1.209 & 0.383 & Al separator plates, borated $\mathrm{H}_{2} \mathrm{O}$ & 17 \\
\hline 26 & $\mathrm{BW} 1645 \mathrm{~S} 2$ & 2.46 & 1.209 & 0.383 & Al separator plates, borated $\mathrm{H}_{2} \mathrm{O}$ & 17 \\
\hline 27 & BW1645T1 & 2.46 & 1.209 & 0.148 & Hexagonal lattice, $\mathrm{Al}$ separator plates, borated $\mathrm{H}_{2} \mathrm{O}$ & 17 \\
\hline 28 & BW1645T2 & 2.46 & 1.209 & 0.148 & Hexagonal lattice, $\mathrm{Al}$ separator plates, borated $\mathrm{H}_{2} \mathrm{O}$ & 17 \\
\hline 29 & BW1645T3 & 2.46 & 1.209 & 0.148 & Hexagonal lattice, $\mathrm{Al}$ separator plates, borated $\mathrm{H}_{2} \mathrm{O}$ & 17 \\
\hline 30 & BW1645T4 & 2.46 & 1.209 & 0.148 & Hexagonal lattice, $\mathrm{Al}$ separator plates, borated $\mathrm{H}_{2} \mathrm{O}$ & 17 \\
\hline 31 & BW1810A & 2.46 & 1.636 & 1.841 & $\mathrm{UO}_{2}-\mathrm{Gd}_{2} \mathrm{O}_{3}$ rods, water holes, borated $\mathrm{H}_{2} \mathrm{O}$ & 24 \\
\hline 32 & $\mathrm{BW} 1810 \mathrm{~B}$ & 2.46 & 1.636 & 1.841 & $\mathrm{UO}_{2}-\mathrm{Gd}_{2} \mathrm{O}_{3}$ rods, water holes, borated $\mathrm{H}_{2} \mathrm{O}$ & 24 \\
\hline 33 & $\mathrm{BW} 1810 \mathrm{C}$ & $2.46 \& 4.02$ & 1.636 & $1.841 \& 1.532$ & $\mathrm{UO}_{2}-\mathrm{Gd}_{2} \mathrm{O}_{3}$ rods, water holes, borated $\mathrm{H}_{2} \mathrm{O}$ & 24 \\
\hline
\end{tabular}


Table 2.1 (continued)

\begin{tabular}{|c|c|c|c|c|c|c|}
\hline $\begin{array}{l}\text { Case } \\
\text { No. }\end{array}$ & $\begin{array}{c}\text { Case } \\
\text { designation } \\
\end{array}$ & $\begin{array}{l}\text { Enrich. } \\
\text { (wt \%) }\end{array}$ & $\begin{array}{l}\text { Pitch } \\
(\mathrm{cm})\end{array}$ & $\begin{array}{l}\mathrm{H}_{2} \mathrm{O} / \text { fuel } \\
\text { volume }\end{array}$ & Description & $\begin{array}{c}\text { Reference } \\
\text { No. } \\
\end{array}$ \\
\hline 34 & BW1810D & $2.46 \& 4.02$ & 1.636 & $1.841 \& 1.532$ & $\mathrm{UO}_{2}-\mathrm{Gd}_{2} \mathrm{O}_{3}$ rods, water holes, borated $\mathrm{H}_{2} \mathrm{O}$ & 24 \\
\hline 35 & BW1810E & $2.46 \& 4.02$ & 1.636 & $1.841 \& 1.532$ & $\mathrm{UO}_{2}-\mathrm{Gd}_{2} \mathrm{O}_{3}$ rods, water holes, borated $\mathrm{H}_{2} \mathrm{O}$ & 24 \\
\hline 36 & BW1810F & 2.46 & 1.636 & 1.841 & Water holes, borated $\mathrm{H}_{2} \mathrm{O}$ & 24 \\
\hline 37 & BW1810G & $2.46 \& 4.02$ & 1.636 & $1.841 \& 1.532$ & Water holes, borated $\mathrm{H}_{2} \mathrm{O}$ & 24 \\
\hline 38 & BW1810H & $2.46 \& 4.02$ & 1.636 & $1.841 \& 1.532$ & Water holes, borated $\mathrm{H}_{2} \mathrm{O}$ & 24 \\
\hline 39 & BW1810I & 2.46 & 1.636 & 1.841 & Water holes, Ag-In-Cd rods, borated $\mathrm{H}_{2} \mathrm{O}$ & 24 \\
\hline 40 & BW1810J & $2.46 \& 4.02$ & 1.636 & $1.841 \& 1.532$ & Water holes, $\mathrm{B}_{4} \mathrm{C}$ rods, borated $\mathrm{H}_{2} \mathrm{O}$ & 24 \\
\hline 41 & CR1071AS & 4.46 & 2.43 & 0.77 & $\mathrm{U}_{3} \mathrm{O}_{8}$ fuel cans & 28 \\
\hline 42 & CR1653AS & 4.48 & 2.43 & 1.25 & $\mathrm{U}_{3} \mathrm{O}_{8}$ fuel cans & 28 \\
\hline 43 & CR2500S & 4.48 & 2.43 & 2.03 & $\mathrm{U}_{3} \mathrm{O}_{8}$ fuel cans & 28 \\
\hline 44 & DSN399-1 & 4.74 & 1.6 & 3.807 & Hafnium separator plates & 18 \\
\hline 45 & DSN399-2 & 4.74 & 1.6 & 3.807 & Hafnium separator plates & 18 \\
\hline 46 & DSN399-3 & 4.74 & 1.6 & 3.807 & Hafnium separator plates & 18 \\
\hline 47 & DSN399-4 & 4.74 & 1.6 & 3.807 & Hafnium separator plates & 18 \\
\hline 48 & EPRU65 & 2.35 & 1.562 & 1.196 & Simple square lattice & 7 \\
\hline 49 & EPRU65B & 2.35 & 1.562 & 1.196 & Borated $\mathrm{H}_{2} \mathrm{O}$ & 7 \\
\hline 50 & EPRU75 & 2.35 & 1.905 & 2.408 & Simple square lattice & 7 \\
\hline 51 & EPRU75B & 2.35 & 1.905 & 2.408 & Borated $\mathrm{H}_{2} \mathrm{O}$ & 7 \\
\hline 52 & EPRU87 & 2.35 & 2.210 & 3.687 & Simple square lattice & 7 \\
\hline 53 & EPRU87B & 2.35 & 2.210 & 3.687 & Borated $\mathrm{H}_{2} \mathrm{O}$ & 7 \\
\hline 54 & NSE71H1 & 4.74 & 1.35 & 2.302 & Simple hexagonal lattice & 8 \\
\hline 55 & NSE71H2 & 4.74 & 1.72 & 4.619 & Simple hexagonal lattice & 8 \\
\hline 56 & NSE71H3 & 4.74 & 2.26 & 9.004 & Simple hexagonal lattice & 8 \\
\hline 57 & NSE71SQ & 4.74 & 1.26 & 1.823 & Simple square lattice & 8 \\
\hline 58 & NSE71W1 & 4.74 & 1.26 & 1.823 & Water holes & 8 \\
\hline 59 & NSE71W2 & 4.74 & 1.26 & 1.823 & Water holes & 8 \\
\hline 60 & P2438AL & 2.35 & 2.032 & 2.918 & Aluminum separator plates & 9 \\
\hline 61 & P2438BA & 2.35 & 2.032 & 2.918 & Boral-A separator plates & 9 \\
\hline 62 & P2438CU & 2.35 & 2.032 & 2.918 & Copper separator plates & 9 \\
\hline
\end{tabular}


Table 2.1 (continued)

\begin{tabular}{|c|c|c|c|c|c|c|}
\hline $\begin{array}{c}\text { Case } \\
\text { No. }\end{array}$ & $\begin{array}{c}\text { Case } \\
\text { designation }\end{array}$ & $\begin{array}{l}\text { Enrich. } \\
\text { (wt \%) }\end{array}$ & $\begin{array}{l}\text { Pitch } \\
\text { (cm) }\end{array}$ & $\begin{array}{l}\mathrm{H}_{2} \mathrm{O} / \text { fuel } \\
\text { volume }\end{array}$ & Description & $\begin{array}{c}\text { Reference } \\
\text { No. }\end{array}$ \\
\hline 63 & P2438SLG & 2.35 & 2.032 & 2.918 & Simple square lattice & 9 \\
\hline 64 & P2438SS & 2.35 & 2.032 & 2.918 & Stainless steel separator plates & 9 \\
\hline 65 & P2438ZR & 2.35 & 2.032 & 2.918 & Zircaloy-4 separator plates & 9 \\
\hline 66 & P2615AL & 4.31 & 2.540 & 3.883 & Aluminum separator plates & 19 \\
\hline 67. & P2615BA & 4.31 & 2.540 & 3.883 & Boral-A separator plates & 19 \\
\hline 68 & $\mathrm{P} 2615 \mathrm{CD} 1$ & 4.31 & 2.540 & 3.883 & Cadmium separator plates & 19 \\
\hline 69 & $\mathrm{P} 2615 \mathrm{CD} 2$ & 4.31 & 2.540 & 3.883 & Cadmium separator plates & 19 \\
\hline 70 & $\mathrm{P} 2615 \mathrm{CU}$ & 4.31 & 2.540 & 3.883 & Copper separator plates & 19 \\
\hline 71 & P2615SS & 4.31 & 2.540 & 3.883 & Stainless steel separator plates & 19 \\
\hline 72 & P2615ZR & 4.31 & 2.540 & 3.883 & Zircaloy-4 separator plates & 19 \\
\hline 73 & P2827Ll & 2.35 & 2.032 & 2.918 & Lead reflecting walls & 10 \\
\hline 74 & P2827L2 & 2.35 & 2.032 & 2.918 & Lead reflecting walls & 10 \\
\hline 75 & P2827L3 & 4.31 & 2.540 & 3.883 & Lead reflecting walls & 10 \\
\hline 76 & P2827L4 & 4.31 & 2.540 & 3.883 & Lead reflecting walls & 10 \\
\hline 77 & P2827SLG & 2.35 & 2.032 & 2.918 & Simple square lattice & 10 \\
\hline 78 & P2827U1 & 2.35 & 2.032 & 2.918 & Uranium reflecting walls & 10 \\
\hline 79 & P2827U2 & 2.35 & 2.032 & 2.918 & Uranium reflecting walls & 10 \\
\hline 80 & P2827U3 & 4.31 & 2.540 & 3.883 & Uranium reflecting walls & 10 \\
\hline 81 & P2827U4 & 4.31 & 2.540 & 3.883 & Uranium reflecting walls & 10 \\
\hline 82 & P3314AL & 4.31 & 1.892 & 1.60 & Aluminum separator plates & 11 \\
\hline 83 & P3314BA & 4.31 & 1.892 & 1.60 & Boral-A separator plates & 11 \\
\hline 84 & P3314BC & 4.31 & 1.892 & 1.60 & Boral-C separator plates & 11 \\
\hline 85 & P3314BF1 & 4.31 & 1.892 & 1.60 & Boroflex separator plates & 11 \\
\hline 86 & P3314BF2 & 4.31 & 1.892 & 1.60 & Boroflex separator plates & 11 \\
\hline 87 & P3314BS1 & 2.35 & 1.684 & 1.60 & Borated steel separator plates & 11 \\
\hline 88 & P3314BS2 & 2.35 & 1.684 & 1.60 & Borated steel separator plates & 11 \\
\hline 89 & P3314BS3 & 4.31 & 1.892 & 1.60 & Borated steel separator plates & 11 \\
\hline 90 & P3314BS4 & 4.31 & 1.892 & 1.60 & Borated steel separator plates & 11 \\
\hline 91 & P3314CD1 & 4.31 & 1.892 & 1.60 & Cadmium separator plates & 11 \\
\hline 92 & $\mathrm{P} 3314 \mathrm{CD} 2$ & 2.35 & 1.684 & 1.60 & Cadmium separator plates & 11 \\
\hline 93 & P3314CU1 & 4.31 & 1.892 & 1.60 & Copper separator plates & 11 \\
\hline 94 & P3314CU2 & 4.31 & 1.892 & 1.60 & Copper separator plates & 11 \\
\hline
\end{tabular}


Table 2.1 (continued)

\begin{tabular}{|c|c|c|c|c|c|c|}
\hline $\begin{array}{l}\text { Case } \\
\text { No. }\end{array}$ & $\begin{array}{c}\text { Case } \\
\text { designation }\end{array}$ & $\begin{array}{l}\text { Enrich. } \\
\text { (wt \%) }\end{array}$ & $\begin{array}{l}\text { Pitch } \\
(\mathrm{cm})\end{array}$ & $\begin{array}{l}\mathrm{H}_{2} \mathrm{O} / \text { fuel } \\
\text { volume }\end{array}$ & Description & $\begin{array}{c}\text { Reference } \\
\text { number }\end{array}$ \\
\hline 95 & P3314CU3 & 4.31 & 1.892 & 1.60 & Copper separator plates & 11 \\
\hline 96 & P3314CU4 & 4.31 & 1.892 & 1.60 & Copper-cadmium separator plates & 11 \\
\hline 97 & P3314CU5 & 2.35 & 1.684 & 1.60 & Copper separator plates & 11 \\
\hline 98 & P3314CU6 & 2.35 & 1.684 & 1.60 & Copper-cadmium separator plates & 11 \\
\hline 99 & P3314SLG & 4.31 & 1.892 & 1.60 & Simple square lattice & 11 \\
\hline 100 & P3314SS1 & 4.31 & 1.892 & 1.60 & Steel separator plates & 11 \\
\hline 101 & P3314SS2 & 4.31 & 1.892 & 1.60 & Steel separator plates & 11 \\
\hline 102 & P3314SS3 & 4.31 & 1.892 & 1.60 & Steel separator plates & 11 \\
\hline 103 & P3314SS4 & 4.31 & 1.892 & 1.60 & Steel separator plates & 11 \\
\hline 104 & P3314SS5 & 2.35 & 1.684 & 1.60 & Steel separator plates & 11 \\
\hline 105 & P3314SS6 & 4.31 & 1.892 & 1.60 & Steel separator plates & 11 \\
\hline 106 & P3314W1 & 4.31 & 1.892 & 1.60 & Water holes & 11 \\
\hline 107 & P3314W2 & 2.35 & 1.684 & 1.60 & Water holes & 11 \\
\hline 108 & P3314ZR & 4.31 & 1.892 & 1.60 & Zircaloy -4 separator plates & 11 \\
\hline 109 & P3602BA & 4.31 & 1.892 & 1.60 & Boral-B separator plates, steel reflecting walls & 22 \\
\hline 110 & P3602BS1 & 2.35 & 1.684 & 1.60 & Borated steel separator plates, steel reflecting walls & 22 \\
\hline 111 & P3602BS2 & 4.31 & 1.892 & 1.60 & Borated steel separator plates, steel reflecting walls & 22 \\
\hline 112 & $\mathrm{P} 3602 \mathrm{CD} 1$ & 2.35 & 1.684 & 1.60 & Cadmium separator plates, steel reflecting walls & 22 \\
\hline 113 & P3602CD2 & 4.31 & 1.892 & 1.60 & Cadmium separator plates, steel reflecting walls & 22 \\
\hline 114 & P3602CU1 & 2.35 & 1.684 & 1.60 & Copper separator plates, steel reflecting walls & 22 \\
\hline 115 & P3602CU2 & 2.35 & 1.684 & 1.60 & Copper-cadmium separator plates, steel reflecting walls & 22 \\
\hline 116 & P3602CU3 & 4.31 & 1.892 & 1.60 & Copper separator plates, steel reflecting walls & 22 \\
\hline 117 & P3602CU4 & 4.31 & 1.892 & 1.60 & Copper-cadmium separator plates, steel reflecting walls & 22 \\
\hline 118 & $\mathrm{P} 3602 \mathrm{~N} 11$ & 2.35 & 1.684 & 1.60 & Steel reflecting walls & 22 \\
\hline 119 & $\mathrm{P} 3602 \mathrm{~N} 12$ & 2.35 & 1.684 & 1.60 & Steel reflecting walls & 22 \\
\hline 120 & P3602N13 & 2.35 & 1.684 & 1.60 & Steel reflecting walls & 22 \\
\hline 121 & P3602N14 & 2.35 & 1.684 & 1.60 & Steel reflecting walls & 22 \\
\hline 122 & P3602N21 & 2.35 & 2.032 & 2.918 & Steel reflecting walls & 22 \\
\hline 123 & P3602N22 & 2.35 & 2.032 & 2.918 & Steel reflecting walls & 22 \\
\hline 124 & P3602N31 & 4.31 & 1.892 & 1.60 & Steel reflecting walls & 22 \\
\hline
\end{tabular}


Table 2.1 (continued)

\begin{tabular}{|c|c|c|c|c|c|c|}
\hline $\begin{array}{l}\text { Case } \\
\text { No. }\end{array}$ & $\begin{array}{c}\text { Case } \\
\text { designation }\end{array}$ & $\begin{array}{l}\text { Enrich. } \\
\text { (wt \%) }\end{array}$ & $\begin{array}{l}\text { Pitch } \\
(\mathrm{cm})\end{array}$ & $\begin{array}{l}\mathrm{H}_{2} \mathrm{O} / \text { fuel } \\
\text { volume }\end{array}$ & Description & $\begin{array}{c}\text { Reference } \\
\text { number }\end{array}$ \\
\hline 125 & $\mathrm{P} 3602 \mathrm{~N} 32$ & 4.31 & 1.892 & 1.60 & Steel reflecting walls & 22 \\
\hline 126 & P3602N33 & 4.31 & 1.892 & 1.60 & Steel reflecting walls & 22 \\
\hline 127 & P3602N34 & 4.31 & 1.892 & 1.60 & Steel reflecting walls & 22 \\
\hline 128 & $\mathrm{P} 3602 \mathrm{~N} 35$ & 4.31 & 1.892 & 1.60 & Steel reflecting walls & 22 \\
\hline 129 & P3602N36 & 4.31 & 1.892 & 1.60 & Steel reflecting walls & 22 \\
\hline 130 & P3602N41 & 4.31 & 2.540 & 3.883 & Steel reflecting walls & 22 \\
\hline 131 & $\mathrm{P} 3602 \mathrm{~N} 42$ & 4.31 & 2.540 & 3.883 & Steel reflecting walls & 22 \\
\hline 132 & $\mathrm{P} 3602 \mathrm{~N} 43$ & 4.31 & 2.540 & 3.883 & Steel reflecting walls & 22 \\
\hline 133 & P3602SS1 & 2.35 & 1.684 & 1.60 & Steel separator plates, steel reflecting walls & 22 \\
\hline 134 & P3602SS2 & 4.31 & 1.892 & 1.60 & Steel separator plates, steel reflecting walls & 22 \\
\hline 135 & P3926L1 & 2.35 & 1.684 & 1.60 & Lead reflecting walls & 12 \\
\hline 136 & P3926L2 & 2.35 & 1.684 & 1.60 & Lead reflecting walls & 12 \\
\hline 137 & P3926L3 & 2.35 & 1.684 & 1.60 & Lead reflecting walls & 12 \\
\hline 138 & P3926L4 & 4.31 & 1.892 & 1.60 & Lead reflecting walls & 12 \\
\hline 139 & P3926L5 & 4.31 & 1.892 & 1.60 & Lead reflecting walls & 12 \\
\hline 140 & P3926L6 & 4.31 & 1.892 & 1.60 & Lead reflecting walls & 12 \\
\hline 141 & P3926SLl & 2.35 & 1.684 & 1.60 & Simple lattice & 12 \\
\hline 142 & P3926SL2 & 4.31 & 1.892 & 1.60 & Simple lattice & 12 \\
\hline 143 & P3926U1 & 2.35 & 1.684 & 1.60 & Uranium reflecting walls & 12 \\
\hline 144 & P3926U2 & 2.35 & 1.684 & 1.60 & Uranium reflecting walls & 12 \\
\hline 145 & P3926U3 & 2.35 & 1.684 & 1.60 & Uranium reflecting walls & 12 \\
\hline 146 & P3926U4 & 4.31 & 1.892 & 1.60 & Uranium reflecting walls & 12 \\
\hline 147 & P3926U5 & 4.31 & 1.892 & 1.60 & Uranium reflecting walls & 12 \\
\hline 148 & P3926U6 & 4.31 & 1.892 & 1.60 & Uranium reflecting walls & 13 \\
\hline 149 & P4267B1 & 4.31 & 1.8901 & 1.59 & Borated $\mathrm{H}_{2} \mathrm{O}$ & 13 \\
\hline 150 & P4267B2 & 4.31 & .890 & 1.59 & Borated $\mathrm{H}_{2} \mathrm{O}$ & 13 \\
\hline 151 & P4267B3 & 4.31 & 1.715 & 1.090 & Borated $\mathrm{H}_{2} \mathrm{O}$ & 13 \\
\hline 152 & P4267B4 & 4.31 & 1.715 & 1.090 & Borated $\mathrm{H}_{2} \mathrm{O}$ & 13 \\
\hline 153 & P4267B5 & 4.31 & 1.715 & 1.090 & Borated $\mathrm{H}_{2} \mathrm{O}$ & 13 \\
\hline
\end{tabular}


Table 2.1 (continued)

\begin{tabular}{|c|c|c|c|c|c|c|c|}
\hline & $\begin{array}{l}\text { Case } \\
\text { No. }\end{array}$ & $\begin{array}{c}\text { Case } \\
\text { designation }\end{array}$ & $\begin{array}{l}\text { Enrich. } \\
\text { (wt \%) }\end{array}$ & $\begin{array}{l}\text { Pitch } \\
(\mathrm{cm})\end{array}$ & $\begin{array}{l}\mathrm{H}_{2} \mathrm{O} / \text { fuel } \\
\text { volume }\end{array}$ & Description & $\begin{array}{c}\text { Reference } \\
\text { number }\end{array}$ \\
\hline & 154 & P4267SL1 & 4.31 & 1.890 & 1.59 & Borated $\mathrm{H}_{2} \mathrm{O}$ & 13 \\
\hline & 155 & P4267SL2 & 4.31 & 1.715 & 1.090 & Borated $\mathrm{H}_{2} \mathrm{O}$ & 13 \\
\hline & 156 & P49-194 & 4.31 & 1.598 & 0.509 & Simple hexagonal lattice & 14 \\
\hline & 157 & P62FT231 & 4.31 & 1.891 & 1.60 & Boral flux traps, no void material & 20 \\
\hline & 158 & P71F14F3 & 4.31 & 1.891 & 1.60 & Boral flux traps, void material fuel rods & 21 \\
\hline & 159 & P71F14V3 & 4.31 & 1.891 & 1.60 & Boral flux traps, void material Al plates & 21 \\
\hline & 160 & P71F14V5 & 4.31 & 1.891 & 1.60 & Boral flux traps, void material Al rods & 21 \\
\hline & 161 & P71F214R & 4.31 & 1.891 & 1.60 & Boral flux traps, no void material & 21 \\
\hline & 162 & PAT80L1 & 4.74 & 1.6 & 3.807 & Boral separator plates, lead reflecting walls & 23 \\
\hline & 163 & PAT80L2 & 4.74 & 1.6 & 3.807 & Boral separator plates, lead reflecting walls & 23 \\
\hline & 164 & PAT80SS1 & 4.74 & 1.6 & 3.807 & Boral separator plates, steel reflecting walls & 23 \\
\hline & 165 & PAT80SS2 & 4.74 & 1.6 & 3.807 & Boral separator plates, steel reflecting walls & 23 \\
\hline & 166 & W3269A & 5.7 & 1.422 & 1.93 & Ag-In-Cd rods & 15 \\
\hline N & 167 & W3269B1 & 3.7 & 1.105 & 1.432 & Ag-In-Cd rods & 15 \\
\hline & 168 & W3269B2 & 3.7 & 1.105 & 1.432 & Ag-In-Cd rods & 15 \\
\hline & 169 & W3269B3 & 3.7 & 1.105 & 1.432 & $\mathrm{Ag}-\mathrm{In}-\mathrm{Cd}$ rods & 15 \\
\hline & 170 & W3269C & 2.72 & 1.524 & 1.494 & Ag-In-Cd rods & 15 \\
\hline & 171 & W3269SL1 & 2.72 & 1.524 & 1.494 & Simple square lattice & 15 \\
\hline & 172 & W3269SL2 & 5.7 & 1.422 & 1.93 & Simple square lattice & 15 \\
\hline & 173 & W3269W1 & 2.72 & 1.524 & 1.494 & Water holes & 15 \\
\hline & 174 & W3269W2 & 5.7 & 1.422 & 1.93 & Water holes & 15 \\
\hline & 175 & W3385SL1 & 5.74 & 1.422 & 1.932 & Simple square lattice & 16 \\
\hline & 176 & W3385SL2 & 5.74 & 2.012 & 5.067 & Simple square lattice & 16 \\
\hline & 177 & YDR14PL2 & 2.00 & - & 8.23 & Homogenized $\mathrm{U}(2) \mathrm{F}_{4}$ in paraffin & 27 \\
\hline & 178 & YDR14PL3 & 3.00 & - & 4.05 & Homogenized $\mathrm{U}(3) \mathrm{F}_{4}$ in paraffin & 27 \\
\hline 己 & 179 & YDR14UN2 & 2.00 & - & 5.95 & Homogenized $\mathrm{U}(2) \mathrm{F}_{4}$ in paraffin & 27 \\
\hline$\stackrel{T}{Q}$ & 180 & YDR14UN3 & 3.00 & - & 4.05 & Homogenized $\mathrm{U}(3) \mathrm{F}_{4}$ in paraffin & 27 \\
\hline
\end{tabular}


Physical Description

Table 2.2 Properties of $\mathrm{UO}_{2}$ fuel rods from ANS Transactions, Vol. $33^{a}$

\begin{tabular}{ll}
\hline Parameter & Value \\
\hline Outside diameter, cm & 0.94 \\
Wall thickness, cm & 0.06 \\
Wall material & aluminum \\
Pellet diameter, $\mathrm{cm}$ & 0.790 \\
Total fuel length, cm & $100.0^{\circ}$ \\
Active fuel length, cm & 90.0 \\
Enrichment, ${ }^{23} \mathrm{U} / \mathrm{U}$ wt \% & 4.742 \\
Fuel density, $\mathrm{g} / \mathrm{cm}^{3}$ & 10.38 \\
\hline
\end{tabular}

${ }^{a}$ See Ref. 5.

b 98.85 wt \% Al, 0.47 wt \% Mg, 0.43 wt \% Si, 0.22 wt \% Fe, 0.03 wt \% Zn.

'Includes 1.8-cm-long bottom end plug, 1.3-cm-long top end plug, and $6.9-\mathrm{cm}$-long spring above the pellet stack.

Table 2.3 Critical data for simple lattice experiment ANS33SLG ${ }^{a}$

\begin{tabular}{|c|c|c|c|c|c|}
\hline Experiment & $\begin{array}{c}\text { Enrichment } \\
\text { (wt \%) }\end{array}$ & $\begin{array}{l}\text { Pitch } \\
(\mathrm{cm})\end{array}$ & $\begin{array}{l}\text { Separation of } \\
\text { assemblies }^{b}\end{array}$ & $\begin{array}{c}\text { No. of } \\
\text { assemblies- } \\
\text { assembly size } \\
\text { (No. of rods) }\end{array}$ & $\begin{array}{l}\text { Moderator } \\
\text { height, }{ }^{c} \mathrm{H}_{\mathrm{c}} \\
\text { (cm) }\end{array}$ \\
\hline ANS33SLG & 4.742 & 1.35 & $5.0 \mathrm{~cm}$ & $4-18 \times 18$ & 31.47 \\
\hline
\end{tabular}

${ }^{a}$ See Ref. 5.

${ }^{b}$ Perpendicular distance between cell boundaries of assemblies.

${ }^{\circ}$ Measured from lower end of fuel rod pellet column. 
Table 2.4 Properties of $\mathrm{UO}_{2}$ fuel rods from BAW-1484

\begin{tabular}{ll}
\hline Parameter & Value \\
\hline Outside diameter, cm & 1.206 \\
Wall thickness, cm & 0.081 \\
Wall material & aluminum \\
Pellet diameter, cm & 1.030 \\
Total length, cm & $156.44^{b}$ \\
Active fuel length, cm & 153.34 \\
Enrichment, ${ }^{235} \mathrm{U} / \mathrm{U}$ wt \% & 2.459 \\
Fuel density, $\mathrm{g} / \mathrm{cm}^{3}$ & 10.22 \\
\hline
\end{tabular}

a See Ref. 6.

${ }^{b}$ Includes $0.3175-\mathrm{cm}$-long aluminum top and bottom end plugs and $2.464 \mathrm{~cm}$ of Kaowool above the pellet stack.

Table 2.5 Critical data for simple lattice experiment BW1484SL from BAW-1484 ${ }^{a}$

\begin{tabular}{cccccc}
\hline Experiment & $\begin{array}{c}\text { Enrichment } \\
(\mathrm{wt} \%)\end{array}$ & $\begin{array}{c}\text { Pitch } \\
(\mathrm{cm})\end{array}$ & $\begin{array}{c}\text { Separation of } \\
\text { assemblies }^{b}\end{array}$ & $\begin{array}{c}\text { No. of } \\
\text { assemblies- } \\
\text { assembly size } \\
\text { (No. of rods) }\end{array}$ & $\begin{array}{c}\text { Moderator } \\
\text { height, }{ }^{c} \mathrm{H}_{\mathrm{c}} \\
(\mathrm{cm})\end{array}$ \\
\hline BW1484SL & 2.459 & 1.636 & $6.544 \mathrm{~cm}$ & $9-14 \times 14^{d}$ & 129.65 \\
\hline
\end{tabular}

${ }^{a}$ See Ref. 6.

${ }^{b}$ Believed to be the perpendicular distance between cell boundaries of assemblies.

${ }^{c}$ Measured from the top of the aluminum base plate.

${ }^{d}$ Four corner rods of assemblies are 0.635 -cm-radius aluminum rods. 
Physical Description

Table 2.6 Critical data for simple lattice experiments from EPRI NP-196 ${ }^{a}$

\begin{tabular}{lccccc}
\hline Experiment & $\begin{array}{c}\text { Enrichment } \\
(\text { wt \%) }\end{array}$ & $\begin{array}{c}\text { Pitch } \\
(\mathrm{cm})\end{array}$ & $\begin{array}{c}\text { No. of } \\
\text { rods }\end{array}$ & $\begin{array}{c}\text { Moderator } \\
\text { height } \\
\text { (cm) }\end{array}$ & $\begin{array}{c}\text { Boron } \\
\text { concentration } \\
\text { (ppm) }\end{array}$ \\
\hline EPRU65 & 2.35 & 1.562 & 708 & 111.76 & 0.9 \\
EPRU75 & 2.35 & 1.905 & 383 & 112.08 & 0.5 \\
EPRU87 & 2.35 & 2.210 & 342 & 111.76 & 0.9 \\
\hline
\end{tabular}

a See Ref. 7.

${ }^{b}$ Measured from the top of the aluminum solid plate.

Table 2.7 Critical data for simple lattice experiments from NS\&E, Vol. $71^{a}$

\begin{tabular}{ccccc}
\hline Experiment & $\begin{array}{c}\text { Enrichment } \\
(\mathrm{wt} \%)\end{array}$ & $\begin{array}{c}\text { Pitch } \\
(\mathrm{cm})\end{array}$ & $\begin{array}{c}\text { No. of } \\
\text { assemblies- } \\
\text { assembly size } \\
\text { (No. of rods) }\end{array}$ & $\begin{array}{c}\text { Moderator } \\
\text { height, }{ }^{b} \mathrm{H}_{\mathrm{c}} \\
(\mathrm{cm})\end{array}$ \\
\hline NSE71H1 & 4.742 & $1.35^{c}$ & $\begin{array}{c}1-14 \text { per side } \\
(547)\end{array}$ & 60.93 \\
NSE71H2 & 4.742 & $1.72^{c}$ & $\begin{array}{c}1-10 \text { per side } \\
(271)\end{array}$ & 68.06 \\
NSE71H3 & 4.742 & $2.26^{c}$ & $\begin{array}{c}1-9 \text { per side } \\
(217)\end{array}$ & 79.50 \\
NSE71SQ & 4.742 & 1.26 & $1-22 \times 22$ & 90.69 \\
\hline
\end{tabular}

a See Ref. 8.

${ }^{b}$ Measured from lower end of fuel rod pellet column.

${ }^{c}$ Hexagonal lattice. 
Table 2.8 Critical data for simple lattice experiment P2438SLG from PNL-2438 ${ }^{a}$

\begin{tabular}{ccccc}
\hline Experiment & $\begin{array}{c}\text { Enrichment } \\
(\mathrm{wt} \%)\end{array}$ & $\begin{array}{c}\text { Pitch } \\
(\mathrm{cm})\end{array}$ & $\begin{array}{c}\text { Separation of } \\
\text { assemblies, }{ }^{b} \mathrm{X}_{\mathrm{c}} \\
(\mathrm{cm})\end{array}$ & $\begin{array}{c}\text { No. of assemblies- } \\
\text { assembly size } \\
\text { (No. of rods) }\end{array}$ \\
\hline P2438SLG & 2.35 & 2.032 & 8.39 & $3-20 \times 16$ \\
\hline
\end{tabular}

${ }^{a}$ See Ref. 9.

${ }^{b}$ Perpendicular distance between cell boundaries of assemblies.

Table 2.9 Critical data for simple lattice experiment P2827SLG from PNL-2827 ${ }^{a}$

\begin{tabular}{ccccc}
\hline Experiment & $\begin{array}{c}\text { Enrichment } \\
(\mathrm{wt} \%)\end{array}$ & $\begin{array}{c}\text { Pitch } \\
(\mathrm{cm})\end{array}$ & $\begin{array}{c}\text { Separation of } \\
\text { assemblies, }{ }^{b} \mathrm{X}_{\mathrm{c}} \\
(\mathrm{cm})\end{array}$ & $\begin{array}{c}\text { No. of assemblies- } \\
\text { assembly size } \\
\text { (No. of rods) }\end{array}$ \\
\hline P2827SLG & 2.35 & 2.032 & 8.31 & $3-19 \times 16$ \\
\hline
\end{tabular}

a See Ref. 10.

${ }^{b}$ Perpendicular distance between cell boundaries of assemblies.

Table 2.10 Critical data for simple lattice experiment P3314SLG from PNL-3314 ${ }^{a}$

\begin{tabular}{lcccc}
\hline Experiment & $\begin{array}{c}\text { Enrichment } \\
(\mathrm{wt} \%)\end{array}$ & $\begin{array}{c}\text { Pitch } \\
(\mathrm{cm})\end{array}$ & $\begin{array}{c}\text { Separation of } \\
\text { assemblies, }{ }^{b} \mathrm{X}_{\mathrm{c}} ; \mathrm{Y}_{\mathrm{c}} \\
(\mathrm{cm})\end{array}$ & $\begin{array}{c}\text { No. of assemblies- } \\
\text { assembly size } \\
\text { (No. of rods) }\end{array}$ \\
\hline P3314SLG & 4.31 & 1.892 & $2.83 ; 10.86$ & $2-9 \times 12 ; 2-9 \times 1$ \\
\hline
\end{tabular}

a See Ref. 11.

${ }^{b}$ Perpendicular distance between cell boundaries of assemblies. 
Table 2.11 Critical data for simple lattice experiments from PNL-3926 ${ }^{a}$

\begin{tabular}{lcccc}
\hline Experiment & $\begin{array}{c}\text { Enrichment } \\
(\text { wt \%) }\end{array}$ & $\begin{array}{c}\text { Pitch } \\
(\mathrm{cm})\end{array}$ & $\begin{array}{c}\text { Separation of } \\
\text { assemblies, }^{b} \mathrm{X}_{\mathrm{c}} \\
(\mathrm{cm})\end{array}$ & $\begin{array}{c}\text { No. of assemblies- } \\
\text { assembly size } \\
\text { (No. of rods) }\end{array}$ \\
\hline P3926SL1 & 2.35 & 1.684 & 6.59 & $2-20 \times 18 ; 1-23 \times 18^{c}$ \\
P3926SL2 & 4.31 & 1.892 & 12.79 & $3-12 \times 16$ \\
\hline
\end{tabular}

a See Ref. 12.

${ }^{b}$ Perpendicular distance from outer rod surface to outer rod surface.

- Center assembly.

Table 2.12 Critical data for simple lattice experiments from PNL-4267 ${ }^{a}$

\begin{tabular}{ccccccc}
\hline & & & & \multicolumn{2}{c}{ Total No. of rods } \\
\cline { 5 - 6 } Experiment & $\begin{array}{c}\text { Enrichment } \\
(\text { wt \%) }\end{array}$ & $\begin{array}{c}\text { Pitch } \\
(\mathrm{cm})\end{array}$ & $\begin{array}{c}\text { Array width } \\
\text { (No. of rods) }\end{array}$ & Predicted $^{b}$ & Modeled \\
\hline P4267SL1 & 4.31 & 1.890 & 40 & 356.8 & 357 \\
P4267SL2 & 4.31 & 1.715 & 44 & 509.08 & 509 \\
\hline
\end{tabular}

${ }^{a}$ See Ref. 13.

${ }^{b}$ Obtained by approach-to-critical method.

Table 2.13 Critical data for simple lattice experiment P49-194 from PNL-4976a

\begin{tabular}{cccc}
\hline Experiment & $\begin{array}{c}\text { Enrichment } \\
(\mathrm{wt} \mathrm{\% )}\end{array}$ & $\begin{array}{c}\mathrm{Pitch}^{b} \\
(\mathrm{~cm})\end{array}$ & $\begin{array}{c}\text { No. of assemblies- } \\
\text { assembly size } \\
\text { (No. of rods) }\end{array}$ \\
\hline P49-194 & 4.31 & 1.598 & $1-1185$ \\
\hline
\end{tabular}

a See Ref. 14.

${ }^{b}$ Hexagonal lattice. 
Table 2.14 Properties of $\mathrm{UO}_{2}$ fuel rods from WCAP-3269

\begin{tabular}{llll}
\hline & 2.72 & 3.7 & 5.7 \\
\hline Outer diameter, cm & 1.1895 & 0.8600 & 0.9931 \\
Wall thickness, cm & 0.0799 & 0.0406 & 0.0762 \\
Wall material & Zircaloy-4 & 304 -stainless steel & \\
Pellet diameter, cm & 1.0160 & 0.7544 & 304 -stainless steel \\
Total fuel length, cm & $139.65^{d}$ & $-{ }^{e}$ & 0.9068 \\
Active fuel length, cm & 121.92 & 121.92 & $97.155^{f}$ \\
Enrichment, ${ }^{235} \mathrm{U} / \mathrm{U}, \mathrm{wt} \%$ & 2.72 & 3.7 & 92.964 \\
Fuel density, g/cm & 10.412 & 10.4120 & 5.7 \\
\hline
\end{tabular}

a See Ref. 15.

${ }^{b} 98.20$ wt \% $\mathrm{Zr}, 1.4 \mathrm{wt} \% \mathrm{Sn}, 0.21 \mathrm{wt} \% \mathrm{Fe}, 0.10 \mathrm{wt} \% \mathrm{Cr}$.

${ }^{\circ} 18.54$ wt \% Cr, 9.44 wt \% Ni, 1.76 wt \% Mn, 69.46 wt \% Fe.

${ }^{d}$ Includes 3.81-cm-long bottom and at least 13.92-cm-long top 304-stainless steel end plugs.

- No data available on end plug lengths.

${ }^{f}$ Includes $1.905-\mathrm{cm}-$ long bottom and at least $2.286-\mathrm{cm}$-long top Zircaloy-4 end plugs.

Table 2.15 Critical data for simple lattice experiments from WCAP-3269

\begin{tabular}{ccccc}
\hline Experiment & $\begin{array}{c}\text { Enrichment } \\
(\mathrm{wt} \%)\end{array}$ & $\begin{array}{c}\text { Pitch } \\
(\mathrm{cm})\end{array}$ & $\begin{array}{c}\text { No. of assemblies- } \\
\text { assembly size } \\
\text { (No. of rods) }\end{array}$ & $\begin{array}{c}\text { Moderator } \\
\text { height }^{b} \\
\text { (cm) }\end{array}$ \\
\hline W3269SL1 & 2.72 & 1.524 & $1-31 \times 31$ & 38.58 \\
W3269SL2 & 5.7 & 1.422 & $1-27 \times 17$ & 52.97 \\
\hline
\end{tabular}

${ }^{a}$ See Ref. 15.

${ }^{b}$ Believed to have been measured from the bottom of fuel, see Figs. 20 and 21. 
Table 2.16 Properties of $\mathrm{UO}_{2}$ fuel rods from WCAP-3385 ${ }^{a}$

\begin{tabular}{ll}
\hline Parameter & Value \\
\hline Outside diameter, cm & 0.993 \\
Wall thickness, cm & 0.038 \\
Wall material & 304 -stainless steel \\
Pellet diameter, cm & 0.907 \\
Total fuel length, cm & $97.155^{b}$ \\
Active fuel length, cm & 92.96 \\
Enrichment, ${ }^{235} \mathrm{U} / \mathrm{Ut}$ wt $\%$ & 5.74 \\
Fuel density, $\mathrm{g} / \mathrm{cm}^{3}$ & 10.19 \\
\hline
\end{tabular}

${ }^{a}$ See Ref. 16.

${ }^{b}$ Includes $1.905-\mathrm{cm}$-long bottom and $2.286-\mathrm{cm}$-long top end plugs believed to be of 304-stainless steel.

Table 2.17 Critical data for simple lattice experiments from WCAP-3385 ${ }^{a}$

\begin{tabular}{ccccc}
\hline Experiment & $\begin{array}{c}\text { Enrichment } \\
(\mathrm{wt} \%)\end{array}$ & $\begin{array}{c}\text { Pitch } \\
(\mathrm{cm})\end{array}$ & $\begin{array}{c}\text { No. of assemblies- } \\
\text { assembly size }^{b} \\
\text { (No. of rods) }\end{array}$ & $\begin{array}{c}\text { Moderator }_{\text {height }^{c}} \\
(\mathrm{~cm})\end{array}$ \\
\hline W3385SL1 & 5.74 & 1.42 & $1-19 \times 19$ & 83.71 \\
W3385SL2 & 5.74 & 2.012 & $1-13 \times 14$ & 90.60 \\
\hline
\end{tabular}

a See Ref. 16.

${ }^{b}$ Obtained by approach-to-critical method.

${ }^{c}$ Reference from bottom of fuel rod pellet column. 
Table 2.18 Critical data for separator plate experiments from ANS Transactions, Vol. $33^{a}$

\begin{tabular}{|c|c|c|c|c|c|c|c|c|c|}
\hline Experiment & $\begin{array}{c}\text { Enrichment } \\
\text { (wt \%) }\end{array}$ & $\begin{array}{l}\text { Pitch } \\
\text { (cm) }\end{array}$ & $\begin{array}{l}\text { Plate } \\
\text { material }\end{array}$ & $\begin{array}{l}\text { Plate } \\
\text { thickness } \\
(\mathrm{cm})\end{array}$ & $\begin{array}{l}\text { Separation of } \\
\text { assemblies }^{b} \\
\text { (cm) }\end{array}$ & $\begin{array}{l}\text { No. of } \\
\text { assemblies- } \\
\text { assembly size } \\
\text { (No. of rods) }\end{array}$ & $\begin{array}{l}\text { Moderator } \\
\text { height, }{ }^{c} \mathrm{H}_{c} \\
\quad(\mathrm{~cm})\end{array}$ & $\begin{array}{l}\text { Interstitial } \\
\text { compound }\end{array}$ & $\begin{array}{l}\text { Interstitial } \\
\text { Compound } \\
\text { density } \\
\left(\mathrm{g} / \mathrm{cm}^{3}\right)\end{array}$ \\
\hline ANS33AL1 & 4.742 & 1.35 & aluminum & 0.3 & 2.5 & $4-18 \times 18$ & 25.66 & $\mathrm{H}_{2} \mathrm{O}$ & 1.0 \\
\hline ANS33AL2 & 4.742 & 1.35 & aluminum & 0.3 & 5.0 & $4-18 \times 18$ & 32.78 & $\mathrm{H}_{2}^{2} \mathrm{O}$ & 1.0 \\
\hline ANS33AL3 & 4.742 & 1.35 & aluminum & 0.3 & 10.0 & $4-18 \times 18$ & 64.12 & $\mathrm{H}_{2} \mathrm{O}$ & 1.0 \\
\hline ANS33EB1 & 4.742 & 1.35 & aluminum & 0.3 & 2.5 & $4-18 \times 18$ & 25.54 & $\left(\mathrm{CH}_{2}\right)_{\mathrm{n}}$ & 0.5540 \\
\hline ANS33EB2 & 4.742 & 1.35 & aluminum & 0.3 & 5.0 & $4-18 \times 18$ & 30.73 & $\left(\mathrm{CH}_{2}\right)_{\mathrm{n}}$ & 0.5796 \\
\hline ANS33EP1 & 4.742 & 1.35 & aluminum & 0.3 & 2.5 & $4-18 \times 18$ & 26.98 & $\left(\mathrm{CH}_{2}\right)_{\mathrm{n}}$ & 0.2879 \\
\hline ANS33EP2 & 4.742 & 1.35 & aluminum & 0.3 & 5.0 & $4-18 \times 18$ & 30.16 & $\left(\mathrm{C}_{8} \mathrm{H}_{8}\right)_{n}$ & 0.3335 \\
\hline ANS33STG & 4.742 & 1.35 & aluminum & 0.3 & 2.5 & $4-18 \times 18$ & 28.61 & $\left(\mathrm{C}_{8} \mathrm{H}_{8}\right)_{\mathrm{n}}$ & 0.0323 \\
\hline
\end{tabular}

${ }^{a}$ See Ref. 5.

${ }^{b}$ Perpendicular distance between cell boundaries of assemblies.

'Measured from lower end of fuel rod pellet column. 
Table 2.19 Critical data for separator plate experiments from BAW-1484 ${ }^{a}$

\begin{tabular}{|c|c|c|c|c|c|c|c|c|c|}
\hline Experiment & $\begin{array}{c}\text { Enrichment } \\
(\text { wt \%) }\end{array}$ & $\begin{array}{l}\text { Pitch } \\
(\mathrm{cm})\end{array}$ & $\begin{array}{c}\text { Plate } \\
\text { material }\end{array}$ & $\begin{array}{c}\text { Plate } \\
\text { dimensions } \\
\text { (cm) }\end{array}$ & $\begin{array}{l}\text { Separation } \\
\text { of } \\
\text { assemblies }^{b} \\
(\mathrm{~cm})\end{array}$ & $\begin{array}{l}\text { Plate-to-center } \\
\text { assembly } \\
\text { distance }^{c}(\mathrm{~cm})\end{array}$ & $\begin{array}{l}\text { No. of } \\
\text { assemblies- } \\
\text { assembly size } \\
\text { (No. of rods) }\end{array}$ & $\begin{array}{l}\text { Moderator } \\
\text { height, } \mathrm{H} \\
\quad(\mathrm{cm})\end{array}$ & $\begin{array}{c}\text { Boron } \\
\text { concentration } \\
\text { (ppm) }\end{array}$ \\
\hline BW1484A1 & 2.459 & 1.636 & $\begin{array}{l}1.614 \mathrm{wt} \% \mathrm{~B} \\
\text { aluminum }\end{array}$ & $91.6 \times 155.6 \times 0.645$ & 1.636 & 0.3725 & $9-14 \times 14$ & 150.27 & 15 \\
\hline BW1484A2 & 2.459 & 1.636 & $\begin{array}{c}0.100 \text { wt } \% \mathrm{~B} \\
\text { aluminum }\end{array}$ & $91.6 \times 155.6 \times 0.645$ & 4.908 & 0.3725 & $9-14 \times 14$ & 151.69 & 72 \\
\hline BW1484S1 & 2.459 & 1.636 & $\begin{array}{c}304-\mathrm{L} \\
\text { stainless steel }\end{array}$ & $91.6 \times 155.6 \times 0.462$ & 1.636 & 0.4525 & $9.14 \times 14$ & 149.90 & 514 \\
\hline BW1484S2 & 2.459 & 1.636 & $\begin{array}{c}304-\mathrm{L} \\
\text { stainless steel }\end{array}$ & $91.6 \times 155.6 \times 0.462$ & 1.636 & 0.4525 & $9-14 \times 14$ & 100.89 & 432 \\
\hline
\end{tabular}

aSee Ref. 6.

${ }^{6}$ Believed to be the perpendicular distance between cell boundaries of assemblies.

'Perpendicular distance between cell boundary of assembly and near surface of plate.

"Four corner rods of assemblies are 0.635 -cm-radius aluminum rods.

Measured from the top of the aluminum base plate

Density of $2.7 \mathrm{~g} / \mathrm{cm}^{3}$. 
Table 2.20 Critical data for separator plate experiments from BAW-1645

\begin{tabular}{|c|c|c|c|c|c|c|c|c|c|}
\hline Experiment & $\begin{array}{c}\text { Enrichment } \\
(w t \%)\end{array}$ & $\begin{array}{l}\text { Pitch } \\
\text { (cm) }\end{array}$ & $\begin{array}{c}\text { Plate }^{b} \\
\text { material }\end{array}$ & $\begin{array}{c}\text { Plate } \\
\text { dimensions } \\
(\mathrm{cm})\end{array}$ & $\begin{array}{l}\text { Separation of } \\
\text { assemblies } \\
\text { (cm) }\end{array}$ & $\begin{array}{l}\text { Plate-to- } \\
\text { assembly } \\
\text { distance } \\
\text { (cm) }\end{array}$ & $\begin{array}{l}\text { No. of } \\
\text { assemblies- } \\
\text { assemby size } \\
\text { (No. of rods) }\end{array}$ & $\begin{array}{l}\text { Moderator } \\
\text { height, }{ }^{c} \mathrm{H}_{c} \\
\text { (cm) }\end{array}$ & $\begin{array}{c}\text { Boron } \\
\text { concentration } \\
(\mathrm{ppm})\end{array}$ \\
\hline BW1645S1 & 2.459 & 1.2090 & aluminum & $15.24 \times 159.32^{e} \times 0.0762$ & $1.778 \times 1.778^{f}$ & 0.0 & $9-15 \times 15$ & 100.24 & 746 \\
\hline BW1645S2 & 2.459 & 1.2090 & aluminum & $15.24 \times 159.32^{e} \times 0.0762$ & $1.778 \times 1.778^{\prime}$ & 0.0 & $9-15 \times 15$ & 145.00 & 886 \\
\hline B1645SO1 & 2.459 & 1.4097 & aluminum & $15.24 \times 159.32^{e} \times 0.0762$ & $1.792 \times 1.792^{\checkmark}$ & 0.0 & $9-15 \times 15$ & 101.81 & 1068 \\
\hline B1645SO2 & 2.459 & 1.4097 & aluminum & $15.24 \times 159.32^{e} \times 0.0762$ & $1.792 \times 1.792^{f}$ & 0.0 & $9-15 \times 15$ & 144.85 & 1156 \\
\hline BW1645T1 & 2.459 & $1.2093^{d}$ & aluminum & $15.24 \times 159.32^{e} \times 0.0762$ & $1.778 \times 1.945^{8}$ & 0.0 & $25-247^{h}$ & 104.82 & 335 \\
\hline BW1645T2 & 2.459 & $1.2093^{d}$ & aluminum & $15.24 \times 159.32^{e} \times 0.0762$ & $1.778 \times 1.945^{8}$ & 0.0 & $25-247^{h}$ & 143.96 & 435 \\
\hline BW1645T3 & 2.459 & $1.2093^{d}$ & aluminum & $15.24 \times 159.32^{e} \times 0.0762$ & $2.539 \times 2.7098$ & 0.0 & $25-247^{h}$ & 142.54 & 361 \\
\hline BW1645T4 & 2.459 & $1.2093^{d}$ & aluminum & $15.24 \times 159.32^{e} \times 0.0762$ & $3.807 \times 3.976^{8}$ & 0.0 & $25-247^{h}$ & 145.64 & 121 \\
\hline
\end{tabular}

${ }^{a}$ See Ref. 17.

${ }^{b}$ Assumed to be aluminum.

Measured from lower end of pellet column of fuel rods.

Hexagonal lattice.

Minimum height.

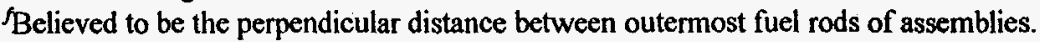

8Perpendicular distance between outermost fuel rods of assemblies, see Fig. 2.27.

Nine rows of 15,8 rows of 14, 4 corner rods are aluminum, see Fig. 2.27. 
Table 2.21 Critical data for separator plate experiments from DSN No. 399/80

\begin{tabular}{|c|c|c|c|c|c|c|c|c|}
\hline Experiment & $\begin{array}{l}\text { Enrichment } \\
\quad(w t \%)\end{array}$ & $\begin{array}{l}\text { Pitch } \\
\text { (cm) }\end{array}$ & $\begin{array}{c}\text { Plate } \\
\text { material }^{b}\end{array}$ & $\begin{array}{c}\text { Plate } \\
\text { dimensions } \\
\text { (cm) }\end{array}$ & $\begin{array}{l}\text { Separation } \\
\text { of } \\
\text { assemblies }^{d} \\
(\mathrm{~cm})\end{array}$ & $\begin{array}{l}\text { Plate-to- } \\
\text { assembly } \\
\text { distance } \\
\text { (cm) }\end{array}$ & $\begin{array}{c}\text { No. of } \\
\text { assemblies- } \\
\text { assembly size } \\
\text { (No. of rods) }\end{array}$ & $\begin{array}{l}\text { Moderator } \\
\text { height, }{ }^{d} \mathrm{H}_{\mathrm{c}} \\
\text { (cm) }\end{array}$ \\
\hline DSN399-1 & 4.742 & 1.35 & hafnium & $100 \times 100 \times 0.1046$ & 1.8 & $0.9^{e}$ & $4-18 \times 18$ & 37.66 \\
\hline DSN399-2 & 4.742 & 1.35 & hafnium & $100 \times 100 \times 0.1046$ & 5.8 & $2.9^{e}$ & $4-18 \times 18$ & 53.40 \\
\hline DSN399-3 & 4.742 & 1.60 & hafnium & $50 \times 100 \times 0.1046$ & - & $0.8^{f}$ & $1-21 \times 21$ & 49.99 \\
\hline DSN399-4 & 4.742 & 1.60 & hafnium & $50 \times 100 \times 0.1046$ & _- & $2.4^{5}$ & $1-20 \times 20$ & 52.83 \\
\hline
\end{tabular}

${ }^{a}$ See Ref. 18.

${ }^{b}$ Density of $13.29 \mathrm{~g} / \mathrm{cm}^{3}$.

${ }^{c}$ Believed to be the perpendicular distance between cell boundaries of assemblies.

'Measured from lower end of fuel rod pellet column.

'Believed to be the perpendicular distance between cell boundaries of assemblies and center of plate.

Perpendicular distance between cell boundary of assembly and near surface of plate. 
Table 2.22 Description of separator plates from PNL-2438

\begin{tabular}{|c|c|c|c|c|c|c|c|}
\hline \multirow[t]{2}{*}{ Element } & \multicolumn{7}{|c|}{ Separator plate, wt \% } \\
\hline & $\begin{array}{l}\text { Boral-A, Boral-B, } \\
\text { Boral-C } \\
\end{array}$ & $\mathrm{Cu}-\mathrm{Cd}, \mathrm{Cu}$ & $\mathrm{Al}$ & $\mathrm{Zr}-4$ & $\begin{array}{l}\text { Steel: } 0.0 \mathrm{wt} \% \mathrm{~B}, \\
1.1 \mathrm{wt} \% \mathrm{~B}, 1.6 \mathrm{wt} \% \mathrm{~B}\end{array}$ & $\mathrm{Cd}$ & Boroflex \\
\hline $\mathrm{Al}$ & $62.39,61.21,59.26$ & & 97.15 & & & & \\
\hline B & $28.70,30.36,31.88$ & $0.005,0.0$ & & & $0.0,1.05,1.62$ & & 32.74 \\
\hline $\begin{array}{l}\mathrm{C} \\
\mathrm{H}\end{array}$ & $7.97,8.43,8.86$ & $0.002,0.340$ & & & & & $\begin{array}{l}21.13 \\
2.65\end{array}$ \\
\hline $\mathrm{Cd}$ & & $0.989,0.0$ & & & & 99.7 & \\
\hline $\mathrm{Cr}$ & $0.05,0.0,0.0$ & & 0.21 & 0.13 & $18.56,19.03,19.60$ & & 0.03 \\
\hline $\mathrm{Cu}$ & $0.09,0.0,0.0$ & $98.685,99.60$ & 0.12 & & $0.27,0.28,0.26$ & & \\
\hline $\mathrm{Fe}$ & $0.33,0.02,0.05$ & $0.020,0.004$ & 0.82 & 0.21 & $68.24,68.04,66.40$ & & 0.05 \\
\hline $\mathrm{Mg}$ & $0.05,0.01,0.01$ & $0.0,0.002$ & & & & & \\
\hline $\mathrm{Mn}$ & $0.05,0.0,0.0$ & $0.009,0.0$ & 0.21 & & $1.58,1.58,1.69$ & & \\
\hline Mo & & & & & $0.26,0.49,0.31$ & & \\
\hline $\mathrm{Na}$ & $0.02,0.02,0.02$ & $0.0,0.002$ & & & & & \\
\hline $\mathrm{Ni}$ & $0.02,0.0,0.0$ & $0.01,0.0$ & & & $11.09,9.53,10.12$ & & \\
\hline $\mathrm{O}$ & & $0.019,0.03$ & & & & & 21.01 \\
\hline $\mathrm{Si}$ & $0.2,0.0,0.06$ & $0.004,0.02$ & 0.82 & & & & 22.39 \\
\hline $\mathrm{Sn}$ & & $0.25,0.0$ & & 1.5 & & & \\
\hline $\mathrm{S}$ & $0.03,0.0,0.0$ & $0.0,0.002$ & 0.06 & & & & \\
\hline $\mathrm{Ti}$ & & & 0.61 & & & & \\
\hline $\mathrm{Zn}$ & $0.10,0.0,0.0$ & $0.007,0.0$ & & & & 0.3 & \\
\hline $\begin{array}{l}\mathrm{Zr} \\
\text { Density } \mathrm{g} / \mathrm{cm}\end{array}$ & & & & $\begin{array}{l}98.16 \\
6.32\end{array}$ & 703070007770 & & \\
\hline Density, g/cm & $2.49,2.50,2.47$ & $8.910,8.913$ & 2.692 & 6.32 & $7.930,7.900,7.770$ & 8.650 & 1.731 \\
\hline Thickness, cm & $\begin{array}{l}0.713^{\mathrm{c}}, 0.292^{\mathrm{d}} \\
0.231^{\mathrm{e}}\end{array}$ & $\begin{array}{l}0.357,0.646 \text { or } \\
0.337^{f}\end{array}$ & 0.625 & 0.652 & $\begin{array}{l}0.485 \text { or } 0.302, \\
0.298,0.298\end{array}$ & 0.061 & $0.546,{ }^{8} 0.772^{8}$ \\
\hline \multicolumn{8}{|c|}{$\begin{array}{l}\text { "See Ref. } 9 . \\
\text { "Separator plates are } 91.5 \mathrm{~cm} \text { long by } 35.6 \mathrm{~cm} \text { wide. } \\
\text { 'Includes } 0.102-\mathrm{cm} \text {-thick aluminum on sides of Boral-A. } \\
\text { dIncludes } 0.038-\mathrm{cm} \text {-thick aluminum on sides of Boral-B. } \\
\text { 'Includes } 0.025-\mathrm{cm} \text {-thick aluminum on sides of Boral-C. } \\
\text { fSeparator plate is } 30.6 \mathrm{~cm} \text { wide. } \\
\text { BIncludes } 0.160-\mathrm{cm} \text {-thick Plexiglas on sides of Boroflex. }\end{array}$} \\
\hline
\end{tabular}


Table 2.23 Critical data for separator plate experiments from PNL-2438 ${ }^{a}$

\begin{tabular}{|c|c|c|c|c|c|c|c|}
\hline Experiment & $\begin{array}{l}\text { Enrichment } \\
\text { (wt \%) }\end{array}$ & $\begin{array}{l}\text { Pitch } \\
(\mathrm{cm})\end{array}$ & $\begin{array}{l}\text { Plate } \\
\text { material }\end{array}$ & $\begin{array}{c}\text { Plate } \\
\text { thickness } \\
\text { (cm) }\end{array}$ & $\begin{array}{l}\text { Separation of } \\
\text { assemblies, }{ }^{b} X_{c} \\
(\mathrm{~cm})\end{array}$ & $\begin{array}{l}\text { Plate-to-assembly } \\
\text { distance, }{ }^{c} \mathrm{G} \\
(\mathrm{cm})\end{array}$ & $\begin{array}{c}\text { No. of } \\
\text { assemblies- } \\
\text { assembly size } \\
\text { (No. of rods) }\end{array}$ \\
\hline P2438AL & 2.35 & 2.032 & aluminum & 0.625 & 8.67 & 0.645 & $3-20 \times 16$ \\
\hline P2438BA & 2.35 & 2.032 & Boral-A & 0.713 & 5.05 & 0.645 & $2-22 \times 16 ; 1-20 \times 16^{d}$ \\
\hline P2438CU & 2.35 & 2.032 & copper & 0.646 & 6.62 & 0.645 & $3-20 \times 16$ \\
\hline P2438SS & 2.35 & 2.032 & stainless steel & 0.485 & 6.88 & 0.645 & $3.20 \times 16$ \\
\hline P2438ZR & 2.35 & 2.032 & Zircaloy-4 & 0.652 & 8.79 & 0.645 & $3-20 \times 16$ \\
\hline
\end{tabular}

aSee Ref. 9.

${ }^{b}$ Perpendicular distance between cell boundaries of assemblies.

Perpendicular distance between cell boundary of center assembly and near surface of plate.

${ }^{d}$ Center assembly. 
Table 2.24 Critical data for separator plate experiments from PNL-2615 ${ }^{a}$

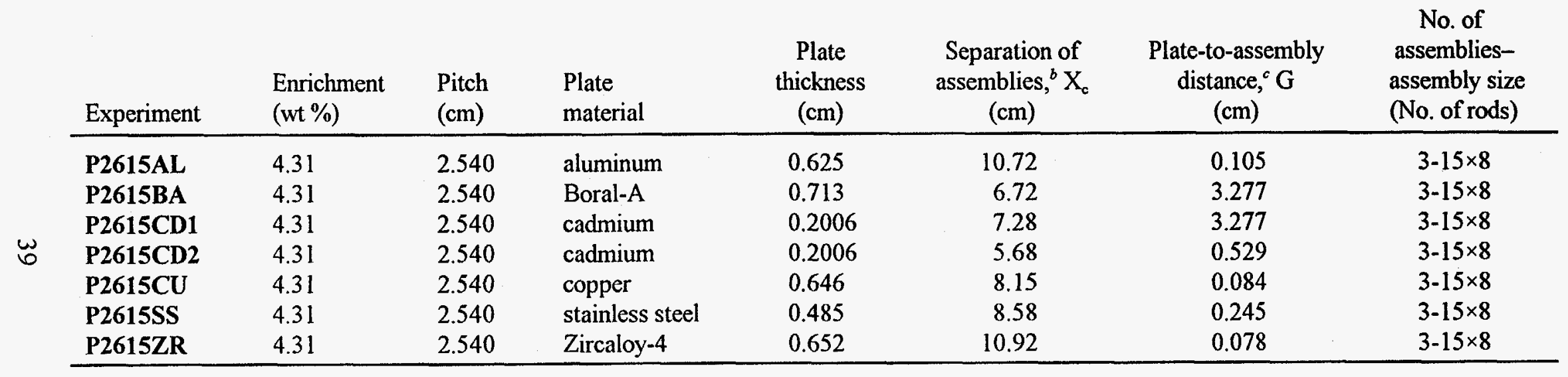

${ }^{a}$ See Ref. 19.

${ }^{b}$ Perpendicular distance between cell boundaries of assemblies.

'Perpendicular distance between cell boundary of center assembly and near surface of plate. 
Table 2.25 Criticality data for separator plate experiments from PNL-3314

\begin{tabular}{|c|c|c|c|c|c|c|}
\hline Experiment & $\begin{array}{c}\text { Enrichment } \\
(w t \%)\end{array}$ & $\begin{array}{l}\text { Pitch } \\
\text { (cm) }\end{array}$ & Plate material & $\begin{array}{l}\text { Plate thickness } \\
\text { (cm) }\end{array}$ & $\begin{array}{c}\text { Separation of assemblies, } \\
\mathrm{X}_{c} ; \mathrm{Y}_{c}(\mathrm{~cm})\end{array}$ & $\begin{array}{l}\text { No. of assemblies- } \\
\text { assembly size } \\
\text { (no. of rods) }\end{array}$ \\
\hline P3314CU5 & 2.35 & 1.684 & Copper & 0.337 & 5.24 & $2-20 \times 18 ; 1-25 \times 18^{c}$ \\
\hline P3314CU6 & 2.35 & 1.684 & Copper-Cd & 0.357 & 2.60 & $2-20 \times 18 ; 1-25 \times 18^{c}$ \\
\hline P3314CD2 & 2.35 & 1.684 & Cadmium & 0.061 & 3.04 & $2-17 \times 20 ; 1-25 \times 20^{c}$ \\
\hline P3314SS5 & 2.35 & 1.684 & Steel, 0.0 wt \% B & 0.302 & 7.80 & $2-17 \times 20 ; 1-25 \times 20^{\circ}$ \\
\hline P3314BS1 & 2.35 & 1.684 & Steel, 1.1 wt \% B & 0.298 & 3.86 & $2-17 \times 20 ; 1-25 \times 20^{\circ}$ \\
\hline P3314BS2 & 2.35 & 1.684 & Steel, 1.6 wt \% B & 0.298 & 3.46 & $2-17 \times 20 ; 1-25 \times 20^{c}$ \\
\hline P3314CU3 & 4.31 & 1.892 & Copper & 0.337 & 10.36 & $3-12 \times 16$ \\
\hline P3314CU4 & 4.31 & 1.892 & Copper-Cd & 0.357 & 7.61 & $3.12 \times 16$ \\
\hline P3314SS6 & 4.31 & 1.892 & Steel, 0.0 wt \% B & 0.302 & 10.52 & $3-12 \times 16$ \\
\hline P3314BS3 & 4.31 & 1.892 & Steel, 1.1 wt \% B & 0.298 & 7.23 & $3-12 \times 16$ \\
\hline P3314BS4 & 4.31 & 1.892 & Steel, 1.6 wt \% B & 0.298 & 6.63 & $3-12 \times 16$ \\
\hline P3314CU1 & 4.31 & 1.892 & Copper & 0.337 & $2.83,5.94$ & $2-9 \times 12 ; 2-9 \times 7$ \\
\hline P3314CU2 & 4.31 & 1.892 & Copper & 0.646 & $2.83,2.67$ & $2-9 \times 12 ; 2-9 \times 5$ \\
\hline P3314CD1 & 4.31 & 1.892 & Cadmium & 0.061 & $2.83,5.30$ & $2-11 \times 14 ; 2-11 \times 13$ \\
\hline P3315SS1 & 4.31 & 1.892 & Steel, 0.0 wt \% B & 0.302 & $2.83,3.38$ & $2-9 \times 12 ; 2-9 \times 2$ \\
\hline P3314SS2 & 4.31 & 1.892 & Steel, 0.0 wt $\%$ B & 0.302 & $2.83,11.55$ & $2-9 \times 12 ; 2-9 \times 13$ \\
\hline P3314SS3 & 4.31 & 1.892 & Steel, 0.0 wt $\%$ B & 0.485 & $2.83,4.47$ & $2-9 \times 12 ; 2-9 \times 5$ \\
\hline P3314SS4 & 4.31 & 1.892 & Steel, 0.0 wt $\%$ B & 0.485 & $2.83,8.36$ & $2-9 \times 12 ; 2-9 \times 12$ \\
\hline P3314AL & 4.31 & 1.892 & Aluminum & 0.625 & $2.83,9.04$ & $2-9 \times 12 ; 2-9 \times 1$ \\
\hline P3314ZR & 4.31 & 1.892 & Zircaloy-4 & 0.652 & $2.83,11.04$ & $2-9 \times 12 ; 2-9 \times 1$ \\
\hline P3314BF1 & 4.31 & 1.892 & Boroflex & 0.546 & $2.83,3.60$ & $2-11 \times 14 ; 2-11 \times 14$ \\
\hline P3314BF2 & 4.31 & 1.892 & Boroflex & 0.772 & $2.83,4.94$ & $2-11 \times 14 ; 2-11 \times 16$ \\
\hline P3314BC & 4.31 & 1.892 & Boral-C & 0.231 & $2.83,3.53$ & $2-11 \times 14 ; 2-11 \times 14$ \\
\hline P3314BA & 4.31 & 1.892 & Boral-A & 0.713 & $2.83,4.80$ & $2-11 \times 14 ; 2 \times 11 \times 16$ \\
\hline
\end{tabular}

aSee Ref. 11.

'Perpendicular distance between cell boundaries of assemblies.

${ }^{c}$ Center assembly. 
Table 2.26 Description of Boral separator plates in experiments from PNL-6205 and PNL-7167 ${ }^{a}$ Separator plate, wt \%

\begin{tabular}{lll}
\cline { 2 - 3 } Element & PNL-6205 & PNL-7167 \\
\hline Al & 54.33 & 62.54 \\
$\mathrm{~B}$ & 35.63 & 29.22 \\
$\mathrm{C}$ & 9.95 & 8.16 \\
$\mathrm{O}$ & 0.07 & 0.06 \\
$\mathrm{Fe}$ & 0.02 & 0.02 \\
Core density, g/cm & 2.64 & 2.64 \\
Core thickness, cm & $0.683^{b}$ & $0.673^{b}$ \\
Length, cm & 96 & 96 \\
Width, cm & 45 & 45 \\
\hline
\end{tabular}

${ }^{a}$ See Refs. 21 and 22.

${ }^{b}$ Includes two 0.1015 -cm-thick aluminum side plates. 
Table 2.27 Critical data for separator plate experiments from PNL-6205 and PNL-7167 ${ }^{a}$

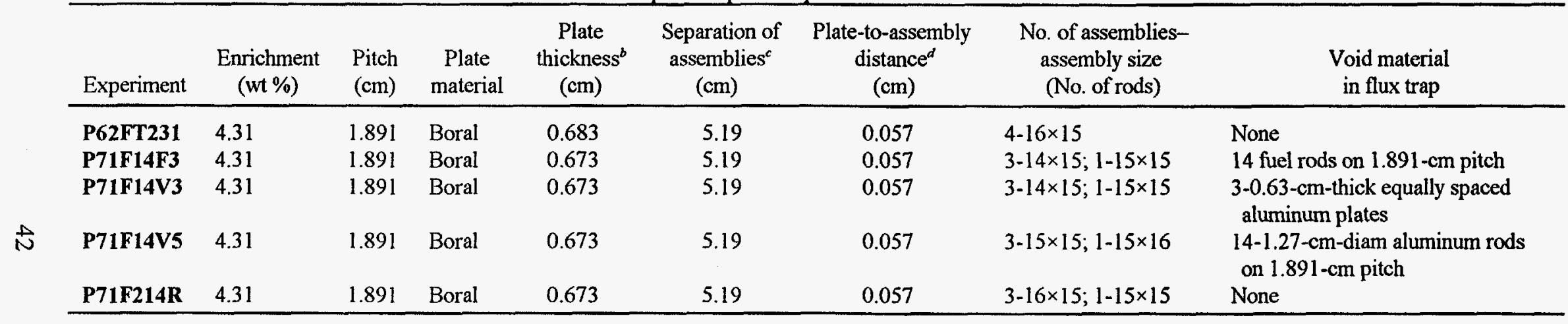

${ }^{a}$ See Refs. 20 and 21

${ }^{b}$ Includes two 0.1015 -cm-thick aluminum side plates.

cPerpendicular distance between cell boundaries of assemblies.

aperpendicular distance between cell boundaries of assemblies and near surface of plate. 
Table 2.28 Modeled assemblies of PNL-6205 and PNL-7167 separator plate experiments

\begin{tabular}{rlllllll} 
& & & & \multicolumn{3}{c}{ Modeled assemblies (No. rows $\times$ fuel rods per row) } \\
\cline { 6 - 8 } & $\begin{array}{l}\text { No. of rods, } \\
\text { experiment }\end{array}$ & $\begin{array}{l}\text { No. of rods, delayed } \\
\text { critical (predicted) }\end{array}$ & $\begin{array}{l}\text { No. of rods } \\
\text { modeled }\end{array}$ & Top left & Top right & Bottom left & Bottom right \\
\hline \multirow{\omega}{*}{ P62FT231 } & 960 & 963 & 963 & $15 \times 16$ & $15 \times 16$ & $15 \times 16$ & $12 \times 16,3 \times 17^{a}$ \\
P71F14F3 & 855 & 858.5 & 858 & $12 \times 14,3 \times 15^{a}$ & $15 \times 14$ & $15 \times 15$ & $15 \times 14$ \\
P71F14V3 & 855 & 862.1 & 862 & $15 \times 14$ & $15 \times 15$ & $15 \times 14$ & $8 \times 14,7 \times 15^{a}$ \\
P71F14V5 & 915 & 924.9 & 925 & $1 \times 10^{b}, 15 \times 15^{a}$ & $16 \times 15$ & $15 \times 15$ & $15 \times 15$ \\
P71F214R & 945 & 952 & 952 & $15 \times 16$ & $15 \times 16$ & $15 \times 16$ & $7 \times 16,8 \times 15^{a}$ \\
\hline
\end{tabular}

${ }^{a}$ Bottom rows.

${ }^{b}$ Aligned against right assembly boundary. 
Table 2.29 Critical data for reflecting wall experiments from PNL-2827

\begin{tabular}{|c|c|c|c|c|c|c|}
\hline Experiment & $\begin{array}{c}\text { Enrichment } \\
\text { (wt \%) }\end{array}$ & $\begin{array}{l}\text { Pitch } \\
(\mathrm{cm})\end{array}$ & $\begin{array}{c}\text { Reflector } \\
\text { material }\end{array}$ & $\begin{array}{l}\text { Separation of } \\
\text { assemblies, }^{b} \\
\mathrm{X}_{\mathrm{c}}(\mathrm{cm})\end{array}$ & $\begin{array}{l}\text { Wall-to-assembly } \\
\text { distance, }{ }^{c} Y(\mathrm{~cm})\end{array}$ & $\begin{array}{l}\text { No. of assemblies- } \\
\text { assembly size } \\
\text { (No. of rods) }\end{array}$ \\
\hline P2827L1 & 2.35 & 2.032 & lead & 13.27 & 0.66 & $3-19 \times 16$ \\
\hline P2827L2 & 2.35 & 2.032 & lead & 11.25 & 2.616 & $3-19 \times 16$ \\
\hline P2827L3 & 4.31 & 2.540 & lead & 20.78 & 0.66 & $3-13 \times 8$ \\
\hline P2827L4 & 4.31 & 2.540 & lead & 19.04 & 1.321 & $3-13 \times 8$ \\
\hline P2827U1 & 2.35 & 2.032 & depleted uranium & 11.83 & 0.0 & $3-19 \times 16$ \\
\hline P2827U2 & 2.35 & 2.032 & depleted uranium & 14.11 & 1.956 & $3-19 \times 16$ \\
\hline P2827U3 & 4.31 & 2.540 & depleted uranium & 15.38 & 0.0 & $3-13 \times 8$ \\
\hline P2827U4 & 4.31 & 2.540 & depleted uranium & 15.32 & 1.956 & $3-13 \times 8$ \\
\hline
\end{tabular}

${ }^{a}$ See Ref. 10.

${ }^{b}$ Perpendicular distance from outer rod surface to outer rod surface of assemblies.

' Perpendicular distance between cell boundaries of assemblies and near surface of reflecting walls. 
Table 2.30 Critical data for reflecting wall experiments from PNL-3602 ${ }^{a}$

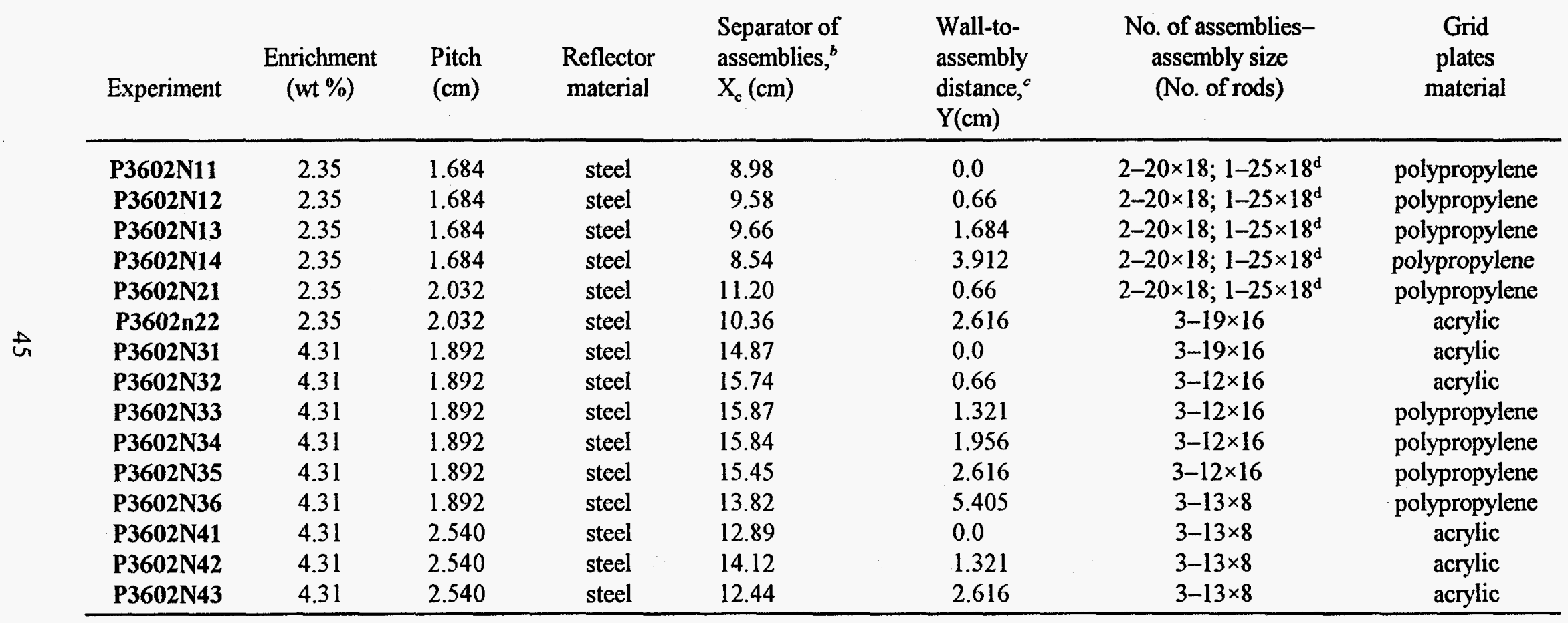

a See Ref. 22.

${ }^{b}$ Perpendicular distance from outer rod surface to outer rod surface of assemblies.

c Perpendicular distance between cell boundary of assembly and near surface of reflecting wall.

${ }^{d}$ Center assembly. 
Table 2.31 Critical data for reflecting wall experiments from PNL-3926 ${ }^{a}$

\begin{tabular}{lcccccc}
\hline Experiment & $\begin{array}{c}\text { Enrichment } \\
(\text { wt \%) }\end{array}$ & $\begin{array}{c}\text { Pitch } \\
(\mathrm{cm})\end{array}$ & $\begin{array}{c}\text { Reflector } \\
\text { material }\end{array}$ & $\begin{array}{c}\text { Separation of } \\
\text { assemblies, } \\
\mathrm{X}_{c}(\mathrm{~cm})\end{array}$ & $\begin{array}{c}\text { Wall-to- } \\
\text { assembly } \\
\left.\text { distance, }{ }^{c} \mathrm{Ycm}\right)\end{array}$ & $\begin{array}{c}\text { No. of assemblies- } \\
\text { assembly size } \\
\text { (No. of rocis) }\end{array}$ \\
\hline P3926L1 & 2.35 & 1.684 & lead & 10.06 & 0.0 & $2-20 \times 18 ; 1-23 \times 18^{\mathrm{d}}$ \\
P3926L2 & 2.35 & 1.684 & lead & 10.11 & 0.66 & $2-20 \times 18 ; 1-23 \times 18^{\mathrm{d}}$ \\
P3926L3 & 2.35 & 1.684 & lead & 8.50 & 3.276 & $2-20 \times 18 ; 1-23 \times 18^{\mathrm{d}}$ \\
P3926L4 & 4.31 & 1.892 & lead & 17.74 & 0.0 & $3-12 \times 16$ \\
P3926L5 & 4.31 & 1.892 & lead & 18.18 & 0.66 & $3-12 \times 16$ \\
क3926L6 & 4.31 & 1.892 & lead & 17.43 & 1.956 & $3-12 \times 16$ \\
P3926U1 & 2.35 & 1.684 & depleted uranium & 8.06 & 0.0 & $2-20 \times 18 ; 1-23 \times 18^{\text {d }}$ \\
P3926U2 & 2.35 & 1.684 & depleted uranium & 9.50 & 1.321 & $2-20 \times 18 ; 1-23 \times 18^{\text {d }}$ \\
P3926U3 & 2.35 & 1.684 & depleted uranium & 9.19 & 3.912 & $2-20 \times 18 ; 1-23 \times 18^{\text {d }}$ \\
P3926U4 & 4.31 & 1.892 & depleted uranium & 15.33 & 0.0 & $3-12 \times 16$ \\
P3926U5 & 4.31 & 1.892 & depleted uranium & 19.24 & 1.956 & $3-12 \times 16$ \\
P3926U6 & 4.31 & 1.892 & depleted uranium & 18.78 & 3.276 & $3-12 \times 16$ \\
\hline
\end{tabular}

${ }^{a}$ See Ref. 12.

${ }^{b}$ Perpendicular distance from outer rod surface to outer rod surface of assemblies.

c Perpendicular distance between cell boundary of assemblies and near surface of reflecting walls.

${ }^{d}$ Center assembly. 
Table 2.32 Critical data for reflecting wall-separator plate experiments from PNL-3602 ${ }^{a}$

\begin{tabular}{|c|c|c|c|c|c|c|c|c|}
\hline Experiment & $\begin{array}{c}\text { Enrichment } \\
\text { (wt \%) }\end{array}$ & $\begin{array}{l}\text { Pitch } \\
\text { (cm) }\end{array}$ & Plate material & $\begin{array}{l}\text { Plate } \\
\text { thickness } \\
\text { (cm) }\end{array}$ & $\begin{array}{l}\text { Reflecting } \\
\text { wall } \\
\text { material }\end{array}$ & $\begin{array}{l}\text { Separation of } \\
\text { assemblies, } \\
X_{c}(\mathrm{~cm})\end{array}$ & $\begin{array}{c}\text { Wall-to-assembly } \\
\text { distance, }{ }^{c} Y \\
\text { (cm) }\end{array}$ & $\begin{array}{c}\text { No. of assemblies- } \\
\text { assembly size } \\
\text { (No. of rods) }\end{array}$ \\
\hline P3602BB & 4.31 & 1.892 & Boral-B & 0.292 & steel & 8.30 & 1.956 & $3-12 \times 16$ \\
\hline P3602BS1 & 2.35 & 1.684 & $1.1 \mathrm{wt} \% \mathrm{~B}$ steel & 0.298 & steel & 4.80 & 1.321 & $2-20 \times 18 ; 1-25 \times 18^{d}$ \\
\hline P3602BS2 & 4.31 & 1.892 & $1.1 \mathrm{wt} \% \mathrm{~B}$ steel & 0.298 & steel & 9.83 & 1.956 & $3-12 \times 16$ \\
\hline P3602CD1 & 2.35 & 1.684 & cadmium & 0.061 & steel & 3.86 & 1.321 & $2-20 \times 18 ; 1-25 \times 18^{d}$ \\
\hline P3602CD2 & 4.31 & 1.892 & cadmium & 0.061 & steel & 8.94 & 1.956 & $3-12 \times 16$ \\
\hline P3602CU1 & 2.35 & 1.684 & copper & 0.337 & steel & 7.79 & 1.321 & $2-20 \times 18 ; 1-25 \times 18^{d}$ \\
\hline P3602CU2 & 2.35 & 1.684 & $\begin{array}{l}99 \text { wt } \% \text { copper- } \\
1 \text { wt } \% \text { cadmium }\end{array}$ & 0.357 & steel & 5.43 & 1.321 & $2-20 \times 18 ; 1-25 \times 18^{d}$ \\
\hline P3602CU3 & 4.31 & 1.892 & copper & 0.337 & steel & 13.47 & 1.956 & $3-12 \times 16$ \\
\hline P3602CU4 & 4.31 & 1.892 & $\begin{array}{l}99 \text { wt } \% \text { copper- } \\
1 \text { wt } \% \text { cadmium }\end{array}$ & 0.357 & steel & 10.57 & 1.956 & $3-12 \times 16$ \\
\hline P3602SS1 & 2.35 & 1.684 & steel & 0.302 & steel & 8.28 & 1.321 & $2-20 \times 18 ; 1-25 \times 18^{d}$ \\
\hline P3602SS2 & 4.31 & 1.892 & steel & 0.302 & steel & 13.75 & 1.956 & $3-12 \times 16$ \\
\hline
\end{tabular}

${ }^{a}$ See Ref. 22.

${ }^{b}$ Perpendicular distance from outer rod surface to outer rod surface of assemblies.

' Perpendicular distance between cell boundary of assemblies and near surface of reflecting walls.

${ }^{d}$ Center assembly. 
Table 2.33 Critical data for reflecting wall-separator plate experiments ${ }^{a}$

\begin{tabular}{|c|c|c|c|c|c|c|c|c|c|c|c|}
\hline Experiment & $\begin{array}{c}\text { Enrichment } \\
(\mathbf{w t} \%)\end{array}$ & $\begin{array}{l}\text { Pitch } \\
(\mathrm{cm})\end{array}$ & $\begin{array}{c}\text { Plate } \\
\text { material }^{b}\end{array}$ & $\begin{array}{l}\text { Plate } \\
\text { thickness } \\
(\mathrm{cm})\end{array}$ & $\begin{array}{c}\text { Reflector } \\
\text { material }\end{array}$ & $\begin{array}{c}\text { Reflector } \\
\text { thickness, } \\
\text { E (cm) }\end{array}$ & $\begin{array}{l}\text { Separation } \\
\text { of } \\
\text { assemblies }^{d} \\
(\mathrm{~cm})\end{array}$ & $\begin{array}{l}\text { Plate-to- } \\
\text { assembly } \\
\text { distance } \\
\text { (cm) }\end{array}$ & $\begin{array}{l}\text { Wall-to- } \\
\text { assembly } \\
\text { distance } \\
\text { (cm) }\end{array}$ & $\begin{array}{c}\text { No. of } \\
\text { assemblies- } \\
\text { assembly size } \\
\text { (No. of rods) }\end{array}$ & $\begin{array}{l}\text { Moderator } \\
\text { height, }{ }^{h} \mathrm{H}_{\mathrm{c}} \\
(\mathrm{cm})\end{array}$ \\
\hline PAT80L1 & 4.742 & 1.6 & Boral & 0.65 & lead' & 10.0 & 4.9 & 0.8 & 1.68 & $4-18 \times 18$ & 53.98 \\
\hline PAT80L2 & 4.742 & 1.6 & Boral & 0.65 & lead ${ }^{\prime}$ & 10.0 & 4.9 & 0.8 & 3.95 & $4-18 \times 18$ & 63.85 \\
\hline PAT80SS1 & 4.742 & 1.6 & Boral & 0.65 & steel ${ }^{j}$ & 15.0 & 4.9 & 0.8 & 1.68 & $4-18 \times 18$ & 51.55 \\
\hline PAT80SS2 & 4.742 & 1.6 & Boral & 0.65 & steel $f^{\prime}$ & 15.0 & 4.9 & 0.8 & 3.95 & $4-18 \times 18$ & 61.84 \\
\hline
\end{tabular}

${ }^{a}$ See Ref. 23.

${ }^{b}$ Boral $\left(2.6189 \mathrm{~g} / \mathrm{cm}^{3}\right): 71.63 \mathrm{wt} \% \mathrm{Al}, 22.20 \mathrm{wt} \% \mathrm{~B}$, and $6.17 \mathrm{wt} \% \mathrm{C}$.

${ }^{c}$ Includes 0.11 -cm-thick aluminum $\left(2.651 \mathrm{~g} / \mathrm{cm}^{3}\right)$ sides.

${ }^{d}$ Perpendicular distance between the center of outer rods of assemblies.

- Perpendicular distance between the center of outer rods of assemblies and near surface of plates.

$\delta^{\prime}$ Perpendicular distance between the center of outer rods of assemblies and near surface of reflecting walls.

8 Includes four 1.0 -cm-diam stainless steel corner rods.

${ }^{h}$ Measured from lower end of fuel rod pellet column.

'Lead $\left(11.34 \mathrm{~g} / \mathrm{cm}^{3}\right)$.

${ }^{\prime}$ Steel $\left(7.8 \mathrm{~g} / \mathrm{cm}^{3}\right): 99.66 \mathrm{wt} \% \mathrm{Fe}, 0.20 \mathrm{wt} \% \mathrm{Si}$, and $0.14 \mathrm{wt} \% \mathrm{C}$. 
Table 2.34 Physical properties of $\mathrm{UO}_{2}$ fuel rods from BAW-1810

\begin{tabular}{ll}
\hline Parameter & Value \\
\hline Outside diameter, cm & 1.2078 \\
Wall thickness, cm & 0.0406 \\
Wall material & 304 stainless steel \\
Fuel diameter, cm & 1.128 \\
Total length, cm & $181.6^{\mathrm{b}}$ \\
Active fuel length, cm & 169.4 \\
Enrichment, $235 \mathrm{U} / \mathrm{U}, \mathrm{wt} \%$ & 4.020 \\
Fuel density, $\mathrm{g} / \mathrm{cm}^{3}$ & 9.46 \\
\hline
\end{tabular}

${ }^{\text {aS }}$ ee Ref. 24.

bIncludes approximately $5.967-\mathrm{cm}$-long stainless steel end plugs.

Table 2.35 Physical properties of $\mathrm{UO}_{2}-\mathrm{Gd}_{2} \mathrm{O}_{3}$ fuel rods from BAW-1810

\begin{tabular}{ll}
\hline Parameter & Value \\
\hline Outside diameter, cm & 1.207 \\
Wall thickness, cm & 0.081 \\
Wall material & aluminum \\
Fuel pellet diameter, cm & 1.030 \\
Total length, cm & $160.02^{\mathrm{b}}$ \\
Active fuel length, cm & 153.42 \\
Enrichment, ${ }^{23 \mathrm{U} / \mathrm{U}, \mathrm{wt} \%}$ & 1.944 \\
$\mathrm{Gadolinia}, \mathrm{wt} \%$ & 4.00 \\
$\mathrm{UO} \mathrm{O}_{2}$ density, $\mathrm{g} / \mathrm{cm}^{3}$ & 10.24 \\
$\mathrm{Gd} \mathrm{O}_{3}$ density, $\mathrm{g} / \mathrm{cm}^{3}$ & 0.410 \\
$\mathrm{Gd}$ density, $\mathrm{g} / \mathrm{cm}^{3}$ & 0.356 \\
$\mathrm{O}$ density, $\mathrm{g} / \mathrm{cm}^{3}$ & 0.054 \\
\hline
\end{tabular}

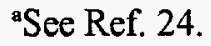

'Includes $0.3175-\mathrm{cm}-$ long aluminum end plugs and $5.97 \mathrm{~cm}$ of void above the 153.42-cm-long pellet stack (active fuel). 
Table 2.36 Critical data for $\mathrm{UO}_{2}-\mathrm{Gd}_{2} \mathrm{O}_{3}$ rod experiments from BAW-1810 ${ }^{a}$

\begin{tabular}{|c|c|c|c|c|c|c|c|c|}
\hline Experiment & $\begin{array}{c}\text { Enrichment } \\
\text { (wt \%) }\end{array}$ & $\begin{array}{l}\text { Pitch } \\
(\mathrm{cm}) \\
\end{array}$ & $\begin{array}{c}\text { No. of } \\
2.459 \text { wt } \% \\
{ }^{235} \mathrm{U} \text { rods }\end{array}$ & $\begin{array}{c}\text { No. of } \\
4.020 \text { wt \% } \\
{ }^{235} \mathrm{U} \text { rods }\end{array}$ & $\begin{array}{c}\text { No. of } \\
\mathrm{UO}_{2}-\mathrm{Gd}_{2} \mathrm{O}_{3} \\
\text { rods }\end{array}$ & $\begin{array}{l}\text { No. of } \\
\text { water } \\
\text { holes }\end{array}$ & $\begin{array}{c}\text { Moderator } \\
\text { height, }^{b} \\
\mathrm{H}(\mathrm{cm})\end{array}$ & $\begin{array}{c}\text { Boron } \\
\text { concentration } \\
(\mathrm{ppm})\end{array}$ \\
\hline BW1810A & 2.459 & 1.636 & 4788 & 0 & 20 & 153 & 145.0 & 1239.3 \\
\hline BW1810B & 2.459 & 1.636 & 4772 & 0 & 36 & 153 & 145.0 & 1170.7 \\
\hline BW1810C & 2.459 and 4.020 & 1.636 & 3676 & $912^{\circ}$ & 32 & 180 & 145.0 & 1499.0 \\
\hline BW1810D & 2.459 and 4.020 & 1.636 & 3920 & $860^{d}$ & 28 & 153 & 145.0 & 1653.8 \\
\hline BW1810E & 2.459 and 4.020 & 1.636 & 3920 & $852^{\circ}$ & 36 & 153 & 145.0 & 1579.4 \\
\hline
\end{tabular}

"See Ref. 24.

'Measured from the top of the 5.08-cm-thick aluminum base plate.

'Inner $32 \times 32$ lattice positions including 80 water holes and $32 \mathrm{Gd}_{2} \mathrm{O}_{3}$ rods.

Inner $31 \times 31$ lattice positions including 73 water holes and $28 \mathrm{Gd}_{2} \mathrm{O}_{3}$ rods

Inner $31 \times 31$ lattice positions including 73 water holes and $36 \mathrm{Gd}_{2} \mathrm{O}_{3}$ rods. 
Table 2.37 Critical data for water hole experiments from BAW-1810 ${ }^{\mathrm{a}}$

\begin{tabular}{|c|c|c|c|c|c|c|c|}
\hline Experiment & $\begin{array}{c}\text { Enrichment } \\
(\text { wt \%) }\end{array}$ & $\begin{array}{l}\text { Pitch } \\
(\mathrm{cm})\end{array}$ & $\begin{array}{c}\text { No. of } \\
2.459 \text { wt } \% \\
{ }^{235} \mathrm{U} \text { rods }\end{array}$ & $\begin{array}{c}\text { No. of } \\
4.020 \text { wt } \% \\
{ }^{235} \mathrm{U} \text { rods }\end{array}$ & $\begin{array}{l}\text { No. of } \\
\text { water } \\
\text { holes }\end{array}$ & $\begin{array}{c}\text { Moderator } \\
\text { height, } \\
\mathrm{H}(\mathrm{cm})\end{array}$ & $\begin{array}{c}\text { Boron } \\
\text { concentration } \\
(\mathrm{ppm})\end{array}$ \\
\hline BW1810F & 2.459 & 1.636 & 4808 & 0 & 153 & 145.0 & 1337.9 \\
\hline BW1810G & 2.459 and 4.020 & 1.636 & 3676 & $944^{c}$ & 180 & 145.0 & 1776.8 \\
\hline BW1810H & 2.459 and 4.020 & 1.636 & 3920 & $888^{d}$ & 153 & 145.0 & 1899.3 \\
\hline
\end{tabular}

See Ref. 24.

'Measured from the top of the 5.08 -cm-thick aluminum base plate.

Inner $32 \times 32$ lattice positions including 80 water holes.

Inner $31 \times 31$ lattice positions including 73 water holes. 
Physical Description

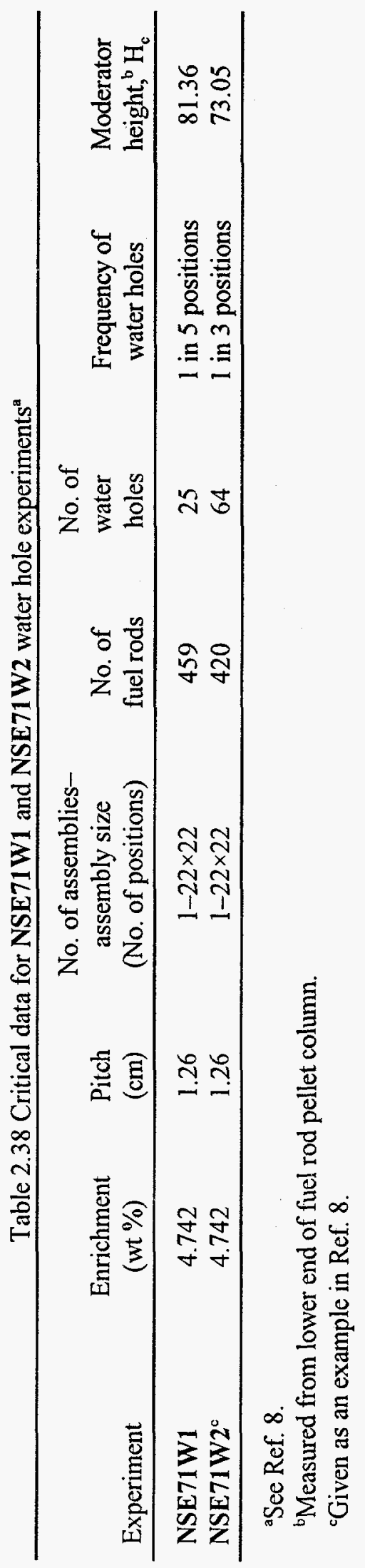


Table 2.39 Critical data for water hole experiments from PNL-3314

\begin{tabular}{|c|c|c|c|c|c|c|}
\hline \multirow[b]{2}{*}{ Experiment } & \multirow{2}{*}{$\begin{array}{l}\text { Enrichment } \\
\text { (wt \%) }\end{array}$} & \multirow{2}{*}{$\begin{array}{l}\text { Pitch } \\
(\mathrm{cm})\end{array}$} & \multirow{2}{*}{$\begin{array}{l}\text { No. of assemblies- } \\
\text { assembly size } \\
\text { (No. of positions) }\end{array}$} & \multicolumn{2}{|c|}{ No. of fuel rods } & \multirow{2}{*}{$\begin{array}{c}\text { No. of } \\
\text { water holes }\end{array}$} \\
\hline & & & & Predicted $^{\mathrm{b}}$ & Modeled & \\
\hline P3314W1 & 4.31 & 1.892 & $1-14 \times 14$ & 167.6 & 168 & 25 \\
\hline P3314W2 & 2.35 & 1.684 & $1-23 \times 23$ & 485.8 & 486 & 25 \\
\hline
\end{tabular}

${ }^{2}$ See Ref. 11.

bobtained by approach-to-critical method.

Table 2.40 Critical data for water hole experiments from WCAP $-3269^{\mathrm{a}}$

\begin{tabular}{lcccccc}
\hline Experiment & $\begin{array}{c}\text { Enrichment } \\
(\mathrm{wt} \mathrm{\% )}\end{array}$ & $\begin{array}{c}\text { Pitch } \\
(\mathrm{cm})\end{array}$ & $\begin{array}{c}\text { No. of assemblies- } \\
\text { assembly size } \\
\text { (No. of positions) }\end{array}$ & $\begin{array}{c}\text { No. of } \\
\text { fuel rods }\end{array}$ & $\begin{array}{c}\text { No. of } \\
\text { water } \\
\text { holes }\end{array}$ & $\begin{array}{c}\text { Moderator } \\
\text { height, }^{\mathrm{b}} \mathrm{H}_{\mathrm{c}}\end{array}$ \\
\hline W3269W1 & 2.72 & 1.524 & $1-31 \times 31$ & 945 & 16 & 37.21 \\
W3269W2 & 5.7 & 1.422 & $1-27 \times 17$ & 453 & 6 & 51.93 \\
\hline
\end{tabular}

'See Ref. 15.

believed to have been measured from the bottom of fuel, see Figs. 2.20 and 2.21 .

Table 2.41 Properties of $\mathrm{B}_{4} \mathrm{C}$ absorber rods from BAW-1484

\begin{tabular}{lc}
\hline Parameter & Value \\
\hline Outside diameter, cm & 1.113 \\
Wall thickness, cm & 0.089 \\
Wall material & aluminum \\
Total length, $\mathrm{cm}$ & $177.5^{\mathrm{b}}$ \\
Active length, $\mathrm{cm}$ & 177.18 \\
$\mathrm{~B}_{4} \mathrm{C}$ powder density, $\mathrm{g} / \mathrm{cm}^{3}$ & 1.28 \\
\hline & \\
\hline aSee Ref. 6. & \\
'blucludes $0.3175-\mathrm{cm}$-long aluminum bottom end plug.
\end{tabular}


Table 2.42 Critical data for $\mathrm{B}_{4} \mathrm{C}$ absorber rod experiments from BAW-1484

\begin{tabular}{|c|c|c|c|c|c|c|}
\hline Experiment & $\begin{array}{c}\text { Enrichment } \\
\quad(w t \%)\end{array}$ & $\begin{array}{l}\text { Pitch } \\
\text { (cm) }\end{array}$ & $\begin{array}{l}\text { Separation of } \\
\text { assemblies }^{\mathrm{b}} \\
(\mathrm{cm})\end{array}$ & $\begin{array}{l}\text { No. of assemblies- } \\
\text { assembly size } \\
\text { (No. of rods) }\end{array}$ & $\begin{array}{l}\text { No. of } \\
\text { absorber } \\
\text { rods }\end{array}$ & $\begin{array}{l}\text { Moderator } \\
\text { height, }{ }^{d} \mathrm{H} \\
\quad(\mathrm{cm})\end{array}$ \\
\hline BW1484C1 & 2.459 & 1.636 & 1.636 & $9-14 \times 14$ & 84 & 145.68 \\
\hline BW1484C2 & 2.459 & 1.636 & 4.908 & $9-14 \times 14$ & 34 & 111.49 \\
\hline
\end{tabular}

${ }^{a}$ See Ref. 6.

believed to be the perpendicular distance between cell boundaries of assemblies.

${ }^{c}$ Four corner rods of assemblies are 0.635 -cm-radius aluminum rods.

${ }^{\mathrm{d}}$ Measured from the top of the aluminum base plate. 
Table 2.43 Properties of Ag-In-Cd absorber rods from BAW-1810

\begin{tabular}{ll}
\hline Parameter & \multicolumn{1}{c}{ Value } \\
\hline Outside diameter, cm & 1.207 \\
Wall thickness, cm & 0.081 \\
Wall material & aluminum \\
Length, cm & $157.56^{\mathrm{b}}$ \\
$\mathrm{Wt} \% \mathrm{Ag}$ & 79.68 \\
$\mathrm{Wt} \% \mathrm{In}$ & 15.09 \\
$\mathrm{Wt} \% \mathrm{Cd}$ & 5.2 \\
$\mathrm{Wt} \% \mathrm{Cu}$ & 0.05 \\
$\mathrm{Wt} \% \mathrm{Zr}$ & 0.02 \\
Density, g/cm & 10.15
\end{tabular}

aSee Ref. 25.

${ }^{b}$ Minus the $0.3175-\mathrm{cm}-$ long aluminum bottom end plug. 
Table 2.44 Critical data for absorber rod experiments from BAW-1810

\begin{tabular}{|c|c|c|c|c|c|c|c|c|c|}
\hline Experiment & $\begin{array}{c}\text { Enrichment } \\
\text { (wt \%) }\end{array}$ & $\begin{array}{l}\text { Pitch } \\
(\mathrm{cm})\end{array}$ & $\begin{array}{l}\text { No. of } \\
2.459 \text { wt } \% \\
{ }^{235} \mathrm{U} \text { rods }\end{array}$ & $\begin{array}{l}\text { No. of } \\
4.020 \text { wt \% } \\
{ }^{235} \mathrm{U} \text { rods }\end{array}$ & $\begin{array}{l}\text { Absorber } \\
\text { rod }\end{array}$ & $\begin{array}{l}\text { No. of } \\
\text { absorber } \\
\text { rods }\end{array}$ & $\begin{array}{l}\text { No. of } \\
\text { water } \\
\text { holes }\end{array}$ & $\begin{array}{c}\text { Moderator } \\
\text { height, } \\
\text { H (cm) }\end{array}$ & $\begin{array}{c}\text { Boron } \\
\text { concentration } \\
(\mathrm{ppm})\end{array}$ \\
\hline BW1810I & 2.459 & 1.636 & 4808 & 0 & $\mathrm{Ag}-\mathrm{In}-\mathrm{Cd}$ & 16 & 137 & 145.0 & 1250.0 \\
\hline BW1810J & 2.459 and 4.020 & 1.636 & 3920 & $888^{\circ}$ & $\mathrm{B}_{4} \mathrm{C}$ & 16 & 137 & 145.0 & 1635.4 \\
\hline
\end{tabular}

"See Ref. 24.

beasured from the top of the 5.08-cm-thick aluminum base plate

'Inner $31 \times 31$ positions include 57 water holes and $16 \mathrm{~B}_{4} \mathrm{C}$ rods. 
Table 2.45 Critical data for Ag-In-Cd absorber rod experiments from WCAP-3269 ${ }^{\mathrm{a}}$

\begin{tabular}{lccccccc}
\hline Experiment & $\begin{array}{c}\text { Enrichment } \\
\text { (wt \%) }\end{array}$ & $\begin{array}{c}\text { Pitch } \\
\text { (cm) }\end{array}$ & $\begin{array}{c}\text { No. of assemblies- } \\
\text { assembly size } \\
\text { (No. of rods) }\end{array}$ & $\begin{array}{c}\text { No. of } \\
\text { fuel rods }\end{array}$ & $\begin{array}{c}\text { No. of } \\
\text { Ag-In-Cd } \\
\text { rods }^{b}\end{array}$ & $\begin{array}{c}\text { Ag-In-Cd } \\
\text { rod diameter } \\
\text { (cm) }\end{array}$ & $\begin{array}{c}\text { Moderator } \\
\text { height } \\
\text { (cm) }\end{array}$ \\
\hline W3269A & 5.7 & 1.422 & $1-27 \times 17$ & 453 & 6 & 1.024 & 88.27 \\
W3269B1 & 3.7 & 1.105 & $1-47 \times 47^{\text {d }}$ & 2221 & 12 & 0.838 & 55.41 \\
W3269B2 & 3.7 & 1.105 & $1-47 \times 47^{\text {d }}$ & 2209 & 24 & 0.838 & 64.56 \\
W3269B3 & 3.7 & 1.105 & $1-47 \times 47^{\text {d }}$ & 2185 & 48 & 0.838 & 57.00 \\
W3269C & 2.72 & 1.524 & $1-31 \times 31$ & 945 & 16 & 1.024 & 89.75 \\
\hline
\end{tabular}

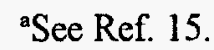

bods taken to be $79.6 \mathrm{wt} \% \mathrm{Ag}, 15.17 \mathrm{wt} \% \mathrm{In}, 5.08 \mathrm{wt} \% \mathrm{Cd}$ with a density of $9.91 \mathrm{~g} / \mathrm{cm}^{3}$.

'Believed to have been measured from the bottom of the fuel, see Figs. 2.20 and 2.21.

Includes 24 fuel follower rods positioned outside the array (see Figs. 2.58, 2.59, and 2.60). 
Table 2.46 Critical data for soluble boron experiments from BAW-1231 ${ }^{\mathrm{a}}$

\begin{tabular}{lccccc}
\hline Experiment & $\begin{array}{c}\text { Enrichment } \\
(\text { wt \%) }\end{array}$ & $\begin{array}{c}\text { Pitch } \\
(\mathrm{cm})\end{array}$ & $\begin{array}{c}\text { No. of } \\
\text { fuel rods }\end{array}$ & $\begin{array}{c}\text { Moderator } \\
\text { height } \\
(\mathrm{cm})\end{array}$ & $\begin{array}{c}\text { Boron } \\
\text { concentration } \\
(\mathrm{ppm})\end{array}$ \\
\hline BW1231B1 & 4.020 & 1.511 & 936 & 167.5 & 1152 \\
BW1231B2 & 4.020 & 1.511 & 4904 & 146.65 & 3389 \\
\hline
\end{tabular}

${ }^{\text {aSee Ref. } 25 .}$

${ }^{b}$ Measured from the bottom of the active fuel or reference plane of Fig. 2.62.

Table 2.47 Critical data for soluble boron experiment BW1273M ${ }^{\mathrm{a}}$

\begin{tabular}{lccccc}
\hline Experiment & $\begin{array}{c}\text { Enrichment } \\
(\text { wt \%) }\end{array}$ & $\begin{array}{c}\text { Pitch } \\
(\mathrm{cm})\end{array}$ & $\begin{array}{c}\text { No. of } \\
\text { fuel rods }\end{array}$ & $\begin{array}{c}\text { Moderator } \\
\text { height, }{ }^{b} \mathrm{H} \\
(\mathrm{cm})\end{array}$ & $\begin{array}{c}\text { Boron } \\
\text { concentration } \\
\text { (ppm) }\end{array}$ \\
\hline BW1273M & 2.459 & 1.511 & 5137 & 93.2 & 1675 \\
\hline
\end{tabular}

${ }^{\text {a See Ref. } 26 .}$

beasured from the bottom of the active fuel or reference plane in Fig. 2.63.

Table 2.48 Critical data for soluble boron experiments from BAW-1484 ${ }^{\mathrm{a}}$

\begin{tabular}{lcccccc}
\hline Experiment & $\begin{array}{c}\text { Enrichment } \\
(\mathrm{wt} \%)\end{array}$ & $\begin{array}{c}\text { Pitch } \\
(\mathrm{cm})\end{array}$ & $\begin{array}{c}\text { Separation of } \\
\text { assemblies }^{\mathbf{b}} \\
(\mathrm{cm})\end{array}$ & $\begin{array}{c}\text { No. of } \\
\text { assemblies- } \\
\text { assembly size }^{\mathrm{c}} \\
\text { (No. of rods) }\end{array}$ & $\begin{array}{c}\text { Moderator }_{\text {height }^{\mathrm{d}}} \\
(\mathbf{c m})\end{array}$ & $\begin{array}{c}\text { Boron } \\
\text { concentration } \\
\text { (ppm) }\end{array}$ \\
\hline BW1484B1 & 2.459 & 1.636 & 0.0 & $9-14 \times 14$ & 144.29 & 1037 \\
BW1484B2 & 2.459 & 1.636 & 1.636 & $9-14 \times 14$ & 100.32 & 702 \\
BW1484B3 & 2.459 & 1.636 & 4.908 & $9-14 \times 14$ & 149.12 & 143 \\
\hline
\end{tabular}

${ }^{a}$ See Ref. 6.

believed to be the perpendicular distance between cell boundaries of assemblies.

'Four corner rods of assemblies are 0.635 -cm-radius aluminum rods.

Measured from the top of the aluminum base plate.

Table 2.49 Critical data for soluble boron experiments from EPRI NP-196 ${ }^{\mathrm{a}}$

\begin{tabular}{lccccc}
\hline Experiment & $\begin{array}{c}\text { Enrichment } \\
(\mathrm{wt} \%)\end{array}$ & $\begin{array}{c}\text { Pitch } \\
(\mathrm{cm})\end{array}$ & $\begin{array}{c}\text { No. of } \\
\text { rods }\end{array}$ & $\begin{array}{c}\text { Moderator } \\
\text { height } \\
\text { (cm) }\end{array}$ & $\begin{array}{c}\text { Boron } \\
\text { concentration } \\
(\mathrm{ppm})\end{array}$ \\
\hline EPRU65B & 2.35 & 1.562 & 1201 & 111.76 & 463.8 \\
EPRU75B & 2.35 & 1.905 & 1201 & 112.08 & 568 \\
EPRU87B & 2.35 & 2.210 & 885 & 111.76 & 285.8 \\
\hline
\end{tabular}

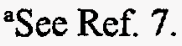

${ }^{b}$ Measured from the base of the aluminum solid plate. 
Physical Description

Table 2.50 Critical data for solúble boron experiments PNL-4267

\begin{tabular}{|c|c|c|c|c|c|c|}
\hline \multirow[b]{2}{*}{ Experiment } & \multirow{2}{*}{$\begin{array}{c}\text { Enrichment } \\
(\text { wt \%) }\end{array}$} & \multirow{2}{*}{$\begin{array}{l}\text { Pitch } \\
(\mathrm{cm})\end{array}$} & \multirow{2}{*}{$\begin{array}{l}\text { Array width } \\
\text { (No. of rods) }\end{array}$} & \multicolumn{2}{|c|}{ Total No. of rods } & \multirow{2}{*}{$\begin{array}{c}\text { Boron } \\
\text { concentration } \\
(\mathrm{g} / \mathrm{l})\end{array}$} \\
\hline & & & & Predicted $^{b}$ & Modeled & \\
\hline P4267B1 & 4.31 & 1.890 & 40 & 922.8 & 923 & $2.15 \pm 0.35$ \\
\hline P4267B2 & 4.31 & 1.890 & 40 & 1236.8 & 1237 & $2.55 \pm 0.07$ \\
\hline P4267B3 & 4.31 & 1.715 & 44 & 737.0 & 737 & $1.03 \pm 0.05$ \\
\hline P4267B4 & 4.31 & 1.715 & 44 & 916.96 & 917 & $1.82 \pm 0.30$ \\
\hline P4267B5 & 4.31 & 1.715 & 44 & 1191.96 & 1192 & $2.55 \pm 0.21$ \\
\hline
\end{tabular}

${ }^{\text {aSee Ref. } 13 .}$

bobtained by approach-to-critical method.

Table 2.51 Atom densities (atoms/b-cm) of fuel in critical experiments from Y-DR-14 ${ }^{a}$

\begin{tabular}{llllll}
\hline Experiment & ${ }^{235} \mathrm{U}$ & ${ }^{238} \mathrm{U}$ & $\mathrm{H}$ & $\mathrm{C}$ & $\mathrm{F}$ \\
\hline YDR14UN2 & $1.330 \mathrm{E}-4$ & $6.437 \mathrm{E}-3$ & $3.910 \mathrm{E}-2$ & $1.880 \mathrm{E}-2$ & $2.628 \mathrm{E}-2$ \\
YDR14PL2 & $1.119 \mathrm{E}-4$ & $5.415 \mathrm{E}-3$ & $4.547 \mathrm{E}-2$ & $2.186 \mathrm{E}-2$ & $2.211 \mathrm{E}-2$ \\
YDR14PL3 & $2.349 \mathrm{E}-4$ & $7.500 \mathrm{E}-3$ & $3.134 \mathrm{E}-2$ & $1.507 \mathrm{E}-2$ & $3.094 \mathrm{E}-2$ \\
YDR14UN3 & $2.349 \mathrm{E}-4$ & $7.500 \mathrm{E}-3$ & $3.134 \mathrm{E}-2$ & $1.507 \mathrm{E}-2$ & $3.094 \mathrm{E}-2$ \\
\hline
\end{tabular}

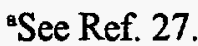

Table 2.52 Criticality data for homogenized uranium experiments from Y-DR-14

\begin{tabular}{|c|c|c|c|c|}
\hline Experiments & $\begin{array}{c}\text { Enrichment } \\
\text { (wt \%) }\end{array}$ & $\begin{array}{l}\text { Fuel } \\
\text { form }\end{array}$ & $\begin{array}{l}\text { Fuel dimensions } \\
\qquad(\mathrm{cm})\end{array}$ & Reflector \\
\hline YDR14PL2 & 2.00 & $\mathrm{U}(2) \mathrm{F}_{4}$ & $53.67 \times 53.67 \times 54.29$ & $\begin{array}{l}\text { 15.2-cm paraffin on top and sides } \\
\text { 15.2-cm Plexiglas }{ }^{\mathrm{b}} \text { on bottom }\end{array}$ \\
\hline YDR14PL3 & 3.00 & $\mathrm{U}(3) \mathrm{F}_{4}$ & $43.47 \times 43.47 \times 86.39$ & $\begin{array}{l}\text { 15.2-cm paraffin on top and sides } \\
15.2-\mathrm{cm} \text { Plexiglas }{ }^{\mathrm{b}} \text { on bottom }\end{array}$ \\
\hline YDR14UN2 & 2.00 & $\mathrm{U}(2) \mathrm{F}_{4}$ & $56.22 \times 56.22 \times 122.47$ & none \\
\hline YDR14UN3 & 3.00 & $\mathrm{U}(3) \mathrm{F}_{4}$ & $56.47 \times 56.47 \times 86.64$ & none \\
\hline
\end{tabular}

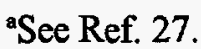

bolume fraction: 0.918 Plexiglas, 0.062 aluminum, 0.01 void. 
Table 2.53 Cuboid fuel can descriptions for CR1071AS, CR1653AS, CR2500S

\begin{tabular}{|c|c|c|c|}
\hline Parameter & CR1071AS & CR1653AS & CR2500S \\
\hline \multicolumn{4}{|l|}{ Can } \\
\hline Material $^{\mathrm{b}}$ & aluminum & aluminum & aluminum \\
\hline Can volume, $\mathrm{cm}^{3}$ & $(15.28)^{3}$ & $(15.24)^{3}$ & $(15.24)^{3}$ \\
\hline Wall thickness, ${ }^{c} \mathrm{~cm}$ & 0.15 & 0.16 & 0.16 \\
\hline Cavity volume, $\mathrm{cm}^{3}$ & $(14.98)^{3}$ & $(14.92)^{3}$ & $(14.92)^{3}$ \\
\hline Vinyl tape mass, $\mathrm{g}$ & 3 & 3.0 & 3.4 \\
\hline Mylar tape mass, $\mathrm{g}$ & 4 & 4.0 & 5.9 \\
\hline \multicolumn{4}{|l|}{ Cavity contents } \\
\hline $\mathrm{U}_{3} \mathrm{O}_{8}$ mass, $\mathrm{g}$ & 15088 & 15084 & 15081 \\
\hline $\mathrm{H}_{2} \mathrm{O}$ mass, $\mathrm{g}$ & 314 & 533.4 & 905.3 \\
\hline $\mathrm{C}_{2} \mathrm{H}_{4}$ mass, $\mathrm{g}$ & 53 & 53.0 & 53.0 \\
\hline \multicolumn{4}{|l|}{$\mathrm{U}_{3} \mathrm{O}_{8}$ enrichment } \\
\hline${ }^{234} \mathrm{U}$, wt \% & 0.03 & 0.030 & 0.03 \\
\hline${ }^{235} \mathrm{U}$, wt $\%$ & 4.46 & 4.481 & 4.48 \\
\hline${ }^{236} \mathrm{U}$, wt \% & 0.08 & 0.089 & 0.09 \\
\hline${ }^{238} \mathrm{U}$, wt $\%$ & 95.43 & 95.399 & 95.40 \\
\hline
\end{tabular}

${ }^{a}$ See Refs. 28-31.

bDensity of $2.713 \mathrm{~g} / \mathrm{cm}^{3}$.

'Walls have water injection holes $(56 \mathrm{total})$ of $0.64 \mathrm{~cm}$ diameter. 
Table 2.54 Materials for damp uranium oxide experiments $\mathrm{s}^{\mathrm{a}}$

\begin{tabular}{|c|c|c|c|c|c|c|c|}
\hline $\begin{array}{c}\text { Element, } \\
\text { wt } \%\end{array}$ & $\begin{array}{c}\text { Fire- } \\
\text { retardant } \\
\text { Plexiglas }\end{array}$ & Glue $^{b}$ & $\begin{array}{l}\text { Vinyl } \\
\text { tape }\end{array}$ & $\begin{array}{l}\text { Mylar } \\
\text { tape }\end{array}$ & $\begin{array}{l}\text { Nonfire- } \\
\text { retardant } \\
\text { Plexiglas }\end{array}$ & Paperb & Polyethylene \\
\hline $\mathrm{Al}$ & & & 0.5 & & & & \\
\hline $\mathrm{Br}$ & 7.10 & & & & & & \\
\hline $\mathrm{C}$ & 52.03 & 86.29 & 45.91 & 65.50 & 59.52 & 42.17 & 84.90 \\
\hline $\mathrm{Ca}$ & & & 6.9 & & & & \\
\hline $\mathrm{Cl}$ & 1.81 & & 25.73 & & & & \\
\hline $\mathrm{H}$ & 7.16 & 11.67 & 5.92 & 6.83 & 7.84 & 6.48 & 14.01 \\
\hline$N$ & 0.16 & & 0.16 & & & & \\
\hline 0 & 29.82 & 1.20 & 10.82 & 27.02 & 32.23 & 49.50 & 1.20 \\
\hline$P$ & 1.02 & & & & & & \\
\hline $\mathrm{Pb}$ & & & 1.1 & & & & \\
\hline $\mathrm{Si}$ & & & 0.6 & & & & \\
\hline $\mathrm{Ti}$ & & & 1.6 & & & & \\
\hline $\mathrm{Zn}$ & & & 0.1 & & & & \\
\hline $\begin{array}{l}\text { Density, } \\
\mathrm{g} / \mathrm{cm}^{3}\end{array}$ & 1.284 & 0.728 & 1.310 & 1.11 & 1.185 & 0.766 & 0.824 \\
\hline
\end{tabular}

aSee Ref. 29.

bPlates of both Plexiglas types covered with paper $(0.49 \mathrm{wt} \%)$ and glue $(0.16 \mathrm{wt} \%)$. 
Table 2.55 Cuboid dimensions ${ }^{\mathrm{a}}(\mathrm{cm})$ of Fig. 2.69 for CR1071 AS

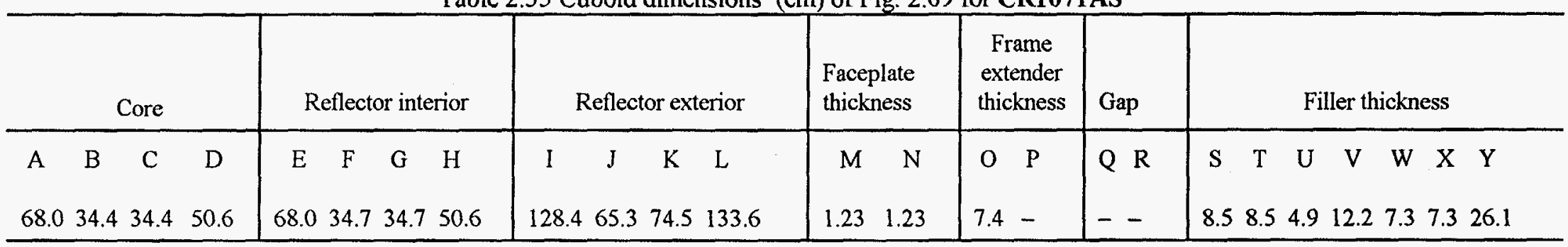

i

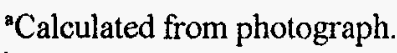

bSee Ref. 29.

'Dimensions for plastic reflector of shape seen in Figure 2.72. 


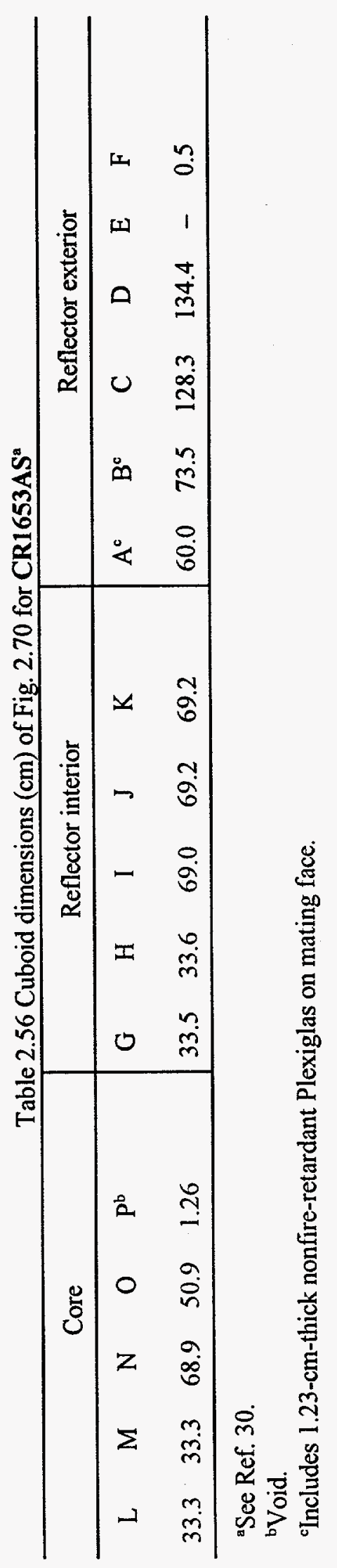

Physical Description 
Table 2.57 Cuboid dimensions for CR2500S

\begin{tabular}{|c|c|c|c|}
\hline & Cuboid & & Dimension $(\mathrm{cm})$ \\
\hline Core $^{b}$ & $\begin{array}{l}\text { North table } \\
\text { South table }\end{array}$ & $\begin{array}{l}\text { East/west } \\
\text { North/south } \\
\text { Vertical } \\
\text { East/west } \\
\text { North/south } \\
\text { Vertical }\end{array}$ & $\begin{array}{l}50.8 \\
33.3 \\
51.1 \\
\\
51.1 \\
33.4 \\
51.1\end{array}$ \\
\hline Reflector interior ${ }^{\circ}$ & $\begin{array}{l}\text { North table } \\
\text { South table }\end{array}$ & $\begin{array}{l}\text { East/west } \\
\text { North/south } \\
\text { Vertical } \\
\text { East/west } \\
\text { North/south } \\
\text { Vertical }\end{array}$ & $\begin{array}{l}77.6 \\
33.5 \\
51.1 \\
\\
77.4 \\
33.9 \\
51.1 \\
\end{array}$ \\
\hline Reflector exterior & $\begin{array}{l}\text { North table } \\
\text { South table }\end{array}$ & $\begin{array}{l}\text { East/west } \\
\text { North/south } \\
\text { Vertical } \\
\text { East/west } \\
\text { North/south } \\
\text { Vertical }\end{array}$ & $\begin{array}{c}128.3 \\
59.7^{\text {de }} \\
132.5 \\
128.4 \\
74.4^{\text {d }} \\
134.6\end{array}$ \\
\hline Core location $^{\mathfrak{f}}$ & $\begin{array}{l}\text { North table } \\
\text { South table }\end{array}$ & $\begin{array}{l}\text { West } \\
\text { Bottom } \\
\text { West } \\
\text { Bottom }\end{array}$ & $\begin{array}{l}25.3 \\
55.1 \\
\\
25.8 \\
55.4\end{array}$ \\
\hline
\end{tabular}

${ }^{8}$ See Ref. 31.

'Planar and elevation views of core seen in Fig. 2.70.

'Dimensions for plastic reflector of shape seen in Fig. 2.72.

Includes 1.215 -cm-thick faceplate.

Includes thickness of plastic inserted between frame and end panel.

Locates the lower west corner of the core relative to the reflector exterior. 
Physical Description

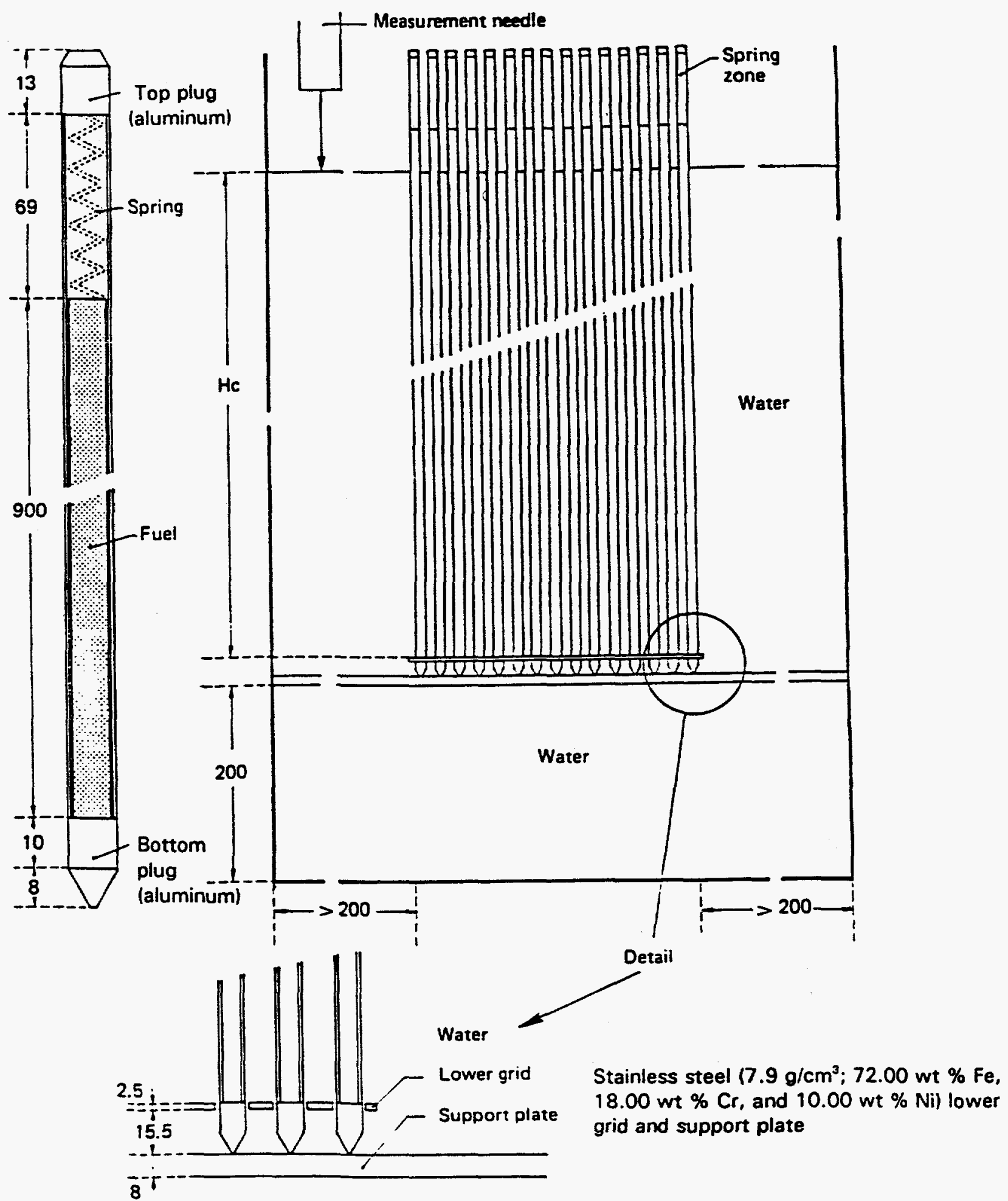

Figure 2.1 Principal dimensions (in centimeters) of $4.742 \mathrm{wt} \% \mathrm{UO}_{2}$ fuel rods for ANS Transactions, Vol. 33, and NS\&E, Vol. 71, and assembly for NS\&E, Vol. 71. Source: Refs. 5 and 8 (Reprinted with permission by author) 
Physical Description

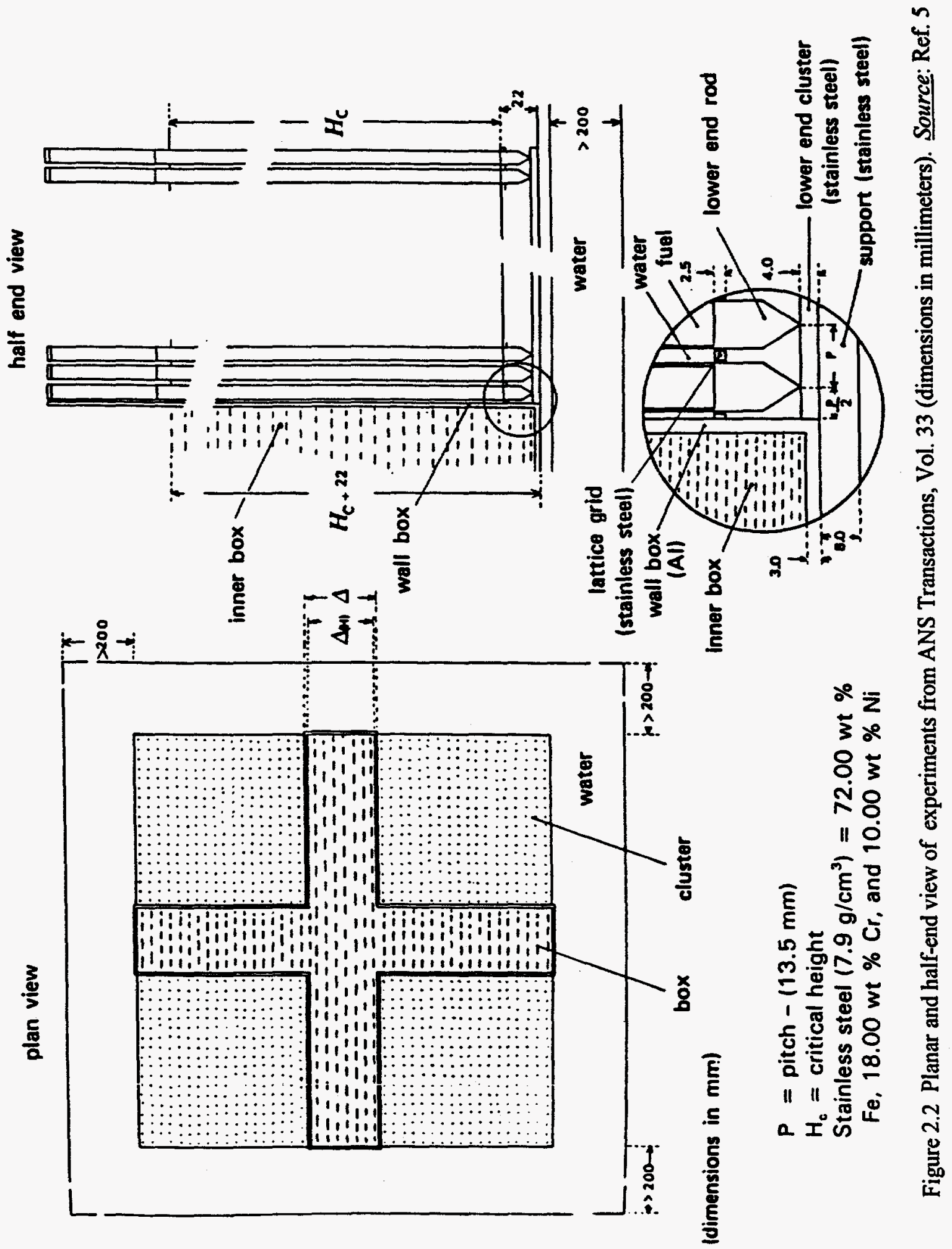


Physical Description

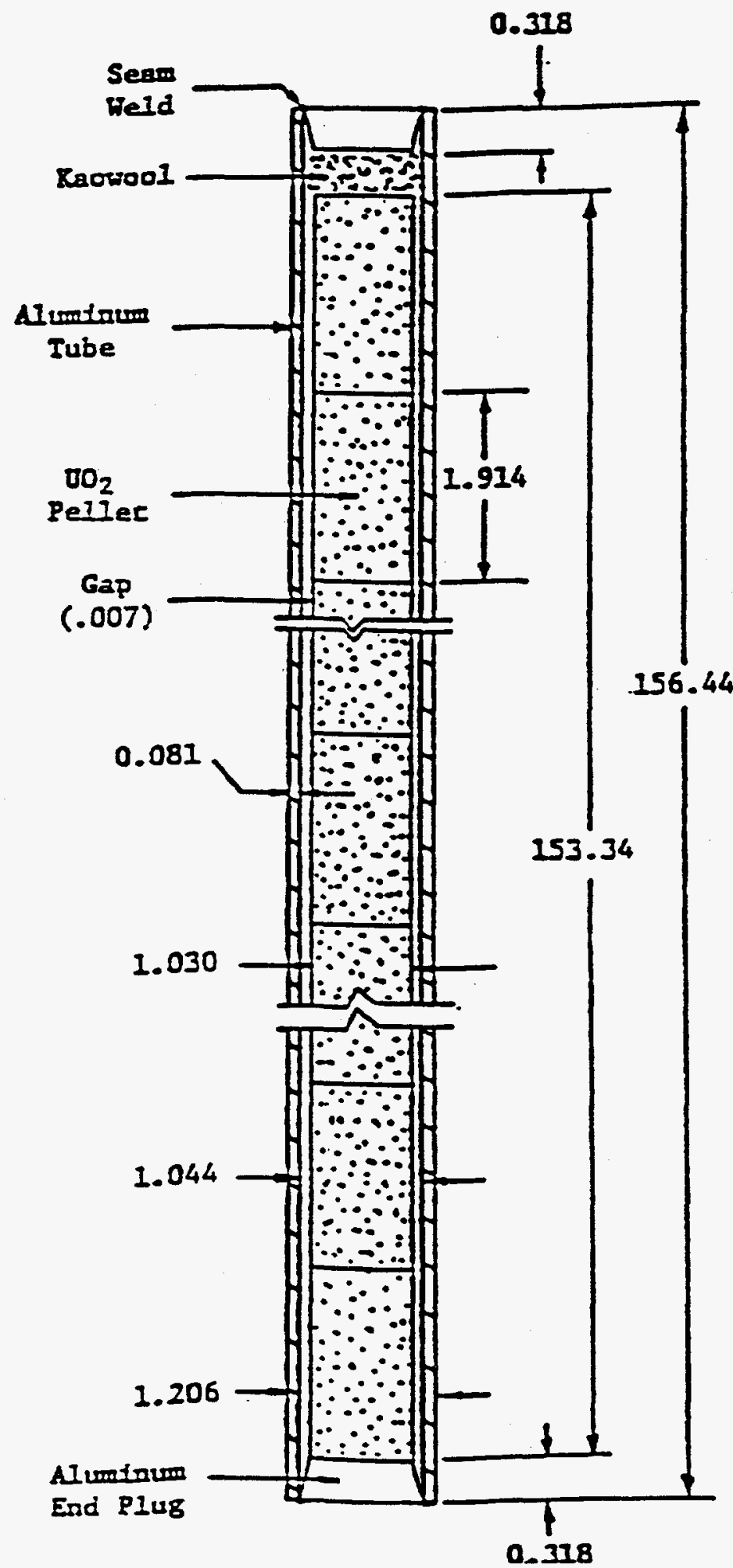

Figure 2.3 Dimensions (in centimeters) of $2.459 \mathrm{wt} \% \mathrm{UO}_{2}$ fuel rods for BAW-1484 experiments. Source: Ref. 6 
Physical Description

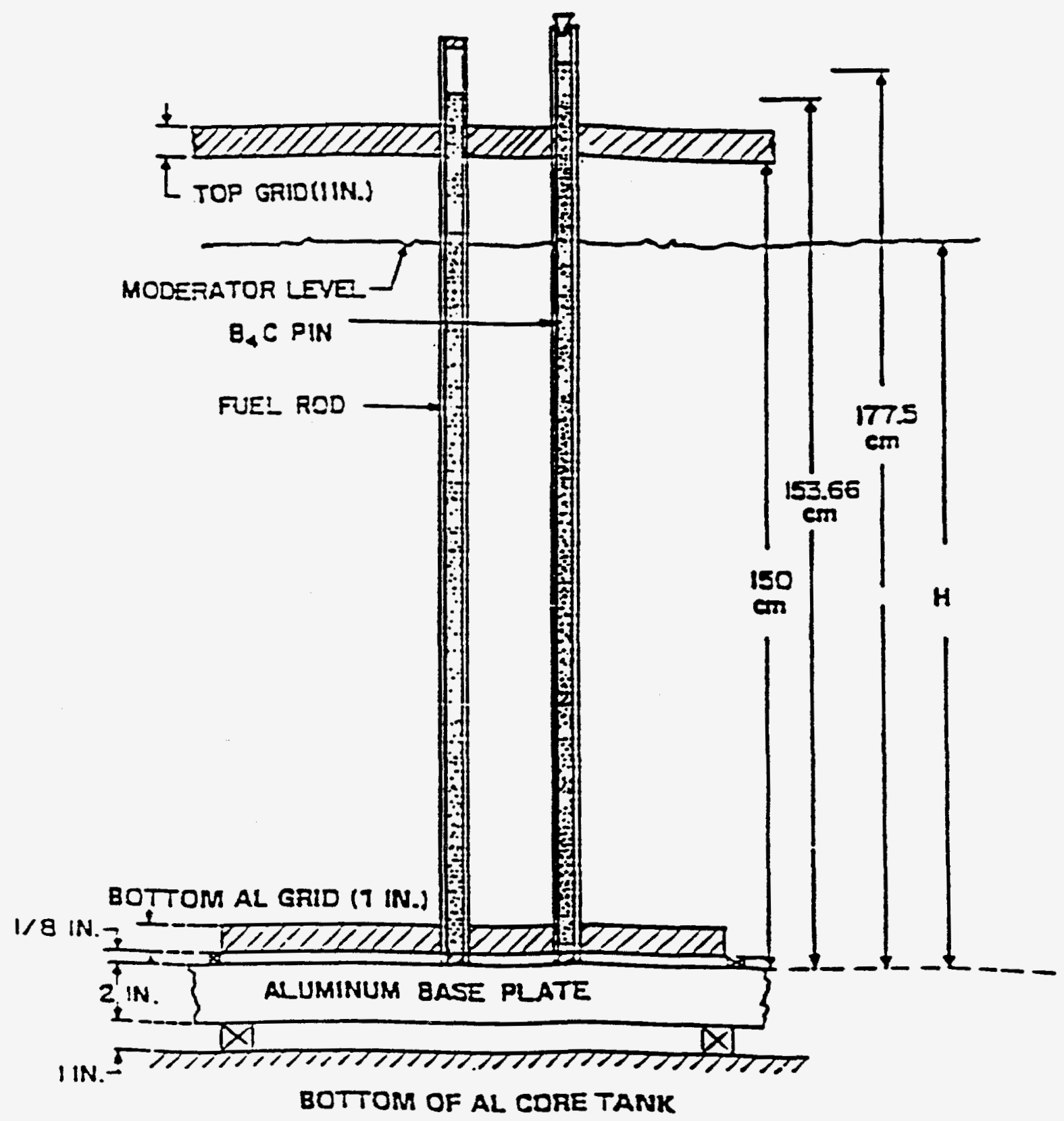

Figure 2.4 Experiment BW1484SL vertical dimensions. Source: Ref. 6 
DESCRIPTION OF 2.35 wt\% ${ }^{235} \mathrm{U}$ ENRICHED UO 2 RODS

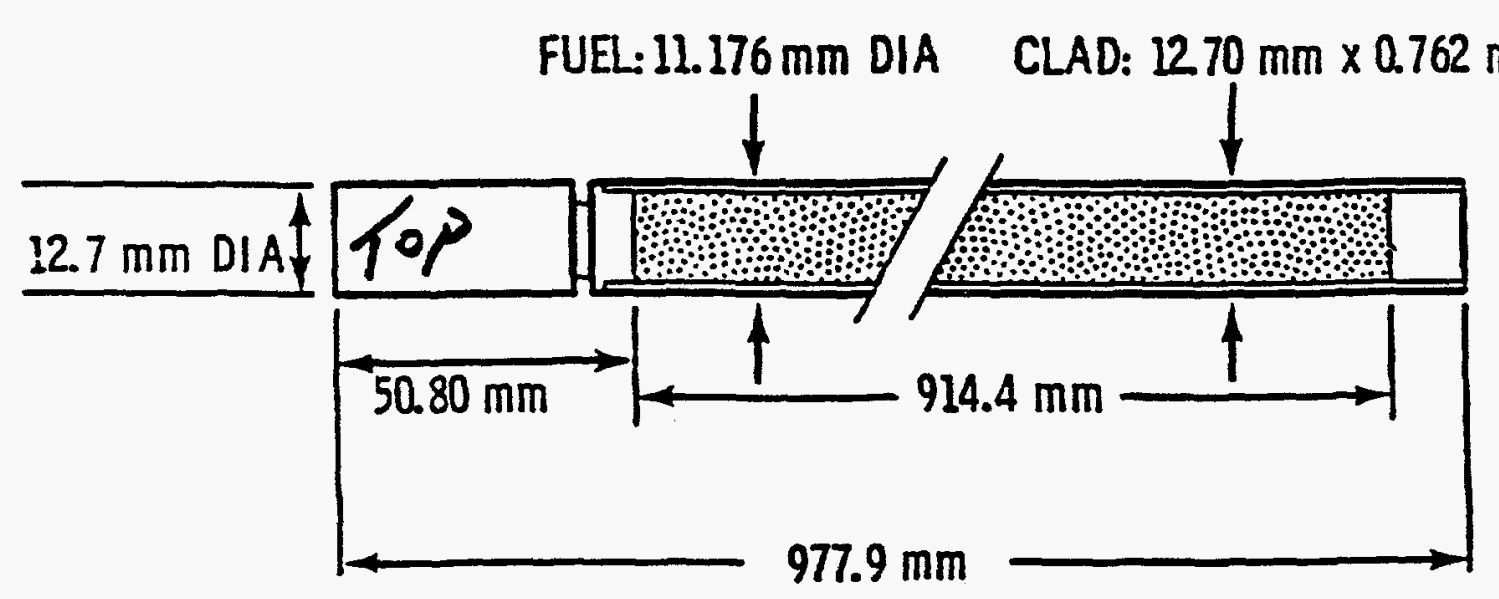

CLADDING: 6061 ALUMINUM TUBING SEAL WELDED WITH A LOWER END PLUG OF 5052 +132 ALUMINUM AND A TOP PLUG OF 1100 ALUMINUM

TOTAL WEIGHT OF LOADED FUEL RODS: $917 \mathrm{gm}$ (AVERAGE)

LOADING:

$825 \mathrm{gm}$ OF UO2 2 POWDER / ROD, $726 \mathrm{gm}$ OF U/ROD, $17.08 \mathrm{gm}$ OF U-235/ROD ENRI CHMENT $-235 \pm 0.05$ W/O U-235

FUEL DENSITY $-9.20 \mathrm{mg} / \mathrm{mm}^{3}$ (84\% THEORETICAL DENSITY)

Figure 2.5 Description of $2.35 \mathrm{wt} \% \mathrm{UO}_{2}$ fuel rods used in experiments from EPRI NP-196. Source: Ref. 7. (Copyright 0 1976. Electric Power Research Institute. EPRI NP-196. Clean Critical Experiment Benchmarks for Plutonium Recycle in LWRs. Reprinted with Permission.) 
Physical Description

\section{FULLY REFLCTED}

IYIODERATOR HEIGHT

106

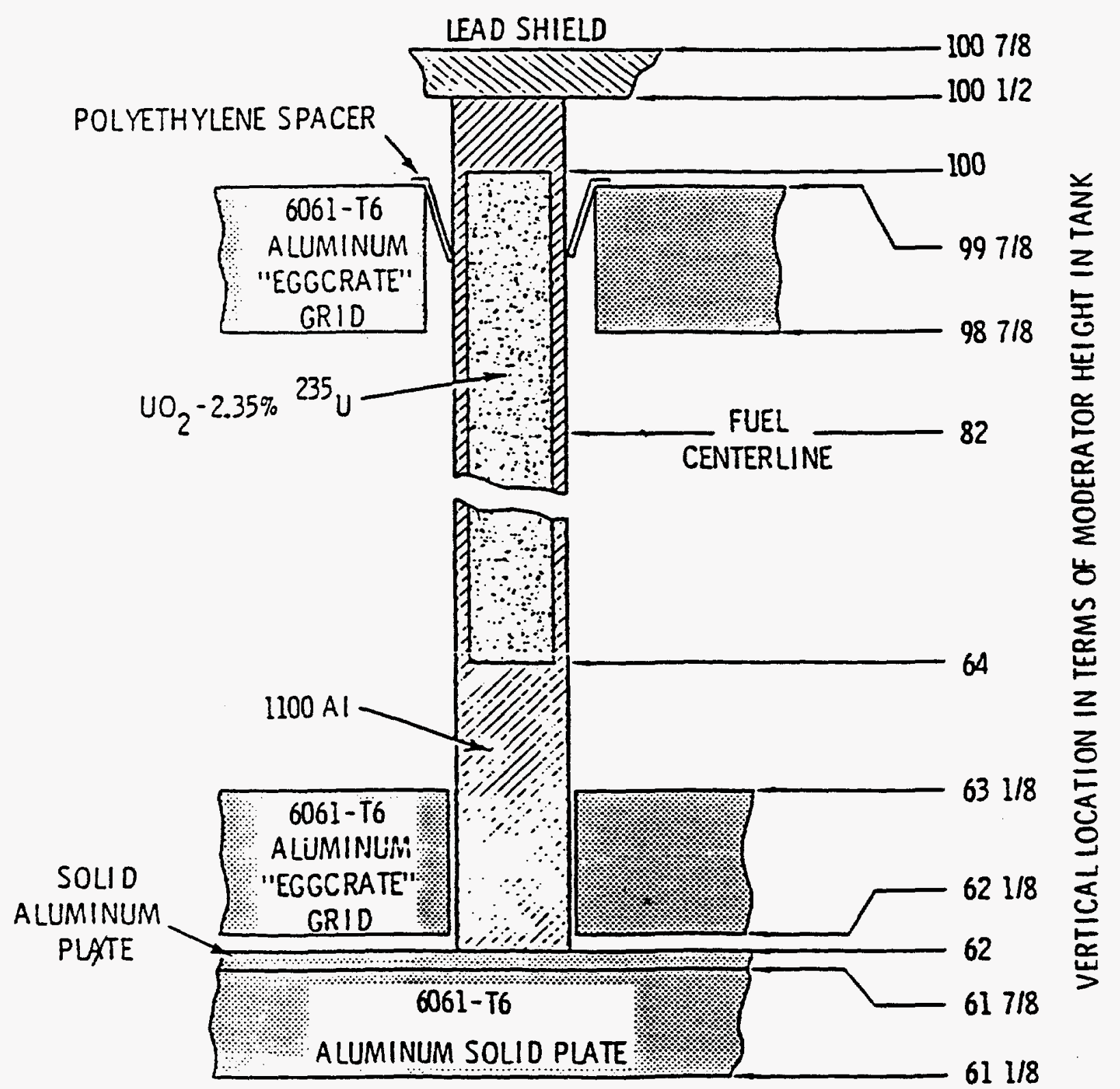

Figure 2.6 Axial core structure dimensions (in inches) for EPRU65 and EPRU87. Source: Ref. 7 (Copyright $\odot$ 1976. Electric Power Research Institute. EPRI NP-196. Clean Critical Experiment Benchmarks for Plutonium Recycle in LWRs. Reprinted with Permission.) 
Physical Description

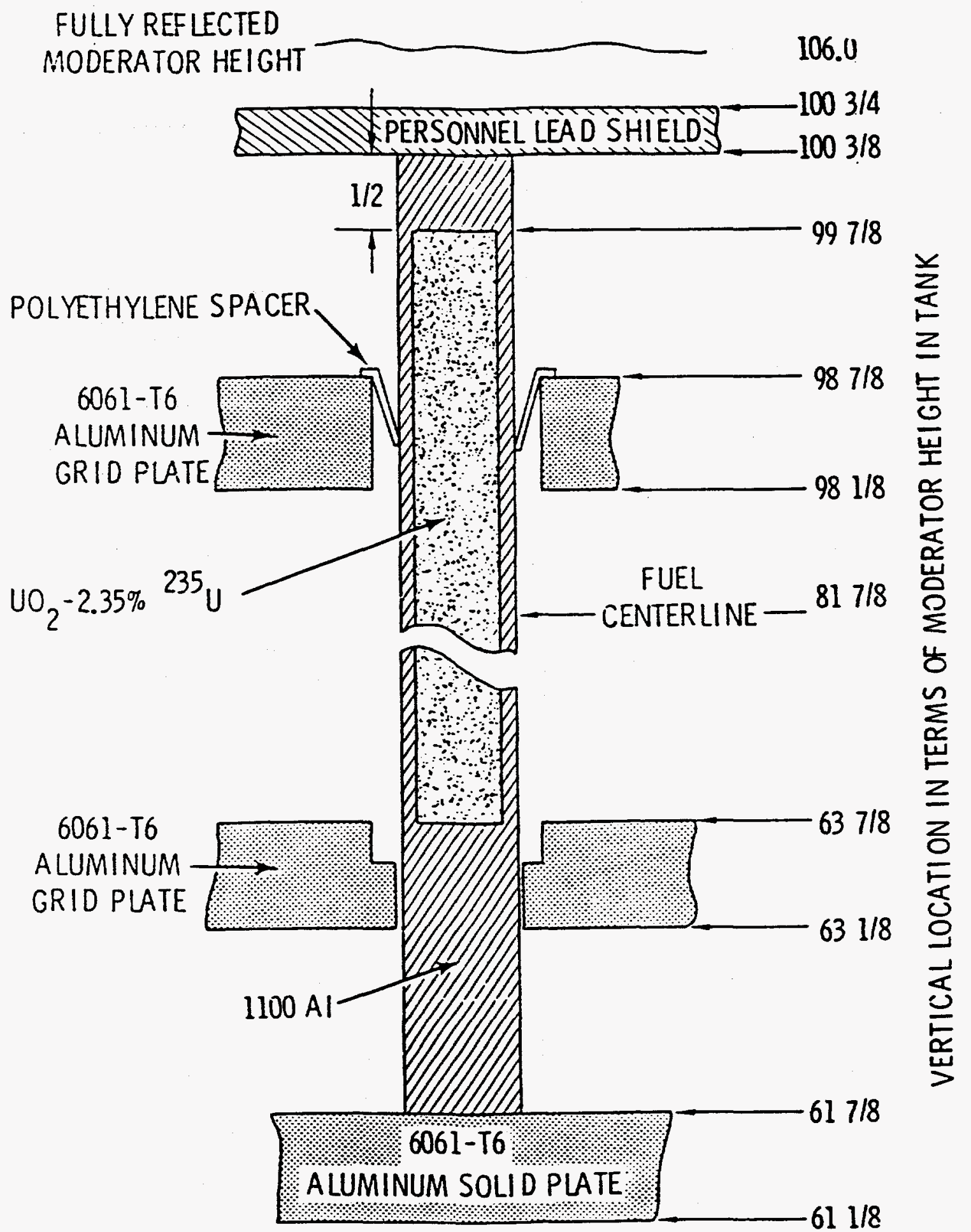

Figure 2.7 Axial core structure dimensions (in inches) for EPRU75. Source: Ref. 7 (Copyright $@ 1976$. Electric Power Research Institute. EPRI NP-196. Clean Critical Experiment Benchmarks for Plutonium Recycle in LWRs. Reprinted with Permission.) 
Physical Description

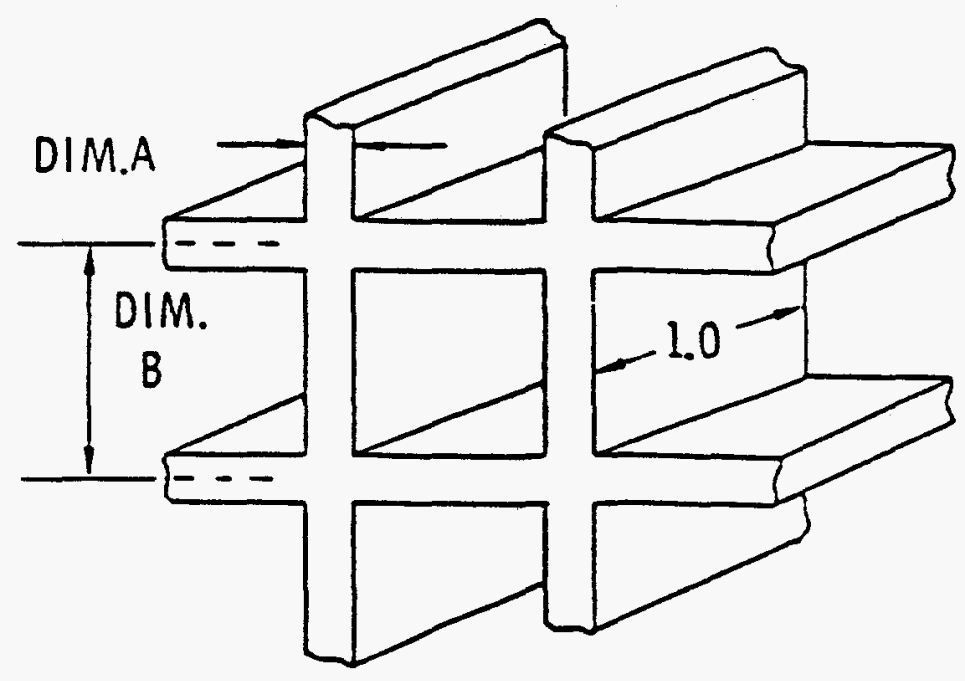

FUEL TYPE

PITCH

GRID

DIM.A

DIM B

\begin{tabular}{|l|l|l|l|l|}
\hline $\mathrm{UO}_{2}-2.35 \%{ }^{235} \mathrm{U}$ & $\begin{array}{l}0.615 \\
0.87\end{array}$ & UPPEP & 0.032 & 0.615 \\
\hline
\end{tabular}

Figure 2.8 Dimensions (in inches) of "eggcrate" grids for EPRU65 and EPRU87. Source: Ref. 7 (Copyright 1 1976. Electric Power Research Institute. EPRI NP-196. Clean Critical Experiment Benchmarks for Plutonium Recycle in LWRs. Reprinted with Permission.) 


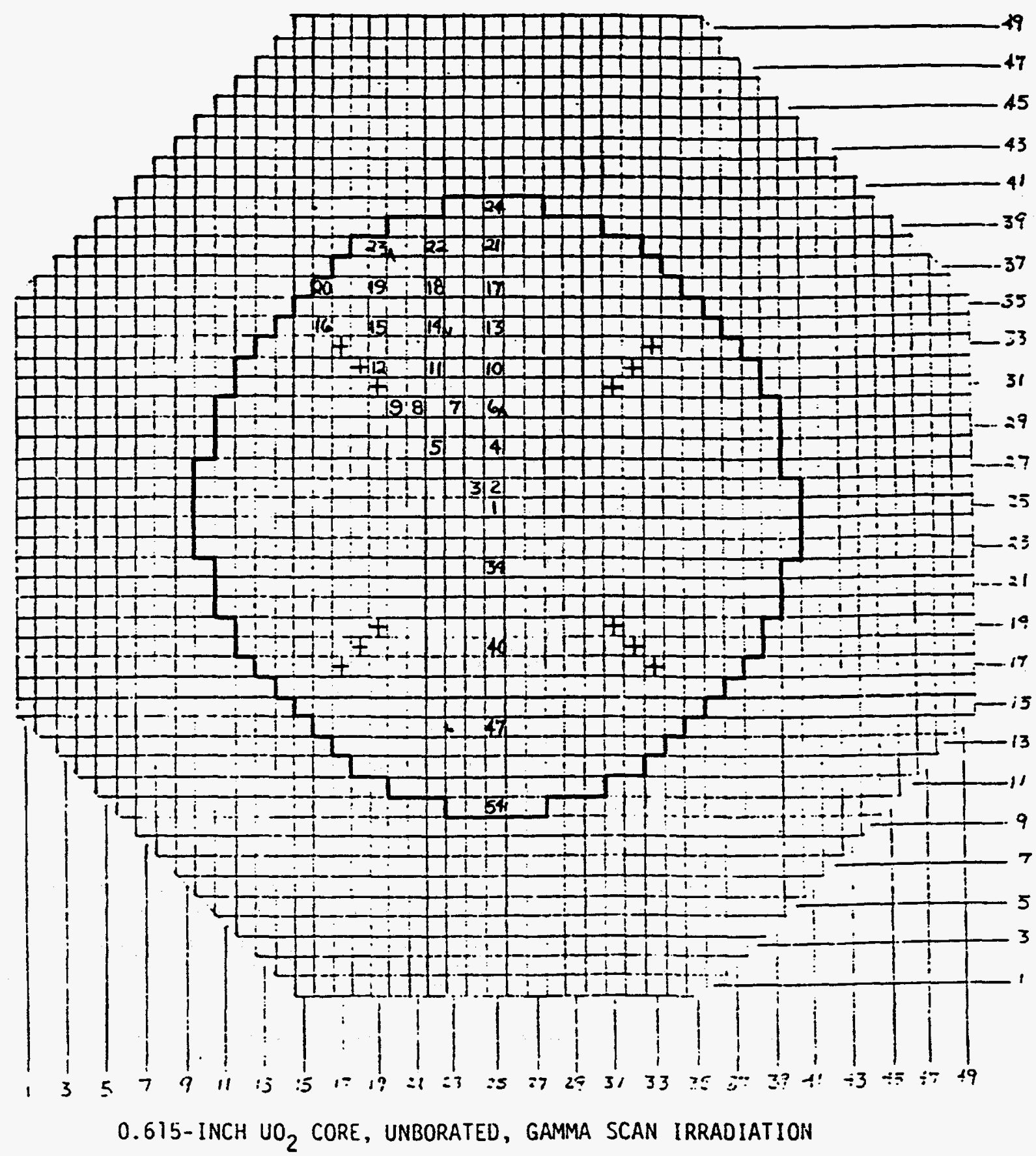

Figure 2.9 Core layout for EPRU65. Source: Ref. 7 (Copyright (C) 1976. Electric Power Research Institute. EPRINP-196. Clean Critical Experiment Benchmarks for Plutonium Recycle in LWRs. Reprinted with Permission.) 
Physical Description

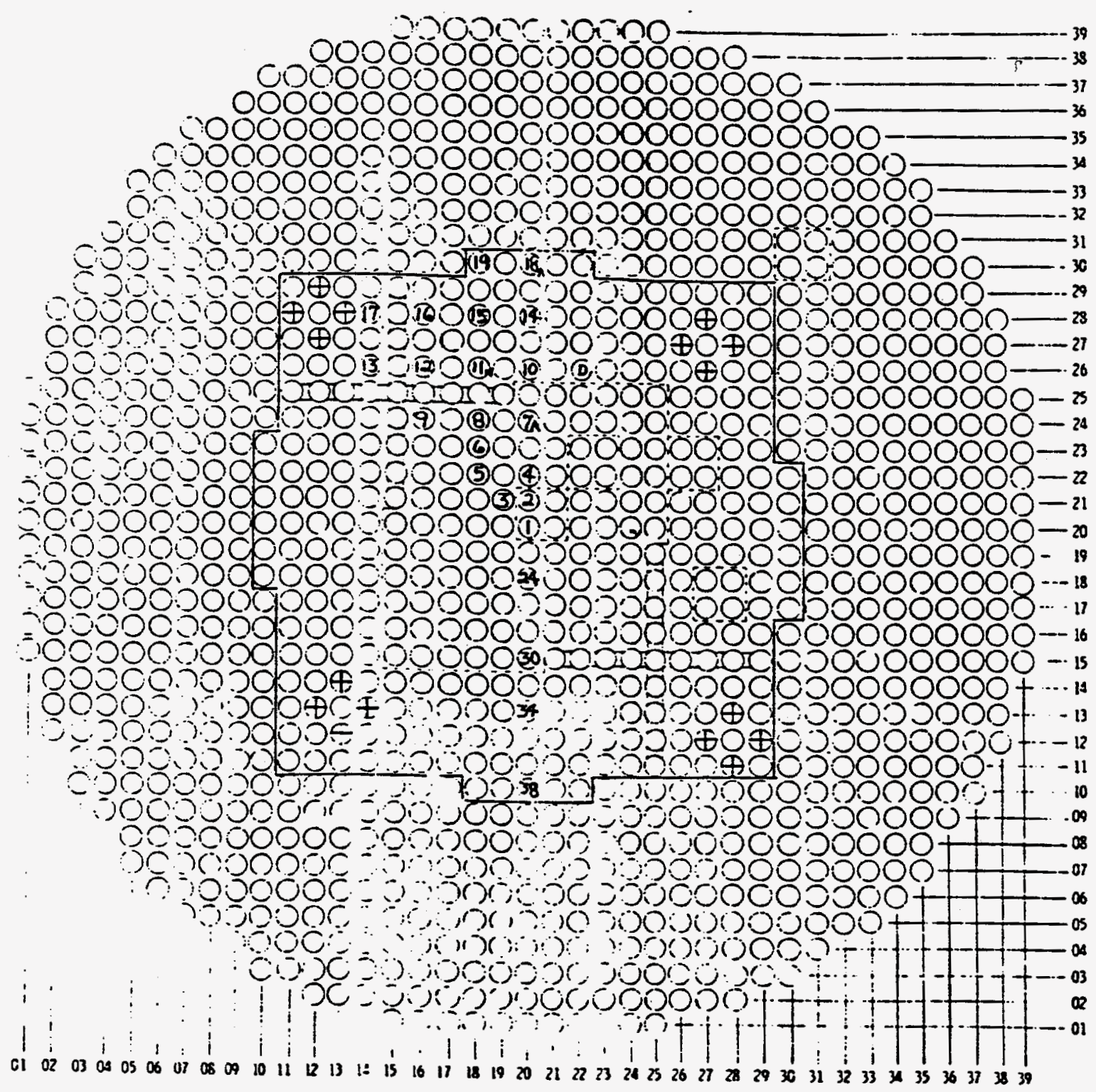

0.75-INCH UO 2 CORE, UNBORATED, GAMMA SCAN IRRADIATION

Figure 2.10 Core layout for EPRU75. Source: Ref. 7 (Copyright $\subset$ 1976. Electric Power Research Institute. EPRI NP-196. Clean Critical Experiment Benchmarks for Plutonium Recycle in LWRs. Reprinted with Permission.) 
Physical Description

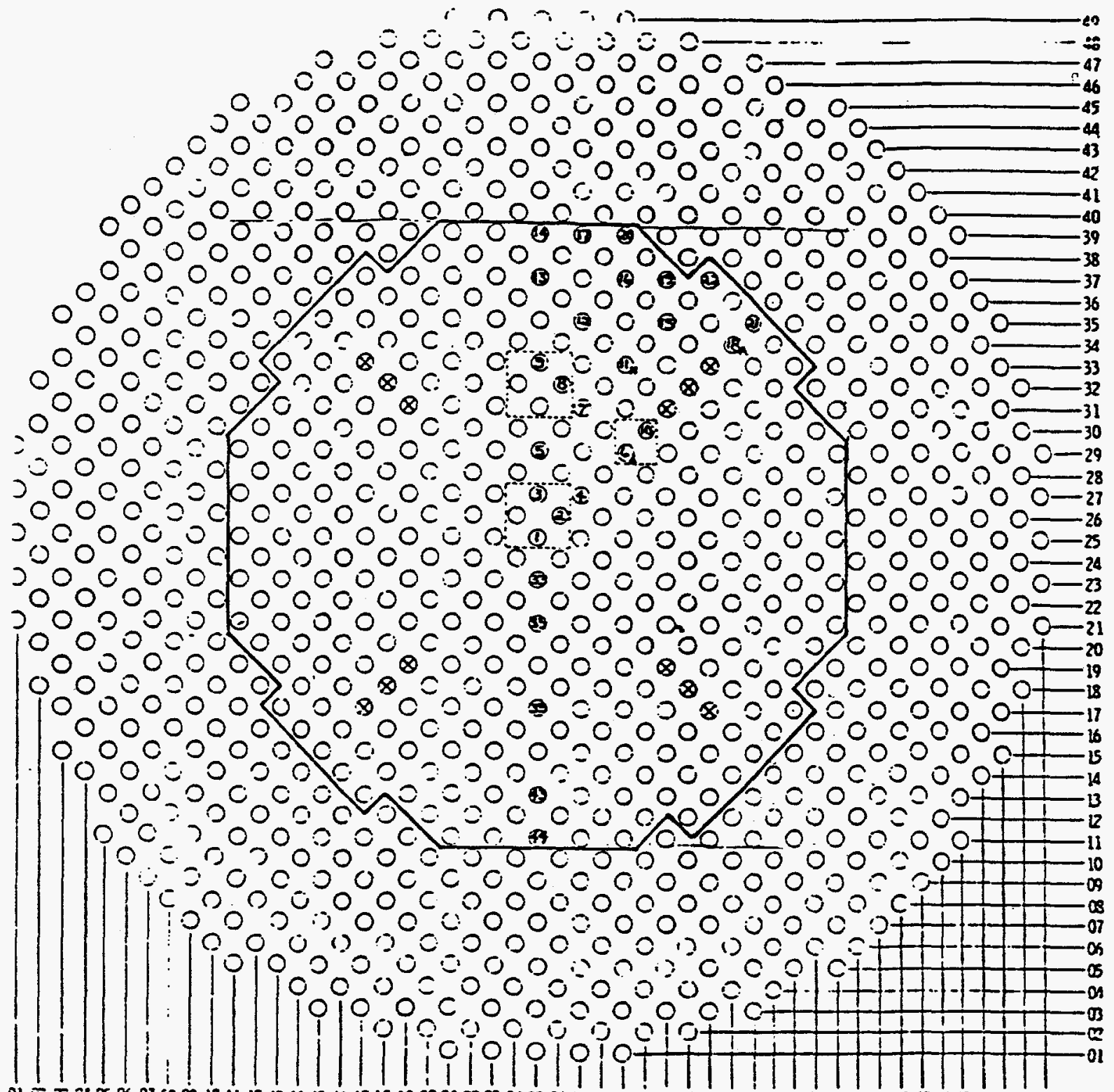

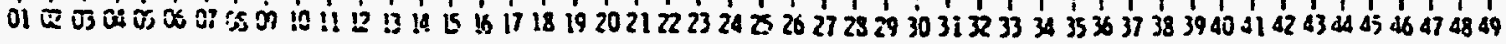

\subsection{7-INCH $\mathrm{NO}_{2}$ CORE, UNBORATED, GAMMA SCAN IRRADIATION}

Figure 2.11 Core layout for EPRU87. Source: Ref. 7 (Copyright $(1976$. Electric Power Research Institute. EPRI NP-196. Clean Critical Experiment Benchmarks for Plutonium Recycle in LWRs. Reprinted with Permission.) 
Physical Description

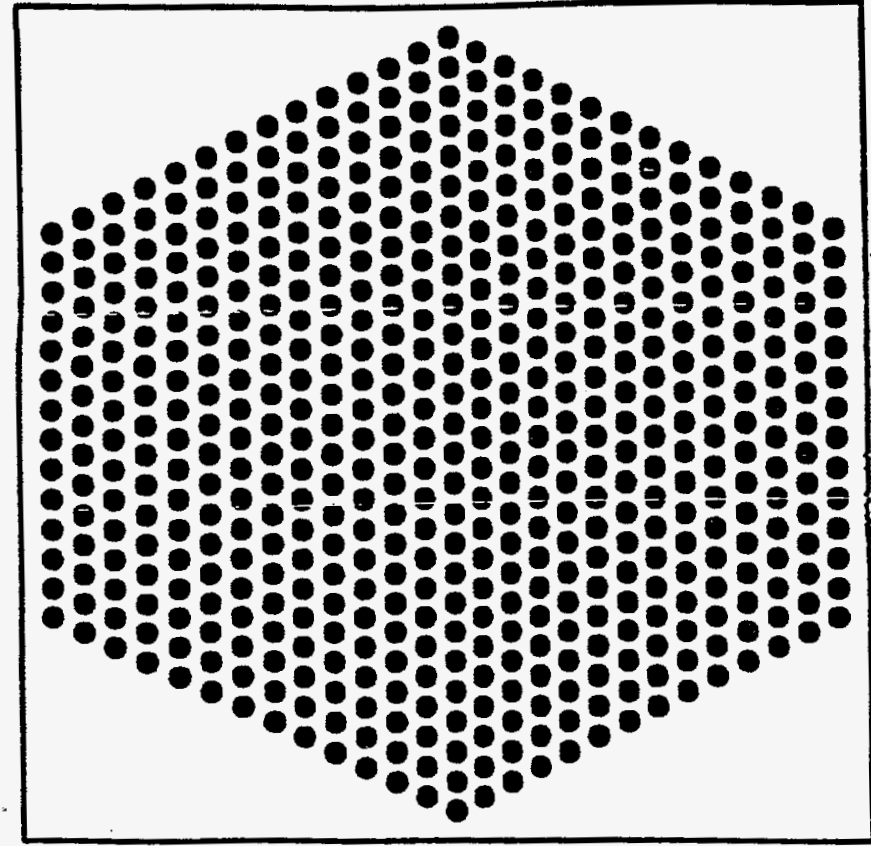

14 pins per side

Pitch $=1.35 \mathrm{~cm}$

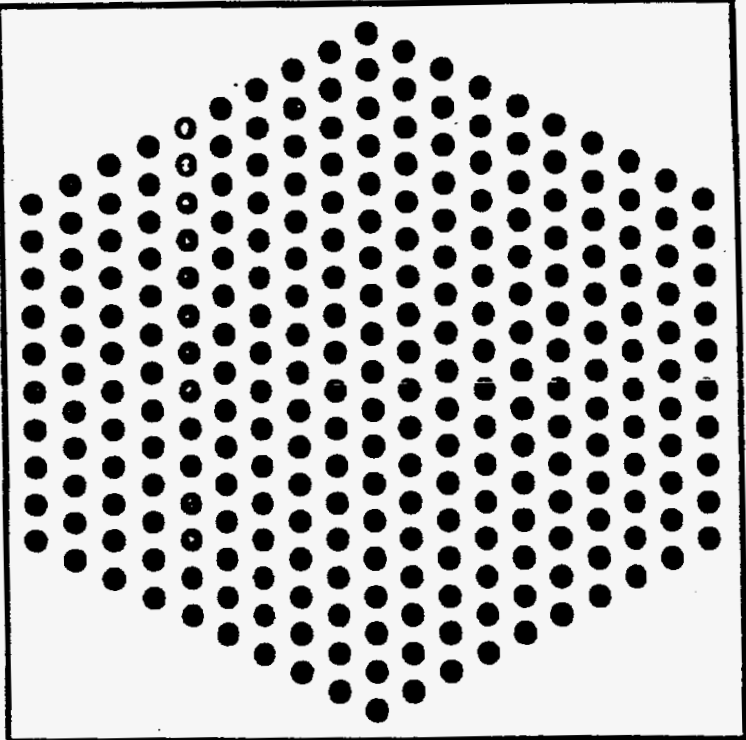

10 pins per side

Pitch $=1.72 \mathrm{~cm}$

(b) NSE71H2

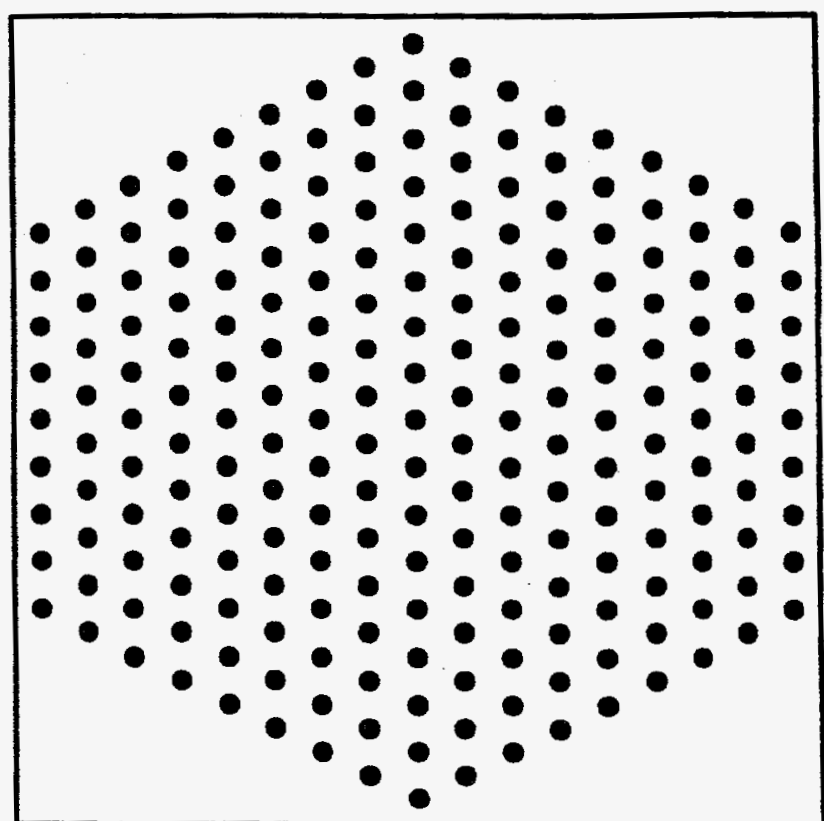

9 pins per side

Pitch $=2.26 \mathrm{~cm}$

(c) NSET1H3

Figure 2.12 Core configurations for three hexagonal lattice experiments of NS\&E, Vol. 71. Source: Ref. 8 (Reprinted with permission by author) 


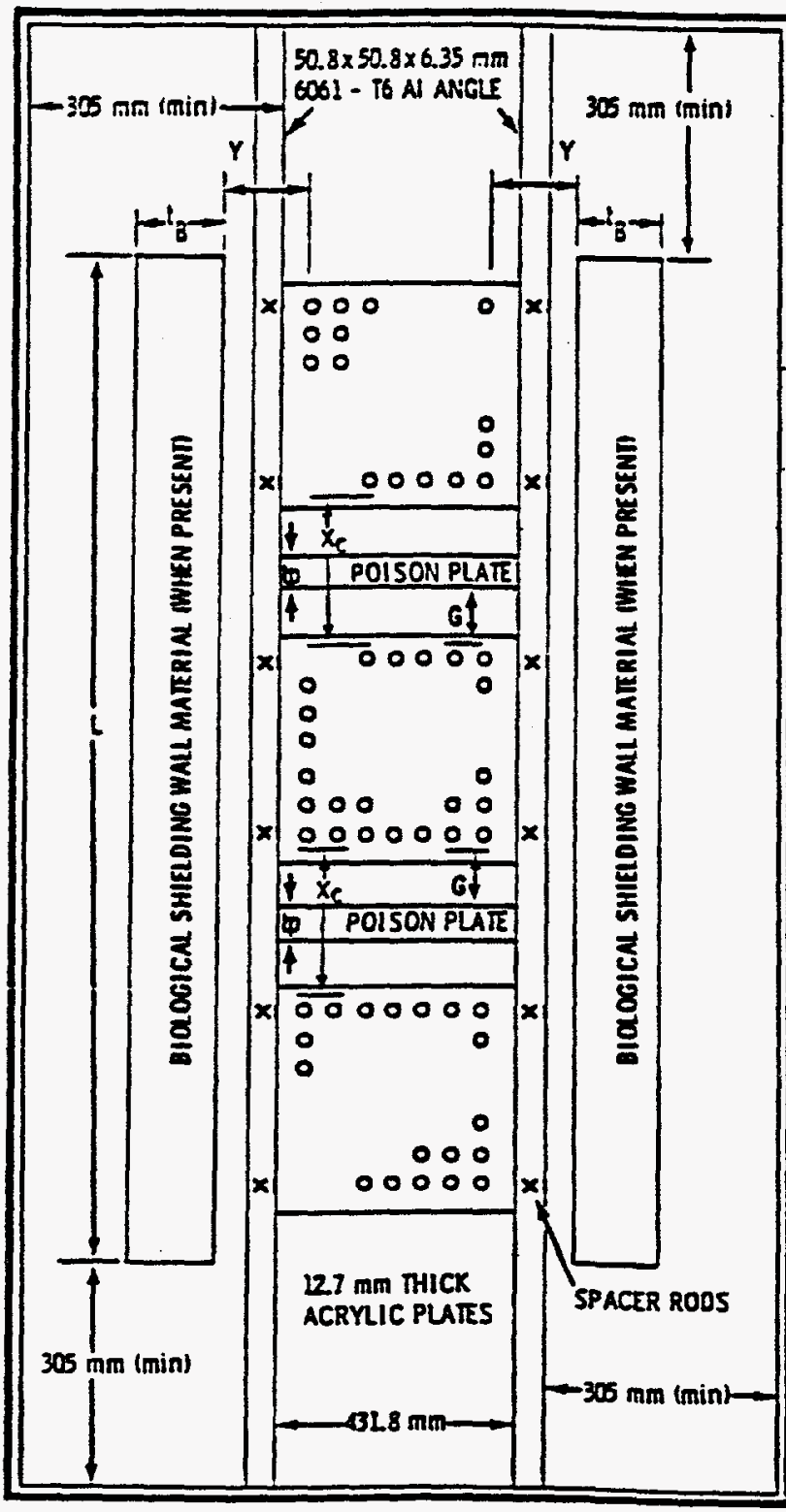

ACRYLIC $\left(1.185 \mathrm{~g} / \mathrm{cm}^{3}\right): 60 \mathrm{wt} \% \mathrm{C}$, $32 \mathrm{wt} \% \mathrm{O}$, and $8 \mathrm{wt} \% \mathrm{H}$.

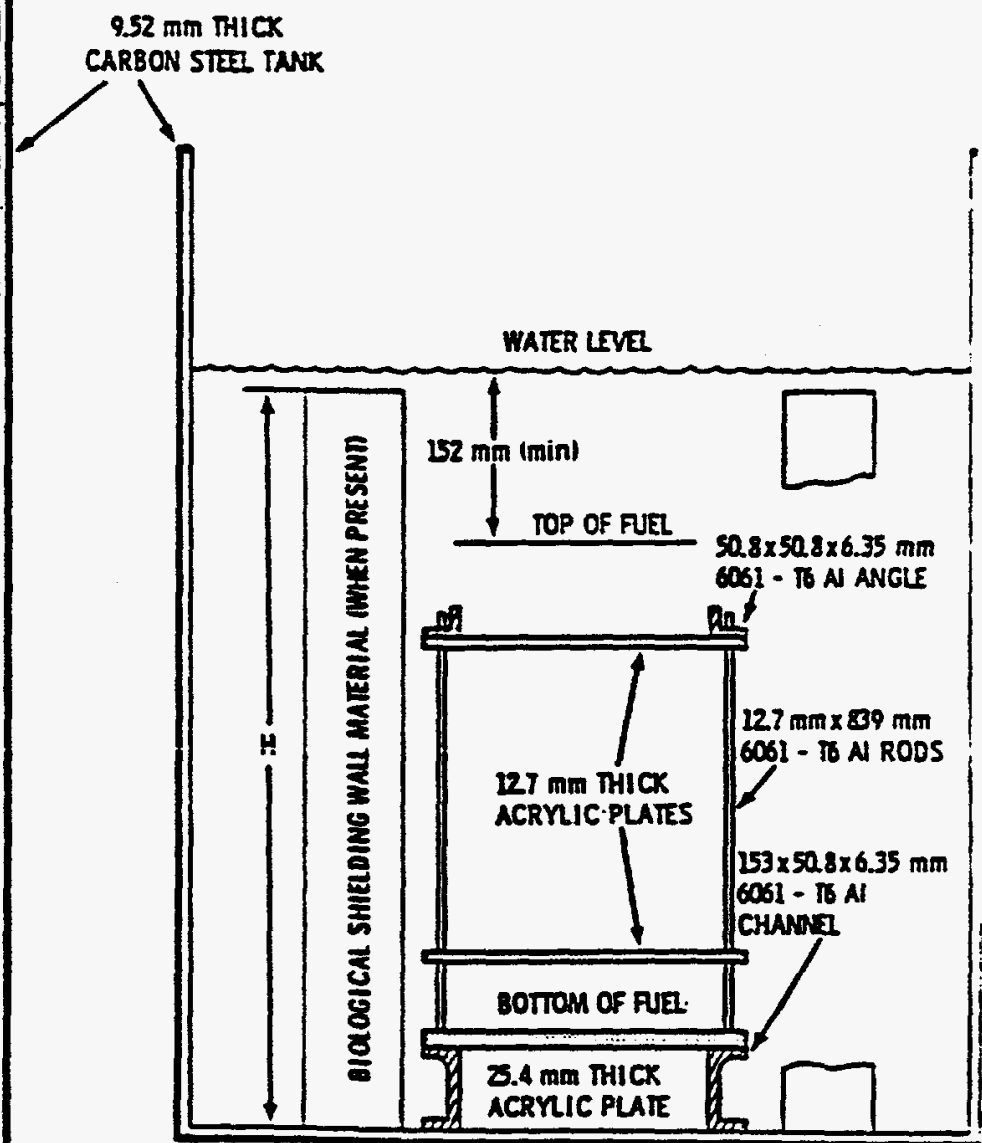

avo VIEW

PLAN VIEW

Figure 2.13 Planar and end views of experiments from PNL-2438. Source: Ref. 9 (Reprinted with permission from authors) 
Physical Description

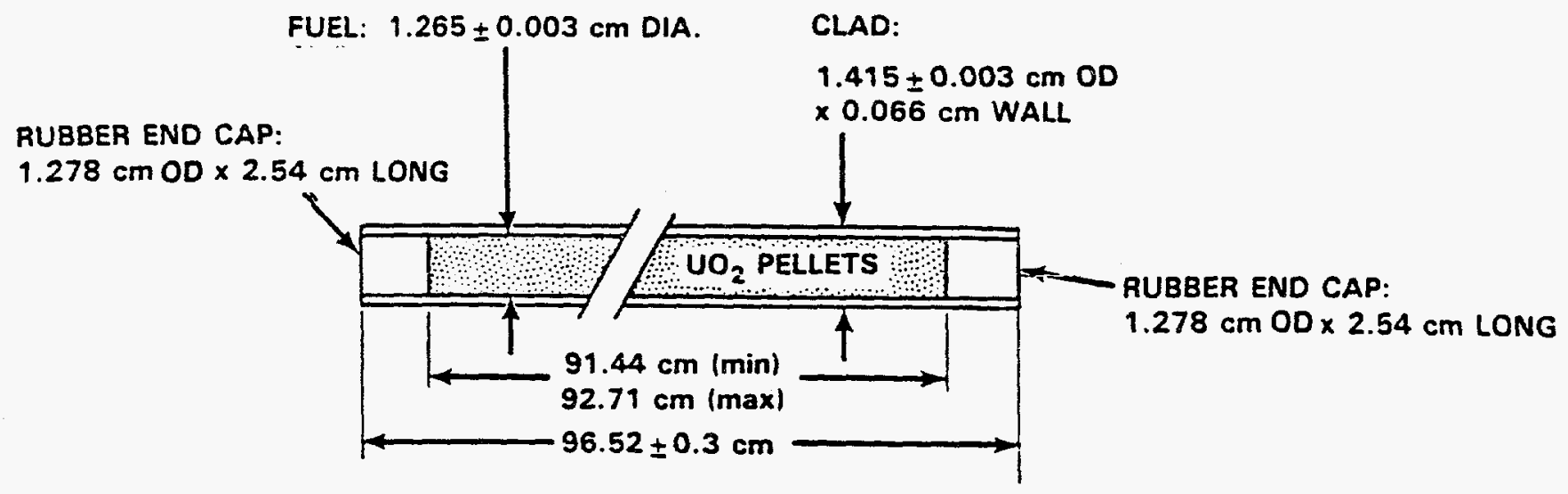

CLADDING: 6061 ALUMINUM TUBINE

LOADING

ENRICHMENT $-4.306 \pm 0.013 \% 235 \mathrm{U}$

OXIDE DENSITY $-10.40 \pm 0.06 \mathrm{~g} / \mathrm{cm}^{3}$

$\mathrm{UO}_{2} \cdot 1203.38 \pm 4.12 \mathrm{~g} / \mathrm{ROD}$

$\mathrm{U} \cdot 1059.64 \pm 4.80 \mathrm{~g} / \mathrm{ROD}$

URANIUM COMPOSITION:

$234 U-0.022 \pm 0.002$
$235 U-4.306 \pm 0.013$
$236 U-0.022 \pm 0.002$
$238 U-95.650 \pm 0.017$

END CAP:

C. $58 \pm 1 W T \%$

H-6.5 $\pm 0.3 W T \%$

S-1.7 $\pm 0.2 W T \%$

Ca-11.4 \pm 1.8 WT\%

0.22 .1 WT\% (BALANCE)

Si-0.3 \pm 0.1 WT\%

NOTES:

1. ERROR LIMITS ARE ONE STANDARD DEVIATION

2. END CAP DENSITY IS $1.321 \mathrm{~g} / \mathrm{cm}^{3}$

Figure 2.14 Description of $4.31 \mathrm{wt} \% \mathrm{UO}_{2}$ fuel rods used in experiments from PNL-3314. Source: Refs. 11 and 14 (Reprinted with permission from authors) 
Physical Description
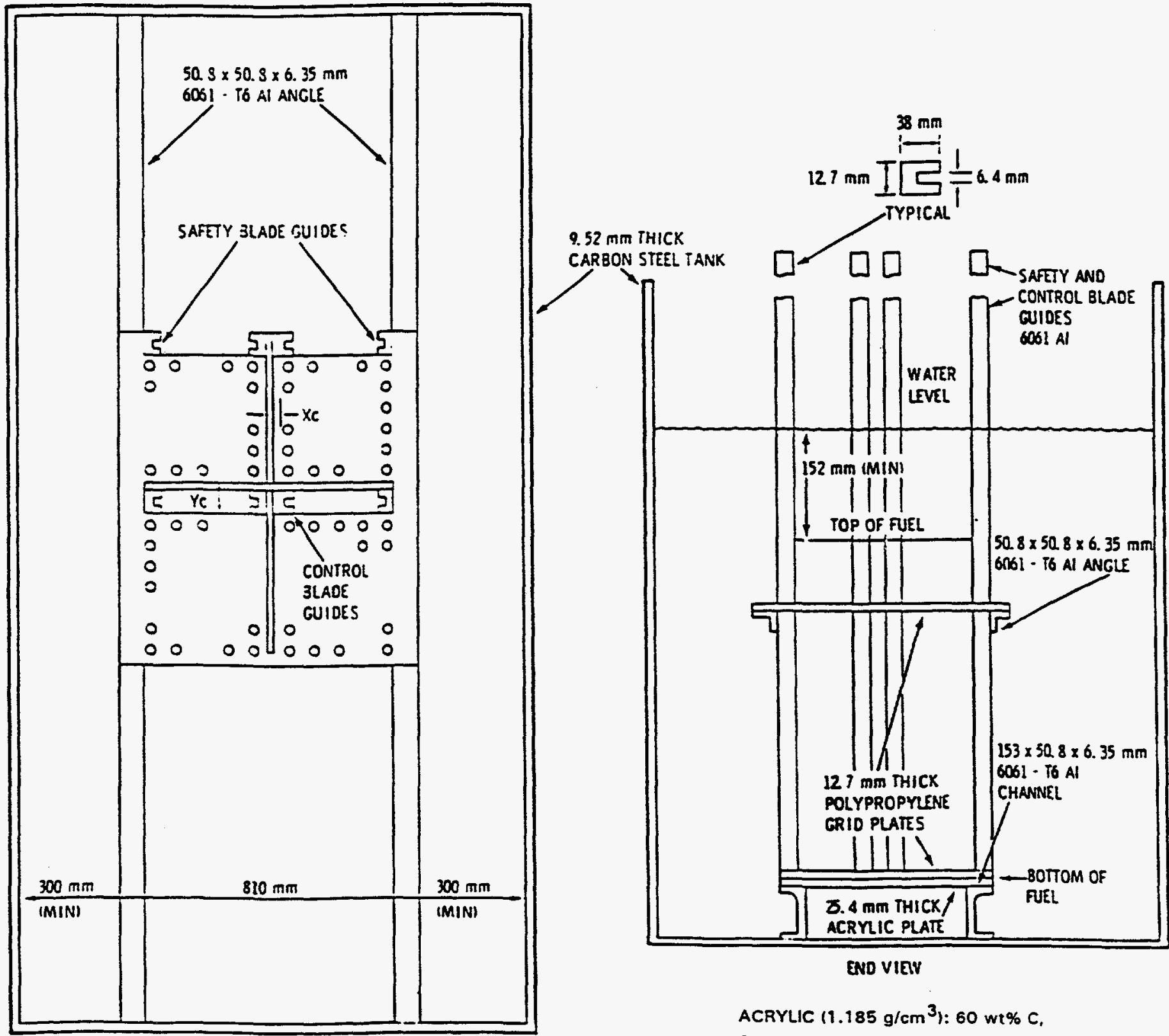

END VIBV

ACRYLIC $\left(1.185 \mathrm{~g} / \mathrm{cm}^{3}\right): 60 \mathrm{wt} \% \mathrm{C}$,

32 wt\% $O$, and 8 wt\% H.

POLYPROPYLENE $10.904 \mathrm{~g} / \mathrm{cm}^{3}$ )

Figure 2.15 Planar and end view of experiments with four-assembly geometry from PNL-3314. Source:

Ref. 11 (Reprinted with permission from author) 


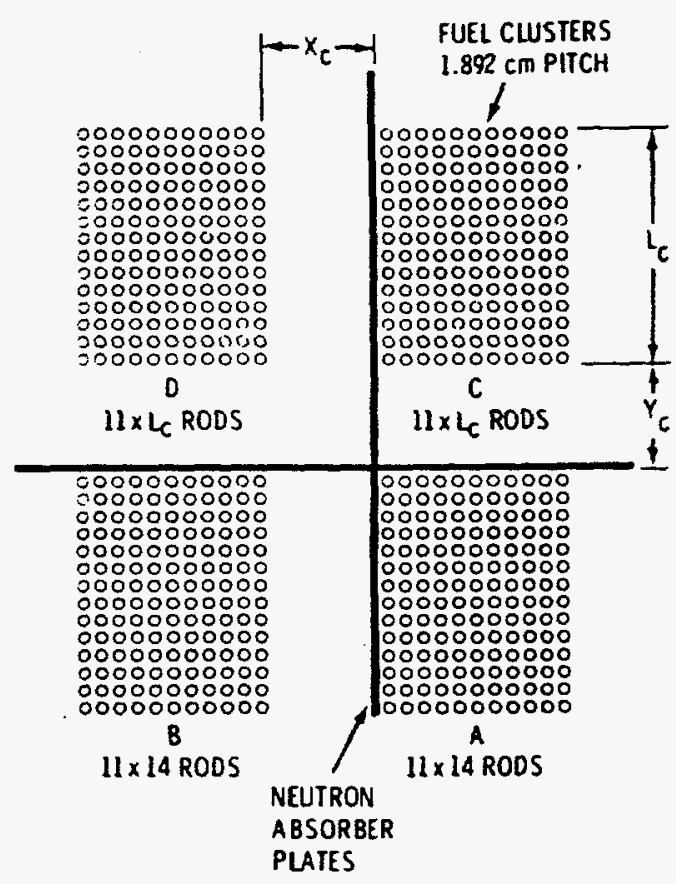

(a) $2 \times 2$ assemblies and separator plates: boral, boroflex, cadmium

(c) $2 \times 2$ assemblies and separator plates: aluminum, Zircaloy-4, 304-L steel, copper

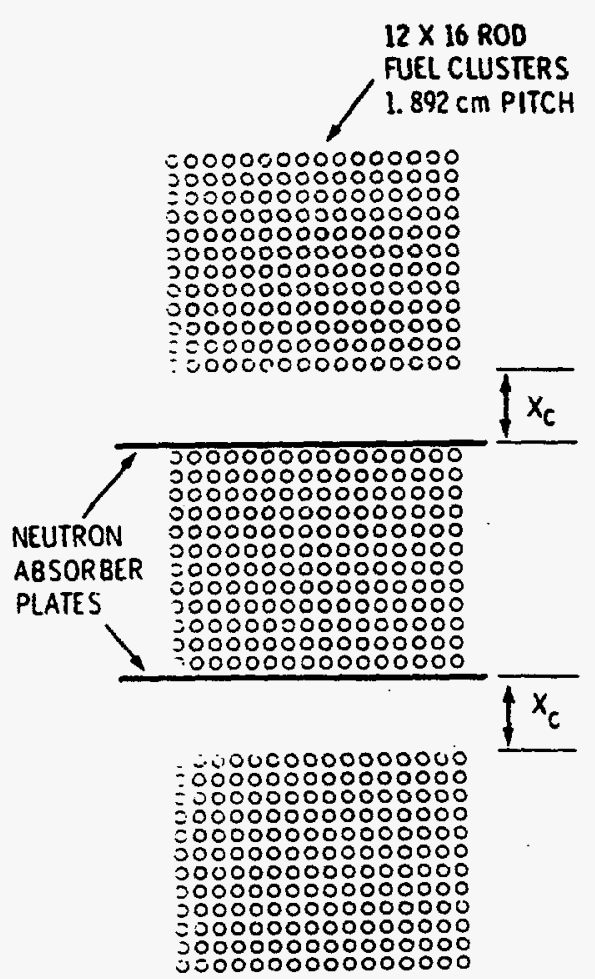

(b) $3 \times 1$ assemblies

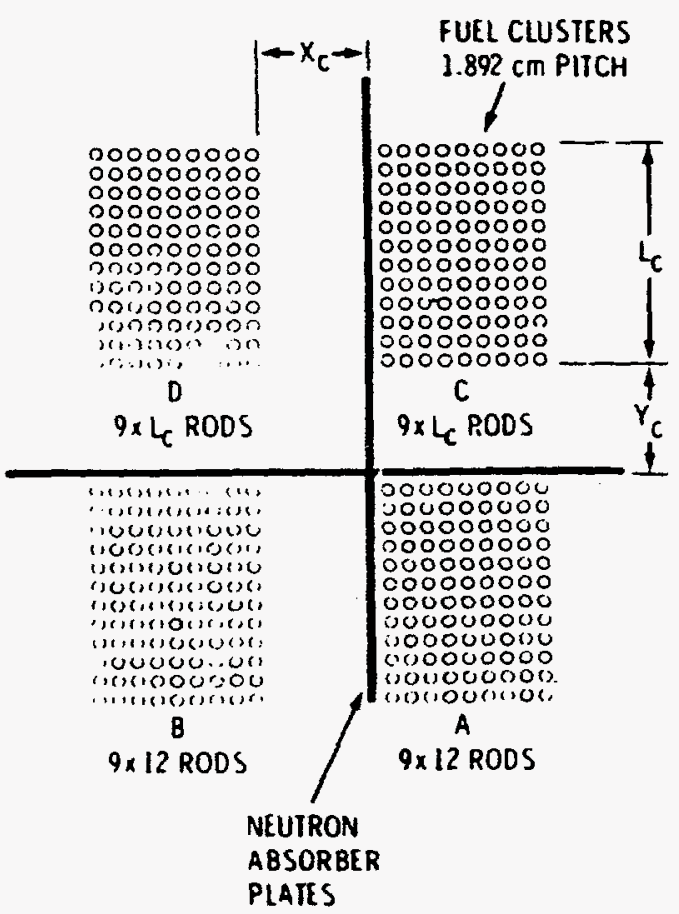

Figure 2.16a Fuel assemblies and absorber plate configurations for experiments with $4.31 \mathrm{wt} \% \mathrm{UO}_{2}$ fuel rods from PNL-3314. Source: Ref. 11 (Reprinted with permission from author) 
Physical Description
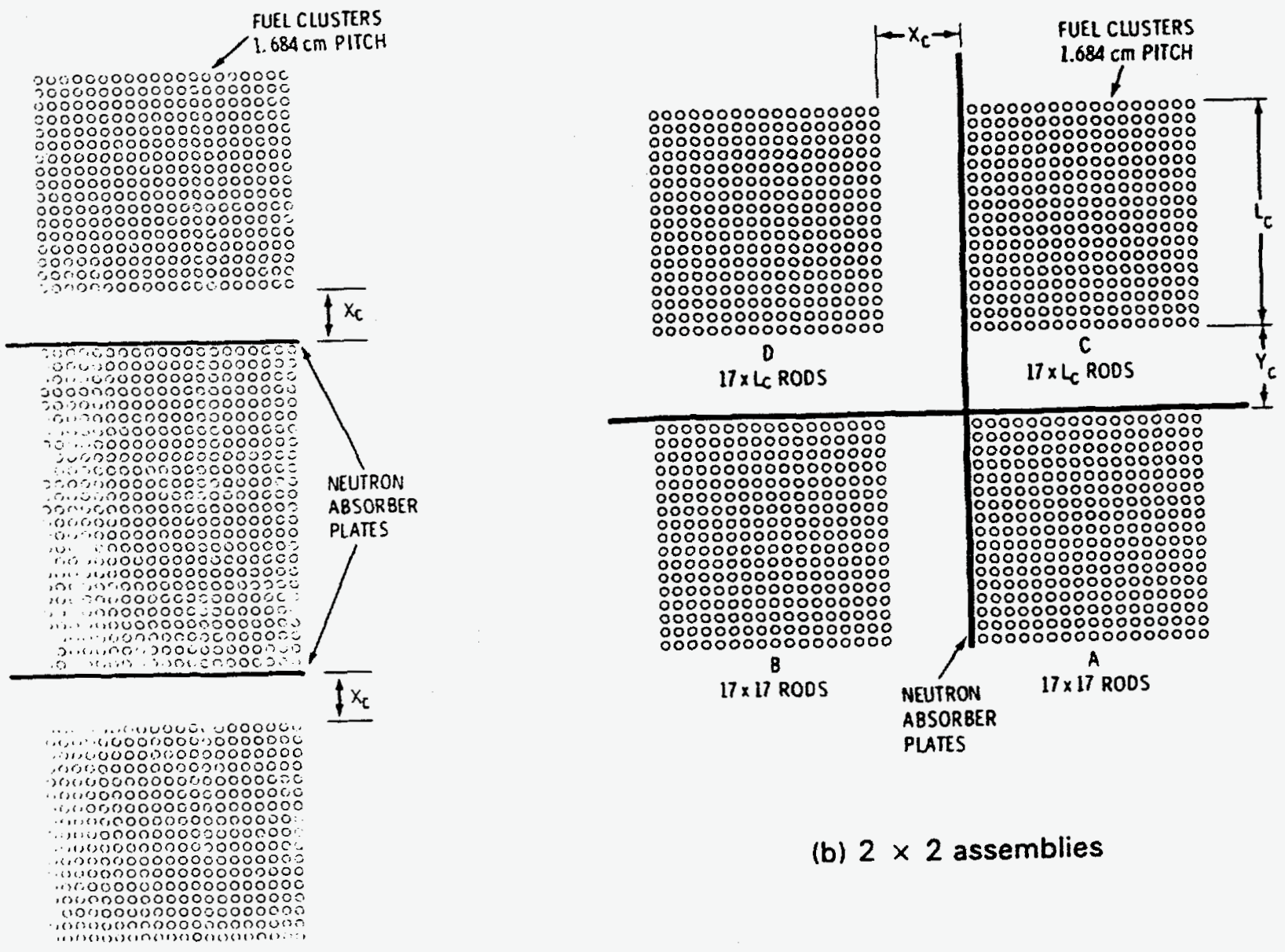

(a) $3 \times 1$ assemblies

(b) $2 \times 2$ assemblies

Figure 2.16b Fuel assemblies and absorber plate configurations for experiments with $2.35 \mathrm{wt} \% \mathrm{UO}_{2}$ fuel rods from PNL-3314. Source: Ref. 11 (Reprinted with permission from author) 
Physical Description
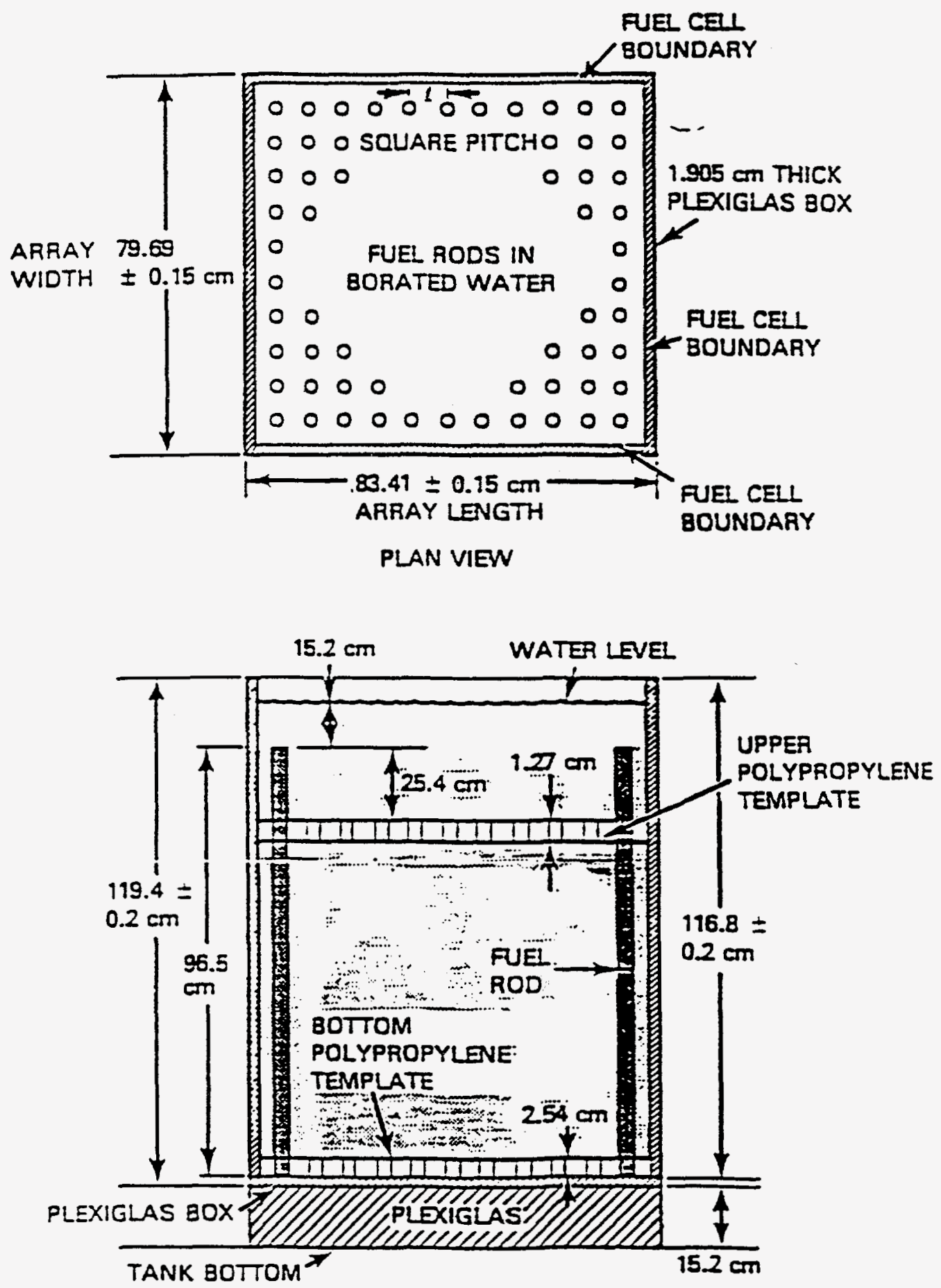

ELEVATION VIEN

Figure 2.17 Experimental setup for experiments from PNL-4267. Source: Ref. 13 (Reprinted with permission from author) 
Physical Description

II

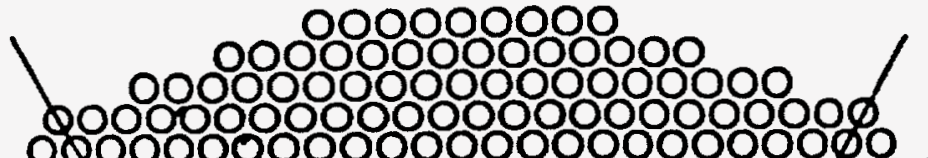

000000000000000000000000

o009000000000000000000\%000

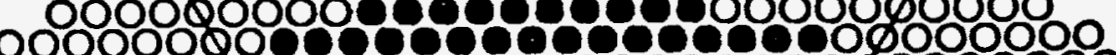

0000000101000000000000000

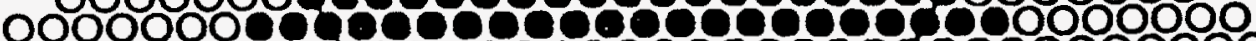

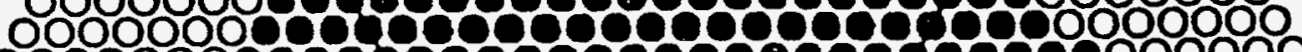

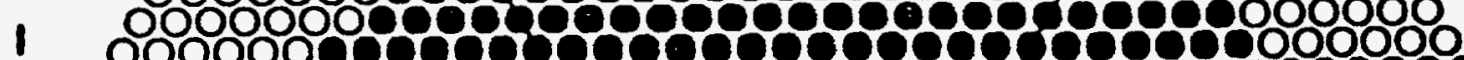

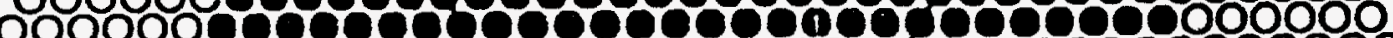

0000001190101001000000000

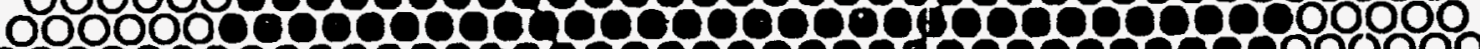

000000000000010000000100000000000

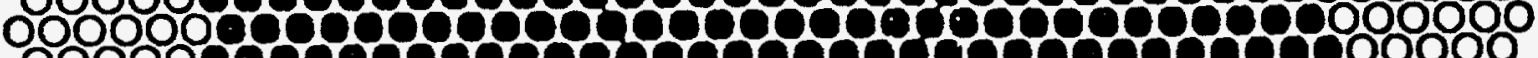

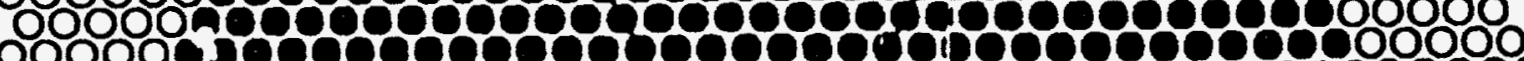

ooooof

0000010000000000100100000000000

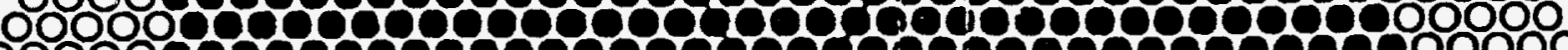

00000000000000001000000000000

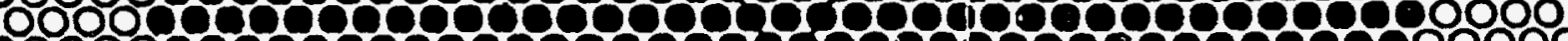

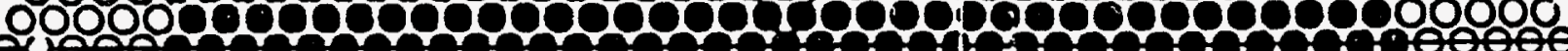

00000000000000000000000000000

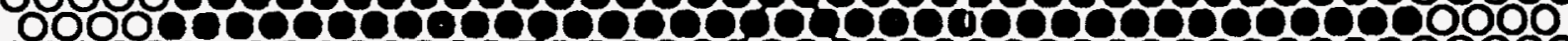

000000000000000000010000000000

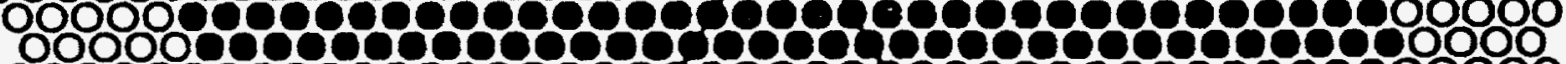

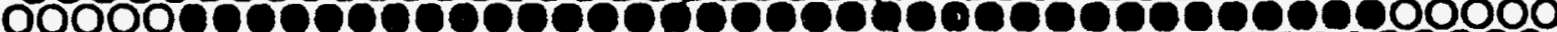

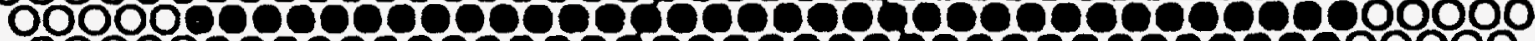

00000000000000000000000000000

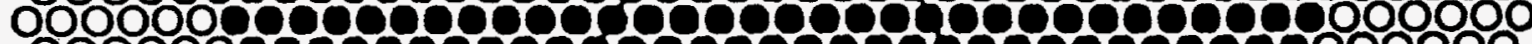

000000000000000100000001000000000000

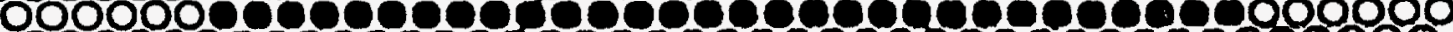

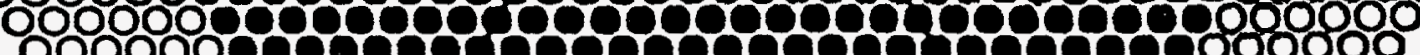

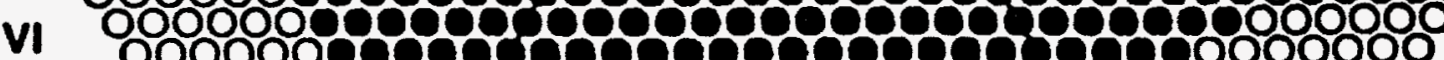

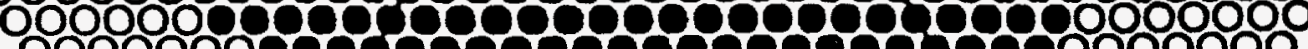

IV

000000000000000000100000000

0000000000000000000000000

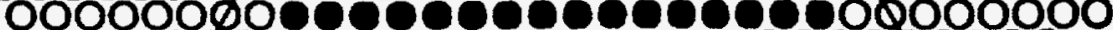

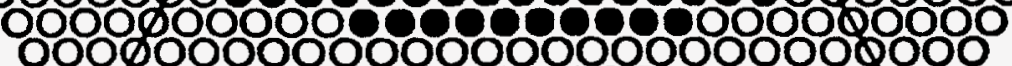

$0 \varnothing 00000000000000000000000$

$\varnothing 00000000000000000000004$

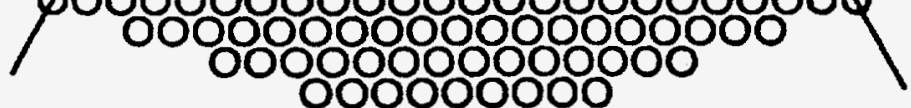

V

Figure 2.18 Core assembly for P49-194. Source: Ref. 14 (Reprinted with permission from author) 
Physical Description

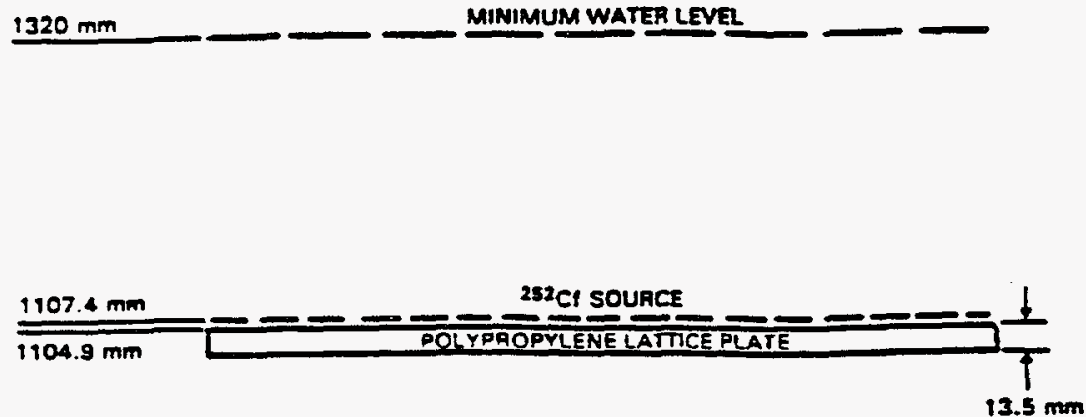

$848.49 \mathrm{~mm} \quad-\quad$ BOTTOM OF $8.35 \mathrm{~mm}$ THICX TVEE 6081 AI SPACEAS

$701.68 \mathrm{~mm}$
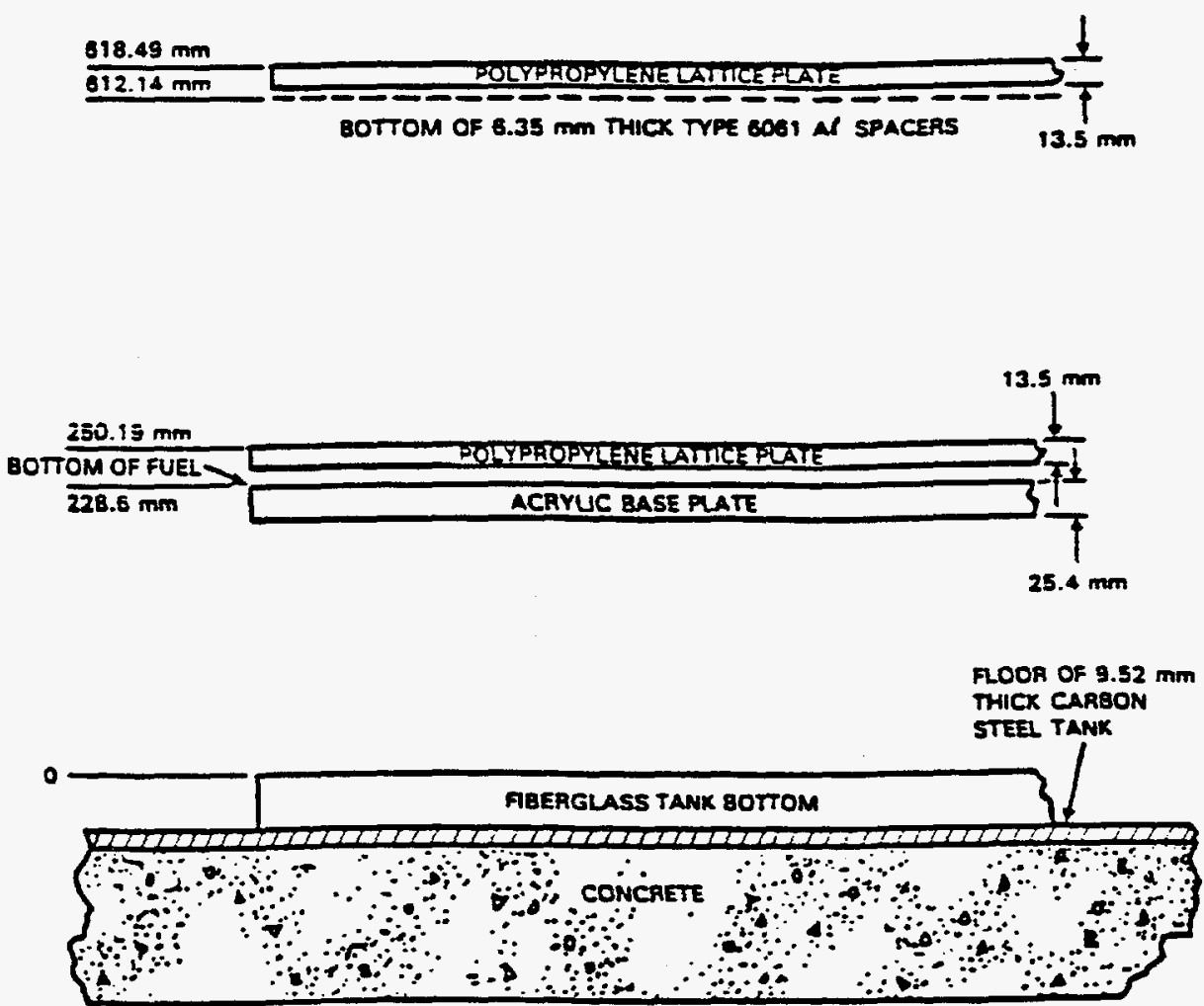

Figure 2.19 Experimental assembly elevations for P49-194. Source: Ref. 14 (Reprinted with permission from author) 
Physical Description

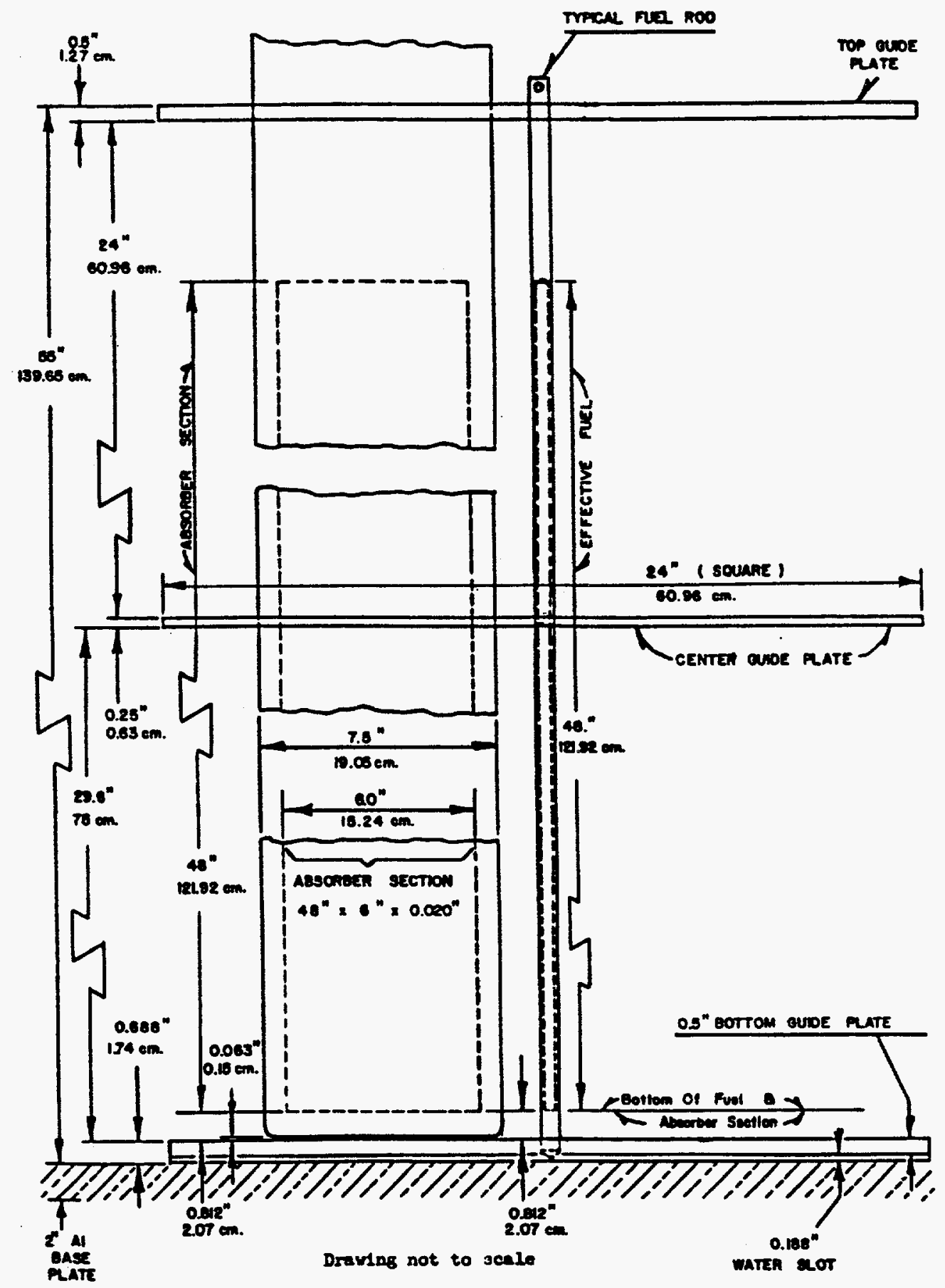

Figure 2.20 Vertical dimensions of experimental assembly for W3269SL1. Source: Ref. 15 
Physical Description

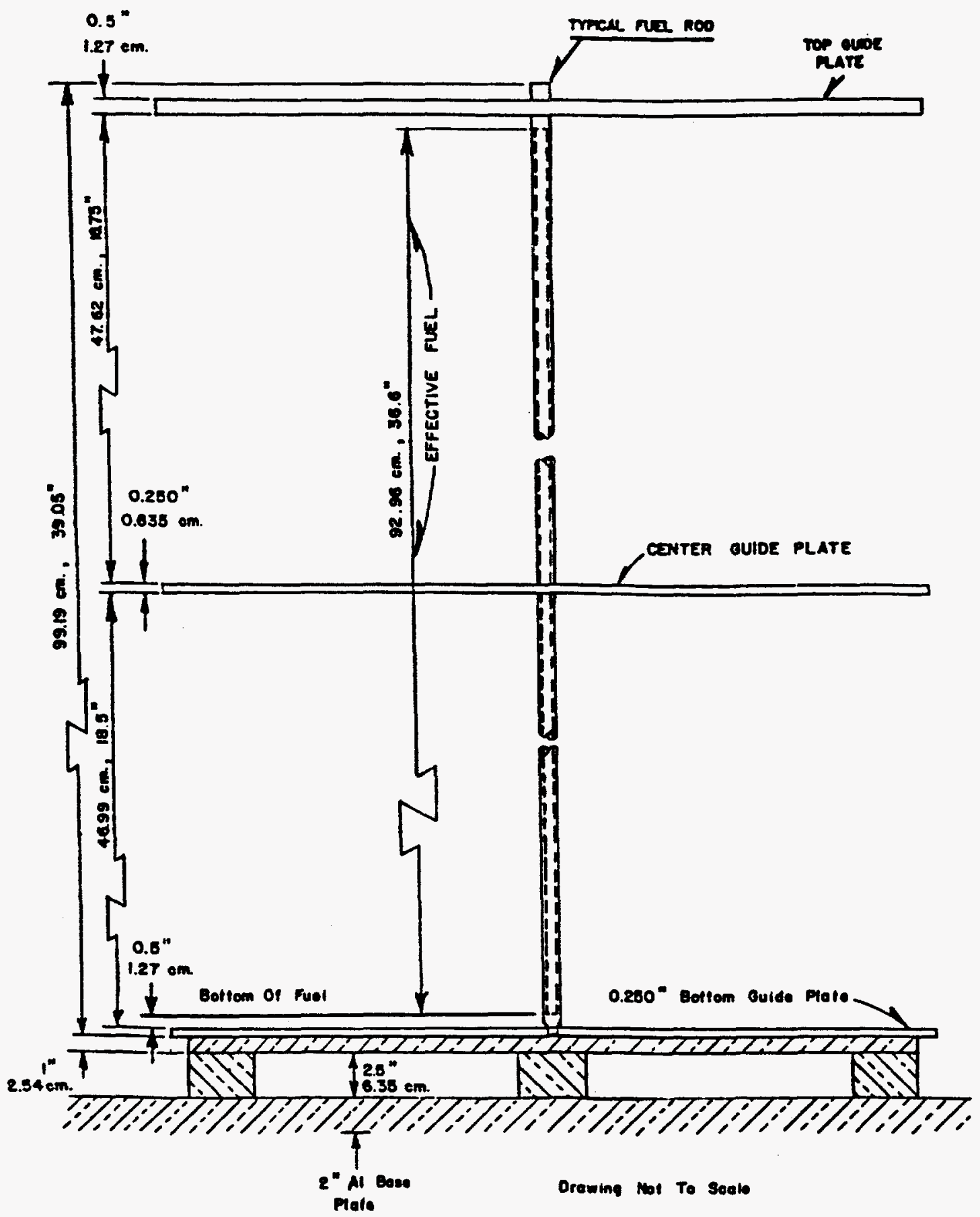

Figure 2.21 Vertical dimensions of experimental assembly for W3269SL2. Source: Ref. 15 
Physical Description

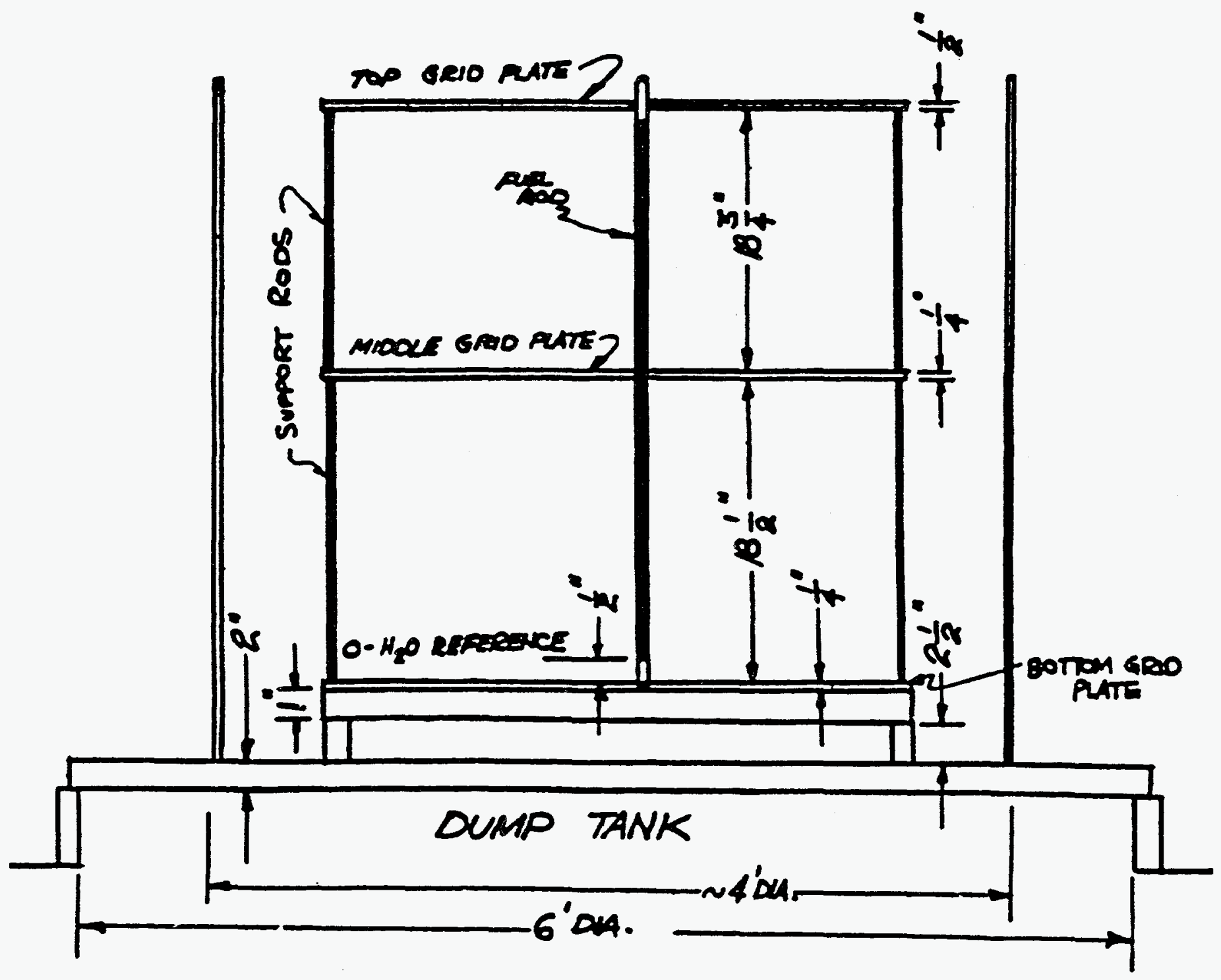

Figure 2.22 Vertical dimensions of experiments from WCAP-3385. Source: Ref. 16 
Physical Description

Top and bottom grid plate

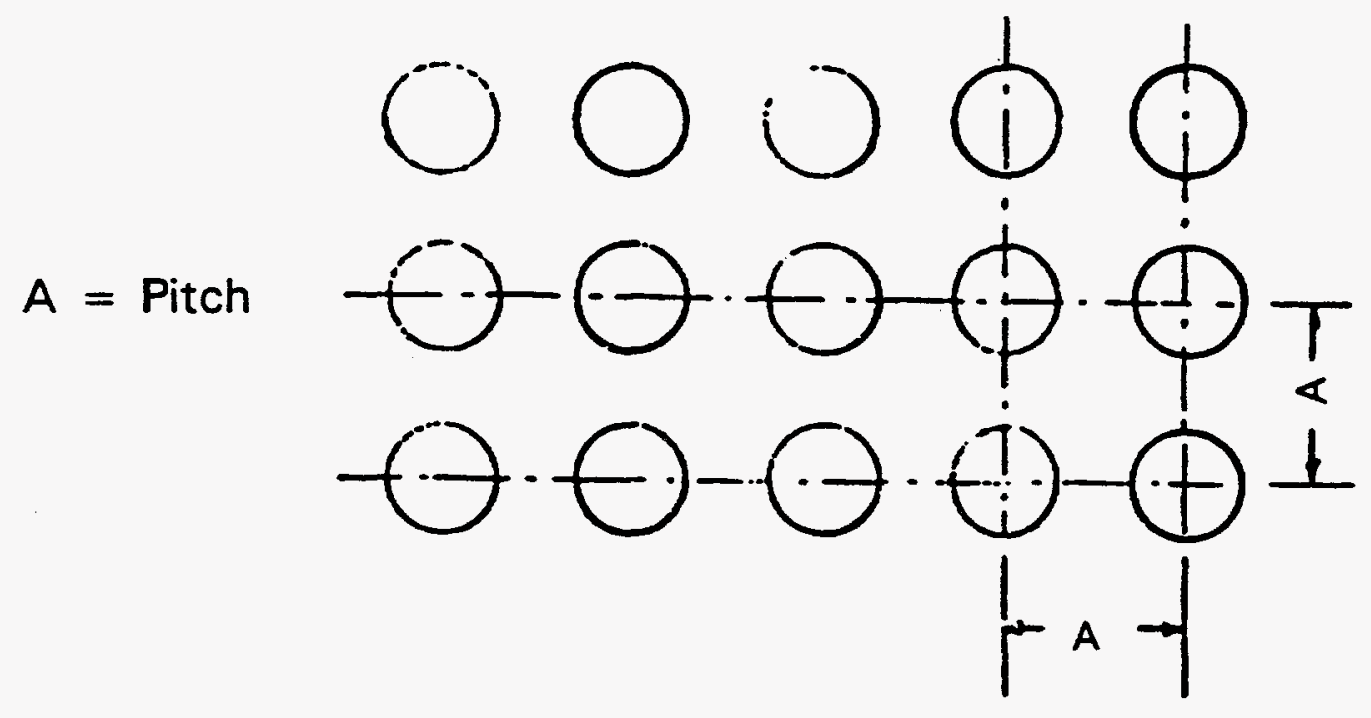

Fuel rod holes $=0.397 \pm 0.002$ in. diam

Center grid plate

$A=$ Pitch

$B=1 / 2 A$

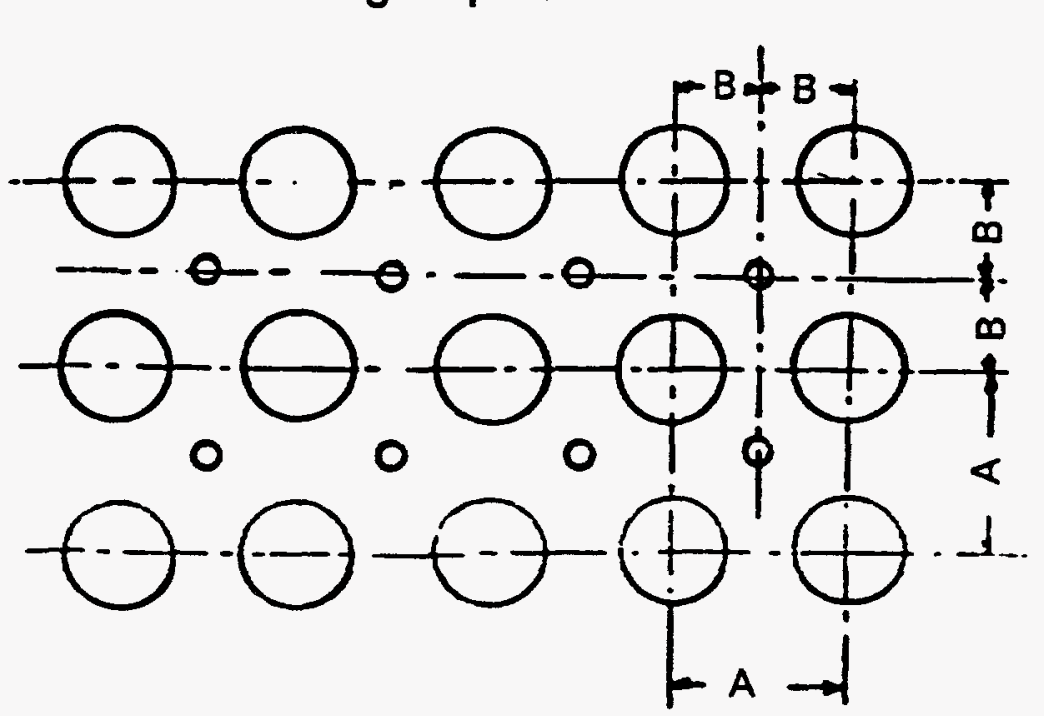

Fuel rod holes $=0.397 \pm 0.002$ in. diam Circulation holes $=0.193 \pm .0 .001$ in. diam

Figure 2.23 Grid plate layouts for experiments from WCAP-3385. Source: Ref. 16 
Physical Description

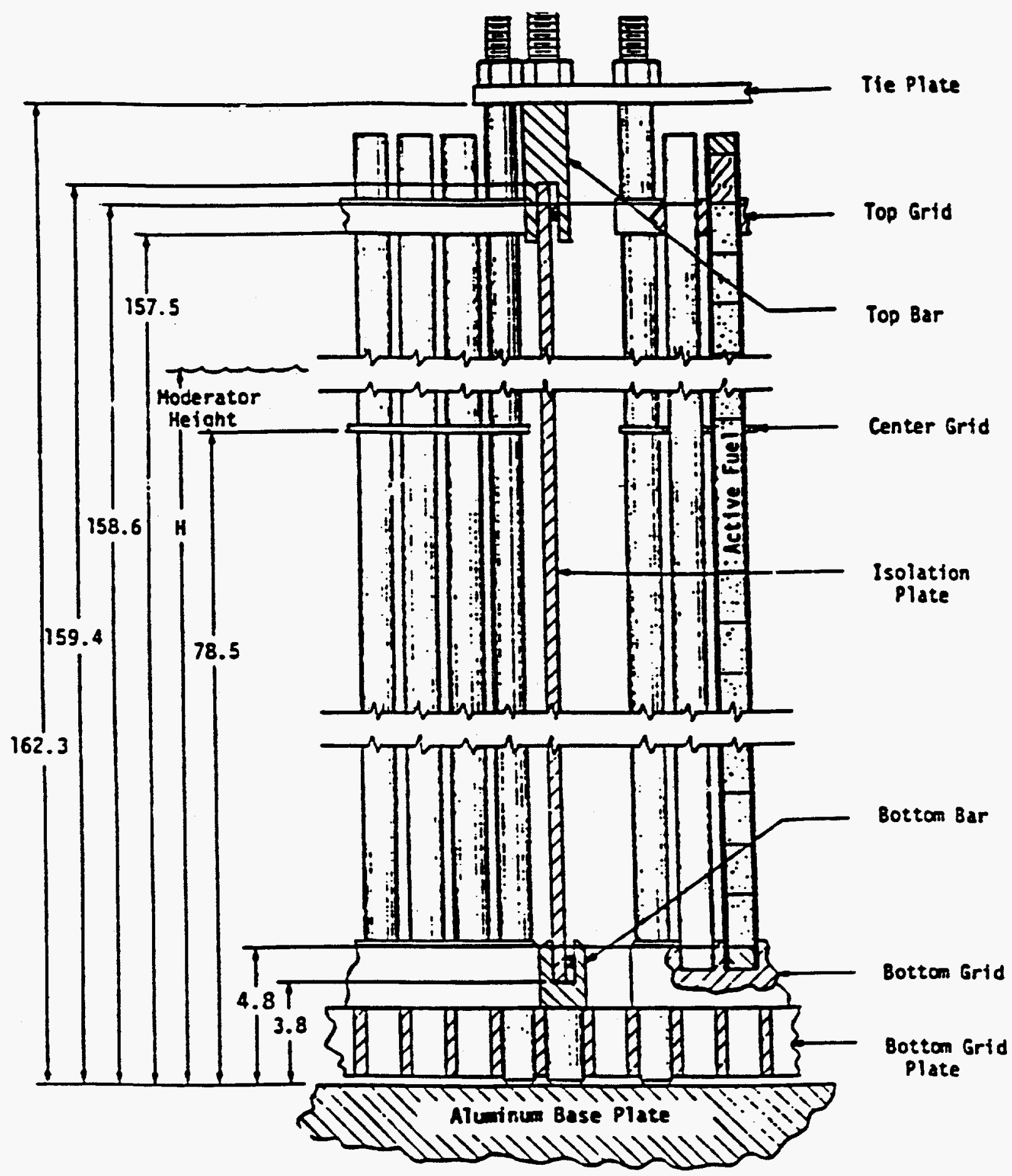

Note: Dimensions given In centimeters.

Figure 2.24 Vertical dimensions for separator plate experiments from BAW-1484. Source: Ref. 6 
Physical Description

SIDE SHEET LOCATIONS (24 PLACES)

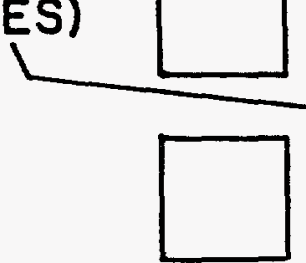

W
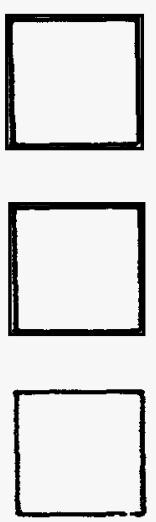

$N$
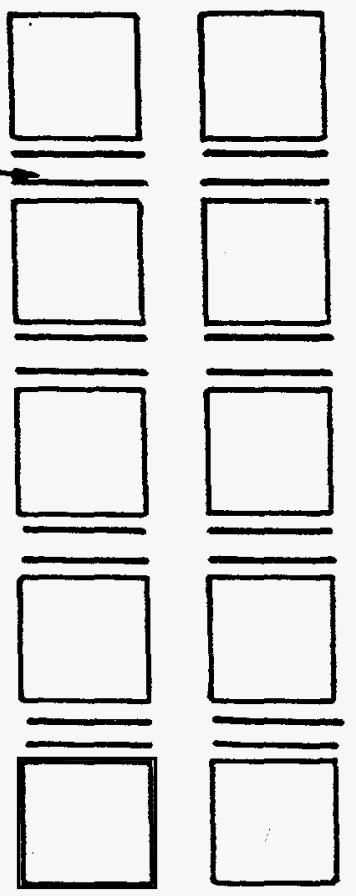
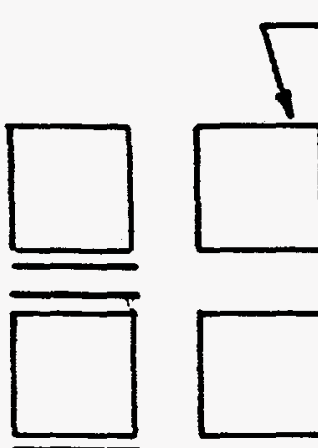

FUEL MODULE
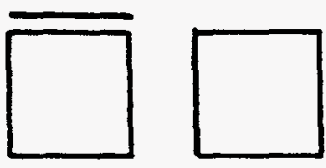

$\mathbf{E}$
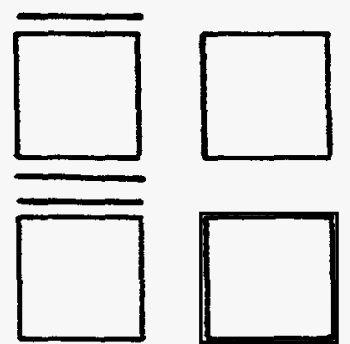

S

STAGGERED-ROW EDGES ALWAYS FACED EAST - WEST ( $T$-TYPE MODULES ONLY)

Figure 2.25 Side sheet locations for separator plate experiments from BAW-1645. Source: Ref. 17 
Physical Description

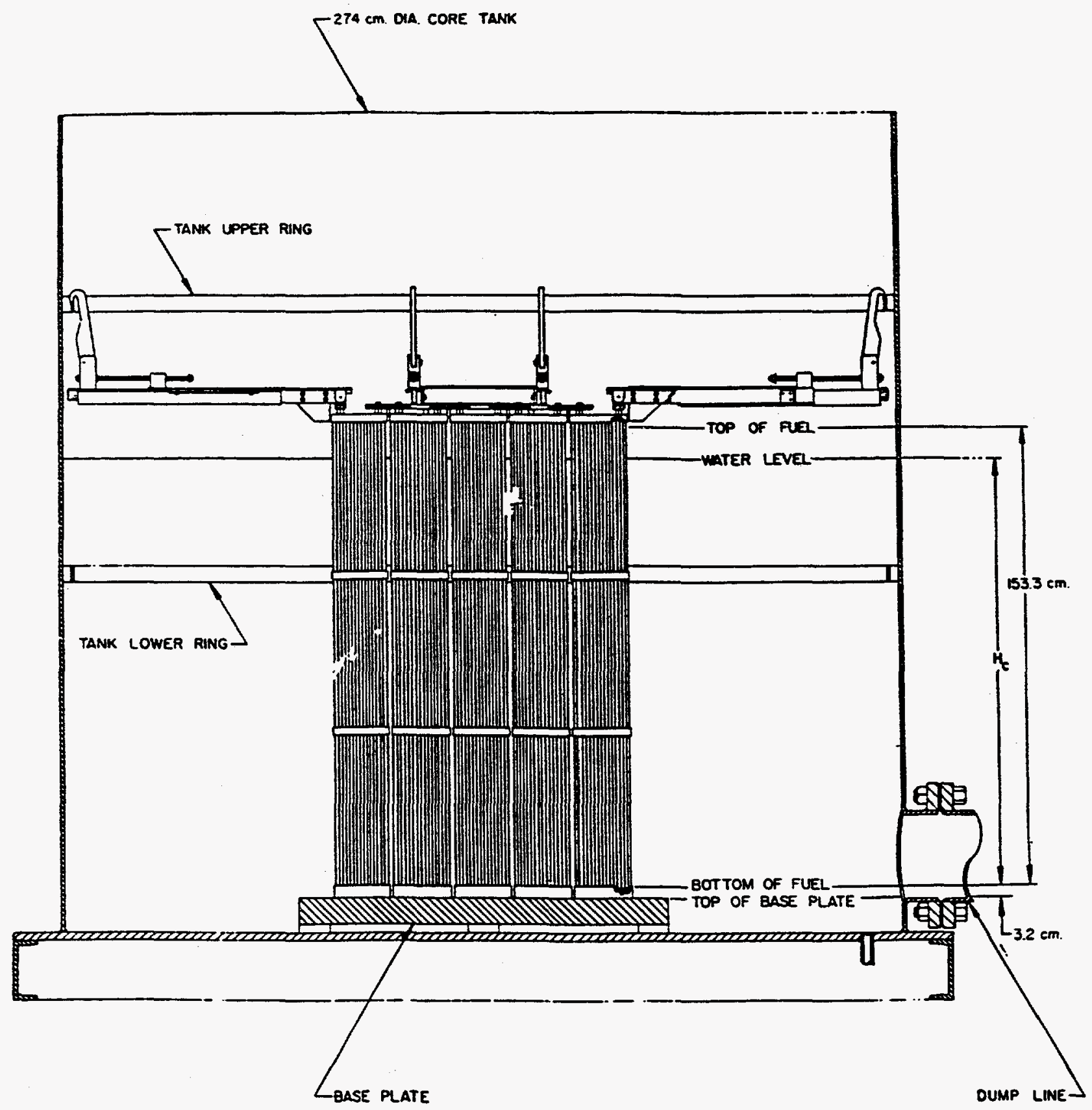

Figure 2.26 Vertical dimensions of experiments from BAW-1645. Source: Ref. 17 
Physical Description

$T$ - TYPE INTERMODULAR SPACING QUOTED AS $a \times b$
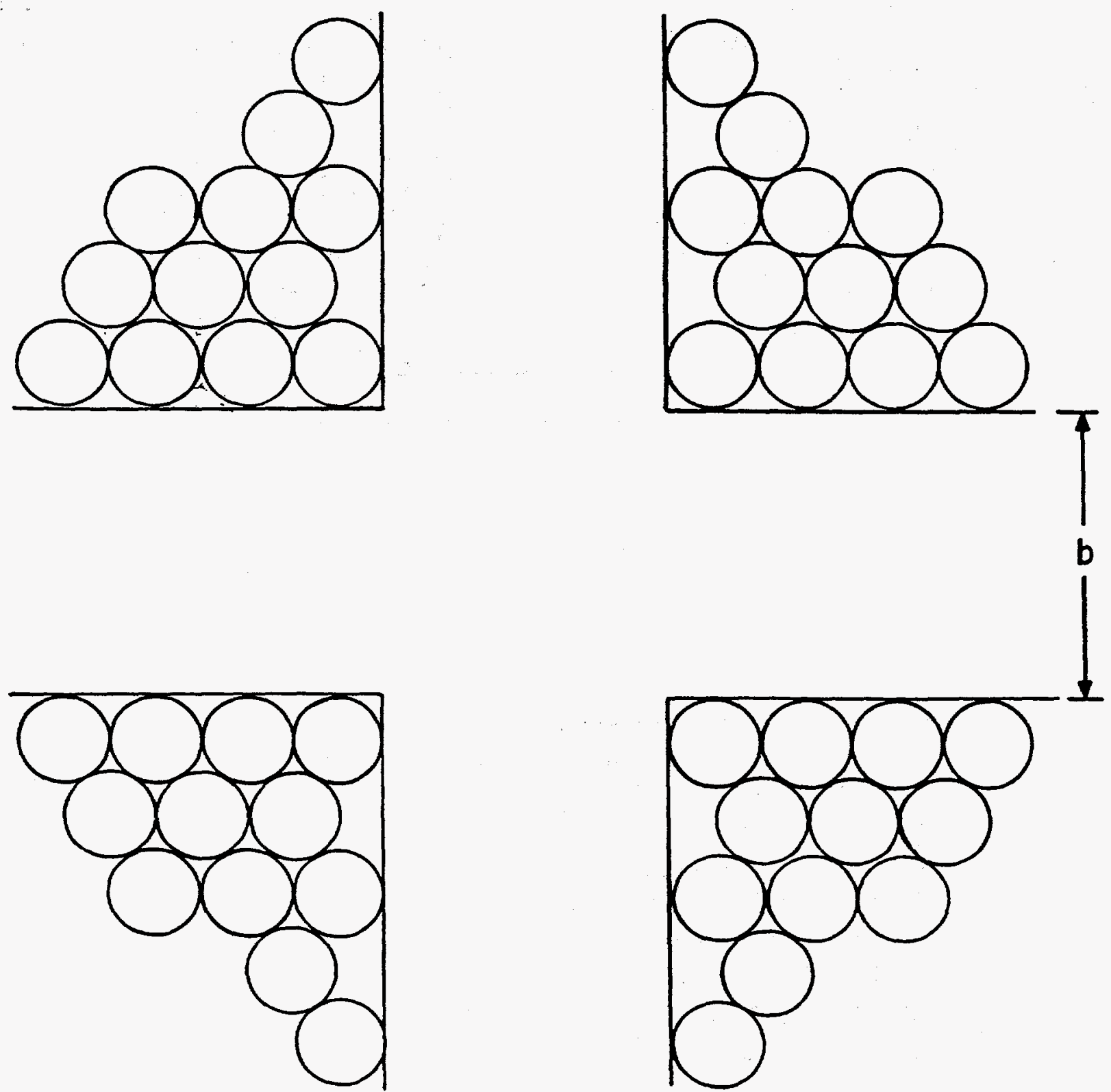

10

Figure 2.27 Intermediate spacing for BW1645T1, BW1645T2, BW1645T3, and BW1645T4. Source: Ref. 17 


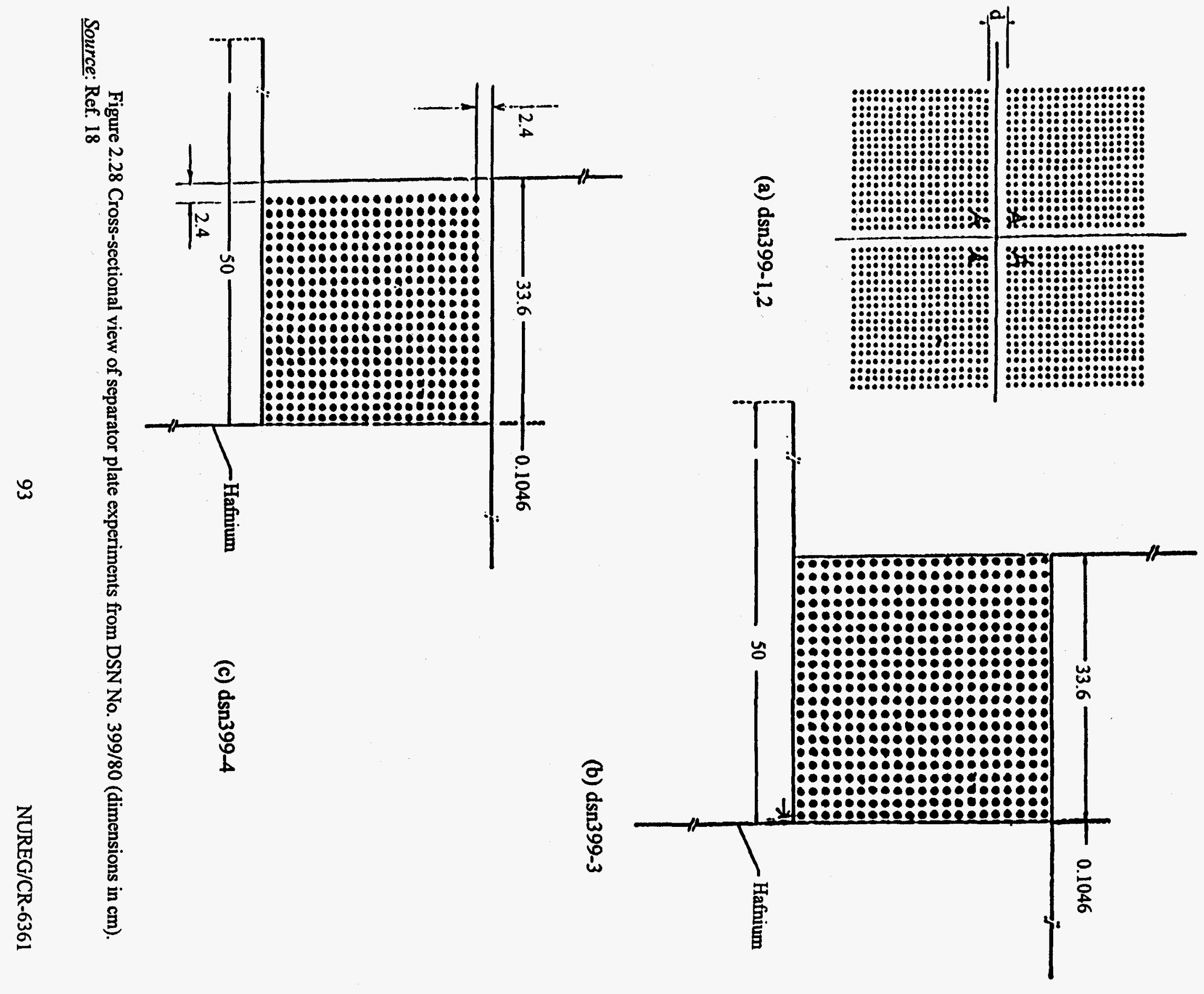


Physical Description

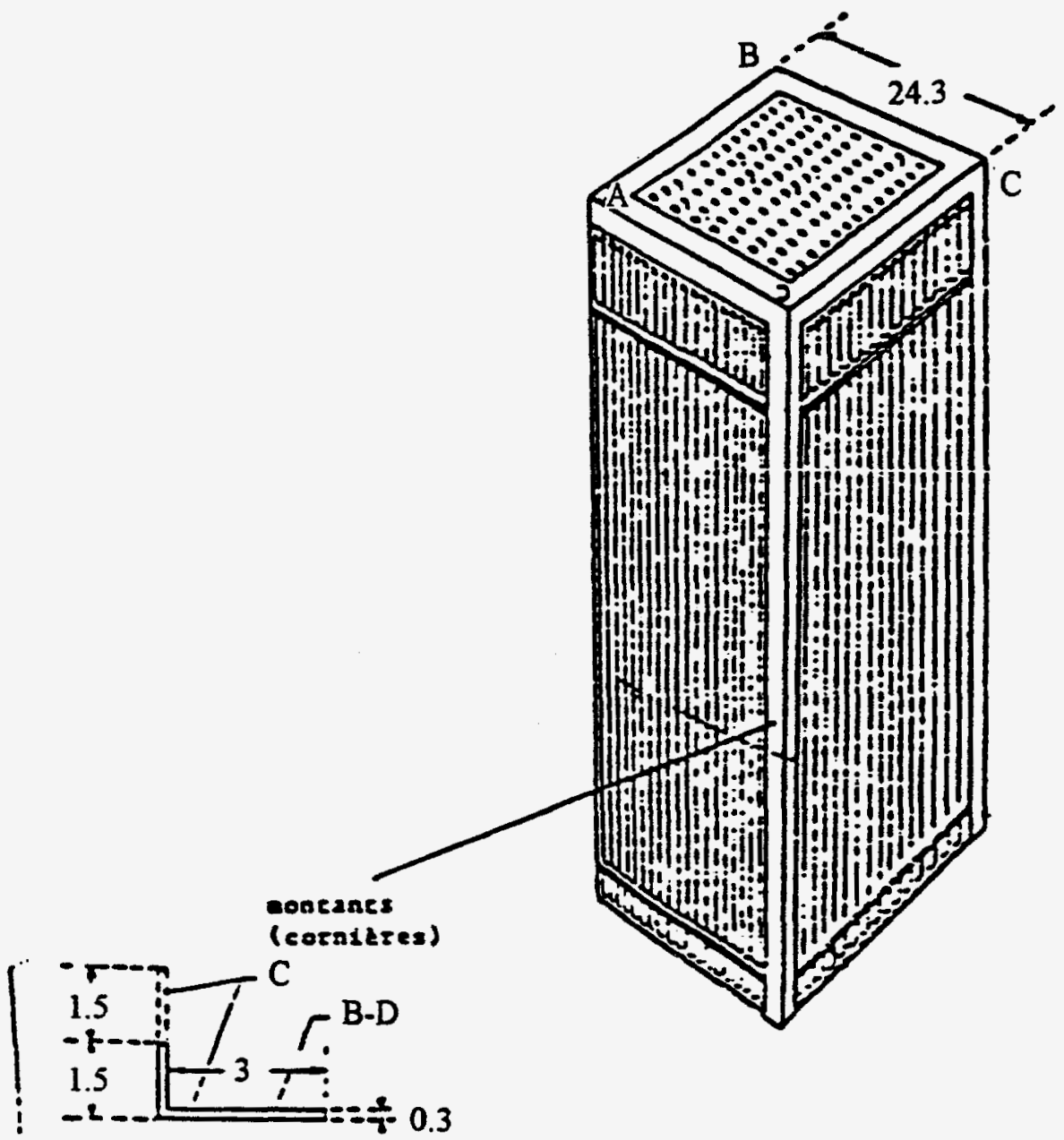

Figure 2.29 Fuel cluster and steel angle iron for DSN399-1 and DSN399-2 (dimensions in cm). Source: Ref. 18 
Physical Description

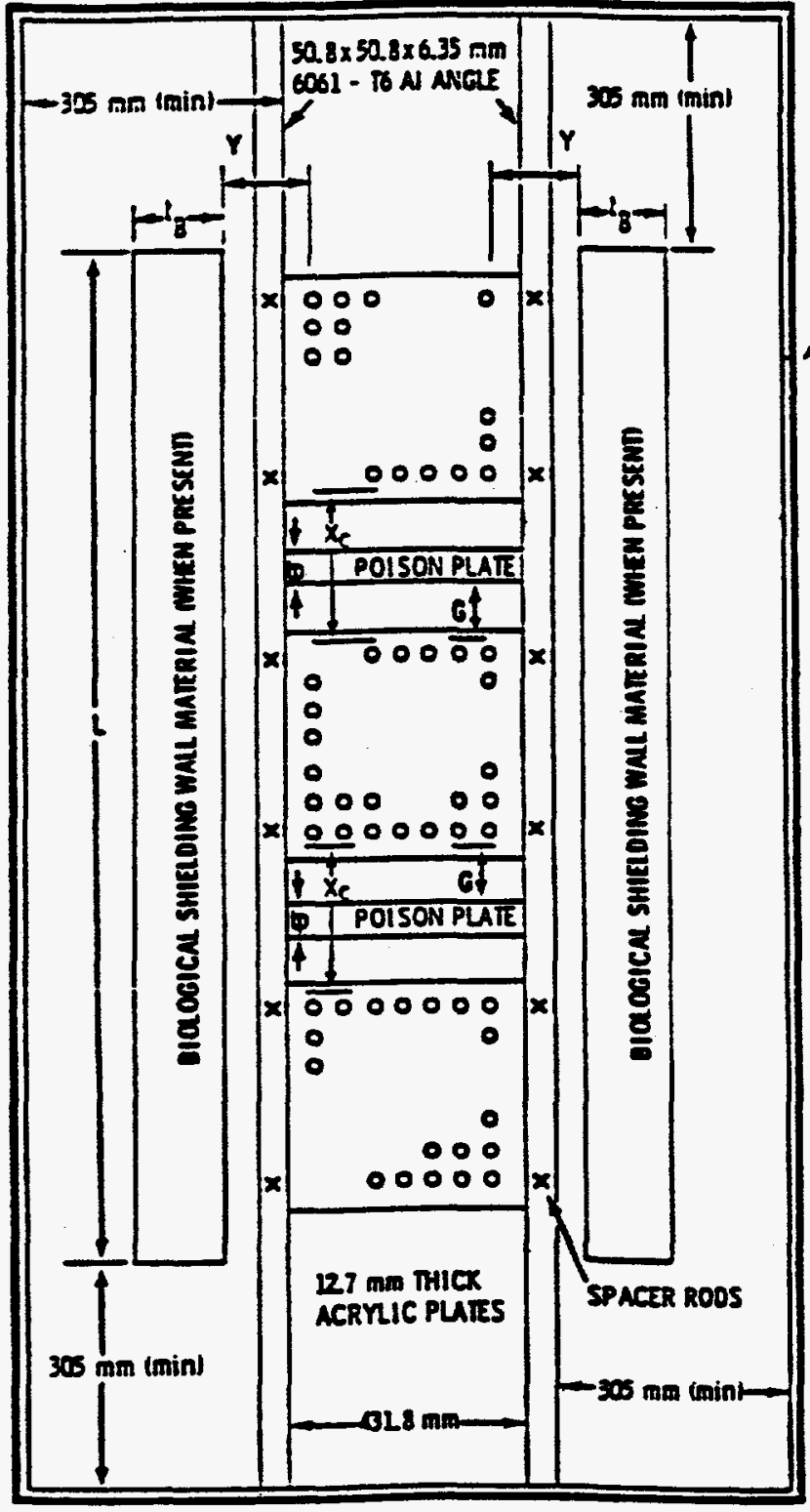

ruW VIaW

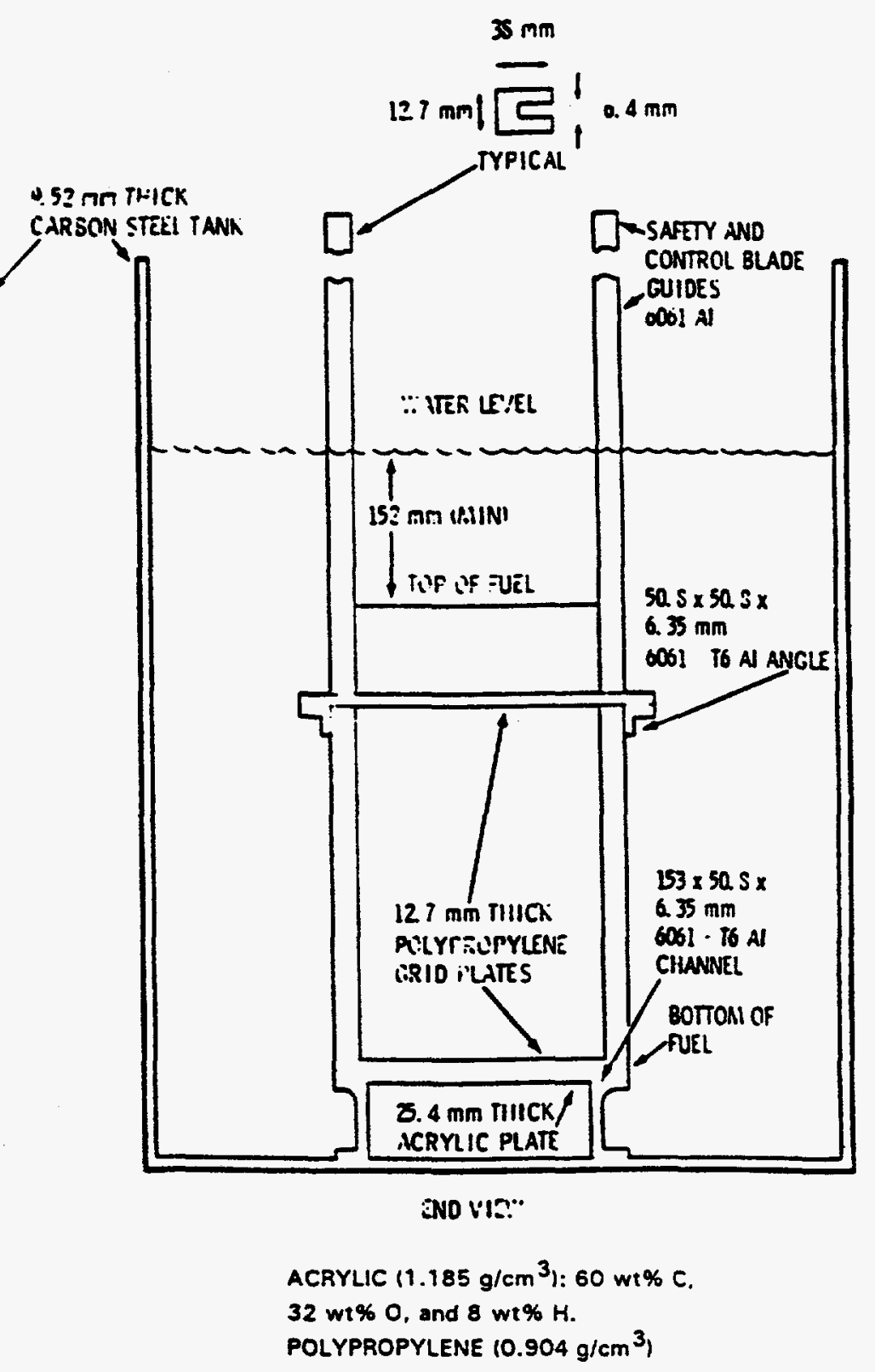

Figure 2.30 Planar and end view of experiments with three-assembly geometry from PNL-3314.

Source: Ref. 11 
Physical Description

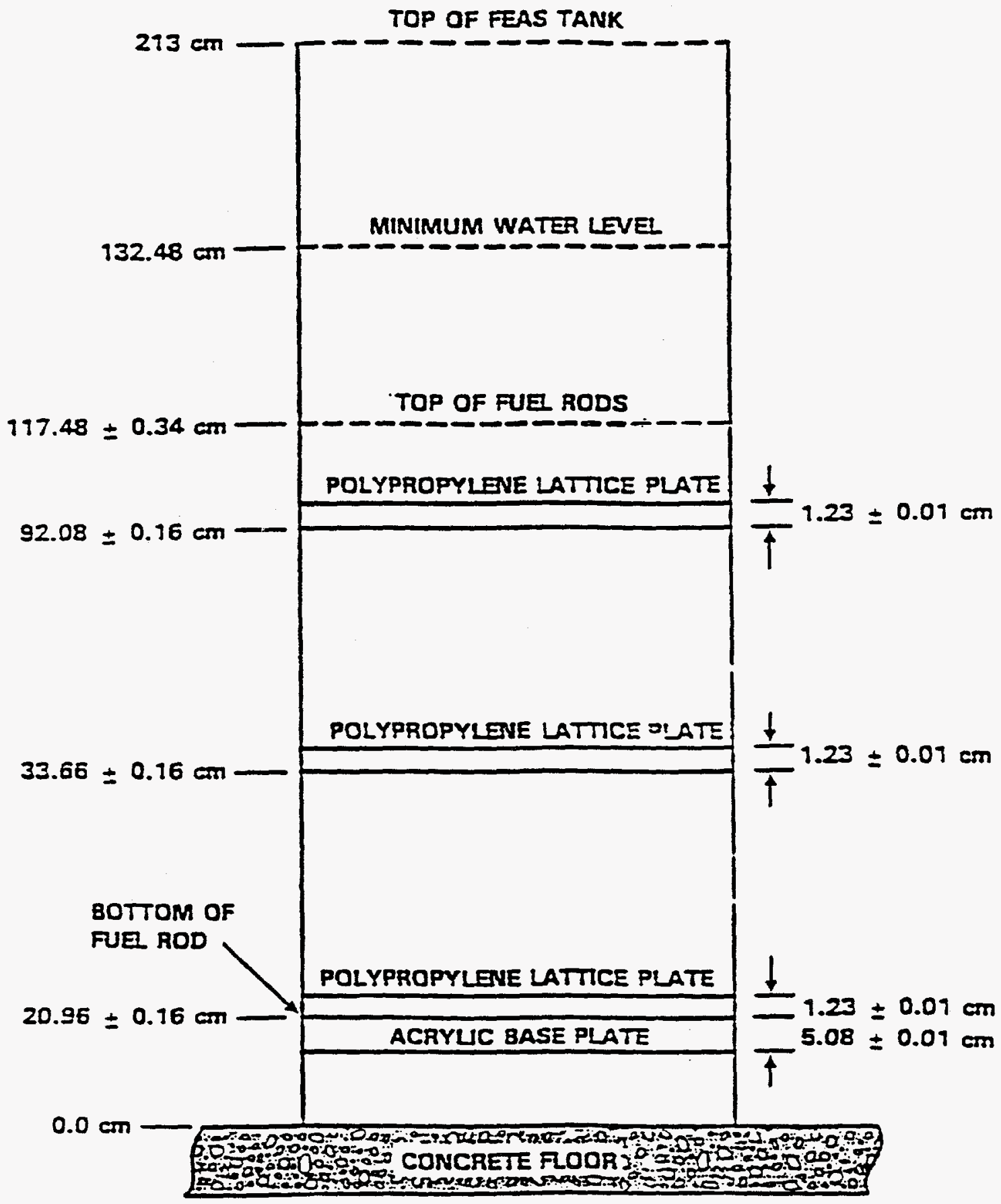

Figure 2.31 Experimental assembly evaluations for experiments from PNL-6205 and PNL-7176. Source: Refs. 20 and 21 (Reprinted with permission from authors) 


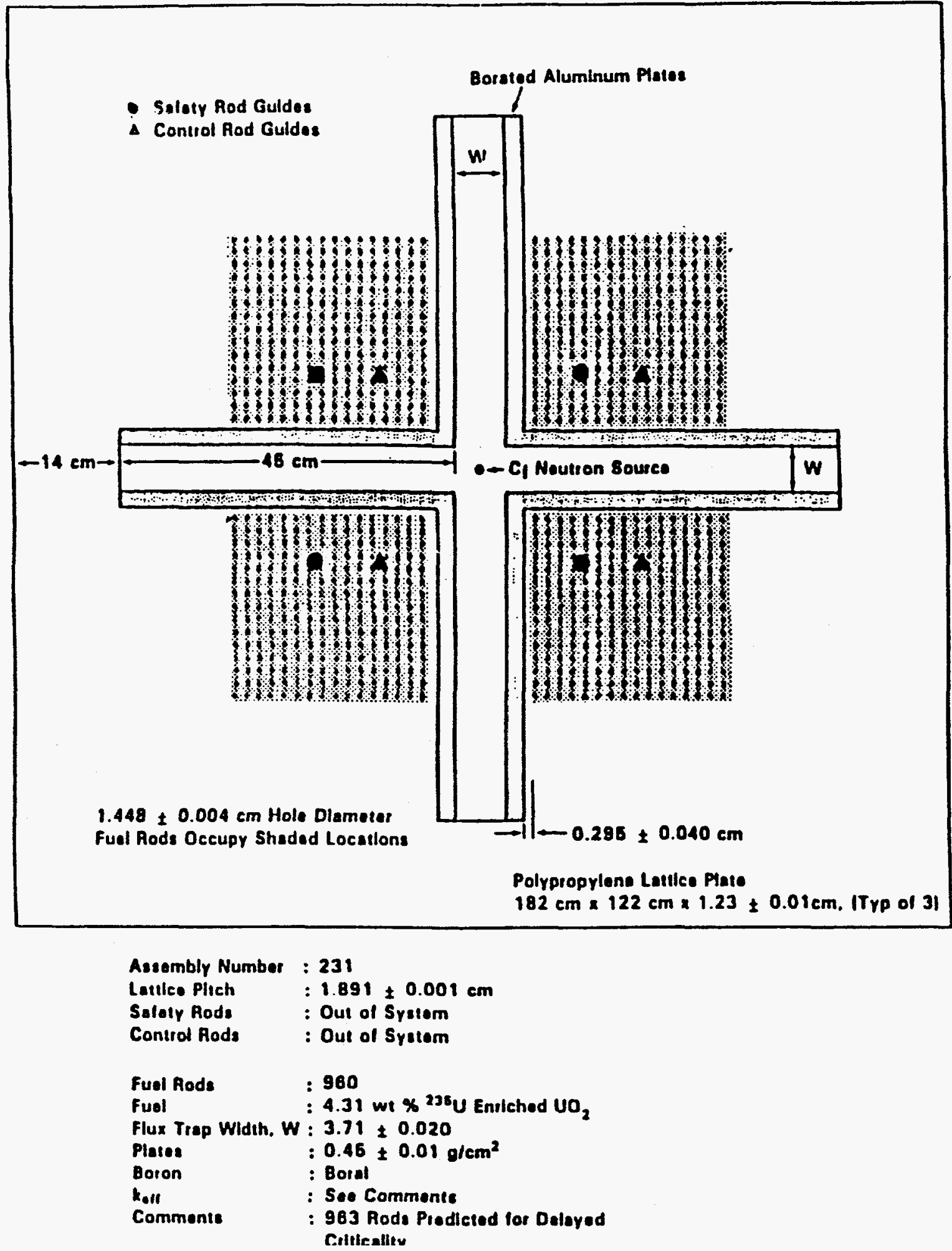

Figure 2.32 Experimental assembly for P62FT231. Source: Ref. 20 (Reprinted with permission from author) 
Physical Description

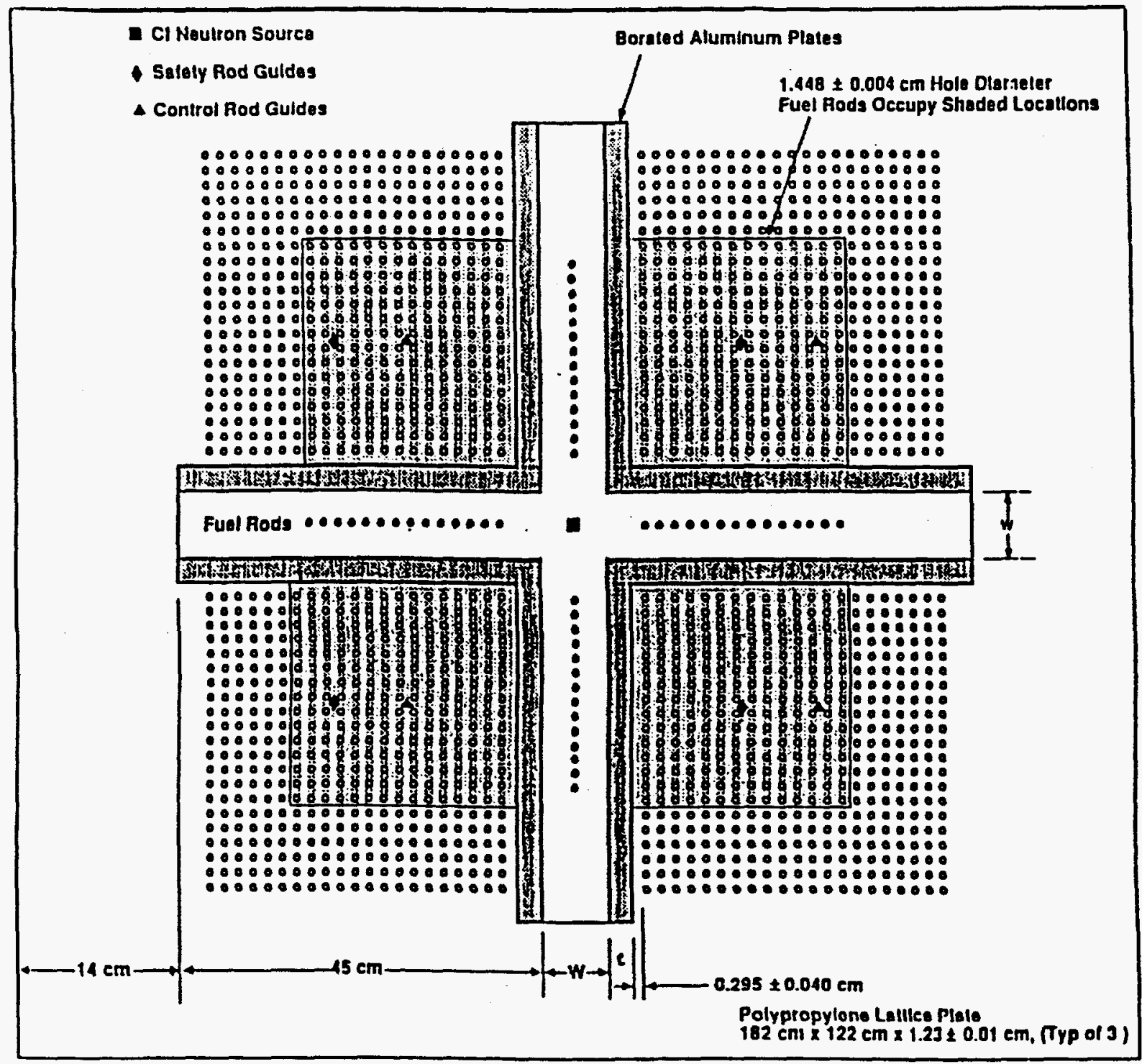

\begin{tabular}{|c|c|}
\hline $\begin{array}{l}\text { Assemoly Mumber } \\
\text { Lattlee Plich } \\
\text { Salely fods } \\
\text { Conlrol Rods }\end{array}$ & $\begin{array}{l}: 2191-3 \\
1.891 \pm 0.001 \mathrm{~cm} \\
\text { Oul of System } \\
\text { : Out of System }\end{array}$ \\
\hline $\begin{array}{l}\text { Fuel Rods } \\
\text { Fuel } \\
\text { Flux Trap Widlh, W } \\
\text { Plates } \\
\text { Goron } \\
\text { Volding Materlal } \\
\text { Commenis }\end{array}$ & 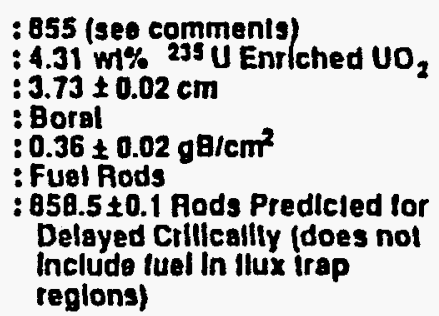 \\
\hline
\end{tabular}

Figure 2.33 Experimental assembly for P71F14F3. Source: Ref. 21 (Reprinted with permission from authors) 

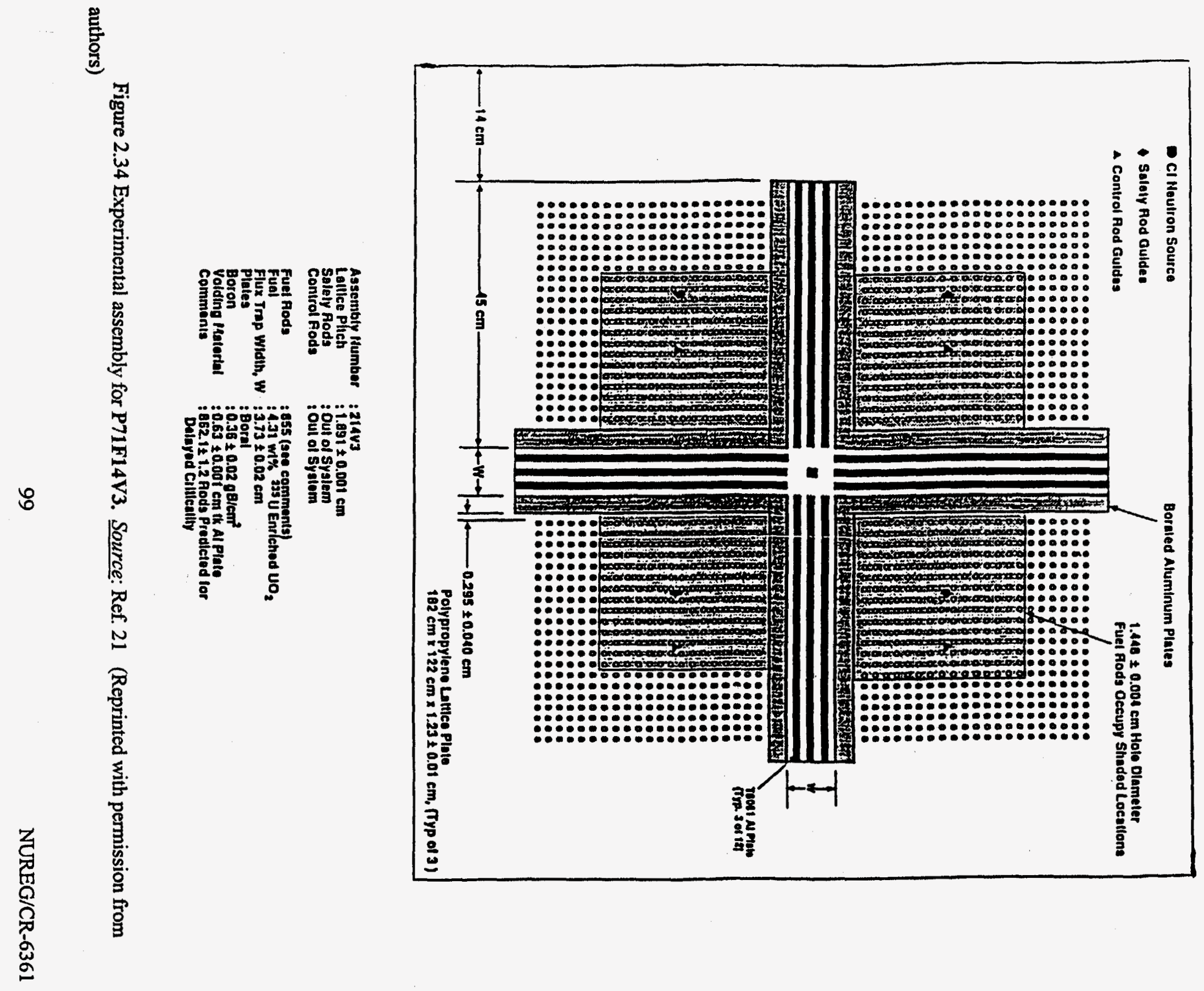
Physical Description

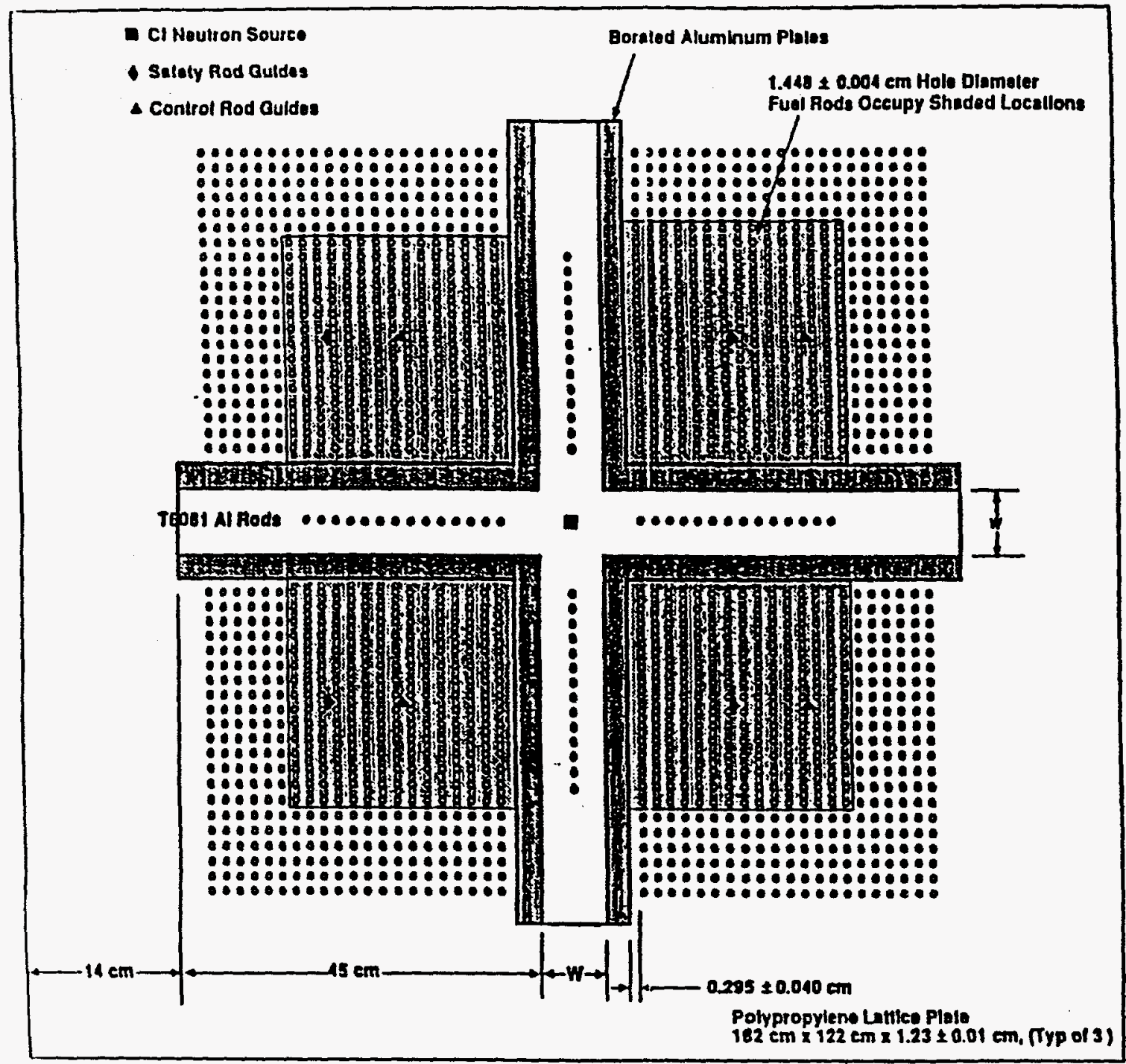

\begin{tabular}{|c|c|}
\hline $\begin{array}{l}\text { Assembly Numbat } \\
\text { Lellice Flleh } \\
\text { Salety Rods } \\
\text { Conirol Fods }\end{array}$ & $\begin{array}{l}: 214 V 5 \\
: 1.891 \pm 0.001 \mathrm{~cm} \\
: \text { Oul of System } \\
\text { : Oul of Syatem }\end{array}$ \\
\hline $\begin{array}{l}\text { Fuel Rods } \\
\text { Fuel } \\
\text { Flux Trip Wndth, W } \\
\text { Plates } \\
\text { Boron } \\
\text { Yolding Hatertal } \\
\text { Comments }\end{array}$ & 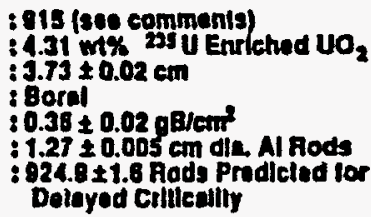 \\
\hline
\end{tabular}

Figure 2.35 Experimental assembly for P71F14V5 


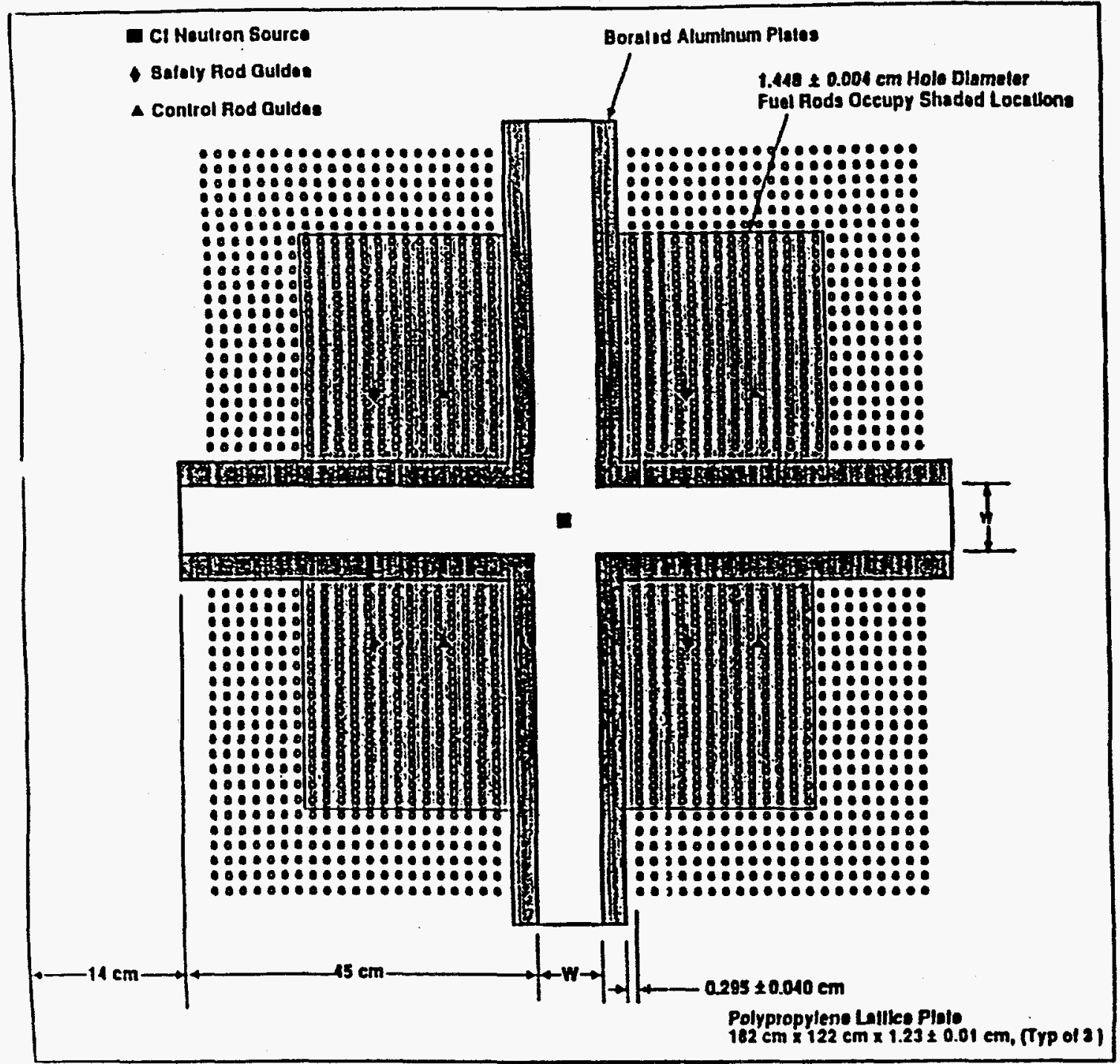

\begin{tabular}{|c|c|}
\hline $\begin{array}{l}\text { Assembly Number } \\
\text { Lsilice Plich } \\
\text { Salely Rods } \\
\text { Control Rods }\end{array}$ & $\begin{array}{l}\text { :214R } \\
1.891 \text { } \pm 0.001 \mathrm{~cm} \\
\text { : Out of Syglem } \\
\text { Oul of Systom }\end{array}$ \\
\hline 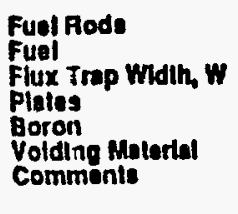 & 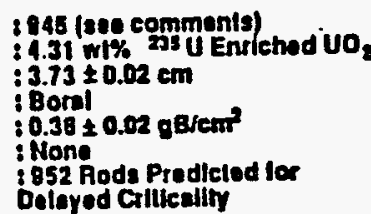 \\
\hline
\end{tabular}

Figure 2.36 Experimental assembly for P71F214R 
Physical Description

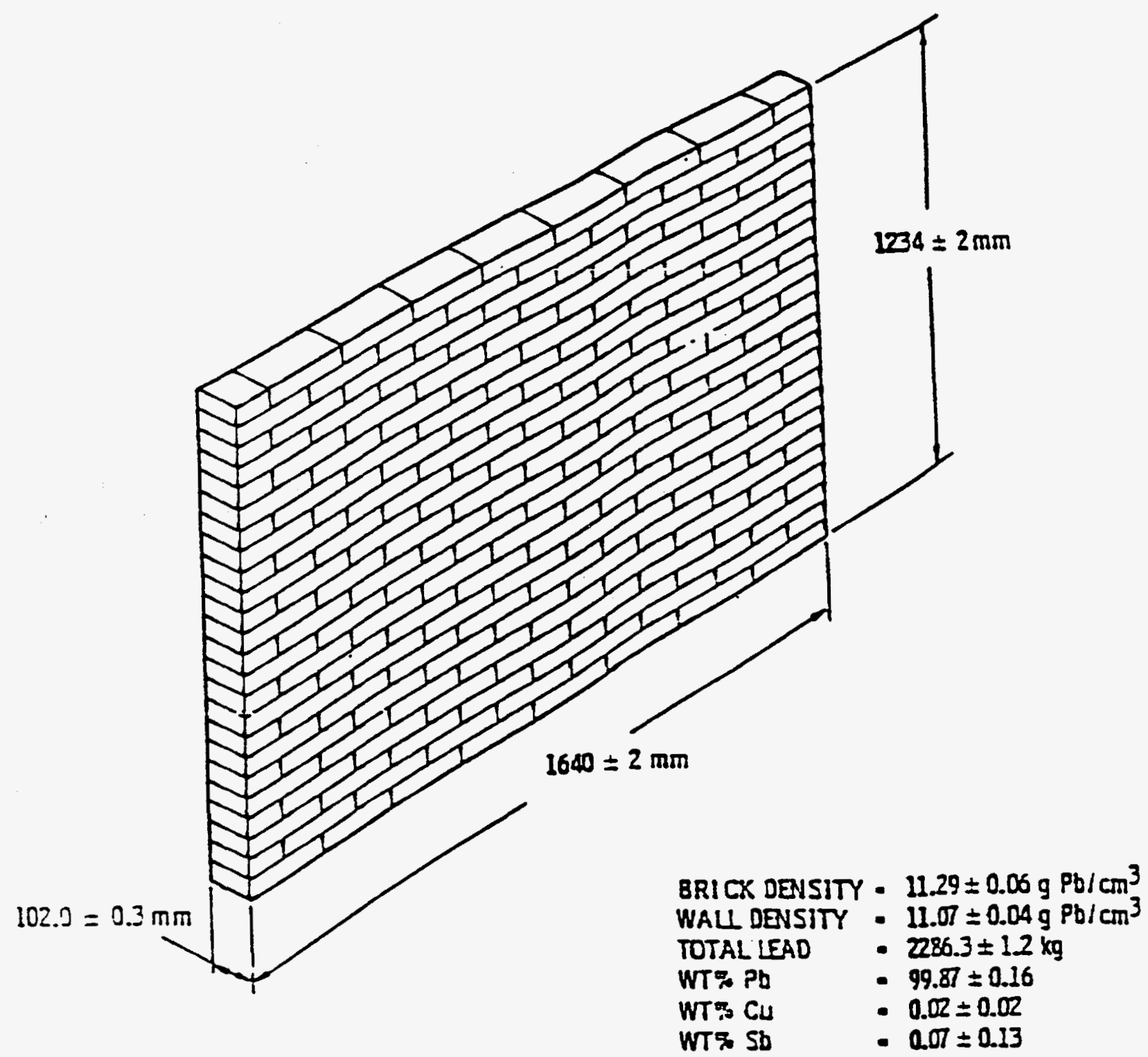

Figure 2.37 Assembled lead wall for experiments from PNL-2827. Source: Ref. 10 (Reprinted with permission from authors) 
Physical Description

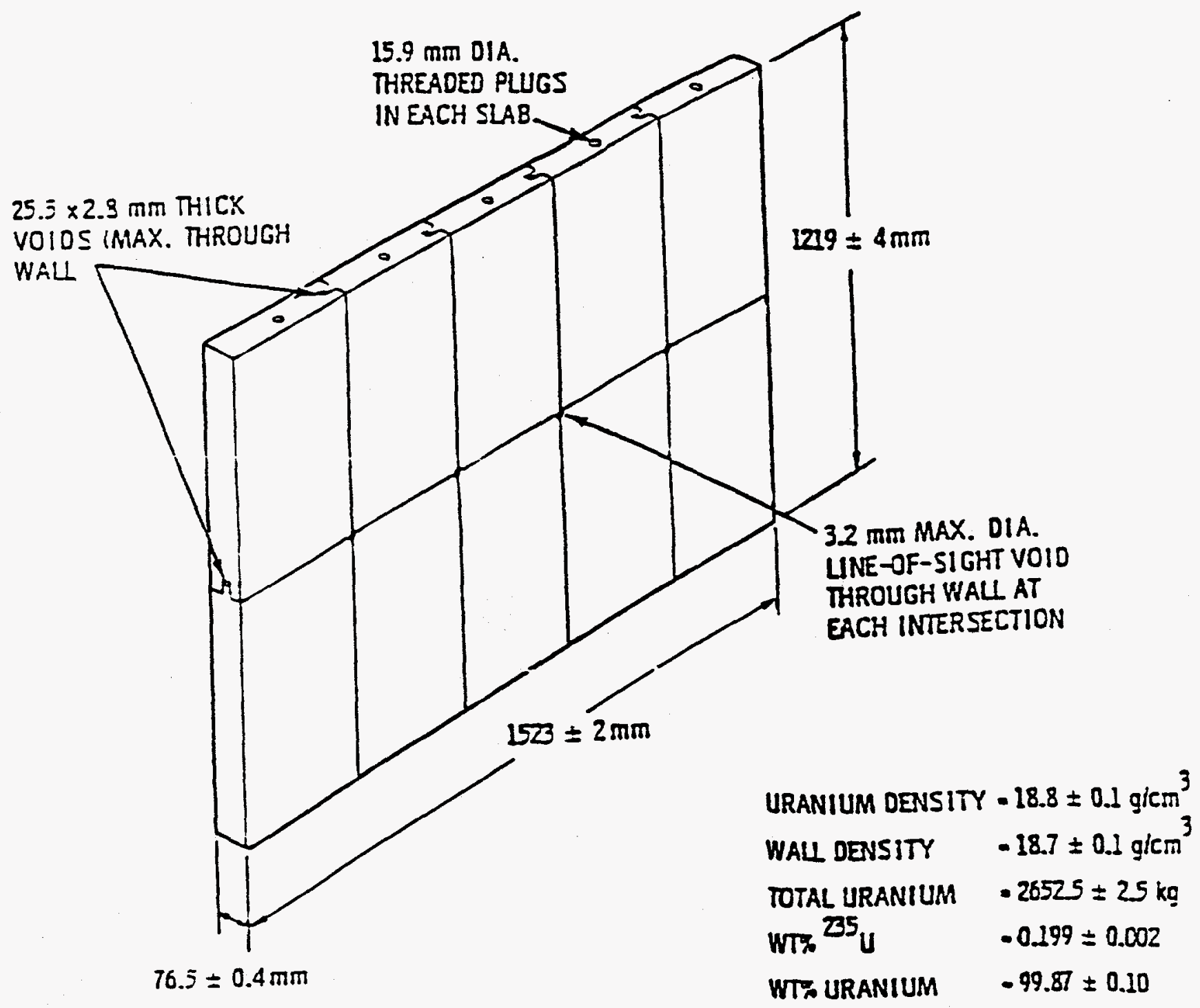

Figure 2.38 Assembled depleted uranium wall for experiments from PNL-2827. Source: Ref. 10 (Reprinted with permission from authors) 
Physical Description

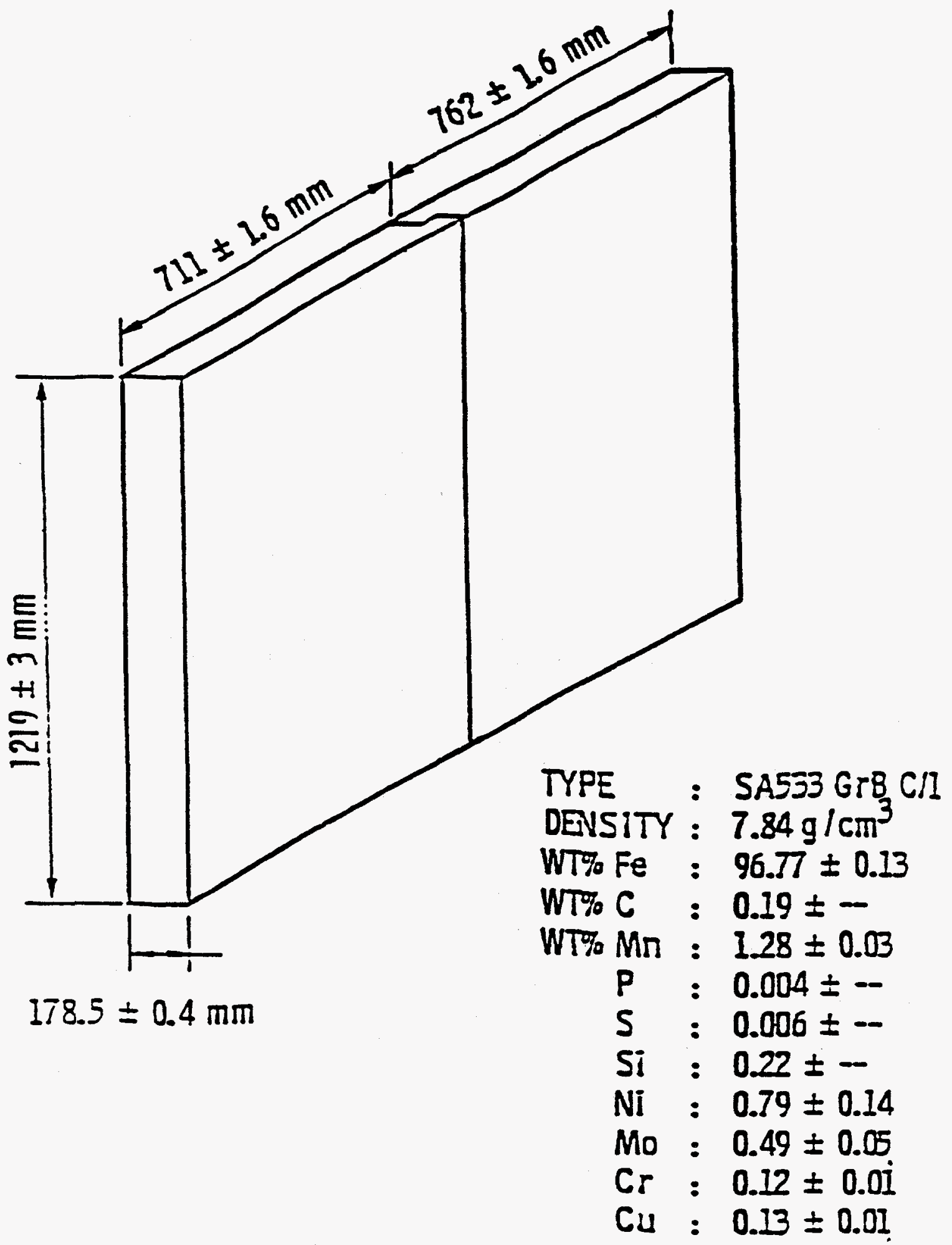

Figure 2.39 Assembled steel wall for experiments from PNL-3602. Source: Ref. 22 (Reprinted with permission from authors) 
Physical Description

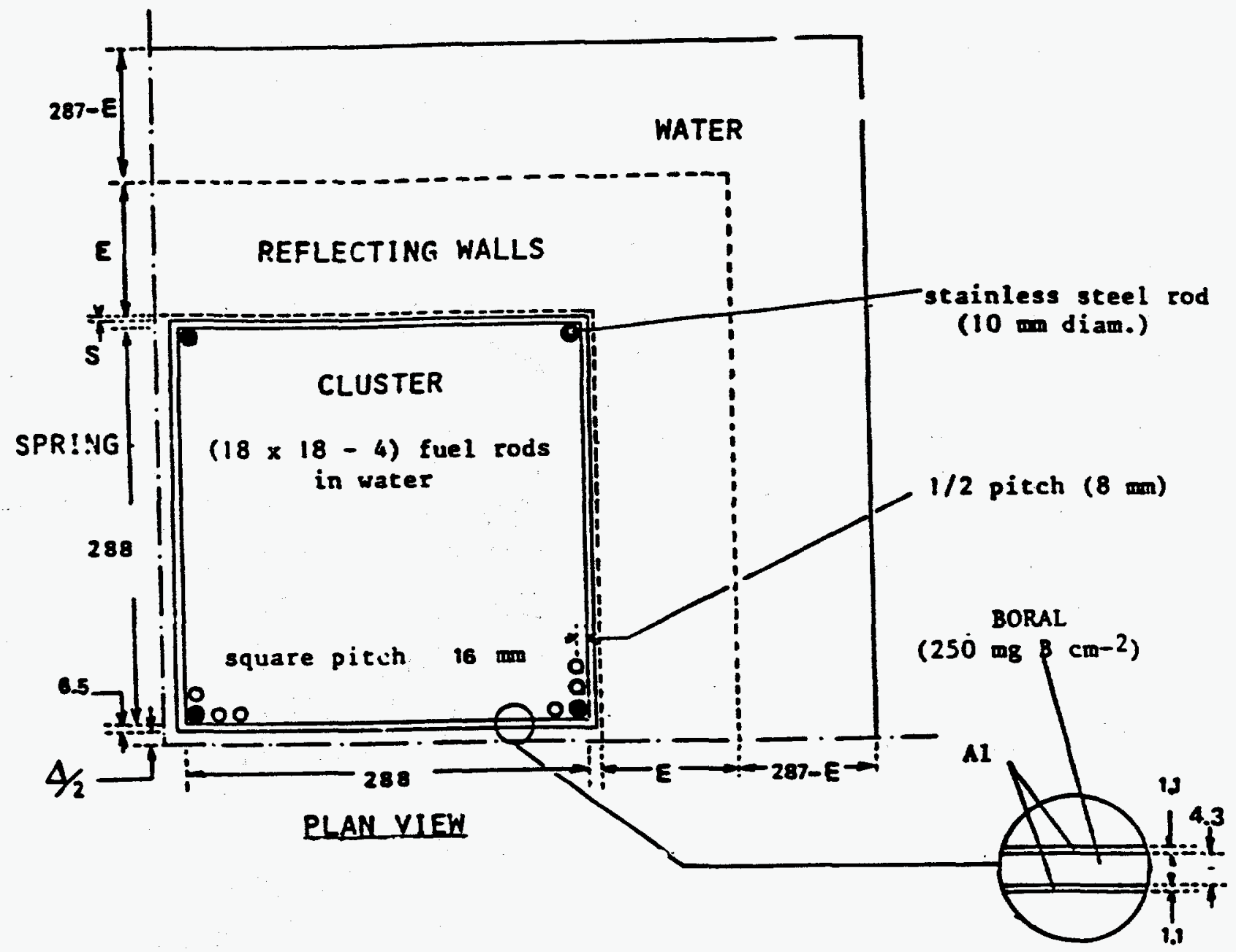

Figure 2.40 Quarter-assembly configuration for experiments from PATRAM'80. Source: Ref. 23 (Reprinted with permission by author) 
Physical Description

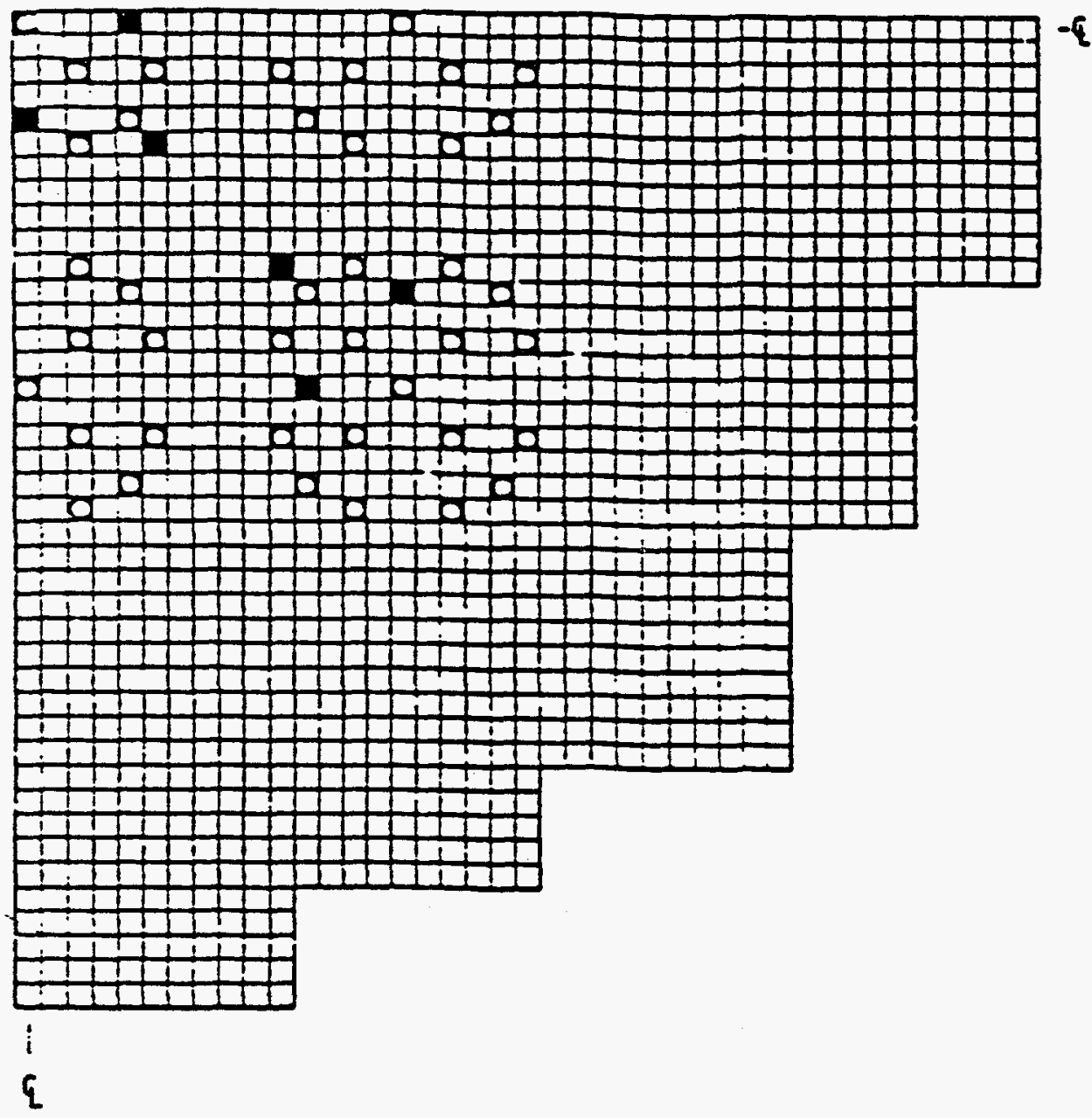

D VACANT WATER-FHLLED POSITIOH

$\square 2.46$ We : U-235 ENRICHED FUEL

$4.00 \mathrm{wt}=\mathrm{Gd}_{2} \mathrm{O}_{3} / 1.94 \mathrm{wt}=U-235$ EKRICHED FUEL

Figure 2.41 Quarter-core loading diagram for BW1810A. Source: Ref. 24 
Physical Description

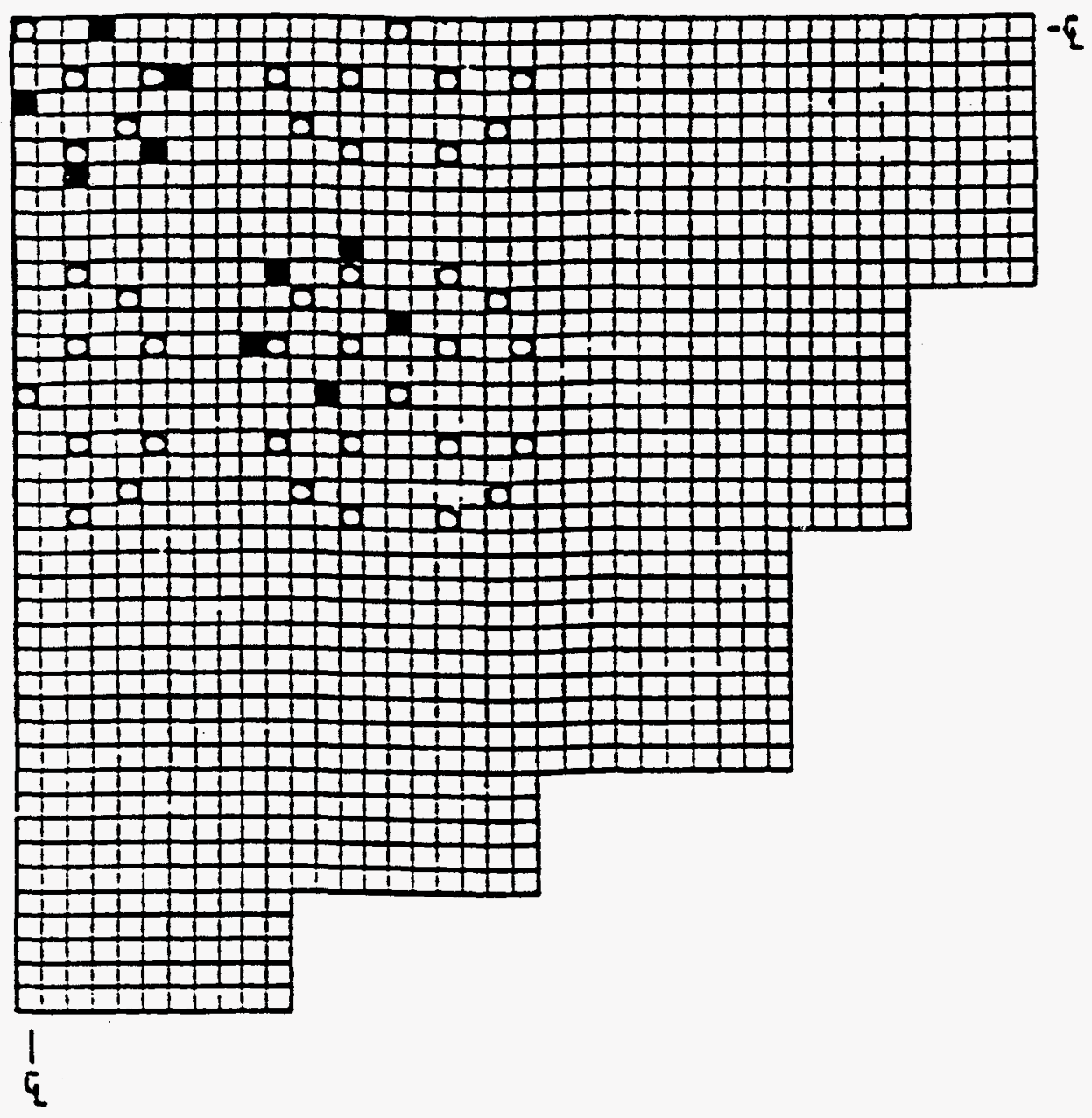

Q VRCAKT IATER-FILLED POSITION

2.46 wt I U-235 EHRICHED FUEL

$4 . C 0 \mathrm{wt}: \mathrm{Cd}_{2} \mathrm{O}_{3} / 1.34$ wt $2 \mathrm{U}-235$ ENRICHED FUEL

Figure 2.42 Quarter-core loading diagram for BW1810B. Source: Ref. 24 
Physical Description

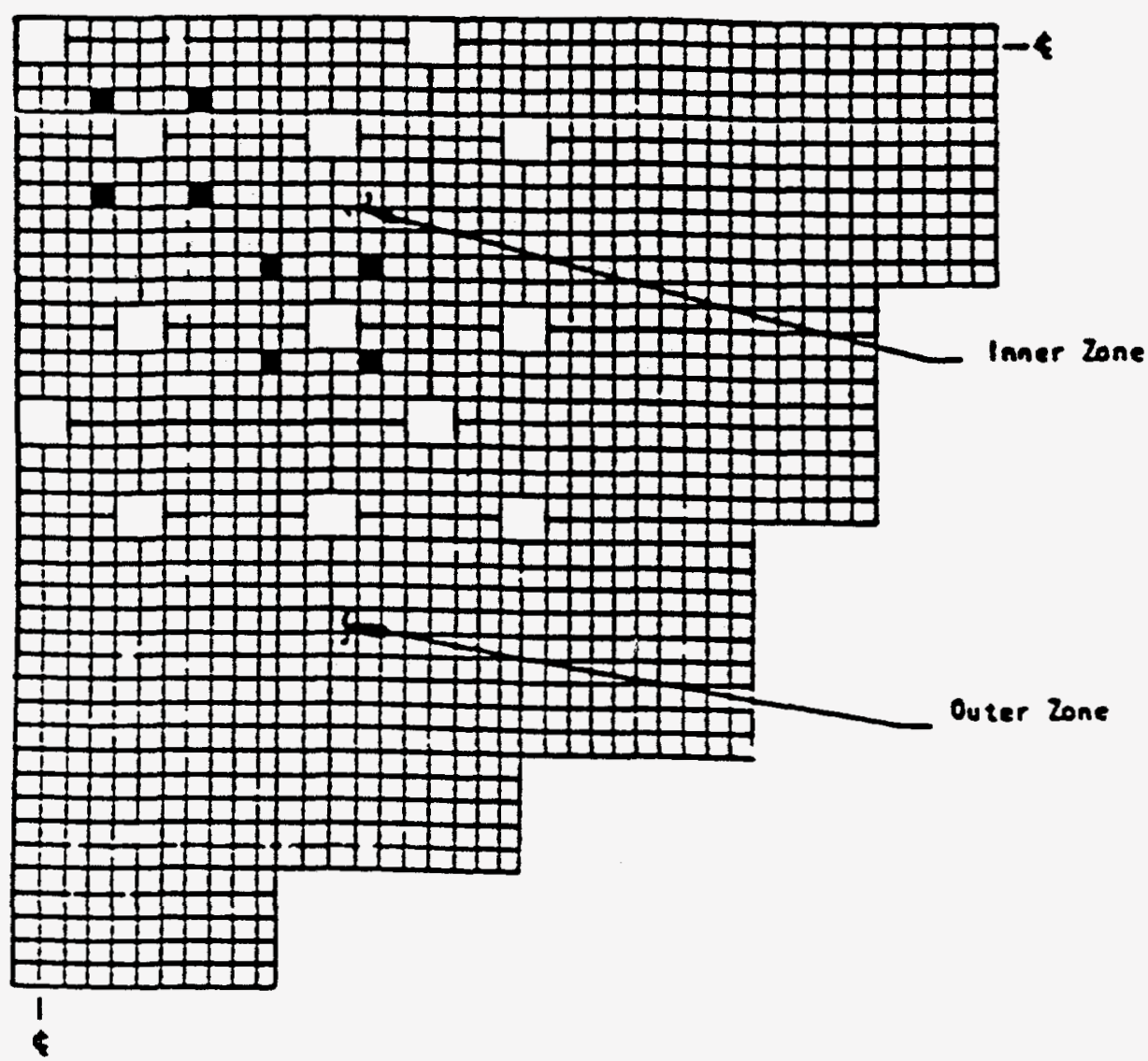

VACANT WATER-FILLED POSITION

$\square 2.46$ WE $\&$ U-235 ENRICHED FUEL: OUTER ZONE

Q 4.02 WE I U-235 EMRICHED FUEL: IWHER ZONE

L 4.00 we $: \mathrm{Gd}_{2} \mathrm{O}_{3} / 1.94: \mathrm{*t}: \mathrm{U}-235$ EXRICHED FUEl

Figure 2.43 Quarter-core loading diagram for BW1810C. Source: Ref. 24 


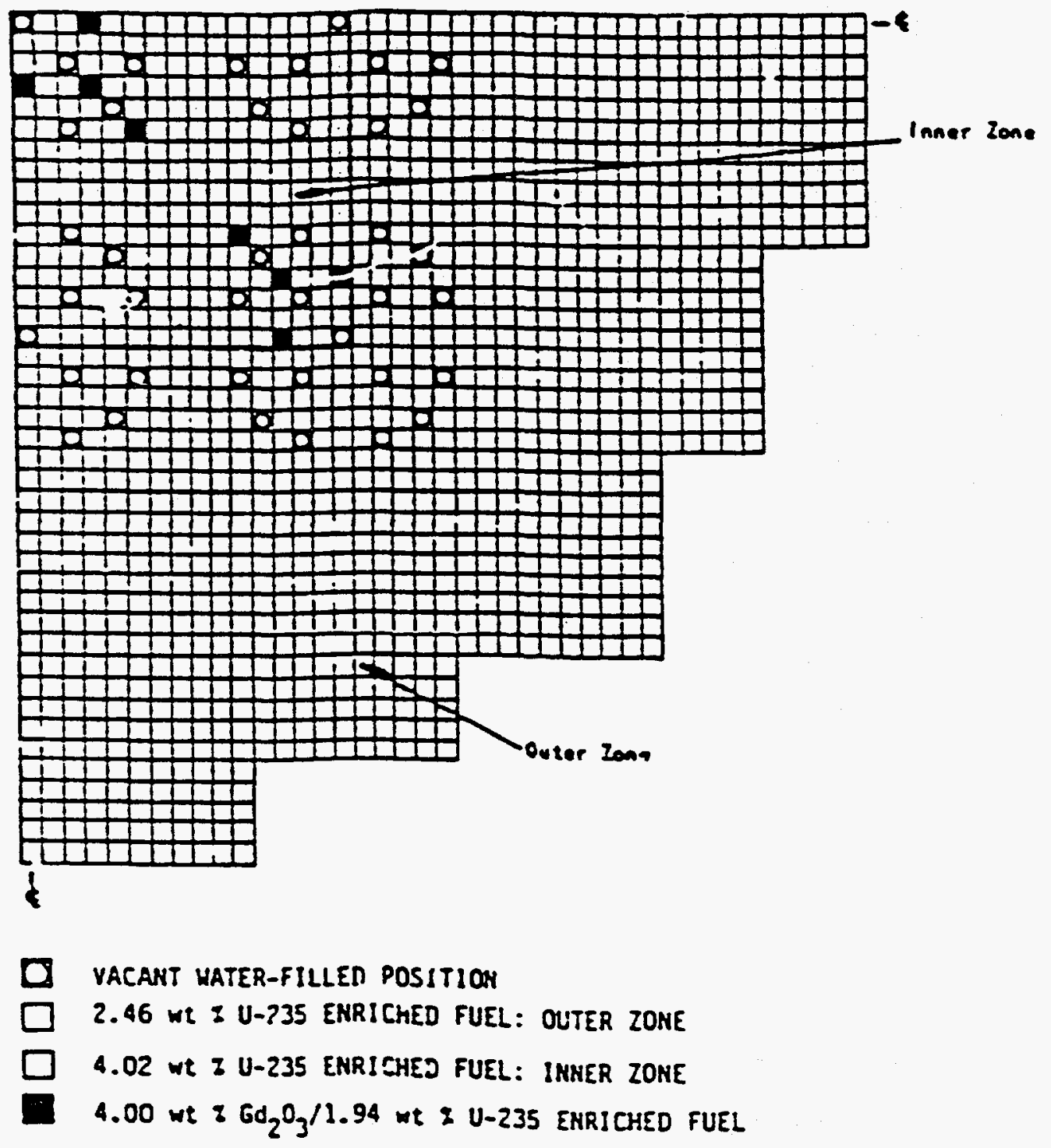

Figure 2.44 Quarter-core loading diagram for BW1810D. Source: Ref. 24 
Physical Description

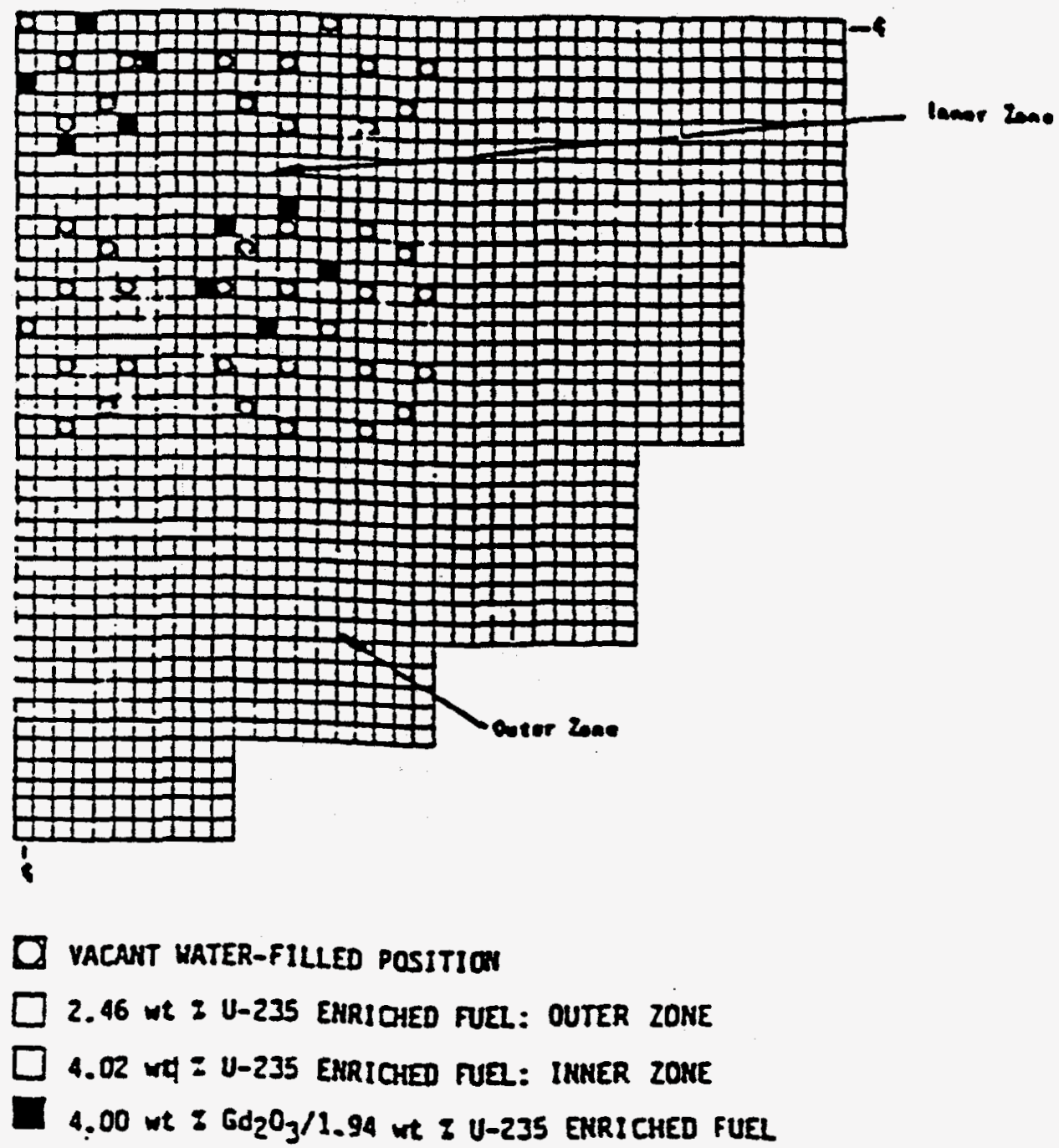

Figure 2.45 Quarter-core loading diagram for BW1810E. Source: Ref. 24 
Physical Description

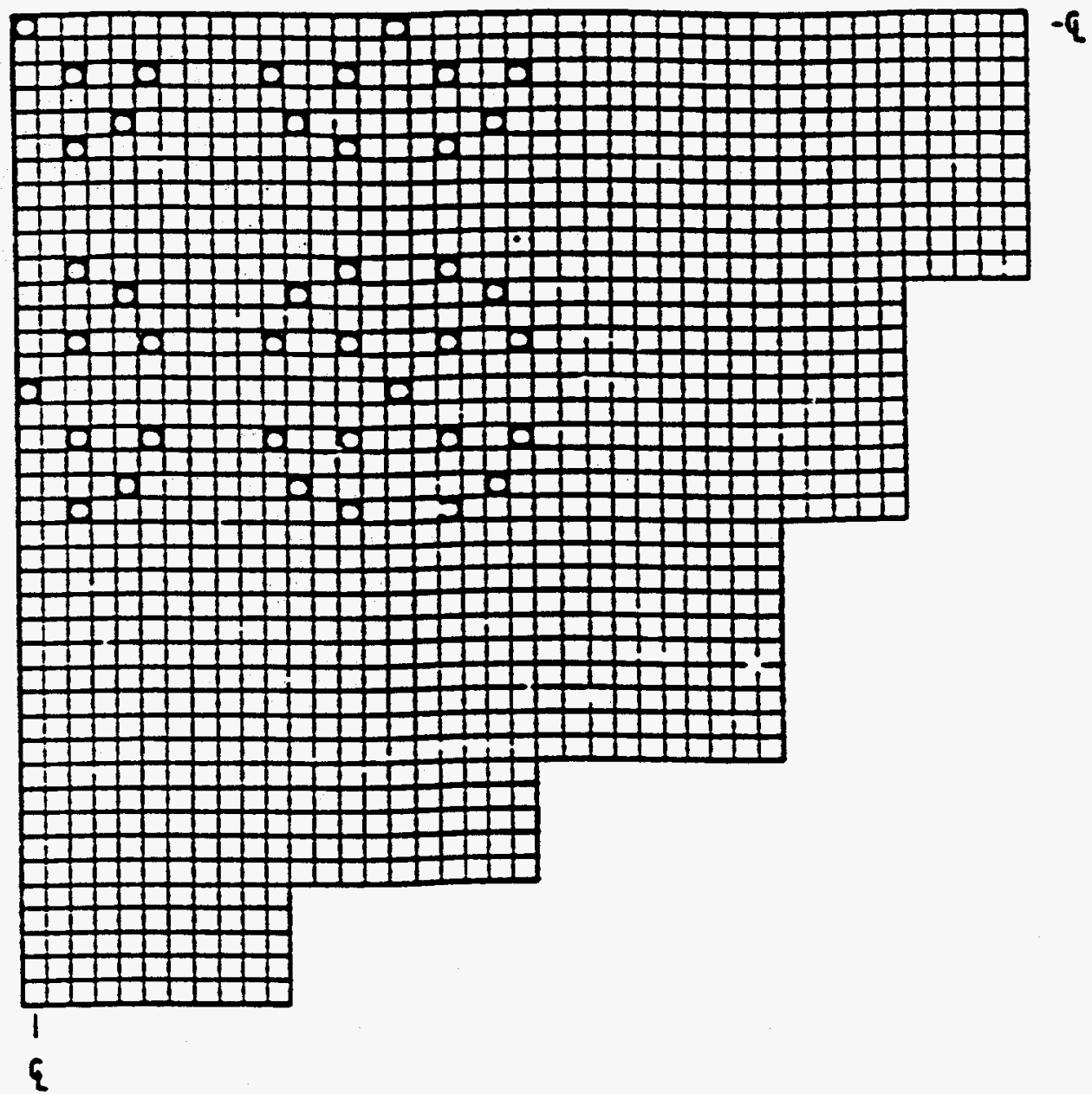

D LCANT IATER-FILLEO POSITION

D 2.46 W $U$-235 EHRICHED FUEL

Figure 2.46 Quarter-core loading diagram for BW1810F. Source: Ref. 24 
Physical Description

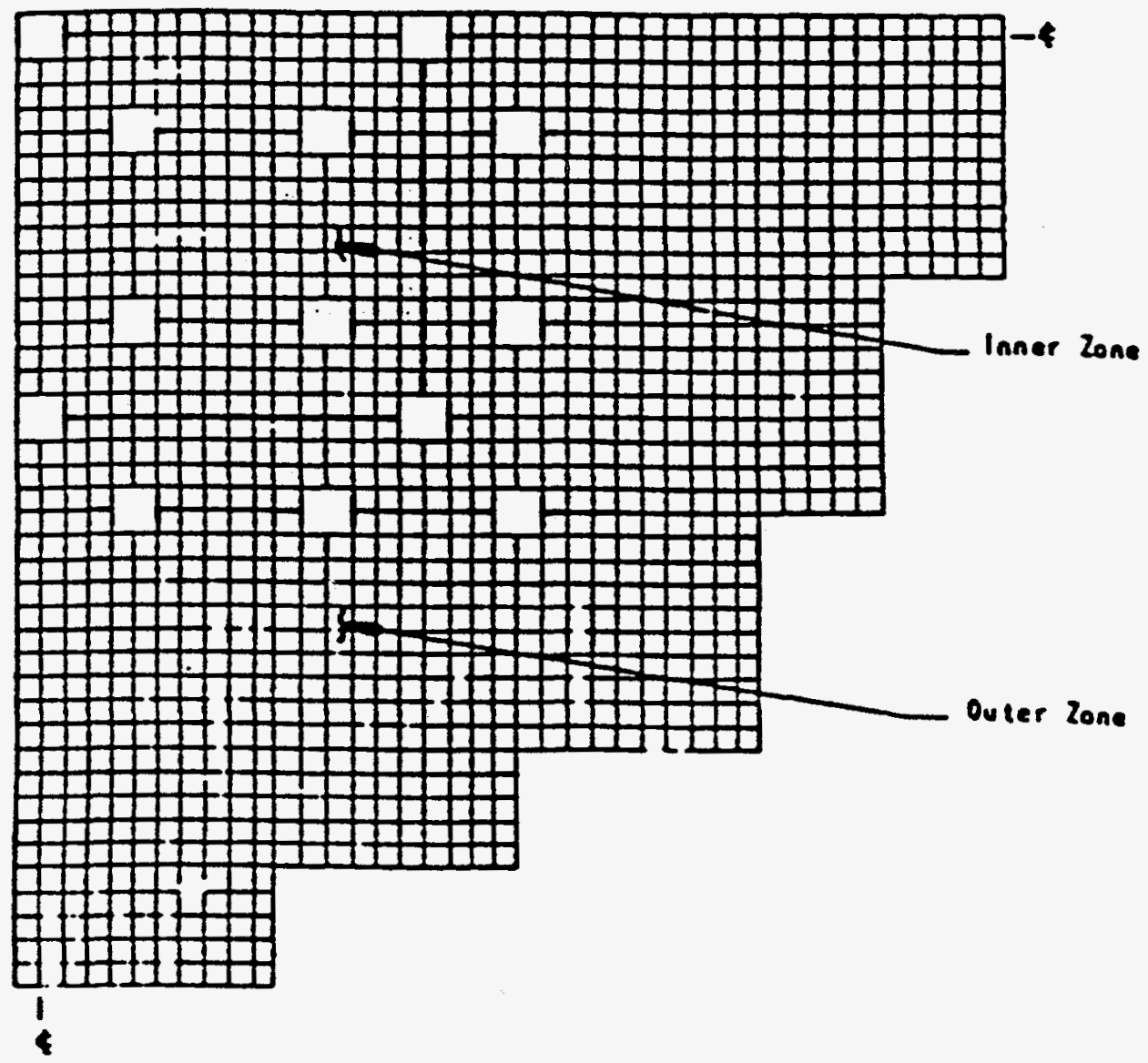

VACANT MATER-FILLED PCSITIOH

2.46 W I U-235 ERRICHED FUEL: OUTER ZONE

4.02 Wt I U-235 ERRICHED FUEL: IHNER ZONE

Figure 2.47 Quarter-core loading diagram for BW1810G. Source: Ref 24 
Physical Description

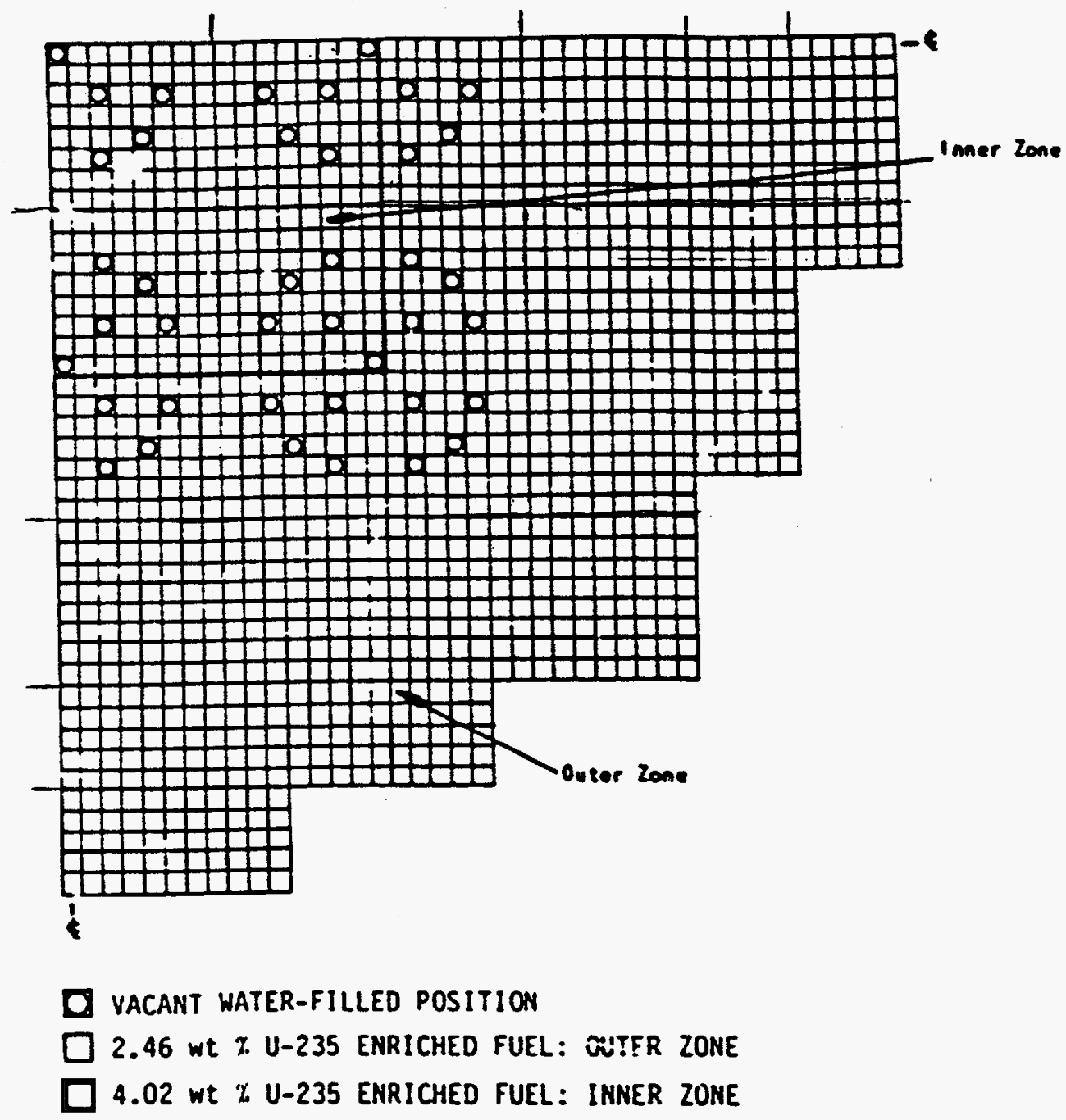

Figure 2.48 Quarter-core loading diagram for BW1810H. Source: Ref. 24 
Physical Description

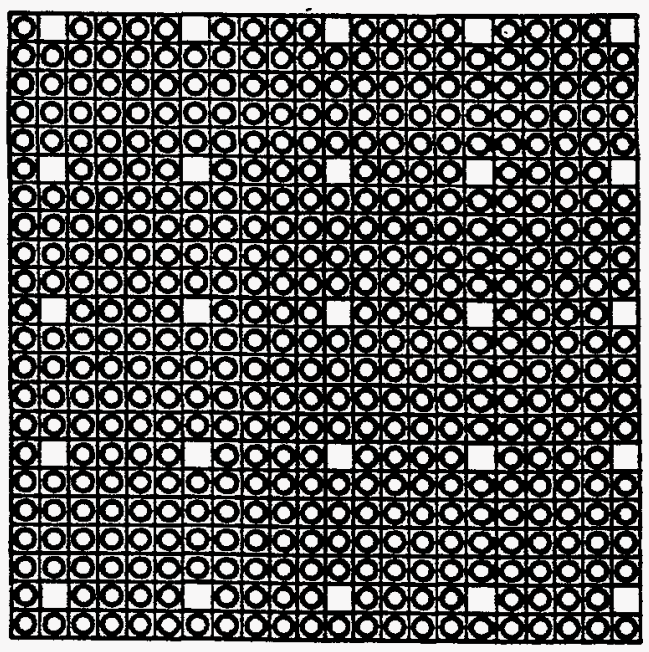

(a) NSE71W1

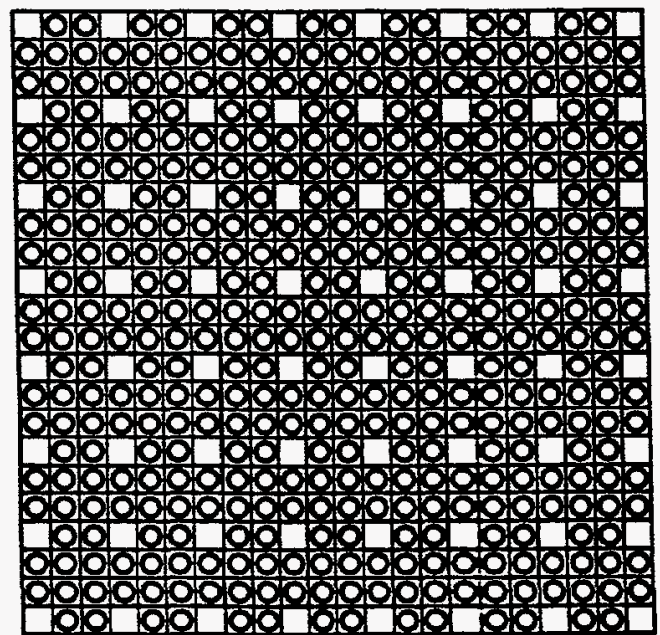

b) NSE71 W2

Figure 2.49 Cross-sectional view of modeled NSE71W1 and NSE71 W2 assemblies with water holes 
Physical Description

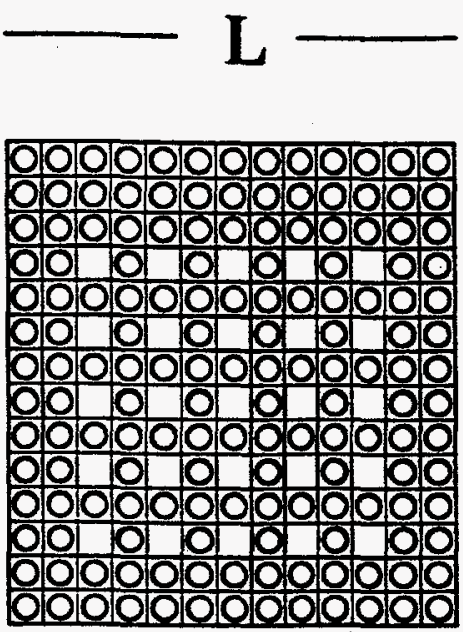

(a) 4.31 wt $\% \mathrm{uO}_{2}$ rods

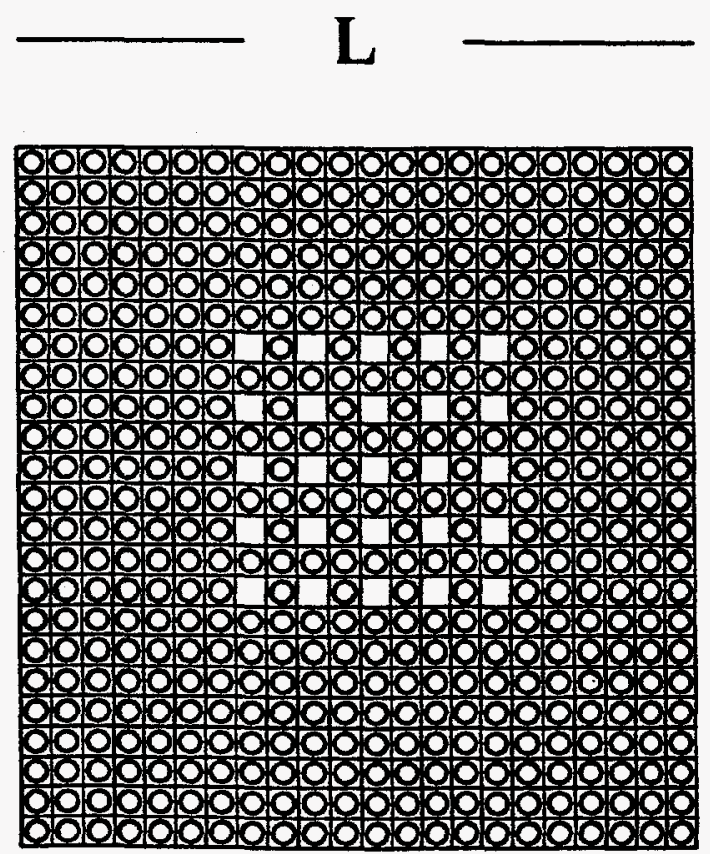

(b) 2.35 wt $\% \mathrm{UO}_{2}$ rods

Figure 2.50 Cross-sectional view of water hole experiments from PNL-3314 
Physical Description

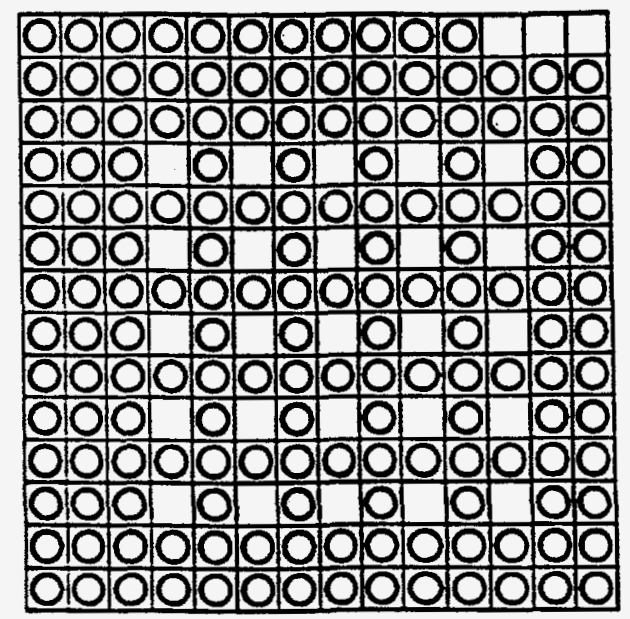

(a) P3314W1

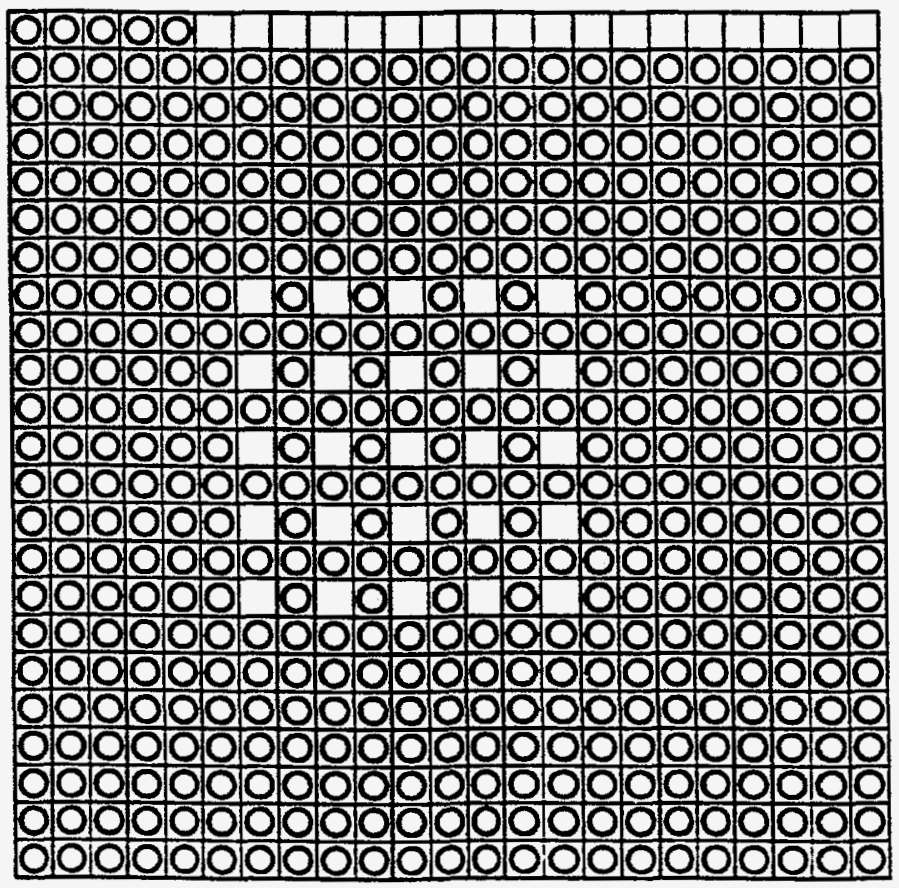

(b) P3314W2

Figure 2.51 Cross-sectional view of modeled P3314W1 and P3314W2 assemblies with water holes 


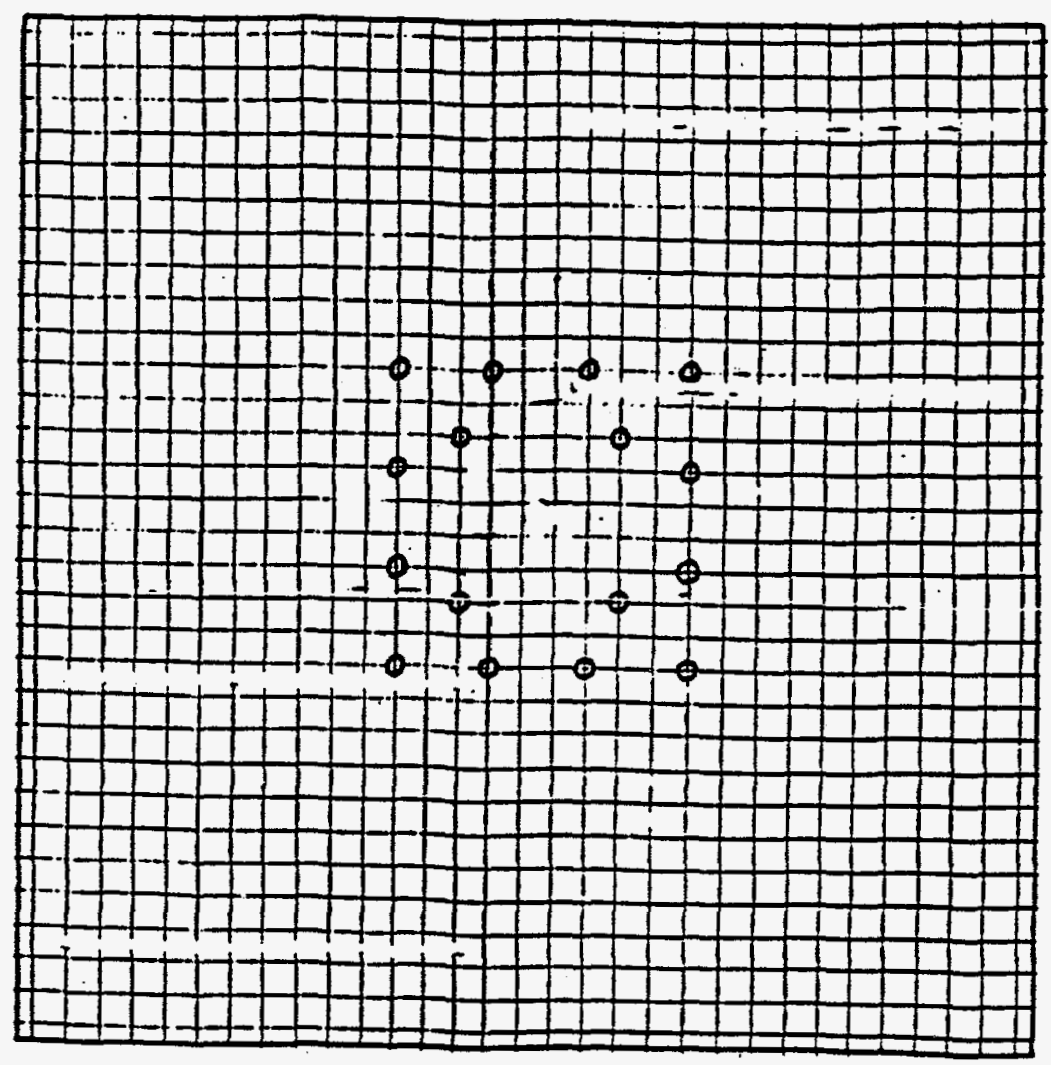

(a) W3269W1

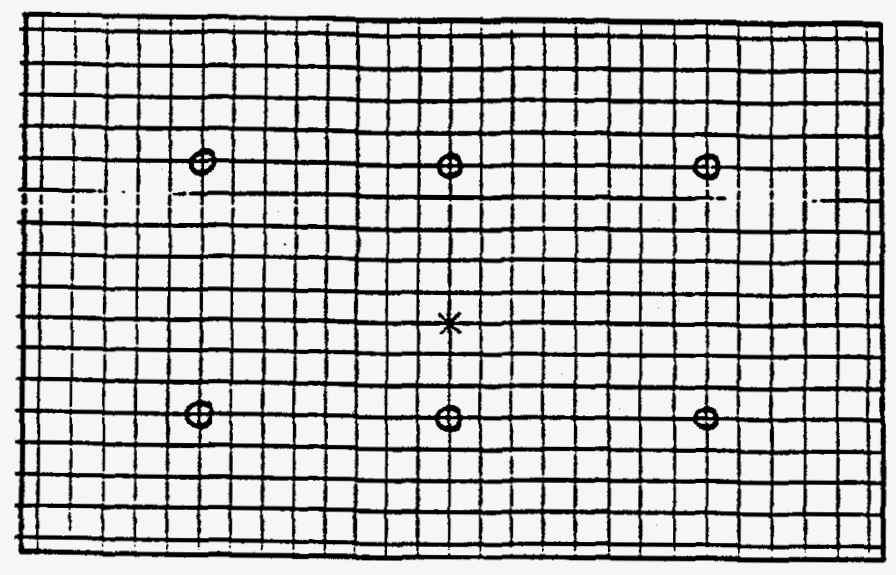

(b) W3269W2

Figure 2.52 Core layouts of W3269W1 and W3269W2 with water hole locations (circles) 
Physical Description

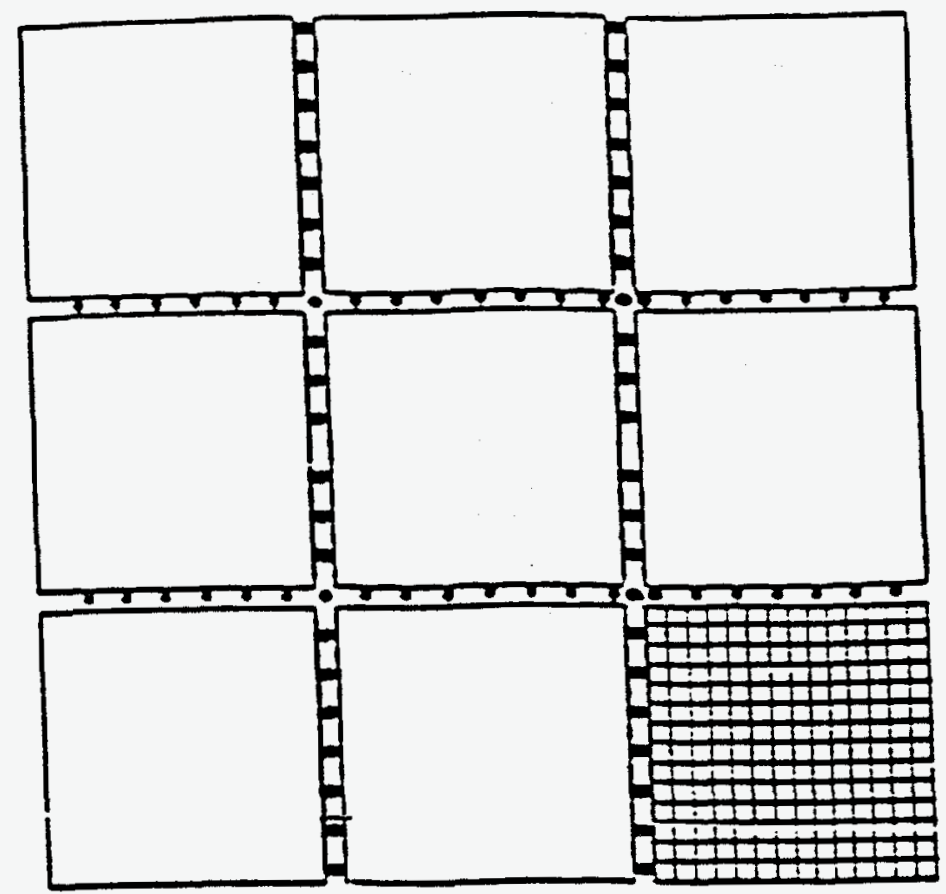

- Fucl Rad Pasizlon
- Bz Cin

Note: The large squares represent $14 \times 14$ fue! assemblies as shown in the lower righthand comes.

Figure 2.53 Experiment BW1484C1 core loading diagram. Source: Ref. 6 
Physical Description
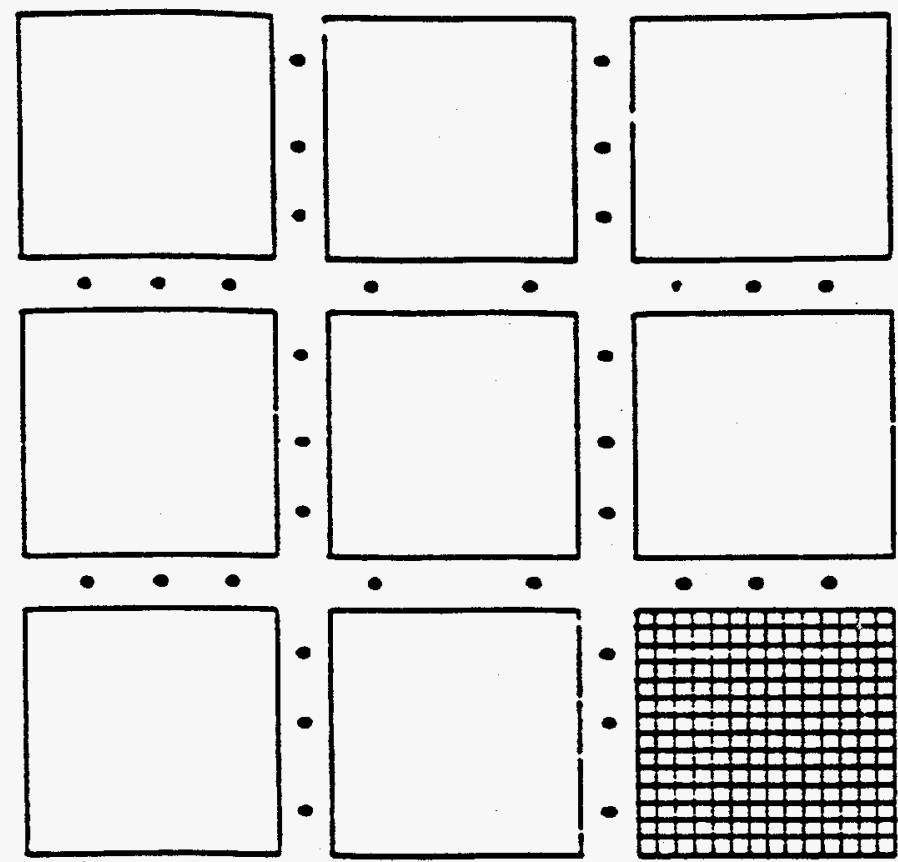

$$
\begin{aligned}
& \text { - Fucl Rad Pasizion } \\
& \text { - } z_{6} \text { C Pin }
\end{aligned}
$$
Note: The large squares represent $14 \times 14$ fuel assemblies as shown in the lower right-
hand comer.

Figure 2.54 Experiment BWS1484C2 core loading diagram. Source: Ref. 6 
Physical Description

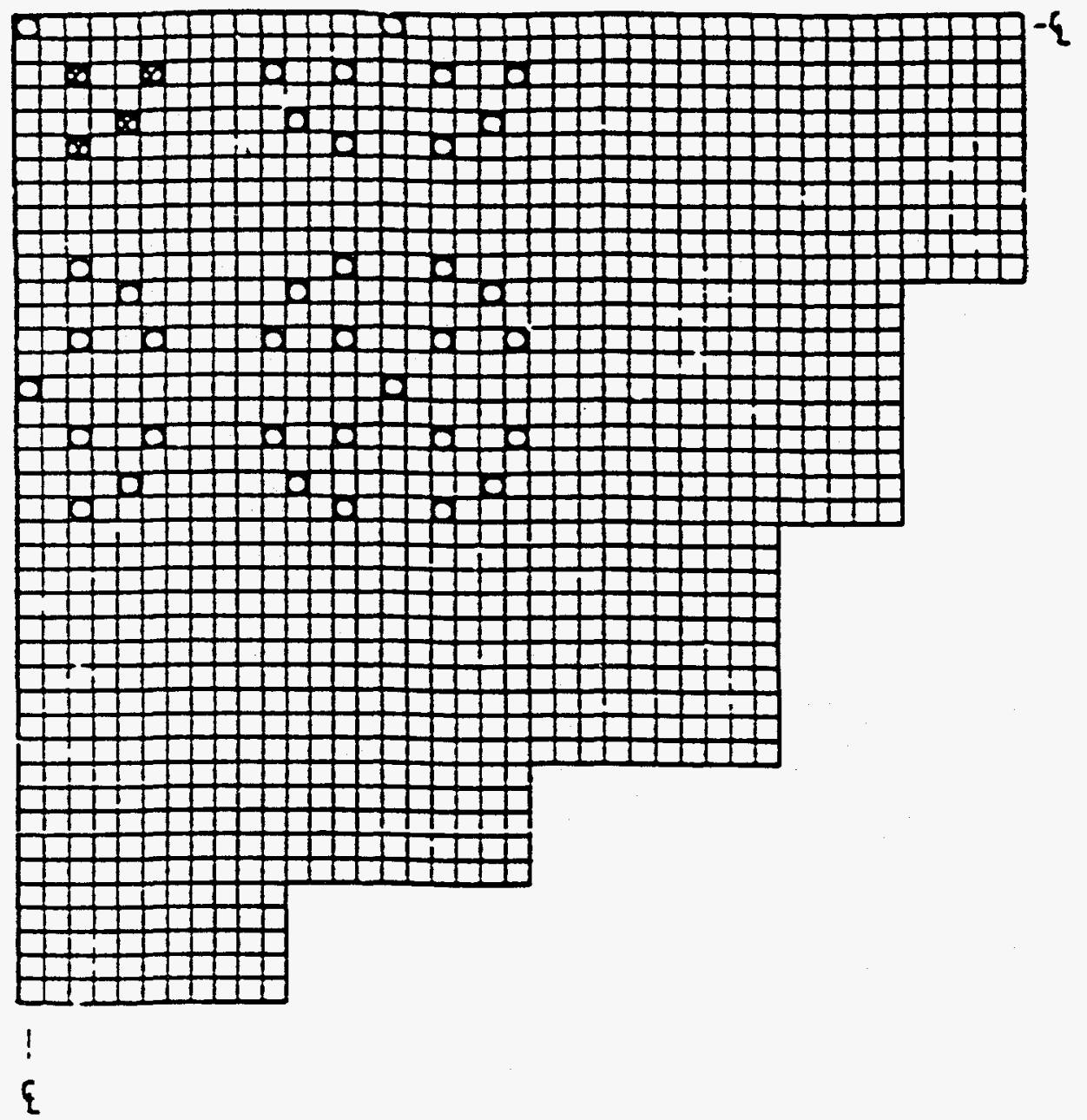

D VACANT TATER -FILLEO POSITIOH

$\square 2.46$ wE 8 U-235 EMRICHED FUEL

(8) Ag-In-Cd ROD POSITION

Figure 2.55 Core loading diagram for BW1810I. Source: Ref. 24 
Physical Description

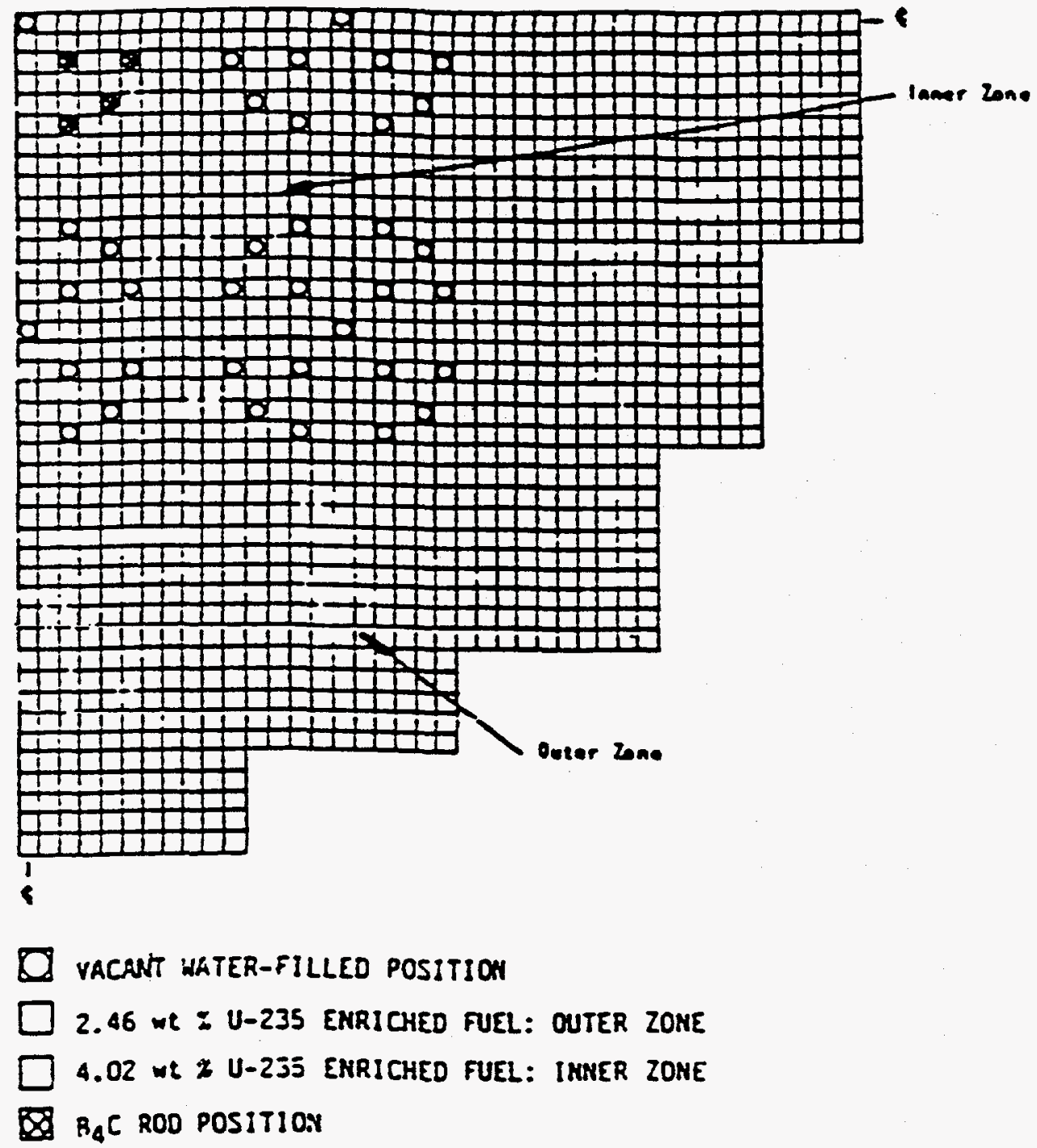

Figure 2.56 Core loading diagram for BW1810J. Source: Ref. 24 
Physical Description

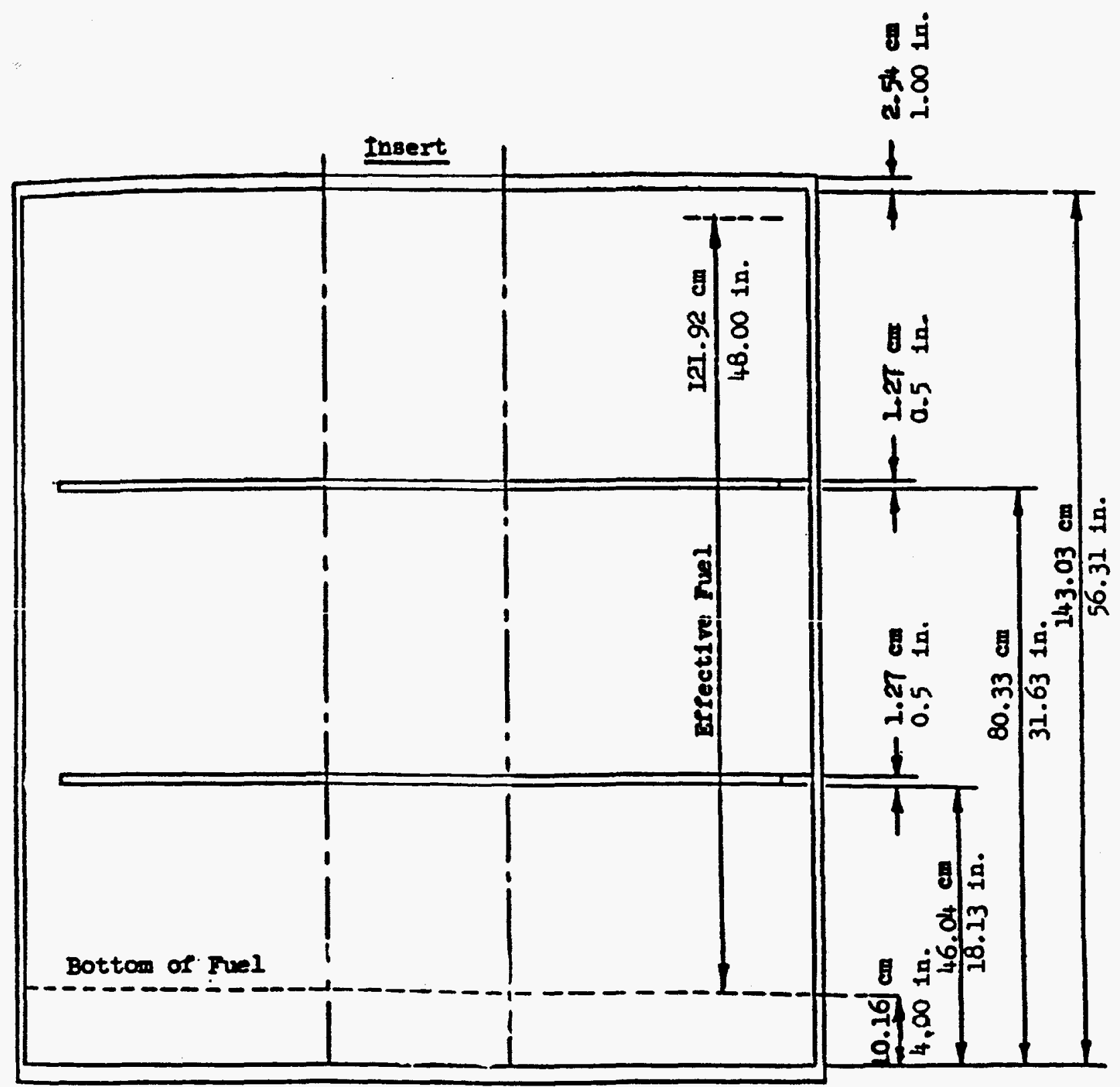

Figure 2.57 Vertical cross section of core assembly for experiments W3269B1, W3269B2, and W3269B3. Source: Ref. 15 
Physical Description

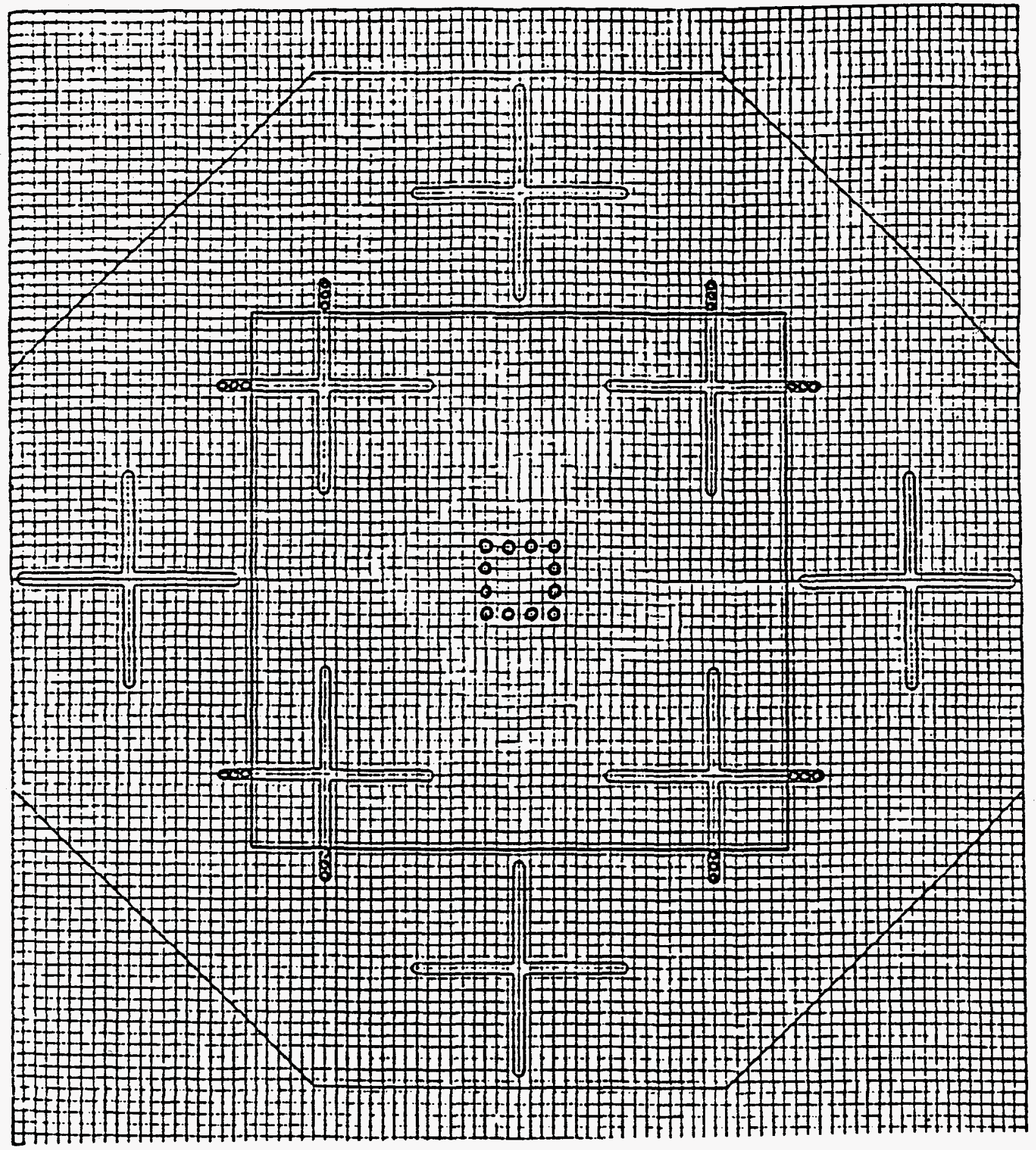

Figure 2.58 Core configuration for experiment W3269B1. Source: Ref. 15 
Physical Description

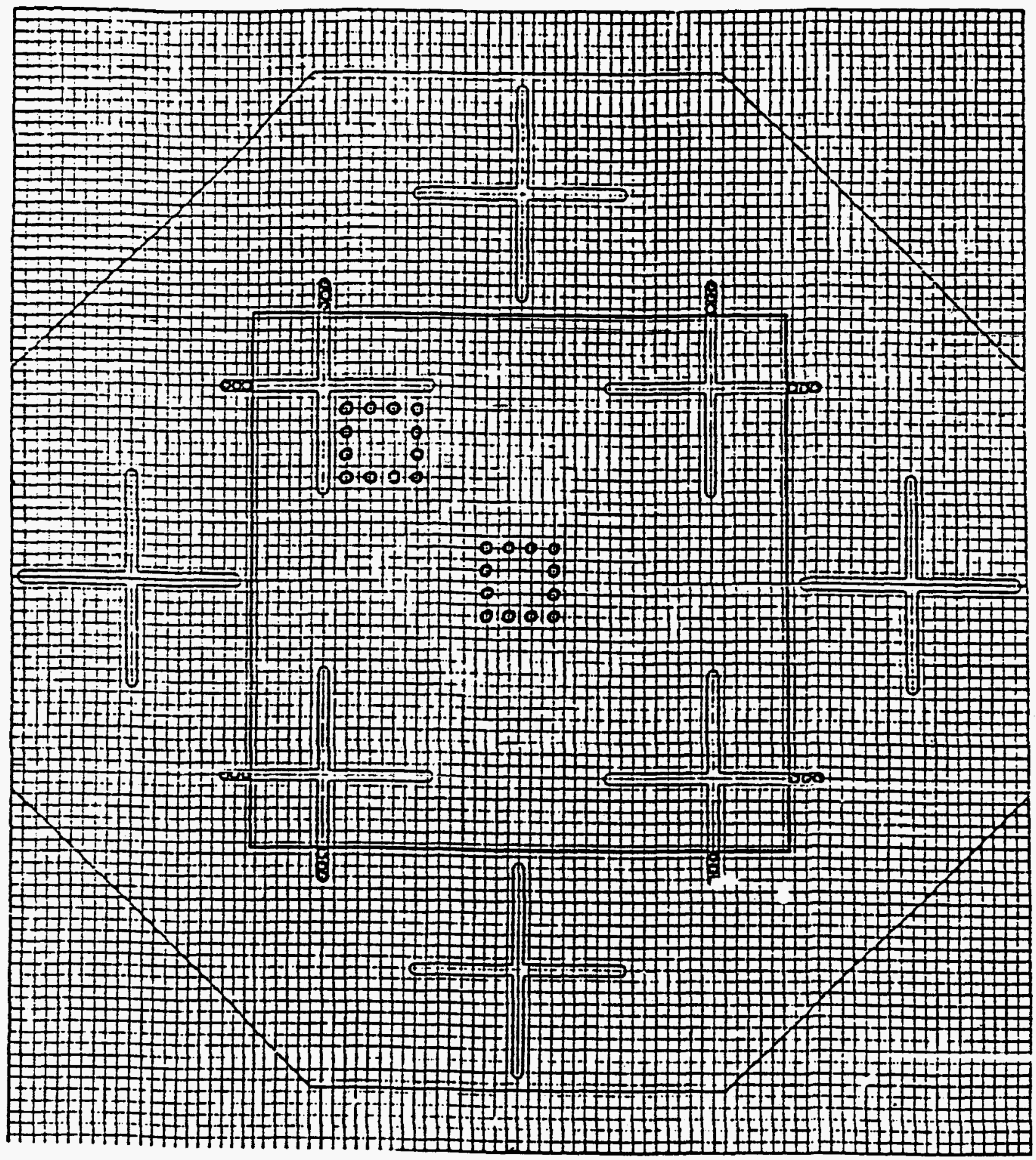

Figure 2.59 Core configuration for experiment W3796B2. Source: Ref. 15 


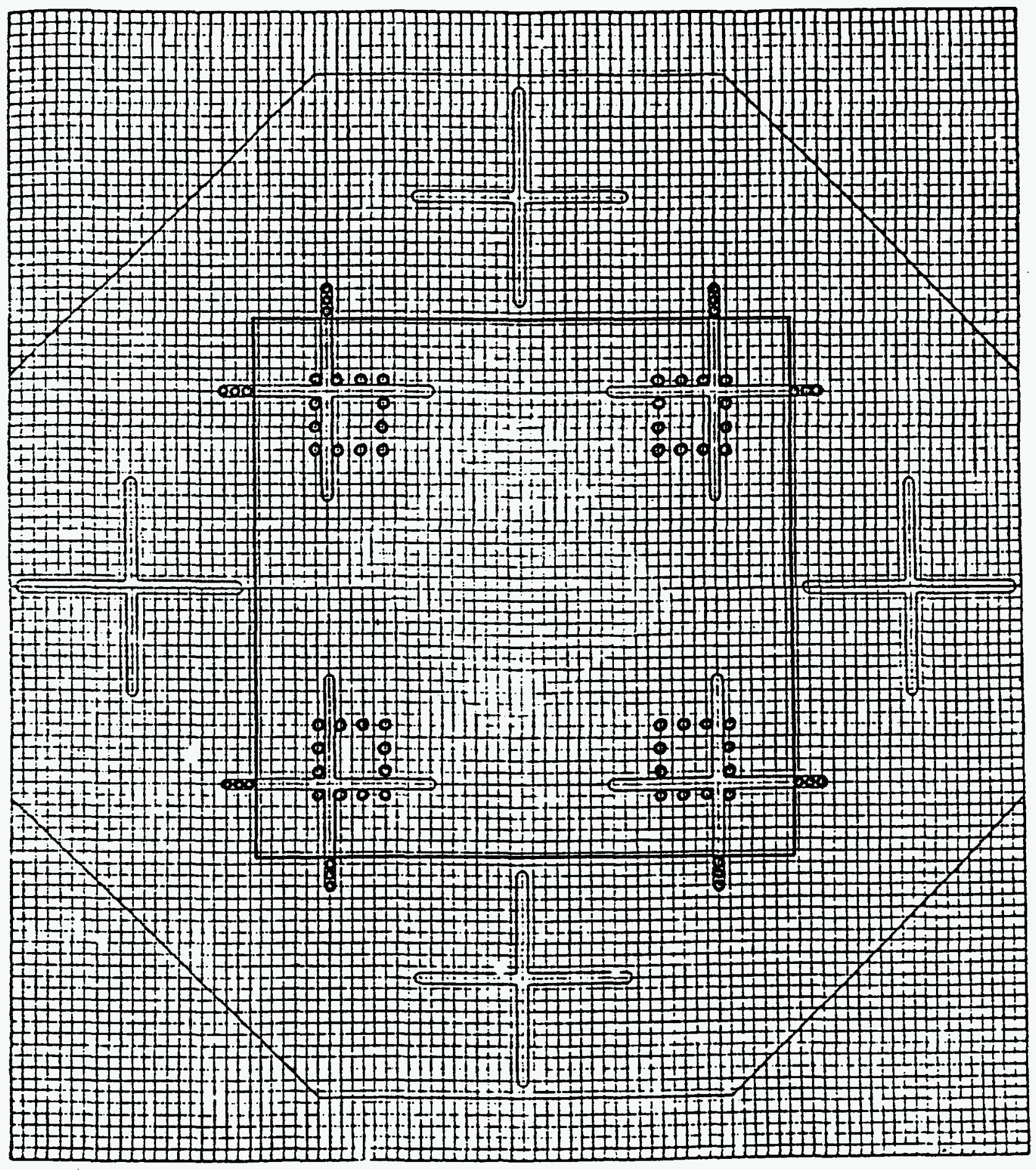

Figure 2.60 Core configuration for experiment W3269B3. Source: Ref. 15 
Physical Description

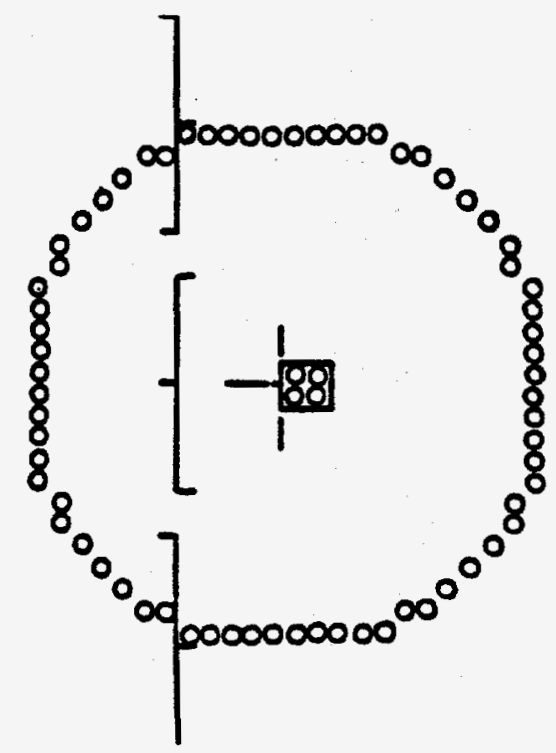

(a) BW1231B1

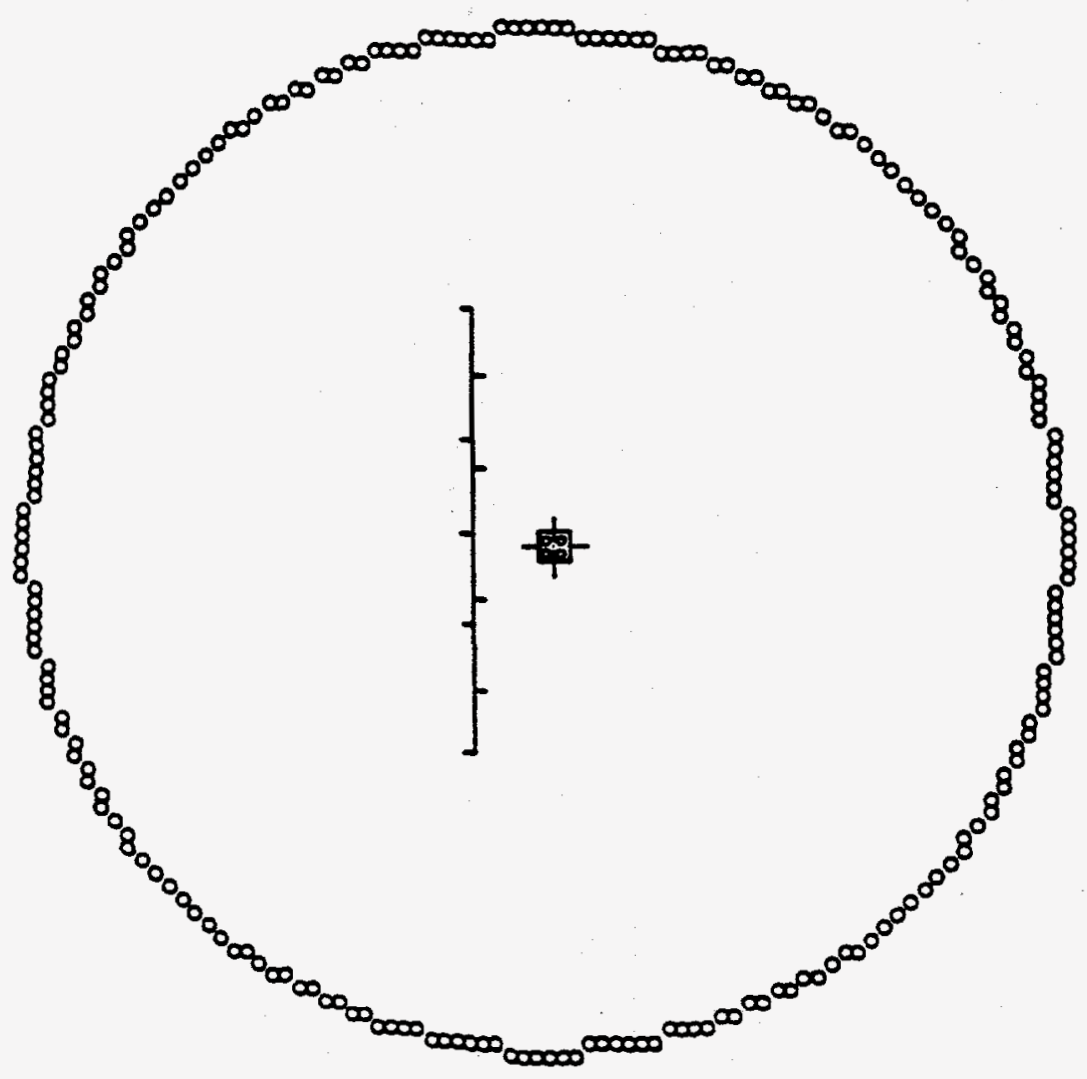

(b) BW1231B2

Figure 2.61 Core diagrams for experiments from BAW-1231 
Physical Description

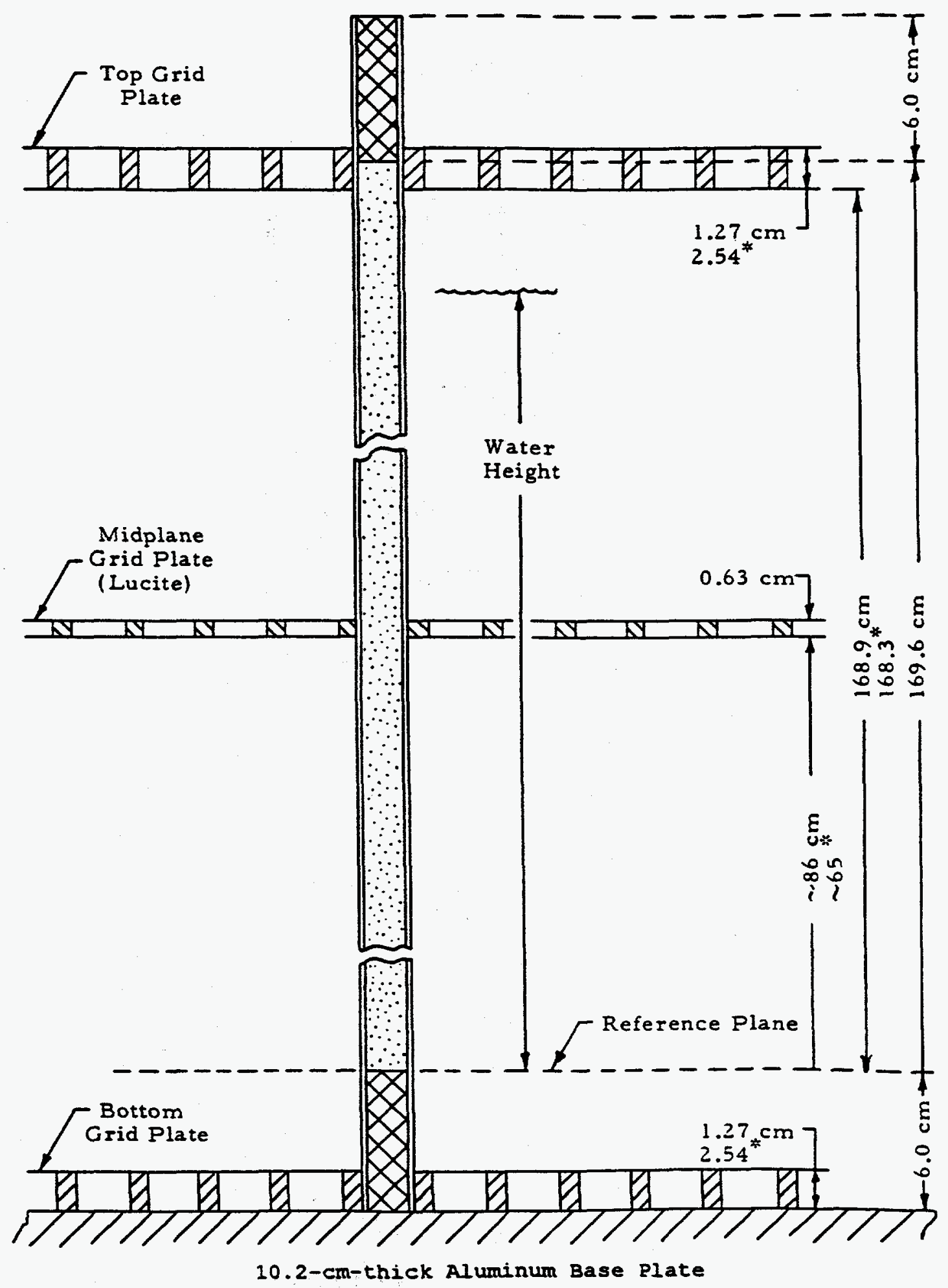

Figure 2.62 Vertical dimensions for BW1231B1 and BW1231B2 
Physical Description

Aluminum
top grid
$2.54-\mathrm{cm}$.thick

Aluminum bottom grid $2.54 \mathrm{~cm}$.thick

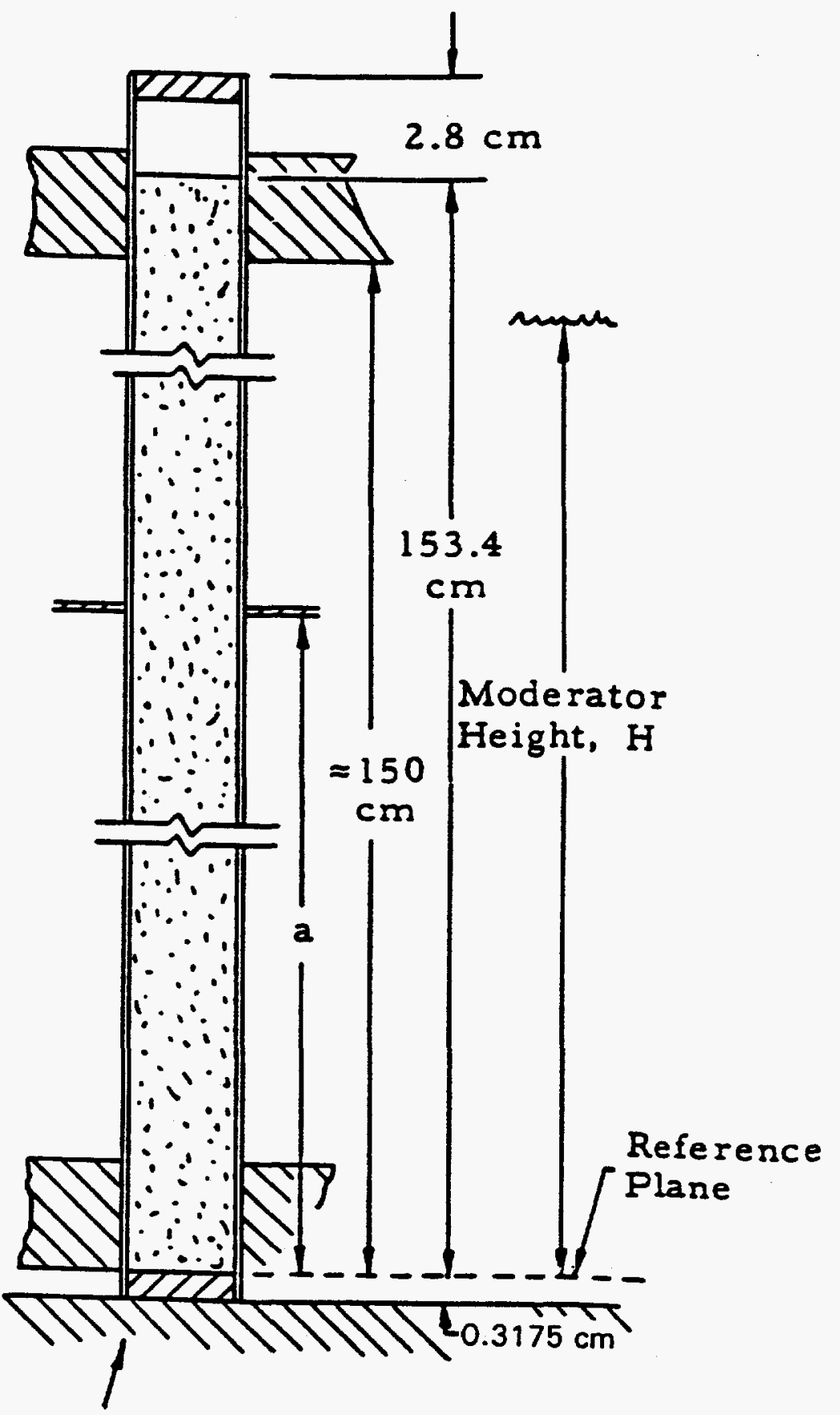

Aluminum base plate 5.08-cm-thick

Figure 2.63 Vertical dimensions for BW1273M experiment 
Physical Description

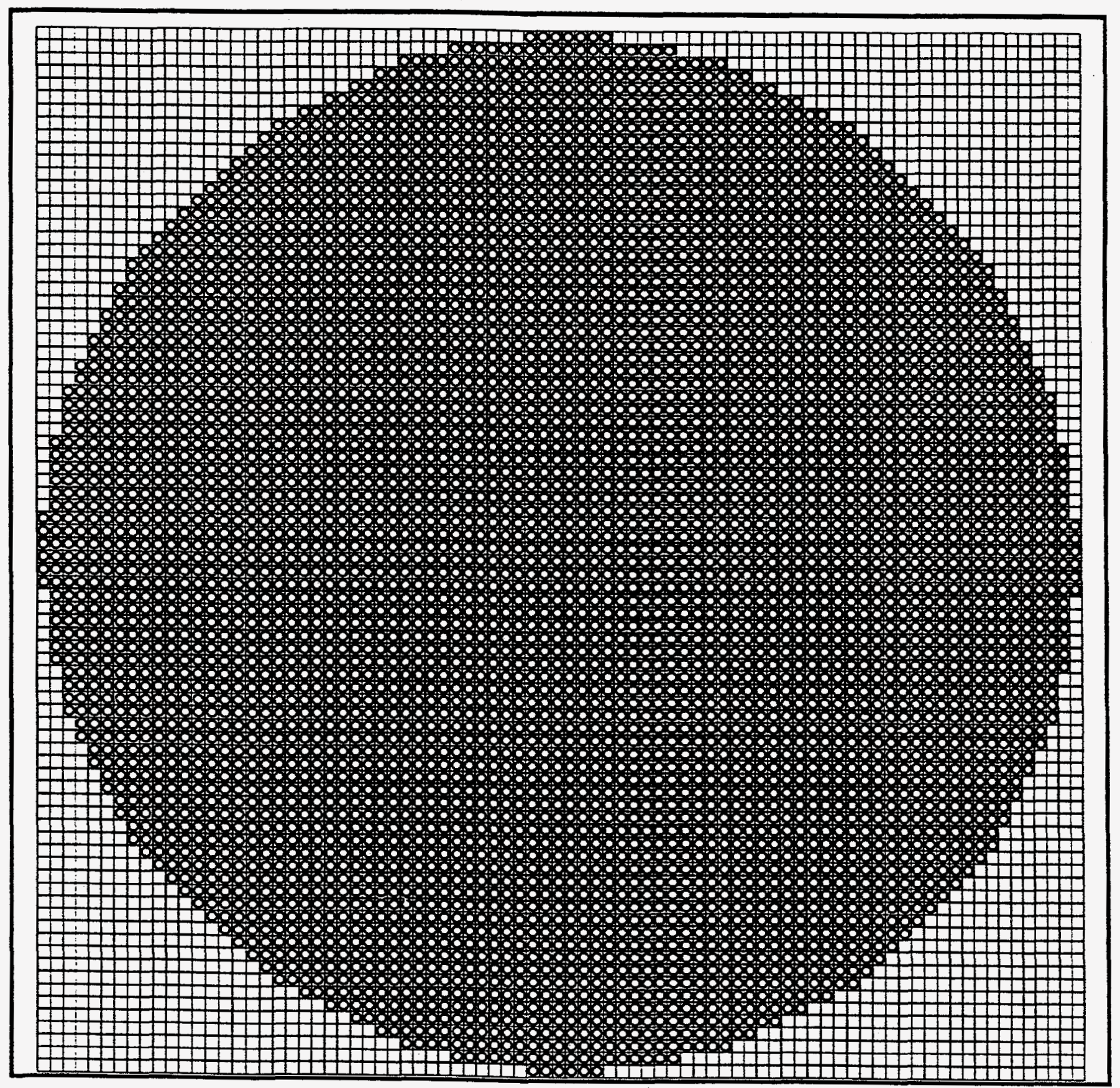

Figure 2.64 Cross-sectional view of modeled BW1273M core 
Physical Description

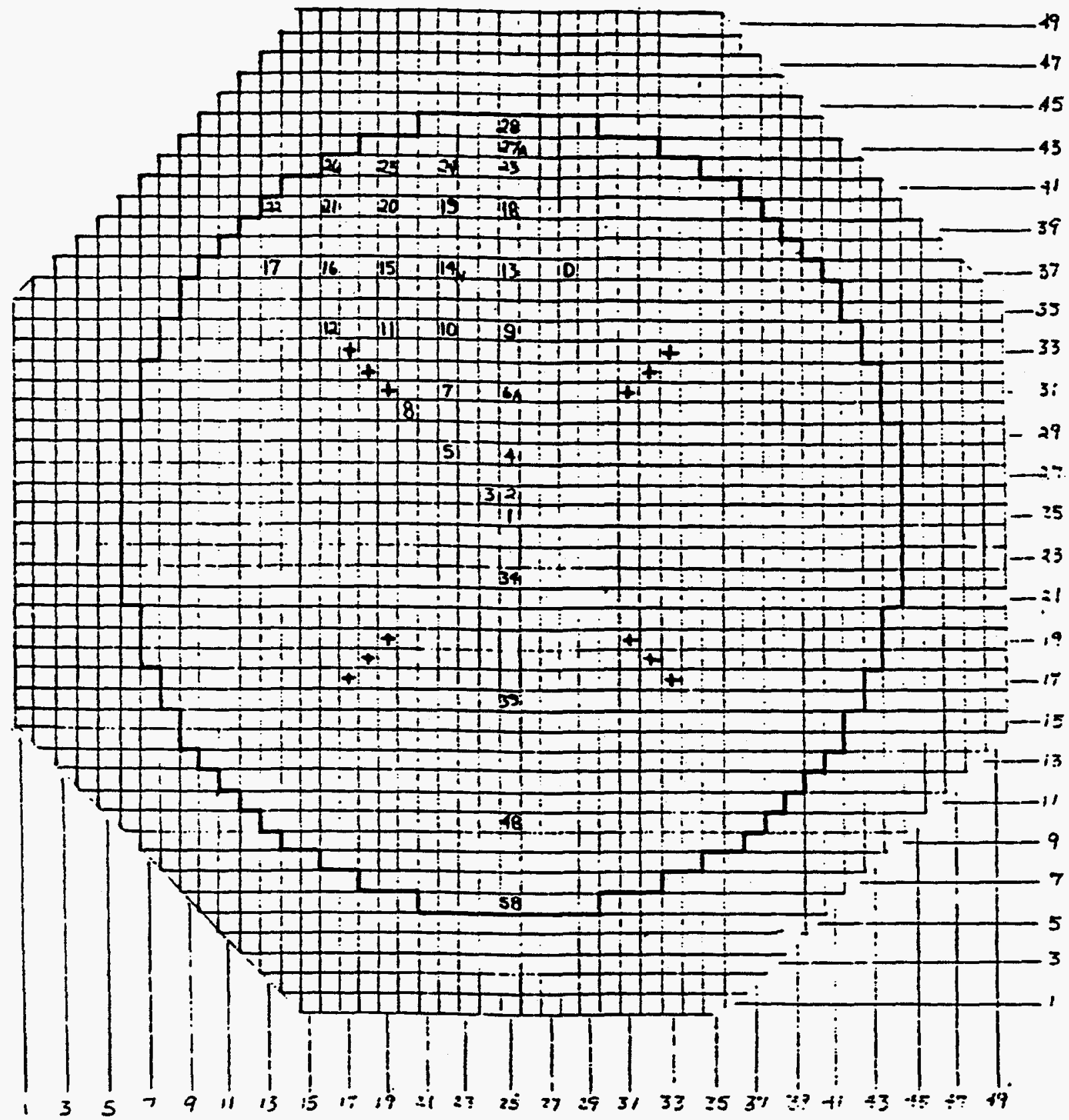

Figure 2.65 Core layout for EPRU65B. Source: Ref. 7 (Copyright $\odot$ 1976. Electric Power Research Institute. EPRI NP-196. Clean Critical Experiment Benchmarks for Plutonium Recycle in LWRs. Reprinted with Permission.) 
Physical Description

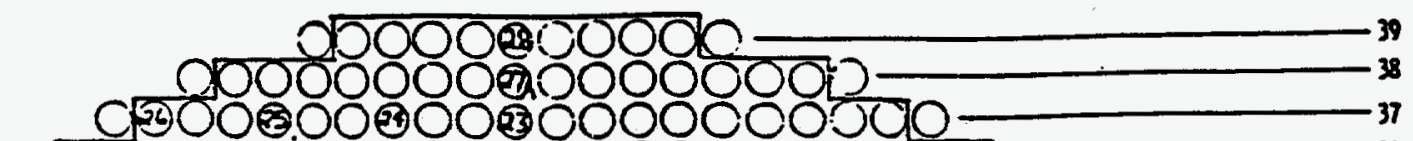
00000000000000000000000

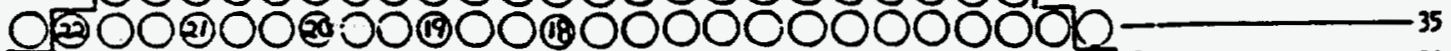
d0000000000000000000000000000 - - *

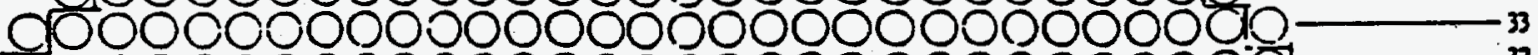

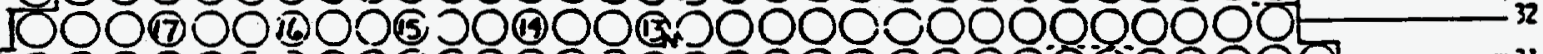
1000000:

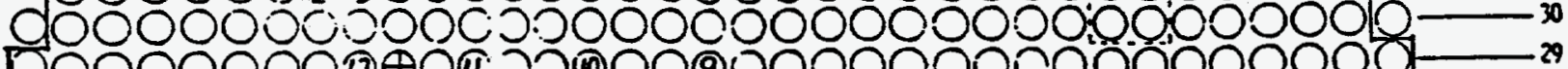

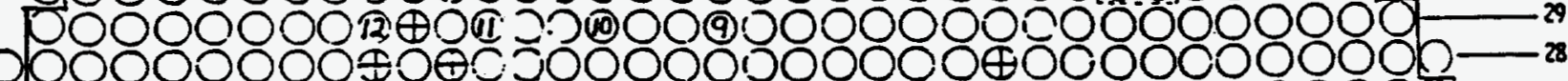

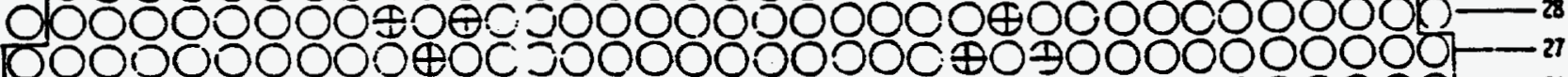

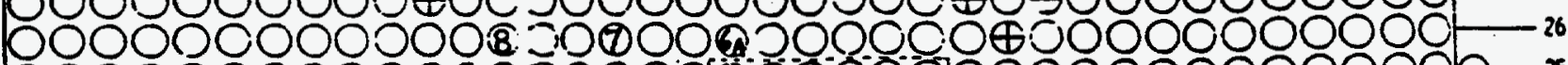

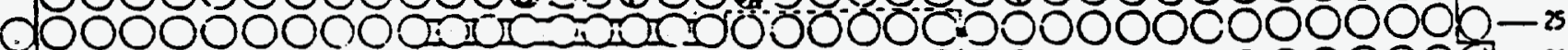
$00000000000000300000000000000000000000 y^{24}$ $5000000000000 \mathrm{cos} 0040000000000000000000$ -

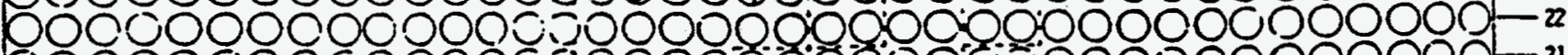

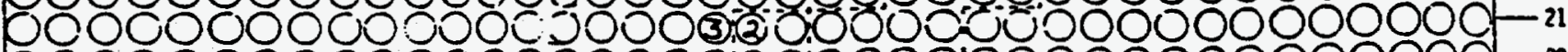

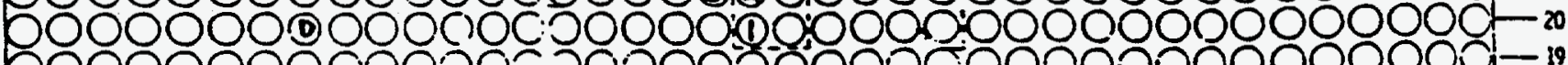

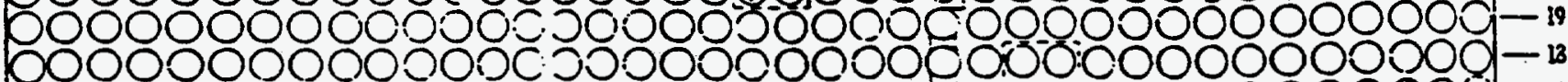

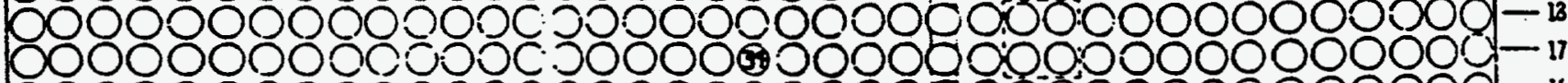

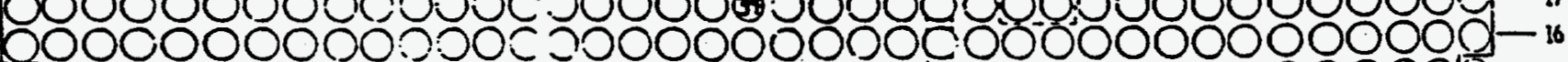
$0000000000000 \% 500000000000000000000000-$ 00000000000 =

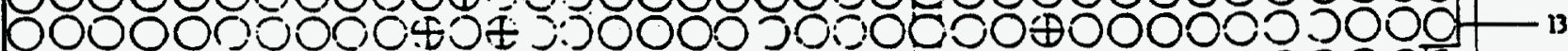
000000000005: $00500000000 \approx 0000300000000000000000+1-11$ 90000000.00 = =

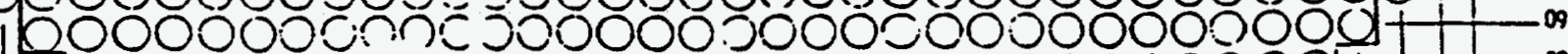
b000000000.0000000000000000000001 C00000000:5000000000000000000000 00000000

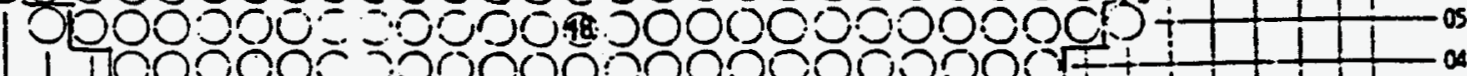
1000000 jobs

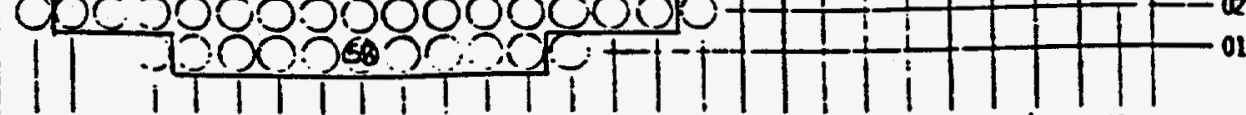

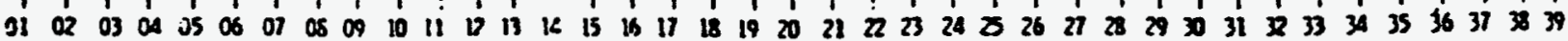

Figure 2.66 Core layout for EPRU75B. Source: Ref. 7 (Copyright $(\mathcal{C}$ 1976. Electric Power Research Institute. EPRI NP-196. Clean Critical Experiment Benchmarks for Plutonium Recycle in LWRs. Reprinted with Permission.) 
Physical Description

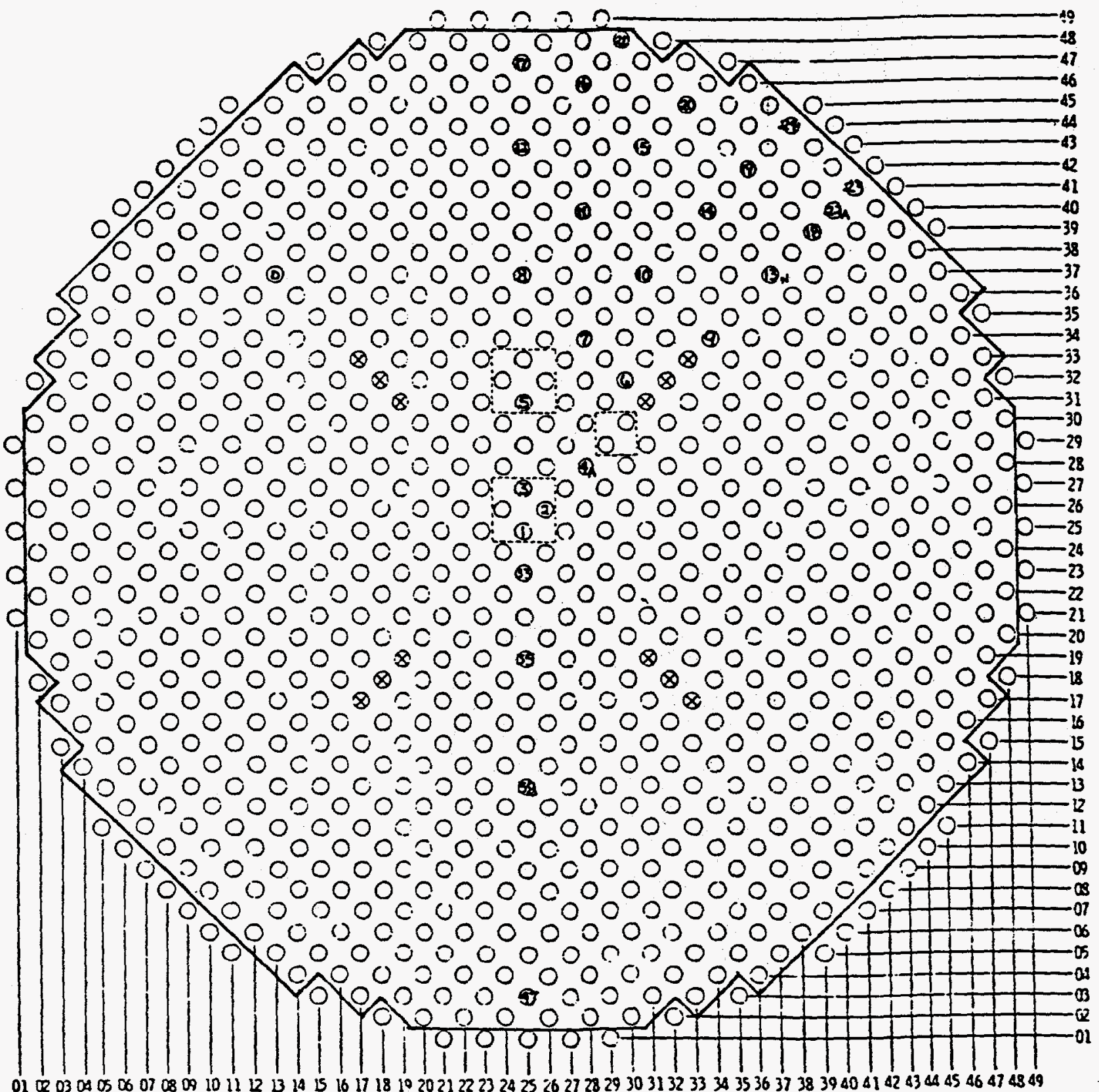

Figure 2.67 Core layout for EPRU87B. Source: Ref. 7 (Copyright $(C$ 1976. Electric Power Research Institute. EPRI NP-196. Clean Critical Experiment Benchmarks for Plutonium Recycle in LWRs. Reprinted with Permission.) 
Physical Description

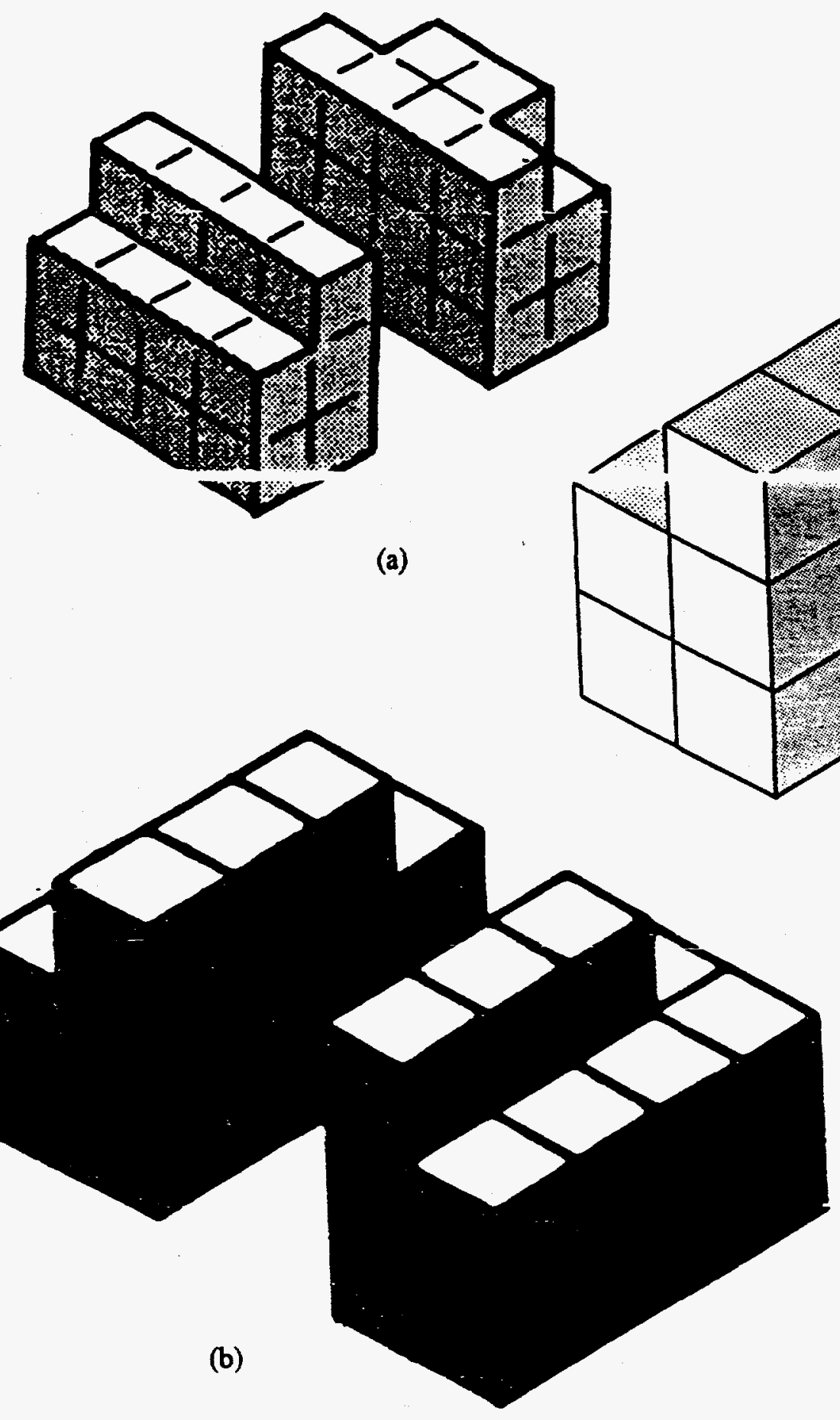

(c)

- Cans separated by 2.43 -cm-thick nonfire-retardant Plexiglas blocks

- Two arrays of cans separated by 0.31 , 1.26 , and $0.57 \mathrm{~cm}$ of void for CR1071AS, CR1653AS, and CR2500S

- Moderator above second level is present in the vicinity of absent cans as well as behind the top layer can.

Figure 2.68 Isometric drawings of the (a) 42 , (b) 38 , and (c) 30 can configurations of experiments CR1071AS, CR1653AS, CR2500S. Source: Refs. 29-31. 
Physical Description
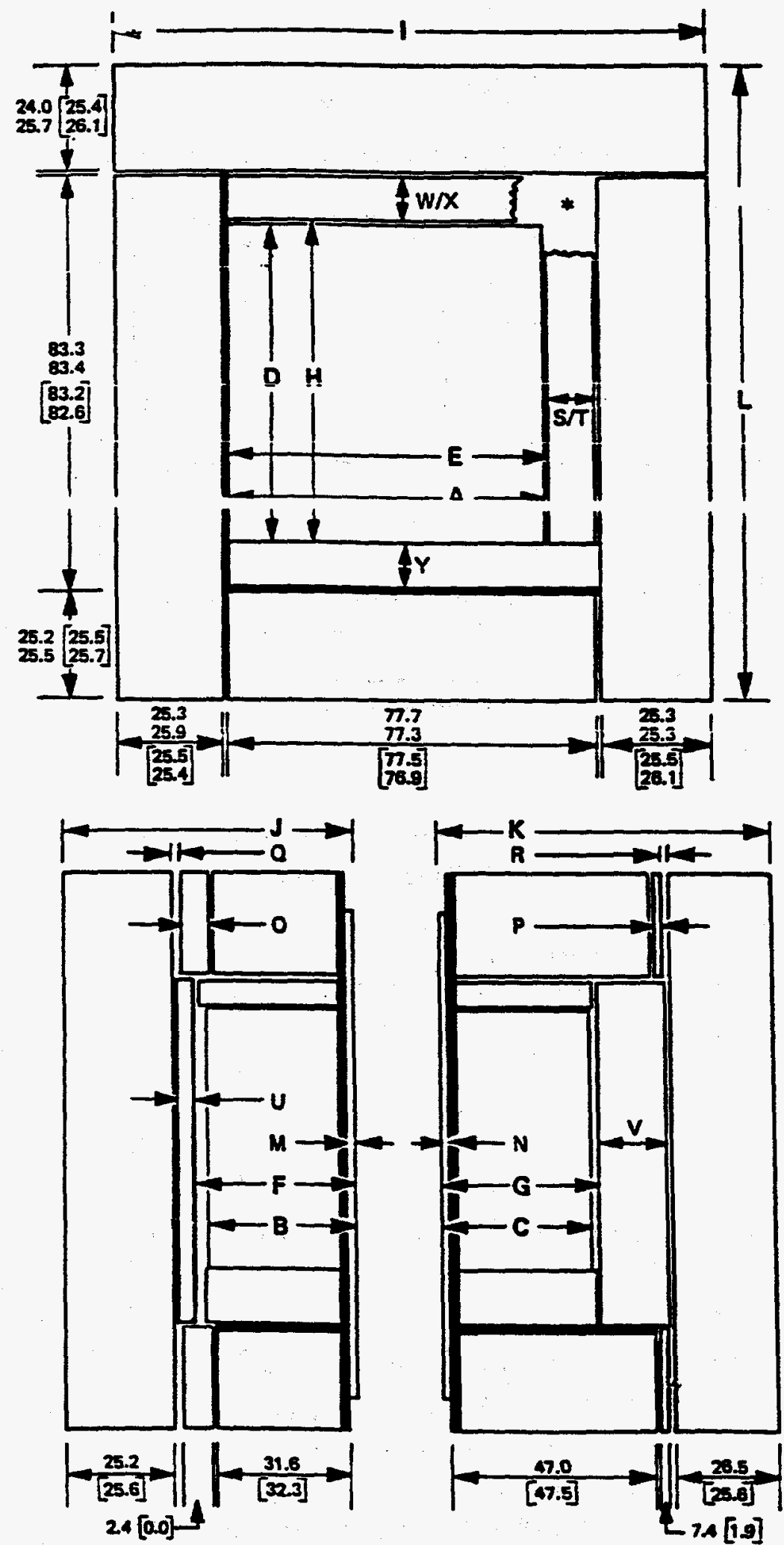

Figure 2.69 Planar and elevation view for CR1071AS (dimensions in $\mathrm{cm}$, paired dimensions are for north and south tables). Source: Ref. 29 
Physical Description
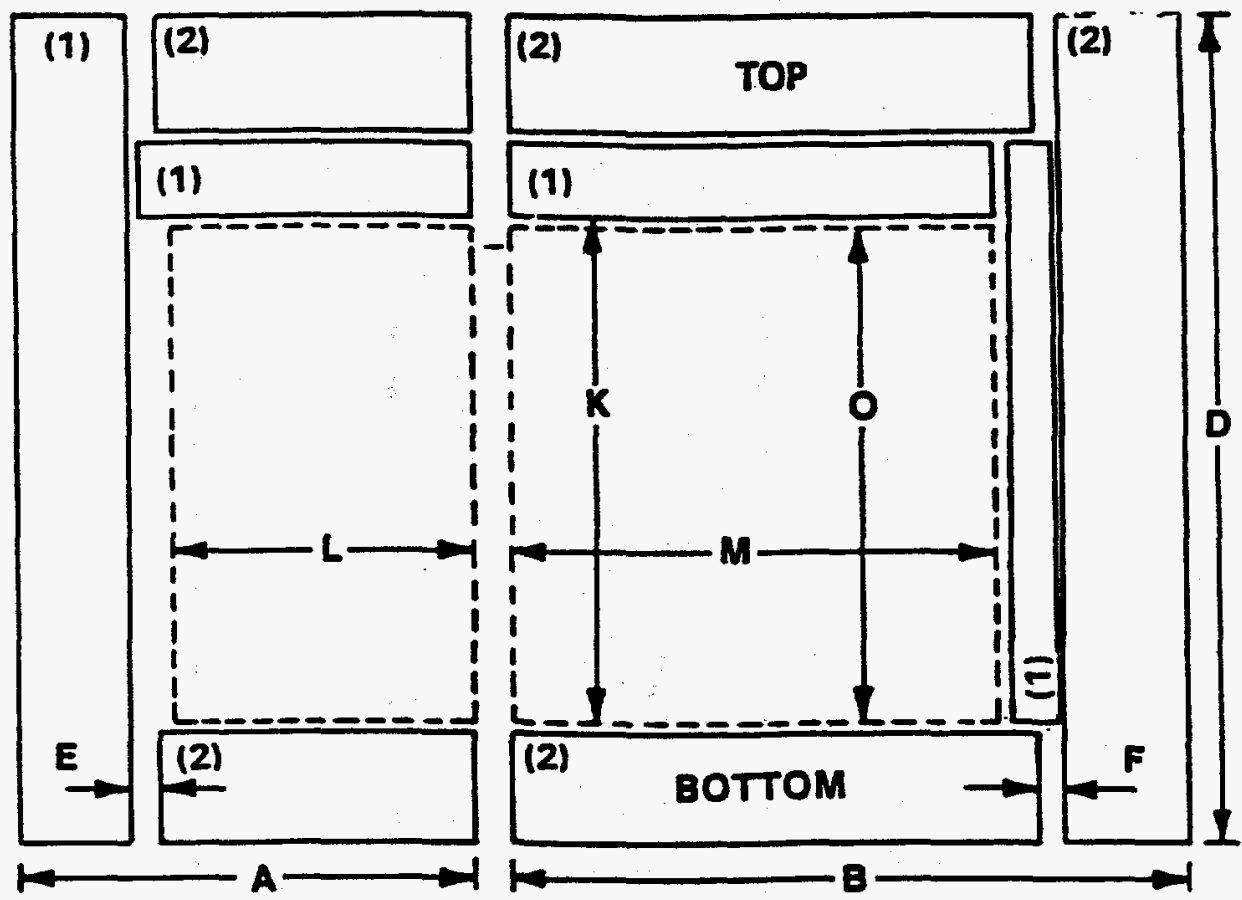

ELEVATION

NORTH

SOUTH $\longrightarrow$
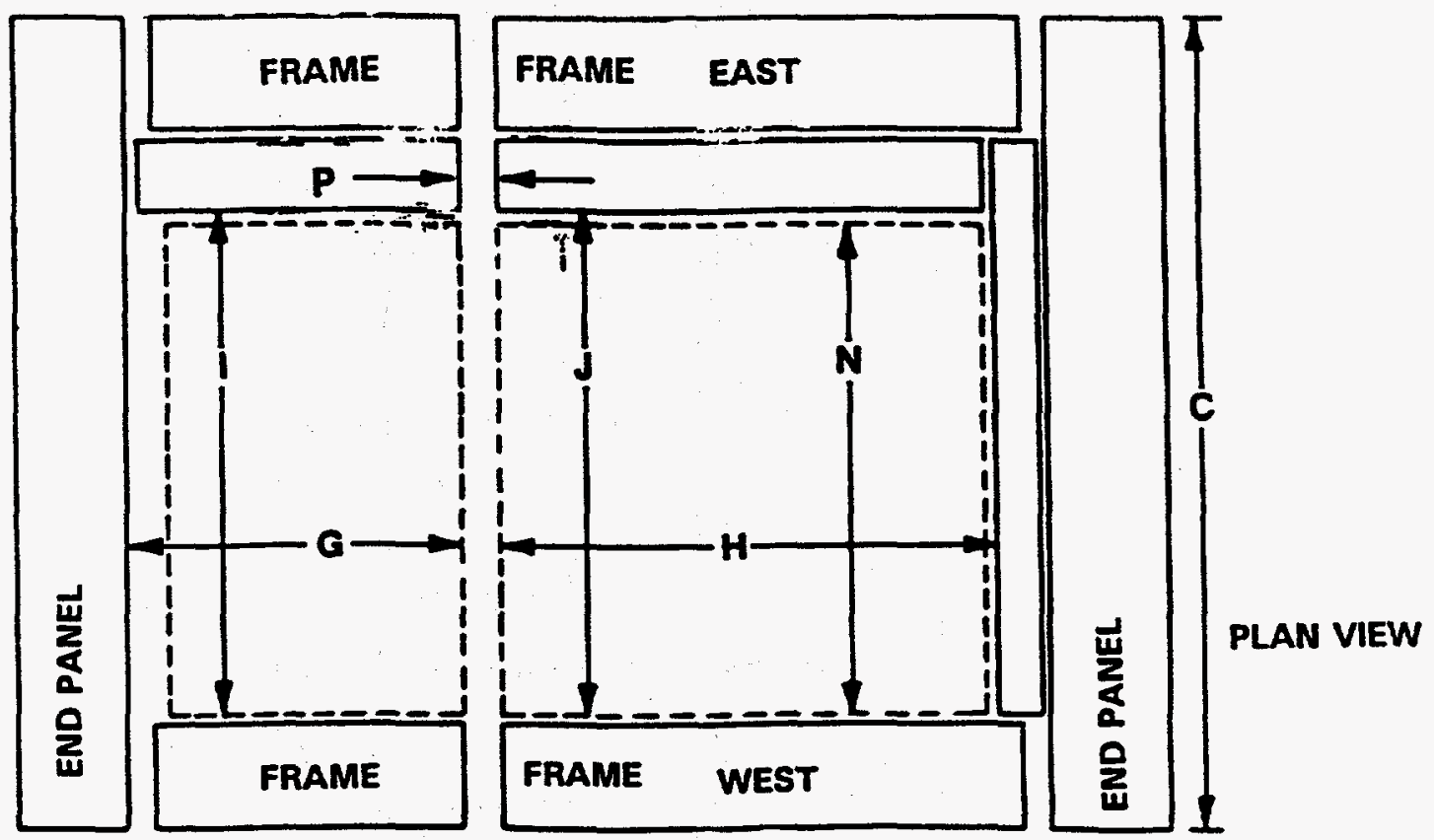

(1) Nonfire-retardent Plexiglas + paper $(0.49$ wt $\%)+$ glue $(0.16$ wt $\%)$

(2) Fire-retardent Plexiglas + paper $(0.49$ wt \%) + glue $(0.16$ wt \%)

Figure 2.70 Planar and elevation view of core boundary (dashed lines) and reflector boundaries (solid lines) for CR1653AS and CR2500AS. Source: Ref. 30. 
Physical Description

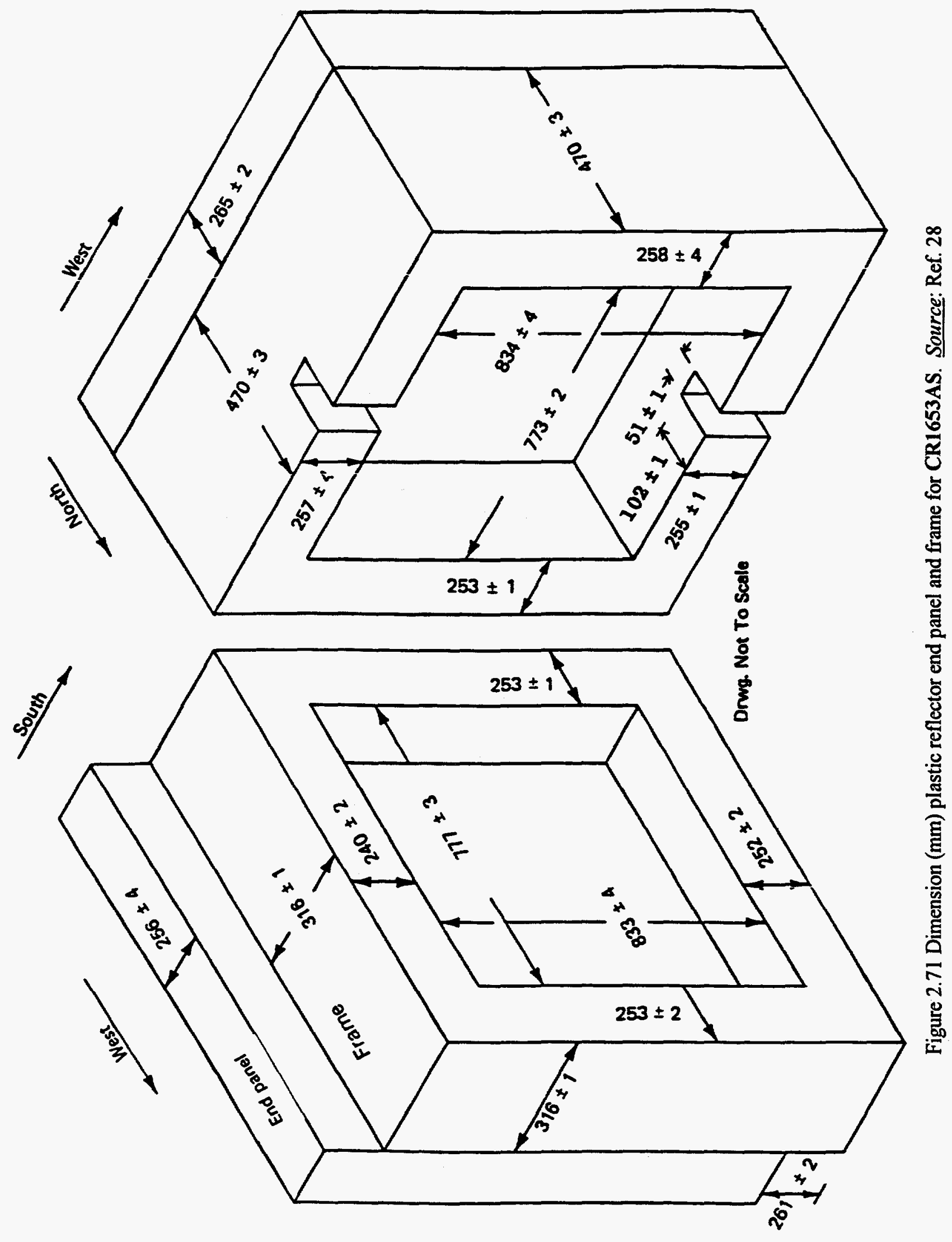

NUREG/CR-6361 
Physical Description

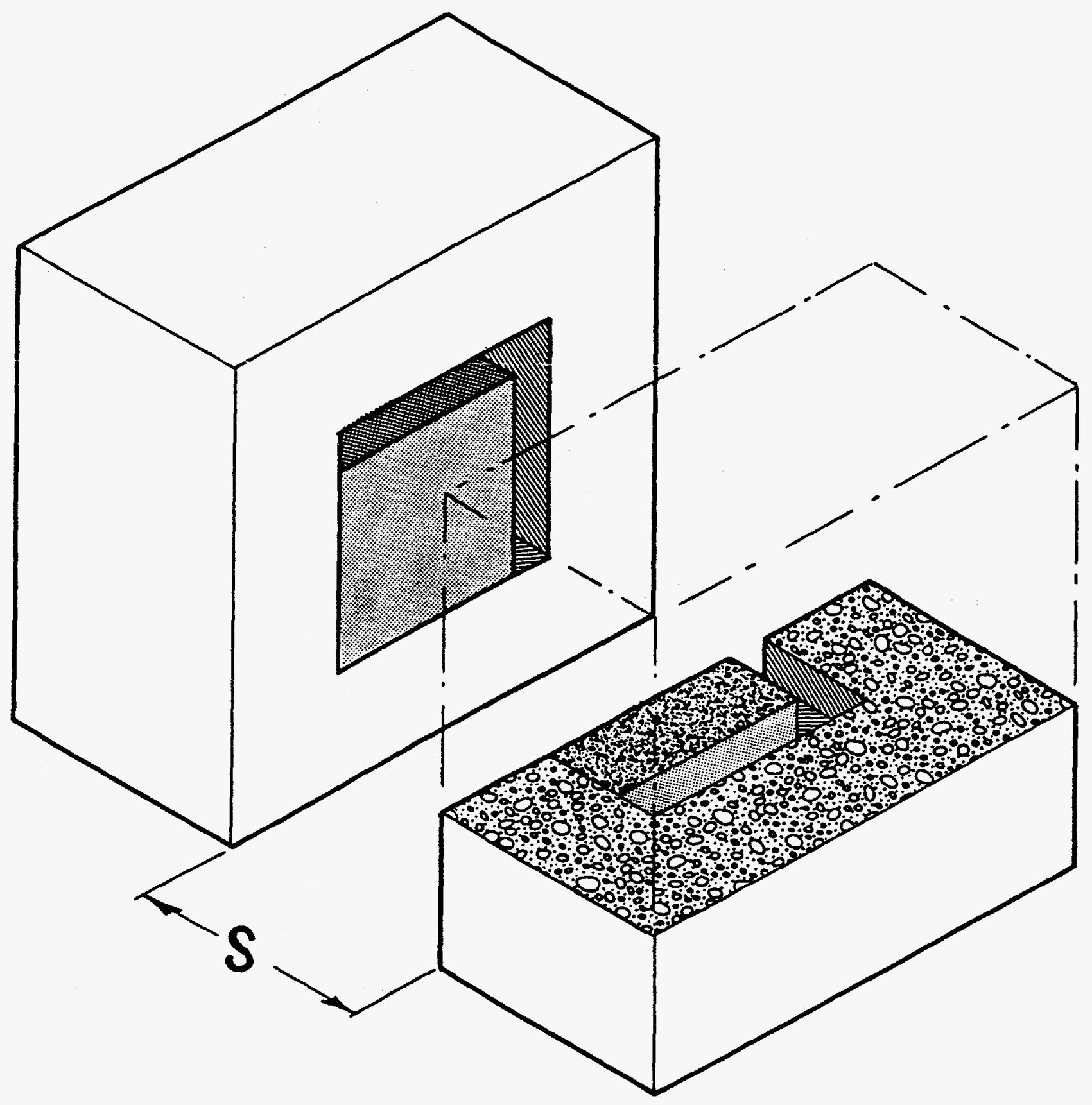

Figure 2.72 Three nested cuboids of CR1071AS and CR2500S: core, reflector interior, reflector exterior. Source: Ref. 29 



\section{Validation of the SCALE 44-Group ENDF/B-V Library with the LWR Critical Experiments}

In order to demonstrate the applicability of the SCALE 44-group ENDF/B-V cross-section library (created on $5 / 16 / 96)$ for the range of conditions spanned by the fresh fuel experiments described in the previous section, computational models have been developed based on each experimental configuration. Structural components such as control and safety rod guides, support angles and channels, and tanks were neglected because they had no significant impact on the system $k_{\text {eff }}$. Specific modeling assumptions were noted in detail in Sect. 2. Chemical elements that appeared in materials in trace amounts were also considered negligible in the models. For reference, the SCALE-4.3 input files for these experiments are included in Appendix A.

The following subsection describes the sequences and modules of the SCALE-4.3 system used in the analysis of these computational models. This description is followed by a discussion of the results of calculations performed with SCALE-4.3 using these models, in terms of the classes of experiments described in Sect. 2.

\subsection{Description of the SCALE Code System}

SCALE is a computational system consisting of a set of well-established codes and data libraries suitable for analyses of nuclear fuel facility and package designs in the areas of criticality safety, radiation shielding, source term characterization, and heat transfer. The codes are compiled in a modular fashion and called by control modules that provide automated sequences for standard system analyses in each area.

The CSAS control module contains automated sequences that perform problem-dependent cross-section processing and three-dimensional (3-D) Monte Carlo calculations of neutron multiplication. Three CSAS control sequences, CSASN, CSAS25, and CSAS2X, were used to perform resonance processing and/or calculate effective neutron multiplication factors for the critical benchmark experiments supplied in this document. All neutronic control sequences use the SCALE Material Information Processor (MIP) to calculate material number densities and prepare geometry data for resonance self-shielding and optional flux-weighting cell calculations and to create data input files for the cross-section processing codes. The BONAMI ${ }^{32}$ and NITAWL-II ${ }^{33}$ codes are then used to perform problemspecific (resonance- and temperature-corrected) cross-section processing. BONAMI applies the Bondarenko method of resonance self-shielding for nuclides that have Bondarenko data included in the cross-section library. NITAWL-II uses the Nordheim integral treatment to perform resonance self-shielding corrections for nuclides that have resonance parameters included with their cross-section data. The CSASN sequence terminates at this point; the cross-section library produced from this calculation can be used as is or combined with other cross-section libraries and used in a subsequent criticality calculation.

The CSAS25 sequence then invokes KENO V.a, ${ }^{34}$ a three-dimensional (3-D) multigroup Monte Carlo criticality code to determine the effective multiplication factor $\left(k_{\mathrm{eff}}\right)$ from the problem-dependent cross-section data and the userspecified geometry data. Other calculated KENO V.a quantities include average neutron lifetime and generation time, energy-dependent leakages, energy- and region-dependent absorptions, fissions, fluxes, and fission densities. The CSAS2X sequence invokes the one-dimensional (1-D) discrete-ordinates code XSDRNPM ${ }^{35}$ to prepare cell-weighted cross sections prior to the execution of KENO V.a.

SCALE-4.3 includes the 238-group AMPX master-format neutron cross-section library ${ }^{3}$ which contains data for all the nuclides available in ENDF/B-V and a broad structure 44-group neutron cross-section library ${ }^{2}$ collapsed from the 238group library. The group structure specification for each library was based on knowledge gained from the definition and use of the 218-group and 27-group libraries developed for the SCALE code system using ENDF/B-IV data. 


\section{Validation}

The 238-group library has Bondarenko shielding factors included for nuclides with unresolved resonances, nuclides with Adler-Adler resonance data $\left({ }^{233} \mathrm{U}\right.$ and $\left.{ }^{241} \mathrm{Pu}\right)$, and the nonresonance nuclides ${ }^{7} \mathrm{Li},{ }^{19} \mathrm{~F},{ }^{27} \mathrm{Al}$, and ${ }^{28} \mathrm{Si}$. Bondarenko factors are used for the latter nuclides because they exhibit resonance structure in the point data. This library includes $s-, p-$, and d-wave resonance data in the resonance parameters that are passed to NITAWL-II. The calculational effect of including these higher-order resonance data is addressed in Appendix B.

The broad-structure 44-group derivative of the fine-structure 238-group library was collapsed using a fuel cell spectrum based on a $17 \times 17$ Westinghouse pressurized-water-reactor (PWR) fuel assembly. The broad-group structure was designed to accommodate two windows in the oxygen cross-section spectrum, a window in the iron cross-section spectrum, the Maxwellian peak in the thermal range, and the $0.3-\mathrm{eV}$ resonance in ${ }^{239} \mathrm{Pu}$ (which, due to low energy and lack of resonance data, cannot be modeled by the Nordheim Integral Treatment in NITAWL-II). The 44-group library has a group structure similar to the 27-group library, as shown in Table 3.1. All boundaries in the 27-group ENDF/ BIV library correspond to those in the 44-group ENDF/B-V library except for the upper bound of group 23. Based on an analysis of a set of 93 thermal and fast critical experiments, the ENDF/B-V 44-group library demonstrated markedly improved performance over similar analyses performed with the ENDF/B-IV 27-group library. ${ }^{2}$

\subsection{Modeling and Calculational Techniques}

Several techniques for modeling the fuel region of particular experiments are addressed in Sect. 3.2.1. KENO V.a input parameters are discussed in Sect. 3.2.2.

\subsubsection{Fuel Region Modeling}

The experiments listed in Table 3.2 were explicitly modeled by considering the fuel rod regions submerged in and protruding from the moderator region. To accomplish this in CSAS, the Dancoff factors and resonance data had to be calculated for the fuel in the region void of moderator using a CSASN "PARM=CHECK" case and then explicitly specified in the CSAS25 calculation using the "MORE DATA" option. In all other experiments with fuel rods above the moderator, the contribution of the fuel in the region void of moderator was considered negligible and was not modeled.

In some instances more than one fissile mixture was present per experiment. Since only one CSAS unit cell specification is allowed per problem, the Dancoff factors and resonance data had to be calculated for the other unit cell descriptions using a CSASN "PARM=CHECK" case and then explicitly specified in the CSAS25 calculation using the "MORE DATA" option. The experiments with two or three fissile mixtures modeled using this method are listed in Table 3.3.

The experiments in Table 3.4 were modeled with CSAS2X in order to account for eccentricities of the control rod cruciform followers (the nine fuel rods of the follower veins were on a slightly different pitch than the core lattice). The followers were represented as nine core lattice positions containing mixture 500 that uses the XSDRNPM cellweighted cross sections for the fuel and moderator. For the fuel rods of the core, the Dancoff factors and resonance data had to be calculated for the regions submerged in and protruding from the moderator region using CSASN and then explicitly specified in the CSAS25 calculation as in the other experiments above.

\subsubsection{KENO V.a Parameter Data}

All criticality calculations were run with 405 generations and 600 neutrons per generation for a total of 245,000 histories. The first five generations were omitted when calculating the average eigenvalue of the system. Default values 
were used for all other parameters including the start option of flat neutron distribution over the entire system. No albedo boundary conditions were applied; the default for each face is vacuum and is applied to the outermost region of every problem. No biasing was used to track neutrons in the water reflector region of each problem.

The calculated $k_{\text {eff }}$ values throughout this report are estimates of the eigenvalues of systems and have associated uncertainties due to the statistical nature of the Monte Carlo codes.

\subsection{Calculational Results Overview}

The calculational results for the $173 \mathrm{LWR}$ fuel pin lattice critical experiments described in Sect. 2.1 are presented and discussed in Sect. 3.4. The results for the seven homogeneous uranium systems described in Sect. 2.2 are presented and discussed in Sect. 3.5.

Two important calculated parameters in the presentation of results are the average energy group causing fission (AEG) and the energy of the average lethargy causing fission (AEF). AEG is a pseudo-neutron-spectra parameter calculated by KENO V.a that has been used historically as a single global parameter for correlating experiments to safety evaluation applications. ${ }^{36}$ AEF has been added to the KENO V.a output in version 4.3 of SCALE and offers a single parameter in physical units (eV). Due to group structure and the techniques used to determine the average values, the energy that corresponds to AEG and the value of AEF may not be equivalent. AEG is a spectral index that is useful in validation but is not valid when comparing different cross-section libraries. On the other hand, AEF is more useful in interlibrary comparisons since it is a physically real parameter and not as dependent on the group structure of a library. For this reason, the remainder of this report will focus on AEF rather than AEG. Table 3.1 gives the group structure for the 44-group library used in this validation and is useful in locating an energy range corresponding to an AEG value. The table provides the upper energy bound of each broad group and the number of fine groups collapsed into it from the 238-group cross-section library.

In the following sections of this report, the validation results have been analyzed by grouping the experiments in terms of physical or neutronic characteristics as set forth in Sect. 2. For each group, the mean $k_{\text {eff }}\left(\bar{k}_{\text {eff }}\right)$ and the range of calculated $k_{\text {eff }}$ and AEF $(e V)$ values are reported. The reported mean is the average of the nominal values of $k_{\text {eff }}$ computed for each group of experiments. The standard deviation $\left(\sigma_{k}\right)$ characterizes the distribution of the nominal values around this mean; this statistic does not include uncertainties associated with each individual Monte Carlo calculation.

In the discussions that follow, the term "positive bias" denotes a calculated $k_{\text {eff }}$ value greater than the measured critical condition $\left(k_{\text {eff }}=1\right)$ and "negative bias" conversely denotes a calculated $k_{\text {eff }}$ value less than 1 . The remainder of this section presents the results of these analyses by group. A comparison of these results to those obtained with the 238 group library is provided in Appendix B.

\subsection{Results for LWR Fuel Pin Lattices}

This section reports the calculational results for 173 critical experiments classified as containing LWR fuel pin lattices. Table 3.5 contains nominal computed values of the effective neutron multiplication factor, $\mathrm{k}_{\text {eft }}$, with associated standard deviation ( $\sigma$ ), average energy group of neutrons causing fission (AEG), and energy of the average lethargy causing fission (AEF) for the LWR critical experiments. Figures 3.1 and 3.2 are histograms showing the frequency of $k_{\text {eff }}$ and AEF for all 173 experiments in Table 3.5. Note the clustering of calculated $k_{\text {eff }}$ near $k_{\text {eff }}=1$ and the narrow range of AEF values that this validation encompasses. This distribuiton is remarkably normal, indicating no systematic 


\section{Validation}

biases on a global level. The nominal calculated $k_{\text {eff }}$ values range from 0.9909 to 1.0129 , with an average $k_{\text {eff }}$ value of $0.9994 \pm 0.0035$ and an average bias of $-0.06 \%$. Sixty-eight percent of all $k_{\text {eff }}$ values fall within 0.0035 of 0.9994 while $95 \%$ of all $k_{\text {eff }}$ values fall within 0.0070 of 0.9994 . AEF values ranged from 3.4451 to $0.0702 \mathrm{eV}$ with more than $75 \%$ of the values within the range of 0.1 to $0.562 \mathrm{eV}$.

\subsubsection{Simple Lattices}

Descriptive statistics of the 21 simple (i.e., no absorbers, poisons, reflecting walls, etc.) square or hexagonal lattice calculations are given in Table 3.6. As can be seen from the table, the 17 square lattice calculations exhibited an average bias of $-0.20 \%$ and spanned a relatively large range of $A E F$ values, attesting to the variety of square lattice experiments used in the validation. The limited set of four hexagonal lattice calculations has a positive bias of $0.27 \%$. Note that the hexagonal lattice experiments tend to have harder spectra than the square lattices and span a much larger energy range.

\subsubsection{Lattices and Separator Plates Only}

The calculated $k_{\text {eff }}$ results for the 45 lattice with separator plate calculations are given in Table 3.7. The results of calculations of simple lattices with separator plates and borated moderator are given in Table 3.8, while Table 3.9 gives statistics of the calculational results of experiments with separator plates and voiding materials (i.e., flux traps). From Tables 3.7,3.8, and 3.9, one can see that calculations involving aluminum separator plates have larger standard deviations associated with the average $\mathrm{k}_{\mathrm{eff}}$ values, indicating a larger variation in results for these experiments. In all three tables, the highest $k_{\text {eff }}$ values are recorded for calculations involving aluminum separator plates.

\subsubsection{Lattices and Reflecting Walls Only}

The calculational results of 35 lattice experiments with reflecting walls are summarized in Table 3.10. The largest positive bias in the validation of LWR-type fuel pin lattices for a group of more than four experiments is seen in the lead reflecting wall experiment calculations. For this group of experiments, the calculational bias is $+0.37 \%$. Only very small positive biases are observed for the calculations of the steel reflecting wall and the uranium reflecting wall experiments. The overall bias for all reflecting wall experiment calculations is $+0.13 \%$.

\subsubsection{Lattices with Separator Plates and Reflecting Walls}

The calculational results of 15 lattice experiments with separator plates and reflecting walls are presented in Table 3.11. The calculated $k_{\text {eff }}$ values were scattered over a broad range from 0.9920 to 1.0040 . As a group, the average calculational bias was $-0.08 \%$, and a narrow range of AEF values was observed $(0.144-0.305 \mathrm{eV})$.

\subsubsection{Lattices with Urania Gadolinia Rods}

Table 3.12 gives descriptive statistics of the five lattices containing interspersed fuel rods with gadolinium in the form of $\mathrm{UO}_{2}-\mathrm{Gd}_{2} \mathrm{O}_{3}$. This set of five calculations yielded nominal $\mathrm{k}_{\text {eff }}$ values over a small range from 0.9971 to 1.0005 with an average $\mathrm{k}_{\mathrm{eff}}$ value of 0.9986 (Table 3.12). All of these experiments contained water holes and soluble boron. These cases give very consistent results but provide a very limited validation for urania gadolinia because the set consisted of only five critical experiments from one report with a small range in AEF values. 
Validation

\subsubsection{Lattices with Water Holes}

A negative calculational bias of $0.15 \%$ is observed in the results in Table 3.13 for the six water-hole experiments with no soluble boron. For the three water-hole calculations where soluble boron was used in the moderator, a small bias of $-0.08 \%$ and an increased average AEF (i.e., harder spectrum) were observed.

\subsubsection{Lattices with $\mathrm{B}_{4} \mathrm{C}$ or Ag-In-Cd Absorber Rods}

Calculations of experiments with $\mathrm{B}_{4} \mathrm{C}$ or $\mathrm{Ag}$-In- $\mathrm{Cd}$ rods had average biases of $-0.49 \%$ and $-0.30 \%$, respectively. One experiment in each group contained soluble boron and water holes. The total bias for the set of nine absorber rod calculations was $-0.37 \%$, given in Table 3.14 with other descriptive statistics of these calculations.

\subsubsection{Simple Lattices with Soluble Boron}

Fourteen simple lattice experiments contained soluble boron in the moderator. This set of calculations yielded calculated $k_{\text {eff }}$ values ranging from 0.9947 to 1.0035 with an average $k_{\text {eff }}$ value of 0.9992 (Table 3.15). AEF values spanned from 0.92 to $1.189 \mathrm{eV}$, a significant span of thermalized systems.

\subsection{Results for Homogeneous Uranium Systems}

This section reports the calculational results for seven low-enriched homogeneous uranium critical experiments. These experiments are included because some transportation packages may contain low-enriched fuel in geometric configurations other than the typical LWR fuel pin lattice geometry. Individual results are tabulated in Table 3.16. Group statistics are presented in Table 3.17.

The nominal calculated $k_{\text {eff }}$ values ranged from 1.0011 to 1.0207 with an average bias of $+1.29 \%$. This bias is attributed to the manner in which the library was generated. The 44-group library was collapsed to encompass the key spectral aspects of a different type of system, that of a typical LWR spent fuel package. Results obtained for these cases with the 238-group library show a much smaller bias, as can be seen in Table B.3. Only a small number of homogeneous systems have been considered in this validation. These few results for homogeneous systems indicate a larger bias than demonstrated for lattice systems. If the 44-group library is to be used for low-enriched fuel in other geometric configurations, care should be taken to ensure that adequate validation is performed for the particular application.

\subsubsection{Homogeneous $\mathrm{UF}_{4}$ in Paraffin}

This set of calculations consisted of four experiments. Note that the $2.00 \mathrm{wt} \%{ }^{235} \mathrm{U}$ cases agree well with the LWR fuel results (i.e., $+0.29 \%$ bias). However, the $3.00 \mathrm{wt} \%$ cases exhibit a bias of approximately $+1.4 \%$. Although the reasons for this discrepancy are unknown, it is consistent with previous results for these cases. ${ }^{37}$

\subsubsection{Damp Oxide}

This set of calculations consisted of three experiments. The average calculated $\mathrm{k}_{\text {eff }}$ value is $1.9 \%$ high. This bias is consistent with previous results for these cases. ${ }^{37}$ 
Validation

Table 3.1 Broad-group structure of the 44GROUPNDF5 library

\begin{tabular}{|c|c|c|c|c|c|}
\hline \multicolumn{3}{|c|}{ Fast groups } & \multicolumn{3}{|c|}{ Thermal groups } \\
\hline Group & $\begin{array}{l}\text { No. fine } \\
\text { groups }\end{array}$ & $\begin{array}{c}\text { Upper energy } \\
(\mathrm{eV})\end{array}$ & Group & $\begin{array}{l}\text { No. fine } \\
\text { groups }\end{array}$ & $\begin{array}{c}\text { Upper energy } \\
(\mathrm{eV})\end{array}$ \\
\hline 1 & (7) & $2.0000 \times 10^{7 a}$ & 23 & (14) & $3.0000 \times 10^{0 b}$ \\
\hline 2 & (1) & $8.1873 \times 10^{6}$ & 24 & (27) & $1.7700 \times 10^{0 a}$ \\
\hline 3 & (1) & $6.4340 \times 10^{6 a}$ & 25 & (10) & $1.0000 \times 10^{0 a}$ \\
\hline 4 & (2) & $4.8000 \times 10^{6}$ & 26 & (5) & $6.2500 \times 10^{-1}$ \\
\hline 5 & (1) & $3.0000 \times 10^{6 a}$ & 27 & (1) & $4.0000 \times 10^{-1 a}$ \\
\hline 6 & (1) & $2.4790 \times 10^{6}$ & 28 & (1) & $3.7500 \times 10^{-1}$ \\
\hline 7 & (1) & $2.3540 \times 10^{6}$ & 29 & (1) & $3.5000 \times 10^{-1}$ \\
\hline 8 & (2) & $1.8500 \times 10^{6 a}$ & 30 & (2) & $3.2500 \times 10^{-1 a}$ \\
\hline 9 & (8) & $1.4000 \times 10^{6 a}$ & 31 & (1) & $2.7500 \times 10^{-1}$ \\
\hline 10 & (14) & $9.0000 \times 10^{5 a}$ & 32 & (1) & $2.5000 \times 10^{-1}$ \\
\hline 11 & (6) & $4.0000 \times 10^{5 a}$ & 33 & (1) & $2.2500 \times 10^{-1 a}$ \\
\hline 12 & (10) & $1.0000 \times 10^{5 a}$ & 34 & (2) & $2.0000 \times 10^{-1}$ \\
\hline 13 & (1) & $2.5000 \times 10^{4}$ & 35 & (2) & $1.5000 \times 10^{-1}$ \\
\hline 14 & (7) & $1.7000 \times 10^{4 a}$ & 36 & (3) & $1.0000 \times 10^{-1 a}$ \\
\hline 15 & (11) & $3.0000 \times 10^{3 a}$ & 37 & (2) & $7.0000 \times 10^{-2}$ \\
\hline 16 & (12) & $5.5000 \times 10^{2 a}$ & 38 & (1) & $5.0000 \times 10^{-2 a}$ \\
\hline 17 & (30) & $1.0000 \times 10^{2 a}$ & 39 & (1) & $4.0000 \times 10^{-2}$ \\
\hline 18 & (16) & $3.0000 \times 10^{1 a}$ & 40 & (1) & $3.0000 \times 10^{-2 a}$ \\
\hline 19 & (2) & $1.0000 \times 10^{1 a}$ & 41 & (1) & $2.5300 \times 10^{-2}$ \\
\hline 20 & (6) & $8.1000 \times 10^{0}$ & 42 & (1) & $1.0000 \times 10^{-2 a}$ \\
\hline 21 & (3) & $6.0000 \times 10^{0}$ & 43 & (3) & $7.5000 \times 10^{-3}$ \\
\hline \multirow[t]{2}{*}{22} & (6) & $4.7500 \times 10^{0}$ & 44 & (9) & $3.0000 \times 10^{-3}$ \\
\hline & & & \multicolumn{2}{|c|}{44 Lower Energy } & $1.0000 \times 10^{-5}$ \\
\hline
\end{tabular}

27-group boundaries.

${ }^{b}$ Adjusted 27-group boundary.

Table 3.2 Explicit fuel rod models for fuel above the moderator

$\begin{array}{llll}\text { ANS33AL1 } & \text { ANS33STY } & \text { NSE71H1 } & \text { W3269C } \\ \text { ANS33AL2 } & \text { BW1231B1 } & \text { NSE71H2 } & \text { W3269SL1 } \\ \text { ANS33AL3 } & \text { BW1231B2 } & \text { NSE71H3 } & \text { W3269SL2 } \\ \text { ANS33EB1 } & \text { BW1273M } & \text { PAT80L1 } & \text { W3269W1 } \\ \text { ANS33EB2 } & \text { DSN399-1 } & \text { PAT80L2 } & \text { W3269W2 } \\ \text { ANS33EP1 } & \text { DSN399-2 } & \text { PAT80SS1 } & \text { W3385SL1 } \\ \text { ANS33EP2 } & \text { DSN399-3 } & \text { PAT80SS2 } & \text { W3385SL2 } \\ \text { ANS33SLG } & \text { DSN399-4 } & \text { W3269A } & \end{array}$


Table 3.3 Experiments with multiple fissile mixtures

\begin{tabular}{cc}
\hline Two fissile mixtures & Three fissile mixtures \\
\hline BW1810A & BW1810J \\
BW1810B & BW1810C \\
BW1810G & BW1810D \\
BW1810H & BW1810E \\
\hline
\end{tabular}

Table 3.4 Experiments modeled with CSAS2X

W3269B1

W3269B2

W3269B3 


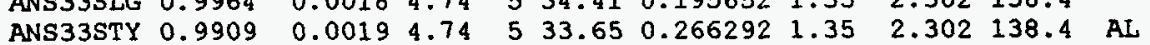

$10 \quad$ B1645SO1 $0.9976 \quad 0.0013 \quad 2.46 \quad 17 \quad 32.82 \quad 0.401634 \quad 1.410 \quad 1.015 \quad 119.4$ AL

$11 \mathrm{~B} 16455021.0007$

$\begin{array}{lllllllllll}\text { B1645SO2 } & 1.0007 & 0.0014 & 2.46 & 17 & 32.76 & 0.411180 & 1.410 & 1.015 & 119.4 \text { AL } \\ \text { BW1231B1 } & 0.9969 & 0.0014 & 4.02 & 25 & 31.13 & 0.727147 & 1.511 & 1.139 & 88.6 & -\end{array}$

13 BW1231B2 0.9985

14 BW1273M 0.9947

15 BW1 484A1 0.9965

16 BW1 484A2 0.9954

17 BW1 484B1 0.9973

18 BW1484B2 0.9973

19 BW1484B3 0.9992

20 BN1484C1 0.9937

21.

.

BW1 $484 \mathrm{C} 20.993$

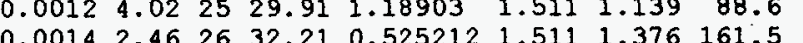

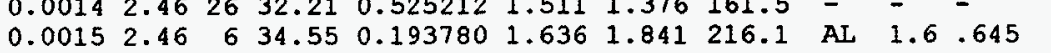

$\begin{array}{llllllllllll}0.0016 & 2.46 & 6 & 35.16 & 0.152035 & 1.636 & 1.841 & 216.1 & \text { AL } & 1.1 .645\end{array}$

$\begin{array}{llllllll}0.0014 & 2.46 & 6 & 33.95 & 0.247151 & 1.636 & 1.841 & 216.1\end{array}$

$\begin{array}{llllllll}0.0012 & 2.46 & 6 & 34.55 & 0.193879 & 1.636 & 1.841 & 216.1\end{array}$

$\begin{array}{lllllllll}0.0014 & 2.46 & 6 & 35.25 & 0.146615 & 1.636 & 1.841 & 216.1\end{array}$

$\begin{array}{lllllllll}0.0014 & 2.46 & 6 & 34.64 & 0.188354 & 1.636 & 1.841 & 216.1\end{array}$

$\begin{array}{llllllllll}0.9981 & 0.0015 & 2.46 & 6 & 34.52 & 0.194990 & 1.636 & 1.841 & 216.1 & \text { SS }\end{array}$

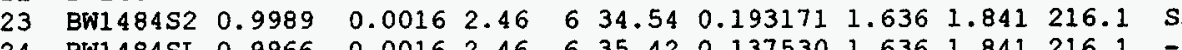

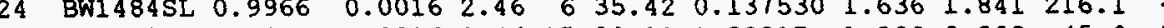

$\begin{array}{lllllllllllll}25 & \text { BW1645S1 } & 1.0010 & 0.0016 & 2.46 & 17 & 30.11 & 1.32817 & 1.209 & 0.383 & 45.0 & \text { AL }\end{array}$

$\begin{array}{llllllllllll}26 & \text { BW1645S2 } & 1.0035 & 0.0014 & 2.46 & 17 & 29.99 & 1.39167 & 1.209 & 0.383 & 45.0 & \text { AL }\end{array}$

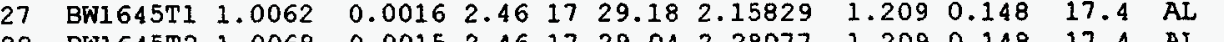

29 BW1645T 31.0007

30 BW1645T4 0.998

31 BW1B1OA 0.9971

32 BW1810B 0.9980

$\begin{array}{lllllllll}0.0015 & 2.46 & 17 & 29.04 & 2.28077 & 1.209 & 0.148 & 17.4 & \text { AL }\end{array}$

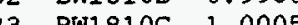

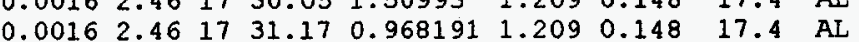

$0.0013 \quad 2.46 \quad 2433.960 .2447831 .6361 .841216 .1$

-

30

30

4 BW1810D 0.9999

$0.0012 \quad 2.4624 \quad 33.140 .3313691 .6361 .841216 .1$ $4.02 \quad 1.532119 .2$

$\begin{array}{llllll}35 & 4.02 & & 0.3369401 .636 & 1.841 & 216.1 \\ & 4.532 & 119.2\end{array}$

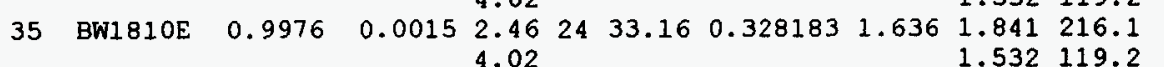

$\begin{array}{lllllllllll}36 & \mathrm{BW} 1810 \mathrm{~F} & 1.0032 & 0.0010 & 2.46 & 24 & 33.96 & 0.244782 & 1.636 & 1.841 & 216.1\end{array}$

$\begin{array}{lllllllllll}37 & \text { BW1810G } & 0.9971 & 0.0012 & 2.46 & 24 & 32.91 & 0.360952 & 1.636 & 1.841 & 216.1\end{array}$

$38 \mathrm{BW} 181 \mathrm{OH} \quad 0.9973 \quad 0.0013 \quad 2.46 \quad 24 \quad 32.95 \quad 0.354969 \quad 1.636 \quad 1.841 \quad 216.1$

$4.02 \quad 1.532119 .2$

$\begin{array}{llllllllllll}39 & \text { BW1810I } & 1.0022 & 0.0014 & 2.46 & 24 & 33.96 & 0.245038 & 1.636 & 1.841 & 216.1\end{array}$

40 BW1810J $0.9980 \quad 0.0013 \quad 2.4624 \quad 33.130 .3323941 .6361 .532 \quad 216.1$

4.0221 .532119 .2

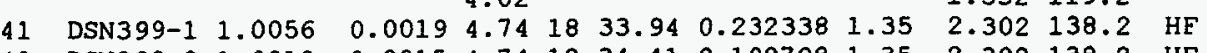

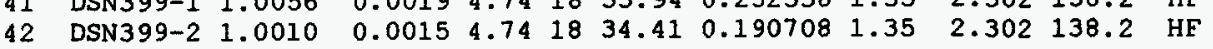

\begin{tabular}{|c|c|c|c|}
\hline- & $\mathbf{N}$ & - & $\mathrm{AL}$ \\
\hline - & $\mathrm{N}$ & - & $A I$ \\
\hline - & $\mathrm{N}$ & - & AL \\
\hline - & $\mathbf{N}$ & - & AL \\
\hline - & $\mathbf{N}$ & - & $\mathrm{AL}$ \\
\hline - & $\mathbf{N}$ & - & $A L$ \\
\hline - & $\mathrm{N}$ & - & AL \\
\hline- & $\mathbf{N}$ & - & $A L$ \\
\hline - & $\mathbf{N}$ & - & AL \\
\hline - & $Y$ & 1068 & $\mathrm{AL}$ \\
\hline - & $Y$ & 1156 & $\mathrm{AL}$ \\
\hline - & $Y$ & 1152 & SS \\
\hline - & $Y$ & 3389 & sS \\
\hline - & $Y$ & 1675 & AL \\
\hline - & $\mathbf{Y}$ & 15 & $\mathrm{AL}$ \\
\hline - & $\mathbf{Y}$ & 72 & AL \\
\hline - & $\mathbf{Y}$ & 1037 & AL \\
\hline - & $\mathbf{Y}$ & 769 & $\mathrm{AL}$ \\
\hline - & $\mathbf{Y}$ & 143 & $A L$ \\
\hline - & $\mathbf{N}$ & - & $A L$ \\
\hline - & $\mathrm{N}$ & - & AL \\
\hline - & $Y$ & 432 & AL \\
\hline - & $Y$ & 514 & $A L$ \\
\hline - & $\mathbf{N}$ & - & $A L$ \\
\hline 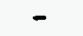 & $Y$ & 746 & AL \\
\hline- & $Y$ & 886 & $A L$ \\
\hline - & $\mathbf{Y}$ & 335 & $A L$ \\
\hline - & $Y$ & 435 & $A L$ \\
\hline - & $Y$ & 361 & $A L$ \\
\hline - & $Y$ & 121 & $\mathrm{AL}$ \\
\hline .032 & $Y$ & 1239 & AL \\
\hline .032 & $\mathbf{Y}$ & 1170 & $A L$ \\
\hline .039 & $\mathbf{Y}$ & 1499 & ${ }_{S S}^{A L}$ \\
\hline .032 & $Y$ & 1654 & $A L$ \\
\hline .032 & $Y$ & 1579 & AL \\
\hline & & & SS \\
\hline .032 & $Y$ & 1337 & AL \\
\hline .039 & $Y$ & 1776 & $\begin{array}{l}\text { AL } \\
\text { SS }\end{array}$ \\
\hline .032 & $\mathbf{Y}$ & 1899 & $\mathrm{AL}$ \\
\hline & 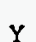 & 1250 & $\begin{array}{l}\text { SS } \\
\text { AL }\end{array}$ \\
\hline .028 & $\mathrm{Y}$ & 1635 & $A L$ \\
\hline- & $\mathbf{N}$ & - & AL \\
\hline . & $\mathbf{N}$ & & AL \\
\hline
\end{tabular}

\begin{tabular}{|c|c|c|c|}
\hline other* & Lattice & $\begin{array}{l}\text { Assembly } \\
\text { separ. } \\
\text { (cm) }\end{array}$ & $\begin{array}{l}\text { Dancoff } \\
\text { factor }\end{array}$ \\
\hline - & $\bar{s}$ & 5.0 & .20096 \\
\hline 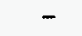 & $s$ & 2.5 & .20096 \\
\hline - & $\mathbf{s}$ & 10.0 & .20096 \\
\hline F3 & $s$ & 2.5 & .20096 \\
\hline E3 & $s$ & 5.0 & .20096 \\
\hline F3 & $s$ & 2.5 & .20096 \\
\hline F3 & $s$ & 5.0 & .20096 \\
\hline - & $s$ & 5.0 & .20096 \\
\hline F3 & $s$ & 2.5 & .20096 \\
\hline - & $s$ & 1.78 & .34784 \\
\hline - & $s$ & 1.78 & .34784 \\
\hline - & $s$ & - & .25722 \\
\hline - & $\mathbf{s}$ & - & .25699 \\
\hline - & $s$ & - & .26414 \\
\hline - & $s$ & 1.64 & .19071 \\
\hline - & $\mathbf{s}$ & 4.92 & .19071 \\
\hline - & $s$ & - & .19062 \\
\hline - & $s$ & 1.64 & .19065 \\
\hline - & $s$ & 4.92 & .19070 \\
\hline B & $s$ & 1.64 & .19072 \\
\hline B & $\mathbf{s}$ & 1.64 & .19072 \\
\hline - & $s$ & 1.64 & .19069 \\
\hline - & $s$ & 1.64 & .19068 \\
\hline - & $s$ & 6.54 & .19072 \\
\hline - & $s$ & 1.78 & .61533 \\
\hline - & $s$ & 1.78 & .61532 \\
\hline - & $\mathrm{H}$ & 1.78 & .76438 \\
\hline - & H & 1.78 & .76437 \\
\hline - & H & 2.54 & .76438 \\
\hline - & $\mathrm{H}$ & 3.81 & .76439 \\
\hline G & $s$ & - & .19043 \\
\hline G & $s$ & - & .19043 \\
\hline & $s$ & - & .18973 \\
\hline & & & .18527 \\
\hline G & $s$ & - & $\begin{array}{r}.18973 \\
.18527\end{array}$ \\
\hline G & $s$ & - & $\begin{array}{l}.18973 \\
.18527\end{array}$ \\
\hline - & $\mathrm{s}$ & - & .18973 \\
\hline . & $s$ & - & $\begin{array}{r}.18973 \\
.18527\end{array}$ \\
\hline - & $s$ & - & $\begin{array}{l}.18973 \\
.18527\end{array}$ \\
\hline A & $s$ & - & .18973 \\
\hline B & $s$ & - & $\begin{array}{l}.18973 \\
.18527\end{array}$ \\
\hline - & $s$ & 1.8 & .20101 \\
\hline . & $\mathbf{s}$ & 5.8 & .20101 \\
\hline
\end{tabular}


Table 3.5 (continued)

\begin{tabular}{|c|c|c|c|c|c|c|c|c|c|c|c|c|c|c|c|c|c|c|c|c|c|c|c|}
\hline $\begin{array}{l}\text { Case } \\
\text { No. }\end{array}$ & $\begin{array}{l}\text { Case } \\
\text { name }\end{array}$ & $k_{* t t}$ & to & $\begin{array}{l}\text { Enrich. } \\
\text { (wt ") }\end{array}$ & Ref. & AEG & $\operatorname{AEE}(\mathrm{eV})$ & $\begin{array}{c}\text { Pitch } \\
(\mathrm{cm})\end{array}$ & $\begin{array}{l}\mathrm{H}_{2} \mathrm{O} / \text { fuel } \\
\text { vol. }\end{array}$ & $H / X$ & $\begin{array}{c}\text { Plate } \\
\text { mat1. }\end{array}$ & $\begin{array}{l}\text { Boron } \\
\text { concen. } \\
\text { (wt i) }\end{array}$ & $\begin{array}{l}\text { Plate } \\
\text { thick. } \\
\text { (cm) }\end{array}$ & $\begin{array}{l}\text { Wall } \\
\text { mat1. }\end{array}$ & $\begin{array}{l}\text { Wall } \\
\text { separ. } \\
\text { (cm) }\end{array}$ & $\begin{array}{l}\text { No. of } \\
\text { holes' } \\
\text { pin }\end{array}$ & $\begin{array}{l}\text { sol. } \\
\text { boron }\end{array}$ & $\begin{array}{l}\text { Boron } \\
\text { concen } \\
\text { (ppm) C }\end{array}$ & cladd & Other" & - Lattices & $\begin{array}{l}\text { Assembly } \\
\text { separ. } \\
\text { (cm) }\end{array}$ & $\begin{array}{l}\text { Dancoff } \\
\text { factor }\end{array}$ \\
\hline 43 & DSN399-3 & 1.0024 & 0.0017 & 4.74 & 18 & 35.32 & 0.130849 & 1.6 & 3.807 & 228.6 & $H F$ & - & .105 & - & - & - & N & - & $A L$ & - & $s$ & - & .10503 \\
\hline 44 & DSN399-4 & 0.9965 & 0.0017 & 4.74 & 18 & 35.36 & 0.128298 & 1.6 & 3.807 & 228.6 & $\mathrm{HF}$ & - & .105 & - & - & - & $\mathrm{N}$ & - & $A L$ & - & $s$ & - & .10503 \\
\hline 45 & EPRU65 & 0.9977 & 0.0018 & 2.35 & 7 & 33.90 & 0.258691 & 1.562 & 1.196 & 163.6 & - & - & - & - & - & - & $\mathrm{N}$ & - & $A L$ & - & $s$ & - & .27720 \\
\hline 46 & EPRU65B & 0.9985 & 0.0018 & 2.35 & 7 & 33.41 & 0.315549 & 1.562 & 1.196 & 163.6 & - & - & - & - & - & - & $Y$ & 463 & AL & - & $s$ & - & .27715 \\
\hline 47 & EPRU75 & 0.9974 & 0.0016 & 2.35 & 7 & 35.86 & 0.113352 & 1.905 & 2.408 & 329.4 & - & - & - & - & - & - & $\mathrm{N}$ & - & AL & - & $\mathbf{s}$ & - & .11669 \\
\hline 48 & EPRU75B & 1.0023 & 0.0011 & 2.35 & 7 & 35.28 & 0.143515 & 1.905 & 2.408 & 329.4 & - & - & - & - & - & - & $\mathbf{Y}$ & 568 & $\mathrm{AL}$ & - & $\mathbf{s}$ & - & .11665 \\
\hline 49 & EPRU 87 & 0.9986 & 0.0014 & 2.35 & 7 & 36.61 & 0.082692 & 2.210 & 3.687 & 504.2 & - & - & - & - & - & - & $\mathrm{N}$ & - & AL & - & $\mathrm{s}$ & - & .05727 \\
\hline 50 & EPRU87B & 0.9985 & 0.0014 & 2.35 & 7 & 36.33 & 0.092090 & 2.210 & 3.687 & 504.2 & - & - & - & - & - & - & $Y$ & 286 & AL & - & $s$ & - & .05726 \\
\hline 51 & NSE71H1 & 0.9982 & 0.0019 & 4.74 & 8 & 33.52 & 0.272528 & 1.35 & 1.804 & 108.3 & - & - & - & - & - & - & $\mathrm{N}$ & - & $\overline{A L}$ & - & $\mathrm{H}$ & - & .25747 \\
\hline 52 & NSE71H2 & 1.0009 & 0.0017 & 4.74 & 8 & 35.74 & 0.110404 & 1.72 & 3.811 & 228.8 & - & - & - & - & - & - & $\mathrm{N}$ & - & AL & - & H & - & .10167 \\
\hline 53 & NSE $71 \mathrm{H} 3$ & 1.0036 & 0.0021 & 4.74 & 8 & 36.88 & 0.070193 & 2.26 & 7.608 & 456.8 & 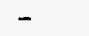 & - & - & - & - & - & $\mathrm{N}$ & - & $A L$ & - & H & - & .02984 \\
\hline 54 & NSE7 1SQ & 1.0014 & 0.0017 & 4.74 & 8 & 33.79 & 0.243524 & 1.26 & 1.823 & 110.0 & - & - & - & - & - & - & N & - & AL & - & $s$ & - & .25719 \\
\hline 55 & NSE71W1 & 0.9993 & 0.0020 & 4.74 & 8 & 33.99 & 0.224204 & 1.26 & 1.823 & 110.0 & - & - & - & - & - & .054 & $\mathrm{~N}$ & - & $A I$ & - & $s$ & - & .25719 \\
\hline 56 & NSE71W2 & 0.9976 & 0.0016 & 4.74 & 8 & 34.37 & 0.192011 & 1.26 & 1.823 & 110.0 & - & - & - & - & - & .152 & $\mathrm{~N}$ & - & AL & - & s & - & .25719 \\
\hline 57 & P2438AL & 0.9951 & 0.0016 & 2.35 & 9 & 36.30 & 0.094226 & 2.032 & 2.918 & 398.7 & AL & - & .625 & - & - & - & $\mathrm{N}$ & - & $A L$ & - & $s$ & 8.67 & .08629 \\
\hline 58 & P2438BA & 0.9972 & 0.0015 & 2.35 & 9 & 36.22 & 0.097404 & 2.032 & 2.918 & 398.7 & B & 28.7 & .713 & - & - & - & $\mathrm{N}$ & - & $A L$ & - & $s$ & 5.05 & .08629 \\
\hline 59 & P2438CU & 0.9957 & 0.0015 & 2.35 & 9 & 36.25 & 0.095990 & 2.032 & 2.918 & 398.7 & $\mathrm{CU}$ & - & .646 & - & - & - & $\mathrm{N}$ & - & $A L$ & - & $s$ & 6.62 & .08629 \\
\hline 60 & P2438SLG & 0.9997 & 0.0012 & 2.35 & 9 & 36.29 & 0.094440 & 2.032 & 2.918 & 398.7 & - & - & - & - & - & - & $\mathrm{N}$ & - & $A L$ & - & s & 8.39 & .08629 \\
\hline 61 & P2438SS & 0.9978 & 0.0014 & 2.35 & 9 & 36.26 & 0.095690 & 2.032 & 2.918 & 398.7 & sS & - & .485 & - & - & - & $\mathrm{N}$ & - & $A L$ & - & $\mathbf{s}$ & 6.88 & .08629 \\
\hline 62 & P2438ZR & 0.9956 & 0.0014 & 2.35 & 9 & 36.28 & 0.095115 & 2.032 & 2.918 & 398.7 & $\mathrm{ZR}$ & - & .652 & - & - & - & $\mathrm{N}$ & - & AL & - & $s$ & 8.79 & .08629 \\
\hline 63 & P2615AL & 1.0004 & 0.0016 & 4.31 & 19 & 35.74 & 0.114069 & 2.540 & 3.883 & 256.1 & AL & - & .625 & - & - & - & $N$ & - & AL & - & $s$ & 10.72 & .03889 \\
\hline 64 & P2615BA & 0.9970 & 0.0017 & 4.31 & 19 & 35.72 & 0.115080 & 2.540 & 3.883 & 256.1 & B & 28.7 & .713 & - & - & - & $\mathrm{N}$ & - & $\overline{A L}$ & - & $\mathbf{s}$ & 6.72 & .03889 \\
\hline 65 & $\mathrm{P} 2615 \mathrm{CD} 1$ & 0.9985 & 0.0017 & 4.31 & 19 & 35.74 & 0.114253 & 2.540 & 3.883 & 256.1 & $C D$ & - & .201 & - & - & - & $\mathrm{N}$ & - & $A L$ & - & $\mathrm{s}$ & 7.82 & .03889 \\
\hline 66 & P2615CD2 & 0.9990 & 0.0018 & 4.31 & 19 & 35.72 & 0.115015 & 2.540 & 3.883 & 256.1 & $C D$ & - & .201 & - & - & - & $\mathrm{N}$ & - & $A L$ & - & $\mathrm{s}$ & 5.68 & .03889 \\
\hline 67 & P261.5CU & 1.0003 & 0.0016 & 4.31 & 19 & 35.73 & 0.114411 & 2.540 & 3.883 & 256.1 & $\mathrm{CU}$ & - & .646 & - & - & - & $\mathrm{N}$ & - & $A L$ & - & $s$ & 8.15 & .03889 \\
\hline 68 & P2615ss & 0.9998 & 0.0017 & 4.31 & 19 & 35.74 & 0.113821 & 2.540 & 3.883 & 256.1 & ss & - & .485 & - & - & - & $\mathrm{N}$ & - & AL & - & $s$ & 8.58 & .03889 \\
\hline 69 & P26152R & 0.9980 & 0.0018 & 4.31 & 19 & 35.75 & 0.113465 & 2.540 & 3.883 & 256.1 & $2 R$ & - & .652 & - & - & - & $N$ & - & $A L$ & - & $s$ & 10.92 & .03889 \\
\hline 70 & P2827L1 & 1.0023 & 0.0014 & 2.35 & 10 & 36.24 & 0.096202 & 2.032 & 2.918 & 398.7 & - & - & - & L & 0.66 & - & $\mathrm{N}$ & - & $A I$ & - & s & 13.72 & .08629 \\
\hline 71 & P2827L2 & 0.9997 & 0.0016 & 2.35 & 10 & 36.28 & 0.095061 & 2.032 & 2.918 & 398.7 & - & - & - & $\mathrm{L}$ & 2.62 & - & $\mathrm{N}$ & - & $\overline{A L}$ & - & $s$ & 11.25 & .08629 \\
\hline 72 & P2827L3 & 1.0081 & 0.0020 & 4.31 & 10 & 35.66 & 0.116654 & 2.540 & 3.883 & 256.1 & - & - & - & I & 0.66 & - & $\mathrm{N}$ & - & $A L$ & - & $s$ & 20.78 & .03889 \\
\hline 73 & P2827L4 & 1.0073 & 0.0019 & 4.31 & 10 & 35.72 & 0.114400 & 2.540 & 3.883 & 256.1 & - & - & - & L & 1.32 & - & N & - & AL & - & $s$ & 19.04 & .03889 \\
\hline 74 & P2827SLG & 0.9948 & 0.0015 & 2.35 & 10 & 36.29 & 0.094400 & 2.032 & 2.918 & 398.7 & - & - & - & - & - & - & $\mathrm{N}$ & - & $\mathrm{AL}$ & & $\mathrm{s}$ & 8.31 & .08629 \\
\hline 75 & $\mathrm{P} 2827 \mathrm{U}_{1}$ & 1.0004 & 0.0015 & 2.35 & 10 & 34.74 & 0.212926 & 2.032 & 2.918 & 398.7 & - & - & - & $U$ & - & - & $\mathrm{N}$ & - & $A L$ & - & $\mathrm{s}$ & 11.83 & .08629 \\
\hline 76 & P2827U2 & 0.9998 & 0.0014 & 2.35 & 10 & 35.15 & 0.173574 & 2.032 & 2.918 & 398.7 & - & - & - & $\mathrm{U}$ & 1.96 & - & $\mathrm{N}$ & - & $A L$ & - & $s$ & 14.11 & .0 \\
\hline 77 & P2827U3 & 1.0018 & 0.0018 & 4.31 & 10 & 33.43 & 0.385311 & 2.540 & 3.883 & 256.1 & - & - & - & U & - & - & $\mathrm{N}$ & - & $A L$ & & $s$ & 15.38 & .0 \\
\hline 78 & P2827U4 & 1.0042 & 0.0017 & 4.31 & 10 & 34.09 & 0.280030 & 2.540 & 3.883 & 256.1 & - & - & - & $\mathrm{U}$ & 1.96 & - & $\mathrm{N}$ & - & $\mathrm{AL}$ & - & $s$ & 15.32 & .03889 \\
\hline 79 & P3314AL & 0.9973 & 0.0020 & 4.31 & 11 & 33.96 & 0.237442 & 1.892 & 1.60 & 105.4 & $A L$ & - & .6 & - & - & - & $\mathrm{N}$ & - & $\mathrm{AL}$ & & $s$ & 9.049 & .17284 \\
\hline 80 & P3314BA & 1.0002 & 0.0017 & 4.31 & 11 & 33.17 & 0.324124 & 1.892 & 1.60 & 105.4 & B & 28.7 & .713 & - & - & - & $\mathrm{N}$ & - & $A L$ & - & $s$ & $4.80^{\circ}$ & .17284 \\
\hline 81 & P3314BC & 1.0008 & 0.0019 & 4.31 & 11 & 33.23 & 0.316489 & 1.892 & 1.60 & 105.4 & B & 31.9 & .231 & - & - & - & $\mathrm{N}$ & - & AL & - & $s$ & $3.53^{\circ}$ & .17284 \\
\hline 82 & P $3314 \mathrm{BF} 1$ & 1.0022 & 0.0017 & 4.31 & 11 & 33.22 & 0.317435 & 1.892 & 1.60 & 105.4 & $\mathrm{BE}$ & - & .546 & - & - & - & $\mathrm{N}$ & - & $A L$ & & $s$ & $3.60^{\circ}$ & .17284 \\
\hline 83 & $\mathrm{P} 3314 \mathrm{BF} 2$ & 0.9981 & 0.0019 & 4.31 & 11 & 33.21 & 0.318470 & 1.892 & 1.60 & 105.4 & $\mathrm{BE}$ & - & .772 & - & - & - & $\mathrm{N}$ & - & $A L$ & - & $s$ & $4.94^{9}$ & .17284 \\
\hline 84 & P3314BS1 & 0.9970 & 0.0016 & 2.35 & 11 & 34.84 & 0.174267 & 1.684 & 1.60 & 218.6 & SS & 1.1 & .298 & - & - & - & $N$ & - & $A I$ & & $s$ & 3.86 & .20179 \\
\hline 85 & P3314BS2 & 0.9959 & 0.0016 & 2.35 & 11 & 34.84 & 0.173578 & 1.684 & 1.60 & 218.6 & sS & 1.6 & .298 & - & - & - & $\mathrm{N}$ & - & $\overline{A L}$ & - & $s$ & 3.46 & .20179 \\
\hline 86 & P3314BS3 & 0.9972 & 0.0016 & 4.31 & 11 & 33.41 & 0.294518 & 1.892 & 1.60 & 105.4 & ss & 1.1 & .298 & - & - & - & $\mathrm{N}$ & - & $A L$ & - & $s$ & 7.23 & .17284 \\
\hline 87 & P3314BS4 & 1.0005 & 0.0016 & 4.31 & 11 & 33.41 & 0.294168 & 1.892 & 1.60 & 105.4 & ss & 1.6 & .298 & - & - & - & $\mathrm{N}$ & - & $\overline{A L}$ & & $s$ & 6.63 & .17284 \\
\hline 88 & P331 4CD1 & 1.0001 & 0.0016 & 4.31 & 11 & 33.30 & 0.305451 & 1.892 & 1.60 & 105.4 & $C D$ & - & .061 & - & - & - & $\mathrm{N}$ & - & AL & - & $s$ & $5.30^{9}$ & .17284 \\
\hline 89 & P3314CD2 & 0.9929 & 0.0018 & 2.35 & 11 & 34.80 & 0.176886 & 1.684 & 1.60 & 218.6 & $C D$ & - & .061 & - & - & - & $\mathrm{N}$ & - & $A L$ & - & $s$ & 3.04 & .20179 \\
\hline 90 & P3314CU1 & 0.9941 & 0.0017 & 4.31 & 11. & 33.88 & 0.244311 & 1.892 & 1.60 & 105.4 & $\mathrm{cu}$ & - & .337 & - & - & - & $\mathrm{N}$ & - & $A L$ & - & $s$ & $5.94^{9}$ & .17284 \\
\hline
\end{tabular}


Table 3.5 (continued)

\begin{tabular}{|c|c|c|c|c|c|c|c|c|c|c|c|c|c|c|c|c|c|c|c|c|c|c|c|}
\hline $\begin{array}{l}\text { Case } \\
\text { No. }\end{array}$ & $\begin{array}{l}\text { Case } \\
\text { name }\end{array}$ & $k_{\text {ott }}$ & \pm 0 & $\begin{array}{l}\text { Enrich. } \\
\text { (wt } 1 \text { ) }\end{array}$ & Ref. & AEG & $\operatorname{AEF}(\theta V)$ & $\begin{array}{c}\text { Pitch } \\
\text { (cm) }\end{array}$ & $\begin{array}{l}\mathrm{H}_{2} \mathrm{O} / \mathrm{fuel} \\
\text { vol. }\end{array}$ & $H / X$ & $\begin{array}{l}\text { Plate } \\
\text { mat1." }\end{array}$ & $\begin{array}{l}\text { Boron } \\
\text { conean. } \\
\text { (wt i) }\end{array}$ & $\begin{array}{l}\text { Plate } \\
\text { thick. } \\
\text { (cr) }\end{array}$ & $\begin{array}{l}\text { Wall } \\
\text { mat1." }\end{array}$ & $\begin{array}{l}\text { Wall } \\
\text { separ. } \\
\text { (cm) }\end{array}$ & $\begin{array}{l}\text { No. of } \\
\text { holes/ } \\
\text { pin b }\end{array}$ & $\begin{array}{l}\text { Sol. } \\
\text { boron }\end{array}$ & $\begin{array}{l}\text { Boron } \\
\text { concen. } \\
\text { (ppm) C }\end{array}$ & $\mathrm{Clad}^{4}$ & Other" & ticet & $\begin{array}{l}\text { Assembly } \\
\text { separ. } \\
\text { (cm) }\end{array}$ & $\begin{array}{l}\text { Dancolf } \\
\text { factor }\end{array}$ \\
\hline 91 & P3314CU2 & 1.0005 & 0.0017 & 4.31 & 11 & 33.70 & 0.260474 & 1.892 & 1.60 & 105.4 & $\mathrm{CU}$ & - & .646 & - & - & - & $\mathrm{N}$ & - & $\overline{A L}$ & - & $\bar{s}$ & $2.67^{9}$ & .17284 \\
\hline 92 & P3314 CU3 & 0.9972 & 0.0016 & 4.31 & 11 & 33.50 & 0.283800 & 1.892 & 1.601 & 105.4 & $\mathrm{CU}$ & - & .337 & - & - & - & $\mathrm{N}$ & - & $A L$ & - & $\mathrm{s}$ & 10.36 & .17284 \\
\hline 93 & P3314CU 4 & 0.9992 & 0.0018 & 4.31 & 11 & 33.43 & 0.292385 & 1.892 & 1.60 & 105.4 & UD & - & .357 & - & - & - & N & - & $A L$ & - & $s$ & 7.61 & .17284 \\
\hline 94 & P3314CU5 & 0.9938 & 0.0017 & 2.35 & 11 & 34.98 & 0.164629 & 1.684 & 1.60 & 218.6 & $\mathrm{CU}$ & - & .337 & - & - & - & $\mathrm{N}$ & - & $A L$ & - & $s$ & 5.24 & .20179 \\
\hline 95 & P3314CU6 & 0.9958 & 0.0016 & 2.35 & 11 & 34.86 & 0.172536 & 1.684 & 1.60 & 218.6 & UD & - & .357 & - & = & - & N & - & AL & - & $s$ & 2.60 & .20179 \\
\hline 96 & P3314SLG & 0.9982 & 0.0017 & 4.31 & 11 & 33.97 & 0.235686 & 1.892 & 1.60 & 105.4 & - & - & - & - & - & - & N & - & $A L$ & - & $s$ & $10.86^{g}$ & .17284 \\
\hline 97 & P3314SS1 & 0.9969 & 0.0015 & 4.31 & 11 & 33.95 & 0.235781 & 1.892 & 1.60 & 105.4 & ss & - & .302 & - & - & - & $\mathbf{N}$ & - & AL & - & $s$ & $3.38^{\circ}$ & .17284 \\
\hline 98 & P3314SS2 & 1.0011 & 0.0016 & 4.31 & 11 & 33.77 & 0.255004 & 1.892 & 1.60 & 105.4 & ss & - & .302 & - & - & - & $\mathrm{N}$ & - & AL & - & $s$ & $11.55^{\circ}$ & .17284 \\
\hline 99 & P3314SS3 & 0.9990 & 0.0018 & 4.31 & 11 & 33.88 & 0.243407 & 1.892 & 1.60 & 105.4 & ss & - & .485 & - & - & - & $\mathrm{N}$ & - & AL & - & $s$ & 4.479 & .17284 \\
\hline 100 & P3314SS4 & 0.9957 & 0.0018 & 4.31 & 11 & 33.74 & 0.257511 & 1.892 & 1.60 & 105.4 & sS & - & .485 & - & - & - & $\mathrm{N}$ & - & AL & - & $\mathrm{s}$ & $8.36^{\circ}$ & .17284 \\
\hline 101 & P3314SS5 & 0.9944 & 0.0018 & 2.35 & 11 & 34.95 & 0.166761 & 1.684 & 1.60 & 218.6 & ss & - & .302 & - & - & - & $\mathrm{N}$ & - & $A L$ & - & $s$ & 7.80 & .20179 \\
\hline 102 & P3314SS6 & 1.0003 & 0.0018 & 4.31 & 11 & 33.52 & 0.282203 & 1.892 & 1.60 & 105.4 & ss & - & .302 & - & - & - & $\mathrm{N}$ & - & $\mathrm{AL}$ & - & $s$ & 10.52 & .17284 \\
\hline 103 & P3314W1 & 1.0015 & 0.0017 & 4.31 & 11 & 34.38 & 0.199212 & 1.892 & 1.60 & 105.4 & - & - & - & - & - & .149 & $9 \mathrm{~N}$ & - & AI & - & $s$ & - & .17284 \\
\hline 104 & P3314W2 & 0.9958 & 0.0015 & 2.35 & 11 & 35.22 & 0.148243 & 1.684 & 1.602 & 218.6 & - & - & - & - & - & .051 & $1 \mathrm{~N}$ & - & AI & - & $s$ & - & .20179 \\
\hline 105 & P33142R & 0.9978 & 0.0016 & 4.31 & 11 & 33.97 & 0.235701 & 1.892 & 1.60 & 105.4 & ZR & - & .652 & - & - & - & $\mathrm{N}$ & - & AL & - & $s$ & $11.04^{\circ}$ & .20179 \\
\hline 106 & P3602BB & 0.9988 & 0.0015 & 4.31 & 22 & 33.31 & 0.304578 & 1.892 & 1.60 & 105.4 & B & 30.4 . & .292 & SS & 1.96 & - & $\mathrm{N}$ & - & AL & - & $s$ & 8.30 & .17284 \\
\hline 107 & P3602BS1 & 1.0000 & 0.0017 & 2.35 & 22 & 34.76 & 0.177973 & 1.684 & 1.602 & 218.6 & sS & 1.1 & .298 & ss & 1.32 & - & $\mathrm{N}$ & - & AL & - & $s$ & 4.80 & .20179 \\
\hline 108 & P3602BS2 & 1.0001 & 0.0018 & 4.31 & 22 & 33.36 & 0.298546 & 1.892 & 1.60 & 105.4 & SS & 1.1. & .298 & ss & 1.96 & - & $\mathrm{N}$ & - & AL & - & $s$ & 9.83 & .17284 \\
\hline 109 & P3602CD1 & 0.9988 & 0.0016 & 2.35 & 22 & 34.72 & 0.181191 & 1.684 & 1.60 & 218.6 & $C D$ & - & .061 & ss & 1.32 & - & $\mathrm{N}$ & - & AL & - & $s$ & 3.86 & .20179 \\
\hline 110 & P3602CD2 & 1.0002 & 0.0017 & 4.31 & 22 & 33.33 & 0.301071 & 1.892 & 1.60 & 105.4 & $C D$ & - & .061 & ss & 1.96 & - & $\mathrm{N}$ & - & AL & - & $s$ & 8.94 & .17284 \\
\hline 111 & P3602CU1 & 0.9964 & 0.0014 & 2.35 & 22 & 34.84 & 0.172511 & 1.684 & 1.60 & 218.6 & $\mathrm{CU}$ & - & .337 & SS & 1.32 & - & $\mathrm{N}$ & - & AL & . & $s$ & 7.79 & .20179 \\
\hline 112 & P3602CU2 & 0.9980 & 0.0014 & 2.35 & 22 & 34.78 & 0.176071 & 1.684 & 1.60 & 218.6 & UD & - & .357 & SS & 1.32 & - & $\mathbf{N}$ & - & AL & - & $s$ & 5.43 & .20179 \\
\hline 113 & P3602CU3 & 1.0039 & 0.0016 & 4.31 & 22 & 33.38 & 0.296714 & 1.892 & 1.60 & 105.4 & $\mathrm{CU}$ & - & .337 & ss & 1.96 & - & $\mathrm{N}$ & - & AL & - & $s$ & 13.47 & .17284 \\
\hline 114 & $\mathrm{P} 3602 \mathrm{CU} 4$ & 1.0040 & 0.0016 & 4.31 & 22 & 33.37 & 0.297224 & 1.892 & $1.60 \mathrm{~J}$ & 105.4 & UD & - & .357 & SS & 1.96 & - & $\mathbf{N}$ & - & $A L$ & - & $s$ & 10.57 & .17284 \\
\hline 115 & $\mathrm{P} 3602 \mathrm{~N} 11$ & 1.0003 & 0.0018 & 2.35 & 22 & 34.73 & 0.180388 & 1.684 & 1.60 & 218.6 & - & - & - & ss & - & - & $\mathrm{N}$ & - & AL & - & $s$ & 8.98 & .20179 \\
\hline 116 & P3602N12 & 0.9963 & 0.0017 & 2.35 & 22 & 34.80 & 0.175283 & 1.684 & 1.60 & 218.6 & - & - & - & ss & 0.66 & - & $\mathrm{N}$ & - & $A L$ & - & $s$ & 9.58 & .20179 \\
\hline 117 & $83602 \mathrm{~N} 13$ & 0.9957 & 0.0016 & 2.35 & 22 & 34.92 & 0.167197 & 1.684 & 1.60 & 218.6 & - & - & - & ss & 1.68 & - & $\mathrm{N}$ & - & $A L$ & - & $s$ & 9.66 & .20179 \\
\hline 118 & P3602N14 & 0.9984 & 0.0015 & 2.35 & 22 & 35.03 & 0.160446 & 1.684 & 1.60 & 218.6 & - & - & - & ss & 3.91 & - & $\mathrm{N}$ & - & AL & - & $s$ & 8.54 & .20179 \\
\hline 119 & P3602N21 & 0.9995 & 0.0015 & 2.35 & 22 & 36.27 & 0.094794 & 2.032 & 2.918 & 398.7 & - & - & - & ss & 2.62 & - & $\mathrm{N}$ & - & $A L$ & - & $s$ & 10.36 & .08290 \\
\hline 120 & P3602N22 & 0.9967 & 0.0015 & 2.35 & 22 & 36.18 & 0.098047 & 2.032 & 2.918 & 398.7 & - & - & - & ss & 0.66 & - & $\mathrm{N}$ & - & $\mathrm{AL}$ & - & $S$ & 11.20 & .08290 \\
\hline 121 & P $3602 N 31$ & 1.0010 & 0.0018 & 4.31 & 22 & 33.19 & 0.317009 & 1.892 & 1.60 & 105.4 & - & - & - & SS & 0.00 & - & $\mathrm{N}$ & - & $A L$ & - & $s$ & 14.87 & .17284 \\
\hline 122 & $\mathrm{P} 3602 \mathrm{~N} 32$ & 1.0015 & 0.0019 & 4.31 & 22 & 33.29 & 0.304586 & 1.892 & 1.60 & 105.4 & - & - & - & ss & 0.66 & - & $\mathrm{N}$ & - & $\mathrm{AL}$ & - & $s$ & 15.74 & .17284 \\
\hline 123 & P3602N33 & 1.0045 & 0.0019 & 4.31 & 22 & 33.37 & 0.295430 & 1.892 & 1.60 & 105.4 & - & - & - & sS & 1.32 & - & $\mathbf{N}$ & - & AL & - & $s$ & 15.87 & .17284 \\
\hline 124 & P3602N34 & 1.0027 & 0.0016 & 4.31 & 22 & 33.43 & 0.289835 & 1.892 & 1.60 & 105.4 & - & - & - & ss & 1.96 & - & $\mathrm{N}$ & - & AL & - & $s$ & 15.84 & .17284 \\
\hline 125 & P3602N 35 & 1.0036 & 0.0018 & 4.31 & 22 & 33.48 & 0.284370 & 1.892 & 1.60 & 105.4 & - & - & - & ss & 2.62 & - & $\mathrm{N}$ & - & $A L$ & - & $s$ & 15.45 & .17284 \\
\hline 126 & $\mathrm{P} 3602 \mathrm{~N} 36$ & 1.0009 & 0.0017 & 4.31 & 22 & 33.58 & 0.275421 & 1.892 & 1.60 & 105.4 & - & - & - & SS & 5.41 & - & $\mathrm{N}$ & - & AL & - & $\mathrm{s}$ & 13.82 & .17284 \\
\hline 127 & P3602N41 & 1.0002 & 0.0016 & 4.31 & 22 & 35.50 & 0.123377 & 2.540 & 3.883 & 256.1 & - & - & - & SS & - & - & $\mathrm{N}$ & - & $A L$ & - & $\mathrm{s}$ & 12.89 & .03885 \\
\hline 128 & P3602N 42 & 1.0019 & 0.0017 & 4.31 & 22 & 35.63 & 0.117741 & 2.540 & 3.883 & 256.1 & - & - & - & ss & 1.32 & - & $\mathrm{N}$ & - & AL & - & $s$ & 14.12 & .03885 \\
\hline 129 & $\mathrm{P} 3602 \mathrm{~N} 43$ & 0.9994 & 0.0017 & 4.31 & 22 & 35.74 & 0.113378 & 2.540 & 3.883 & 256.1 & - & - & . & sS & 2.62 & - & $\mathrm{N}$ & - & $A L$ & - & $s$ & 12.44 & .03885 \\
\hline 130 & P3602SS1 & 0.9978 & 0.0016 & 2.35 & 22 & 34.87 & 0.170525 & 1.684 & 1.60 & 218.6 & ss & - & .302 & SS & 1.32 & - & $\mathrm{N}$ & - & $\mathrm{AL}$ & - & $s$ & 8.28 & .20179 \\
\hline 131 & P3602SS2 & 1.0012 & 0.0018 & 4.31 & 22 & 33.40 & 0.292861 & 1.892 & 1.60 & 105.4 & ss & - & .302 & SS & 1.96 & - & $\mathrm{N}$ & - & $A L$ & - & $s$ & 13.75 & .17284 \\
\hline 132 & P3926L1 & 0.9996 & 0.0014 & 2.35 & 12 & 34.85 & 0.172514 & 1.684 & 1.60 & 218.6 & - & - & - & $\mathrm{L}$ & - & - & $\mathrm{N}$ & - & AI & - & $s$ & 10.06 & .20179 \\
\hline 133 & P3926L2 & 1.0028 & 0.0016 & 2.35 & 12 & 34.92 & 0.167446 & 1.684 & 1.60 & 218.6 & - & - & - & $\mathrm{L}$ & 0.66 & - & $\mathrm{N}$ & - & AL & - & $s$ & 10.11 & .20179 \\
\hline 134 & P3926L 3 & 1.0017 & 0.0015 & 2.35 & 12 & 35.06 & 0.158275 & 1.684 & 1.60 & 218.6 & - & - & - & $\mathrm{L}$ & 3.28 & - & $\mathrm{N}$ & - & AL & - & s & 8.50 & .20179 \\
\hline 135 & P3926L4 & 1.0055 & 0.0019 & 4.31 & 12 & 33.31 & 0.304464 & 1.892 & 1.60 & 105.4 & - & - & - & $L$ & - & - & $\mathrm{N}$ & - & AL & - & $s$ & 17.74 & .17284 \\
\hline 136 & P3926L5 & 1.0070 & 0.0017 & 4.31 & 12 & 33.37 & 0.296797 & 1.892 & 1.60 & 105.4 & - & - & - & $\mathrm{L}$ & 0.66 & - & $\mathrm{N}$ & - & AL & - & $s$ & 18.18 & .17284 \\
\hline 137 & P3926L6 & 1.0026 & 0.0016 & 4.31 & 12 & 33.51 & 0.281808 & 1.892 & 1.60 & 105.4 & - & - & - & L & 1.96 & - & $\mathrm{N}$ & - & AL & - & $s$ & 17.43 & .17284 \\
\hline 138 & P3926SL1 & 0.9942 & 0.0015 & 2.35 & 12 & 35.07 & 0.159108 & 1.684 & 1.60 & 218.6 & - & - & - & W & - & - & $\mathrm{N}$ & - & $A L$ & - & $s$ & 6.59 & .20179 \\
\hline
\end{tabular}


Table 3.5 (continued)

\begin{tabular}{|c|c|c|c|c|c|c|c|c|c|c|c|c|c|c|c|c|c|c|c|c|c|c|c|c|}
\hline $\begin{array}{l}\text { Case } \\
\text { No. }\end{array}$ & $\begin{array}{l}\text { Case } \\
\text { name }\end{array}$ & k.ez & \pm 0 & $\begin{array}{l}\text { Enrich. } \\
\text { (wt i) }\end{array}$ & Ref. & - $\quad A E G$ & $\operatorname{AEF}(\theta \mathrm{V})$ & $\begin{array}{l}\text { Pitch } \\
\text { (cm) }\end{array}$ & $\begin{array}{l}\mathrm{H}_{2} \mathrm{OI} \\
\text { fue1/vol. }\end{array}$ & $\mathrm{H} / \mathrm{X}$ & $\begin{array}{l}\text { Plate } \\
\text { mat1. }\end{array}$ & $\begin{array}{l}\text { Boron } \\
\text { concen. } \\
\text { (wt i) }\end{array}$ & $\begin{array}{l}\text { Plate } \\
\text { thick. } \\
\text { (cm) }\end{array}$ & $\begin{array}{l}\text { Wa11 } \\
\text { mat1." }\end{array}$ & $\begin{array}{l}\text { Wall } \\
\text { separ. } \\
\text { (cm) }\end{array}$ & . Hole/ & $\begin{array}{l}\text { sol. } \\
\text { boron }\end{array}$ & $\begin{array}{c}\text { Borc } \\
\text { conc } \\
\text { (ppar) }\end{array}$ & $\mathrm{Cl}$ & & other ${ }^{*}$ & Lattice & $\begin{array}{l}\text { Assemb } \\
\text { separ } \\
\text { (cm) }\end{array}$ & $\begin{array}{c}\text { ably } \\
x \cdot \begin{array}{c}\text { Dancoff } \\
\text { factor }\end{array}\end{array}$ \\
\hline 139 & P3926SL2 & 0.9990 & 0.0019 & 4.31 & 12 & 33.55 & .279386 & 1.892 & 1.60 & 105.4 & - & - & - & - & - & - & $\mathrm{N}$ & $=$ & AL & - & $\bar{s}$ & & 12.97 & .17284 \\
\hline 140 & P3926U1 & 0.9979 & 0.0017 & 2.35 & $12=$ & 33.28 & .403558 & 1.684 & 1.60 & 218.6 & - & - & - & $U$ & - & - & $\mathrm{N}$ & - & $A L$ & - & s & & 8.06. & .20179 \\
\hline 141 & P3926U2 & 0.9980 & 0.0017 & 2.35 & 123 & 33.62 & .341429 & 1.684 & 1.60 & 218.6 & - & - & - & $\mathrm{U}$ & 1.32 & - & $\mathrm{N}$ & - & $A L$ & - & $s$ & & 9.50 & .20179 \\
\hline 142 & P3926U3 & 1.0001 & 0.0015 & 2.35 & 12 & 34.15 & .263699 & 1.684 & 1.60 & 218.6 & - & - & - & $\mathrm{U}$ & 3.91 & - & $\mathrm{N}$ & - & AL & - & $s$ & ; & 9.19 & .20179 \\
\hline 143 & P3926U4 & 1.0026 & 0.0017 & 4.31 & 12 & 31.93 & .655172 & 1.892 & 1.60 & 105.4 & - & - & - & $\mathrm{u}$ & - & - & $\mathrm{N}$ & - & $A L$ & - & $\mathrm{s}$ & & 15.33. & .17284 \\
\hline 144 & P3926U5 & 0.9999 & 0.0017 & 4.31 & 12 & 32.45 & .511391 & 1.892 & 1.60 & 105.4 & - & - & - & u & 1.96 & - & $\mathrm{N}$ & - & $A L$ & - & $\mathrm{s}$ & 1 & 19.24 . & .17284 \\
\hline 145 & P3926U6 & 1.0024 & 0.0016 & 4.31 & 12 & 32.74 & .444529 & 1.892 & 1.60 & 105.4 & - & - & - & U & 3.28 & - & $\mathrm{N}$ & - & AL & - & $\mathrm{s}$ & 1 & 18.78 . & .17284 \\
\hline 146 & P4267B1 & 0.9969 & 0.0015 & 4.31 & 13 & 31.80 & .548827 & 1.890 & 1.59 & 105.1 & - & - & - & - & - & - & $Y$ & 2150 & AL & - & $s$ & & - & .17349 \\
\hline 147 & $\mathrm{P} 4267 \mathrm{~B} 2$ & 1.0035 & 0.0013 & 4.31 & $13:$ & 31.54 & .608525 & 1.890 & 1.59 & 105.1 & - & - & - & - & - & - & $Y$ & 2550 & AL & - & $s$ & & - & .17346 \\
\hline 148 & P4267B3 & 1.0025 & 0.0015 & 4.31 & $13:$ & 30.97 & .794676 & 1.715 & 1.090 & 71.9 & - & - & - & - & - & - & $\gamma$ & 1030 & AL & - & $s$ & & - & .27021 \\
\hline 149 & P4267B4 & 0.9993 & 0.0016 & 4.31 & $13:$ & 30.51 & .955273 & 1.715 & 1.090 & 71.9 & - & - & - & - & - & - & $Y$ & 1820 & $A L$ & - & $s$ & & - & .27012 \\
\hline 150 & P4267B5 & 1.0031 & 0.0014 & 4.31 & 13 & 30.08 & 1.12803 & 1.715 & 1.090 & 71.9 & - & - & - & - & - & - & $Y$ & 2550 & AL & - & $s$ & & - & 100 \\
\hline 151 & P4267SL1 & 0.9974 & 0.0018 & 4.31 & 13 & 33.44 & .291929 & 1.890 & 1.59 & 105.1 & - & - & - & - & - & - & $\mathrm{N}$ & - & $A L$ & - & $s$ & & - & .17369 \\
\hline 152 & P4267SL2 & 0.9993 & 0.0017 & 4.31 & 13 & 31.95 & .543594 & 1.715 & 1.090 & 71.9 & - & - & - & - & - & - & $\mathrm{N}$ & - & $A L$ & - & $s$ & & - & .27032 \\
\hline 153 & P49-194 & 1.0081 & 0.0016 & 4.31 & 14 & 27.62 & 3.44514 & 1.598 & 0.509 & 33.6 & - & - & - & - & - & - & $\mathbf{N}$ & - & AL & - & & & - & .47372 \\
\hline 154 & Р62FT231 & 1.0005 & 0.0018 & 4.31 & 20 & 32.92 & .359262 & 1.891 & 1.60 & 105.0 & B & 35. & .683 & - & - & - & $\mathrm{N}$ & - & AL & - & $s$ & & 5.67 . & .17338 \\
\hline 155 & P71E14E3 & 1.0029 & 0.0018 & 4.31 & 21 & 32.81 & .376130 & 1.891 & 1.60 & 105.0 & B & 29 & .673 & - & - & - & $\mathrm{N}$ & - & $\mathrm{AL}$ & F1 & $\mathrm{s}$ & & 5.19 . & .17388 \\
\hline 156 & P71E14V3 & 0.9973 & 0.0017 & 4.31 & 21 & 32.88 & .364695 & 1.891 & 1.60 & 105.0 & B & 29 & .673 & - & - & - & $\mathrm{N}$ & - & AI & E2 & $s$ & & 5.19 & .17388 \\
\hline 157 & P71E14V5 & 0.9977 & 0.0016 & 4.31 & 21 & 32.86 & .367506 & 1.891 & 1.60 & 105.0 & B & 29 & .673 & - & - & - & $\mathrm{N}$ & - & $A L$ & $\mathrm{~F}$ & $s$ & & 5.19 . & .17388 \\
\hline 158 & P71E214R & 1.0010 & 0.0020 & 4.31 & 21 & 32.88 & .365283 & 1.891 & 1.60 & 105.0 & B & 29 & .673 & - & - & - & $\mathrm{N}$ & - & AL & - & $s$ & & 5.19 & .17388 \\
\hline 159 & PAT8OL1 & 1.0031 & 0.0018 & 4.74 & 23 & 35.03 & .148022 & 1.6 & 3.807 & 228.6 & B & 39 & .111 & L & 0.23 & - & N & - & $A L$ & - & $s$ & & 2.0 & .10503 \\
\hline 160 & PAT 8OL 2 & 0.9920 & 0.0018 & 4.74 & 23 & 35.08 & .143839 & 1.6 & 3.807 & 228.6 & B & 39 & .11 & $\mathrm{~L}$ & 2.50 & - & $\mathrm{N}$ & - & AL & - & & & 2.0 & .10503 \\
\hline 161 & PAT80SS1 & 1.0004 & 0.0017 & 4.74 & 23 & 35.00 & .149442 & 1.6 & 3.807 & 228.6 & B & 39. & .11 & ss & 0.23 & - & $\mathrm{N}$ & - & $\mathrm{AL}$ & - & $s$ & & 2.0 & .10503 \\
\hline 162 & PAT80SS2 & 0.9926 & 0.0021 & 4.74 & 23 & 35.09 & .143903 & 1.6 & 3.807 & 228.6 & B & 39 & .1 & ss & 2.50 & - & $\cdot \mathrm{N}$ & - & $\mathrm{AL}$ & - & $s$ & & 2.0 & 03 \\
\hline 163 & W3269A & 0.9934 & 0.0016 & 5.70 & 15 & 33.11 & .311261 & 1.422 & 1.932 & 98.3 & - & - & - & - & - & - & $\mathrm{N}$ & - & ss & - & $s$ & & - & .18534 \\
\hline 164 & W3269B1 & 0.9963 & 0.0015 & 3.70 & 15 & 32.36 & .452602 & 1.105 & 1.447 & 111.0 & - & - & - & - & - & - & $\mathrm{N}$ & - & ss & - & $\mathrm{s}$ & & - & 346 \\
\hline 165 & W3269B2 & 0.9964 & 0.0014 & 3.70 & 15 & 32.39 & .447071 & 1.105 & 1.447 & 111.0 & - & - & - & - & - & - & $N$ & - & ss & $A$ & G & & - & .18 \\
\hline 166 & W3269B3 & 0.9948 & 0.0016 & 3.70 & 15 & 32.26 & .471281 & 1.105 & 1.447 & 111.0 & - & - & - & - & - & - & $\mathrm{N}$ & - & ss & A & $s$ & & - & .37720 \\
\hline 167 & W3269C & 0.9986 & 0.0015 & 2.72 & 15 & 33.75 & .269691 & 1.524 & 1.495 & 156.1 & - & - & - & - & - & - & $\mathrm{N}$ & - & SS & $\triangle$ & $s$ & & - & .37720 \\
\hline 168 & W3269SL1 & 0.9952 & 0.0017 & 2.72 & 15 & 33.35 & .329668 & 1.524 & 1.495 & 156.1 & - & - & - & - & - & - & N & - & ss & A & $s$ & & - & .37720 \\
\hline 169 & W3269SL2 & 1.0040 & 0.0020 & 5.70 & 15 & 33.08 & .319337 & 1.422 & 1.932 & 98.3 & - & - & - & - & - & - & $\mathrm{N}$ & - & $2 R$ & A & $s$ & & - & .23385 \\
\hline 170 & W3269W1 & 0.9957 & 0.0018 & 5.70 & 15 & 33.50 & .31 & 1.524 & 1.495 & 156.1 & - & - & - & - & - & - & $\mathrm{N}$ & - & ZR & - & $s$ & &. & .23388 \\
\hline 171 & W3269W2 & 1.0009 & 0.0017 & 5.70 & 15 & 33.18 & .305719 & 1.422 & 1.932 & 98.3 & - & - & - & - & - & - & $\mathrm{N}$ & - & ss & - & $s$ & & - & .18534 \\
\hline 172 & W3385S11 & 0.9964 & 0.0017 & 5.74 & 16 & 33.21 & .29 & 1.422 & 1.933 & 97.6 & - & - & - & - & - & & $\mathrm{N}$ & - & $\mathrm{ZR}$ & - & $\mathrm{s}$ & 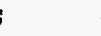 & - & .23388 \\
\hline 173 & W3385S12 & 1.0005 & 0.0020 & 5.74 & 16 & 35.86 & .104227 & 2.011 & 5.067 & 255.9 & - & 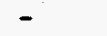 & - & - & - & .013 & $\mathrm{~N}$ & - & SS & - & $\mathrm{s}$ & & - & .18534 \\
\hline
\end{tabular}

"AL - aluminum; B - boral; BF - boroflex; CD - cadmium; CU - copper; HF - hafnium; SS - stainless steel; UD - copper-cadmium; $\mathrm{ZR}$ - Zircaloy.

DL - lead; SS - stainless steel; U - depleted uranium.

oY - Yes: $\mathrm{N}$ - no.

Z AAL - aluminum; SS - stainless steel; ZR - Zircaloy.

eA - Ag-In-Cd rods; B - B $B_{4}$ rods; F1 - flux trap containing fuel rods; F2 - flux trap containing aluminum plates or rods; 33 - flux trap $\mathrm{m}$ containing polyethylene or polystyrene; $\mathrm{G}-\mathrm{UO}_{2}-\mathrm{Gd}_{2} \mathrm{O}_{3}$ rods.

${ }^{{ }^{2}} \mathrm{H}$ - hexagonal; $\mathrm{S}$ - square.

9Other separation distance is $2.83 \mathrm{~cm}$. 
Validation

Table 3.6 Descriptive statistics of simple lattice calculations

\begin{tabular}{lccccc}
\hline Description & No. of exp. & $\mathbf{k}_{\text {ef }}$ range & $\overline{\mathbf{k}}_{\text {eff }}$ & $\pm \sigma_{\mathrm{k}}$ & AEF range $(\mathrm{eV})$ \\
\hline Square & 17 & $0.9942-1.0004$ & 0.9980 & 0.0025 & $0.083-0.544$ \\
Hexagonal & 4 & $0.9982-1.0081$ & 1.0027 & 0.0042 & $0.070-3.445$ \\
TOTAL & 21 & $0.9942-1.0081$ & 0.9989 & 0.0033 & $0.070-3.445$ \\
\hline
\end{tabular}

Table 3.7. Descriptive statistics of separator plate only calculations

\begin{tabular}{lccccc}
\hline Description & No. of exp. & $\mathbf{k}_{\text {off }}$ range & $\overline{\mathbf{k}}_{\text {eff }}$ & $\pm \sigma_{\mathbf{k}}$ & $\begin{array}{c}\text { AEF range } \\
(\mathrm{eV})\end{array}$ \\
\hline Aluminum & 6 & $0.9951-1.0129$ & 1.0020 & 0.0062 & $0.094-0.237$ \\
Boral & 6 & $0.9970-1.0010$ & 0.9995 & 0.0018 & $0.097-0.365$ \\
Boroflex & 2 & $0.9981-1.0022$ & 1.0002 & 0.0029 & $0.317-0.318$ \\
Cadmium & 4 & $0.9929-1.0001$ & 0.9976 & 0.0032 & $0.114-0.305$ \\
Copper & 6 & $0.9938-1.0005$ & 0.9969 & 0.0029 & $0.096-0.283$ \\
Cu-Cd & 2 & $0.9938-0.9992$ & 0.9965 & 0.0038 & $0.173-0.292$ \\
Hafnium & 4 & $0.9965-1.0056$ & 1.0014 & 0.0038 & $0.128-0.232$ \\
Borated steel & 4 & $0.9959-1.0005$ & 0.9977 & 0.0020 & $0.174-0.295$ \\
Steel & 8 & $0.9944-1.0011$ & 0.9981 & 0.0023 & $0.097-0.282$ \\
Zircaloy & 3 & $0.9956-0.9980$ & 0.9971 & 0.0013 & $0.095-0.236$ \\
TOTAL & 45 & $0.9929-1.0129$ & 0.9989 & 0.0035 & $0.094-0.365$ \\
\hline
\end{tabular}

Table 3.8 Descriptive statistics of separator plate-soluble boron calculations

\begin{tabular}{lccccc}
\hline Plate lattice & No. of exp. & $\mathrm{k}_{\text {eff }}$ range & $\overline{\mathrm{k}}_{\mathrm{eff}}$ & $\pm \mathrm{o}_{\mathrm{k}}$ & $\begin{array}{c}\mathrm{AEF} \text { range } \\
(\mathrm{eV})\end{array}$ \\
\hline $\begin{array}{l}\text { Hexagonal } \\
\quad \text { Aluminum }\end{array}$ & 4 & $0.9986-1.0068$ & 1.0031 & 0.0041 & $0.968-2.281$ \\
$\quad$ Square & & & & & \\
$\quad$ Borated aluminum & 2 & $0.9954-0.9965$ & 0.9960 & 0.0008 & $0.152-0.194$ \\
$\quad$ Aluminum & 4 & $0.9976-1.0035$ & 1.0007 & 0.0024 & $0.402-1.392$ \\
$\quad$ Steel & 2 & $0.9981-0.9989$ & 0.9985 & 0.0006 & $0.193-0.195$ \\
$\quad$ TOTAL & 12 & $0.9954-1.0068$ & 1.0003 & 0.0036 & $0.152-2.281$ \\
\hline
\end{tabular}


Table 3.9 Descriptive statistics of separator plate-void material (flux trap) calculations

\begin{tabular}{lcllll}
\hline Description & No. of exp. & $\mathbf{k}_{\mathrm{eff}}$ range & $\overline{\mathrm{k}}_{\mathrm{eff}}$ & $\pm \sigma_{\mathrm{k}}$ & AEF range $(\mathrm{eV})$ \\
\hline Al-polyethylene & 4 & $0.9964-1.0096$ & 1.0015 & 0.0059 & $0.206-0.250$ \\
Al-polystyrene & 1 & 0.9909 & 0.9909 & 0.0000 & 0.266 \\
Boral-Al & 2 & $0.9973-0.9977$ & 0.9975 & 0.0003 & $0.365-0.368$ \\
Boral-fuel rods & 1 & 1.0029 & 1.0029 & 0.0000 & 0.376 \\
TOTAL & 8 & $0.9909-1.0096$ & 0.9993 & 0.0055 & $0.206-0.368$ \\
\hline
\end{tabular}

Table 3.10 Descriptive statistics of reflecting wall calculations

\begin{tabular}{lccccc}
\hline Description & No. of exp. & $\mathrm{k}_{\text {eff }}$ range & $\overline{\mathrm{k}}_{\text {eff }}$ & $\pm \mathrm{\sigma}_{\mathrm{k}}$ & AEF range (eV) \\
\hline Lead & 10 & $0.9996-1.0081$ & 1.0037 & 0.0031 & $0.095-0.304$ \\
Steel & 15 & $0.9957-1.0045$ & 1.0002 & 0.0026 & $0.095-0.317$ \\
Uranium & 10 & $0.9979-1.0042$ & 1.0007 & 0.0020 & $0.173-0.655$ \\
TOTAL & 35 & $0.9957-1.0081$ & 1.0013 & 0.0030 & $0.095-0.655$ \\
\hline
\end{tabular}

Table 3.11 Descriptive statistics of reflecting wall-separator plate calculations

\begin{tabular}{lccccc}
\hline $\begin{array}{l}\text { Wall/plate } \\
\text { material }\end{array}$ & No. of exp. & $\mathrm{k}_{\text {eff }}$ range & $\overline{\mathrm{k}}_{\mathrm{eff}}$ & $\pm \sigma_{\mathrm{k}}$ & $\begin{array}{c}\text { AEF range } \\
(\mathrm{eV})\end{array}$ \\
\hline Lead-Boral & 2 & $0.9920-1.0031$ & 0.9976 & 0.0078 & $0.144-0.148$ \\
Steel-Boral & 3 & $0.9926-1.0004$ & 0.9973 & 0.0041 & $0.144-0.305$ \\
Steel-Cadmium & 2 & $0.9988-1.0002$ & 0.9995 & 0.0010 & $0.181-0.301$ \\
Steel-Copper & 2 & $0.9964-1.0039$ & 1.0002 & 0.0053 & $0.173-0.297$ \\
Steel-Cu-Cd & 2 & $0.9980-1.0040^{-}$ & 1.0010 & 0.0042 & $0.176-0.297$ \\
Steel-Borated steel & 2 & $1.0000-1.0001$ & 1.0001 & 0.0001 & $0.178-0.299$ \\
Steel-Steel & 2 & $0.9978-1.0012$ & 0.9995 & 0.0024 & $0.171-0.293$ \\
TOTAL & 15 & $0.9920-1.0040$ & 0.9992 & 0.0035 & $0.144-0.305$ \\
\hline
\end{tabular}

Table 3.12 Descriptive statistics of urania gadolinia rod calculations

\begin{tabular}{lccccc} 
Description & $\begin{array}{c}\text { No. of } \\
\text { exp. }\end{array}$ & $\mathrm{k}_{\text {eff }}$ range & $\overline{\mathrm{k}}_{\mathrm{eff}}$ & $\pm \sigma_{\mathrm{k}}$ & AEF range (eV) \\
\hline Urania-gadolinia rods & 5 & $0.9971-1.0005$ & 0.9986 & 0.0015 & $0.243-0.337$ \\
\hline
\end{tabular}


Validation

Table 3.13 Descriptive statistics of water hole calculations

\begin{tabular}{lccccc}
\hline Description & $\begin{array}{c}\text { No. of } \\
\text { exp. }\end{array}$ & $\mathbf{k}_{\text {eff }}$ range & $\overline{\mathbf{k}}_{\text {eff }}$ & $\pm \sigma_{\mathrm{k}}$ & AEF range (eV) \\
\hline $\begin{array}{l}\text { Water holes } \\
\begin{array}{l}\text { Water holes- } \\
\text { soluble boron }\end{array}\end{array}$ & 6 & $0.9957-1.0015$ & 0.9985 & 0.0025 & $0.148-0.310$ \\
TOTAL & 3 & $0.9971-1.0032$ & 0.9992 & 0.0035 & $0.245-0.355$ \\
\hline
\end{tabular}

Table 3.14 Descriptive statistics of absorber rod calculations

\begin{tabular}{lccccc}
\hline Description & $\begin{array}{c}\text { No. of } \\
\text { exp. }\end{array}$ & $\mathrm{k}_{\mathrm{eff}}$ range & $\overline{\mathrm{k}}_{\mathrm{eff}}$ & $\pm \mathrm{\sigma}_{\mathrm{k}}$ & AEF range (eV) \\
\hline $\mathrm{B}_{4} \mathrm{C}$ & 3 & $0.9936-0.9980$ & 0.9951 & 0.0025 & $0.148-0.332$ \\
Ag-In-Cd & 6 & $0.9934-1.0022$ & 0.9970 & 0.0031 & $0.245-0.471$ \\
TOTAL & 9 & $0.9936-1.0022$ & 0.9963 & 0.0029 & $0.148-0.471$ \\
\hline
\end{tabular}

Table 3.15 Descriptive statistics of soluble boron calculations

\begin{tabular}{lccccc}
\hline Description & $\begin{array}{c}\text { No. of } \\
\text { exp. }\end{array}$ & $\mathbf{k}_{\text {eff }}$ range & $\bar{k}_{\text {eff }}$ & $\pm \sigma_{\mathrm{k}}$ & AEF range (eV) \\
\hline Borated water & 14 & $0.9947-1.0035$ & 0.9992 & 0.0027 & $0.92-1.189$ \\
\hline
\end{tabular}


Table 3.16 Calculational results and experimental parameters of homogenized uranium experiments

\begin{tabular}{|c|c|c|c|c|c|c|c|c|c|c|c|c|}
\hline $\begin{array}{l}\text { Case } \\
\text { No. } \\
\end{array}$ & $\begin{array}{r}\text { Case } \\
\text { desig. }\end{array}$ & $k_{\text {eff }}$ & $\pm \sigma$ & Enrichment & Ref. & $\mathrm{AEG}$ & $\mathrm{AEF}$ & $\mathrm{H} / \mathrm{X}$ & $\begin{array}{l}\text { Fuel } \\
\text { form }\end{array}$ & Moderator & $\begin{array}{c}\text { Moderator } \\
\text { thickness }\end{array}$ & Reflector \\
\hline 174 & CRI071AS & 1.0207 & 0.0016 & 4.46 & $28,29,31$ & 32.45 & 0.45405 & 17.12 & $\mathrm{U}_{3} \mathrm{O}_{8}$ & Plexiglas & 2.43 & Plexiglas \\
\hline 176 & CR2500S & 1.0194 & 0.0018 & 4.48 & 28,31 & 33.20 & 0.31665 & 44.27 & $\mathrm{U}_{3} \mathrm{O}_{8}$ & Plexiglas & 2.43 & Plexiglas \\
\hline 177 & YDR14PL2 & 1.0011 & 0.0015 & 2.00 & 27 & 36.49 & 0.08319 & 406.3 & $\mathrm{UF}_{4}$ & Paraffin & 0.0 & Paraffin \\
\hline 179 & YDR14UN2 & 1.0047 & 0.0015 & 2.00 & 27 & 35.39 & 0.13273 & 293.9 & $\mathrm{UF}_{4}$ & Paraffin & 0.0 & None \\
\hline 180 & YDR14UN3 & 1.0163 & 0.0017 & 3.00 & 27 & 33.10 & 0.32152 & 133.4 & $\mathrm{UF}_{4}$ & Paraffin & 0.0 & None \\
\hline
\end{tabular}


Validation

Table 3.17 Descriptive statistics of homogenized uranium calculations

\begin{tabular}{lccccc}
\hline Description & $\begin{array}{c}\text { No. of } \\
\text { exp. }\end{array}$ & $\mathbf{k}_{\text {eff }}$ range & $\overline{\mathrm{k}}_{\mathrm{eff}}$ & $\pm \sigma_{\mathrm{k}}$ & AEF range $(\mathrm{eV})$ \\
\hline $\mathrm{UF}_{4}$ in paraffin & 4 & $1.0011-1.0163$ & 1.0084 & 0.0068 & $0.083-0.322$ \\
Damp oxide $\left(\mathrm{U}_{3} \mathrm{O}_{8}\right)$ & 3 & $1.0169-1.0207$ & 1.0190 & 0.0019 & $0.317-0.454$ \\
TOTAL & 7 & $1.0011-1.0207$ & 1.0129 & 0.0075 & $0.083-0.454$ \\
\hline
\end{tabular}


Validation

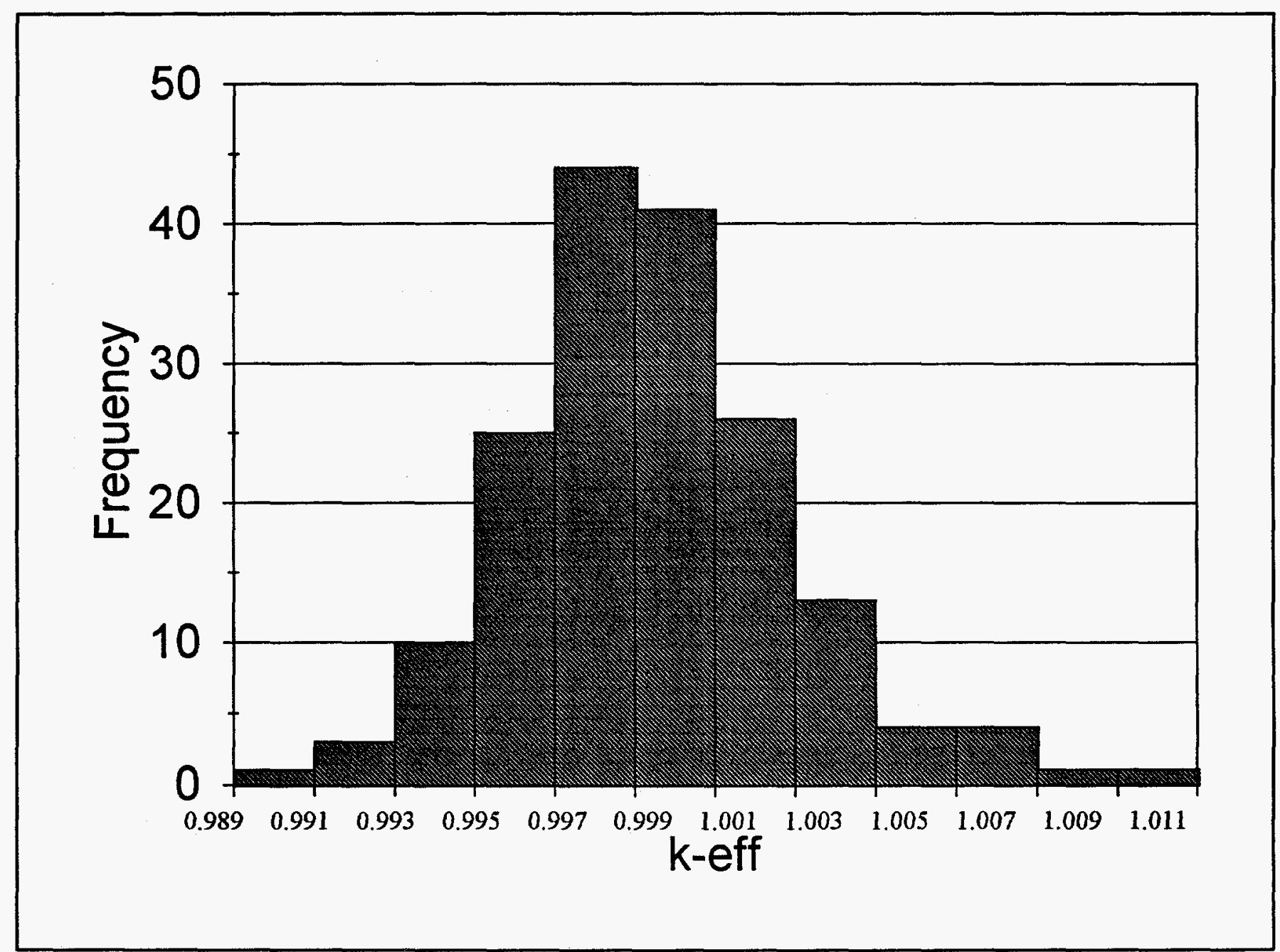

Figure 3.1 Frequency chart for calculated $k_{\text {eff }}$ of $173 \mathrm{LWR}$ fuel pin lattice experiments 
Validation

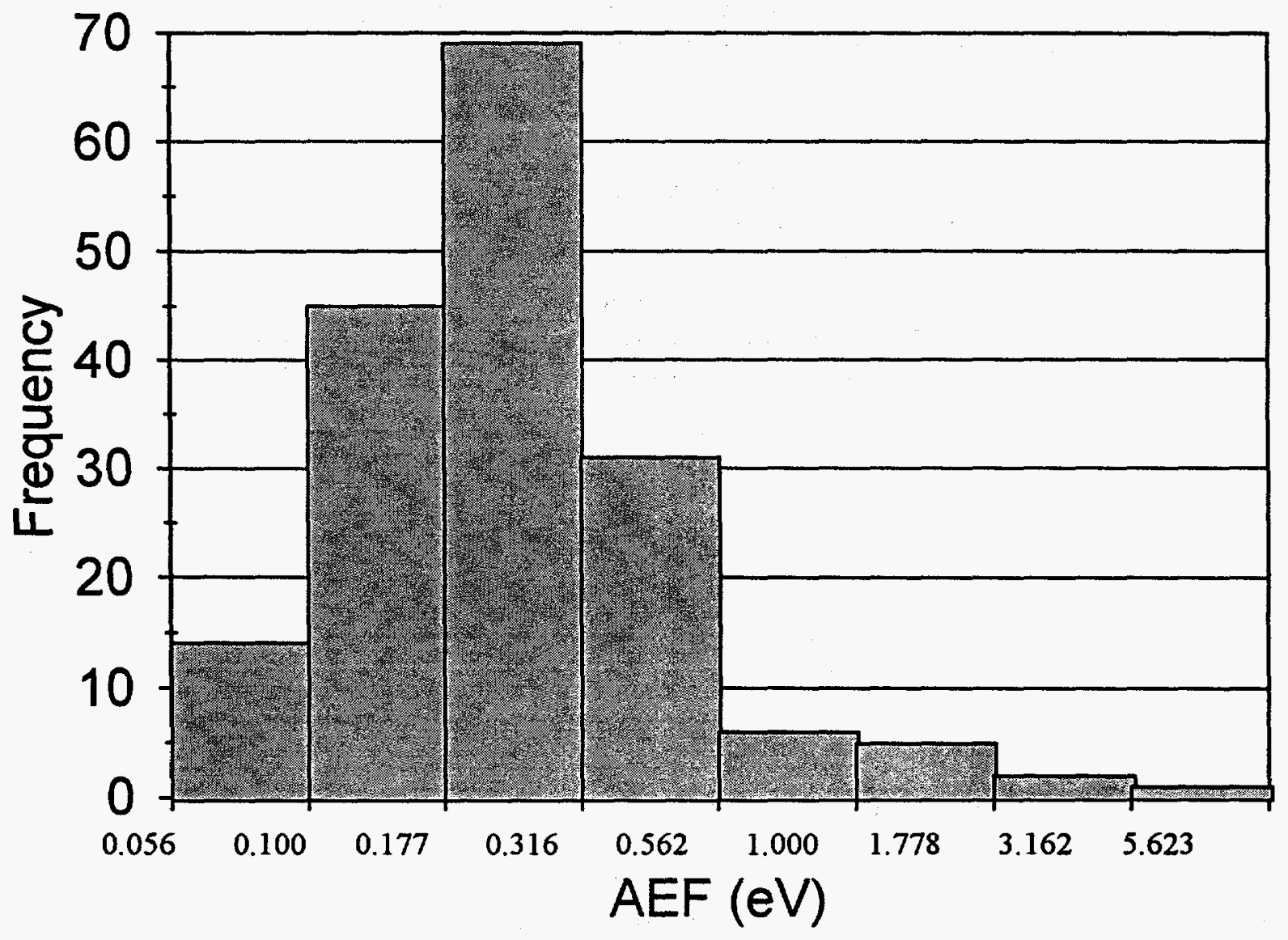

Figure 3.2 Frequency chart for calculated AEF of 173 LWR fuel pin lattice experiments 


\section{Determination of Bias and Subcritical Limits}

Essential elements in the validation of a calculational method for criticality safety applications include the determination of calculational biases and the incorporation of these biases into subcritical limits. This section presents two methods for performing this task and then applies the methods to the validation of the SCALE 44-group library for the LWR lattice results in Sect. 3.

\subsection{Establishment of an Upper Subcritical Limit}

For a subcritical configuration, it is desirable to possess a confidence that the calculation of $k_{\text {eff }}$ for a system guarantees sufficient subcriticality. This assurance of subcriticality requires the determination of an acceptable margin based on known biases and uncertainties associated with the codes and data used to calculate $k_{\text {eff }}$. This subsection describes two methods for the determination of an Upper Subcritical Limit (USL) from the bias and uncertainty terms associated with the calculation of criticality. These approaches are used to help define uncertainty and bias terms as they relate to criticality experiments and calculations.

The recommended approach for establishing subcriticality based on the numerical calculation of the neutron multiplication factor is prescribed in Sect. 5.1 of ANSI/ANS-8.17 (Ref. 38). The following paragraphs describe the recommended approach as set forth in the Standard.

The criteria to establish subcriticality requires that for a system to be considered subcritical the calculated multiplication factor for the system, $k_{b}$, must be less than or equal to an established maximum allowable multiplication factor based on benchmark calculations and uncertainty terms, that is,

$$
\mathrm{k}_{\mathrm{s}} \leq \mathrm{k}_{\mathrm{c}}-\Delta \mathrm{k}_{\mathrm{s}}-\Delta \mathrm{k}_{\mathrm{c}}-\Delta \mathrm{k}_{\mathrm{m}}
$$

where

$$
\begin{aligned}
& \mathrm{k}_{\mathrm{c}}=\quad \text { mean value of } \mathrm{k}_{\text {eff }} \text { resulting from the calculation of benchmark criticality experiments using a specific } \\
& \Delta \mathrm{k}_{\mathrm{s}}=\quad \text { uncertainty in the value of } \mathrm{k}_{\mathrm{s}} \\
& \Delta \mathrm{k}_{\mathrm{c}}=\text { uncertainty in the value of } \mathrm{k}_{\mathrm{c}} \\
& \Delta \mathrm{k}_{\mathrm{rm}}=\text { additional margin to ensure subcriticality. }
\end{aligned}
$$

Often $\Delta \mathrm{k}_{\mathrm{m}}$ is arbitrarily assigned an administrative limit of 0.05 ; however, a value for this margin can also be estimated statistically.

If the calculational bias $\beta$ is defined as $\beta=k_{c}-1$, then the uncertainty in the bias is identical to the uncertainty in $k_{c}$ (i.e., $\Delta k_{c}=\Delta \beta$ ). According to this definition of bias, the bias is negative if $k_{c}<1$ and positive if $k_{c}>1$. Thus the subcriticality condition may be rewritten as

$$
\mathrm{k}_{\mathrm{s}}+\Delta \mathrm{k}_{\mathrm{s}}+\Delta \mathrm{k}_{\mathrm{m}}-\beta+\Delta \beta \leq 1
$$

The value $k_{c}$ and thus the bias $\beta$ are not necessarily constant over the range of a parameter of interest. If trends exist which cause the benchmark values of $k_{\text {eff }}$ to vary with one or more parameters (e.g., enrichment, AEF, etc.), then $\beta$ can be determined from a best fit for the calculated $k_{\text {eff }}$ values as a function of each of the parameters upon which it is dependent. Trends must be taken into account if extrapolation outside the range of validation is to be performed.

The set of critical experiments used as benchmarks in the computation of $\beta$ should be representative of the composition, configuration, and nuclear characteristics of the system for which $\mathrm{k}_{3}$ is to be determined. However, 


\section{Determination of Limits}

ANSV/ANS-8.1 (Ref. 39) allows that the range of applicability may be extended beyond the range of conditions represented by the benchmark experiments by extrapolating the trends established for the bias. When the extrapolation is large relative to the range of data ("large" is not defined by the ANSI standard), the calculational method applied should be supplemented by other methods in order to better estimate the extrapolated bias.

In addition to the bias $\beta$ determined based on a given computational method, data, and a suite of benchmarks, there is an uncertainty in the bias $\Delta \beta$. This uncertainty may include uncertainties in the critical experiments, statistical and/or convergence uncertainties in the benchmark calculations, uncertainties due to extrapolation beyond the range of experimental data, and uncertainties due to limitations or weaknesses in the geometrical or nuclear modeling of the critical experiments. Similarly, for a given subcritical system, there is an uncertainty, $\Delta \mathrm{k}_{\mathrm{s}}$, associated with the calculated $k_{\text {eff }}$ value for the system, $k_{2}$. This uncertainty may include statistical/convergence and modeling uncertainties, as well as uncertainties in the materials and fabrication.

Based on the criteria for subcriticality set forth in ANSU/ANS-8.17 and described above, a USL may be determined based on the analysis of a number of critical systems. The USL is determined such that there is a high degree of confidence that a calculated result is subcritical; a system is considered acceptably subcritical if a calculated $k_{\text {eff }}$ plus calculational uncertainties lies at or below this limit (i.e., $k_{s}+\Delta k_{s} \leq$ USL). Thus the USL is the magnitude of the sum of the biases, uncertainties, and administrative and/or statistical margins applied to a set of critical benchmarks, such that with a high degree of confidence

$$
\mathrm{USL}=1-\Delta \mathrm{k}_{\mathrm{m}}+\beta-\Delta \beta
$$

Based on a given set of critical experiments, USLs are determined as a function of key system parameters, such as $\mathrm{AEF}$, fuel enrichment, or fuel/moderator ratio. Because both $\Delta \beta$ and $\beta$ can vary with a given parameter, the USL is typically expressed as a function of the parameter, within an appropriate range of applicability derived from the parameter bounds.

In the two methods presented in the following sections, a minimum of 25 data points (i.e., calculated $k_{\text {eff }}$ values) are required to verify normality, which is assumed in these methods. The software discussed below which implements these methods performs a crude test for whether or not the $k_{\text {eff }}$ values as a group are normal around the mean value of $k_{\text {eff }}$. The test is commonly known as the "goodness of fit." The distribution of $k_{\text {eff }}$ values around the mean are pooled into five equally probable bins. The test may not be reliable if there are fewer than 5 observations in each bin. Thus the test will always be flagged as not being reliable for cases where there are fewer than 25 observations and may be flagged for more observations if there are fewer than 5 observations in any bin. If there is an insufficient number of observations to ensure normality with this test, other statistical methods should be employed to determine normality or additional uncertainties to account for the paucity of data should be considered. Note in the example applications in Sect. 4.1.3 that contain less than 25 points, no additional uncertainty has been applied. These examples are only for illustration of the methods presented in Sects. 4.1.1 and 4.1.2.

\subsubsection{USL Method 1: Confidence Band with Administrative Margin}

The first method applies a statistical calculation of the bias and its uncertainty plus an administrative margin to a linear fit of critical experiment benchmark data. This approach is illustrated in Fig. 4.1. In this figure, the upper line (solid) represents a linear regression fit to a set of calculations based on critical experiments, $k_{c}(x)$. As indicated in the figure, $\beta(x)$ is given as $k_{c}(x)-1$. The middle line (long dashes) represents the lower confidence band for a single additional calculation. The width of this band is determined statistically based on the existing data and a specified level of confidence; the greater the standard deviation in the data or the larger the confidence desired, the larger the band width will be. This confidence band, $\mathrm{W}$, accounts for uncertainties in the experiments, the calculational approach, and in 
calculational data (e.g., cross sections), and is therefore a statistical basis for $\Delta \beta$, the uncertainty in the value of $\beta$. W is defined for a confidence level of $\left(1-\gamma_{1}\right)$ using the relationship

$$
\mathrm{W}=\max \left\{\left.w(\mathrm{x})\right|_{\mathrm{x}_{\min }, \mathrm{x}_{\max }}\right\}
$$

where

$$
w(x)=t_{1-\gamma_{1}} s_{p}\left[1+\frac{1}{n}+\frac{(x-\bar{x})^{2}}{\sum_{i=1, n}\left(x_{i}-\bar{x}\right)^{2}}\right]^{\frac{1}{2}}
$$

and

$$
\begin{aligned}
n & =\text { the number of critical calculations used in establishing } k_{c}(x), \\
t_{1-\gamma_{1}} & =\text { the Student-t distribution statistic for } 1-\gamma_{1} \text { and } n-2 \text { degrees of freedom (obtained } \\
& \text { from } t \text { tables }^{40} \text { ) } \\
\bar{x} \quad= & \text { the mean value of parameter } x \text { in the set of calculations, } \\
s_{p} & =\text { the pooled standard deviation for the set of criticality calculations. }
\end{aligned}
$$

The function $w(x)$ is a curvilinear function. For simplicity, it is desirable to obtain a constant width margin. Hence, for conservatism, the confidence band width $W$ is defined as the maximum of $\left(w\left(x_{\min }\right), w\left(x_{\max }\right)\right)$, where $x_{\min }$ and $x_{\max }$ are the minimum and maximum values of the independent parameter $x$, respectively. Typically, $W$ is determined at a $95 \%$ confidence level.

The pooled standard deviation is obtained from the pooled variance $\left(s_{p}=\sqrt{s_{p}^{2}}\right)$, where $s_{p}^{2}$ is given as

$$
s_{p}^{2}=s_{k(x)}^{2}+s_{w}^{2}
$$

where $s_{k(x)}^{2}$ is the variance (or mean-square error) of the regression fit, and is given by:

$$
s_{k(x)}^{2}=\frac{1}{(n-2)}\left[\sum_{i=1, n}\left(k_{i}-\bar{k}\right)^{2}-\frac{\left\{\sum_{i=1, n}\left(x_{i}-\bar{x}\right)\left(k_{i}-\bar{k}\right)\right\}^{2}}{\sum_{i=1, n}\left(x_{i}-\bar{x}\right)^{2}}\right],
$$

and $s_{w}^{2}$ is the within-variance of the data:

$$
s_{w}^{2}=\frac{1}{n} \sum_{i=1, n} \sigma_{i}^{2}
$$

where $\sigma_{i}$ is the standard deviation associated with $k_{i}$ for a Monte Carlo calculation. For best results, it is recommended that the individual standard deviations for the Monte Carlo calculations be roughly uniform in value. For deterministic 


\section{Determination of Limits}

codes that do not have a standard deviation associated with a computed value of $k$, the standard deviation is zero. However, this term could be used as a mechanism to include known uncertainties in experimental data.

The bottom line (short dashes) in Fig. 4.1 represents the USL, based on an additional margin of subcriticality. Note in Fig. 4.2 that a "positive bias adjustment" line has been added to the line $k_{c}(x)-W$ at the point where $k_{c}(x)=1$. This adjustment has been made to prevent taking credit for a positive bias $[\beta(x)>0]$ by assuming $k_{c}(x)=1$ everywhere that $k_{c}(x)>1$. Likewise, the USL has been adjusted so that the margin of subcriticality is constant with respect to the positive-bias-adjusted form of $k_{c}(x)-W$. This additional margin provides further assurance of subcriticality, and represents the quantity $\Delta \mathrm{k}_{\mathrm{m}}$ defined earlier. In USL Method $1, \Delta \mathrm{k}_{\mathrm{m}}$ is given an arbitrary administrative value; a minimum of 0.05 is recommended for application to transportation and storage packages. Using these formulations, the USL Method 1 is defined as

$$
\mathrm{USL}_{1}(\mathrm{x})=1-\Delta \mathrm{k}_{\mathrm{m}}-\mathrm{W}+\beta(\mathrm{x})
$$

In application to transportation and storage packages, the USL(x) value should not be greater than 0.95 .

\subsubsection{USL Method 2: Single-Sided Uniform Width Closed Interval Approach}

In this method, sometimes referred to as a lower tolerance band (LTB) approach, statistical techniques with a rigorous basis ${ }^{41-44}$ are applied in order to determine a combined lower confidence band plus subcritical margin. In other words, in the administrative margin approach $\Delta \mathrm{k}_{\mathrm{m}}$ and $\Delta \beta$ are determined independently, while in the LTB method, a combined statistical lower bound is determined. The following discussion of Method 2 is taken from Ref. 45 and is based on equations and/or definitions from Refs. 43 and 44.

The purpose of this method is to determine a uniform tolerance band over a specified closed interval for a linear least-squares model. The level of confidence in the limit being calculated is $\alpha$ and is typically in the range from 0.90 to 0.999 .

The USL Method 2 is defined as

$$
\mathrm{USL}_{2}(\mathrm{x})=1.0-\left(\mathrm{C}_{\alpha / \mathrm{P}} \cdot \mathrm{S}_{\mathrm{p}}\right)+\beta(\mathrm{x}),
$$

where $s_{p}$ is the pooled variance of $k_{c}$ described earlier. The term $C_{\alpha / P} \cdot s_{p}$ provides a band for which there is a probability $P$ with a confidence $\alpha$ that an additional calculation of $k_{\text {eff }}$ for a critical system will lie within the band. For example, a $\mathrm{C}_{95 / 99.5}$ multiplier produces a USL for which there is a $95 \%$ confidence that 995 out of 1000 future calculations of critical systems will yield a value of $\mathbf{k}_{\text {eff }}$ above the USL.

The analysis is over the closed interval from $x=a$ to $x=b . C_{\alpha / p}$ is calculated according to the following equations:

$$
g=\sqrt{\frac{1}{n}+\frac{(a-\bar{x})^{2}}{\sum_{i=1}^{n}\left(x_{i}-\bar{x}\right)^{2}}}
$$




$$
\begin{gathered}
h=\sqrt{\frac{1}{n}+\frac{(b-\bar{x})^{2}}{\sum_{i=1}^{n}\left(x_{i}-\bar{x}\right)^{2}}} \\
\rho=\frac{1}{g h} \cdot\left\{\begin{array}{c}
\frac{1}{n}+\frac{(a-\bar{x})(b-\bar{x})}{\sum_{i=1}^{n}\left(x_{i}-\bar{x}\right)^{2}} \\
A=\frac{g}{h}
\end{array}\right.
\end{gathered}
$$

It should be noted that there is an inconsistency in the notation used in Refs. 43 and 44 . In Ref. 44 , the notation for the summation of $x^{2}$ actually means the summation of the quantity $(x-\bar{x})^{2}$.

$A, \rho$, and $(n-2)$ are used to determine the value of $D$ from Table 3 in Ref. 43 , which covers values of $0.5 \leq A \leq 1.5$. The procedure to follow when $A$ is in this range is

$$
\mathrm{C}^{*}=\mathrm{D} \cdot \mathrm{g}
$$

When $A$ is outside the above range, $A$ is replaced by $1 / A$ for the determination of $D$, and $C^{*}$ is given by

$$
\mathrm{C}^{*}=\mathrm{D} \cdot \mathrm{h} \text {. }
$$

Next,

$$
C_{\alpha / \mathrm{P}}=C^{*}+z_{p} \cdot \sqrt{\frac{n-2}{\chi^{2}}},
$$

where

$z_{\mathrm{P}}$ is the Student $\mathbf{t}$ statistic depending on $\mathbf{n}$ and $\mathbf{P}$, and

$\chi^{2}$ is the chi square distribution, a function of $n-2$ and $\alpha$.

This approach provides a more statistically based subcritical margin, $\Delta \mathrm{k}_{\mathrm{tp}}$, which can be determined as the difference $\left(C_{\alpha / \rho} \cdot s_{p}\right)-W$. In criticality safety applications, such a statistically determined approach generally, but not necessarily, yields a margin of less than 0.05 , which serves to illustrate the adequacy of the administrative margin. The recommended purpose of Method 2 is to apply it in tandem with Method 1 to verify that the administrative margin is conservative relative to a purely statistical basis. This concurrent application of Method $\mathbf{2}$ is especially important when a limited number of data points are used in determination of $k_{c}(x)$, or when the 
Determination of Limits

Table 4.1 Subsets of LWR-type fuel experiments

\begin{tabular}{|c|c|c|c|c|c|c|c|c|c|}
\hline \multirow[b]{2}{*}{$\begin{array}{l}\text { Table } \\
\text { No. }\end{array}$} & \multirow[b]{2}{*}{ Class } & \multicolumn{8}{|c|}{ Number of experiments in subsets } \\
\hline & & $\begin{array}{c}\text { All } \\
\text { expts. }\end{array}$ & $\begin{array}{c}\text { Separator } \\
\text { plates }\end{array}$ & $\begin{array}{l}\text { Reflecting } \\
\text { walls }\end{array}$ & $\begin{array}{c}\text { Soluble } \\
\text { boron }\end{array}$ & $\begin{array}{c}\text { Plates } \\
\text { and } \\
\text { walls }\end{array}$ & $\begin{array}{l}\text { Plates } \\
\text { and } \\
\text { boron }\end{array}$ & $\begin{array}{l}\text { Water } \\
\text { holes }\end{array}$ & $\begin{array}{c}\text { Borated } \\
\text { plates } \\
\text { and walls }\end{array}$ \\
\hline \multirow{11}{*}{$\begin{array}{l}3.6 \\
3.7 \\
3.8 \\
3.9 \\
3.10 \\
3.11 \\
3.12 \\
3.13 \\
3.14 \\
3.15\end{array}$} & Simple lattice & 21 & & \multirow{10}{*}{$\begin{array}{l}35 \\
15\end{array}$} & \multirow{10}{*}{$\begin{array}{l}2 \\
1 \\
1 \\
4\end{array}$} & \multirow{10}{*}{15} & \multirow{10}{*}{12} & \multirow{10}{*}{$\begin{array}{l}2 \\
7 \\
1\end{array}$} & \multirow{10}{*}{7} \\
\hline & Separator plate & 45 & 45 & & & & & & \\
\hline & Plates w/soluble boron & 12 & 12 & & & & & & \\
\hline & Flux trap & 8 & 8 & & & & & & \\
\hline & Reflecting wall & 35 & & & & & & & \\
\hline & Plates w/walls & 15 & 15 & & & & & & \\
\hline & Urania gadolinia $^{a}$ & 2 & & & & & & & \\
\hline & Water holes ${ }^{a}$ & 7 & & & & & & & \\
\hline & Absorber rods ${ }^{\circ}$ & 8 & & & & & & & \\
\hline & Simple lattice w/boron & 14 & & & & & & & \\
\hline & TOTAL & 167 & 80 & 50 & 30 & & 12 & 10 & 7 \\
\hline
\end{tabular}

${ }^{a}$ One or more experiments were not included because they consisted of two types of fuel rods with different enrichments.

Table 4.2 Statistical summary of LWR-type fuel experiments

\begin{tabular}{lccccc}
\hline Variable & $\begin{array}{c}\text { No. of } \\
\text { experiments }\end{array}$ & Mean & Median & Minimum & Maximum \\
\hline $\mathrm{k}_{\text {eff }}$ & 173 & 0.9994 & 0.9992 & 0.9909 & 1.0129 \\
$\mathrm{AEG}$ & 173 & 33.94 & 33.95 & 27.62 & 36.88 \\
$\mathrm{AEF}$ & 173 & 0.3267 & 0.2443 & 0.0702 & 3.4451 \\
Pitch & 173 & 1.771 & 1.684 & 1.105 & 2.540 \\
Enrichment & 167 & 3.62 & 4.31 & 2.35 & 5.74 \\
Water/fuel & 167 & 2.100 & 1.6 & 0.148 & 9.004 \\
Dancoff factor & 167 & 0.1916 & 0.1739 & 0.0298 & 0.7644 \\
H/X & 167 & 183.5 & 155.9 & 17.2 & 541.1 \\
Assembly separation & 110 & 8.11 & 7.81 & 1.64 & 20.78 \\
Plate thickness & 76 & 0.371 & 0.302 & 0.061 & 0.772 \\
Wall separation & 50 & 1.51 & 1.32 & 0.00 & 5.41 \\
Soluble boron conc. & 36 & 1132 & 1110 & 15 & 3389 \\
Plate boron conc. & 22 & 21.3 & 29.0 & 1.1 & 39.3 \\
Water hole/fuel pin & 16 & 0.048 & 0.032 & 0.013 & 0.152 \\
& & & & & \\
\hline
\end{tabular}


Determination of Limits

Table 4.3 Independent variables by subset

\begin{tabular}{|c|c|c|c|c|c|c|c|c|}
\hline Variable & $\begin{array}{l}\text { All } \\
\text { expts. }\end{array}$ & $\begin{array}{l}\text { Separator } \\
\text { plates }\end{array}$ & $\begin{array}{l}\text { Reflecting } \\
\text { walls }\end{array}$ & $\begin{array}{c}\text { Soluble } \\
\text { boron }\end{array}$ & $\begin{array}{c}\text { Plates } \\
\text { and } \\
\text { walls }\end{array}$ & $\begin{array}{l}\text { Water } \\
\text { holes }\end{array}$ & $\begin{array}{c}\text { Plates } \\
\text { and } \\
\text { boron }\end{array}$ & $\begin{array}{c}\text { Borated } \\
\text { plates } \\
\text { and walls }\end{array}$ \\
\hline Enrichment & $\mathbf{x}$ & $\mathrm{x}$ & $\mathrm{x}$ & $\mathrm{X}$ & $\mathrm{x}$ & $\mathrm{x}$ & $\mathrm{X}$ & $\mathrm{X}$ \\
\hline AEF & $\mathbf{x}$ & $\mathrm{x}$ & $\mathrm{X}$ & $\mathrm{X}$ & $\mathrm{X}$ & $\mathrm{X}$ & $\mathrm{X}$ & $\mathrm{X}$ \\
\hline Pitch & $\mathbf{X}$ & $\mathrm{X}$ & $\mathrm{x}$ & $\mathrm{X}$ & $\mathrm{X}$ & $\mathrm{x}$ & $x$ & $\mathrm{X}$ \\
\hline $\mathrm{H}_{2} \mathrm{O} /$ fuel & $\mathbf{x}$ & $\mathrm{x}$ & $\mathrm{x}$ & $\mathrm{x}$ & $\mathrm{x}$ & $\mathrm{x}$ & $\mathrm{X}$ & $\mathrm{X}$ \\
\hline $\mathrm{H} / \mathrm{X}$ & $\mathbf{x}$ & $\mathrm{x}$ & $\mathrm{x}$ & $\mathrm{x}$ & $\mathrm{X}$ & $\mathrm{X}$ & $X$ & $\mathrm{X}$ \\
\hline Dancoff & $\mathbf{X}$ & $\mathrm{x}$ & $\mathrm{X}$ & $\mathrm{X}$ & $\mathrm{x}$ & $\mathrm{X}$ & $\mathrm{X}$ & $\mathrm{X}$ \\
\hline $\begin{array}{l}\text { Assembly separation } \\
\text { distance }\end{array}$ & & & $\mathrm{x}$ & & $\mathrm{X}$ & & $\mathrm{X}$ & $\mathrm{X}$ \\
\hline $\begin{array}{l}\text { Assembly-to-wall } \\
\text { separation distance }\end{array}$ & & & $\mathrm{X}$ & & $\mathrm{X}$ & & & $\mathrm{x}$ \\
\hline $\begin{array}{l}\text { Soluble boron } \\
\text { concentration }\end{array}$ & & & & $\mathrm{X}$ & & & $\mathrm{X}$ & \\
\hline Plate thickness & & $\mathbf{x}$ & & & $\mathrm{x}$ & & $\mathrm{x}$ & $\mathrm{x}$ \\
\hline $\begin{array}{l}\text { Plate boron } \\
\text { concentration }\end{array}$ & & & & & & & & $\mathrm{x}$ \\
\hline Water holes/fuel pin & & & & & & $\mathrm{x}$ & & \\
\hline
\end{tabular}


Table 4.4 Regression analyses results for subsets of LWR-type fuel experiments

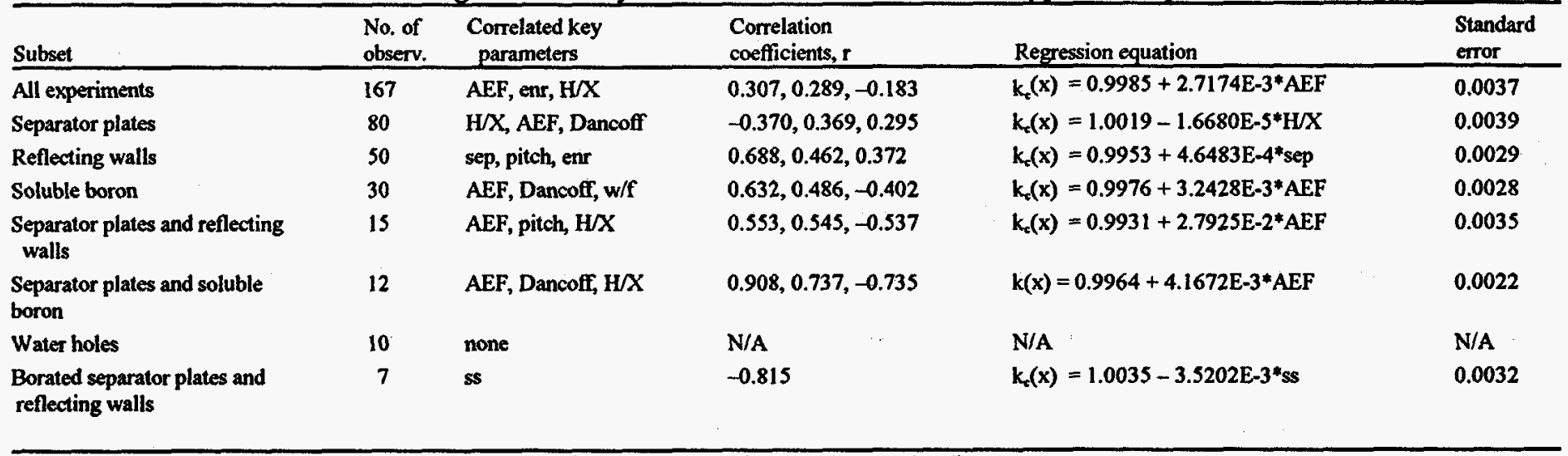

enr - enrichment; sep - assembly separation; w/f - water/fuel volume; ss - reflector wall separation distance. 
Table 4.5 Summary of USL calculations of Figs. 4.4-4.13

\begin{tabular}{|c|c|c|c|c|c|c|c|c|c|}
\hline Figure & $\begin{array}{l}\text { No. of } \\
\text { exp. }\end{array}$ & $k_{c}(x)$ & $\begin{array}{l}\text { Correlation } \\
\text { coefficient, } r\end{array}$ & $\begin{array}{c}\text { Most } \\
\text { negative } \\
\text { bias }\left(\beta_{\mathrm{m},}\right)\end{array}$ & W & $\begin{array}{l}\Delta k_{m} \\
\left(\mathrm{USL}_{2}\right)\end{array}$ & $\begin{array}{l}\text { Parameter } \\
\text { bounds }\end{array}$ & $\begin{array}{c}\text { Confidence band } \\
\text { with administrative } \\
\text { margin of } 0.05, \text { USL }\end{array}$ & $\begin{array}{c}\text { Single-sided uniform } \\
\text { width closed interval, } \\
\text { USL }\end{array}$ \\
\hline $\begin{array}{l}4.2, \mathrm{k}_{\text {ofd }} \text { Vs AEF (all } \\
\text { experiments) }\end{array}$ & 167 & $0.9985+2.7174 \mathrm{E}-3 * \mathrm{AEF}$ & 0.307 & -0.0091 & $7.2146 \mathrm{E}-3$ & 0.015 & $0.070-3.445$ & $0.9415-0.9428$ & $0.9770-0.9782$ \\
\hline $\begin{array}{l}4.3, k_{\text {aft }} v s \text { enrichment } \\
\text { (all experiments) }\end{array}$ & 167 & $0.9959+9.6691 E-4^{*} \mathrm{enr}$ & 0.289 & -0.0091 & $6.2707 \mathrm{E}-3$ & 0.012 & $2.35-5.74$ & $0.9419-0.9437$ & $0.9796-0.9814$ \\
\hline $\begin{array}{l}4.4, k_{\text {ws }} \text { vs } \mathrm{H} / \mathrm{X} \\
\text { (separator plates) }\end{array}$ & 80 & $1.0019-1.6680 \mathrm{E}-5 * \mathrm{H} / \mathrm{X}$ & -0.370 & -0.0091 & $6.6660 \mathrm{E}-3$ & 0.010 & $17.2-398.5$ & $0.9433-0.9386$ & $0.9836-0.9789$ \\
\hline $\begin{array}{l}4.5, \mathrm{k}_{\text {en }} \text { vs assembly } \\
\text { separation (reflecting } \\
\text { walls) }\end{array}$ & 50 & $0.9953+4.6483 E-4 * \operatorname{sep}$ & 0.688 & -0.0080 & $5.1381 \mathrm{E}-3$ & 0.008 & $2.00-20.78$ & $0.9411-0.9449$ & $0.9829-0.9867$ \\
\hline $\begin{array}{l}4.6, k_{\text {edr }} \text { vs AEF (soluble } \\
\text { boron) }\end{array}$ & 30 & $0.9976+3.2428 \mathrm{E}-3 * \mathrm{AEF}$ & 0.632 & -0.0053 & $5.4354 \mathrm{E}-3$ & 0.009 & $0.092-2.281$ & $0.9425-0.9446$ & $0.9831-0.9851$ \\
\hline $\begin{array}{l}\text { 4.7, } k_{\text {ws AEF (separator }} \text { A } \\
\text { plates and reflecting walls) }\end{array}$ & 15 & $0.9931+2.7925 \mathrm{E}-2 * \mathrm{AEF}$ & 0.553 & -0.0080 & $6.7130 \mathrm{E}-3$ & 0.014 & $0.144-0.305$ & $0.9403-0.9433$ & $0.9761-0.9791$ \\
\hline $\begin{array}{l}4.8, \mathrm{k}_{\mathrm{d}} \text { vs AEF (separator } \\
\text { plates and soluble boron) }\end{array}$ & 12 & $0.9964+4.1672 \mathrm{E}-3 * \mathrm{AEF}$ & 0.908 & -0.0046 & $4.6206 \mathrm{E}-3$ & 0.010 & $0.152-2.281$ & $0.9425-0.9454$ & $0.9822-0.9851$ \\
\hline $\begin{array}{l}4.9, \mathrm{k}_{\text {ex }} \text { Vs reflecting wall } \\
\text { separation (borated } \\
\text { separator plates and } \\
\text { reflecting walls) }\end{array}$ & 7 & $1.0035-3.5202 \mathrm{E}-3 * \mathrm{ss}$ & -0.815 & -0.0080 & $7.7476 \mathrm{E}-3$ & 0.023 & $0.23-2.50$ & $0.9423-0.9370$ & $0.9696-0.9644$ \\
\hline
\end{tabular}


Determination of Limits

Table 4.6 USL equations for Figs. 4.4-4.12

\begin{tabular}{|c|c|c|}
\hline $\begin{array}{l}\text { Figure } \\
\text { No. }\end{array}$ & $\begin{array}{l}\text { Confidence band with administrative } \\
\text { margin of } 0.05, \text { USL, }\end{array}$ & $\begin{array}{l}\text { Single-sided uniform width closed interval, } \\
\text { USL, }\end{array}$ \\
\hline 4.2 & $\begin{array}{l}0.9413+2.7174 \mathrm{E}-3 * \mathrm{AEF}(\mathrm{AEF}<0.535) \\
0.9428(\mathrm{AEF} \geq 0.535)\end{array}$ & $\begin{array}{l}0.9768+2.7174 \mathrm{E}-3 * \mathrm{AEF}(\mathrm{AEF}<0.535) \\
0.9782(\mathrm{AEF} \geq 0.535)\end{array}$ \\
\hline 4.3 & $\begin{array}{l}0.9397+9.6691 \mathrm{E}-4 * \mathrm{enr}(\mathrm{enr}<4.209) \\
0.9437 \text { (enr. } \geq 4.209)\end{array}$ & $\begin{array}{l}0.9773+9.6691 \mathrm{E}-4 * \mathrm{enr}(\mathrm{enr}<4.209) \\
0.9814(\mathrm{enr} \geq 4.209)\end{array}$ \\
\hline 4.4 & $\begin{array}{l}0.9433(\mathrm{H} / \mathrm{X} \leq 116.8) \\
0.9453-1.6680 \mathrm{E}-5 * \mathrm{H} / \mathrm{X}(\mathrm{H} / \mathrm{X}>116.8)\end{array}$ & $\begin{array}{l}0.9836(\mathrm{H} / \mathrm{X} \leq 116.8) \\
0.9856-1.6680 \mathrm{E}-5 * \mathrm{H} / \mathrm{X}(\mathrm{H} / \mathrm{X}>116.8)\end{array}$ \\
\hline 4.5 & $\begin{array}{l}0.9402+4.6483 \mathrm{E}-4 * \operatorname{sep}(\operatorname{sep}<10.073) \\
0.9449(\operatorname{sep} \geq 10.073)\end{array}$ & $\begin{array}{l}0.9820+4.6483 E-4 * \operatorname{sep}(\text { sep }<10.073) \\
0.9867(\operatorname{sep} \geq 10.073)\end{array}$ \\
\hline 4.6 & $\begin{array}{l}0.9422+3.2428 \mathrm{E}-3^{*} \mathrm{AEF}(\mathrm{AEF}<0.731) \\
0.9446(\mathrm{AEF} \geq 0.731)\end{array}$ & $\begin{array}{l}0.9828+3.2428 \mathrm{E}-3 * \mathrm{AEF}(\mathrm{AEF}<0.731) \\
0.9851(\mathrm{AEF} \geq 0.731)\end{array}$ \\
\hline 4.7 & $\begin{array}{l}0.9364+2.7925 \mathrm{E}-2 * \mathrm{AEF}(\mathrm{AEF}<0.247) \\
0.9433(\mathrm{AEF} \geq 0.247)\end{array}$ & $\begin{array}{l}0.9722+2.7925 \mathrm{E}-2 * \mathrm{AEF}(\mathrm{AEF}<0.247) \\
0.9791(\mathrm{AEF} \geq 0.247)\end{array}$ \\
\hline 4.8 & $\begin{array}{l}0.9418+4.1672 \mathrm{E}-3 * \mathrm{AEF}(\mathrm{AEF}<0.852) \\
0.9454(\mathrm{AEF} \geq 0.852)\end{array}$ & $\begin{array}{l}0.9816+4.1672 \mathrm{E}-3 * \mathrm{AEF}(\mathrm{AEF}<0.852) \\
0.9851(\mathrm{AEF} \geq 0.852)\end{array}$ \\
\hline 4.9 & $\begin{array}{l}0.9423(\mathrm{ss} \leq 1.001) \\
0.9458-3.5202 \mathrm{E}-3 * \mathrm{ss}(\mathrm{ss}>1.001)\end{array}$ & $\begin{array}{l}0.9696(\mathrm{ss} \leq 1.001) \\
0.9732-3.5202 \mathrm{E}-3 * \mathrm{ss}(\mathrm{ss}>1.001)\end{array}$ \\
\hline
\end{tabular}


Determination of Limits

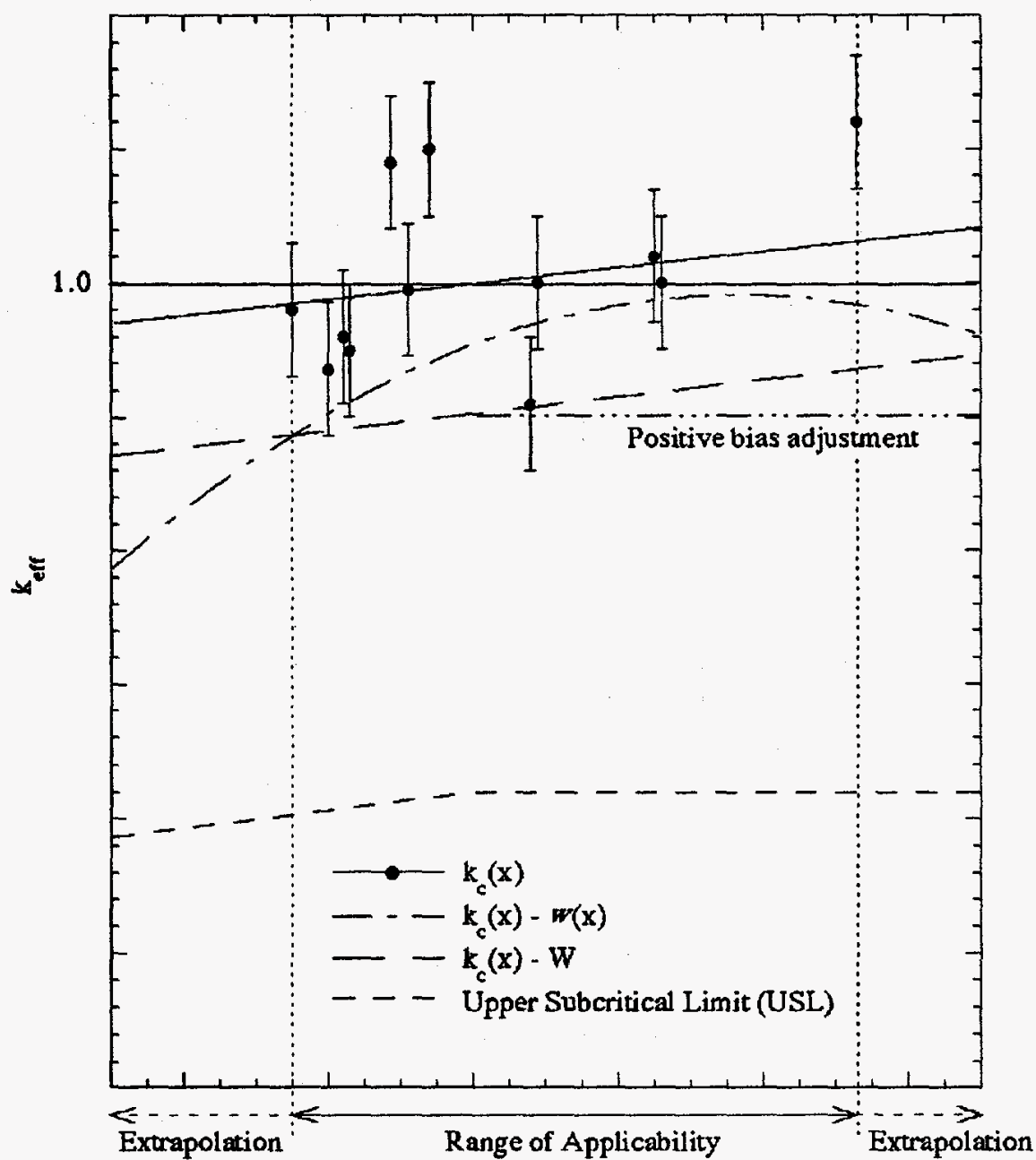

Figure 4.1 Confidence band and additional margin applied to a set of criticality calculations 


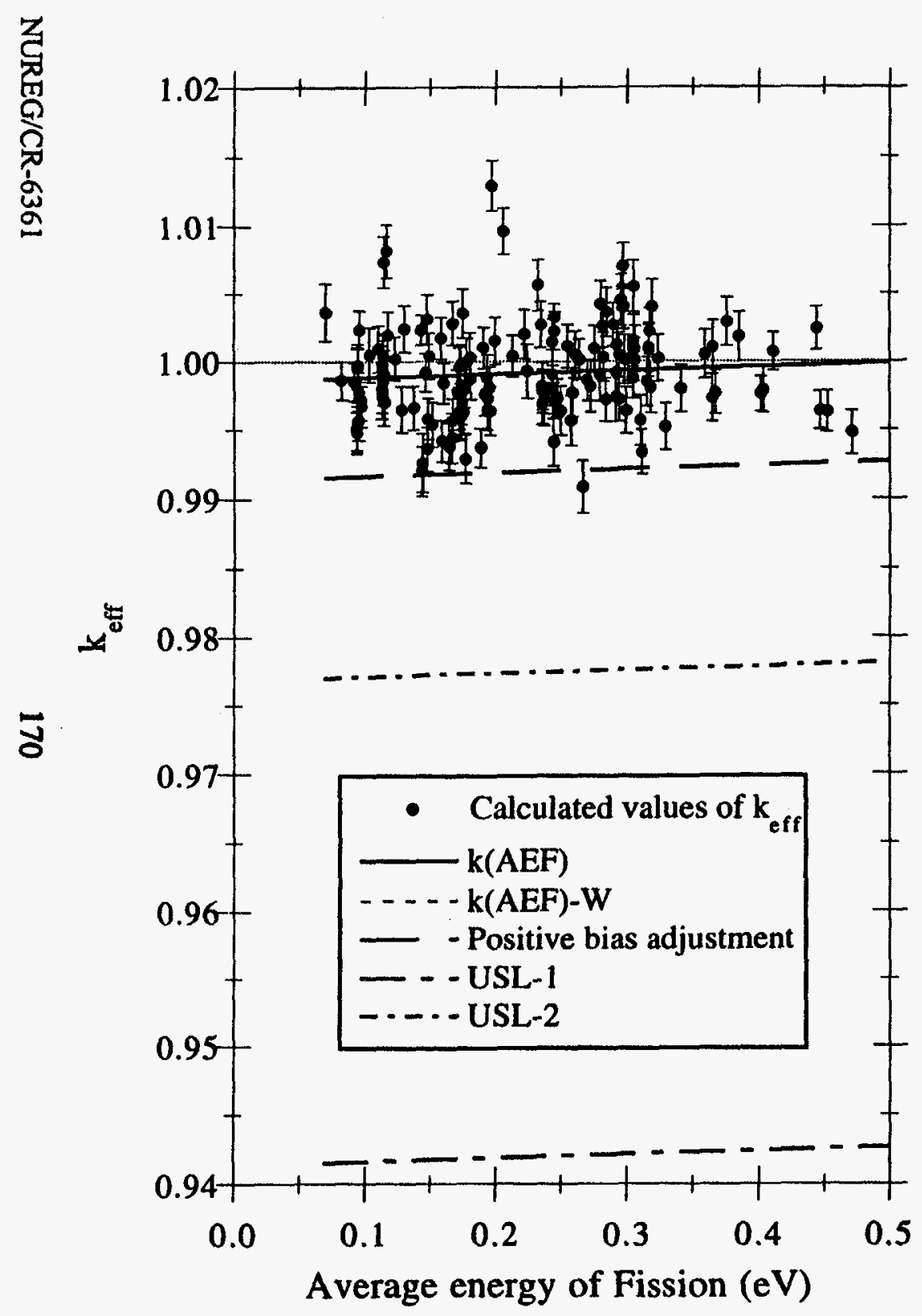

(a)

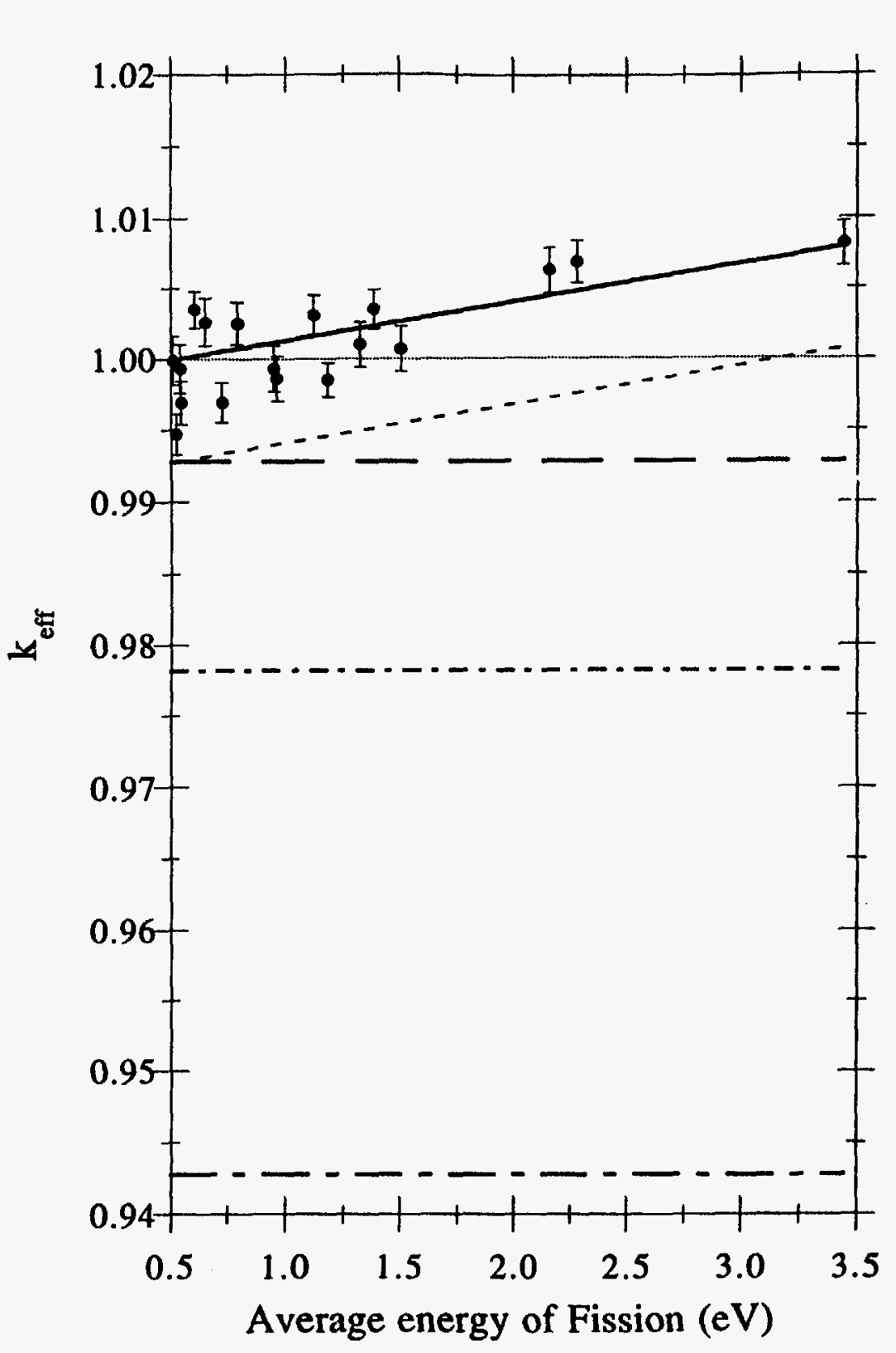

(b)

Figure 4.2 USLs for 167 LWR-type fuel critical experiments, $k_{\text {off }} v s$ enrichment 
Determination of Limits

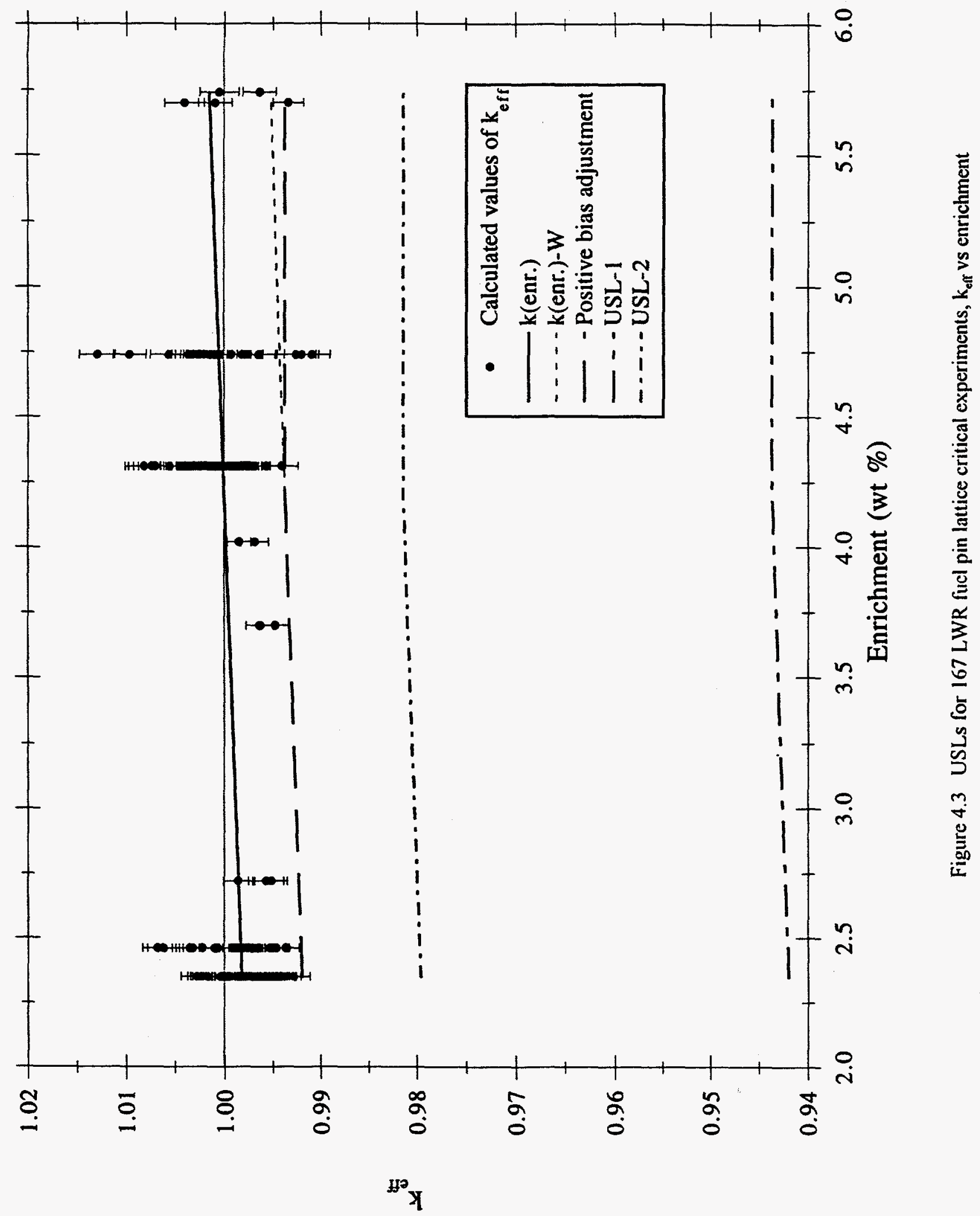




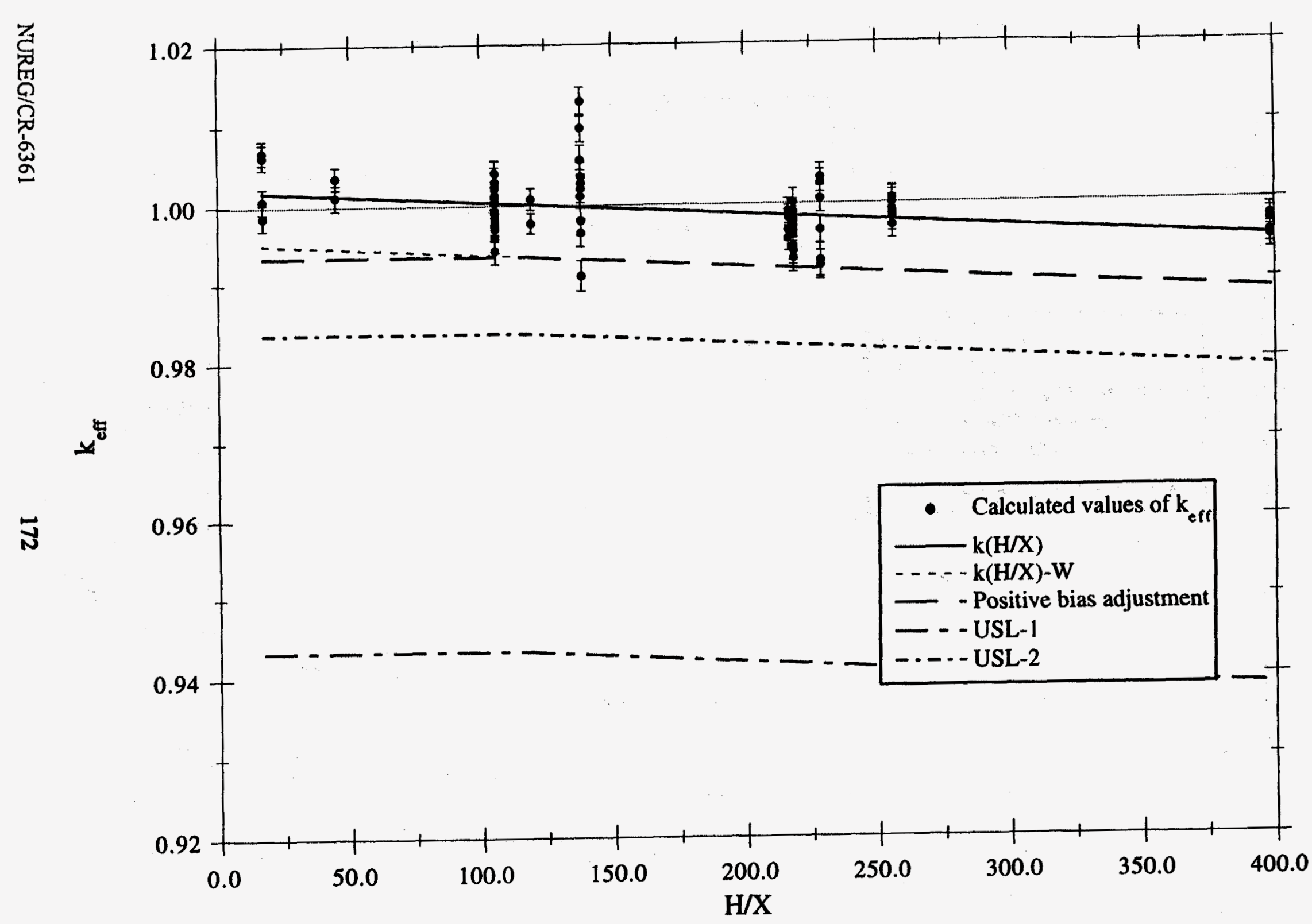

Figure 4.4 USLs for 80 separator plate critical experiments, $k_{\text {eff }} v s \mathrm{H} / \mathrm{X}$ 
Determination of Limits

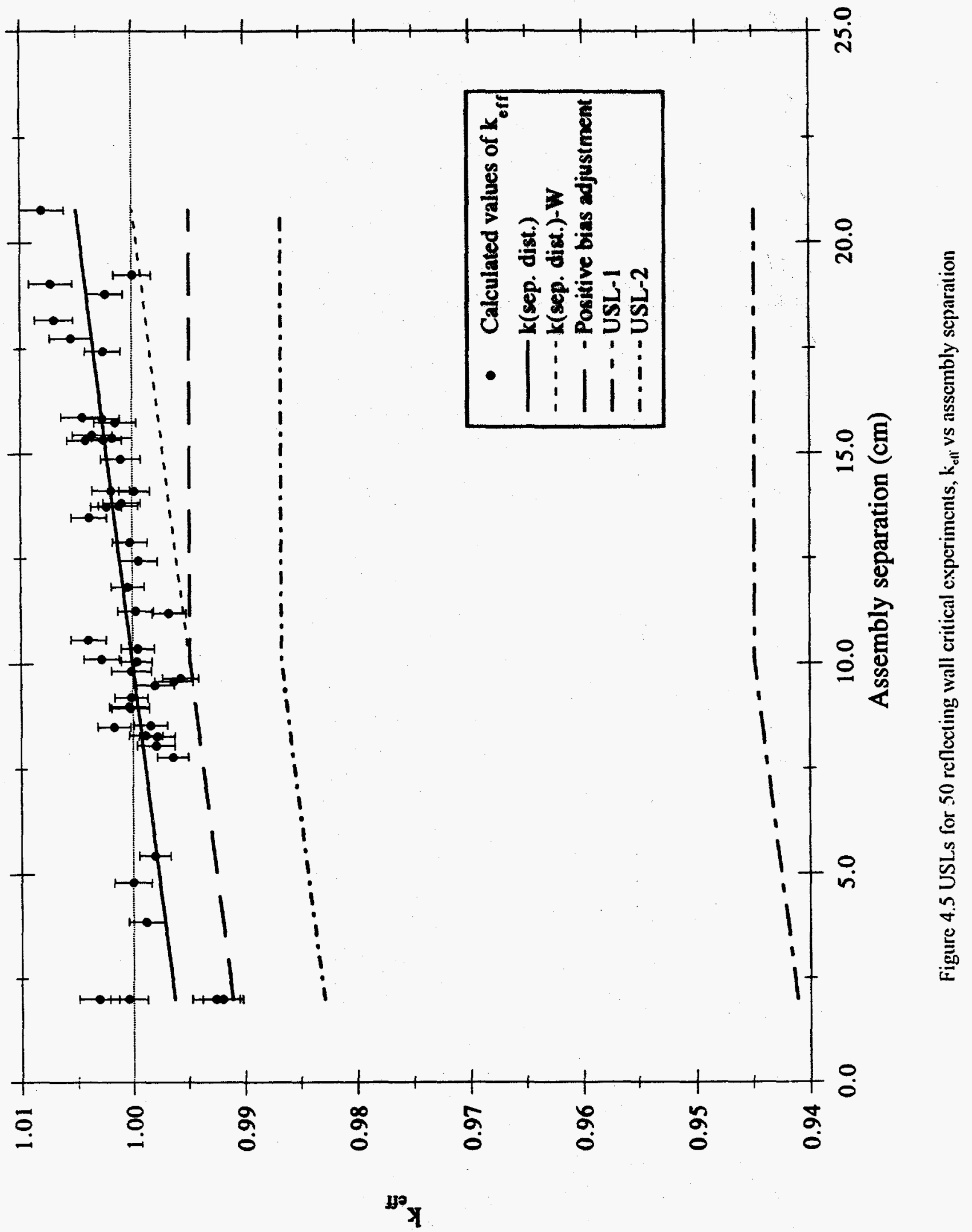




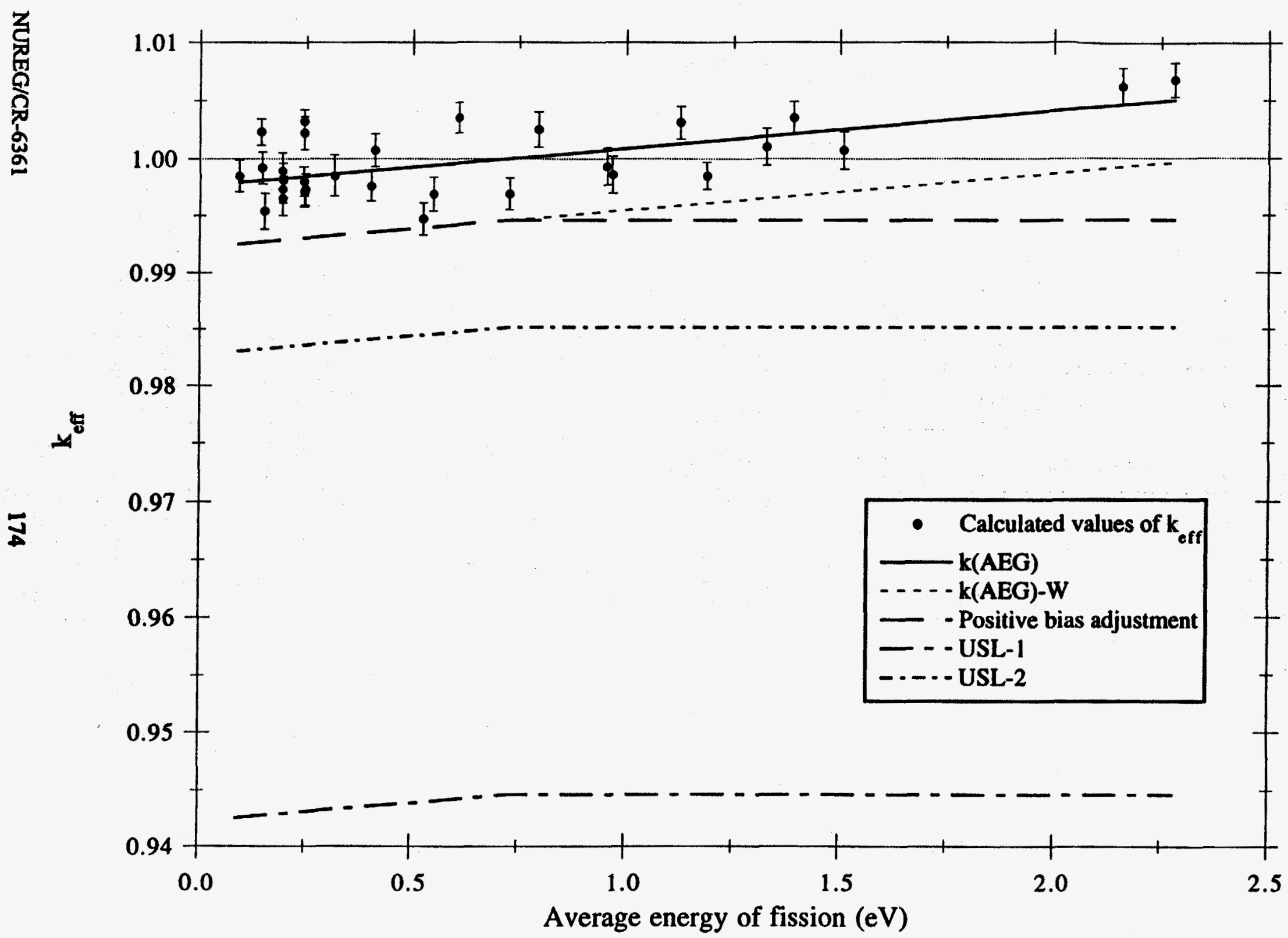

Figure 4.6 USLs for 30 soluble boron critical experiments, $k_{\text {eff }}$ vs AEF 


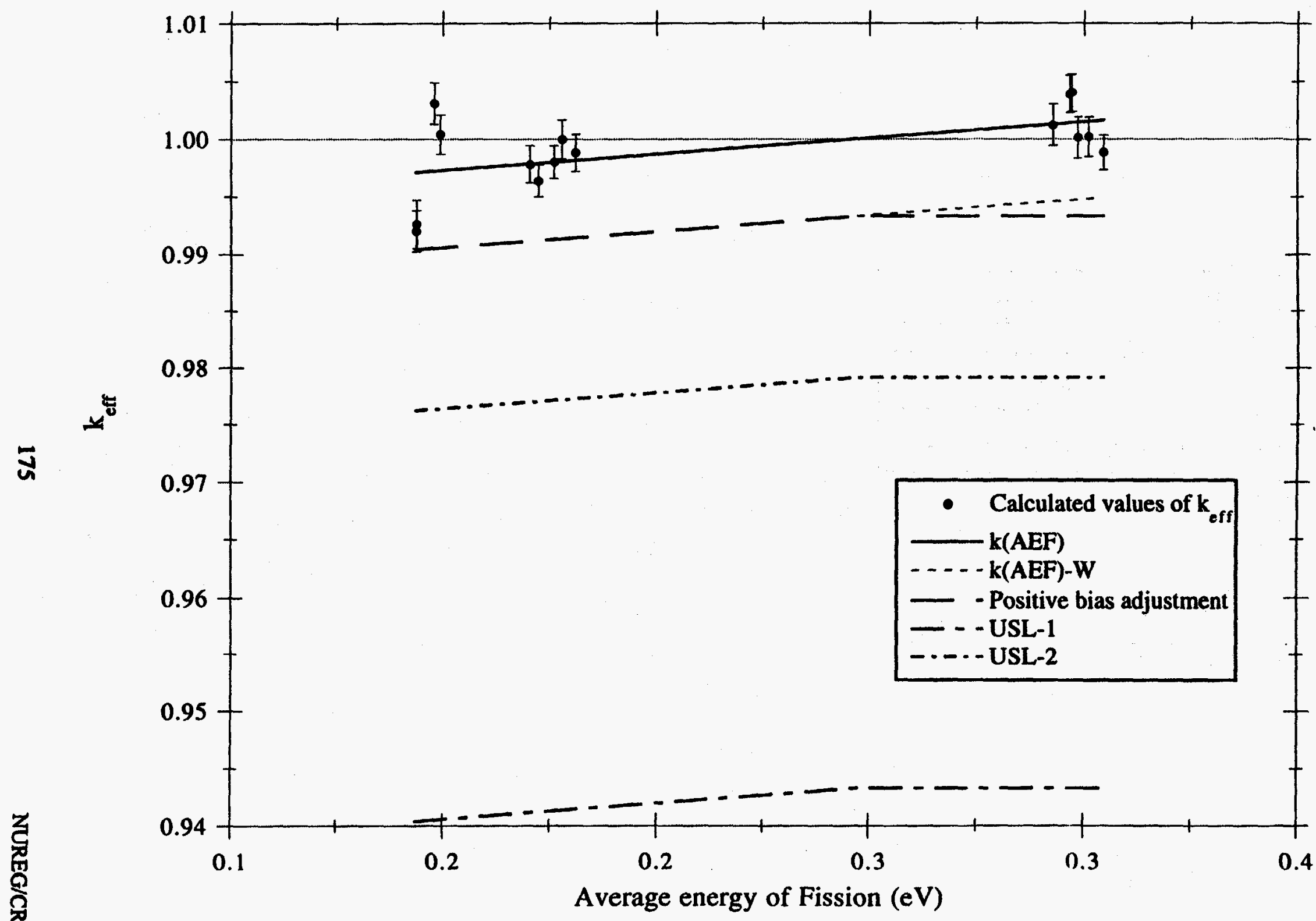

Figure 4.7 USL s for 15 separalor plate and reflecting wall critical experiments, $k_{\text {ofl }}$ vs AEF 
Determination of Limits

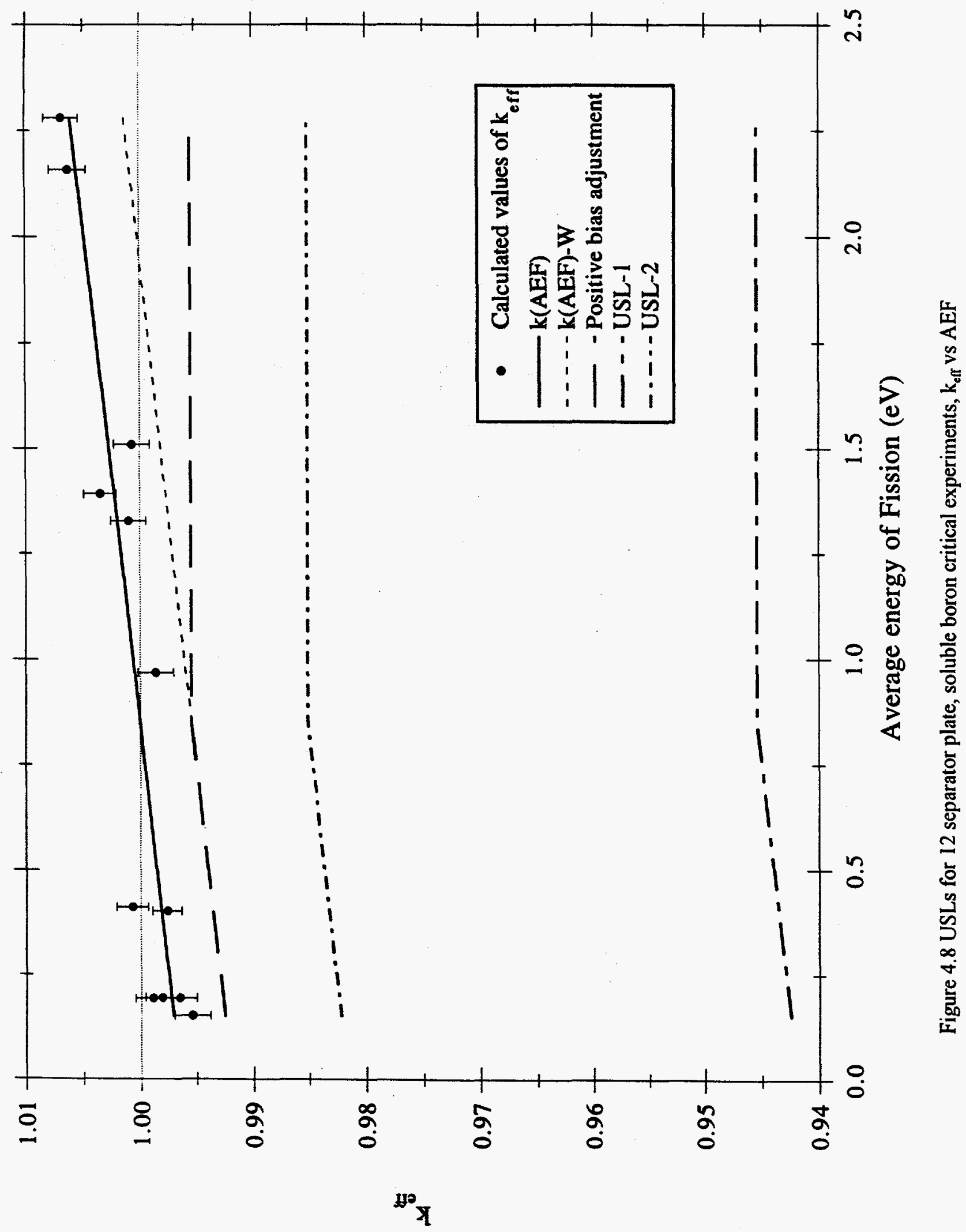




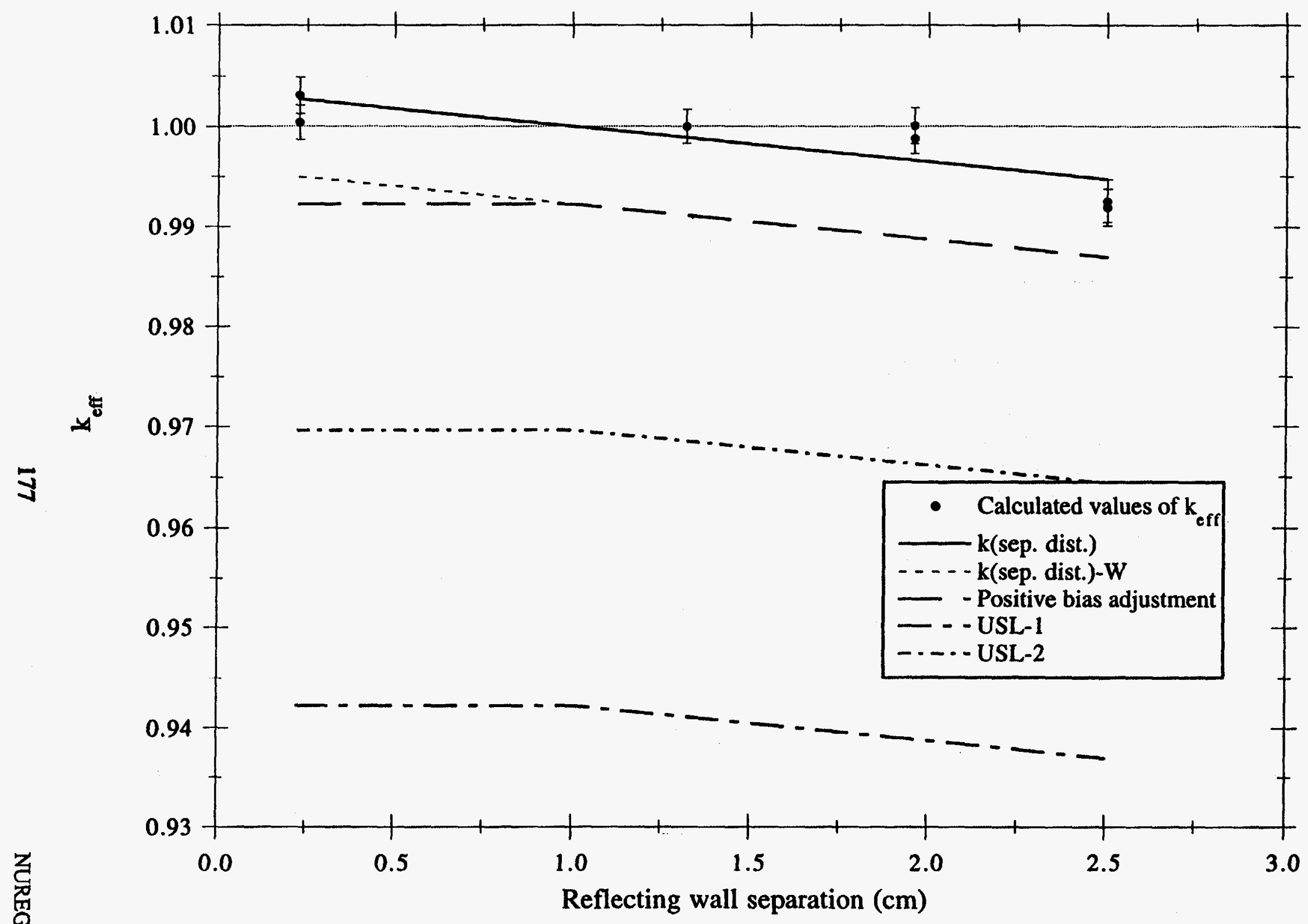

Figure 4.9 USLs for 7 borated separator plates and reflecting wall critical experiments, $k_{\text {eff }}$ vs reflecting wall separation distance 



\section{Guidelines for Experiment Selection and Areas of Applicability}

The calculation method used to establish the criticality safety of transportation and storage packages needs to be validated against measured data that have been shown to be applicable to the package under consideration. This section provides guidance in determining the applicable range for a validation study. Section 5.1 provides guidelines for selecting suitable experiments to use in the evaluation of transportation and storage package designs. The experiments used in the validation are parameterized in Sect. 5.2 for easy comparison to package designs under consideration.

\subsection{Guidelines for Selecting Suitable Experiments}

Historically, nuclear criticality experiments were performed to address specific fissionable material, reactor, fabrication/process, storage or transport design concerns. The experiments generally consisted of the same, or very nearly the same, configurations and materials requiring safety evaluation for the designs of interest. Aside from performing diagnostic measurements (e.g., flux wire traverses, threshold detector measurements, reaction rate ratios, replacement measurements, etc.), little effort was expended or needed to characterize the experiment for its intended application because the experiment itself was a "mockup" of the configuration of materials needing a safety evaluation. Many of the experiments were subsequently characterized according to elemental constituents, densities, and various parametric ratios. The use of uranium metal-to-water mass-, atom- and volume-ratios; uranium metal-to-carbon atomratios \{e.g., $[\mathrm{C} /(\mathrm{Pu}+\mathrm{U})],[\mathrm{H} /(\mathrm{Pu}+\mathrm{U})], \mathrm{C} / \mathrm{X}, \mathrm{H} / \mathrm{X}$, where $\mathrm{X}$ refers to the fissile isotope\}; fuel lattice pitches; solid angles; surface densities; and others came into broad usage with fair success. With the advent of more sophisticated computational techniques, parameters such as neutron leakage fraction and neutron absorption fraction became useful characterization parameters. Subsequently, a pseudo-neutron-spectra parameter, the computed average neutron energy group causing fission (AEG), was used as a single global parameter for correlating experiments to safety evaluation applications $^{36}$ for systems of similar fissile species, enrichments, degree of heterogeneity or homogeneity, and chemical form. Additionally, various neutron energy-weighted parameters, such as thermal neutron absorptions versus total neutron absorption, total neutron fissions versus fast neutron fissions, thermal neutron fissions versus thermal neutron absorptions, and the average neutron energy group weighted by fissions, were used for the characterization of systems and their concomitant computational biases. ${ }^{46}$ A two-group diffusion theory parameter ${ }^{47}$ for characterizing the neutron slowing down, resonance absorption characteristics, and thermal absorption characteristics has also been applied.

Most neutronics computer codes provide integral and energy dependent parameters that can be used for characterizing the spectra of a calculated system. Computer codes that have been historically used for nuclear criticality safety benchmarking and evaluations provide simplistic integral values such as average neutron energy group causing fission (AEG) ${ }_{3}^{34}$ and more complex parametric categories ${ }^{48}$ such as nonfuel absorption, leakage fraction, resonance absorption probability, fast fission probability, energy distribution of neutron absorption, and geometry effects as measured by the energy weighted ratios of neutron collisions in all materials divided by neutron collisions in fissile and fissionable materials.

There are three fundamental parameters that should be considered in the selection of suitable experiments ${ }^{49}$ for use in the evaluation of transportation and storage package designs. They are the materials of construction (including fissionable material), the geometry of construction, and the inherent neutron energy spectrum affecting the fissionable material(s). There are substantial variances within each of these three categories that require further considerations.

Regarding materials of construction (including fissionable material): 
Guidelines

- The fissionable species (e.g. ${ }^{233} \mathrm{U},{ }^{235} \mathrm{U},{ }^{238} \mathrm{U},{ }^{238} \mathrm{Pu},{ }^{239} \mathrm{Pu},{ }^{240} \mathrm{Pu},{ }^{241} \mathrm{Pu}$, etc.) used in the benchmark experiments should be as similar as possible, including enrichment and relative masses and volumes, as those materials in the package design under normal and hypothetical accident conditions.

- The nonfissionable species (e.g., lead, stainless steel, boron, cadmium, phenolic foam, depleted uranium, water, etc.) used in the benchmark experiments should be as similar as possible, including relative masses and volumes, as those materials in the package design under normal and hypothetical accident conditions.

- Reflector and moderator materials in experiments should be as similar as possible to those in the package design under normal and hypothetical accident conditions.

- Physical form (e.g., metal, solution, compound) and temperature of materials should be as similar as possible.

- Ratio of nonfissionable materials to fissionable material should be similar as well.

Important items to consider regarding geometry of construction (including fissionable material):

- The relative geometric position and size of the construction materials

- Homogeneity or heterogeneity of system

- Shape of units and system

- Lattice pattern, spacing, and interstitial materials in arrays

- Reflection

Regarding the neutron energy spectrum, the neutron density versus energy should be considered by comparison of the following items from the benchmark experiments and the package design:

- $\quad$ Leakage

- Absorption

- Production

- Flux

The specific quantization of the above three categories of parameters is difficult at best and generally requires the use of alternative approaches by using benchmark experiments that are characterized by a limited set of physical and computed neutronic parameters that are then compared with the safety design computed neutronic parameters.

\subsection{Parameterization of Validation Experiments}

Several principal parameters have been identified for controlling the criticality of transportation packages: ${ }^{50}$ (1) type, mass, and form of fissile material; (2) degree of moderation; (3) amount and distribution of absorber materials; (4) internal and external package geometry; and (5) reflector effectiveness. The fissile material, degree of moderation, and 
package geometry controls the neutron flux energy and spatial distributions. The degree of moderation, absorber materials, and package geometry are used to control the neutron loss rate through capture and leakage (in conjunction with reflector effectiveness).

These five parameters were used as guidelines in grouping the critical experiments considered by classes in the validation. Table 3.5 presents a parameterization of the $173 \mathrm{LWR}$-type fuel experiments documented in this report. The experiments are listed by case names, reference numbers, and case numbers. Case numbers are alphabetically assigned. The computed effective neutron multiplication factor $\left(k_{\text {eff }}\right)$ and associated standard deviation are given for each case. The number of experiments associated with each characterization used in Table 3.5 is tabulated in Table 5.1 .

The fissile material type, ${ }^{235} \mathrm{U}$, and chemical form of the uranium compound, $\mathrm{UO}_{2}$, were the same for all experiments and were therefore omitted from the table; however, the ${ }^{235} \mathrm{U}$ isotopic enrichment which affects neutron production rates was included since it varied from $2.35 \mathrm{wt} \%$ to $5.74 \mathrm{wt} \%$ across the experiments.

Fissile systems are often characterized by their degree of moderation. A direct characterization can be defined by the effective ratio of hydrogen $(H)$-to-fissile $(X)$ material atom densities, or $H / X$ of the system. Other parameters such as water-to-fuel volume ratio for a unit cell, average energy group for fission (AEG), and energy of average lethargy causing fission (AEF) can also give an indication of the degree of thermalization of a fissile system. These parameters usually correlate to the pitch and the Dancoff factor, which is a function of the pitch. Both of these parameters may be useful in the investigation of system moderation. The thermalization of lattice configurations can also be influenced by the presence of water holes. A parameter giving the ratio of the number of water holes to fuel pin positions is included in Table 3.5 for examination, along with $\mathrm{H} / \mathrm{X}, \mathrm{AEG}, \mathrm{AEF}$, pitch, and Dancoff factor.

Absorber materials are an effective means of criticality control for thermal systems such as those involving LWR-type fuel. Their effectiveness is dependent on the absorber material, distribution, and surface area. Three columns of Table 3.5 give this information in the form of separator plate material, boron weight percent per plate, and plate thickness. Experiments having homogeneous absorbers in the form of soluble boron in water have been singled out with their boron concentration specified. A final column labeled "Other" gives information about other absorbers in the system (e.g., $\mathrm{Ag}$-In-Cd rods, $\mathrm{B}_{4} \mathrm{C}$ rods, $\mathrm{UO}_{2}-\mathrm{Gd}_{2} \mathrm{O}_{3}$ fuel rods). This column also specifies if the separator plates are part of a flux trap with voiding materials.

The geometry of heterogeneous fissile materials, the design and placement of absorber materials (previously discussed), and the separation between fissile materials affect the neutron flux energy and spatial distribution. The fissile material is in the form of fuel rods consisting of $\mathrm{UO}_{2}$ pellets or powder encased in either aluminum, stainless steel, or Zircaloy cladding. Fuel rods are bundled in hexagonal or square lattices of $1.105-\mathrm{cm}$ to $2.540-\mathrm{cm}$ pitch. Table 3.5 lists the lattice type, pitch, cladding material, and separation distance of assemblies for each experiment. Most experiments have water reflection of at least 15 to $20 \mathrm{~cm}(20 \mathrm{~cm}$ of water reflection typically ensures a maximum increase in $k_{\text {eff }}$ due to reflection) on the $x, y$, and $z$ faces of the system. Notable exceptions are those systems that had fuel rods above the moderator surface. In addition to water reflectors, several experiments contained reflectors in the form of shielding materials (e.g., lead, steel, depleted uranium) that minimize neutron leakage. Such experiments were parameterized in Table 3.5 by reflecting wall material and separation distance from fuel assemblies.

The seven homogeneous uranium experiments were also parameterized. Experimental parameters and calculational results for these experiments are compiled in Table 3.16. 
Guidelines

Table 5.1 Characterization variables of LWR-type fuel experiments

\begin{tabular}{|c|c|c|}
\hline Variable & Values & $\begin{array}{l}\text { Number of } \\
\text { experiments }\end{array}$ \\
\hline \multirow{4}{*}{ Other } & $\mathrm{A}: \mathrm{Ag}$-In-Cd rods & 6 \\
\hline & $\mathrm{B}: \mathrm{B}_{4} \mathrm{C}$ rods & 3 \\
\hline & $\begin{array}{l}\text { F: Flux trap with void } \\
\text { materials }\end{array}$ & 8 \\
\hline & $\mathrm{G}: \mathrm{UO}_{2} \mathrm{Gd}_{3}$ rods & 5 \\
\hline Total & & 22 \\
\hline \multirow[t]{2}{*}{ Soluble boron } & Y: yes & 36 \\
\hline & N: no & 137 \\
\hline Total & & 173 \\
\hline \multirow[t]{3}{*}{ Clad } & $\mathrm{AL}:$ aluminum & 154 \\
\hline & SS: stainless steel & 10 \\
\hline & ZR: zircaloy & 3 \\
\hline Total & & 167 \\
\hline \multirow[t]{2}{*}{ Pitch type } & S: square & 165 \\
\hline & $\mathrm{H}$ : hexagonal & 8 \\
\hline Total & & 173 \\
\hline \multirow[t]{9}{*}{ Plate material } & $\mathrm{Al}:$ aluminum & 21 \\
\hline & B: Boral & 14 \\
\hline & BF: Boroflex & 2 \\
\hline & $\mathrm{CD}$ : cadmium & 6 \\
\hline & CU: copper & 8 \\
\hline & HF: hafnium & 4 \\
\hline & SS: stainless steel & 18 \\
\hline & UD: copper-cadmium & 4 \\
\hline & ZR: zircaloy & 3 \\
\hline Total & & 76 \\
\hline Reflecting wall & L: lead & 12 \\
\hline material & U: uranium & 10 \\
\hline & SS: stainless steel & 28 \\
\hline Total & & 50 \\
\hline
\end{tabular}




\section{Summary}

The detailed experiment descriptions in this report provide a useful compendium of criticality benchmark data for validation of LWR fuel transportation and storage applications. Experiments are categorized by common features such as separator plates, reflecting walls, and soluble boron and parameterized by key variables such as enrichment, water-to-fuel volume ratio, $\mathrm{H} / \mathrm{X}, \mathrm{AEF}$, and pitch. These data enable the analyst to readily identify appropriate experiments for a particular application.

Benchmark calculations have been performed for all of these cases using SCALE 4.3 and the 44-group ENDF/B-V cross-section library to provide a sample validation. In addition, the calculations were repeated with the 238-group library to determine the adequacy of the 44-group energy structure as shown in Appendix B. On average, the 238group results were approximately $0.35 \%$ less. The 238 -group results were more accurate for the homogeneous uranium critical experiments.

Two methods for statistical determination of calculational biases and USLs were presented: (1) a $95 \%$ confidence band for a single future calculation with an administrative subcritical margin and (2) a lower tolerance band (LTB) based on a $95 \%$ confidence band on $99.5 \%$ of all future calculations. The methods were demonstrated using several subsets of the 44-group validation results.

Finally, guidance was provided on the selection of appropriate criticality experiments that are applicable to validate a computational method for the system of concern. Important parameters that should be examined to demonstrate areas of applicability were discussed. The parameterization of the included experiments was described to assist the analyst in this process.

Hopefully, the large amount of experimental and calculational data compiled within this report along with the technical guidance provided will prove to be valuable assets for criticality safety analysts and reviewers of LWR fuel applications. Additional LWR-related experiments are available, however, as shown in Table 1.1. A user who may need experiment characterizations not identified in this report should investigate these experiments. 



\section{REFERENCES}

1. SCALE: A Modular Code System for Performing Standardized Computer Analyses for Licensing Evaluation, NUREG/CR-0200, Rev. 4 (ORNL/NUREG/CSD-2/R4), Vols. I, II, and III (April 1995). Available from Radiation Shielding Information Center as CCC-545.

2. M. D. DeHart and S. M. Bowman, Validation of the SCALE Broad Structure 44-Group ENDF/B-V CrossSection Library for Use in Criticality Safety Analyses, NUREG/CR-6102 (ORNL/TM-12460), U.S.Nuclear Regulatory Commission, September 1994.

3. N. M. Greene, J. W. Arwood, R. Q. Wright, and C. V. Parks, The LAW Library - A Multigroup Cross-Section Library for Use in Radioactive Waste Analysis Calculations, ORNL/TM-12370, Martin Marietta Energy Systems, Inc., Oak Ridge Natl. Lab., August 1994.

4. Directory of Certificates of Compliance for Radioactive Materials Packages, NUREG-0383, Vol. 2, Rev. 17, U.S. Nuclear Regulatory Commission, October 1994.

5. J. C. Manaranche, D. Mangin, L. Maubert, G. Colomb, and G. Poullot, "Dissolution and Storage Experimental Program with U[4.75]O $\mathrm{O}_{2}$ Rods," Trans. Am. Nucl. Soc. 33, 362-364 (November 1979).

6. M. N. Baldwin, G. S. Hoovler, R. L. Eng, and F. G. Welfare, Critical Experiments Supporting Close Proximity Water Storage of Power Reactor Fuel, BAW-1484-7, Babcock \& Wilcox Company, July 1979.

7. R. I. Smith and G. J. Konzek, Clean Critical Experiment Benchmarks for Plutonium Recycle in LWR's, Volume I, EPRI NP-196, Electric Power Research Institute, April 1976.

8. J. C. Manaranche, D. Mangin, L. Maubert, G. Colomb, and G. Poullot, "Critical Experiments with Lattices of 4.75-wt\%- ${ }^{235} U-$ Enriched $\mathrm{UO}_{2}$ Rods in Water," Nucl. Sci. Eng. 71, 154-163 (1979).

9. S. R. Bierman, E. D. Clayton, and B. M. Hurst, Critical Separation Between Subcritical Clusters of 2.35 wt $\%{ }^{235} \mathrm{U}$ Enriched $\mathrm{UO}_{2}$ Rods in Water with Fixed Neutron Poisons, PNL-2438, Pacific Northwest Laboratories, October 1977.

10. S. R. Bierman, B. M. Durst, and E. D. Clayton, Critical Experiments with Subcritical Clusters of 2.35 wt \% and 4.31 wt \% ${ }^{235} \mathrm{U}$ Enriched $\mathrm{UO}_{2}$ Rods in Water with Uranium or Lead Reflecting Walls, NUREG/CR0796, Vol. 1 (PNL-2827), U.S. Nuclear Regulatory Commission, April 1979.

11. S. R. Bierman and E. D. Clayton, Criticality Experiments with Subcritical Clusters of 2.35 wt \% and 4.31 $w t \%{ }^{235} U$ Enriched $\mathrm{UO}_{2}$ Rods in Water at a Water-to-Fuel Volume Ratio of 1.6, NUREG/CR-1547 (PNL3314), U.S. Nuclear Regulatory Commission, July 1980.

12. S. R. Bierman, B. M. Durst, and E. D. Clayton, Critical Experiments with Subcritical Clusters of 2.35 wt \% and 4.31 wt \% ${ }^{235} \mathrm{U}$ Enriched $\mathrm{UO}_{2}$ Rods in Water with Uranium or Lead Reflecting Walls, NUREG/CR0796, Vol. 2 (PNL-3926), U.S. Nuclear Regulatory Commission, December 1981.

13. B. M. Durst, S. R. Bierman, and E. D. Clayton, Critical Experiments with 4.31 wt \% ${ }^{235} U$ Enriched $U_{2}$ Rods in Highly Borated Water Lattices, NUREG/CR-2709 (PNL-4267), U.S. Nuclear Regulatory Commission, August 1982. 
References

14. S. R. Bierman, E. S. Murphy, E. D. Clayton, and R. T. Keay, Criticality Experiments with Low Enriched $\mathrm{UO}_{2}$ Fuel Rods in Water Containing Dissolved Gadolinium, PNL-4976, Pacific Northwest Laboratory, February 1984.

15. R. D. Leamer, D. F. Hanlen, G. N. Hamilton, and E. G. Taylor, Critical Experiments Performed with Clustered and Uniform Arrays of Rodded Absorbers, WCAP-3269-39, Westinghouse Electric Corp., November 1965.

16. E. G. Taylor, Saxton Plutonium Program, Critical Experiments for the Saxton Partial Plutonium Core, EURAEC-1493 (WCAP-3385-54), U.S. Atomic Energy Commission, December 1965.

17. G. S. Hoovler, M. N. Baldwin, E. L. Maceda, and F. G. Welfare, Critical Experiments Supporting Underwater Storage of Tightly Packed Configurations of Spent Fuel Pins, BAW-1645-4, Babcock \& Wilcox Company, November 1981.

18. “A.6 Problem Specifications for OECD Benchmarks with Hafnium-Poisoned Fuel Pin Assemblies: Problems 15, 16, 17, and 18," Standard Problem Exercise on Criticality Codes for Dissolving Fissile Oxides in Acids, G. E. Whitesides, Ed., NEACRP-L-306, pp. 121-133 (prepared by Oak Ridge National Laboratory), 1990.

19. Critical Separation Between Subcritical Clusters of 4.31 wt $\%{ }^{235} U$ Enriched $\mathrm{UO}_{2}$ Rods in Water with Fixed Neutron Poisons, NUREG/CR-0073 (PNL-2615), U.S. Nuclear Regulatory Commission, March 1978.

20. S. R. Bierman, Criticality Experiments to Provide Benchmark Data on Neutron Flux Traps, PNL-6205, Pacific Northwest Laboratory, June 1988.

21. S. R. Bierman, Criticality Experiments with Neutron Flux Traps Containing Voids, PNL-7167, Pacific Northwest Laboratory, April 1990.

22. S. R. Bierman and E. D. Clayton, Criticality Experiments with Subcritical Clusters of 2.35 wt \% and 4.31 wt $\%{ }^{235} \mathrm{U}$ Enriched $\mathrm{UO}_{2}$ Rods in Water with Steel Reflecting Walls, NUREG/CR-1784 (PNL-3602), U.S. Nuclear Regulatory Commission, April 1981.

23. D. Haon, J. C. Manaranche, D. Mangin, L. Maubert, and G. Poullot, "Validation of the Apollo-Moret Neutronic Codes on Critical Experimental Configurations simulating the Shipping Casks for Light Water Fuels," PATRAM'80 (1980).

24. L. W. Newman, Urania-Gadolinia: Nuclear Model Development and Critical Experiment Benchmark, DOE/ET/34212-41 (BAW-1810), Babcock \& Wilcox, April 1984.

25. T. C. Engelder et al., Spectral Shift Control Reactor Basic Physics Program,Critical Experiments on Lattices Moderated by $\mathrm{D}_{2} \mathrm{O}-\mathrm{H}_{2} \mathrm{O}$ Mixtures, BAW-1231, Babcock \& Wilcox Company, December 1961.

26. T. C. Engelder et al., Spectral Shift Control Reactor Basic Physics Program, Measurement and Analysis of Uniform Lattices of Slightly Enriched $\mathrm{UO}_{2}$ Moderated by $\mathrm{D}_{2} \mathrm{O}_{-} \mathrm{H}_{2} \mathrm{O}$ Mixtures, BAW-1273, Babcock \& Wilcox Company, November 1963. 
27. S. J. Raffety and J. T. Mihalczo, Homogenized Critical Assemblies of 2 and $3 \%$ Enriched Uranium in Paraffin, Y-DR-14, Union Carbide Corp., Nucl. Div., Oak Ridge Y-12 Plant, 1969.

28. G. Tuck and I. Oh, Benchmark Critical Experiments on Low-Enriched Uranium Oxide Systems with H/U= 0.77, NUREG/CR-0674, U.S. Nuclear Regulatory Commission, August 1979.

29. R. E. Rothe, I. Oh, and G. R. Goebel, Critical Experiments with Interstitially-Moderated Arrays of LowEnriched Uranium Oxide, NUREG/CR-1071 (RFP-3008), U.S. Nuclear Regulatory Commission, September 1980

30. G. R. Goebel, I. Oh, R. E. Rothe, and G. Tuck, Critical Experiments on Low-Enriched Uranium Oxide Systems with $H / U=1.25$, NUREG/CR-1653 (RFP-3129), U.S. Nuclear Regulatory Commission, October 1980.

31. R. E. Rothe and G. R. Goebel, Critical Experiments on Low-Enriched Uranium Oxide Systems with $H / U=$ 2.03, NUREG/CR-2500 (RFP-3277), February 1982.

32. N. M. Greene, "BONAMI-S: Resonance Self-Shielding by the Bondarenko Method," Sect. F1 of SCALE: $A$ Modular Code System for Performing Standardized Computer Analyses for Licensing Evaluation, NUREG/CR-0200, Rev. 4 (ORNL/NUREG/CSD-2/R4), Vols. I, II, and III (April 1995). Available from Radiation Shielding Information Center as CCC-545.

33. N. M. Greene, L. M. Petrie, and R. M. Westfall, "NITAWL-II: SCALE System Module for Performing Resonance Shielding and Working Library Production," Sect. F2 of SCALE: A Modular Code System for Performing Standardized Computer Analyses for Licensing Evaluation, NUREG/CR-0200, Rev. 4 (ORNL/NUREG/CSD-2/R4), Vols. I, II, and III (April 1995). Available from Radiation Shielding Information Center as CCC-545.

34. L. M. Petrie and N. F. Landers, "KENO V.A: An Improved Monte Carlo Criticality Program with Supergrouping," Sect. F11 of SCALE: A Modular Code System for Performing Standardized Computer Analyses for Licensing Evaluation, NUREG/CR-0200, Rev. 4 (ORNL/NUREG/CSD-2/R4), Vols. I, II, and III (April 1995). Available from Radiation Shielding Information Center as CCC-545.

35. N. M. Greene and L. M. Petrie, "XSDRNPM: A One-Dimensional Discrete-Ordinates Code for Transort Analysis," Sect. F3 of SCALE: A Modular Code System for Performing Standardized Computer Analyses for Licensing Evaluation, NUREG/CR-0200, Rev. 4 (ORNL/NUREG/CSD-2/R4), Vols. I, II, and III (April 1995). Available from Radiation Shielding Information Center as CCC-545.

36. G. R. Handley and C. M. Hopper, Validation Checks of the ANISN and KENO Codes by Correlation with Experimental Data, Y-1858, Union Carbide Corp., Nucl. Div., Oak Ridge Y-12 Plant, 1972.

37. W. C. Jordan et al., Validation of KENO V.a-Comparison with Critical Experiments, ORNL/CSD/TM-238, Martin Marietta Energy Systems, Inc., Oak Ridge Natl. Lab., December 1986.

38. American National Standard for Nuclear Criticality Safety Criteria for the Handling, Storage, and Transportation of LWR Fuel Outside Reactors, ANSI/ANS-8.17-1984 (Reaffirmed August 1989). 
References

39. American National Standard for Nuclear Criticality Safety in Operations with Fissionable Materials Outside Reactors, ANSIANS-8.1-1983. (Reaffirmed November 1988).

40. M. G. Natrella, see Table A-4. Percentiles of the $t$ Distribution, p. T-5 of "Experimental Statistics," National Bureau of Standards Handbook 91, August 1, 1963.

41. H. R. Dyer, W. C. Jordan, and V. R. Cain, "A Technique for Code Validation for Criticality Safety Calculations," Trans. Am. Nucl. Soc. 63, 238 (June 1991).

42. M. E. Easter, Validation of KENO V.a and Two Cross-Section Libraries for Criticality Calculations of Low-Enriched Uranium Systems, ORNL/CSD/TM-223, Martin Marietta Energy Systems, Inc., Oak Ridge Natl. Lab., July 1985.

43. D. C. Bowden and F. A. Graybill, "Confidence Bands of Uniform and Proportional Width for Linear Models," Am. Stat. Assoc. J. 61, 182 (March 1966).

44. $\quad$ N. L. Johnson, Ed., "Query," Technometrics 10, 207-209 (February 1968).

45. V. R. Cain, A Computer Code to Perform Analyses of Criticlaity Validation Results, Y/DD-574, Martin Marietta Energy Systems, Inc., Oak Ridge Y-12 Plant, September 1995.

46. Y. Nomura and T. Shimooke, "A Multiple Regression Equation for Calculated $\mathbf{k}_{\mathrm{eff}}$ Bias Errors by Criticality Code System," Nucl. Tech. 65, 340-349 (May 1984).

47. B. B. Bredehoft and R. D. Busch, "Characterization of the Thermalness of a Fissile System with a TwoGroup Diffusion Theory Parameter," Nucl. Tech. 102, 259-269 (May 1993).

48. AEA Technology UK - ANSWERS Service, "MONK6: A Monte Carlo Code for Criticality Calculations, ANSWERS/MONK6, Atomic Energy Establishment, Winfrith, Dorchester, Dorset, United Kingdom, 1995.

49. D. Rutherford, Appendix E - Forecast of Criticality Experiments and Experimental Programs Needed to Support Nuclear Operations in the United States of America: 1994-1999, LA-12683, Los Alamos Natl. Lab., July 1994.

50. C. V. Parks, H. R. Dyer, and G. E. Whitesides, “10. Criticality Safety," Packaging Handbook, ORNL/M5003, Lockheed Martin Energy Research Corp., Oak Ridge Natl. Lab., 1997. 


\section{APPENDIX A}

\section{SCALE Input File Listings}

This appendix contains input files for all cases discussed in Sect. 3. These files are also available in electronic form from the SCALE home page on the World Wide Web (WWW). A pointer for the SCALE home page may be found at http://www.cad.ornl.gov.

\section{INDEX OF CASES}

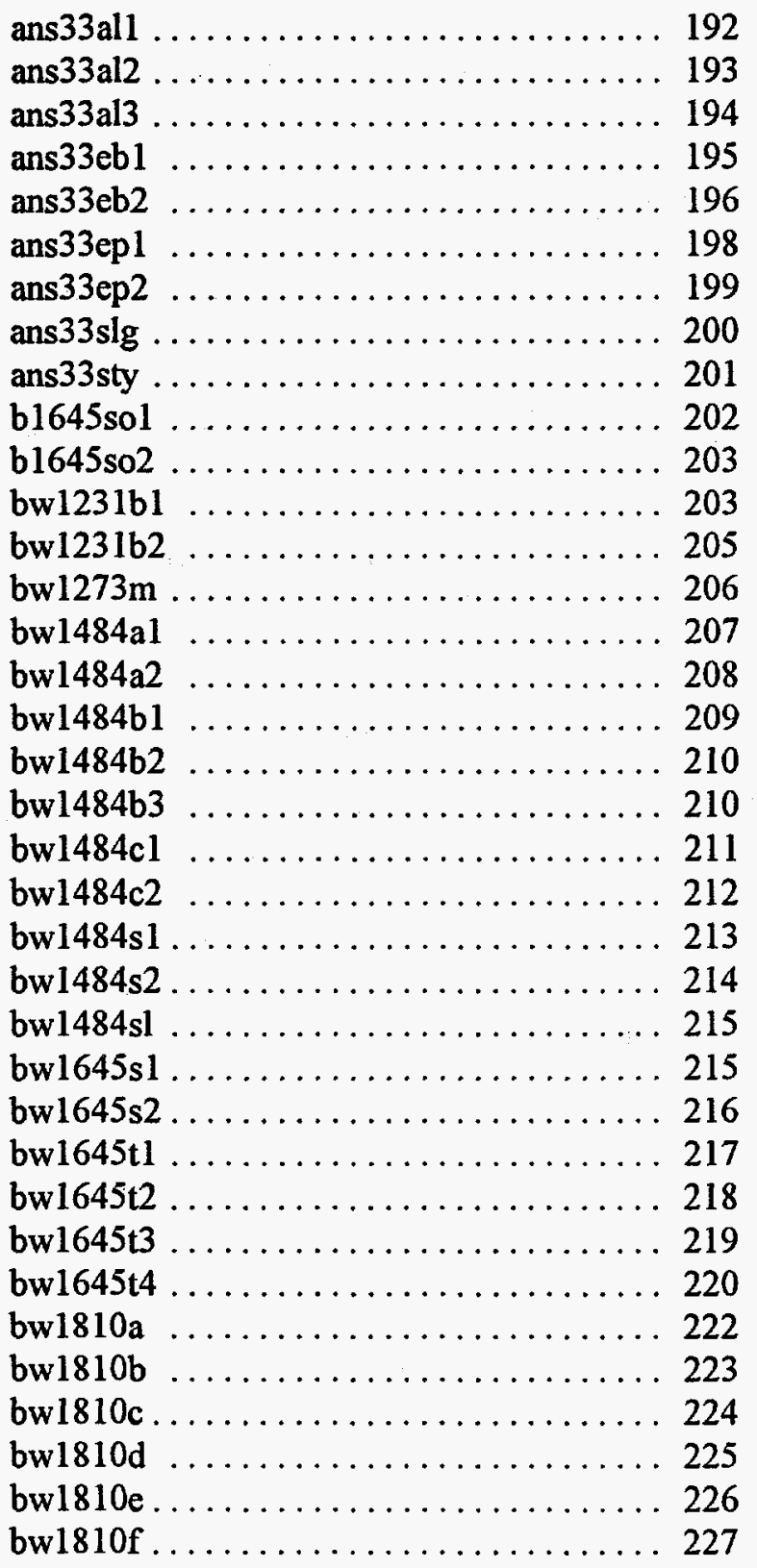

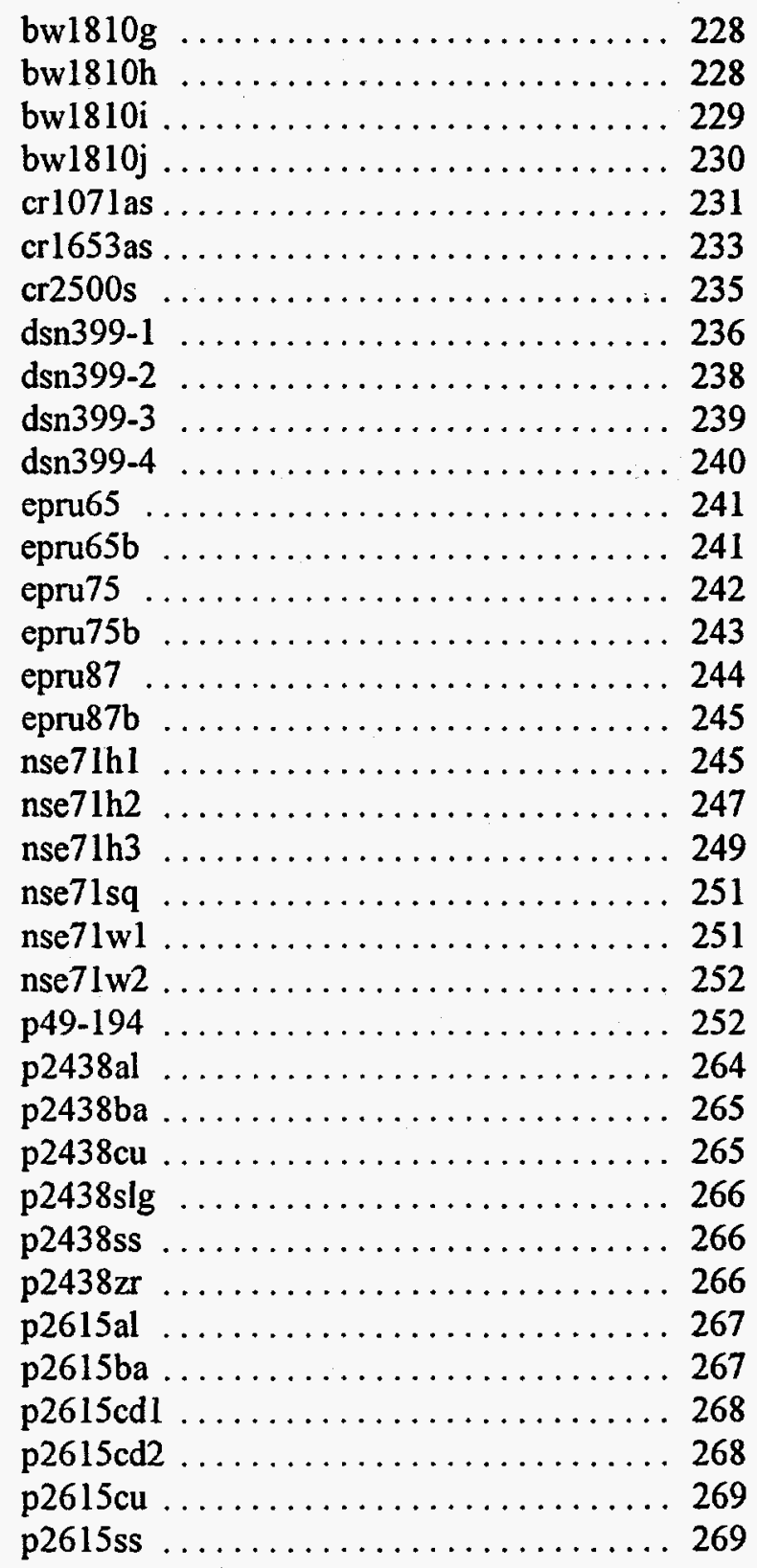


Appendix A

\begin{tabular}{|c|c|}
\hline p2615zr & 270 \\
\hline p282711 & 270 \\
\hline p282712 & 271 \\
\hline p282713 & 271 \\
\hline p282714 & 271 \\
\hline p2827slg & 272 \\
\hline p2827ul & 272 \\
\hline $\mathrm{p} 2827 \mathrm{u} 2$ & 273 \\
\hline p2827u3 & 273 \\
\hline $\mathrm{p} 2827 \mathrm{u} 4$ & 274 \\
\hline p3314al & 274 \\
\hline p3314ba & 275 \\
\hline p3314bc & 276 \\
\hline p3314bfl & 276 \\
\hline p3314bf2 & 277 \\
\hline p3314bs 1 & 278 \\
\hline p3314bs2 & 278 \\
\hline p3314bs3 & 279 \\
\hline p3314bs 4 & 279 \\
\hline p3314cdl & 280 \\
\hline p3314cd2 & 281 \\
\hline p3314cul & 281 \\
\hline p3314cu2 & 282 \\
\hline p3314cu3 & 282 \\
\hline p3314cu4 & 283 \\
\hline p3314cu5 & 283 \\
\hline p3314cu6 & 284 \\
\hline p3314slg & 284 \\
\hline p3314ss 1 & 285 \\
\hline p3314ss2 & 286 \\
\hline p3314ss3 & 286 \\
\hline p3314ss4 & 287 \\
\hline p3314ss5 & 288 \\
\hline p3314ss6 & 288 \\
\hline p3314w1 & 289 \\
\hline p3314w2 & 289 \\
\hline p3314zr & 290 \\
\hline p3602bb & 290 \\
\hline p3602bs 1 & 291 \\
\hline p3602bs 2 & 291 \\
\hline p3602cd1 & 292 \\
\hline p3602cd2 & 292 \\
\hline p3602cul & 293 \\
\hline p3602cu2 & 293 \\
\hline p3602cu3 & 294 \\
\hline
\end{tabular}

p3602cu4 .................... 294

p3602n11 .................... 295

p3602n12 .................... 295

p3602n13 ................... 296

p3602n14 ................... 296

p3602n21 .................... 297

p3602n22 ................... 297

p3602n31 ..................... 298

p3602n32 .................... 298

p3602n33 ....................... 299

p3602n34 ...................... 299

p3602n35 ...................... 300

p3602n36 ..................... 300

p3602n41 ..................... 300

p3602n42 ................... 301

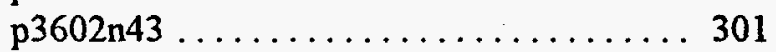

p3602ss1 ...................... 302

p3602ss2 .................. 302

p392611 ..................... 303

p392612 ................... 303

p392613 ................... 304

p392614 .................... 304

p392615 .................... 305

p392616 .................... 305

p3926sll .................. 306

p3926sl2 ................... 306

p3926ul ....................... 306

p3926u2 .................... 307

p3926u3 .................... 307

p3926u4 ...................... 308

p3926u5 ................... 308

p3926u6 .................... 309

p4267b1 .................. 309

p4267b2 .................. 310

p4267b3 ................... 310

p4267b4 ................... 311

p4267b5 ................. 312

p4267sll ................. 312

p4267sl2 ................. 313

p62ft $231 \ldots \ldots \ldots \ldots \ldots \ldots \ldots \ldots . \ldots . \ldots . \ldots$

p71f14f3 ................... 314

p71f14v3 ................... 315

p71f14v5 ................. 316

p71f214r ................ 317

pat8011 ................ 318

$\begin{array}{ll}\text { NUREG/CR-6361 } & 190\end{array}$ 
Appendix A

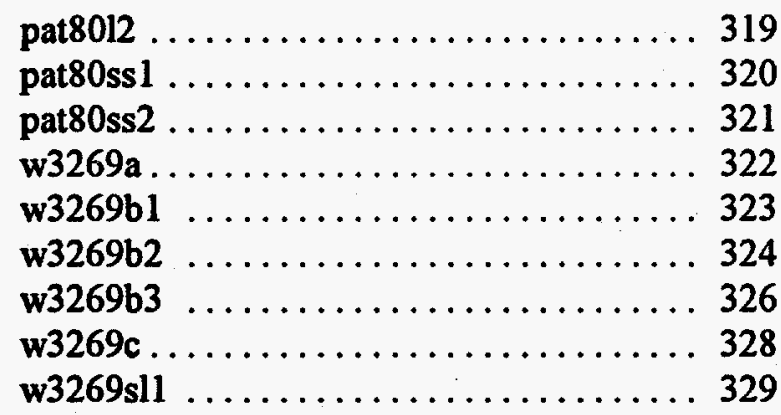

\begin{tabular}{|c|c|}
\hline w3269s12 & 329 \\
\hline $\mathrm{w} 3269 \mathrm{wl}$ & 330 \\
\hline $\mathrm{w} 3269 \mathrm{w} 2$ & 331 \\
\hline w3385sll & 331 \\
\hline w3385s 12 & 332 \\
\hline ydr14pl2 & 333 \\
\hline ydr14pl3 & 333 \\
\hline ydr14un2 & 333 \\
\hline ydr14un3 & 334 \\
\hline
\end{tabular}


ans 33 al1

\section{\#csas25}

ans33al 1

44 roup latticecell

vo2 $\quad 1$ den=10.38 1.0293922354 .7429223895 .258

arbmag5 $2.7040011302798 .8512000 \quad 0.5014000 \quad 0.43$ 260000.2221293

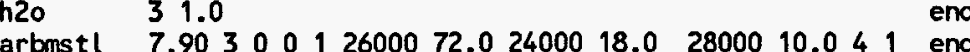

al 5 den=2.651 $1.0 \quad$ end

uo2 6 den=10.38 1.0293922354 .7429223895 .258 end

al $\quad 71.0$

end comp

$\begin{array}{llll}1.0 & & & \\ 1.0 & & \end{array}$

$\begin{array}{lllllllll}\text { squarepitch } & 1.35 & 0.79 & 1 & 3 & 0.94 & 2 & 0.82 & 0\end{array}$

more data res $=6 \mathrm{cyl} 0.395$ dan $(6)=0.989755$

$\mathrm{cm}$ of water, $25.66 \mathrm{~cm}$ water height

read parm run=yes gen $=405$ npg=600 nsk $=5$ nub=yes end parm

read geom

unit 1

$\begin{array}{lllll}\text { cylinder } & 71 & .470 & 0.0 & -1.5\end{array}$

cuboid 31 4p.675 $0.0 \quad-1.55$

6

reflector $415 \mathrm{ro} \quad 0.4$

cylinder $\begin{array}{lllll}7 & 1 & .470 & 0.0 & -0.25\end{array}$

cylinder $31.500 \quad 0.0 \quad-0.25$

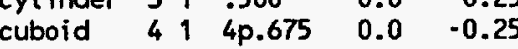

unit 3

$\begin{array}{llllll}\text { cylinder } & 1 & 1 & .395 & 25.66 & 0.0\end{array}$

cylinder $\begin{array}{llllll}0 & 1 & .410 & 25.66 & 0.0\end{array}$

cylinder $21 \quad 470 \quad 25.66 \quad 0.0$

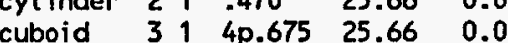

cuit 4

array $13 * 0.0$

reflector $3110.0 \quad 0.3 \quad 0.0 \quad 0.3 \quad 2 r 0$ $\begin{array}{lllllllll} & \text { reflector } 5 & 1 & 0.3 & 0.0 & 0.3 & 0.0 & 2 r 0 & 1\end{array}$

unit 5

array $13 * 0.0$

reflector 310.30 .00 .00 .32 r 01

reflector $51 \quad 0.0 \quad 0.3 \quad .30 .02 \mathrm{r}^{\circ} 1$

unit 6

array $13 * 0.0$

$\begin{array}{llllllll}\text { reflector } 3 & 1 & 0.0 & 0.3 & 0.3 & 0.0 & 2 r 0 & 1\end{array}$ reflector $510.30 .0 \quad 0.0 \quad 0.32$ ro 1

unit 7

array $13 * 0.0$

$\begin{array}{lllllllll}\text { array } 1 & 3 * 0.0 & & 0.0 & & \end{array}$

reflector 51 o.0 $0.3 \quad 0.0 \quad 0.32$ rol

unit 8 $\begin{array}{lllllll}\text { cuboid } \quad 31 & 2 \mathrm{p} 0.95 & 2 \mathrm{p} 12.30 & 27.56 & 0.0\end{array}$

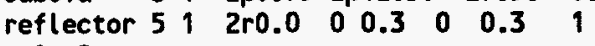

unit 9

$\begin{array}{llllllll}\text { cuboid } & 31 & 2 \mathrm{p} 12.30 & 2 \mathrm{p} 0.95 & 27.56 & 0.0\end{array}$

$\begin{array}{lllllll}\text { reflector } 5 & 1 & 0 & 0.3 & 2 \mathrm{r} 0.0 & 0 & 0.3\end{array}$

unit 10

cuboid $\begin{array}{llllll}3 & 1 & 2 \mathrm{p} 0.95 & 2 \mathrm{p} 0.95 & 27.56 & 0.0\end{array}$

$\begin{array}{llll}\text { reflector } 51 & 5 \mathrm{r0} .0 & 0.35 & 1\end{array}$

unit 11

$\begin{array}{llllll}\text { cuboid } & 31 & 2 \mathrm{p} 12.30 & 2 \mathrm{p} 0.95 & 27.56 & 0.0\end{array}$

reflector $51.0 .3 \quad 0 \quad 2 r 0.0 \quad 0 \quad 0.3 \quad 1$

unit 12

cuboid $31 \quad 2 \mathrm{p} 0.95 \quad 2 \mathrm{p} 12.30 \quad 27.56 \quad 0.0$

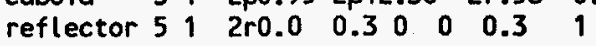

unit 13

$\begin{array}{llllll}\text { cylinder } & 6 & 1 & .395 & 90.00 & 25.66\end{array}$

$\begin{array}{llllll}\text { cylinder } & 0 & 1 & .410 & 96.9 & 25.66\end{array}$

cylinder $\begin{array}{lllll}2 & 1 & .470 & 98.2 & 25.66\end{array}$

cuboid $0140.675 \quad 98.2$

unit 14

array $2 \quad 3 * 0.0$

reflector $0110.0 \quad 0.3 \quad 0.0 \quad 0.32 r 0$

reflector $511 \quad 0.3 \quad 0.0 \quad 0.3 \quad 0.0 \quad 2$ ro 1

unit 15

array $2 \quad 3 * 0.0$

reflector $\begin{array}{lllllllll}0 & 1 & 0.3 & 0.0 & 0.0 & 0.3 & 2 r 0\end{array}$

$\begin{array}{lllllllll}\text { reflector } 5 & 1 & 0.0 & 0.3 & 3 & 0.0 & 2 r 0 & 1\end{array}$

unit 16

array $2 \cdot 3 * 0.0$

$\begin{array}{lllllllllll}\text { reflector } 0 & 1 & 0.0 & 0.3 & .3 & 0.0 & 2 r 0 & 1\end{array}$

$\begin{array}{llllllll}\text { reflector } 5 & 1 & 0.3 & 0.0 & 0.0 & 0.3 & 2 r 0 & 1\end{array}$

unit 17

array $2 \quad 3 * 0.0$

reflector $0910.30 .00 .3 \quad 0.02$ ro

reflector 510.00 .30 .00 .32 ro

unit 18

$\begin{array}{llllll}\text { cuboid } \quad 0 & 1 & 2 \mathrm{p} 0.95 & 2 \mathrm{p} 12.30 & 98.2 & 25.66\end{array}$ reflector 51 2ro.0 $\begin{array}{llllll}0.3 & 2 r 0 & 1\end{array}$

unit 19

$\begin{array}{lllllll}\text { cuboid } & 0 & 1 & 2 \mathrm{p} 12.30 & 2 \mathrm{p} 0.95 & 98.2 & 25.66\end{array}$

reflector $\begin{array}{lllllll}5 & 1 & 0 & 0.3 & 2 \mathrm{r} 0.0 & 2 \mathrm{r} 0 & 1\end{array}$

unit 20

cuboid $0112 \mathrm{p} 0.95 \quad 2 \mathrm{p} 0.95 \quad 98.2 \quad 25.66$

reflector 51 4ro.0 $2 \mathrm{rO}_{1}$

unit 21

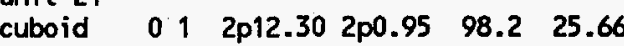

reflector $51 \quad 0.300$ 2ro.0 2 ro 1

unit 22

cuboid $012 p 0.95 \quad 2 p 12.30 \quad 98.2 \quad 25.66$

reflector 51 2ro.0 $\begin{array}{llllll}0.3 & 0 & 2 \mathrm{rO}_{0} & 1\end{array}$

unit 23 
array $33 * 0.0$

reflector 314 r20 $2 r 01$

reflector 415 ro .81

reflector 315 ro 201

unit 24

array $4 \quad 3 * 0.0$

reflector 014 r $20 \quad 2 r 01$

global unit 25

array $5 \quad 3 * 0.0$

end geom

\section{read array}

ara=1 nux =18 nuy=18 nuz=3 fill $324 r 1324 r 2324 \mathrm{r}^{3}$

ara $=2$ nux $=18$ nuy=18 nuz=1 fill $324 r 13$

ara $=3$ nux=3 nuy=3 nuz=1 fill 4855910116127 ara $=5$ nux $=1$ nuy $=1$ nuz=2 fill $2324 \quad$ end fill end array

end

ans33al2

\#csas25

ans 33 al2

4 group latticecel

uo2 1 den=10.38 1.0293922354 .7429223895 .258 end

$\begin{array}{llllllllllll}\text { arbmag5 } 2.7040 & 0 & 1 & 1302798.85 & 12000 & 0.50 & 14000 & 0.4326000\end{array}$ 0.2221293

h20 $\quad 31.0$

end

7.0030012600072 .02400018 .02800010 .041 end

al 5 den=2.651 1.0

uo2 6 den=10.38 1.0293922354 .7429223895 .258

at 71.0

end comp

$\begin{array}{lllllllll}\text { squarepitch } & 1.35 & 0.79 & 1 & 3 & 0.94 & 2 & 0.82 & 0\end{array}$

more data res $=6 \mathrm{cyl} 0.395$ dan $(6)=0.989755$

4-18x18 assemblies separated by $0.3-\mathrm{cm}$-thick aluminum plates and 4.4

$\mathrm{cm}$ of water, $32.78 \mathrm{~cm}$ water height

read parm run=yes gen $=405$ npg $=600$ nsk $=5$ nub=yes end parm

read geom

unit 1

$\begin{array}{lllll}\text { cylinder } & 71 & .470 & 0.0 & -1.55\end{array}$

$\begin{array}{lllll}\text { cuboid } \quad 31 & 4 p .675 & 0.0 & -1.55\end{array}$

reflector $415 \mathrm{r} 0.0 \quad 0.4$

unit 2

cylinder $71.470 \quad 0.0 \quad-0.25$

cylinder $31 \quad .500 \quad 0.0 \quad-0.25$

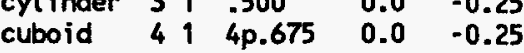

unit 3 cyl inder $1110.395 \quad 32.78 \quad 0.0$

$\begin{array}{llllll}\text { cylinder } & 0 & 1 & .410 & 32.78 & 0.0\end{array}$

$\begin{array}{lllll}\text { cylinder } 2 & 1 & .470 & 32.78 & 0.0\end{array}$

$\begin{array}{lllll}\text { cuboid } & 31 & 4 p .675 & 32.78 & 0.0\end{array}$

unit 4

array $1 \quad 3 * 0.0$

reflector $310.00 .3 \quad 0.00 .32 r 01$

$\begin{array}{lllllllllll}\text { reflector } 5 & 1 & 0.3 & 0.0 & 0.3 & 0.0 & 2 r 0 & 1\end{array}$

unit 5

array $1 \quad 3 * 0.0$

$\begin{array}{llllllllll}\text { reflector } 31 & 0.3 & 0.0 & 0.0 & 0.32 \mathrm{r} 0 & 1\end{array}$ $\begin{array}{lllllll}\text { reflector } 5 & 1 & 0.0 & 0.3 & .3 & 0.0 & 2 r 0\end{array}$

unit 6

array $1 \quad 3 * 0.0$

$\begin{array}{llllllllll}\text { reflector } 3 & 1 & 0.0 & 0.3 & .3 & 0.0 & 2 \mathrm{r} 0\end{array}$

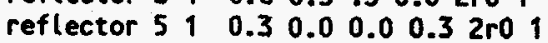

unit 7

array $1 \quad 3 * 0.0$

$\begin{array}{lllllllll} & \text { reflector } 31 & 0.3 & 0.0 & 0.3 & 0.0 & 2 r 0 & 1\end{array}$

reflector $51 \quad 0.0 \quad 0.3 \quad 0.0 \quad 0.32 r 01$

unit 8

$\begin{array}{lllllll}\text { cuboid } 3 \text { I } & 2 p 2.2 & 2 p 12.30 & 34.68 & 0.0\end{array}$

reflector 5 i $2 \mathrm{r} 0.0 \quad 00.3 \quad 0 \quad 0.3 \quad 1$

unit 9

$\begin{array}{llllll}\text { cuboid } 31 & 2 \mathrm{p} 12.30 & 2 \mathrm{p} 2.2 & 34.68 & 0.0\end{array}$

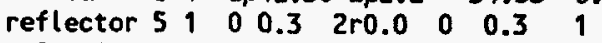

unit 10

$\begin{array}{lllllll}\text { cuboid } \quad 3 & 1 & 2 \mathrm{p} 2.2 & 2 \mathrm{p} 2.2 & 34.68 & 0.0\end{array}$

reflector 51 5r0.0 131

unit 11

$\begin{array}{llllllll}\text { cuboid } & 3 & 1 & 2 \mathrm{p} 12.30 & 2 \mathrm{p} 2.2 & 34.68 & 0.0\end{array}$

reflector $\begin{array}{llllllll}5 & 1 & 0.3 & 0 & 2 r 0.0 & 0 & 0.3 & 1\end{array}$

unit 12

$\begin{array}{lllllll}\text { cuboid } & 3 & 1 & 2 \mathrm{p} 2.2 & 2 \mathrm{p} 12.30 & 34.68 & 0.0\end{array}$

reflector 511 2ro.0 0.30000 .3 .1

unit 13

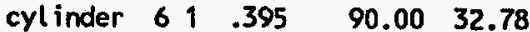

cylinder $0, .410 \quad 96.9 \quad 32.78$

cylinder $21 \quad .470 \quad 98.2 \quad 32.78$

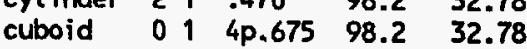

unit 14

array $2 \quad 3 * 0.0$

reflector $0110.0 \quad 0.3 \quad 0.0 \quad 0.3 \quad 2 r 01$

reflector $5110.3 \quad 0.0 \quad 0.3 \quad 0.02 \mathrm{r} 01$

unit 15

array $2 \quad 3 * 0.0$

reflector 0110.30 .00 .00 .3250

reflector $5110.0 \quad 0.3 \quad .30 .02 r_{0} 1$

unit 16

array $2 \quad 3 * 0.0$

reflector $011 \quad 0.0 \quad 0.3 .3 \quad 0.02 r 01$ 
reflector $51 \quad 0.3 \quad 0.0 \quad 0.0 \quad 0.32 r 0$ ? unit 17

array $2 \quad 3 * 0.0$

reflector $0110.3 \quad 0.00 .3 \quad 0.0220$

reflector $5110.0 \quad 0.3 \quad 0.0 \quad 0.32 r 01$

unit 18

cuboid $\begin{array}{llllll}0 & 1 & 2 \mathrm{p} 2.2 & 2 \mathrm{p} 12.30 & 98.2 & 32.78\end{array}$

reflector 511 2ro.0 $\quad 0 \quad 0.3 \quad 2 r 0 \quad 1$

unit 19

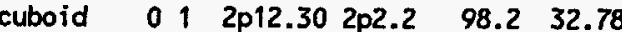

$\begin{array}{lllllll}\text { reflector } 5 & 1 & 0 & 0.3 & 2 r 0.0 & 2 r 0 & 1\end{array}$

unit 20

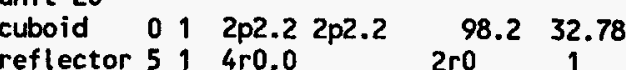

reflector

unit 21

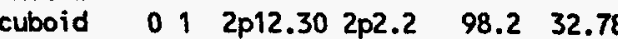

reflector 5110.300 2ro.0 2 ro

unit 22

cuboid $\begin{array}{llllll}0 & 1 & 2 \mathrm{p} 2.2 & 2 \mathrm{p} 12.30 & 98.2 & 32.78\end{array}$

reflector $5,2 \mathrm{rO} 000.30$ 2r0

unit 23

array 3 3*0.0

reflector 314 r20 2ro1

reflector 41 5ro .8

reflector $315 r_{0} \quad 20$

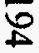

unit 24

array $4 \quad 3 * 0.0$

reflector 014 r20 2ro

global unit 25

array $5 \quad 3 * 0.0$

end geom

read array

ara=1 nux =18 nuy=18 nuz=3 fill 324r1 324r2 324r3

end $\mathrm{fill}$

ara $=2$ nux $=18$ nuy $=18$ nuz $=1$ fill 324 r 13

end fill

ara $=3$ nux $=3$ nuy $=3$ nuz=1 fill 485910116127 end fill

ara $=4$ nux=3 nuy=3 nuz=1 fill $14 \quad 18 \quad 15 \quad 1920 \quad 21 \quad 16 \quad 22 \quad 17$ end fill

ara $=5$ nux=1 nuy $=1$ nuz=2 fill $2324 \quad$ end fill

end array

end

\section{ans 33 al3}

\section{\#cses25}

ans33al3

44group latticecell

vo2 1 den=10.38 1.0293922354 .7429223895 .258

arbmag5 $2.7040011302798 .8512000 \quad 0.5014000 \quad 0.43 \quad 26000$

h20 0.2221293 end arbmstl 7.9030012600072 .002400018 .002800010 .041 end al 5 den $=2.6511 .0$

uo2 6 den=10.38 1.0293922354 .7429223895 .258

71.0

end comp

$\begin{array}{lllllllll}\text { squarepitch } & 1.35 & 0.79 & 1 & 3 & 0.94 & 2 & 0.82 & 0\end{array}$

more data res $=6$ cyl 0.395 dan $(6)=0.989755$

(18x18 assemblies separated by $0.3-\mathrm{cm}$ - thick aluminum plates and 9.4

$\mathrm{cm}$ of water, $64.12 \mathrm{~cm}$ water height

read parm run=yes gen $=405$ npg= 600 nsk $=5$ nub=yes end parm

read geom

unit 1

$\begin{array}{llllll}\text { cylinder } & 7 & 1 & .470 & 0.0 & -1.55\end{array}$

cuboid $31 \quad 4 p .675 \quad 0.0 \quad-1.55$

reflector 41 5ro $0.4 \quad 1$

unit 2

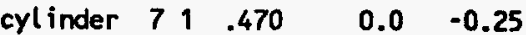

cylinder $31 \% \begin{array}{llll}3 & .500 & 0.0 & -0.25\end{array}$

$\begin{array}{lllll}\text { cuboid } & 41 & 4 \mathrm{p} .675 & 0.0 & -0.25\end{array}$

unit 3

$\begin{array}{llllll}\text { cylinder } & 1 & 1 & .395 & 64.12 & 0.0\end{array}$

cylinder $\begin{array}{llllll}0 & 1 & .410 & 64.12 & 0.0\end{array}$

cylinder $211 \quad .470 \quad 64.12 \quad 0.0$

cuboid $31 \quad 40.675 \quad 64.12 \quad 0.0$

unit 4

array $1 \quad 3 * 0.0$

reflector $3110.0 \quad 0.3 \quad 0.00 .32 r 01$

reflector $5110.3 \quad 0.0 \quad 0.3 \quad 0.0$ 2ro 1

unit 5

array $1 \quad 3 * 0.0$

reflector $31 \quad 0.3 \quad 0.0 \quad 0.00 .32 r 01$

reflector $51 \quad 0.0 \quad 0.3 .3 \quad 0.02 \mathrm{ro} 9$

unit 6

array $13 * 0.0$

reflector $390.0 \quad 0.3 .3 \quad 0.02001$

reflector $51 \quad 0.3 \quad 0.0 \quad 0.0 \quad 0.32 r_{0} 1$

unit 7

array $13 * 0.0$

reflector $310.30 .0 \quad 0.30 .02801$

reflector $51 \quad 0.0 \quad 0.3 \quad 0.0 \quad 0.32 \mathrm{rO} 1$

unit 8

$\begin{array}{lllllllll}\text { cuboid } & 3 & 1 & 2 p 4.7 & 2 p 12.30 & 66.02 & 0.0\end{array}$

reflector 511 2r0.0 $\begin{array}{lllllll}0 & 0.3 & 0 & 0.3 & 1\end{array}$

unit 9

$\begin{array}{lllllll}\text { cuboid } & 3 & 1 & 2 p 12.30 & 2 p 4.7 & 66.02 & 0.0\end{array}$

reflector $51100.3 \quad 2 r 0.0 \quad 0 \quad 0.3 \quad 1$

unit 10

$\begin{array}{lllllll}\text { cuboid } \quad 3 & 1 & 2 p 4.7 & 2 p 4.7 & 66.02 & 0.0\end{array}$

reflector 51 5r0.0 $\quad 0.3 \quad 1$

unit 11 
$\begin{array}{lllllllll}\text { cuboid } \quad 3 & 1 & 2 p 12.30 & 2 p 4.7 & 66.02 & 0.0\end{array}$ $\begin{array}{llllllll}\text { reflector } 5 & 1 & 0.3 & 0 & 2 r 0.0 & 0 & 0.3 & 1\end{array}$ unit 12

cuboid $312 p 4.72 p 12.30 \quad 66.02 \quad 0.0$ reflector 51 2r0.0 $0.3 \quad 0 \quad 00.3 \quad 1$

unit 13

cylinder $61.395 \quad 90.0064 .12$

cylinder $011.410 \quad 96.964 .12$

cylinder $21.470 \quad 98.264 .12$

$\begin{array}{llllll}\text { cuboid } & 0 & 1 & 4 p .675 & 98.2 & 64.12\end{array}$

unit 14

array $2 \quad 3 * 0.0$

reflector $0110.00 .30 .00 .32 r 01$

reflector $5,0.30 .00 .30 .02 \mathrm{r} 01$

unit 15

array $2 \quad 3 * 0.0$

reflector $0110.3 \quad 0.0 \quad 0.0 \quad 0.32 r 0$

reflector $51 \quad 0.0 \quad 0.3 \quad .3 \quad 0.0 \quad 2 r 01$

unit 16

array $2 \quad 3 * 0.0$

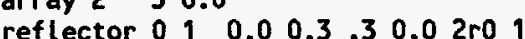

reflector $510.30 .00 .00 .32 \mathrm{ro}$

unit 17

array $2 \quad 3 * 0.0$

$\begin{array}{llllllll}\text { reflector } 0 & 1 & 0.3 & 0.0 & 0.3 & 0.0 & 2 r 0\end{array}$

reflector $5110.0 \quad 0.3 \quad 0.0 \quad 0.32 r 0$

$\begin{array}{llllll}\text { cuboid } \quad 0 & 1 & 2 p 4.7 & 2 p 12.30 & 98.2 & 64.12\end{array}$

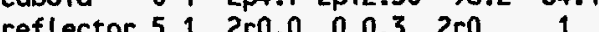

reflector

$\begin{array}{lllllll}\text { cuboid } & 0 & 1 & 2 \mathrm{p} 12.30 & 2 \mathrm{p} 4.7 & 98.2 & 64.12\end{array}$

reflector $5 \begin{array}{lllllll}1 & 0 & 0.3 & 2 r 0.0 & 2 r 0 & 1\end{array}$

unit 20

$\begin{array}{llllll}\text { cuboid } \quad 0 & 1 & 2 p 4.7 & 2 p 4.7 & 98.2 & 64.12\end{array}$

reflector $5,4 \mathrm{r0.0} \quad 2 \mathrm{ro} \quad 1$

reflector

$\begin{array}{lllllll}\text { cuboid } & 0 & 1 & 2 p 12.30 & 2 p 4.7 & 98.2 & 64.12\end{array}$

$\begin{array}{llllll}\text { reflector } 5 & 1 & 0.3 & 0 & 2 r 0.0 & 2 r 0\end{array}$

unit 22

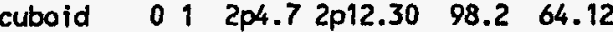

reflector 511 2r0.0 0.300 2ro

unit 23

array $33 * 0.0$

reflector 314 r20 2ro 1

reflector 415 ro $\quad .8$

reflector 315 ro 201

unit 24

array $4 \quad 3 * 0.0$

reflector 014 r $20 \quad 2$ ro 1

global unit 25 array $5 \quad 3 * 0.0$

end geom

read array

ara=1 nux=18 nuy=18 nuz=3 fill 324r1 324r2 324r3 end fill

ara=2 nux=18 nuy=18 nuz=1 fill $324 r 13$ end fill

ara $=3$ nux $=3$ nuy $=3$ nuz=1 fill 485910116127 and fill

$a r a=4$ nux $=3$ ney $=3$ nuz=1 fill $14 \quad 18 \quad 15 \quad 192021 \quad 16 \quad 22 \quad 17$ end fill ara $=5$ nux=1 nuy=1 nuz=2 fill $2324 \quad$ end fill

end array

end data

end

\section{ens33ebl}

\#csas25

ans33eb1

44group latticecel

uo2 I den=10.38 1.0 293922354.7429223895 .258 end

$\begin{array}{llllllllllll}\text { arbmag5 } & 2.7040 & 0 & 1 & 1302798.85 & 12000 & 0.5014000 & 0.4326000\end{array}$

0.2221293 end

h20 31.0 and

arbmstl $\quad 7.9030012600072 .02400018 .02800010 .041$ end

polyethylene 5 den=0.5540 1.0 end

uo2 6 den=10.381.029392235 4.7429223895 .258 end

al 7 den=2.651 $1.0 \quad$ end

end comp

$\begin{array}{llllllllll}\text { squarepitch } & 1.35 & 0.79 & 1 & 3 & 0.94 & 2 & 0.82 & 0 & \text { end }\end{array}$

end

more data res $=6$ cyl 0.395 assemblies separated by $0.3-\mathrm{cm}-$ thick aluminum plates and 1.9

' $\mathrm{cm}$ of polyethylene balls, $25.54 \mathrm{~cm}$ water height

read parm run=yes gen $=405$ npg $=600$ nsk $=5$ nub=yes end parm

read geom

unit

cylinder $81, .470 \quad 0.0 \quad-1.55$

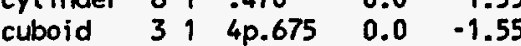

reflector 41 5ro 0.4

unit 2

cylinder $8 \begin{array}{lllll} & 1 & .470 & 0.0 & -0.25\end{array}$

cylinder $3 \quad 1 \quad .500 \quad 0.0 \quad-0.25$

$4140.675 \quad 0.0=0.25$

unit 3

$\begin{array}{llllll}\text { cylinder } & 1 & 1 & .395 & 25.54 & 0.0\end{array}$

cylinder $\begin{array}{lllll}0 & 1 & .410 & 25.54 & 0.0\end{array}$

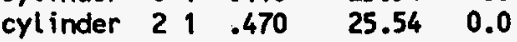

$\begin{array}{llllll}\text { cuboid } & 3 & 1 & 4 p .675 & 25.54 & 0.0\end{array}$

unit 4

array $13 * 0.0$ 
reflector $31 \quad 0.0 \quad 0.3 \quad 0.0 \quad 0.3 \quad 2 r 01$ reflector $7, \quad 0.3 \quad 0.0 \quad 0.3 \quad 0.02201$ unit 5

array $13 * 0.0$

reflector $31 \quad 0.3 \quad 0.0 \quad 0.0 \quad 0.3 \quad 2 r 01$ $\begin{array}{llllllll}\text { reflector } 71 & 0.0 & 0.3 & .3 & 0.0 & 2 r 0 & 1\end{array}$

unit 6

array $1 \quad 3 * 0.0$

$\begin{array}{lllllllll}\text { reflector } 3 & 1 & 0.0 & 0.3 & .3 & 0.0 & 2 \mathrm{r} 0 & 1\end{array}$

reflector 7 i $0.3 \quad 0.0 \quad 0.0 \quad 0.3 \quad 2$ ro

unit 7

array $1 \quad 3 * 0.0$

reflector $3110.3 \quad 0.0 \quad 0.3 \quad 0.02 r 01$

reflector $710.0 \quad 0.3 \quad 0.0 \quad 0.3 \quad 2 r 01$

unit 8

$\begin{array}{llllll}5 & 1 & 2 \mathrm{p} 0.95 & 2 \mathrm{p} 12.30 & 27.44 & 0.0\end{array}$

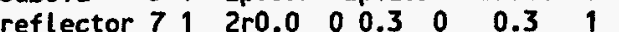

unit 9

cuboid $51 \quad 2 p 12.302 p 0.95 \quad 27.44 \quad 0.0$

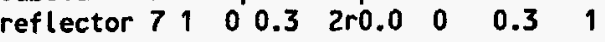

unit 10

$\begin{array}{lllll}\text { cuboid } 51 & 2 \mathrm{p} 0.95 & 2 \mathrm{p} 0.95 & 27.44 & 0.0\end{array}$

$\begin{array}{llll}\text { reflector } 71 & 5 \mathrm{ro} 0 & 0.31\end{array}$

unit 11

$\begin{array}{llllll}\text { cuboid } 5 & 5 & 2 p 12.30 & 2 p 0.95 & 27.44 & 0.0\end{array}$

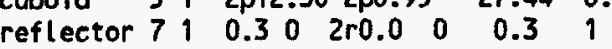

unit 12

$\begin{array}{llllll}\text { cuboid } \quad 51 & 2 \mathrm{p} 0.95 & 2 \mathrm{p} 12.30 & 27.44 & 0.0\end{array}$

reflector 71 2ro.0 0.30000 .31

unit 13

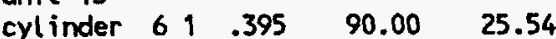

cylinder $0011.410 \quad 96.9 \quad 25.54$

cylinder $21 \quad .470 \quad 98.2 \quad 25.54$

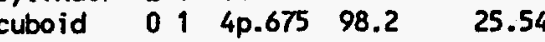

unit 14

array $2 \quad 3 * 0.0$

reflector $0110.0 \quad 0.3 \quad 0.0 \quad 0.3 \quad 2 \mathrm{r} 01$

reflector $7,0.3 \quad 0.0 \quad 0.3 \quad 0.02 \mathrm{ro}$

unit 15

array $2 \quad 3 * 0.0$

reflector $0110.3 \quad 0.0 \quad 0.0 \quad 0.32 r 0$

reflector $71 \quad 0.0 \quad 0.3 .3 \quad 0.022 r 01$

unit 16

array $2 \quad 3 * 0.0$

reflector $010.00 .3 .3 \quad 0.0250$

reflector $710.3 \quad 0.0 \quad 0.00 .32$ ro

unit 17

array $2 \quad 3 * 0.0$

reflector $0110.3 \quad 0.0 \quad 0.3 \quad 0.02$ ro 1

reflector $710.0 \quad 0.3 \quad 0.0 \quad 0.32 r_{0} \quad 1$

unit 18 cuboid $\quad 01 \quad 2 p 0.95 \quad 2 p 12.30 \quad 98.2 \quad 25.54$

reflector 71 2r0.0 $00.3 \quad 2 r$

unit 19

cuboid $0112 \mathrm{p} 12.30 \quad 2 \mathrm{p} 0.95 \quad 98.2 \quad 25.54$

$\begin{array}{lllllll}\text { reflector } 71 & 0 & 0.3 & 2 \mathrm{r0} 0.0 & 2 \mathrm{rO} & 1\end{array}$

unit 20

cuboid $0112 \mathrm{p0} 0.952 \mathrm{po.95} \quad 98.2 \quad 25.54$

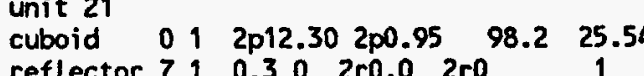

reflector $790.3002 r 0.02$

unit 22

cuboid $\begin{array}{llllll}0 & 1 & 2 p 0.95 & 2 p 12.30 & 98.2 & 25.54\end{array}$

reflector $7,2 r 0.0 \quad 0.302 r 0$

unit 23

array $3 \quad 3 * 0.0$

reflector 3 i 4 r2o 2 ro 1

reflector 4 i 5 ro .8

reflector 315 ro 201

unit 24

array $4 \cdot 3 * 0.0$

reflector 0 i $4 r 20$ 2ro 1

global unit 25

array $5 \quad 3 * 0.0$

end geom

read array

ara=1 nux=18 nuy=18 nuz=3 fill $324 r 1324 r 2324 r 3$ end fill ara $=2$ nux $=18$ nuy=18 nuz=1 fill $324 r 13$.

ara=3 nux=3 nuy=3 nuz=1 fill 485910116127 end fill ara=4 nux=3 nuy=3 nuz=1 fill $14 \quad 18 \quad 15 \quad 192021 \quad 1622 \quad 17$ end fill ara $=5$ nux=1 nuy=1 nuz=2 fill 2324 end fill end array

end data

end

ans33eb?

\#csas 25

ans33eb2

44group latticecell

Uo2 $\quad 1$ den=10.38 1.0293922354 .7429223895 .258

arbmag5 $\quad 2.7040011302798 .85120000 .50140000 .43$

h20 26000

arbmetl 7.0030012600072 .02400018 .02800010 .041 end

polyethylene 5 den=0.5796 1.0

polyethylene 5 den=0.5796 $1.0 \quad 029392354.7429223895 .258$ end

$\begin{array}{lllll}\text { uo2 } & 6 \text { den=10.38 } 1.0293922354 .7429223895 .258 & \text { end } \\ \text { al } & 7 \text { den=2.651 } 1.0 & \text { end }\end{array}$

at $81.0 \quad$ end

end comp end 
squarepitch $1.35 \quad 0.79 \quad 130.94 \quad 2 \quad 0.82 \quad 0$

more data res $=6$ cyl 0.395 dan $(6)=0.989755$

end

4-18x18 assemblies separated by $0.3-\mathrm{cm}$ - thick aluminum plates and 4.4

$\mathrm{cm}$ of polyethylene balls, $30.73 \mathrm{~cm}$ water height

read parm run=yes gen $=405$ npg $=600$ nsk $=5$ nub=yes end parm

read geom

unit 1

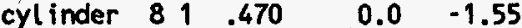

$\begin{array}{lllll}\text { cuboid } & 31 & 4 \mathrm{p} .675 & 0.0 & -1.55\end{array}$

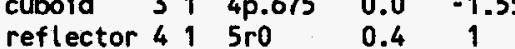

unit 2

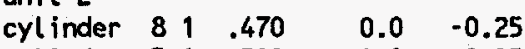

cylinder $\begin{array}{lllll}3 & 1 & .500 & 0.0 & -0.25\end{array}$

cuboid 41 4p.675 $0.0 \quad-0.25$

unit 3

cylinder $111 \quad 395 \quad 30.73 \quad 0.0$

cylinder $01.410=30.73=0.0$

unit 4

array $1 \quad 3 * 0.0$

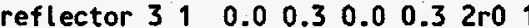

reflector 710.30 .00 .30 .0280

unit 5

array $13 * 0.0$

reflector $3110.3 \quad 0.0 \quad 0.0 \quad 0.32 r 0$

reflector $711 \quad 0.0 \quad 0.3 \quad .30 .02$ r 01

unit 6

array $13 * 0.0$

reflector $310.0 \quad 0.3 \quad 3 \quad 0.02 \mathrm{r}_{0} 1$

reflector $7110.3 \quad 0.0 \quad 0.0 \quad 0.32$ ro

unit 7

array $13 * 0.0$

reflector $3110.30 .0 \quad 0.3 \quad 0.02 r 0$

reflector $711 \quad 0.0 \quad 0.3 \quad 0.0 \quad 0.32 r 0$

unit 8

$\begin{array}{llllll}\text { cuboid } \quad 51 & 2 \mathrm{p} 2.2 & 2 \mathrm{p} 12.30 & 32.63 & 0.0\end{array}$

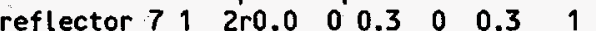

unit 9

cuboid $\begin{array}{llllll}5 & 1 & 2 p 12.30 & 2 p 2.2 & 32.63 & 0.0\end{array}$

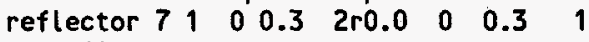

unit 10

$\begin{array}{llllll}\text { cuboid } 5 & 5 & 2 \mathrm{p} 2.2 & 2 \mathrm{p} 2.2 & 32.63 & 0.0\end{array}$

reflector $715 \mathrm{r0.0}$

0.31

unit 11

$\begin{array}{llllll}\text { cuboid } \quad 5 & 1 & 2 \mathrm{p} 12.30 & 2 \mathrm{p} 2.2 & 32.63 & 0.0\end{array}$

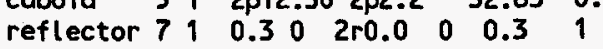

unit 12

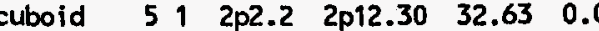

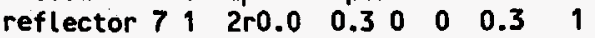

unit 13

$\begin{array}{llllll}\text { cylinder } & 6 & 1 & .395 & 90.00 & 30.73\end{array}$

cylinder $\begin{array}{lllll}0 & 1 & .410 & 96.9 & 30.73\end{array}$

cylinder $21.470 \quad 98.2 \quad 30.73$

$\begin{array}{llllll}\text { cuboid } & 0 & 1 & 4 \mathrm{p} .675 & 98.2 & 30.73\end{array}$

unit 14

array $23 * 0.0$

reflector $0110.0 \quad 0.3 \quad 0.0 \quad 0.3 \quad 2 r 0$

reflector $710.3 \quad 0.0 \quad 0.3 \quad 0.02$ 2ro

unit 15

array $2 \quad 3 * 0.0$

reflector $0.10 .3 \quad 0.0 \quad 0.00 .32 r 01$

reflector $710.0 \quad 0.3 \quad .3 \quad 0.02$ ro 1

unit 16

array $2 \quad 3 * 0.0$

reflector $011 \quad 0.0 \quad 0.3 \quad 3 \quad 0.02 r 01$

reflector $710.30 .00 .00 .32 \mathrm{ro}$

unit 17

array $23 * 0.0$

reflector $0110.3 \quad 0.0 \quad 0.3 \quad 0.02 r 01$

$\begin{array}{llllllll}\text { reflector } 71 & 0.0 & 0.3 & 0.0 & 0.3 & 2 r 0 & 1\end{array}$

unit 18

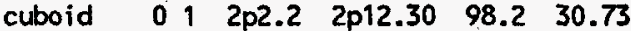

reflector 71 2ro.0 $00.3 \quad 2 r 0$

unit 19

$\begin{array}{lllllll}\text { cuboid } & 0 & 1 & 2 p 12.30 & 2 p 2.2 & 98.2 & 30.73\end{array}$

reflector 71 o $0.3 \quad 2 \mathrm{r} 0.0 \quad 2 \mathrm{ro}$

unit 20

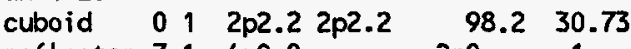

reflector

unit 21

$\begin{array}{lllllll}\text { cuboid } & 0 & 1 & 2 \mathrm{p} 12.30 & 2 \mathrm{p} 2.2 & 98.2 & 30.73\end{array}$

reflector $7110.3002 r_{0.0} \quad 2 r_{0}$

unit 22

cuboid $\begin{array}{llllll}0 & 1 & 2 p 2.2 & 2 p 12.30 & 98.2 & 30.73\end{array}$

reflector 71 2r0.0 0.300 2ro 1

unit 23

array $3 \quad 3 * 0.0$

reflector $394 \mathrm{r} 20 \quad 2 \mathrm{ro} 1$

reflector $415 \mathrm{ro} \quad 81$

reflector 315 ro 201

unit 24

array $4 \quad 3 * 0.0$

reflector $0 \quad 14 r 20 \quad 2 r 01$

global unit 25

array $5 \quad 3 * 0.0$

end geom

read array

ara=1 nux =18 nuy=18 nuz=3 fill 324r1 324r2 324r3 end fill

ara=2 nux=18 nuy=18 nuz=1 fill $324 r 13$ 
ara=3 nux=3 nuy=3 nuz=1 fill 4855910116127 end fill ara=4 nux=3 nuy=3 nuz=1 fill $14 \quad 18 \quad 15 \quad 1920 \quad 21 \quad 16 \quad 22 \quad 17$ end fill ara $=5$ nux $=1$ nuy=1 nuz=2 fill 2324

end fill

end array

end data

end

ens33ep1

\section{\#csas25}

ans33ep1

44group latticecel

Uo2 1 den=10.38 1.0293922354 .7429223895 .258

arbmag5 $2.7040011302798 .8512000 \quad 0.5014000 \quad 0.4326000$ 0.2221293

h20 31.0

poly(h20) 4 den $=.28791 .0$

arbmstl 7.9030012600072 .02400018 .02800010 .051

al 6 den $=2.6511 .0$ uo2 7 den=10.381.0 293922354.7429223895 .258 end

al

end comp 1.0

squarepitch $1.35 \quad 0.79 \quad 130.94 \quad 2 \quad 0.82 \quad 0$

more data res $=7$ cyl 0.395 dan $(7)=0.989755$ ch polyethylene powder, $26.960 \mathrm{~m}$ water height

read geom

unit 1

cyl inder $81 \quad .470 \quad 0.0 \quad-0.75$

$\begin{array}{lllll}\text { cuboid } 3 & 1 & 40.675 & 0.0 & -1.55\end{array}$

reflector $41550.0 \quad 0.4 \quad 1$

unit 2

cylinder $81 \quad .470 \quad 0.0 \quad-0.25$

cylinder $3, \quad .500 \quad 0.0 \quad-0.25$

$\begin{array}{lllll}\text { cuboid } 51 & 4 p .675 & 0.0 & -0.25\end{array}$

unit 3

cylinder $111 \quad .395 \quad 26.98 \quad 0.0$

cylinder $\begin{array}{llllll} & 1 & .410 & 26.98 & 0.0\end{array}$

$\begin{array}{lllll}\text { cyl inder } 21 & 1.470 & 26.98 & 0.0\end{array}$

$\begin{array}{llllll}\text { cuboid } & 3 & 1 & 4 p .675 & 26.98 & 0.0\end{array}$

unit 4

array $1 \quad 3 * 0.0$

reflector $31 \quad 0.0 \quad 0.3 \quad 0.0 \quad 0.32501$

reflector $610.3 \quad 0.0 \quad 0.3 \quad 0.02 r 01$

unit 5

array $13 * 0.0$

reflector $3 ; \quad 0.3 \quad 0.0 \quad 0.0 \quad 0.32501$

reflector $610.0 \quad 0.3 \quad .3 \quad 0.0$ 2ro 1

unit 6 array $1 \quad 3 * 0.0$

reflector $3110.0 \quad 0.3 \cdot 3 \quad 0.0 \quad 2 r 01$ reflector $611 \quad 0.3 \quad 0.0 \quad 0.0 \quad 0.32 r 01$

unit 7

array $1 \quad 3 * 0.0$

$\begin{array}{llllllllll}\text { reflector } 3 & 1 & 0.3 & 0.0 & 0.3 & 0.0 & 2 r 0 & 1\end{array}$

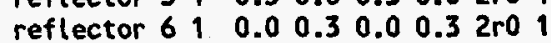

unit 8

$\begin{array}{llllll}\text { cuboid } \quad 4 & 4 & 2 p .95 & 2 p 12.30 & 28.88 & 0.0\end{array}$

reflector $\begin{array}{llllllll}6 & 1 & 2 \mathrm{r} 0.0 & 0 & 0.3 & 0 & 0.3 & 1\end{array}$

unit 9

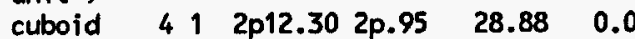

$\begin{array}{lllllll}\text { reflector } 6 & 1 & 0 & 0.3 & 2 r 0.0 & 0 & 0.3\end{array}$

unit 10

$\begin{array}{llllll}\text { cuboid } 4 & 4 & 2 p .95 & 2 p .95 & 28.88 & 0.0\end{array}$

reflector $6115 \mathrm{r0.0} \quad 0.31$

unit 11

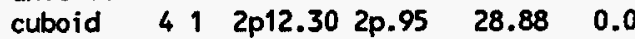

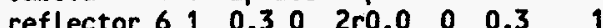

unit 12

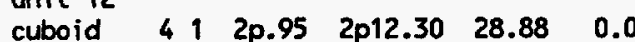

reflector 611 2r0.0 $10.30000 .3 \quad 1$

unit 13

cylinder $\begin{array}{lllll}71 & .395 & 90.00 & 26.98\end{array}$

cylinder $\begin{array}{lllll}0 & 1 & .410 & 96.9 & 26.98\end{array}$

cylinder $21 \quad .470 \quad 98.2 \quad 26.98$

cuboid

cuboid

unit 14

array $2 \quad 3 * 0.0$

reflector $0110.0 \quad 0.3 \quad 0.0 \quad 0.3 \quad 2 r 0$ $\begin{array}{llllllllll}\text { reflector } 6 & 1 & 0.3 & 0.0 & 0.3 & 0.0 & 2 r 0 & 1\end{array}$

unit 15

array $2 \ldots 3 * 0.0$

reflector $0110.3 \quad 0.0 \quad 0.0 \quad 0.32 r 0$

reflector 610.00 .3 .30 .02 ro 1

unit 16

array $2 \quad 3 * 0.0$

reflector $0110.0 \quad 0.3 .3 \quad 0.02201$ reflector $610.3 \quad 0.0 \quad 0.0 \quad 0.32 r 01$

unit 17

array $2 \quad 3 * 0.0$

reflector 010.30 .00 .30 .02501

reflector $610.0 \quad 0.3 \quad 0.0 \quad 0.32 r 01$

unit 18

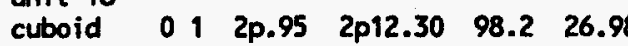

reflector 61 2ro.0 $100.3 \quad 2 r 0 \quad 1$

unit 19

$\begin{array}{lllllll}\text { cuboid } & 0 & 1 & 2012.30 & 20.95 & 98.2 & 26.98\end{array}$

$\begin{array}{lllllll}\text { reflector } 6 & 1 & 0 & 0.3 & 2 r 0.0 & 2 r 0 & 1\end{array}$

unit 20

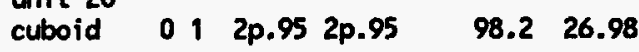


unit 21

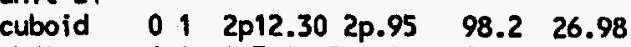

reflector

unit 22

cuboid $\begin{array}{llllll}0 & 1 & 2 p .95 & 2 p 12.30 & 98.2 & 26.98\end{array}$

reflector

unit 23

array $3 \quad 3 * 0.0$

reflector 314 r20 2 ro

reflector $515 r_{0} .81$

reflector $315 r \quad 201$

unit 24

array $4 \quad 3 * 0.0$

reflector 014 r $20 \quad 2 r 01$

global unit 25

array $5 \quad 3 * 0.0$

end geom

read array

ara $=1$ nux $=18$ nuy=18 nuz=3 fill $324 r 1324 r 2 \quad 324 r 3$

end fill

ara $=2$ nux $=18$ nuy=18 nuz=1 fill $324 \mathrm{r} 13$

end fill

ara $=3$ nux $=3$ nuy $=3$ nuz $=1$ fill $4859 \quad 10116127$ end fill

ar $a=4$ nux $=3$ nuy $=3$ nuz=1 fill $14 \quad 18 \quad 15 \quad 1920 \quad 21 \quad 16 \quad 22 \quad 17$ end fill

ara $=5$ nux $=1$ nuy $=1$ nuz $=2$ fill 2324

end fill

end array

end

ans33ep2

\#csas25

ans33ep2

44group latticecell

uo2 1 den=10.38 1.0 293922354.7429223895 .258 end

arbmag5 $2.7040011302798 .8512000 \quad 0.50 \quad 14000 \quad 0.43 \quad 26000$ 0.2221293

h20 31.0

poly(h20) 4 den $=.33351 .0 \quad$ end

arbmstl 7.9030012600072 .02400018 .02800010 .051 end

al 6 den=2.651 1.0 end

uo2 7 den=10.38 1.0293922354 .7429223895 .258 end

at 81.0

end comp

squarepitch $1.35 \quad 0.79 \quad 130.94 \quad 20.82 \quad 0$

more data res=7 cyl 0.395 dan( 7$)=0.989755$

4-18×18 assemblies separated by $0.3-\mathrm{cm}$-thick aluminum plates and 4.4

$\mathrm{cm}$ of polyethylene powder, $30.16 \mathrm{~cm}$ water height

read parm run=yes gen $=405$ npg $=600$ nsk $=5$ nub=yes end parm read geom

unit 1

cyl inder $8 \begin{array}{lllll}8 & 1 & .470 & 0.0 & -0.75\end{array}$

cuboid 314 p.675 $0.0 \quad-1.55$

reflector 415 ro.0 0.41

unit 2

cylinder $811.470 \quad 0.0 \quad-0.25$

cylinder $31.500 \quad 0.0 \quad-0.25$

$\begin{array}{lllll}\text { cuboid } 5 & 1 & \text { 4p.675 } & 0.0 & -0.25\end{array}$

unit 3

cylinder $1110.395 \quad 30.16 \quad 0.0$

cylinder $001 \quad .410 \quad 30.16 \quad 0.0$

cylinder $21 \quad .470 \quad 30.16 \quad 0.0$

cuboid $3140.675 \quad 30.16 \quad 0.0$

unit 4

array $1 \quad 3 * 0.0$

reflector $310.0 \quad 0.3 \quad 0.0 \quad 0.32 r 0$ reflector $61 \quad 0.3 \quad 0.0 \quad 0.3 \quad 0.02 r 0$

unit 5

array $1 \quad 3 * 0.0$

reflector $31 \quad 0.3 \quad 0.0 \quad 0.0 \quad 0.32 r 0$

reflector $610.00 .3 \quad 3 \quad 0.02 r_{0}$ ?

unit 6

array $1 \quad 3 * 0.0$

$\begin{array}{llllllll}\text { reflector } 3 & 1 & 0.0 & 0.3 & .3 & 0.0 & 2 \mathrm{r} 01\end{array}$ reflector $6110.3 \quad 0.0 \quad 0.0 \quad 0.3 \quad 2 r 01$ unit 7

array $1 \quad 3 * 0.0$

reflector $31 \quad 0.3 \quad 0.0 \quad 0.3 \quad 0.02 r 0$

reflector $610.00 .30 .00 .32 \mathrm{ro}$

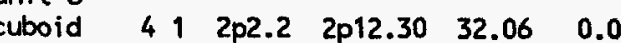

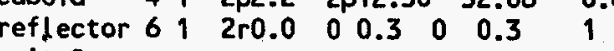

unit 9

$\begin{array}{lllllll}\text { cuboid } & 41 & 2 p 12.30 & 2 p 2.2 & 32.06 & 0.0\end{array}$

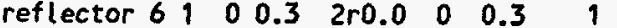

unit 10

$\begin{array}{llllll}\text { cuboid } \quad 4 & 1 & 2 \mathrm{p} 2.2 & 2 \mathrm{p} 2.2 & 32.06 & 0.0\end{array}$

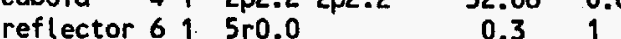

unit 11

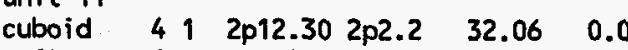

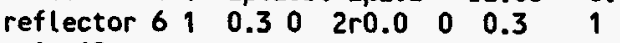

unit 12

$\begin{array}{llllll}\text { cuboid } \quad 4 & 4 & 2 p 2.2 & 2 p 12.30 & 32.06 & 0.0\end{array}$

reflector $\begin{array}{lllllll}6 & 1 & 2 \mathrm{r} 0.0 & 0.3 & 0 & 0 & 0.3\end{array}$

unit 13

cylinder $71 \quad .395 \quad 90.00 \quad 30.16$

cylinder $001 \quad .410 \quad 96.9 \quad 30.16$

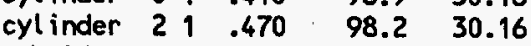

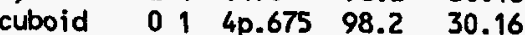


reflector $0110.3 \quad 0.0 \quad 0.3 \quad 0.02$ r.

$\begin{array}{llllllll}\text { reflector } 6 & 1 & 0.0 & 0.3 & 0.0 & 0.3 & 2 r 0\end{array}$

unit 18

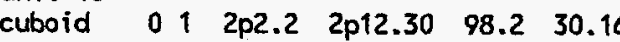

reflector $\begin{array}{lllllll}6 & 1 & 2 \mathrm{r} 0.0 & 0 & 0.3 & 2 \mathrm{r} 0 & 1\end{array}$

unit 19

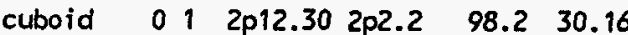

reflector $61100.3 \quad 2 r_{0} 0.0 \quad 2 r_{0}$

reflector

$\begin{array}{lllllll}\text { cuboid } 0 & 1 & 2 p 2.2 & 2 p 2.2 & 98.2 & 30.16\end{array}$

reflector

unit 21

array $3 \quad 3 * 0.0$

reflector 314 r20 2 ro

reflector $5115 \mathrm{ro} \quad .81$

reflector $315 \mathrm{rO} \quad 201$

unit 24

array $4 \quad 3 * 0.0$

reflector 014 r20 2ro 1

global unit 25

array $5 \quad 3 * 0.0$

end geom

\section{read array}

ara $=1$ nux $=18$ nuy=18 nuz=3 fill $324 r 1 \quad 324 r_{2} \quad 324 r 3$

$\begin{array}{ll}\text { ara=1 nux=18 nuy=18 nuz=3 fill } 324 r 1 \quad 324 r 2324 r 3 & \text { end fill } \\ \text { ara=2 nux=18 nuy=18 nuz=1 fill } 324 r 13 & \text { end fill }\end{array}$

ara $=3$ nux $=3$ nuy $=3$ nuz=1 fill 485910116127 end fill

ara=4 nux=3 nuy=3 nuz=1 fill $14 \quad 18 \quad 15 \quad 192021 \quad 16 \quad 22 \quad 17$ end fill

ara $=5$ nux $=1$ nuy=1 nuz=2 fill 2324

end fill

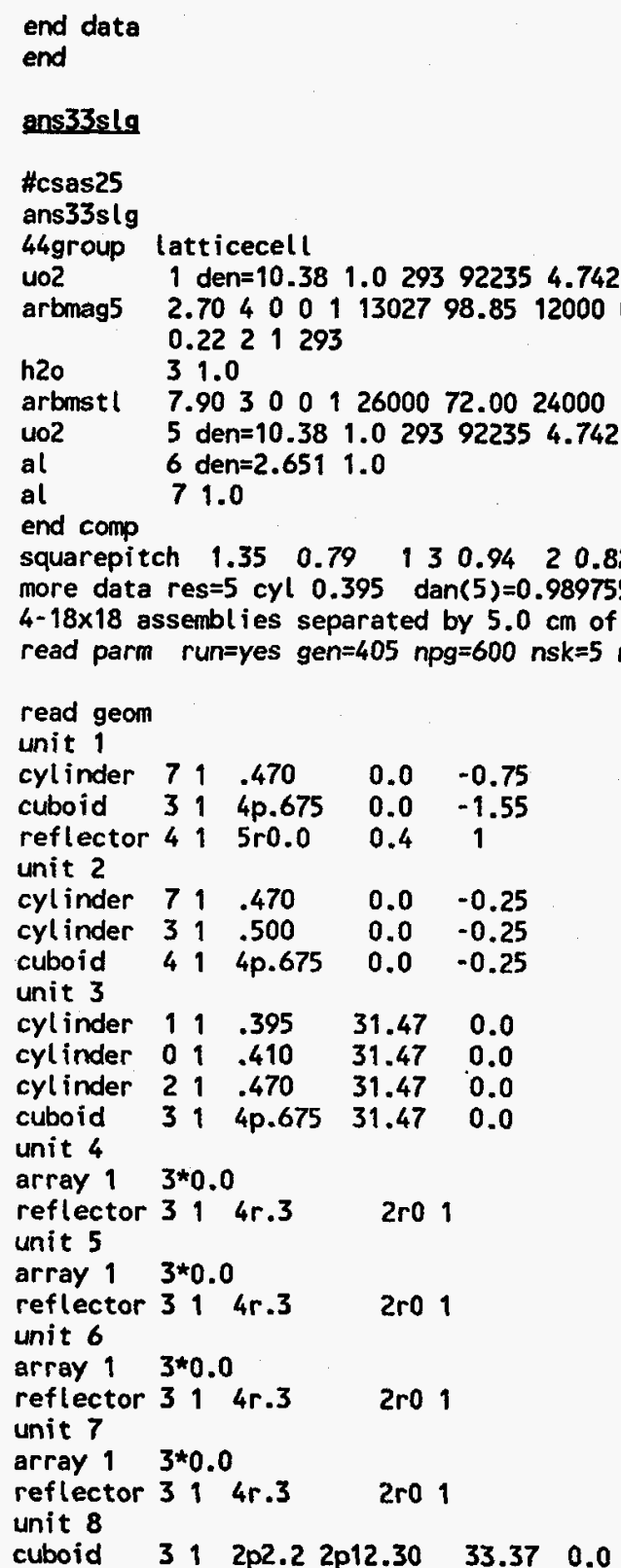


reflector $3112 r 0.0 \quad 0 \quad 0.3 \quad 0 \quad 0.3 \quad 1$ unit 9

$\begin{array}{lllllll}\text { cuboid } & 3 & 1 & 2 p 12.30 & 2 p 2.2 & 33.37 & 0.0\end{array}$

reflector $3100.3 \quad 2 r 0.0 \quad 0 \quad 0.3 \quad 1$

unit 10

$\begin{array}{llllllll}\text { cuboid } & 3 & 1 & 2 p 2.2 & 2 p 2.2 & 33.37 & 0.0\end{array}$

reflector $3115 r 0.0 \quad 0.3 \quad 1$

unit 11

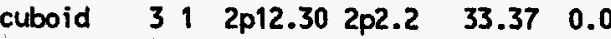

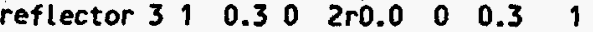

unit 12

$\begin{array}{lllllll}\text { unit } 12 & 3 & 1 & & & & \end{array}$

reflector 311 2r0.0 0.30000 .31

unit 13

$\begin{array}{lllllll}\text { cyl inder } & 5 & 1 & .395 & 90.00 & 31.47\end{array}$

$\begin{array}{lllllll}\text { cylinder } & 0 & 1 & .410 & 96.9 & 31.47\end{array}$

$\begin{array}{llllll}\text { cylinder } & 2 & 1 & .470 & 98.2 & 31.47\end{array}$

$\begin{array}{llllll}\text { cuboid } & 0 & 1 & 4 p .675 & 98.2 & 31.47\end{array}$

array $2 \quad 3 * 0.0$

reflector $014 r .3 \quad 2 r 01$

unit 15

array $2 \quad 3 * 0.0$

reflector 014 r.3 2 ro

array $2 \quad 3 * 0.0$

reflector $0.14 r .3 \quad 2 r 01$

reflector

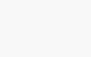

array $2 \quad 3 * 0.0$

reflector $\begin{array}{lll}0 & 1 & 4 r .3\end{array}$

unit 18

cuboid $\begin{array}{llllll}0 & 1 & 2 p 2.2 & 2 p 12.30 & 98.2 & 31.47\end{array}$

reflector 01 2ro.0 $00.3 \quad 2 r 0$

unit 19

unit

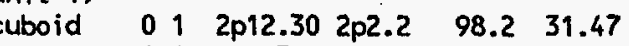

$\begin{array}{lllllll}\text { reflector } 0 & 1 & 0 & 0.3 & 2 \mathrm{r} 0.0 & 2 \mathrm{r} 0 & 1\end{array}$

unit 20

cuboid $01012 p 2.2 \quad 2 p 2.2 \quad 98.2 \quad 31.47$ unit 21

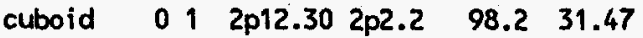

reflector $010.300250 .0 \quad 2 r 0$

unit 22

cuboid $\begin{array}{llllll}0 & 1 & 2 \mathrm{p} 2.2 & 2 \mathrm{p} 12.30 & 98.2 & 31.47\end{array}$

reflector

unit 23

array $3 \quad 3 * 0.0$

reflector $314 \mathrm{r} 202 \mathrm{rO} 1$

reflector $415 \mathrm{ro} \quad .81$

reflector 315 ro 20

unit 24

array $4 \quad 3 * 0.0$ reflector 014 r20 2ro 1

global unit 25

array $5 \quad 3{ }^{*} 0.0$

end geom

\section{read array}

ara=1 nux=18 nuy=18 nuz=3 fill 324r1 324r2 $324 r 3$ end fill

ara=2 nux=18 nuy=18 nuz=1 fill 324r13 end fill

ara=3 nux=3 nuy=3 nuz=1 fill $485591011 \quad 6127$ end fill

ara=4 nux=3 nuy=3 nuz=1 fill $14 \quad 18 \quad 15 \quad 1920 \quad 21 \quad 16 \quad 22 \quad 17$ end fill

ara=5 nux=1 nuy=1 nuz=2 fill $2324 \quad$ end fill

end array

end data

end

ans33sty

\#csas 25

ans33sty

44 group latticecell

U02 1 den=10.38 1.0 293922354.7429223895 .258 end

arbmag5 2.7040011302798 .85120000 .50140000 .4326000 0.2221293

h20 31.0

end

arbmpoly 0.03232010631281901841 end

end

arbmstt 7.9030012600072 .02400018 .02800010 .051 end

al 6 den=2.651 1.0 end

uo2 7 den=10.38 1.0293922354 .7429223895 .258 end

at 81.0

squarepitch $1.35 \quad 0.79 \quad 130.94 \quad 20.82 \quad 0$

$4-18 \times 18$ assemblies separated by $0.3-\mathrm{cm}$ thick aluminum

(1.9 "cm of expanded polystyrene, $28.61 \mathrm{~cm}$ water height

read geom

unit 1

cylinder $8 \begin{array}{lllll}8 & 1 & .470 & 0.0 & -.75\end{array}$

cuboid $314 \mathrm{p} .675 \quad 0.0 \quad-1.55$

reflector 51 5ro.0 $0.4 \quad 1$

unit 2

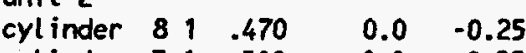

cyl inder $\begin{array}{lllll}3 & 1 & .500 & 0.0 & -0.25\end{array}$

$\begin{array}{lllll}\text { cuboid } 51 & 4 p .675 & 0.0 & -0.25\end{array}$

unit 3

cylinder $111395 \quad 28.61 \quad 0.0$

cylinder $01,410 \quad 28.610 .0$

cylinder $21470 \quad 28.610 .0$

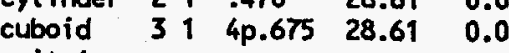

unit 4 
array $1 \quad 3 * 0.0$

reflector $3110.0 \quad 0.3 \quad 0.0 \quad 0.3 \quad 2 r 01$ reflector $6110.3 \quad 0.0 \quad 0.3 \quad 0.02$ 2ro 1 unit 5

array $13 * 0.0$

reflector $310.30 .00 .00 .32 \mathrm{ro} 1$

reflector $6110.00 .3 .30 .02 r 01$

unit 6

array $13 * 0.0$

$\begin{array}{llllllllll}\text { reflector } 3 & 1 & 0.0 & 0.3 & .3 & 0.0 & 2 r 0 & 1\end{array}$

reflector $61 \quad 0.3 \quad 0.0 \quad 0.0 \quad 0.32 \mathrm{ro}$

unit 7

array $1 \quad 3 * 0.0$

$\begin{array}{llllllll}\text { reflector } 3 & 1 & 0.3 & 0.0 & 0.3 & 0.0 & 2 r 0 & 1\end{array}$

reflector $6110.0 \quad 0.3 \quad 0.0 \quad 0.3 \quad 2 r 01$

unit 8

$\begin{array}{lllllll}\text { cuboid } & 4 & 1 & 2 p .95 & 2 p 12.30 & 30.51 & 0.0\end{array}$

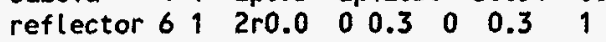

unit 9

cuboid $4 \begin{array}{lllll}4 & 2 \mathrm{p} 12.30 & 2 \mathrm{p} .95 & 30.51 & 0.0\end{array}$

reflector $6 \begin{array}{lllllll}6 & 0 & 0.3 & 2 r 0.0 & 0 & 0.3 & 1\end{array}$

unit 10

$\begin{array}{llllll}\text { cuboid } 4 & 1 & 2 p .95 & 2 p .95 & 30.51 & 0.0\end{array}$

reflector 615 ro.0

$\begin{array}{cl}30.51 & 0.0 \\ 0.3 & 1\end{array}$

unit 11

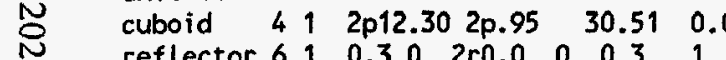

unit 12

cuboid $\begin{array}{llllll}4 & 1 & 2 \mathrm{p} .95 & 2 \mathrm{p} 12.30 & 30.51 & 0.0\end{array}$

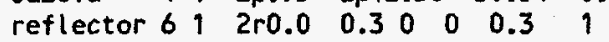

unit 13

$\begin{array}{lllll}\text { cylinder } & 71 & .395 & 90.00 & 28.61\end{array}$

cylinder $\begin{array}{llllll}0 & 1 & .410 & 96.9 & 28.61\end{array}$

cylinder $221 \quad .470 \quad 98.2 \quad 28.61$

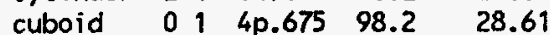

unit 14

array $2 \quad 3 * 0.0$

reflector $0110.0 \quad 0.3 \quad 0.0 \quad 0.32 r 01$ $\begin{array}{lllllllll} & \text { reflector } 6 & 1 & 0.3 & 0.0 & 0.3 & 0.0 & 2 \mathrm{r} 0 & 1\end{array}$ unit 15

array $2 \quad 3 * 0.0$

reflector $011 \quad 0.3 \quad 0.0 \quad 0.00 .32 \mathrm{r} 01$

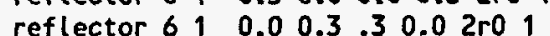

unit 16

array $2 \quad 3 * 0.0$

$\begin{array}{lllllllll}\text { reflector } 0 & 1 & 0.0 & 0.3 & .3 & 0.0 & 2 \mathrm{r} 0 & 1\end{array}$

$\begin{array}{llllllll}\text { reflector } 6 & 1 & 0.3 & 0.0 & 0.0 & 0.3 & 2 \mathrm{rO} & 1\end{array}$

unit 17

array $2 \quad 3 * 0.0$

$\begin{array}{lllllllll}\text { reflector } 0 & 1 & 0.3 & 0.0 & 0.3 & 0.0 & 2 r 0 & 1\end{array}$

$\begin{array}{llllllllll}\text { reflector } 6 & 1 & 0.0 & 0.3 & 0.0 & 0.3 & 2 \mathrm{rO} & 1\end{array}$ unit 18

cuboid $\begin{array}{llllll}0 & 1 & 2 p .95 & 2 p 12.30 & 98.2 & 28.61\end{array}$

reflector

unit 19

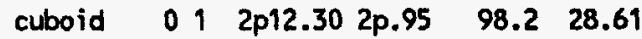

reflector $61100.3 \quad 2 r 0.0 \quad 2 r 0$

unit 20

$2+0$

unit 20

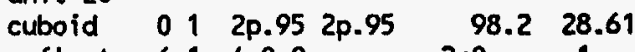

reflector 614 4r0.0

$2 \mathrm{ro} \quad 1$

unit 21

cuboid $\begin{array}{llllll}0 & 1 & 2 p 12.30 & 2 p .95 & 98.2 & 28.61\end{array}$

reflector $6110.3 \quad 0 \quad 2 \mathrm{r} 0.0 \quad 2 \mathrm{r} 0 \quad 1$

unit 22

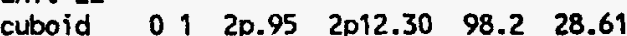

reflector

unit 23

array $3 \quad 3 * 0.0$

reflector 314 r20 2ro

reflector $515 \mathrm{ro} \quad .8$

reflector $315 \mathrm{ro} \quad 201$

unit 24

array $_{4} 3 * 0.0$

reflector 014 r20 2 ro 1

global unit 25

array $5 \quad 3 * 0.0$

end geom

\section{read array}

ara $=1$ nux=18 nuy=18 nuz=3 fill $324 r 1324 r_{2} 324 r 3$

$\begin{array}{lllll}\text { ara }=1 & \text { nux }=18 \text { nuy=18 nuz=3 fill } 324 r 1 \quad 324 r 2 \quad 324 r 3 & \text { end fill } \\ \text { ara } a=2 & \text { nux }=18 & \text { nuy }=18 \text { nuz=1 fill } 324 r 13 & \text { end fill }\end{array}$

ara $=3$ nux $=3$ nuy=3 nuz $=1$ fill 485910116127 and fill ara $=4$ nux $=3$ nuy $=3$ nuz=1 fill $14 \quad 18 \quad 15 \quad 1920 \quad 21 \quad 16 \quad 22 \quad 17$ end fill ara $=5$ nux=1 nuy=1 nuz=2 fill 2324 end fill end array

end data

end

b1645so1

\section{\#csas 25}

b1645so1

44 group

uo2

uor

h2o

boron

carbonstee

$10.9325 \quad 293922352.4599223897 .541$ end

21.0293

$3 \quad 1.0293$

3 den $=1068.0-6$

41.0293

end

end comp

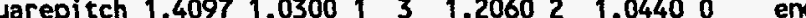

9-15x15 assemblies separated by $0.0762-\mathrm{cm}$-thick aluminum plates and 
$-1.6396 \mathrm{~cm}$ of water, $101.81 \mathrm{~cm}$ water height with $1068 \mathrm{ppm}$ boron read parm gen $=405$ npg $=600$ nsk $=5$ nub=yes run=yes end parm

\section{read geom}

unit 1

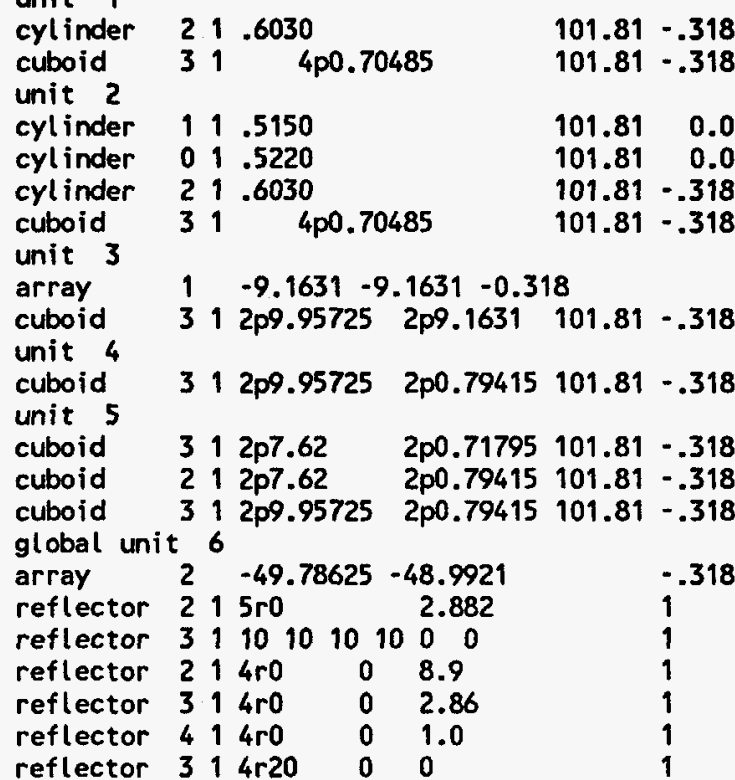

reflector

read array

ara=1 nux=13 nuy=13 nuz=1 fill 111 r $\quad 1 \quad 13 r 2 \quad 10 q 13 \quad 1 \quad 11 r 2 \quad 1$ end fill ara $=2$ nux $=5$ nuy $=9$ nuz $=1$ fill $5 r 3 \quad 4 \quad 3 r 5 \quad 4 \quad 3 q 10 \quad 5 r 3$ and fill end array

end data

end

\section{b1645so?}

\section{\#csas25}

b1645so2

44 group

uo2

al

h20

boron

carbonsteel

end comp

squarepitch 1.40971 .0300131 .206021 .04400 end

9-15x15 assemblies separated by $0.0762-\mathrm{cm}$-thick aluminum plates and
$.1 .6396 \mathrm{~cm}$ of water, $144.85 \mathrm{~cm}$ water height with $1156 \mathrm{ppm}$ boron read parm gen $=405 \mathrm{npg}=600 \mathrm{nsk}=5$ nub=yes run=yes end parm

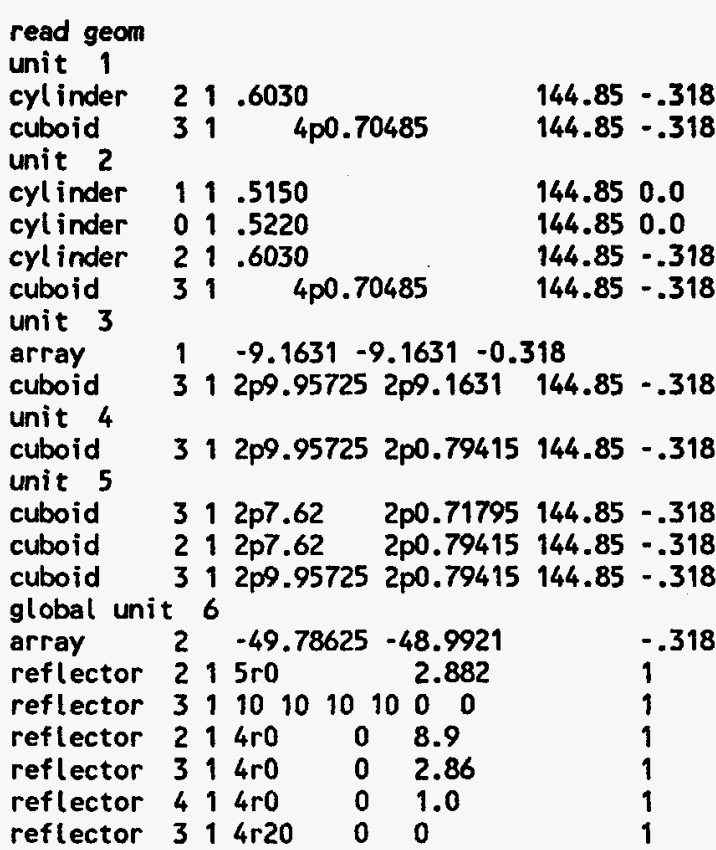

end geon

read array

ara=1 nux=13 nuy=13 nuz=1 fill $111 \mathrm{r} 2,13 \mathrm{r} 210 \mathrm{q} 13,111 \mathrm{r} 21$ end fill $a r a=2$ nux $=5$ nuy=9 nuz=1 fill $5 r 3 \quad 4 \quad 3 r 5 \quad 4 \quad 3 q 10 \quad 5 r 3$ end fill end array

end data

end

\section{bu1231b1}

$=\operatorname{csas} 25$

bu $1231 b 1$

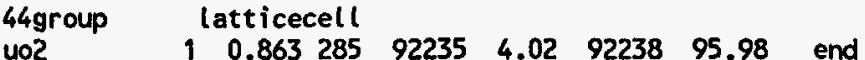

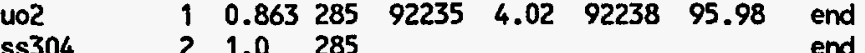

$\begin{array}{llll}55304 & 2 & 1.0 & 285 \\ & 3 & 1.0 & 285\end{array}$

$\begin{array}{llllllll}\text { h20 } & 3 & 1.0 & 285 & & & \text { end } \\ \text { boron } & 3 & \text { den }=.001152 & 1.0 & 285 & \end{array}$

$\begin{array}{llllll} & 3 & \text { den }=.001152 & 1.0 & 285 & \\ \text { boron } & 4 & 1.0 & 285 & & \end{array}$

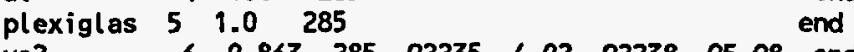




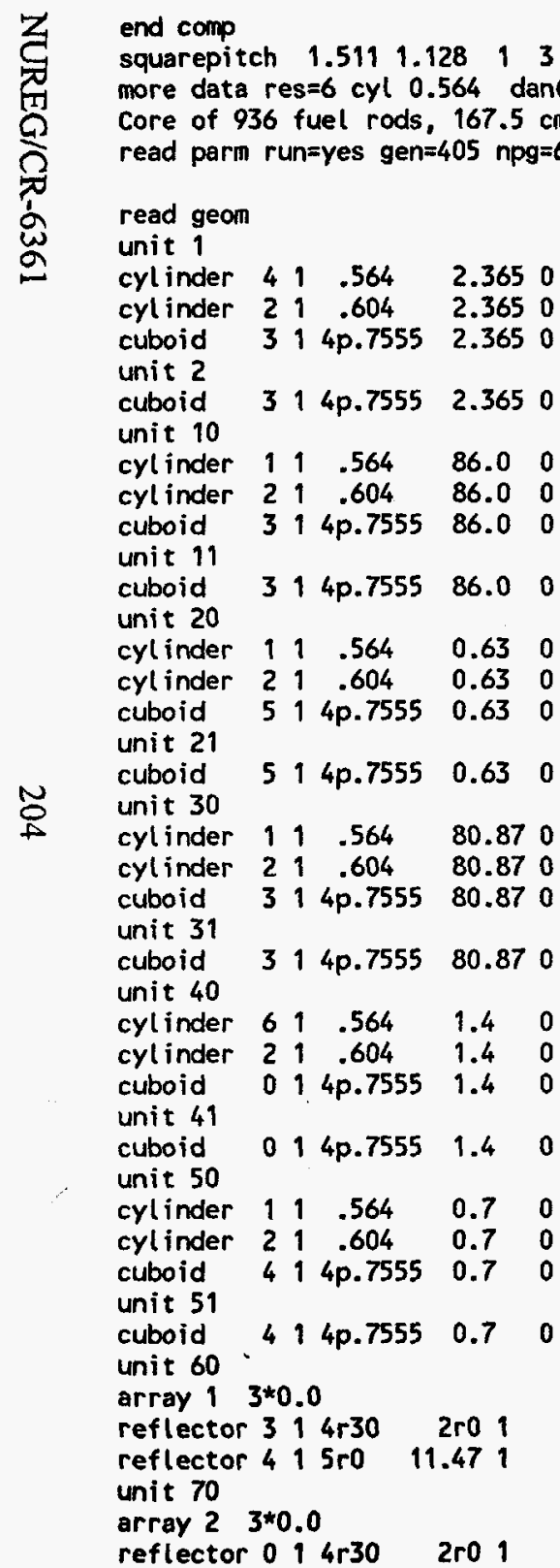

unit 80

array $3 \quad 3 * 0.0$

reflector $414 r 306.5701$

global

unit 90

array $4 \quad 3 * 0.0$

end geom

read array

ara $=1$ nux $=34$ nuy $=34$ nuz $=4$

fill

$12 r 2$ 5r1 n17 9r2 8r1 n17 8r2 9r1 n17

$6 r 211 r 1 n 175 r 212 r 1 n 17 \quad 4 r 213 r 1 n 17$

$3 r 2$ 14r1 n17 3r2 14r1 n17 2r2 15r1 n17 1r2 16r1 n17

$1 r 2$ 16r1 n17 1r2 16r1 n17 170r1

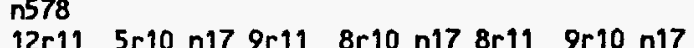

6r11 11 r10 n17 $5 r 11 \quad 12 r 10$ n17 4 r11 $13 r 10$ n17

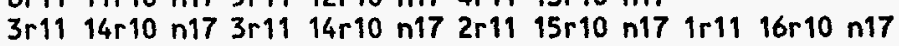
$1 r 11$ 16r10 n17 1r11 16r10 n17 170r10

n578

$\begin{array}{lllllllllll}12 r 21 & 5 r 20 & n 17 & 9 r 21 & 8 r 20 & n 17 & 8 r 21 & 9 r 20 & n 17\end{array}$

6r21 $11 r 20 n 17 \quad 5 r 21 \quad 12 r 20 n 17 \quad 4 r 21 \quad 13 r 20 n 17$

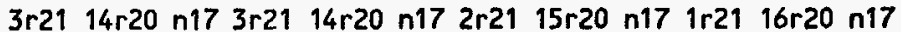
$1 r^{21} 16 r 20$ n17 $1 r^{21} 16 r 20$ n17 170r20

n578

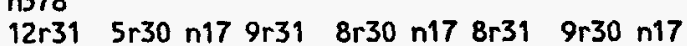

$6 r 3111 r 30 n 175 r 3112 r 30 n 17 \quad 4 r 31 \quad 13 r 30 n 17$

$\begin{array}{llllllllllllllll}3 r 31 & 14 r 30 & n 17 & 3 r 31 & 14 r 30 & n 17 & 2 r 31 & 15 r 30 & n 17 & 1 r 31 & 16 r 30 & n 17\end{array}$

$1 r 3116 r 30$ n17 $1 r 31$ 16r30 n17 170r30

n578 end fill

ara $=2$ nux $=34$ nuy $=34$ nuz $=1$

fill

$12 r 41 \quad 5 r 40$ n17 $9 r 41 \quad 8 r 40 n 17 \quad 8 r 41 \quad 9 r 40 n 17$

$6 r 4111 r 40 n 17 \quad 5 r 41 \quad 12 r 40 n 17 \quad 4 r 41 \quad 13 r 40 n 17$

$3 r 4114 r 40 n 17 \quad 3 r 41 \quad 14 r 40 n 17 \quad 2 r 41 \quad 15 r 40$ n17 $1 r 41 \quad 16 r 40 n 17$

$1 r 4116 r 40$ n17 $1 r 41$ 16r40 n17 170r40

n578 end fill

ara $=3$ nux $=34$ nuy $=34$ nuz $=1$

fill

$\begin{array}{llllllll}12 r 51 & 5 r 50 & n 17 & 9 r 51 & 8 r 50 & n 17 & 8 r 51 & 9 r 50 \\ n 17\end{array}$

$6 r 5111 r 50$ n17 $5 r 5112 r 50$ n17 4 r51 $13 r 50$ n17

$\begin{array}{llllllllllllll}3 r 51 & 14 r 50 & n 17 & 3 r 51 & 14 r 50 & n 17 & 2 r 51 & 15 r 50 & n 17 & 1 r 51 & 16 r 50 & n 17\end{array}$

1 r51 16r50 n17 1r51 16r50 n17 170r50

$n 578$ end fill

$a r a=4$ nux $=1$ nuy $=1$ nuz=3 fill 607080 end fill

end array

end data

end

reflector $014 \mathrm{r} 30 \quad 2 \mathrm{r} 01$ 
b4123162

$=\operatorname{csas} 25$

bw1231b2

44group

no2

ss304

h2o

boron

al

plexiglas

vo2

end comp

squarepitch $1.5111 .128 \quad 1 \quad 3 \quad 1.208 \quad 2$

latticecell

$\begin{array}{lllllll}1 & 0.863 & 287 & 92235 & 4.02 & 92238 & 95.98\end{array}$ end

$2 \quad 1.0287$

$\begin{array}{lll}3 & 1.0 & 287\end{array}$

$1.0 \quad 287$

$\begin{array}{lll}4 & 1.0 & 287\end{array}$

more data res $=6$ cyl 0.564 dan(6) $=0.901122$

read parm run=yes gen $=405$ npg $=600$ nsk $=5$ nub=yes end parm

read geom

unit 1

cylinder $4 \quad 1 \quad .564 \quad 3.46 \quad 0$

cylinder $21 \quad .604 \quad 3.46 \quad 0$

cuboid $314 \mathrm{p} .7555 \quad 3.46 \quad 0$

unit 2

cuboid $\quad 314 p .7555 \quad 3.46 \quad 0$

cylinder $111.564 \quad 65.0 \quad 0$

cylinder $\begin{array}{lllll}2 & 1 & .604 & 65.0 & 0\end{array}$

$\begin{array}{llllll}\text { cuboid } & 3 & 1 & 4 \mathrm{p} .7555 & 65.0 & 0\end{array}$

unit 11

cuboid 314 p.7555 65.0.0

unit 20

cylinder $1110.564 \quad 0.63 \quad 0$

cylinder $21.604 \quad 0.630$

cuboid 514 p.7555 $0.63 \quad 0$

unit 21

cuboid 514 p.7555 $0.63 \quad 0$

unit 30

cylinder $11 \quad .564 \quad 81.020$

cylinder $21 \quad .604 \quad 81.020$

cuboid $3140.7555 \quad 81.020$

unit 31

$314 p .755581 .020$

cubaid

$314 p .755581 .020$

unit 40

cylinder $61 \quad .564 \quad 21.650$

cylinder $21 \quad .604 \quad 21.650$

cuboid 014 p.7555 21.650

unit 41

cuboid

unit 50

014 p.7555 21.650

cylinder $\begin{array}{lllll}1 & 1 & .564 & 1.3 & 0\end{array}$ $\begin{array}{llllll}\text { cylinder } & 2 & 1 & .604 & 1.3 & 0\end{array}$

cuboid $414 \mathrm{p} .7555 \quad 1.3 \quad$

unit 51

cuboid $414 p .7555 \quad 1.3 \quad 0$

unit 60

array $13 * 0.0$

reflector $3 \quad 1 \quad 4 r 30.0 \quad 2 r 0$

reflector $415 \mathrm{ro} \quad 12.74$

unit 70

array $23 * 0.0$

reflector $014 \mathrm{r} 30 \quad 2 \mathrm{r} 01$

unit 80

array $33 * 0.0$

reflector $414 \mathrm{r} 307.2401$

global

unit 90

array $43 * 0.0$

end geom

read array

nux $=80$ nuy $=80$ nuz $=4$

fill

$37 r 23 r 1 \quad n 40 \quad 31 r 2 \quad 9 r 1$ n40 $27 r 2$ 13r1 n40

$25 r 215 r 1$ n40 23r2 17r1 n40 21r2 19r1 n40

$19 r 2$ 21r1 n40 18r2 22r1 n40 16r2 24r1 n40

$15 r 225 r 1 n_{40} 14 r 2 \quad 26 r 1$ n40 13r2 27r1 n40

$12 r 228 r 1 \mathrm{n} 40$ 11r2 $29 r 1$ n40 10r2 30r1 n40

12r2 $28 r 1$ n40 11r2 $29 r 1$ n40 10r2 30r1 n40

$9 r 231 r 1 n 40$ 8r2 32r1 n40 8r2 32r1 n40

$7 r 233 r 1 \quad n 40 \quad 6 r 234 r 1 \quad n 40 \quad 6 r 234 r 1 \quad n 40$

$5 r 235 r 1 n 40 \quad 5 r 235 r 1 n 40$ 4r2 36r1 n40

$4 r 236 r 1$ n40 3r2 37r1 n40 3r2 37r1 n40

$2 r 238 r 1$ n40 $2 r 238 r 1$ n40 $2 r 238 r 1$ n40

2r2 38r1 n40 1r2 39r1 n40 1r2 39r1 n40

$1 r 239 r 1$ n40 1r2 39r1 n40 1r2 39r1 n40

$1 r^{2} 39 r 1$ n40 $40 r 1$ n40 40r1 n40 40r1 n40

n 3200

$37 r 113 r 10$ n40 $31 r 119 r 10$ n40 27r11 $13 r 10$ n40

$25 r 1115 r 10$ n40 $23 r 1117 r 10$ n40 $21 r 11$ 19r10 n40

$19 r 1121 r 10$ n40 18r11 22r10 n40 16r11 24r10 n40

$15 r 1125 r 10$ n40 $14 r 1126 r 10$ n40 $13 r 1127 r 10 n 40$

$12 r 11$ 28r10 n40 11r11 29r10 n40 10r11 30r10 n40

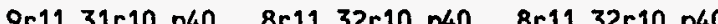

$7 r 1133 r 10 n 40 \quad 6 r 1134 r 10 n 40$ 6r11 $34 r 10 n 40$

$5 r 1135 r 10$ n40 $5 r 1135 r 10$ n40 $4 r 1136 r 10$ n40

$4 r 1136 r 10$ n40 $3 r 1137 r 10$ n40 $3 r 1137 r 10$ n40

$2 r 1138 r 10 n 40 \quad 2 r 1138 r 10 n 40 \quad 2 r 1138 r 10$ n40

$2 r 1138 r 10$ n40 $1 r 1139 r 10$ n40 $1 r 1139 r 10 n 40$

$1 r 1139 r 10$ n40 $1 r 1139 r 10$ n40 $1 r 1139 r 10$ n40

$1 r 1139 r 10$ n40 40r10 n40 40r10 n40 40r10 n40 3200

$37 r 213 r 20 \quad n 40 \quad 31 r 21 \quad 9 r 20 \quad n 40 \quad 27 r 21 \quad 13 r 20 \quad n 40$ 
$25 r 21 \quad 15 r 20$ n40 $23 r 21 \quad 17 r 20$ n40 21r21 19r20 n40 $19 r 2121 r 20$ n40 $18 r 21 \quad 22 r 20$ n40 16r21 $24 r 20$ n40 $15 r 2125 r 20$ n40 $14 r 21 \quad 26 r 20$ n40 13r21 $27 r 20$ n40 $12 r 21 \quad 28 r 20$ n40 $11 r 2129 r 20 n 40 \quad 10 r 21 \quad 30 r 20$ n40 $9 r 2131 r 20$ n40 $8 r 2132 r 20 n 40 \quad 8 r 2132 r 20 n 40$ $7 r 2133 r 20 n 40 \quad 6 r 2134 r 20 n 40$ 6r21 $34 r 20 n 40$ $5 r 2135 r 20$ n40 $5 r 2135 r 20$ n40 $4 r 2136 r 20$ n40 $4 r 2136 r 20$ n40 $3 r 2137 r 20$ n40 3r21 37r20 n40 $2 r 2138 r 20$ n40 $2 r 2138 r 20$ n40 $2 r 2138 r 20$ n40 $2 r 2138 r 20 n 40 \quad 1 r 2139 r 20 n 40 \quad 1 r 2139 r 20 n 40$ $1 r^{21} 39 r 20 n 40 \quad 1 r_{21} 39 r 20 n 40 \quad 1 r 2139 r 20 n 40$ $1 r^{21} 39 r 20$ n40 40r20 n40 40r20 n40 40r20 n40 n3200

$37 r 31 \quad 3 r 30 \quad n 40 \quad 31 r 319 r 30 \quad n 40 \quad 27 r 31 \quad 13 r 30$ n40 $25 r 31 \quad 15 r 30 \quad n 40 \quad 23 r 31 \quad 17 r 30 n 40 \quad 21 r 31 \quad 19 r 30 n 40$ $19 r 3121 r 30$ n40 $18 r 3122 r 30$ n40 $16 r 3124 r 30$ n40 $15 r 3125 r 30 \quad n 40 \quad 14 r 3126 r 30 n 40 \quad 13 r 31 \quad 27 r 30 n 40$ $12 r 3128 r 30 n 40 \quad 11 r 3129 r 30 n 40 \quad 10 r 3130 r 30 n 40$ $9 r 3131 r 30 n 40 \quad 8 r 3132 r 30 n 40$ 8r31 $32 r 30 n 40$ $7 r 3133 r 30 n 40 \quad 6 r 3134 r 30 n 40 \quad 6 r 3134 r 30 n 40$ $5 r 3135 r 30 n 40 \quad 5 r 3135 r 30 n 40 \quad 4 r 3136 r 30 n 40$ $4 r 3136 r 30 n 40 \quad 3 r 3137 r 30 n 40 \quad 3 r 3137 r 30 n 40$ $2 r 3138 r 30$ n40 $2 r 31 \quad 38 r 30 n 40 \quad 2 r 31 \quad 38 r 30$ n40 $2 r 3138 r 30$ n40 $1 r 3139 r 30 n 40 \quad 1 r 3139 r 30 n 40$ $1 r 3139 r 30 n 40 \quad 1 r 3139 r 30 n 40 \quad 1 r 3139 r 30 n 40$

$\stackrel{1}{O} \quad 1 r 3139 r 30 n 40 \quad 40 r 30 n 40 \quad 40 r 30$ n40 $40 r 30 n 40$ n3200

end $f i l l$

ara $=2$ nux $=80$ nuy $=80$ nuz $=1$

fill

$37 r 41 \quad 3 r 40$ n40 $31 r 419 r 40$ n40 $27 r 41 \quad 13 r 40$ n40 $25 r 41 \quad 15 r 40$ n40 $23 r 41 \quad 17 r 40$ n40 $21 r 41 \quad 19 r 40$ n40 $19 r 4121 r 40 n 40 \quad 18 r 4122 r 40 n 40 \quad 16 r 4124 r 40 n 40$ $15 r 4125 r 40 n 40 \quad 14 r 4126 r 40 n 40 \quad 13 r 4127 r 40 n 40$ $12 r 4128 r 40$ n40 $11 r 4129 r 40$ n40 $10 r 4130 r 40$ n40 $9 r 4131 r 40$ n40 $8 r 4132 r 40$ n40 $8 r 4132 r 40$ n40 $7 r 4133 r 40 n 40 \quad 6 r 4134 r 40 n 40$ 6r41 $34 r 40 n 40$ $5 r 4135 r 40$ n40 $5 r 4135 r 40 n 40 \quad 4 r 4136 r 40 n 40$ $4 r 4136 r 40$ n40 $3 r 4137 r 40$ n40 $3 r 4137 r 40$ n40 $2 r 4138 r 40$ n40 $2 r 4138 r 40$ n40 $2 r 4138 r 40 n 40$ $2 r 4138 r 40 n 40 \quad 1 r 4139 r 40 n 40 \quad 1 r 4139 r 40 n 40$ $1 r 4139 r 40$ n40 1r41 39r40 n40 1r41 39r40 n40 $1 r 4139 r 40 n 40 \quad 40 r 40$ n40 $40 r 40 n 40 \quad 40 r 40 \quad n 40$ n3200

and $f i l l$

ara $=3$ nux $=80$ nuy $=80$ nuz $=1$

fill

$\begin{array}{lllllllll}37 r 51 & 3 r 50 & n 40 & 31 r 51 & 9 r 50 & n 40 & 27 r 51 & 13 r 50 & n 40\end{array}$ 25r51 $15 r 50$ n40 $23 r 5117 r 50$ n40 $21 r 51 \quad 19 r 50$ n40 $19 r 51 \quad 21 r 50$ n40 $18 r 5122 r 50$ n40 $16 r 5124 r 50$ n40 $15 r 5125 r 50$ n40 $14 r 5126 r 50$ n40 $13 r 5127 r 50$ n40
$12 r 5128 r 50$ n40 11r51 29r50 n40 10r51 30r50 n40 $9 r 5131 r 50$ n40 8 r51 32r50 n40 8r51 32r50 n40 $7 r 5133 r 50$ n40 $6 r 5134 r 50 n 40$ 6r51 $34 r 50 n_{40}$ $5 r 5135 r 50 \times 40 \quad 5 r 5135 r 50 n 40 \quad 4 r 5136 r 50 n 40$ 5r51 35r50 nu० 5rsi $35 r 50$ n40 4513650 n40 $4 r 5136 r 50$ n40 3r51 37r50 n40 3r51 37r50 n40 $2 r 5138 r 50$ n40 $2 r 5138 r 50$ n40 $2 r 5138 r 50$ n40

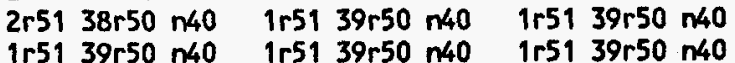
$1 r 5139 r 50$ n40 $40 r 50$ n40 $40 r 50$ n40 40 r50 n40 n3200

end fill

ara $=4$ nux $=1$ nuy $=1$ nuz $=3$ fill 607080 end fill

end array

end data

end

bu1273m

\#csas25

bw $1273 \mathrm{~m}$

44group latticecel

uo2 1 den=10.24 1.0293922352 .4599223897 .541 end

h20 31.0

boron 3 den=1.675e-3

bo2 4 den=10.24 1.0293922352 .4599223897 .541 end plexiglas 51.0

end comp

1.5111 .0301331 .20628 .0440

31.20621 .0440

more data res $=4 \mathrm{cyl} 0.515$ dan( 4$)=0.982883$ with 1675 ppm boron

read parm run $=y e s$ gen $=405 \mathrm{npg}=600 \mathrm{nsk}=5$ nub=yes end parm

read geom

unit 1

cylinder $21 \quad .603 \quad .3175 \quad 0.0$

cuboid $\quad 31 \quad 4 p .7555 \quad .3175 \quad 0.0$

unit 2

cylinder $\begin{array}{lllll}1 & 1 & .515 & 2.54 & 0.0\end{array}$

$\begin{array}{llllll}\text { cylinder } & 0 & 1 & .522 & 2.54 & 0.0\end{array}$

$\begin{array}{lllll}\text { cylinder } 2 & 1 & .603 & 2.54 & 0.0\end{array}$

$\begin{array}{lllll}\text { cuboid } 31 & 4 \mathrm{p} .615 & 2.54 & 0.0\end{array}$

$\begin{array}{lllll}\text { cuboid } 2 & 1 & 4 p .7555 & 2.54 & 0.0\end{array}$

unit 3

cylinder $1110.515 \quad 46.460 .0$

cylinder $011.522 \quad 46.46 \quad 0.0$

cylinder $21.603 \quad 46.460 .0$

cylinder 214035456.460 .0

cuboid

cylinder $11 \quad .515 \quad .1588 \quad 0.0$

cylinder $011.522 \quad .15880 .0$ 


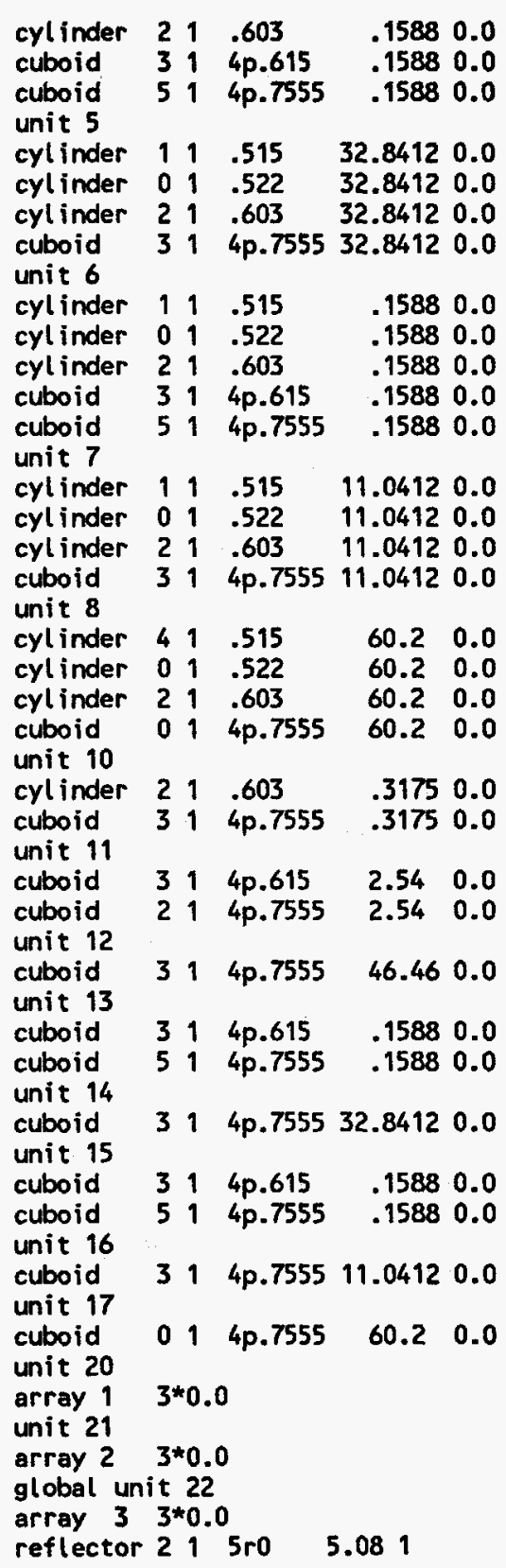

reflector 314 r3o 2 ro 1

end geom

read array

ara $=1$ nux $=1$ nuy=1 nuz=8 fill 123345678 end fill ara=2 nux=1 nuy=1 nuz=8 fill $10 \quad 11 \quad 12 \quad 13 \quad 14 \quad 15 \quad 16 \quad 17$ end fill ara $=3$ nux $=82$ nuy $=82$ nuz $=1$ fill

$38 r 216 r 20 \quad 38 r 2132 r 21$ 18r20 32r21 28r21 26r20 28r21 26r21 30r20 $26 r 21$

$24 r 2134 r 2024 r 2122 r 2138 r 2022 r 2120 r 2142 r 20$ 20r21 18r21 46r20 18 r21

$17 r 2148 r 2017 r 2116 r 2150 r 2016 r 2115 r 2152 r 20 \quad 15 r 2114 r 2154 r 20$ $14 r 21$

$13 r 2156 r 2013 r 2112 r 2158 r 2012 r 2111 r 2160 r 2011 r 2110 r 2162 r 20$ $10 r 21$

$9 r 21 \quad 64 r 20 \quad 9 r 21 \quad 8 r 2166 r 20 \quad 8 r 21 \quad 7 r 21 \quad 68 r 20 \quad 7 r 21 \quad q 82$

$6 r 2170 r 20 \quad 6 r 21982$

$4 r 2174 r 20 \quad 4 r 21982$

$3 r 2176 r 20 \quad 3 r 21$ q82

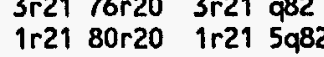

$5 r 2172 r 20 \quad 5 r 21 \quad 982$

n3280

38 r21 7 r20 $37 r 21$ end fill

end array

end data

end

bew1484a1

\#csas 25

bw1484a1

44group latticecell

Uo2 1 den=10.22 1.0293922352 .4599223897 .541 end

al 21.0

h2o 31.0

boron 3 den $=15 e-6$

al 4 den $=2.7 \quad .98386$

boron 4 den $=2.7 \quad 01614$

$2 r 2178 r 20 \quad 2 r 21 \quad 3 q 82$

$82 r 20$

end comp

squarepitch 1.6361 .030131 .20621 .0440 end

9-14x14 assembl ies separated by $0.645-\mathrm{cm}$-thick borated aluminum plates

'and $0.991 \mathrm{~cm}$ of water, plates-to-center assembly distance of 0.3725

$1 \mathrm{~cm}, 150.27 \mathrm{~cm}$ water height with $15 \mathrm{ppm}$ boron

read parm run=yes gen=405 npg=600 nsk=5 nub=yes end parm

read geom

unit $i$

cyl inder $110.515 \quad 0.635 \quad 0.0$

$\begin{array}{llll}\text { cylinder 1 } 1 & 0.515 & 0.635 & 0.0 \\ \text { cyl inder } 01 & 0.522 & 0.635 & 0.0\end{array}$

$\begin{array}{llll}\text { cuboid } 214 \mathrm{p} 0.818 & 0.635 & 0.0\end{array}$ 


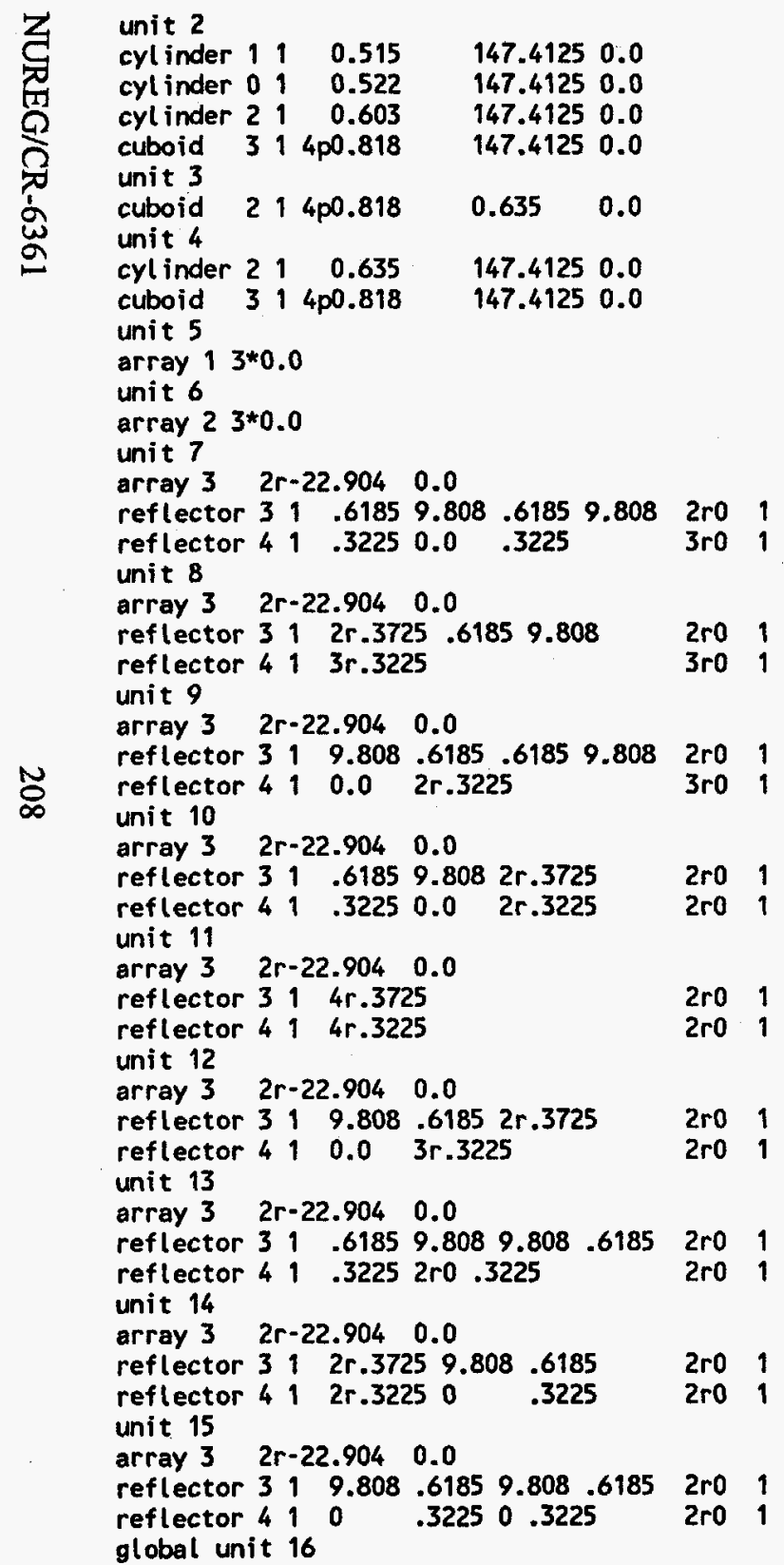

array $42 *-45.808 \quad 0: 0$

reflector $215 r 0.01 .905$

reflector 215 ro 2.54

reflector 31 5ro 0.31751

reflector $2,5 \mathrm{r} 05.08$

reflector $314 \mathrm{r} 20$ 2r0

end geom

read array

ara $=1$ nux $=1$ nuy=1 nuz=2 fill 12 end fill

ara $=2$ nux=1 nuy=1 nuz=2 fill 34 end fill

ara $=3$ nux $=14$ nuy=14 nuz $=1$ fill $6 \quad 12 r 56 \quad 14 r 5 \quad 11914612 r 56$ end fill

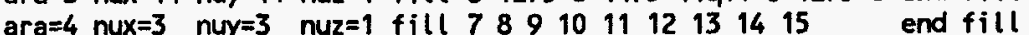

end array

end data

enc

bu1484a2

\#csas25

bw1484a2

44 group latticecell

uo2 1 den=10.22 1.0293922352 .4599223897 .541 end

al 21.0

h2o 31.0

boron 3 den=72e- 6

al 4 den $=2.7 .99899$

boron 4 den $=2.7 \quad .00101$

end comp

$9-14 \times 14$ assemblies separated by $0.645-\mathrm{cm}$-thick borated aluminum plates 'and $4.263 \mathrm{~cm}$ of water, plates-to-center assembly distance of 0.3725

$1 \mathrm{~cm}, 151.69 \mathrm{~cm}$ water height with $72 \mathrm{ppm}$ boron

read parm run=yes gen $=405$ npg $=600$ nsk $=5$ nub $=y e s$ end parm

read geom

unit

cylinder 110.515

cyl inder $011 \quad 0.522 \quad 0.635 \quad 0.0$

cuboid $214 \mathrm{p0} .818$

unit 2

0.6350 .0

cyl inder 110.515

cyl inder 010.522

cylinder 210.603

cuboid $314 \mathrm{p0} .818$

unit 3

cuboid $214 \mathrm{p0} .818$

unit 4

148.83250 .0

148.83250 .0

148.83250 .0

148.83250 .0

0.6350 .0

cyl inder 210.635

cuboid 31400.818

148.83250 .0

unit 5

148.83250 .0 
array $13 * 0.0$

unit 6

array $23 * 0.0$

unit 7

$\begin{array}{lll}\text { array } 3 & 2 r-22.904 & 0.0\end{array}$

reflector $313.8905 \quad 6.536 \quad 3.8905 \quad 6.536 \quad 2 r 0$

reflector $\begin{array}{llllll}4 & 1 & .3225 & 0.0 & .3225 & 3 r 0\end{array}$

unit 8

$\begin{array}{lll}\text { array } 3 & 2 r-22.904 & 0.0\end{array}$

reflector $312 r .3725 \quad 3.8905 \quad 6.536 \quad 2 r 0$

reflector $413 r .3225$

3ro 1

unit

reflector $3 \quad 16.536 \quad 3.8905 \quad 3.8905 \quad 6.536 \quad 2 r 0$

reflector $410.0 \quad 2 r .3225 \quad 3 r 01$

unit 10

$\begin{array}{lll}\text { array } 3 & 2 r-22.904 & 0.0\end{array}$

reflector $313.8905 \quad 6.536 \quad 2 r .3725 \quad 2 r 0$

$\begin{array}{lllll}\text { reflector } 4 & 1.3225 & 0.0 & 2 r .3225 & 2 r 0\end{array}$

unit 11

$\begin{array}{lll}\text { array } 3 & 2 r-22.904 & 0.0\end{array}$

reflector $314 r .3725$

sro

reflector 4 i $4 r .3225$

$\begin{array}{ll}2 r 0 & 1 \\ 2 r 0 & 1\end{array}$

unit 12

$\begin{array}{lll}\text { array } 3 & 2 r-22.904 & 0.0\end{array}$

reflector $316.536 \quad 3.8905 \quad 2 r .3725 \quad 2 r 0 \quad 1$

reflector $410.0 \quad 3 r .3225 \quad 2 r 0$

unit 13

array $3 \quad 2 r-22.904 \quad 0.0$

reflector $313.8905 \quad 6.536 \quad 6.536 \quad 3.8905 \quad 2 r 0 \quad 1$

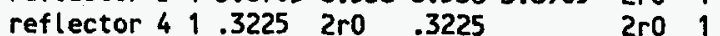

unit 14

$\begin{array}{lll}\text { array } 3 & 2 r-22.904 & 0.0\end{array}$

reflector $312 r .3725 \quad 6.536 \quad 3.8905 \quad$ 2ro 1

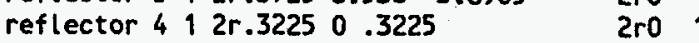

unit 15

array $3 \quad 2 r-22.904 \quad 0.0$

reflector $316.536 \quad 3.8905 \quad 6.536 \quad 3.8905 \quad 2 r 0$

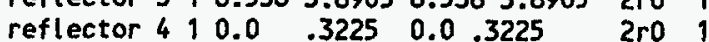

global unit 16

$\begin{array}{lll}\text { array } 4 & 2 *-45.808 & 0.0\end{array}$

reflector $2,5 \mathrm{ro} 1.90$

reflector $21 \quad 5$ ro 2.54

reflector $31 \quad 5 r 00.3175$

reflector $21 \quad 5 r 05.08 \quad 1$

reflector 314 r20 2 ro

end geom

read array

ara $=1$ nux $=1$ nuy=1 nuz=2 fill 12 end fill

ara=2 nux=1 nuy=1 nuz=2 fill 34 end fill ara=3 nux=14 nuy=14 nuz=1 fill $6 \quad 12$ r5 $6 \quad 14 r 5 \quad 11 q 14 \quad 6 \quad 12$ r5 6 end fill ara $=4$ nux=3 nuy=3 nuz=1 fill $78991011 \quad 121314 \quad 15$ end fill end array

end data

end

\section{bu1484b1}

\#csas 25

bw1484b1

44group latticecell

uo2 1 den=10.22 1.0293922352 .4599223897 .541

at 21.0

h2o 31.0

boron 3 den $=1037 \mathrm{e}-6$

end comp

squarepitch $1.6361 .030 \quad 131.20621 .0440$ 'height with 1037 ppm boron

read parm run=yes gen $=405$ npg=600 nsk $=5$ nub=yes end parm

read geom

unit 1

cylinder $2 \begin{array}{llll}2 & 0.603 & .3175 & 0.0\end{array}$

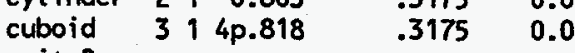

unit 2

$\begin{array}{llllll}\text { cylinder } & 1 & 1 & 0.515 & 2.54 & 0.0\end{array}$

$\begin{array}{llllll}\text { cylinder } & 0 & 1 & 0.522 & 2.54 & 0.0\end{array}$

cylinder $210.603 \quad 2.54 \quad 0.0$

$\begin{array}{lllll}\text { cuboid } \quad 3 & 1 & 4 p .615 & 2.54 & 0.0\end{array}$

$\begin{array}{llll}\text { cuboid } 2 & 14 \mathrm{p} .818 & 2.54 & 0.0\end{array}$

unit 3

cylinder $1110.515 \quad 141.43250 .0$

cylinder $010.522 \quad 141.43250 .0$

cylinder $210.603 \quad 141.43250 .0$

cuboid $314 p .818 \quad 141.43250 .0$

unit 4

array $13 * 0.0$

global unit 5

array $2 \quad 3 * 0.0$

reflector $215 r 0.05 .081$

reflector $315 r 0.02 .54$

reflector $215 r 0.01 .27$

reflector $314 r 24 \quad 2 r 01$

end geom

read array

ara=1 nux=14 nuy=14 nuz=3 fill 196r1 196r2 196r3 end fill

ara $=2$ nux $=3$ nuy $=3$ nuz=1 fill f 4 end fill

end array 
boron 3 den=702e- 6

$9-14 \times 14$ assemblies separated by $1.636 \mathrm{~cm}$ of water, $100.32 \mathrm{~cm}$ water theight with 702 pom boron

read parm run=yes gen $=405$ npg $=600$ nsk $=5$ nub=yes end parm

\section{read geom}

unit 1

$\begin{array}{llllll}\text { cylinder } & 2 & 1 & 0.603 & .3175 & 0.0 \\ \text { cuboid } & 3 & 1 & 4 p .818 & .3175 & 0.0\end{array}$

unit 2

$\begin{array}{llllll}\text { cylinder } & 1 & 1 & 0.515 & 2.54 & 0.0\end{array}$

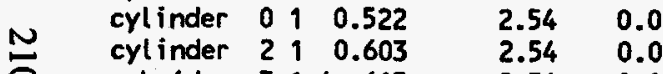

$\begin{array}{llllll}\text { cylinder } & 2 & 1 & 0.603 & 2.54 & 0.0 \\ \text { cuboid } & 3 & 1 & 4 p .615 & 2.54 & 0.0\end{array}$

$\begin{array}{llllll}\text { cuboid } & 3 & 1 & 4 p .615 & 2.54 & 0.0 \\ \text { cuboid } & 2 & 1 & 4 p .818 & 2.54 & 0.0\end{array}$

unit 3

97.46250 .0

$\begin{array}{llllll}\text { cylinder } & 0 & 1 & 0.522 & 97.4625 & 0.0\end{array}$

cylinder $210.603 \quad 97.46250 .0$

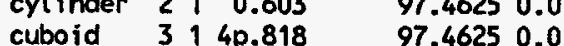

cuboid $314 \mathrm{p} .818$

97.46250 .0

array $13 * 0.0$

reflector 3 i 4 r.818 2 ro

global unit 5

array $2 \quad 3 * 0.0$

reflector $215 r 0.05 .08$

reflector $315 r 0.02 .54$

reflector $315 \mathrm{~s} .02 .541$

$\begin{array}{lllll}\text { reflector } 3 & 1 & 4 \mathrm{r} 24 & 2 \mathrm{r} 0 & 1\end{array}$

end geom

read array

ara=1 nux=14 nuy=14 nuz=3 fill 196r1 196r2 196r3 end fill

ara $=2$ nux $=3$ nuy $=3$ nuz=1 fill f4 end fill

end array

end data

end

latticecell

uo2 1 den=10.22 1.0293922352 .4599223897 .541

al 21.0

h2o 31.0

boron 3 den $=143 e-6$

end comp

squarepitch 1.6361 .030131 .20621 .0440 end

9-14×14 assemblies separated by $4.908 \mathrm{~cm}$ of water, $149.12 \mathrm{~cm}$ water

'height with $143 \mathrm{ppm}$ boron

read parm run=yes gen $=405$ npg $=600$ nsk $=5$ nub=yes end parm

read geom

unit 1

cyl inder $\begin{array}{lllll}1 & 1 & 0.515 & 0.635 & 0.0\end{array}$

cyl inder $\begin{array}{lllll}0 & 1 & 0.522 & 0.635 & 0.0\end{array}$

$\begin{array}{llll}\text { cuboid } 2 & 14 \mathrm{p0} .818 & 0.635 & 0.0\end{array}$

unit 2

cyl inder $1110.515 \quad 148.83250 .0$

cylinder $0110.522 \quad 148.83250 .0$

cyl inder $2110.603 \quad 148.83250 .0$

cuboid $314 \mathrm{p0} 0.818 \quad 148.83250 .0$

unit 3

cuboid $214 \mathrm{p} 0.818 \quad 0.635 \quad 0.0$

unit 4

cylinder $21 \quad 0.635 \quad 148.83250 .0$

cuboid $3,4 \mathrm{p0.818} \quad 148.83250 .0$

unit 5

array $13 * 0,0$

unit 6

array $23 * 0.0$

unit 7

$\begin{array}{lll}\text { array } 3 & 2 r-22.904 & 0.0\end{array}$

$\begin{array}{lllllll}\text { reflector } 31 & 2.454 & 9.808 & 2.454 & 9.808 & 2 r 0 & 1\end{array}$

unit 8

array $3 \quad 2 r-22.904 \quad 0.0$

$\begin{array}{llllll}\text { reflector } 31 & 2 r 2.454 & 2.454 & 9.808 & 2 r 0,\end{array}$

unit 9

$\begin{array}{lll}\text { array } 3 & 2 r-22.904 & 0.0\end{array}$

$\begin{array}{lllllll}\text { reflector } 31 & 9.808 & 2.454 & 2.454 & 9.808 & 2 r 0 & 1\end{array}$

unit 10

$\begin{array}{lll}\text { array } 3 & 2 r-22.904 & 0.0\end{array}$

$\begin{array}{llllll}\text { reflector } 31 & 2.454 & 9.808 & 2 r 2.454 & 2 r 0 & 1\end{array}$

unit 11

array $3 \quad 2 r-22.904 \quad 0.0$

reflector $31 \quad 4 r 2.454$ 
unit 12

$\begin{array}{lll}\text { array } 3 & 2 r-22.904 & 0.0\end{array}$

$\begin{array}{llllll}\text { reflector } 31 & 9.808 & 2.454 & 2 r 2.454 & 2 r 0 & 1\end{array}$

unit 13

$\begin{array}{lll}\text { array } 3 & 2 r-22.904 & 0.0\end{array}$

$\begin{array}{lllllll}\text { reflector } 31 & 2.454 & 9.808 & 9.808 & 2.454 & 2 \text { ro } & 1\end{array}$

unit 14

array $3 \quad 2 r-22.904 \quad 0.0$

$\begin{array}{llllll}\text { reflector } 31 & 2 r 2.454 & 9.808 & 2.454 & 2 r 0 & 1\end{array}$

unit 15

$9.8082 .4542 r 0$

$2 r-22.904 \quad 0.0$

reflector $31 \quad 9.808 \quad 2.454 \quad 9.808 \quad 2.454 \quad 2$ ro

array $4 \quad 2 *-45.808 \quad 0.0$

reflector $21 \quad 5$ ro 1.905

reflector $21 \quad 5 r 02.54$

reflector $31 \quad 5 r 00.31751$

reflector $21 \quad 5 \mathrm{r} 05.08$,

reflector $31 \quad 4$ r20 2 ro

end geom

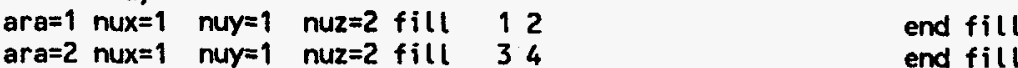

read array

ara $=2$ nux $=1$ nuy=1 nuz=2 fill 34

end $\mathrm{fil}$

$N \quad$ ara=4 nux=3 nuy $=3$ nuz=1 fill $78991011 \quad 121314 \quad 15$ end fill

end array

end data

end

bu1484c1

$=\operatorname{csas} 25$

bw1484c1

44group latticecel

uo2 1 den=10.22 1.0 293922352.4599223897 .541

al 21.0

h20 31.0

b4c 4 den=1.28 0.986

arbmb2031.282110500028016340.001 end

end comp

squarepitch 1.6361 .030131 .20621 .0440 end

-14x14 assemblies separated by $1.636 \mathrm{~cm}$ of water containing $84 \mathrm{~B}, \mathrm{C}$

rods, $145.68 \mathrm{~cm}$ water height

read parm run=yes gen $=405$ npg $=600$ nsk $=5$ nub=yes end parm

read geom

unit 1

cylinder $210.603 \quad .3175 \quad 0.0$

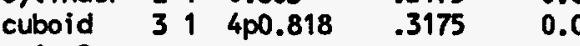

unit 2

.0

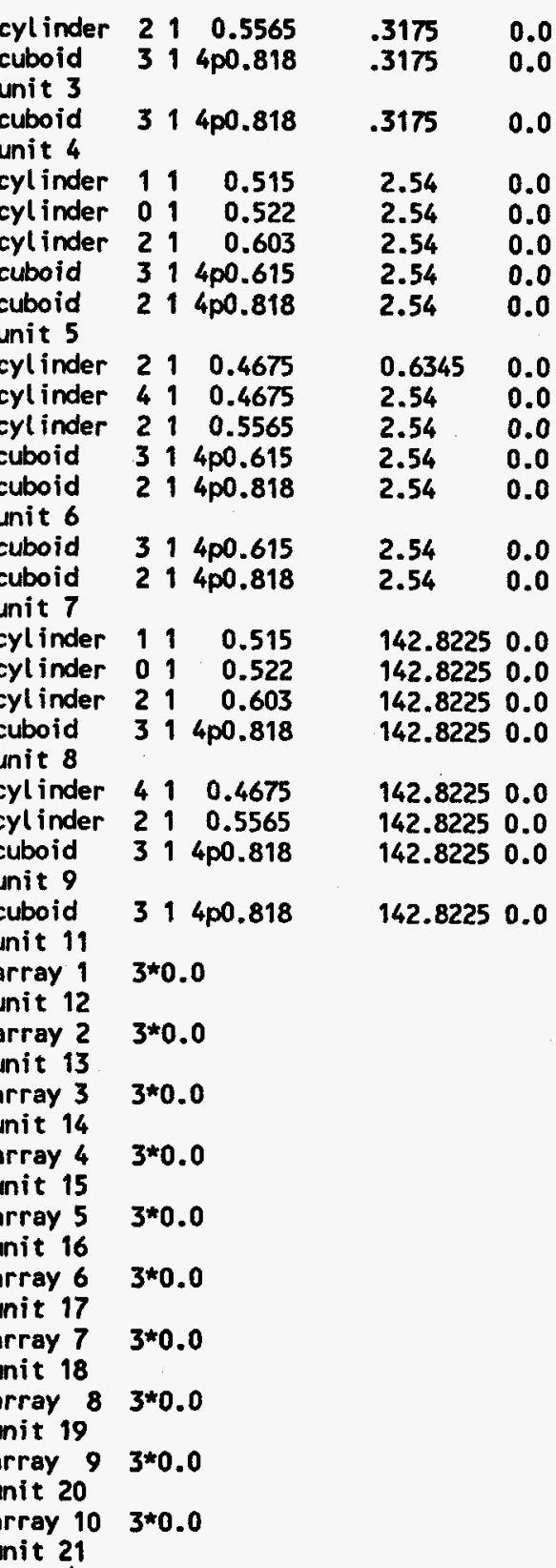


read array

ara=1 nux=1 nuy=1 nuz=3 fill $14 \quad 4 \quad 7$ end fill

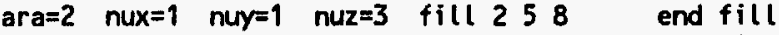
ara $=3$ nux $=1$ nuy=1 nuz $=3$ fill 3699 end fill ara $=4$ nux $=14$ nuy $=14$ nuz $=1$ fill $f 11$ end fill

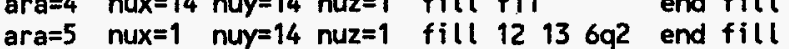
ara $=5$ nux $=1$ nuy=14 nuz=1 fill $12 \quad 13 \quad 6 q 2$ end fill ara $=6$ nux $=1$ nuy $=14$ nuz $=1$

fill $13 \quad 12 \quad 2 q 213 \quad 13 \quad 12 \quad 13 \quad 2 q 2 \quad$ end fill ara $a=7$ nux=1 nuy=14 nuz=1 fill $13 \quad 12 \quad 6 q 2$ end fill ara $=8$ nux $=5$ nuy $=1 \quad n u z=1$

fill $14 \quad 15 \quad 14 \quad 15 \quad 14$

end fill

ara $=9$ nux $=5$ nuy $=1$ nuz $=1$

fill $14 \quad 16 \quad 14 \quad 1614$

end fill

ara $=10$ nux $=5$ nuy $=1$ nuz=1

fill $14 \quad 17 \quad 14 \quad 17 \quad 14$

end fill

ar $a=11$ nux $=44$ nuy $=1$ nuz=1

$\cong \quad$ fill $13 \quad 13 \quad 12 \quad 13 q 2 \quad 12 \quad 12 \quad 13 \quad 6 q 2$

end fill

ara $=12$ nux $=1$ nuy $=5$ nuz=1

fill 1821192120

end fill

end array

end data

end

b-1484c2

$=\operatorname{csas} 25$

bw $1484 \mathrm{c2}$

44 group latticecell

uo2 1 den=10.22 $1.029392235 \quad 2.4599223897 .541$

at 21.0

h20 31.0

b4c 4 den $=1.28 \quad 0.986$

arbmb203 1.282110500028016340 .001 end comp

squarepitch $1.636 \quad 1.030 \quad 131.20621 .0440$

cylinder $210.5565 \quad .3175 \quad 0.0$

$\begin{array}{llllll}\text { cuboid } \quad 312 \mathrm{p} 2.454 & 2 \mathrm{p} .818 & 0.3175 & 0.0\end{array}$

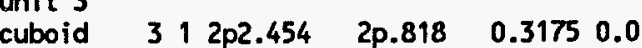

unit 4

cylinder $11 \quad 0.515 \quad 2.54 \quad 0.0$

cylinder $\begin{array}{lllll}0 & 1 & 0.522 & 2.54 & 0.0\end{array}$

cylinder $21 \quad 0.603 \quad 2.54 \quad 0.0$

cuboid 314 p $0.615 \quad 2.54 \quad 0.0$

$\begin{array}{llll}\text { cuboid } 214 \mathrm{p0} .818 & 2.54 & 0.0\end{array}$

unit 5

cylinder $210.4675 \quad 0.6345 \quad 0.0$

$\begin{array}{llllll}\text { cylinder } & 4 & 1 & 0.4675 & 2.54 & 0.0 \\ \text { cylinder } & 2 & 1 & 0.5565 & 2.54 & 0.0\end{array}$

$\begin{array}{llllll}\text { cylinder } & 2 & 1 & 0.5565 & 2.54 & 0.0 \\ \text { cuboid } & 3 & 1 & 4 \mathrm{p} 0.615 & 2.54 & 0.0\end{array}$

$\begin{array}{lllll}\text { cuboid } 212 p 2.454 & 2 p .818 & 2.54 & 0.0\end{array}$

unit 6

cuboid $314 \mathrm{p} 0.615 \quad 2.540 .0$

$\begin{array}{llllll}\text { cuboid } \quad 2 & 1 & 2 \mathrm{p} 2.454 & 2 \mathrm{p} .818 & 2.54 & 0.0\end{array}$

unit 7

cylinder $110.515 \quad 108.6325 \quad 0.0$

cylinder $0110.522 \quad 108.6325 \quad 0.0$

cylinder $210.603108 .6325 \quad 0.0$

$\begin{array}{lllll}\text { cuboid } 314 \mathrm{p0} & 3.818 & 108.6325 & 0.0\end{array}$

cylinder 410.4675108 .6325

cylinder 410.4675108 .6325

108.63250

cuboid $312 \mathrm{p} 2.454 \quad 2 \mathrm{p} .818 \quad 108.63250 .0$

unit 9

$\begin{array}{lllllll}\text { cuboid } \quad 312 p 2.454 & 2 p .818 & 108.6325 & 0.0\end{array}$

unit 10

$\begin{array}{lllll}\text { cyl inder } 21 & 0.5565 & .3175 & 0.0\end{array}$

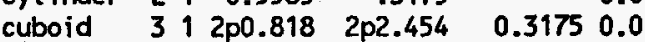

unit 11

cylinder $\begin{array}{lllll}2 & 1 & 0.4675 & 0.6345 & 0.0\end{array}$

cylinder $4 \begin{array}{llll}4 & 0.4675 & 2.54 & 0.0\end{array}$

cylinder $210.5565 \quad 2.54 \quad 0.0$

cuboid 314 p0.615 $2.54 \quad 0.0$

$\begin{array}{llllll}\text { cuboid } \quad 21 & 2 \mathrm{p0} .818 & 2 \mathrm{p} 2.454 & 2.54 & 0.0\end{array}$

unit 12

cylinder $41 \quad 0.4675108 .6325,0.0$

cylinder $210.5565108 .6325 \quad 0.0$

$\begin{array}{lllllll}\text { cuboid } 3 & 1 & 2 \mathrm{p} 0.818 & 2 \mathrm{p} 2.454 & 108.6325 & 0.0\end{array}$

unit 13

$\begin{array}{llllll}\text { cuboid } 31 & 2 \mathrm{p} 0.818 & 2 \mathrm{p} 2.454 & 0.3175 & 0.0\end{array}$

rods, $111.49 \mathrm{~cm}$ water height

read parm run=yes gen $=405 \mathrm{npg}=600 \mathrm{nsk}=5$ nub=yes end parm

read geom

unit

unit 14

cuboid $314 \mathrm{p0} 0.615 \quad 2.54 \quad 0.0$

cuboid $212 p 0.818 \quad 2 p 2.454$

unit 15 
cuboid

unit 16

array 1

unit 17

array 2

array 3

unit 19

array 4

unit 20

array 5

unit 21

array 6

unit 23

array $8 \quad 3 * 0.0$

unit 24

array $93 * 0.0$

unit 25

array $10 \quad 3 * 0.0$

unit 26

array $11 \quad 3 * 0.0$

global unit 27

array $12 \quad 3 * 0.0$

$\begin{array}{llllllll}\text { cuboid } 2 & 1 & 78.528 & 0.0 & 78.528 & 0.0 & 111.49 & -5.08\end{array}$ cuboid $3178.528 \quad 0.0 \quad 78.528 \quad 0.0 \quad 111.49 \quad-7.62$ $\begin{array}{llllllll}\text { cuboid } 2 & 1 & 78.528 & 0.0 & 78.528 & 0.0 & 111.49 & -8.89\end{array}$ reflector $314 \mathrm{r3} 2 \mathrm{rO} 8$

end geom

read array

ara $=1$ nux=1 nuy=1 nuz=3 fill 1447 end fill ara $=2$ nux=1 nuy=1 nuz=3 fill 2558 end fill ara $=3$ nux=1 nuy=1 nuz=3 fill 369 and fill ara $=4$ nux $=1$ nuy=1 nuz=3 fill $10 \quad 11 \quad 12$ end fill ara $=5$ nux $=1$ nuy $=1$ nuz=3 fill 13 14 15 end fill ara $=6$ nux $=14$ nuy $=14$ nuz $=1$ fill $f 16$ end fill ara $=7$ nux=1 nuy=14 nuz=1

fill 2 r18 174 r18 $17 \quad 3 r 18172 r 18 \quad$ end fill ara $=8$ nux $=5$ nuy $=1$ nuz $=1$

fill 2122212221

end fill

ara $=9$ nux $=14$ nuy=1 nuz=1

end fill

ara $=10$ nux $=20$ nuy $=1$ nuz $=1$

fill 5 r20 $198 \mathrm{r} 20195 \mathrm{r} 20$ ara=11 nux=3 nuy=1 nuz=1 fill 242524 end fill ara $=12$ nux $=1$ nuy $=5$ nuz $=1$

fill 2326232623

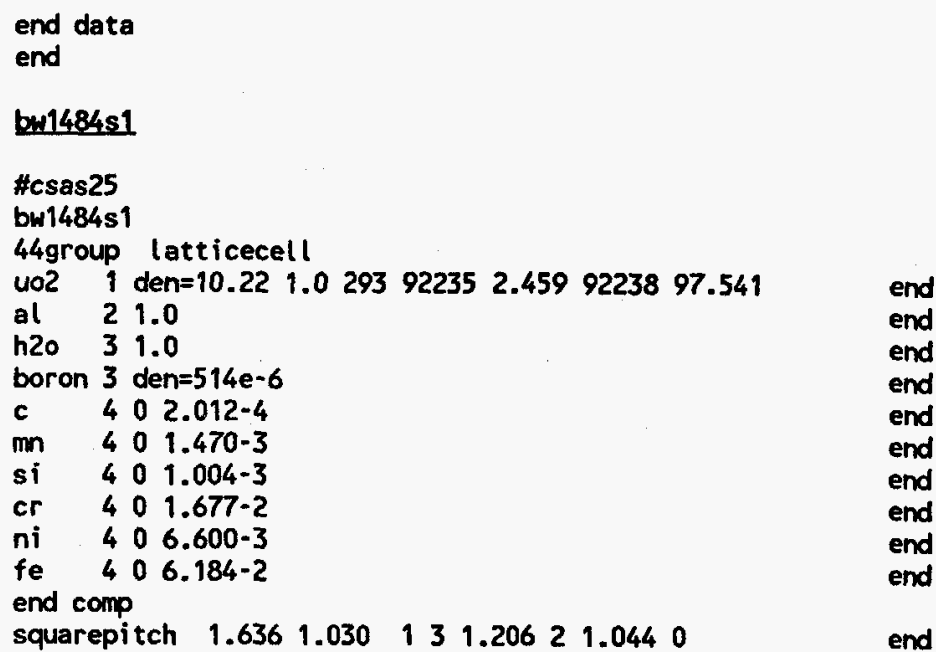

9-14×14 assemblies separated by $0.645-\mathrm{cm}$-thick $304-\mathrm{L}$ stainless steel
'plates and $0.991 \mathrm{~cm}$ of water, plates-to-center assembly distance of $10.4525 \mathrm{~cm}, 149.90 \mathrm{~cm}$ water height with 514 ppm boron
ing read parm run=yes gen $=405 \mathrm{npg}=600 \mathrm{nsk}=5$ nub=yes end parn

read geom

unit 1

cylinder $1110.515 \quad 0.635 \quad 0.0$

cylinder $011.0 .522 \quad 0.635 \quad 0.0$

$\begin{array}{llll}\text { cuboid } 2 & 14 \mathrm{p} 0.818 & 0.635 & 0.0\end{array}$

unit 2

cyl inder $110.515 \quad 147.04250 .0$

cylinder $0110.522 \quad 147.04250 .0$

cylinder $21 \quad 0.603 \quad 147.04250 .0$

cuboid $314 \mathrm{p} 0.818 \quad 147.04250 .0$

unit 3

$\begin{array}{llll}\text { cuboid } 214 \mathrm{p} 0.818 & 0.635 & 0.0\end{array}$

unit 4

cylinder $21 \quad 0.635 \quad 147.04250 .0$

cuboid $314 \mathrm{p0.818} \quad 147.04250 .0$

unit 5

array $13 * 0.0$

unit 6

array $23 * 0.0$

unit 7

$\begin{array}{lll}\text { array } 3 & 2 r-22.904 & 0.0\end{array}$

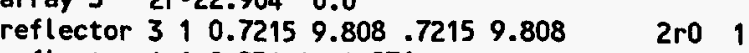

reflector $410.2310 .0 .231 \quad 3 r 0$

unit 8 array $3.2 r-22.904 \quad 0.0$

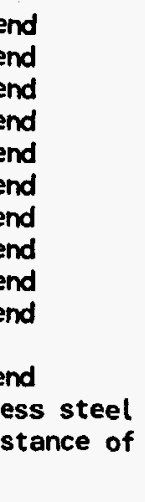


array $3 \quad 2 r-22.904 \quad 0.0$

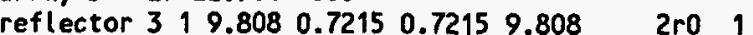

reflector $410.2 \times 0.231$

$\begin{array}{lll}\text { array } 3 & 2 r-22.904 & 0.0\end{array}$

reflector $310.7215 \quad 9.8082 r 0.4525 \quad 2 r 0 \quad 1$

reflector $410.2310 .260 .231 \quad 2 r 0$,

unit 11

array $32 r-22.904 \quad 0.0$

reflector 314 ro.4525

reflector $414 \mathrm{r0} .231$

unit 12

$\begin{array}{lll}\text { array } 3 & 2 r-22.904 & 0.0\end{array}$

reflector $3 \quad 9.808 \quad 0.7215 \quad 2 r 0.4525 \quad 2 r 0 \quad 1$

reflector $4 \uparrow 0.3 r 0.231$

unit 13

reflector 410.2312 2ro. 0.231

unit 14

array $3 \quad 2 r-22.904 \quad 0.0$

reflector $319.808 \quad 0.72159 .808 \quad 0.7215 \quad 2 r 0$

reflector $410.0 .2310 .0 .231 \quad 2 \mathrm{ro} \quad 1$

global unit 16

array $4 \quad 2 *-45.8080 .0$

reflector 215 ro 1.9051

reflector $215 \mathrm{ro} 2.54$

reflector 315 ro 0.3175 i

reflector 215 ro 5.08

reflector 314 r20 2 ro

end geom

\section{read array}

araz=1 nux=1 nuy=1 nuz=2 fill 12

ara $a=2$ nux=1 nuy=1 nuz=2 fill 34

1914612556 and fill ara=4 nux $=3$ nuy $=3$ nuz=1 fill $78891011 \quad 1213 \quad 14 \quad 15$ end fill end array end data

44group latticecell

uo2 1 den=10.22 1.0293922352 .4599223897 .541

al 21.0

h20 31.0

boron 3 den $=432 e-6$

c $402.012-4$

m $401.470-3$

si $409.004-3$

si $401.004-3$

cr $4001.677-2$

ni $406.600-3$

fe $406.184-2$

end comp

squarepitch 1.6361 .030131 .20621 .0440

$9-14 \times 14$ assemblies separated by $0.645-\mathrm{cm}$-thick $304-\mathrm{L}$ stainless steel iplates and $0.991 \mathrm{~cm}$ of water, plates-to-center assembly distance of $10.4525 \mathrm{~cm}, 100.89 \mathrm{~cm}$ water height with $432 \mathrm{ppm}$ boron

read parm run=yes gen $=405 \mathrm{npg}=600$ nsk $=5$ nub=yes end parm

\section{read geom}

unit 1

cylinder $110.515 \quad 0.635 \quad 0.0$

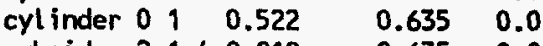

cuboid $21400.818-0.635 \quad 0.0$

cuboid

cylinder $1110.515 \quad 98.03250 .0$

cylinder 0 $1 \quad 0.522 \quad 98.03250 .0$

cylinder $21 \quad 0.603 \quad 98.03250 .0$

cuboid $314 \mathrm{p0.818} 98.03250 .0$

unit 3

$\begin{array}{llll}\text { cuboid } 214 p 0.818 & 0.635 & 0.0\end{array}$

unit 4

cylinder $21 \quad 0.635 \quad 98.03250 .0$

cuboid $314 \mathrm{p0.818} 98.03250 .0$

unit 5

array $13 * 0.0$

unit 6

array $23 * 0.0$

unit 7

array 3

$\begin{array}{lll}3 & 2 r-22.904 & 0.0\end{array}$

reflector $3 \quad 1 \quad 0.7215 \quad 9.808 \quad 0.7215 \quad 9.808 \quad 2 r 0 \quad 1$

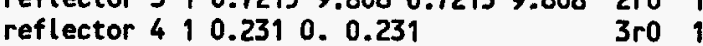

unit 8

array $3 \quad 2 r-22.904 \quad 0.0$

reflector 31 2r0.4525 $0.7215 \quad 9.808 \quad 2 r 0 \quad 1$

reflector $413 \mathrm{ro} .231$ 
unit 9

array $3 \quad 2 r-22.904 \quad 0.0$

reflector $319.808 \quad 0.7215 \quad 0.72159 .808 \quad 2$ ro

reflector $410.2 r 0.231$

unit 10

array $3 \quad 2 r-22.904 \quad 0.0$

reflector 310.72159 .808 2r0.4525 2ro

reflector $4 \begin{array}{lllll}0 & 0.231 & 0.2 r 0.231 & 2 r 0 & 1\end{array}$

unit 11

array $3 \quad 2 r-22.904 \quad 0.0$

reflector $314 r 0.4525 \quad 2 r 0$

reflector $414 \mathrm{r0} .231$

unit 12

$\begin{array}{lll}\text { array } 3 & 2 r-22.904 & 0.0\end{array}$

reflector $319.808 \quad 0.7215 \quad 2 r 0.4525 \quad 2 r 0$

reflector $410.3 r 0.231 \quad 2 r 01$

unit 13

$\begin{array}{lllllll}\text { array } 3 & 2 r-22.904 & 0.0 & & & & \end{array}$

reflector $4 \quad 10.231$ 2ro. $0.231 \quad 0.7215$

unit 14

array $3 \quad 2 r-22.904 \quad 0.0$

reflector $312 r 0.4525 \quad 9.808 \quad 0.7215 \quad 2 r 0$

reflector 412 ro.231 0. $0.231 \quad 2 r 0$

$N$ unit 15

$\begin{array}{lll}\text { array } 3 & 2 r-22.904 & 0.0\end{array}$

reflector $319.808 \quad 0.7215 \quad 9.808 \quad 0.7215 \quad 2$ ro

reflector $410.0 .2310 .0 .231 \quad 2$ ro

global unit 16

array $42 *-45.8080 .0$

reflector $21.5 r 01.9051$

reflector $215 \mathrm{r} 02.541$

reflector 315 ro 0.31751

reflector 215 ro 5.08 ,

reflector $314 \mathrm{r} 202 \mathrm{rO}$

end geom

read array

ara $=1$ nux $=1$ nuy=1 nuz=2 fill 112

end fill

ara $=2$ nux=1 nuy=1 nuz=2 fill $3 \quad 4$

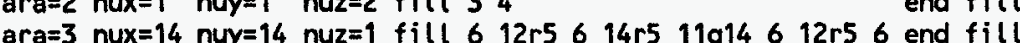
$a_{r a}=4$ nux $=3$ nuy $=3$ nuz $=1$ fill $78991011 \quad 121314 \quad 15$ end fill end array

end data

end

bu1484sl

\#csas 25

bw1484si

44group latticecell uo2 1 den=10.22 1.0 293922352.4599223897 .541

al 21.0

h2o 31.0

end

end comp

squarepitch 1.6361 .030131 .20621 .0440

$9-14 \times 14$ assemblies separated by $6.544 \mathrm{~cm}$ of water, $129.65 \mathrm{~cm}$ water 'height

read parm run=yes gen $=405$ npg=600 nsk $=5$ nub=yes end parm

read geom

unit 1

cylinder $210.603 \quad .31750 .0$

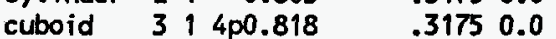

unit 2

cyl inder $1110.515 \quad 2.540 .0$

cylinder $0110.522 \quad 2.54 \quad 0.0$

cyl inder $21 \quad 0.603 \quad 2.540 .0$

cuboid $314 \mathrm{p0} .615 \quad 2.540 .0$

cuboid $214 \mathrm{p0.818} \quad 2.540 .0$

unit 3

cyl inder $110.515 \quad 126.7925 \quad 0.0$

cylinder $0110.522 \quad 126.7925 \quad 0.0$

$\begin{array}{lllll}\text { cylinder } 2 & 1 & 0.603 & 126.7925 & 0.0\end{array}$

$\begin{array}{lllll}\text { cuboid } 314 \mathrm{p} 0.818 & 126.7925 & 0.0\end{array}$

unit 4

reflector $314 r 3.272$ 2ro 1

global unit 5

array $23 * 0.0$

reflector 215 ro.0 5.08

reflector $315 r 0.02 .54$

reflector $215 r 0.01 .27$

reflector 314 r24. 2ro.

end geom

read array

ara=1 nux $=14$ nuy=14 nuz=3 fill 196r1 196r2 196r3 end fill ara $=2$ nux $=3$ nuy $=3$ nuz $=1$ fill $f 4$

end $\mathrm{fill}$ end array

end data

end

bu1645s1

\section{\#csas25}

bw1645s 1

44 group

al

h2o
boron

latticecell

0.9325293922352 .4599223897 .541

21.0293

31.0293

3 den $=746.0-6$ end

end

end 
$\begin{array}{lllll}\text { al } & 4 & .25 & 293 & \text { end } \\ \text { h20 } & 4 & .75 & 293 & \text { end } \\ \text { boron } & 4 & \text { den=746.0-6 } & .75 & \text { end } \\ \text { carbonsteel } & 5 & 1.0293 & \text { end } \\ \text { end comp } & & & \end{array}$

squarepitch $1.20901 .030013 \quad 1.20602 \quad 1.04400$ end

$9-15 \times 15$ assemblies separated by $0.0762-\mathrm{cm}-$ thick aluminum plates and

$-1.6256 \mathrm{~cm}$ of water, $100.24 \mathrm{~cm}$ water height wi th $746 \mathrm{ppm}$ boron

read parm gen $=405 \mathrm{npg}=600 \mathrm{nsk}=5$ nub=yes run=yes end parm

read geom

unit

cylinder 210.6030

cuboid $\quad 314 \mathrm{p} .6045$

unit 2

cylinder $\quad 1110.5150$

cylinder 010.5220

cyl inder 210.6030

cuboid $314 \mathrm{p} .6045$

unit 3

$\begin{array}{lllll}\text { array } \quad 1 & -9.0675 & -9.0675 & -0.318\end{array}$

cuboid $\quad 312 \mathrm{p} 9.9552 \mathrm{p} 9.0675$

unit 4

$100.24-.318$

$100.24-.318$

$100.24 \cdot .318$

$\begin{array}{llllllll}\text { unit } 5 & & & & & & & \\ \text { cuboid } & 3 & 1 & 2 p 7.62 & 2 p 0.8113 & 100.24 & -.318\end{array}$

$\begin{array}{llllll}\text { cuboid } \quad 2 & 1 & 2 \mathrm{p} 7.62 & 2 \mathrm{p} 0.8875 & 100.24 & -.318\end{array}$

cuboid $\quad 312 \mathrm{pg} .9552 \mathrm{p} 0.8875 \quad 100.24 \cdot .318$

global unit 6

$\begin{array}{lllll}\text { array } & 2 & -49.775 & -48.8875 & -.318\end{array}$

reflector $415 r 0 \quad 1.0671$

reflector $215 r_{0} \quad 1.8151$

reflector $\begin{array}{lllllllll}3 & 1 & 10 & 10 & 10 & 10 & 0 & 0 & 1\end{array}$

reflector $214 r 0 \quad 0 \quad 8.91$

reflector 314 ro $\quad 0 \quad 2.861$

$\begin{array}{lllllrl}\text { reflector } & 5 & 1 & 4 \mathrm{r} 0 & 0 & 1.0 & 1 \\ \text { reflector } & 3 & 1 & 4 \mathrm{r} 20 & 0 & 0 & 1\end{array}$

end geom

read array

ara $=1$ nux $=15$ nuy $=15$ nuz=1

fill $113 r 2 \quad 1 \quad 15 r 2 \quad 12 q 15 \quad 1 \quad 13 r 21$

$100.24 \quad 0.0$

$100.24 \quad 0.0$

$\begin{array}{ll}100.24 & -.318 \\ 100.24 & -.318\end{array}$

end array

end data

end

bu1645s?

\#csas 25

bw1645s2
44 group latticecell

no2

al

boron 3 den=886.0-6

al $\quad 4 \quad .25293$

he 4. $\quad .75293$

boron 4 den=886.0-6 .75

carbonsteel 5 1.0.293

end comp

squarepitch 1.20901 .0300131 .206021 .04400

end

end

end

end

end

end

end

$9-15 \times 15$ assemblies separated by $0.0762-\mathrm{cm}$-thick aluminum plates and

$1.6256 \mathrm{~cm}$ of water, $145.00 \mathrm{~cm}$ water height with $886 \mathrm{ppm}$ boron

read parm gen $=405$ npg=600 nsk $=5$ nub=yes run=yes

end parm

read geom

unit 1

cylinder $\quad 210.6030 \quad 145.0 \quad-.318$

cuboid $314 p .6045 \quad 145.0-318$

unit 2

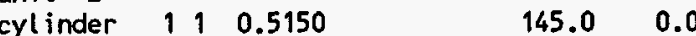

cylinder $010.5220 \quad 145.0 \quad 0.0$

cylinder $210.6030 \quad 145.0 \quad-.318$

$\begin{array}{lllll}\text { cuboid } \quad 314 p .6045 & 145.0 & -.318\end{array}$

unit 3

$\begin{array}{lllll}\text { array } \quad 1 & -9.0675 & -9.0675 & -0.318\end{array}$

$\begin{array}{llllll}\text { cuboid } \quad 312 p 9.955 & 2 \mathrm{p} 9.0675 & 145.0 & -.318\end{array}$

unit 4

cuboid

$312 \mathrm{p} 9.9552 \mathrm{p0} 0.8875$

$145.0-.318$

unit 5

$\begin{array}{lllll}3 & 2 p 7.62 & 2 p 0.8113 & 145.0 & -.318\end{array}$

$\begin{array}{llllll}\text { cuboid } \quad 2 & 1 & 2 \mathrm{p} 7.62 & 2 \mathrm{p} 0.8875 & 145.0 & -.318\end{array}$

$\begin{array}{llllll}\text { cuboid } \quad 312 \mathrm{p} 9.9552 \mathrm{p0.8875} & 145.0 & -.318\end{array}$

global unit 6

$\begin{array}{lllll}\text { array } & 2 & -49.775 & -48.8875 & -.318\end{array}$

reflector $415 \mathrm{ro} \quad 1.0671$

reflector 215 ro $\quad \mathbf{1 . 8 1 5} 1$

reflector $31110 \quad 1010 \quad 10000 \%$

reflector $2 \begin{array}{lllll}1 & 4 r 0 & 0 & 8.9 & 1\end{array}$

reflector $3114 \mathrm{ro} \quad 0 \quad 2.86 \quad 1$

reflector 5 1 4 ro 001.0

reflector 314 r20

end geom

read array

ara $=1$ nux $=15$ nuy $=15$ nuz=1

fill $113 r 2 \quad 1 \quad 15 r 2 \quad 12 q 15 \quad 1 \quad 13 r 21$ end fill

ara $=2$ nux $=5$ nuy $=9$ nuz=1

fill $5 r^{3} 43 r 543 q 105 r^{3}$

end fill 
end array

end data

end

\section{bu1645t1}

\section{\#csas25}

bu1645t 1

44 group

uo2

al

h2o

boron

al

boron

boron

end comp

$\begin{array}{lllllllll}\text { triangpitch } & 1.2093 & 1.030 & 1 & 3 & 1.206 & 2 & 1.044 & 0\end{array}$

latticecell

1 den=10.22 1.0293 .922352 .4599223897 .541

end

21.0293

31.0293

3 den $=335.0-6$

.75293

4 den $=335.0-6 \quad .75$

$5 \times 5$ array of hexagonal lattice assemblies separated by $0.0762-\mathrm{cm}-$ thick

'aluminum plates and $1.6256-$ and $1.7926-\mathrm{cm}$ of water, $104.82 \mathrm{~cm}$ water

'height with 335 ppm boron

read parm gen $=405 \mathrm{npg}=600$ nsk $=5$ nub=yes run=yes end parm

read geom

zhemicyl-y 110.515

zhemicyl-y 010.522

zhemicyl-y $21 \quad 0.603$

unit 2

zhemicylty 110.515

zhemicylty $01 \quad 0.522$

zhemicylty 210.603

unit 3

zhemicyl-x 110.515

zhemicyl-x 010.522

zhemicyl-x $21 \quad 0.603$

unit 4

zhemicyl+x 110.515

zhemicyl+x $01 \quad 0.522$

zhemicylt+x 210.603

unit 5

朵

zhemicyl-y 210.603

unit 6

zhemicylty $21 \quad 0.603$

unit 11

zhemicyl-y 110.515

zhemicyl-y $01 \quad 0.522$

zhemicyl-y $21 \quad 0.603$

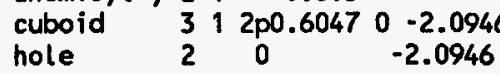

2

0
$104.82 \quad 0.0$

$\begin{array}{ll}104.82 & 0.0 \\ 104.82 & -0.318\end{array}$

$104.82 \quad 0.0$

$104.82 \quad 0.0$

$\begin{array}{ll}104.82 & -0.318\end{array}$

$104.82 \quad 0.0$

$104.82 \quad 0.0$

$104.82-0.318$

$104.82 \quad 0.0$

$104.82 \quad 0.0$

$\begin{array}{ll}104.82 & -0.318\end{array}$

$104.82 \quad-0.318$

$104.82 \quad-0.318$

$\begin{array}{ll}104.82 & 0.0\end{array}$

$104.82 \quad 0.0$

$104.82 \quad-0.318$

$104.82-0.318$ $\begin{array}{lllll}\text { hole } & 3 & +0.6047 & -1.0473 & 0 \\ \text { hole } & 4 & -0.6047 & -1.0473 & 0 \\ \text { unit } 12 & & & & \end{array}$

zhemicyl-y 110.515

zhemicyl-y 010.522

zhemicyl-y $21 \quad 0.603$

cuboid $\quad 312 p 0.60470-2.0946$

$\begin{array}{lllll}\text { hole } & 2 & 0 & -2.0946 & 0 \\ \text { hole } & 3 & +0.6047 & -1.0473 & 0\end{array}$

hole 13

zhemicyl-y 11

zhemicyl-y 010.522

zhemicyl-y $21 \quad 0.603$

cuboid $\quad 312 p 0.60470-2.0946$

$\begin{array}{lllll}\text { hole } & 2 & 0 & -2.0946 & 0\end{array}$

hole 14

zhemicyl-y 110.515

zhemicyl-y 010.522

zhemicyl-y $21 \quad 0.603$

cuboid $\quad 312 \mathrm{p} 0.60470-2.0946$

$\begin{array}{lllll}\text { hole } & 6 & 0 & -2.0946 & 0\end{array}$

hole $3+0.6047-1.04730$

unit 15

zhemicyl-y 110.515

zhemicyl-y $01 \quad 0.522$

zhemicyl-y $21 \quad 0.603$

cuboid $\quad 312 \mathrm{p0} 0.60470-2.0946$

hole $\quad 6 \quad 00.09460$

$\begin{array}{lllll}\text { hole } & 4 & -0.6047 & -1.0473 & 0\end{array}$

unit 16

zhemicyl-y 210.603

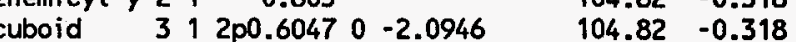

$\begin{array}{llllll}\text { hole } & 2 & 0 & -2.0946 & 0\end{array}$

hole $\quad 3 \quad+0.6047 \begin{array}{llll}-1.0473 & 0\end{array}$

unit 17

zhemicyl-y $21 \quad 0.603$

$\begin{array}{lllll}\text { hole } & 2 & 0 & -2.0946 & 0\end{array}$

$\begin{array}{lllll} & 4 & -0.6047 & -1.0473\end{array}$

unit 18

zhemicyl-y 110.515

zhemicyl-y 010.52

zhemicyl-y $21 \quad 0.603$

cuboid $\quad 312 p 0.60470-.6047$

unit 19

zhemicylty $11 \quad 0.515$

zhemicylty 010.522

zhemicylty 210.603

$\begin{array}{lllllll}\text { zuboid } & 3 & 1 & 2 p 0.6047 .6047 & 0\end{array}$
$104.82 \quad 0.0$

$04.82 \quad 0.0$

$\begin{array}{ll}104.82 & -0.318\end{array}$

$04.82 \quad-0.318$

$104.82 \quad 0.0$

$\begin{array}{ll}104.82 & 0.0\end{array}$

$104.82 \quad-0.318$

$104.82-0.318$

$104.82 \quad 0.0$

$104.82 \quad 0.0$

$\begin{array}{ll}104.82 & -0.318\end{array}$

$104.82-0.318$

$104.82 \quad 0.0$

$104.82 \quad 0.0$

$\begin{array}{ll}104.82 & -0.318\end{array}$

$104.82 \quad-0.318$

$\begin{array}{ll}104.82 & -0.318 \\ 104.82 & -0.318\end{array}$

$104.82 \quad 0.0$

104.820 .0

$104.82-0.318$

$104.82 \quad 0.0$

$\begin{array}{ll}104.82 & 0.0\end{array}$

$104.82-0.318$

$104.82=0.318$ 
unit 6

zhemicyl ty 21.603

unit 11

zhemicyl-y 11 .515

zhemicyl-y 01.522

zhemicyl-y 21.603

$\begin{array}{llllll}142.54 & -0.318 \\ 1 & 2 p .6047 & 0 & -2.0946 & 142.54 & -0.318\end{array}$

$\begin{array}{lllll}\text { hole } & 2 & 0 & -2.0946 & -0.0\end{array}$

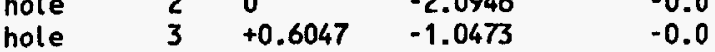

hole $4 \quad-0.6047 \quad-1.0473 \quad-0.0$

unit 12

zhemicyl-y $11.515 \quad 142.540 .0$

zhemicyl-y $011.522 \quad 142.54 \quad 0.0$

$\begin{array}{lllll} & \text { zhemicyl-y } 21.603 & 142.54 & -0.318\end{array}$

$\begin{array}{llllllll}\text { cuboid } & 3 & 1 & 2 \mathrm{p} .6047 & 0 & -2.0946 & 142.54 & -0.318\end{array}$

$\begin{array}{lllll}\text { hole } & 2 & 0 & -2.0946 & -0.0\end{array}$

hole $\quad 3 \quad+0.6047 \quad-1.0473 \quad 0.0$

unit 13

zhemicyl-y $111.515 \quad 142.54 \quad 0.0$

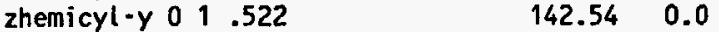

zhemicyl-y $21.603 \quad 142.54 \quad-0.318$

$\begin{array}{llllllll}4 \text { cuboid } & 31 & 2 \mathrm{p} .6047 & 0 & -2.0946 & 142.54 & -0.318\end{array}$

$\begin{array}{lllll}\text { hole } & 2 & 0 & -2.0946 & -0.0\end{array}$

$\begin{array}{lllll}\text { hole } & 4 & -0.6047 & -1.0473 & -0.0\end{array}$

unit 14

N zhemicyl-y $117.515 \quad 142.54 \quad 0.0$

zhemicyl-y $0 \begin{array}{lllll} & 1 & .522 & 142.54 & 0.0\end{array}$

zhemicyl-y $21.603 \quad 142.54 \quad-0.318$

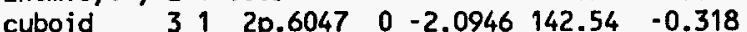

$\begin{array}{lllll}\text { hole } & 6 & 0 & -2.0946 & -0.0\end{array}$

hole $\quad 3 \quad+0.6047 \quad-1.0473 \quad-0.0$

unit 15

zhemicyl-y $111.515 \quad 142.54 \quad 0.0$

$\begin{array}{llllll}\text { zhemicyl-y } & 0 & 1 & .522 & 142.54 & 0.0\end{array}$

zhemicyl-y $21.603 \quad 142.54 \quad-0.318$

$\begin{array}{llllllll} & \text { zheid } & 1 & 2 \mathrm{p} .6047 & 0 & -2.0946 & 142.54 & -0.318\end{array}$

$\begin{array}{lllll}\text { hole } & 6 & 0 & -2.0946 & -0.0\end{array}$

hole $\quad 4 \quad-0.6047 \quad-1.0473 \quad-0.0$

unit 16

zhemicyl-y $21.603 \quad 142.54 \quad-0.318$

$\begin{array}{lllllllll}\text { cuboid } & 3 & 1 & 2 p .6047 & 0 & -2.0946 & 142.54 & -0.318\end{array}$

$\begin{array}{lllll}\text { hole } & 2 & 0 & -2.0946 & -0.0\end{array}$

hole $\quad 3 \quad+0.6047-1.0473 \quad-0.0$

unit 17

zhemicyl-y $21.603 \quad 142.54 \quad-0.318$

$\begin{array}{llllllll}\text { cuboid } & 3 & 1 & 2 p .6047 & 0 & -2.0946 & 142.54 & -0.318\end{array}$

$\begin{array}{lllll}\text { hole } & 2 & 0 & -2.0946 & -0.0\end{array}$

hole $\quad \begin{array}{llll}4 & -0.6047 & -1.0473 & -0.0\end{array}$

unit 18

$\begin{array}{lll}\text { zhemicyl-y } 11.515 & 142.54 & 0.0\end{array}$

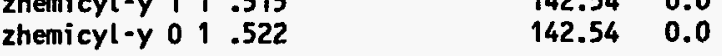

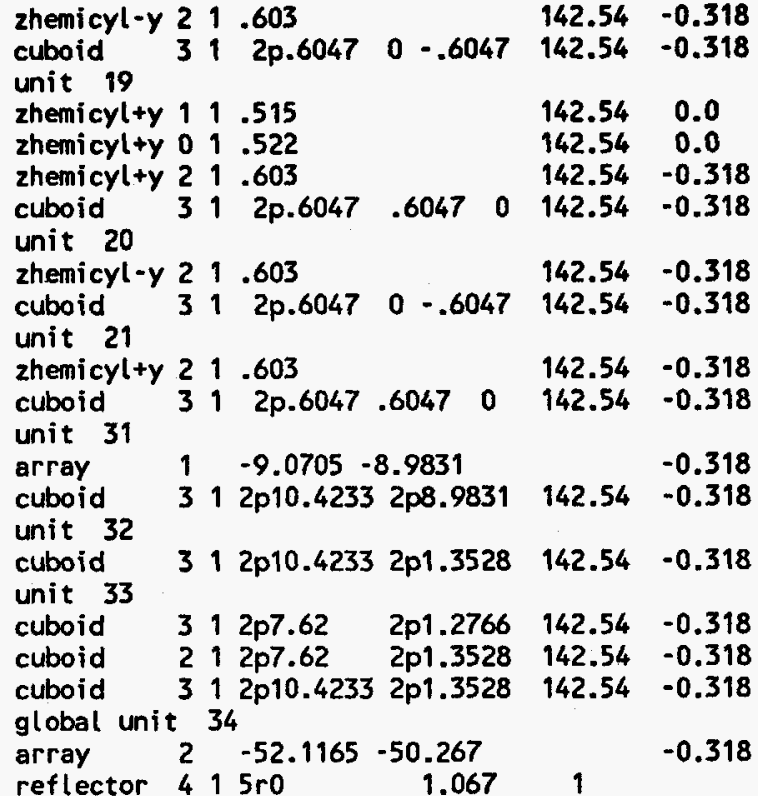

reflector $215 \mathrm{ro} \quad 1.815$

reflector $3112 r 9.3 \quad 2 r 11.0065$ 2ro

reflector 214 ro 008.9

reflector $314 \mathrm{r} 0 \quad 0 \quad 2.86$

reflector $514 \mathrm{ro} \quad 0 \quad 1.0$

reflector 312 r20.7 2r18.9935 2ro

end geom

read array

ara=1 nux=15 nuy=10 nuz=1 fill $20 \quad 13 r 18 \quad 20 \quad 14 \quad 13 r 11 \quad 15 \quad 12 \quad 13 r 11 \quad 13$ $5 q 1516 \quad 13 r 111721 \quad 13 r 1921$

end fill ara $=2$ nux $=5$ nuy=9 nuz=1 fill $5 r 31 \quad 32 \quad 3 r 33 \quad 32 \quad 3 q 10 \quad 5 r 31$ end fill end array

end data

end

bu1645t4

\#csas25

bw1645t4

44 group

402

latticecel

h2o

boron

al end

21.0293

3 1.0293

$\begin{array}{ll}3 & \text { den=121 } \\ 4 & .25293\end{array}$ 
h2o

boron

carbonsteel

$4 \quad .75293$

4 den $=121.0-6.75$

end comp

end

end

$\begin{array}{llllllll}\text { triangpitch } 1.2093 & 1.030 & 1 & 3 & 1.206 & 2 & 1.044 & 0\end{array}$

$5 \times 5$ array of hexagonal lattice assemblies separated by $0.0762-\mathrm{cm}$ end

'aluminum plates and 3.6546 - and $3.8236-\mathrm{cm}$ of water. $145.64 \mathrm{~cm}$ water

'height with 121 ppm boron

read parm gen=405 npg=600 nsk=5 nub=yes run=yes end parm

read geom

unit

zhemicyl-y 110515

zhemicyl-y 001.522

zhemicyl-y 21.603

unit 2

zhemicylty 11 .515

zhemicylty 010.522

zhemicylty 21.603

unit 3

zhemicyl-x 111.515

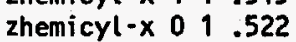

zhemicyl-x 21.603

unit 4

N zhemicyl+x 111.515

zhemicyl+x 011.522

zhemicyltx 21.603

unit 5

zhemicyl-y 21.603

unit 6

zhemicylty 21.603

unit 11

zhemicyl-y 11.515

zhemicyl-y 0101.522

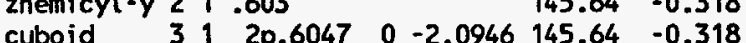

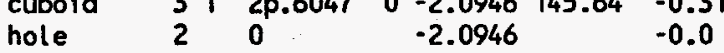

hole $\quad 3 \quad+0.6047 \quad-1.0473 \quad 0.0$

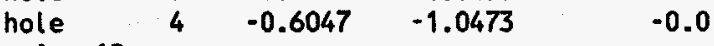

unit 12

zhemicyl-y $11.515 \quad 145.640 .0$

zhemicyl-y $001.522 \quad 145.64 \quad 0.0$

zhemicyl-y $21.603 \quad 145.64-0.318$

$\begin{array}{llllllll}\text { cuboid } & 3 & 1 & 2 p .6047 & 0 & -2.0946 & 145.64 & -0.318\end{array}$

$\begin{array}{lllll}\text { hole } & 2 & 0 & -2.0946 & -0.0\end{array}$

$\begin{array}{lllll}\text { hole } & 3 & +0.6047 & -1.0473 & -0.0\end{array}$

unit 13

zhemicyl-y $11.515 \quad 145.640 .0$

zhemicyl-y $01.522 \quad 145.640 .0$

zhemicyl-y $21.603 \quad 145.64 \quad-0.318$

$\begin{array}{llllllll}\text { cuboid } & 3 & 1 & 2 p .6047 & 0 & -2.0946 & 145.64 & -0.318\end{array}$

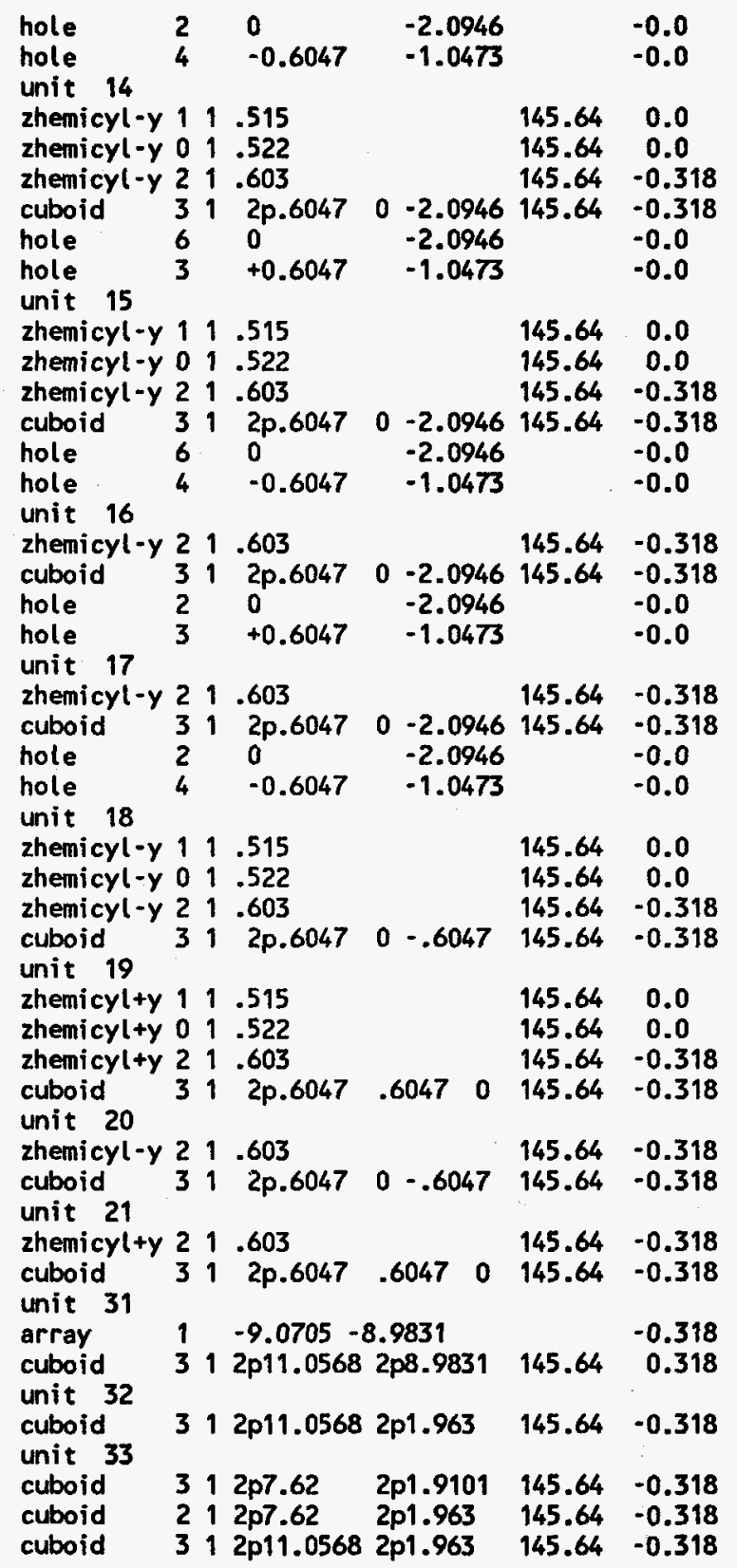


global unit 34

reflector $215 \mathrm{ro}$

$2 \mathrm{r} 6.1252 \mathrm{r} 8.47052 \mathrm{ro}$

reflector $214 \mathrm{ro} \quad 0 \quad 8.9$

reflector 314 ro 002.86

reflector 514 ro 001.0

reflector 312 r23.875 2r21.5295 2ro 1

end geom

\section{read array}

ara=1 nux=15 nuy=10 nuz=1 fill $20 \quad 13 r 18 \quad 20 \quad 14 \quad 13 r 11 \quad 15 \quad 12 \quad 13 r 11 \quad 13$ $5 q 15 \quad 16 \quad 13 r 11 \quad 1721 \quad 13 r 1921 \quad$ end fill ara=2 nux=5 nuy=9 nuz=1 fill $5 r 31 \quad 32 \quad 3 r 33 \quad 32 \quad 3 q 10 \quad 5 r 31$ end fill end array

end data

end

\section{bu1810a}

$=\operatorname{csas} 25$

bw1810a

44 group

$N \quad$ h2o 2 den=10.24 $1.0 \quad 293922352.4599223897 .541$

boron 2 den $=1.0 \quad 0.998761$

al 3

uo2 4 den=10.24 $0.9629392235 \quad 1.9449223898 .056$

gd- 152 \& den $=0.3555 \quad 0.002$

gd $-154 \quad 4$ den $=0.35550 .0218$

d- 1554 den $=0.35550 .148$

gd-156 4 den $=0.3555 \quad 0.2047$

gd-157 4 den=0.3555 0.1565

gd- 1584 den $=0.3555 \quad 0.2484$

gd- 1604 den $=0.35550 .2186$

4 den $=0.05411$

$$
4 \text { den }
$$

squarepitch 1.6361 .0297121 .206031 .04340

more data res $=4$ cylinder .51499 dan $(4)=.189958$ end more dat

Core of $47882.459 \mathrm{wt} \%{ }^{235} \mathrm{U}$ UO $\mathrm{O}_{2}$ fuel rods, $20 \mathrm{UO}_{2}-\mathrm{Gd}_{2} \mathrm{O}_{3}$ fuel rods, 153

water holes, $145.0 \mathrm{~cm}$ water height with $1239.3 \mathrm{ppm}$ boron

read parm gen $=405$ npg=600 nsk $=5$ nub=yes run=yes end parm

read geom

unit 2

cylinder $11.5149 \quad 145.3175$

cylinder $\quad 011.522 \quad 145.317$

cylinder $31.603 \quad 1450$

cuboid $\quad 21.818 \cdot .818 .818 \cdot .8181450$

end

end

end

end

end

end

end

end

end

end

end

nd

end reflector $4,15 r$

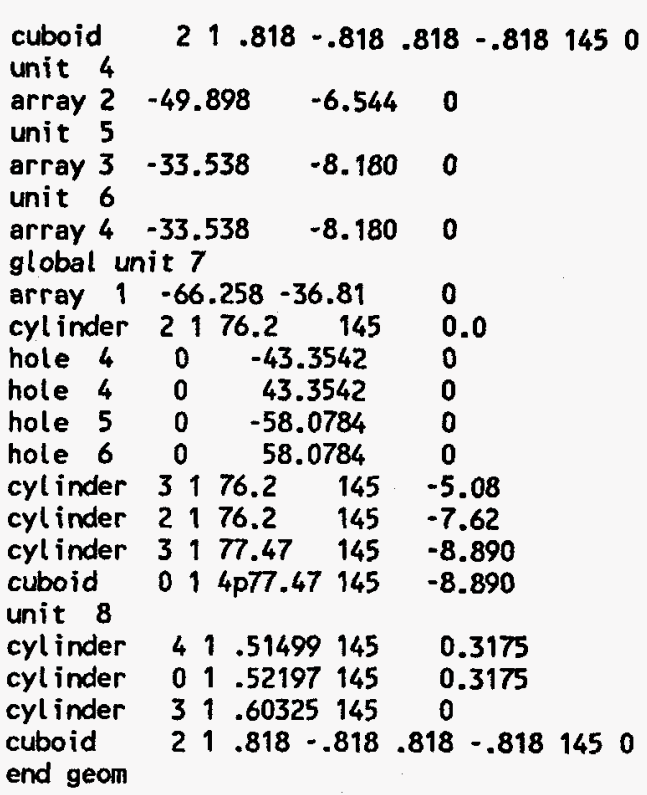

unit 3 read array

ar $a=1$ nux $=81$ nuy $=45$ fill

$10 r^{3} 61 r^{2} 10 r^{3}$

1981

$5 r 3 \quad 15 r 2 \quad 3 r 2 \quad 3 \quad 1 q 4 \quad 10 r 2 \quad 3 \quad 3 r 23 b 39$

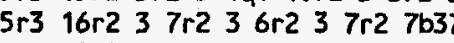

$5 r 371 r 25 r 3$

$\begin{array}{llllllllllllllll}5 r 3 & 15 r 2 & 3 & 2 r 2 & 3 & 3 r 2 & 3 & 2 r 2 & 3 & 4 r 2 & 3 & 2 r 2 & 3 & 3 r 2 & 3 b 39\end{array}$

$5 r 371 r^{2} 5 r 3$

$5 r 320 r 233 r 2810 r 231640$

$5 r 371 r^{2} 5 r^{3}$

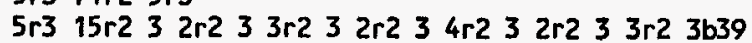

$5 r^{3} 71 r^{2} 5 r^{3}$

$5 r 316 r 233 r 283 r 236 r 237 r 27 b 37$

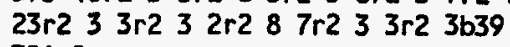

$324 r^{2}$

20r2 3r2 $3 \quad 194 \quad 7 r 2 \quad 8 \quad 2 r 233 r 23 b 39$

$21 r^{2} 37 r 236 r 233 r 281 b 40$

$81 r^{2}$

$\begin{array}{llllllllllllllll}20 r 2 & 3 & 2 r 2 & 3 & 3 r 2 & 3 & 2 r 2 & 3 & 4 r 2 & 3 & 2 r 2 & 3 & 3 r 2 & 3639\end{array}$

$81 \mathrm{r2}$

$25 r 2310 r 283 r 231640$

$81 b 1782$

ara $=2$ nux $=61$ nuy $=8$ fill +2

ara $=3$ nux $=41$ nuy=10 loop

end fill 
$\begin{array}{llllllllll}3 & 1 & 10 & 1 & 1 & 5 & 1 & 1 & 1 & 1\end{array}$

$33241 \quad 1 \quad 1 \quad 5 \quad 1 \quad 111$ end loop ara $=4$ nux $=41$ nuy $=10$ loop

$\begin{array}{llllllllll}2 & 1 & 41 & 1 & 1 & 10 & 1 & 1 & 1 & 1\end{array}$

$\begin{array}{llllllllll}3 & 1 & 10 & 1 & 6 & 10 & 1 & 1 & 1 & 1\end{array}$

$33241,1610,111$ end loop

end array

end data

end

bu1810b

$=\operatorname{csas} 25$

bw $1810 \mathrm{~b}$

44group latticecell

uo2 1 den=10.24 $1.0 \quad 29392235 \quad 2.4599223897 .541$ end

h2o 2 den=1.0 $\quad 0.998829$

boron 2 den=1.0 0.00117

al $3 \quad 1.0$

402 4 den=10.24 $0.96 \quad 29392235 \quad 1.9449223898 .056$

gd-152 4 den $=0.3555 \quad 0.002$

gd-154 4 den $=0.3555 \quad 0.0218$

gd- 1554 den $=0.35550 .148$

gd-155 4 den $=0.3555$ 0.148

gd- 1564 den $=0.35550 .2047$

gd $-158<$ den $=0.35550 .2484$

gd- 1604 den $=0.35550 .2186$

4 den=0.0541 1

end comp

squarepitch 1.6361 .0297121 .206031 .04340 Core of 4772 cylinder .51499 dan $(4)=.189958$ end more data

water hol $2.459 \mathrm{wt} \% \mathrm{U} \mathrm{UO}_{2}$ fuel rods, $36 \mathrm{UO}_{2}-\mathrm{Gd}_{2} \mathrm{O}_{3}$ fuel rods, 153

$145.0 \mathrm{~cm}$ water height with $1170.7 \mathrm{ppm}$ boron

read parm gen $=405 \mathrm{npg}=600$ nsk $=5$ nub=yes run=yes end parm

read geon

unit 2

cylinder 11.5149

cylinder 01.522

cylinder 31.603

cuboid

$1.818-.818 .818-.818$

$145 \quad 0.3175$

unit 3

1450

cuboid

$21.818-.818 .818-.818$

1450

array $2 \quad-49.898 \quad-6.544 \quad 0$

unit 5

array 3

$-33.538 \quad-8.180 \quad 0$

$\begin{array}{llll}\text { array } 4 & -33.538 & -8.180 & 0\end{array}$

global unit 7

array $1-66.258-36.81 \quad 0$ cylinder 2176.2

$145 \quad 0.0$

hole $4 \quad 0 \quad-43.3542$

$\begin{array}{rrrr}\text { hole } & 4 & 0 & 43.3542 \\ \text { hole } & 5 & 0 & -58.0784\end{array}$

hole $6 \quad 0 \quad 58.0784$

cylinder 3176.2

cyl inder 2176.2

cyl inder 3177.47

cuboid $014 \mathrm{p} 77.47$

unit 8

cylinder 41.51499

cylinder 011.52197

cylinder 31.6032

cuboid

$21.818-.818 \quad .818 \cdot .818$

$145 \quad-5.08$

$145 \quad-7.62$

$145-8.890$

$145 \quad-8.890$

end geom

1450.3175

1450

1450

read array

ara $=1$ nux $=81$ nuy $=45$ fill

$10 r 361 r 210 r 3$

1981

$5 r 3 \quad 15 r 23 r 231 \alpha 410 r 233 r 23 b 39$

$5 r^{3} 16 r^{2} 37 r^{2} 36 r^{2} 37 r^{2} 7 b 37$

$5 r 371 r^{2} 5 r 3$

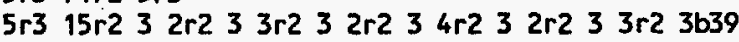

$5 r 371 r 25 r 3$

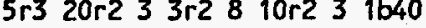

$5 r 371 r 25 r 3$

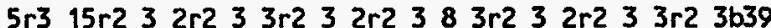

$5 r^{3} 20 r^{2} 829 r^{2} 820 r^{2} 5 r^{3}$

$5 r 3 \quad 16 r 237 r 236 r 237 r 27 b 37$

$23 r 23 \quad 3 r 23$ 2r2 8 7r2 $3 \quad 3 r 23639$

$27 r 28$ 25r2 8 27r2

$162 r^{2}$

$38 r 283 r 2838 r 2$

20r2 $3 r 2 \quad 3 \quad 1 q 4 \quad 7 r 2 \quad 8 \quad 2 r 233 r 23 b 39$

$21 r 237 r 236 r 237 r 27 b 37$

$40 r^{2} 840 r^{2}$

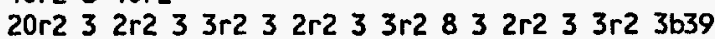

$25 r 23$ 10r2 8 3r2 31640

8161782

and $f i l l$

ara $=2$ nux $=61$ nuy $=8$ fill $f 2$ end fill

ara $=3$ nux $=41$ nuy $=10$ loop

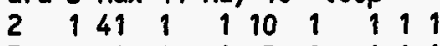

$\begin{array}{llllllllll}3 & 1 & 10 & 1 & 1 & 5 & 1 & 1 & 1 & 1\end{array}$

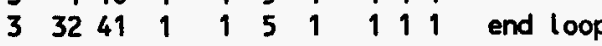

ara $=4$ nux $=41$ nuy $=10$ loop

$\begin{array}{llllllllll}2 & 1 & 41 & 1 & 1 & 10 & 1 & 1 & 1 & 1\end{array}$

$\begin{array}{llllllllll}3 & 1 & 10 & 1 & 6 & 10 & 1 & 1 & 1 & 1\end{array}$

$33241,6101,111$ end loop 


\section{bw1810c}

$=c \operatorname{sas} 25$

bw1810c

44group latticecel

uo2 1 den=9.46 $1.0 \quad 293 \quad 92235 \quad 4.02 \quad 9223895.98 \quad$ end

$\operatorname{ss} 304$

h20 3 den=1.0 0.998501

boron 3 den=1.0 0.001499

uo2 6 den=10.24 0.96293922351 .9449223898 .056

gd -1526 den $=0.35550 .002$

gd-154 6 den $=0.35550 .0218$

gd -1556 den $=0.35550 .148$

gd- 156 o den $=0.35550 .2047$

d -157 den $=0.35550 .2047$

-1586 den $=0.35550 .1565$

gd-160 6 der

6 den $=0.0541$ ?

array $1-66.258-36.81 \quad 0$

cylinder $3176.2 \quad 1450.0$

hole $4 \quad 0 \quad-43.3542 \quad 0$

hole $4 \quad 0 \quad 43.3542 \quad 0$

hole $5 \quad 0 \quad-58.0784 \quad 0$

hole $6 \quad 0 \quad 58.0784 \quad 0$

cylinder $\begin{array}{lllll}5 & 1 & 76.2 & 145 & \mathbf{0 5 . 0 8}\end{array}$

cylinder $31176.2 \quad 145 \quad-7.62$

$\begin{array}{llllll}\text { cylinder } & 5 & 1 & 77.47 & 145 & \mathbf{- 8 . 8 9 0}\end{array}$

$\begin{array}{llllll}\text { cuboid } & 0 & 1 & 4 \mathrm{p} 77.47 & 145 & -8.890\end{array}$

unit 8

cylinder $\quad 61.51499145 .3175$

cylinder $\quad 011.52197 \quad 145 \quad .3175$

cylinder 51.603251450

$\begin{array}{llllll}\text { cuboid } & 3 & 1 & .60325 & 145 & 0 \\ -818 & -.818 & .818 & -.8181450\end{array}$

end geom

read array

ara=1 nux $=81$ nuy $=45$ fill

$10 r 361 r 210 r 3$

$10 r 3 \quad 3 r 26 r 2 \quad 2 r 3 \quad 2 q 8 \quad 7 r 2 \quad 7 b 37$

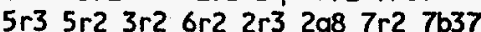

$5 r 371 r^{2} 5 r 3$

1981

$\begin{array}{llllll}5 r 3 & 18 r 2 & 2 r 3 & 14 r 2 & 3 r 3 & 3 b 39\end{array}$

$\begin{array}{lllllll}5 r 3 & 18 r 2 & 2 r 3 & 14 r 1 & 3 r 3 & 3 b 39\end{array}$

$5 r 319 r 233 r 119 r 25 r 3$

$5 r 3$ 19r2 2r1 8 3r1 8 19r1 19b31

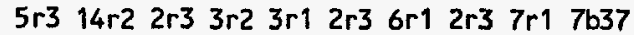

1981

$5 r 319 r 233 r 119 r 25 r 3$

$24 r 2 \quad 2 r 18$ 8 $3 r 18$ 19r1 19b31

$24 r^{2} 33 r_{1} 124 r^{2}$

$1 \mathrm{q} 81$

unit 1

cyl inder $111.5639 \quad 1455.969$

cylinder 21.60391450

$\begin{array}{llllllll}\text { cuboid } \quad 31 & 1.818 & -.818 & .818 & -.818 & 145 & 0\end{array}$

unit 2

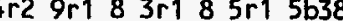

$24 r 233 r 124 r 2$

$\begin{array}{lllllllll}19 r 2 & 2 r 3 & 3 r 2 & 3 r 1 & 2 r 3 & 6 r 1 & 2 r 3 & 7 r 1 & 7637\end{array}$

$1 \mathrm{q} 81$

cylinder $\quad 4 \quad 1.5149 \quad 145 \quad .3175$

$\begin{array}{llllll}\text { cylinder } & 0 & 1 & .5217 & 145 & .3175\end{array}$

cylinder $\quad 51.603 \quad 1450$

cuboid $31.818-.818 .818 \cdot .8181450$

unit 3

cuboid

$31.818-.818 \quad .818-.8181450$

unit 4

$\begin{array}{lll}-49.898 & -6.544 & 0\end{array}$

unit 5

array 3
unit 6

$\begin{array}{lll}-33.538 & -8.180 \quad 0\end{array}$

$\begin{array}{llll}\text { array } 4 & -33.538 & -8.180 & 0\end{array}$

$24 r 2$ 9r1 $83 r 185 r 15 b 38$

$24 r 233 r 124 r 2$

$23 r 2 \quad 2 r 314 r 13 r 33 b 39$

1981

8161782

end fill

ara $=2$ nux $=61$ nuy $=8$ fill $f 2$ and fill

ara $=3$ nux $=41$ nuy $=10$ loop

$\begin{array}{llllllllll}2 & 1 & 41 & 1 & 1 & 10 & 1 & 1 & 1\end{array}$

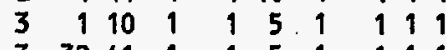

$\begin{array}{llllllllll}3 & 32 & 41 & 1 & 1 & 5 & 1 & 1 & 1 & 1\end{array}$ end loop

ara $=4$ nux $=41$ nuy $=10$ loop 
$2 \begin{array}{llllllllll}2 & 1 & 4 & 1 & 1 & 10 & 1 & 1 & 1 & 1\end{array}$

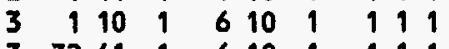

3324116101111 hend loop

\section{end array}

end data

end

\section{but810d}

\section{$=\operatorname{csas} 25$}

bw1810d

44 roup latticecell

wo2 1 den=9.46 $1.0 \quad 293 \quad 92235 \quad 4.02 \quad 9223895.98$ end

ss304

3 den=1.0 0.998346

uo2 4 den=1.0 $\begin{array}{llll}0.001654 & 1.0 & 29392235 & 2.4599223897 .541\end{array}$

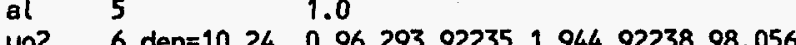

$\begin{array}{lll}\text { gd-152 } 6 \text { den }=0.3555 & 0.002\end{array}$

gd- 1546 den $=0.3555 \quad 0.0218$

gd-155 6 den $=0.3555 \quad 0.148$

gd-156 6 den $=0.3555 \quad 0.2047$

gd- 1576 den $=0.3555 \quad 0.1565$

gd-160 6 d

o 6 den $=0.05411$

end comp

$\begin{array}{lllllll}\text { squarepitch } & 1.636 & 1.128 & 1 & 3 & 1.208 & 2\end{array}$

more data res $=4$ cylinder .51486 dan $(4)=.189732$

end

end

enc

end

end

end

end

end

res $=6$ cyl inder .51499 dan $(6)=.189958$ end more data

Core of $39202.459 \mathrm{wt} \%{ }^{235} \mathrm{U} \mathrm{UO}{ }_{2}$ fuel rods, $8604.020 \mathrm{wt} \%{ }^{235} \mathrm{U} \mathrm{UO}_{2}$ fuel

rods, $28 \mathrm{UO}_{2}-\mathrm{Gd}_{2} \mathrm{O}_{3}$ fuel rods, 153 water holes, $145.0 \mathrm{~cm}$ water height with $1653.8 \mathrm{ppm}$ boron

read parm gen $=405$ npg $=600$ nsk $=5$ nub=yes run=yes end parm

\section{read geom}

unit 1

cylinder $\quad 11.56391455 .969$

cylinder 21.60391450

cuboid $\quad 3, .818-.818 .818 \cdot .8181450$

unit 2

cylinder $\quad 4 \quad 1.5149 \quad 145 \quad .3175$

cylinder $\quad 0 \quad 1.5217 \quad 145.3175$

cylinder $51.603 \quad 1450$

cuboid

$3, .818 \cdot .818 .818 \cdot .8181450$

unit 3

$31.818 \cdot .818 \quad .818 \cdot .8181450$

cuboid

array $2 \quad-49.898 \quad-6.544 \quad 0$

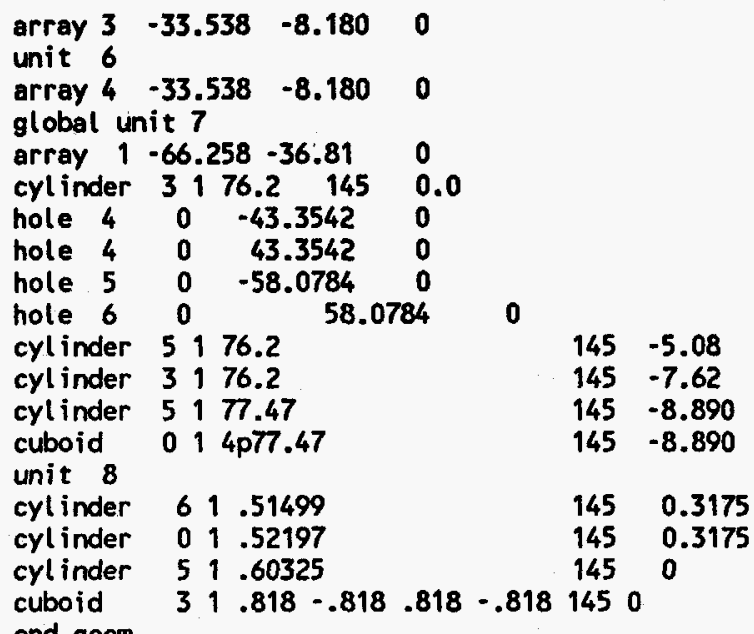

read array

ara $=1$ nux $=81$ nuy $=45$ loop

$\begin{array}{llllllllll}2 & 1 & 81 & 1 & 1 & 45 & 1 & 1 & 1\end{array}$

$\begin{array}{llllllllll}1 & 26 & 56 & 1 & 8 & 38 & 1 & 1 & 1\end{array}$

$\begin{array}{llllllllll}3 & 24 & 54 & 15 & 3 & 33 & 15 & 1 & 1\end{array}$

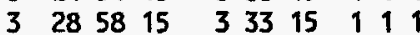

$\begin{array}{llllllllll}3 & 24 & 54 & 15 & 13 & 43 & 15 & 1 & 1 & 1\end{array}$

$\begin{array}{llllllllll}3 & 28 & 58 & 15 & 13 & 43 & 15 & 1 & 1 & 1\end{array}$

$\begin{array}{llllllllll}3 & 22 & 52 & 15 & 4 & 34 & 15 & 1 & 1 & 1\end{array}$

$\begin{array}{llllllllll}3 & 30 & 60 & 15 & 4 & 34 & 15 & 1 & 1 & 1\end{array}$

$\begin{array}{llllllllll}3 & 22 & 52 & 15 & 12 & 42 & 15 & 1 & 1 & 1\end{array}$

$\begin{array}{llllllllll}3 & 30 & 60 & 15 & 12 & 42 & 15 & 1 & 1 & 1\end{array}$

$3015115 \quad 6 \quad 36 \quad 15 \quad 11$

32151 15 636 15 111

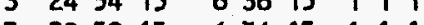

$\begin{array}{llllllllll}3 & 28 & 58 & 15 & 6 & 36 & 15 & 1 & 1 & 1\end{array}$

$\begin{array}{llllllllll}3 & 31 & 61 & 15 & 6 & 36 & 15 & 1 & 1 & 1\end{array}$

$\begin{array}{llllllllll}3 & 21 & 51 & 15 & 10 & 40 & 15 & 1 & 1 & 1\end{array}$

$\begin{array}{llllllllll}3 & 24 & 54 & 15 & 10 & 40 & 15 & 1 & 1 & 1\end{array}$

$\begin{array}{llllllllll}3 & 28 & 58 & 15 & 10 & 40 & 15 & 1 & 1 & 1\end{array}$

$\begin{array}{llllllllll}3 & 31 & 61 & 15 & 10 & 40 & 15 & 1 & 1 & 1\end{array}$

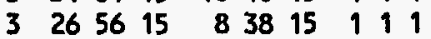

$\begin{array}{llllllllll}3 & 1 & 10 & 1 & 1 & 2 & 1 & 1 & 1 & 1\end{array}$

$\begin{array}{llllllllll}3 & 72 & 81 & 1 & 1 & 2 & 1 & 1 & 1 & 1\end{array}$

$\begin{array}{llllllllll}3 & 1 & 5 & 1 & 3 & 12 & 1 & 1 & 1 & 1\end{array}$

$\begin{array}{llllllllll}3 & 77 & 81 & 1 & 3 & 12 & 1 & 1 & 1 & 1\end{array}$

$\begin{array}{llllllllll}3 & 1 & 10 & 1 & 44 & 45 & 1 & 1 & 1 & 1\end{array}$

$\begin{array}{llllllllll}3 & 72 & 81 & 1 & 44 & 45 & 1 & 1 & 1 & 1\end{array}$

$\begin{array}{llllllllll}3 & 1 & 5 & 1 & 34 & 43 & 1 & 1 & 1 & 1\end{array}$

$\begin{array}{rrrrrrrrrr}3 & 77 & 81 & 1 & 34 & 43 & 1 & 1 & 1 & 1 \\ 8 & 29 & 53 & 24 & 8 & 38 & 30 & 1 & 1 & 1\end{array}$ 
$8 \begin{array}{lllllllll}86 & 56 & 30 & 11 & 35 & 24 & 1 & 1 & 1\end{array}$

$\begin{array}{llllllllll}8 & 29 & 53 & 24 & 11 & 35 & 24 & 1 & 1 & 1\end{array}$

$\begin{array}{lllllllll}8 & 315120 & 13 & 33 & 20 & 1 & 1\end{array}$

$\begin{array}{llllllllll}8 & 36 & 46 & 10 & 18 & 28 & 10 & 1 & 1 & 1\end{array}$

$8 \begin{array}{llllllllll}8 & 38 & 44 & 6 & 20 & 26 & 6 & 1 & 1 & 1\end{array}$

$\begin{array}{llllllllll}8 & 38 & 44 & 6 & 23 & 23 & 1 & 1 & 1 & 1\end{array}$

$\begin{array}{llllllllll}8 & 41 & 41 & 1 & 20 & 26 & 6 & 1 & 1 & 1\end{array}$

end loop

ara $=2$ nux $=61$ nuy $=8$ fill $f 2$ end fill

ara $=3$ nux $=41$ nuy $=10$ loop

$\begin{array}{llllllllll}2 & 1 & 41 & 1 & 1 & 10 & 1 & 1 & 1 & 1\end{array}$

$\begin{array}{rrrrrrrrrr}3 & 1 & 10 & 1 & 1 & 5 & 1 & 1 & 1 & 1 \\ 3 & 32 & 41 & 1 & 1 & 5 & 1 & 1 & 1 & 1\end{array}$

ara $=4$ nux $=41$ nuy=10 loop

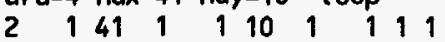

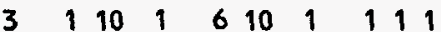

$33241 \quad 1 \quad 610 \quad 1 \quad 111$ end loop

end array

end data

end

bu1810e

$=\operatorname{csas} 25$

bw1810e

N

44group latticecel!

uo2 1 den=9.46 $1.0 \quad 29392235 \quad 4.029223895 .98$ end

ss304

h2o 3 den $=1.0 \quad 0.998421$

boron 3 den=1.0 0.001579

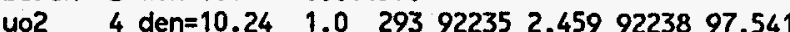

a) 5 1.0

uo2 6 den=10.24 $0.9629392235 \quad 1.9449223898 .056$

gd- 1526 den $=0.3555 \quad 0.002$

gd- 154 6 den $=0.3555 \quad 0.0218$

gd-155 6 den $=0.35550 .148$

gd-156 6 den $=0.3555 \quad 0.2047$

gd-157 6 den 0.35550 .1565

gd 158 o den $=0.35550 .2$

gd- 1586 den=0.3555 0.2484

gd-160 6 den $=0.3555 \quad 0.2186$ 6 den $=0.0541$

end

end

end

end

end

end

end

end

end

end

end

end

squarepitch $1.636 \quad 1.128 \quad 1 \quad 3 \quad 1.208 \quad 2$

end

more data res $=4$ cylinder .51486 dan (4) $=.189732$

res $=6$ cylinder .51499 dan $(6)=.189958$ end more data

Core of $36762.459 \mathrm{wt \%}{ }^{235} \mathrm{U} \mathrm{UO}_{2}$ fuel rods, $8524.020 \mathrm{wt \%}{ }^{233} \mathrm{U} \mathrm{UO}_{2}$ fuel

rods, $36 \mathrm{UO}_{2}-\mathrm{Gd}_{2} \mathrm{O}_{3}$ fuel rods, 153 water holes, $145.0 \mathrm{~cm}$ water height

with $1579.4 \mathrm{ppm}$ boron

read parm gen $=405$ npg $=600$ nsk $=5$ nub=yes run=yes end parm

read geom

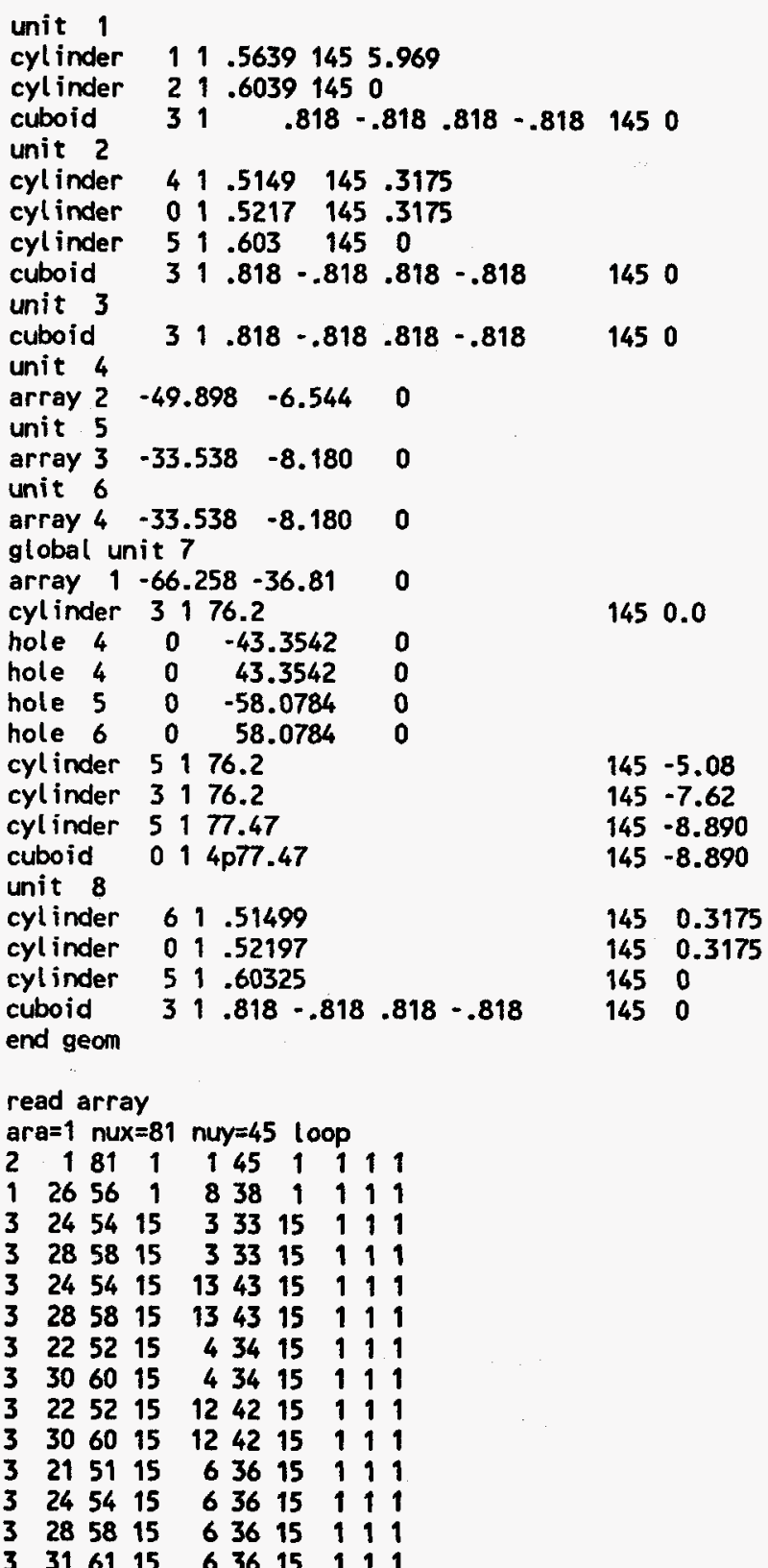

unit 1

cylinder

unit

$49 \quad 145.3175$

unit 3

unit 4

array 2

array 3

unit 6

global unit

cyl inder 5176.2

cylinder 3176.2

0.4077 .47 
$\begin{array}{llllllllll}3 & 21 & 51 & 15 & 10 & 40 & 15 & 1 & 1 & 1\end{array}$

$\begin{array}{llllllllll}3 & 24 & 54 & 15 & 10 & 40 & 15 & 1 & 1 & 1\end{array}$

$\begin{array}{llllllllll}3 & 28 & 58 & 15 & 10 & 40 & 15 & 1 & 1 & 1\end{array}$

$\begin{array}{llllllllll}3 & 31 & 61 & 15 & 10 & 40 & 15 & 1 & 1 & 1\end{array}$

$\begin{array}{llllllllll}3 & 26 & 56 & 15 & 8 & 38 & 15 & 1 & 1 & 1\end{array}$

$\begin{array}{llllllllll}3 & 1 & 10 & 1 & 1 & 2 & 1 & 1 & 1 & 1\end{array}$

$\begin{array}{llllllllll}3 & 72 & 81 & 1 & 1 & 2 & 1 & 1 & 1 & 1\end{array}$

$\begin{array}{llllllllll}3 & 1 & 5 & 1 & 3 & 12 & 1 & 1 & 1 & 1\end{array}$

$\begin{array}{llllllllll}3 & 77 & 81 & 1 & 3 & 12 & 1 & 1 & 1 & 1\end{array}$

$\begin{array}{llllllllll}3 & 1 & 10 & 1 & 44 & 45 & 1 & 1 & 1 & 1\end{array}$

$\begin{array}{llllllllll}3 & 72 & 81 & 1 & 44 & 45 & 1 & 1 & 1 & 1\end{array}$

$\begin{array}{llllllllll}3 & 1 & 5 & 1 & 34 & 43 & 1 & 1 & 1 & 1\end{array}$

$\begin{array}{llllllllll}3 & 77 & 81 & 1 & 34 & 43 & 1 & 1 & 1 & 1\end{array}$

$\begin{array}{llllllllll}8 & 29 & 53 & 24 & 8 & 38 & 30 & 1 & 1 & 1\end{array}$

$\begin{array}{llllllllll}8 & 26 & 56 & 30 & 11 & 35 & 24 & 1 & 1 & 1\end{array}$

$\begin{array}{llllllllll}8 & 28 & 54 & 26 & 14 & 32 & 18 & 1 & 1 & 1\end{array}$

$\begin{array}{llllllllll}8 & 32 & 50 & 18 & 10 & 36 & 26 & 1 & 1 & 1\end{array}$

$\begin{array}{llllllllll}8 & 31 & 51 & 20 & 13 & 33 & 20 & 1 & 1 & 1\end{array}$

$\begin{array}{llllllllll}8 & 36 & 46 & 10 & 18 & 28 & 10 & 1 & 1 & 1\end{array}$

$\begin{array}{llllllllll}8 & 35 & 47 & 12 & 21 & 25 & 4 & 1 & 1 & 1\end{array}$

$\begin{array}{llllllllll}8 & 39 & 43 & 4 & 17 & 29 & 12 & 1 & 1 & 1\end{array}$

$\begin{array}{llllllllll}8 & 38 & 44 & 6 & 23 & 23 & 1 & 1 & 1 & 1\end{array}$

$\begin{array}{llllllllll}8 & 41 & 41 & 1 & 20 & 26 & 6 & 1 & 1 & 1\end{array}$

end loop

ara=2 nux=61 nuy=8 fill +2 end fill

ara $=3$ nux $=41$ nuy=10 loop

$\begin{array}{lllllllll}2 & 1 & 41 & 1 & 1 & 10 & 1 & 1 & 1\end{array}$

$\begin{array}{llllllllll}3 & 1 & 10 & 1 & 1 & 5 & 1 & 1 & 1 & 1\end{array}$

$\begin{array}{llllllllll}3 & 32 & 41 & 1 & 1 & 5 & 1 & 1 & 1\end{array}$ end loop

ara $=4$ nux $=41$ nuy $=10$ loop

$2 \quad 141 \quad 1 \quad 110 \quad 1 \quad 111$

$\begin{array}{lllllllll}3 & 1 & 10 & 1 & 6 & 10 & 1 & 1 & 1\end{array}$

$\begin{array}{llllllllll}3 & 32 & 41 & 1 & 6 & 10 & 1 & 1 & 1 & 1\end{array}$ end loop

end array

end data

end

\section{bu1810f}

\section{$=\operatorname{csas} 25$}

bw1810f

44group

402 1 denticecell

=10.24 $1.029392235 \quad 2.4599223897 .541$

$\begin{array}{lll}\text { al } & 2 & 1.0 \\ \text { h2o } & 3 \text { den }=1.0 & 0.998663\end{array}$

$\begin{array}{lll}\text { h2o } & 3 \text { den=1.0 } & 0.998663 \\ \text { boron } 3 \text { den=1.0 } & 0.001337\end{array}$

end conp

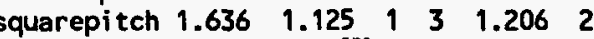

Core of $48082.459 \mathrm{wt} \%{ }^{235} \mathrm{U} \mathrm{UO}_{2}$ fuel roc

read parm gen $=405 \mathrm{npg}=600 \mathrm{nsk}=5$ nub=yes run=yes end parm

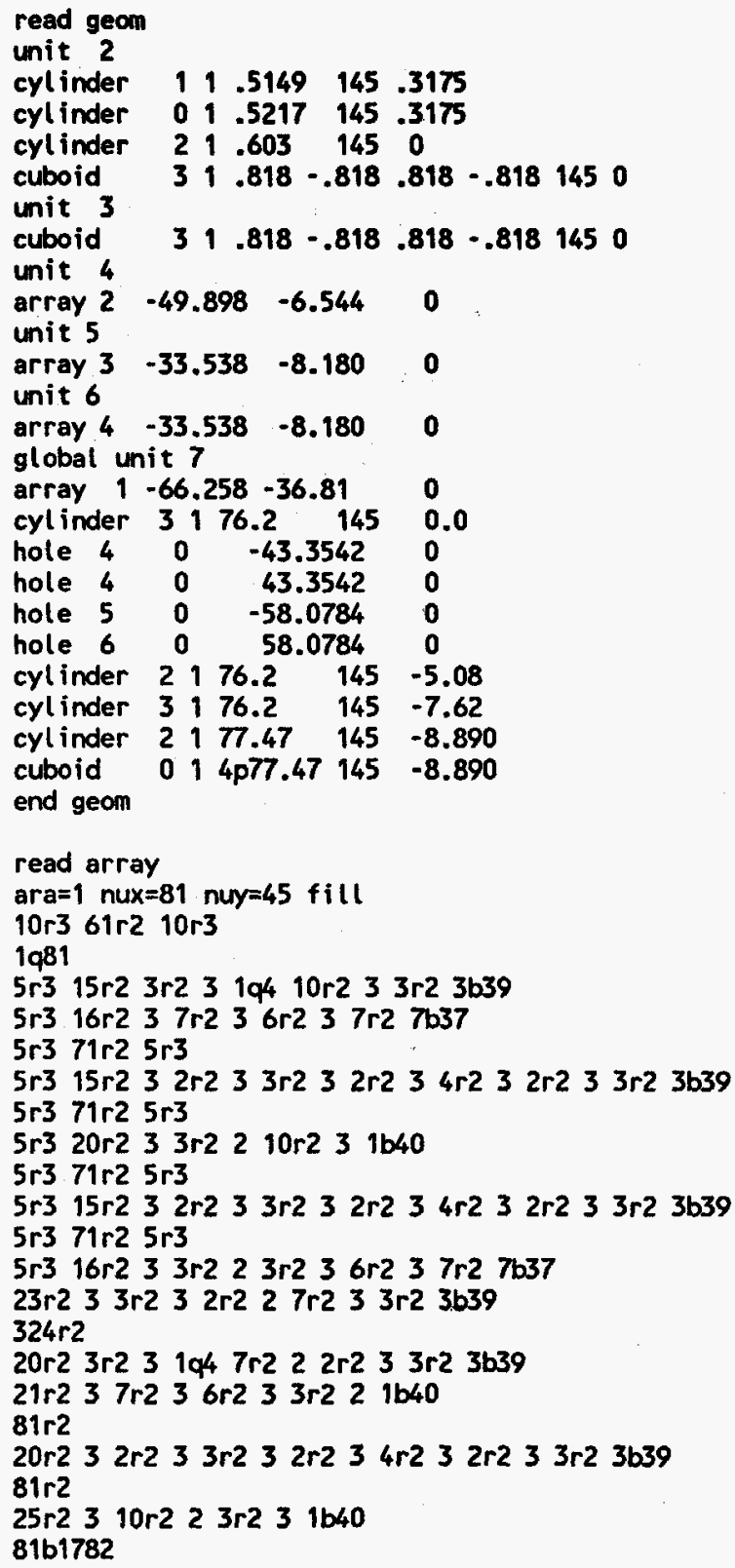


ara $=2$ nux $=61$ nuy=8 fill $f 2$ end fill ara $=3$ nux $=41$ nuy $=10$ loop

$\begin{array}{llllllllll}2 & 1 & 41 & 1 & 1 & 10 & 1 & 1 & 1 & 1\end{array}$

$\begin{array}{llllllllll}3 & 1 & 10 & 1 & 1 & 5 & 1 & 1 & 1 & 1\end{array}$

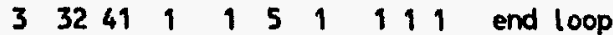

ara $=4$ nux $=41$ nuy $=10$ loop

$2141 \quad 1 \quad 110 \quad 1111$

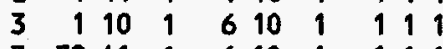

$33241 \quad 1 \quad 610 \quad 1 \quad 111$ end loop

end array

end data

end

bu1810g

$=\operatorname{csas} 25$

bw1810g

44group latticecell

$\begin{array}{llllll}\text { uo2 } 1 \text { den }=9.46 \quad 1.0 & 29392235 \quad 4.02 \quad 9223895.98\end{array}$

553042

h20 3 den $=1.0$

1.0

boron 3 den=1.0 0.001776

uo2 4 den=10.24 1.0293922352 .4599223897 .541

at 5

end comp 1.0

squarepitch $1.636 \quad 1.128 \quad 1 \quad 3 \quad 1.208 \quad 2$

more data res $=4$ cylinder .51486 dan $(4)=.189732$ end more data

Core of $36762.459 \mathrm{wt} \%{ }^{235} \mathrm{U} \mathrm{UO}_{2}$ fuel rods, $9444.020{ }^{235} \mathrm{U} \mathrm{wt} \% \mathrm{UO} \mathrm{O}_{2}$ fuel

r rods, 180 water holes, $145.0 \mathrm{~cm}$ water height with $1776.8 \mathrm{ppm}$ boron read parm gen $=405 \mathrm{npg}=600 \mathrm{nsk}=5$ nub=yes run=yes end parm

\section{read geom}

unit 1

cyl inder 11.56391455 .969

cyl inder 21.60391450

cuboid $\quad 31 \quad .818-.818 .818-.8181450$

unit 2

cylinder $\quad 4 \quad 1.5149 \quad 145 \quad .3175$

cylinder $\quad 01.5217 \quad 145.317$

cylinder $51.603 \quad 145 \quad 0$

$\begin{array}{llllllll}\text { cuboid } & 31 & .818 & -.818 & .818 & -.818 & 1450 & 0\end{array}$

cuboid

$31 \quad .818 \cdot .818 .818 \cdot .8181450$

unit 4

array $2 \quad-49.898 \quad-6.544 \quad 0$

unit 5

arrar 3

$-8.180$

unit 6

array $4-33.538$

global unit 7

array $1-66.258 \quad-36.81 \quad 0$ $\begin{array}{lccccc}\text { cylinder } & 3 & 1 & 76.2 & 145 & 0.0 \\ \text { hole } 4 & 0 & -43.3542 & 0 \\ \text { hole } 4 & 0 & 43.3542 & 0 \\ \text { hole } 5 & 0 & -58.0784 & 0 \\ \text { hole } 6 & 0 & & 58.0784 & 0 \\ \text { cylinder } & 5 & 1 & 76.2 & 145 & -5.08 \\ \text { cylinder } & 3 & 1 & 76.2 & 145 & -7.62 \\ \text { cylinder } & 5 & 1 & 77.47 & 145 & -8.890 \\ \text { cuboid } & 0 & 1 & 4 p 77.47 & 145 & -8.890\end{array}$

end geom

read array

ara $=1$ nux $=81$ nuy $=45$ fill

$10 r 361 r 210 r 3$

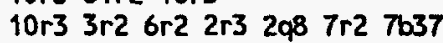

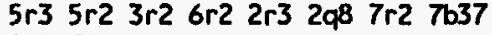

$5 r 371 r 25 r 3$

1981

$5 r 3$ 18r2 $2 r 3 \quad 14 r 23 r 33639$

$5 r 3 \quad 18 r 2 \quad 2 r 3 \quad 14 r 13 r 3 \quad 3 b 39$

$5 r 319 r 233 r 119 r 25 r 3$

$1 q 81$

$\begin{array}{lllllllllll}5 r 3 & 14 r 2 & 2 r 3 & 3 r 2 & 3 r 1 & 2 r 3 & 6 r 1 & 2 r 3 & 7 r 1 & 7 b 37\end{array}$

1981

$24 r 233 r 124 r 2$

$5 q 81$

$\begin{array}{lllllllllllllllll}19 r 2 & 2 r 3 & 3 r 2 & 3 r 1 & 2 r 3 & 6 r 1 & 2 r 3 & 7 r 1 & 7 b 37\end{array}$

1981

$24 r 233 r 124 r 2$

1981

$23 r 2 \quad 2 r 3 \quad 14 r 13 r 3 \quad 3 b 39$

$1 \mathrm{q} 81$

$81 \mathrm{~b} 1782$

ara $=2$ nux $=61$ nuy=8 fill $f 2$ end fill

ara $=3$ nux $=41$ nuy $=10 \quad$ loop

ara $=3$ nux $=41$ nuy=10, loop

$\begin{array}{rrrrrrrrrr}2 & 1 & 41 & 1 & 1 & 10 & 1 & 1 & 1 & 1 \\ 3 & 1 & 10 & 1 & 1 & 5 & 1 & 1 & 1 & 1\end{array}$

$33241 \quad 1 \quad 1511111$ end loop

ara $=4$ nux $=41$ nuy=10 loop

21411111011111

31101961011111

$33241,1610,1,1$ end loop

end array

end data

end

a.1810h

$=\operatorname{csas} 25$

bu1810h 

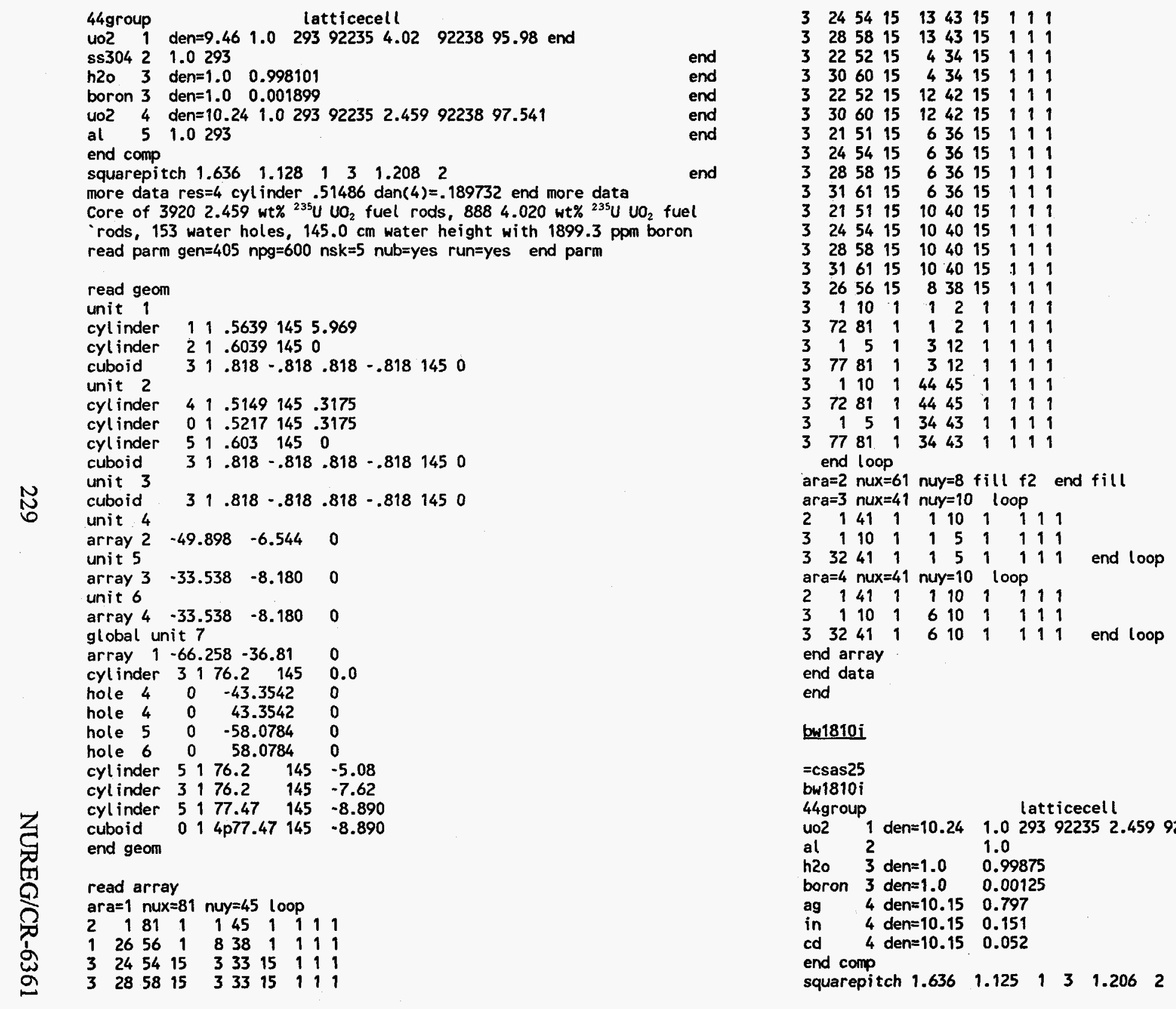

$=\operatorname{csas} 25$

bu1810i

44group latticecell

uo2 1 den $=10.24 \quad 1.0293922352 .4599223897 .541$

$\begin{array}{lll}\text { h20 } & 2 \\ \text { h den }=1.0 & 0.99875\end{array}$

boron 3 den $=1.0 \quad 0.00125$

ag 4 den $=10.15 \quad 0.797$

in 4 den $=10.15 \quad 0.151$

ed 4 den $=10.15 \quad 0.052$

end comp

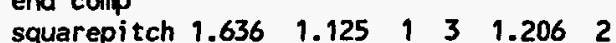


Core of $48082.459 \mathrm{wt}^{235} \mathrm{U} \mathrm{UO}_{2}$ fuel rods, $16 \mathrm{Ag}$-In-Cd rods, 137 water holes, $145.0 \mathrm{~cm}$ water height with $1250.0 \mathrm{ppm}$ boron

read parm gen $=405$ npg $=600$ nsk $=5$ nub=yes run=yes end parm

read geom

unit 1

cylinder $\quad 41.508 \quad 145.3175$

cylinder $01.5219 \quad 145 \quad .317$

cylinder $21.6033 \quad 1450$

cuboid $\quad 31 \quad .818-.818 .818-.8181450$

unit

$\begin{array}{llllll}\text { cylinder } & 1 & 1 & 5149 & 145 & .3175\end{array}$

cyl inder $\quad 001.5217 \quad 145.3175$

cylinder $21.603 \quad 1450$

cuboid $\quad 31 \quad .818 \cdot .818 .818 \cdot .8181450$

unit 3

$1 \quad .818 \cdot .818 .818 \cdot .8181450$

unit 4

array $2 \quad-49.898 \quad-6.544 \quad 0$

unit 5

unit $533.538-8.180-0$

$\begin{array}{llll}\text { array } 3 & -33.538 & -8.180 & 0\end{array}$

array $4-33.538-8.180 \quad 0$

global unit 7

array $1-66.258 \quad-36.81 \quad 0$

心్ర

$\begin{array}{ll}145 & 0.0\end{array}$

hole $4 \quad 0 \quad-43.3542 \quad 0$

$\begin{array}{lllll}\text { hole } & 4 & 0 & 43.3542 & 0\end{array}$

$\begin{array}{lllrl}\text { hole } & 5 & 0 & -58.0784 & 0 \\ \text { hole } & 6 & 0 & 58.0784 & 0\end{array}$

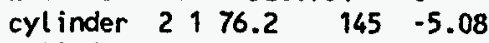

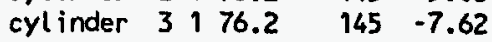

cyl inder $2177.47 \quad 145 \quad-8.890$

cuboid $\quad 014 \mathrm{p} 77.47 \quad 145 \quad-8.890$

end geom

read array

ara $=1$ nux $=81$ nuy $=45 \mathrm{fill}$

$10 r^{3} 61 r^{2} 10 r^{3}$

1981

$5 r 3 \quad 15 r 23 r 23$ 1a4 10r2 $3 \quad 3 r 23639$

$5 r 316 r 237 r 236 r 237 r 27 b 37$

$5 r 371 r^{2} 5 r^{3}$

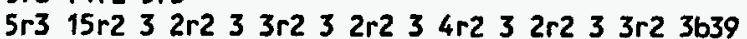

$5 r 371 r 25 r 3$

$5 r 320 r 233 r 2210 r 231640$

$5 r 371 r 25 r 3$

$\begin{array}{lllllllllllllllllll}5 r 3 & 15 r 2 & 3 & 2 r 2 & 3 & 3 r 2 & 3 & 2 r 2 & 3 & 4 r 2 & 3 & 2 r 2 & 3 & 3 r 2 & 3 b 39\end{array}$

$5 r 371 r 25 r 3$

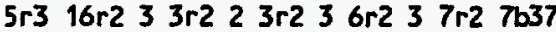

$23 r 23 \quad 3 r 23 \quad 2 r 227 r 233 r 23 b 39$

$324 r^{2}$

20r2 3r2 3 1a4 7r2 2 2r2 $13 r 23 b 39$

$21 r 237 r 23$ 6r2 $13 r 221 b 40$

$81 \mathrm{r} 2$

$20 r 23 \quad 2 r 23 \quad 3 r 23 \quad 2 r 234 r 219 r 2$ 1 $3 r 23 b 39$

$81 r^{2}$

$25 r 2310 r 223 r 231 b 40$

$81 b 1782$

end fill

ara $=2$ nux $=61$ nuy $=8$ fill $f 2$ end fill

ara $=3$ nux $=41$ nuy $=10$ loop

$2 \quad 141 \quad 1 \quad 110 \quad 1 \quad 111$

$\begin{array}{llllllllll}3 & 10 & 1 & 1 & 5 & 1 & 1 & 1\end{array}$

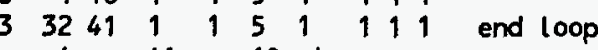

ara $=4$ nux $=41$ nuy $=10$ loop

$\begin{array}{llllllllll}2 & 1 & 41 & 1 & 1 & 10 & 1 & 1 & 1 & 1\end{array}$

$3 \quad 3241 \quad 1 \quad 6 \quad 101111$ end loop

end array

end data

end

bu1810i

\section{$=\operatorname{csas} 25$}

bw1810j

44group latticecell

no2 1 den=9.46 $1.0 \quad 29392235 \quad 4.02 \quad 9223895.98$

ss304 21.0293

h20 3 den=1.0 $\quad 0.998365$

boron 3 den $=1.0 \quad 0.001635$

uo2 4 den=10.24 $1.029392235 \quad 2.4599223897 .541$

at 51.0293

b4c 6 den $=1.28 \quad 0.986$

end comp

$\begin{array}{lllllll}\text { squarepitch } & 1.636 & 1.128 & 1 & 3 & 1.208 & 2\end{array}$

more data res $=4$ cyl inder .51486 dan $(4)=.189732$ end more data

Core of $39202.459 \mathrm{Wt} \%{ }^{235} \mathrm{U} \mathrm{UO}_{2}$ fuel rods, $8884.020 \mathrm{wt} \%{ }^{235} \mathrm{U} \mathrm{UO}_{2}$ fuel

rods, $16 \mathrm{~B}, \mathrm{C}$ rods, 137 water holes, $145.0 \mathrm{~cm}$ water height with 1635.4

ppm boron

read parm gen $=405 \mathrm{npg}=600 \mathrm{nsk}=5$ nub=yes run=yes end parm

read geom

unit 1

cyl inder $\quad 11.56391455 .969$

cylinder 21.60391450

cuboid $31.818 \cdot .818 .818-.8181450$

unit 2

cyl inder $\quad 41.5149 \quad 145.3175$

$\begin{array}{lllllll}\text { cyl inder } & 4 & 1 & .5149 & 145 & .3175 \\ \text { cyl } & 0 & 145 & .3175\end{array}$

cylinder 51.6031450 


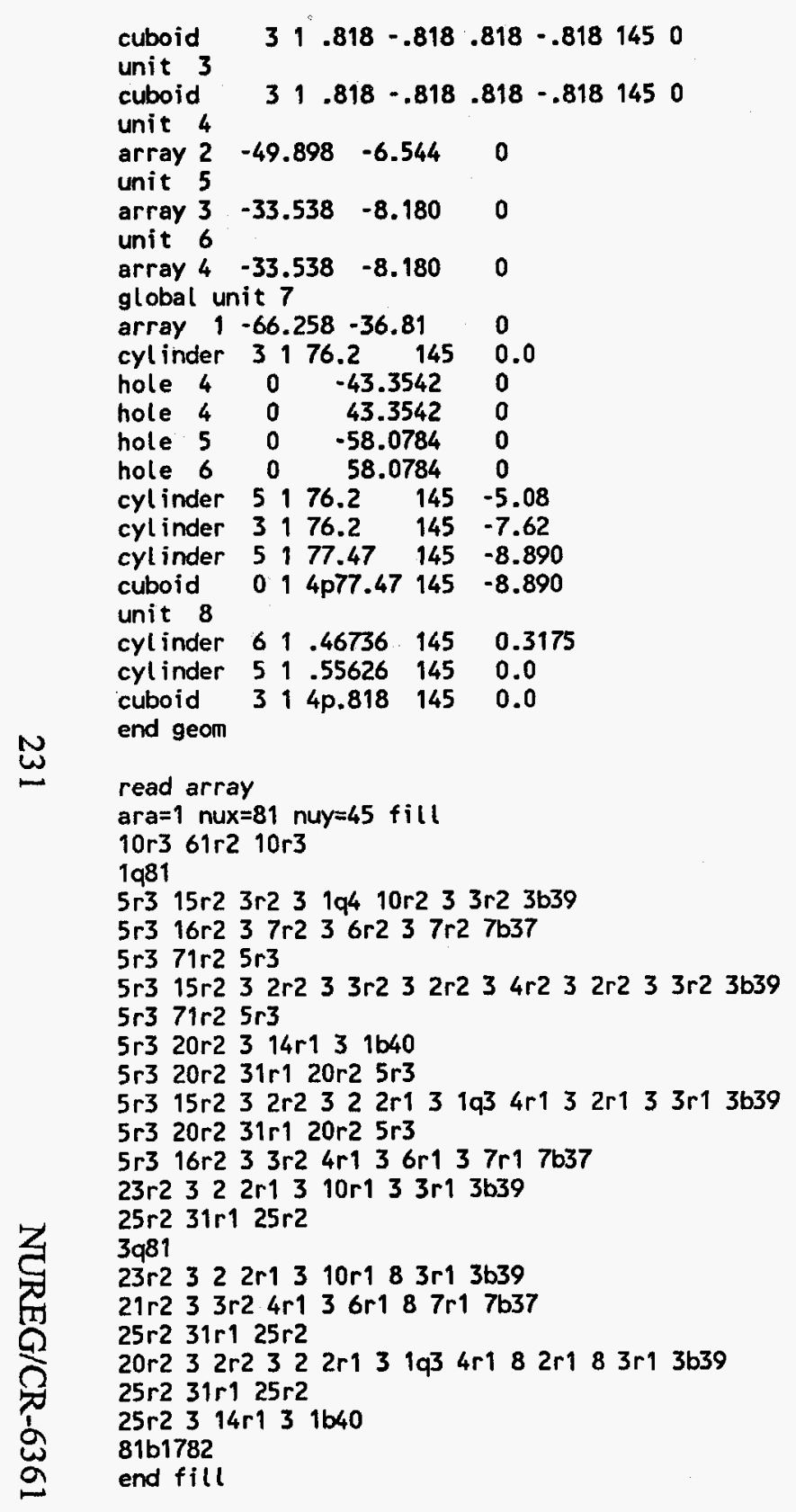

ara $=2$ nux $=61$ nuy $=8$ fill $f 2$ end fill ara $=3$ nux $=41$ nuy $=10$ loop $2 \quad 141,1$ aop $\begin{array}{rrrrrrrrrr}2 & 1 & 41 & 1 & 1 & 10 & 1 & 1 & 1 & 1 \\ 3 & 1 & 10 & 1 & 1 & 5 & 1 & 1 & 1 & 1\end{array}$

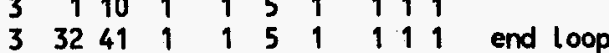
ara $=4$ nux $=41$ nuy $=10$ loop $2 \quad 141 \quad 1 \quad 110 \quad 1 \quad 111$

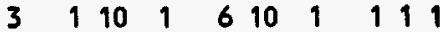
$33241 \quad 1 \quad 61011111$ end loop end array

end data

end

\section{er1071as}

$=\operatorname{csas} 25$ cr1071as 4 croup u308

latticecell

1 den=4.54 1293.0922340 .03922354 .4892236 0.099223895 .4

1 den $=.09316$

h2o

arbm-baggie $\quad 1.596-23000601284.9100114 .0180161 .201$ end arbm-al 1100 140000.12

end arbm-tape(vinyl) $1.374-27000601245.9110015 .92 \quad 8016 \quad 10.82$ 1700025.73200006 .9220001 .6820001 .12 end arbm-tape(mylar) 1.832-2 30000601265.5010016 .83801627 .022 end arbm-moderator $1.185 \quad 3000601259.4910017 .83801632 .483$ end arbm-plex(reg) $\quad 1.185 \quad 3000601259.5910017 .84801632 .234$ $9935,3000601242.1710016 .48801649 .54$ arbm-plex(glue) $\begin{gathered}0.728 \\ 0.0049\end{gathered} \quad 3000601286.29100111 .6780161 .204$ end

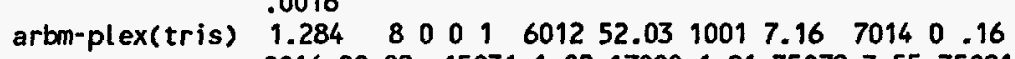
$801629.82 \quad 150311.02 \quad 170001.81350793 .5535081$ 3.555 .9935 arbm-plex(paper) $0.766 \quad 30000601242.17 \quad 10016.48 \quad 8016 \quad 49.5$ 5.0049 end $\begin{array}{llllllllll}\text { arbm-plex(glue) } & 0.728 & 3000 & 0 & 01286.29 & 1001 & 11.678016 & 1.20 & \\ 5 & .0016\end{array}$

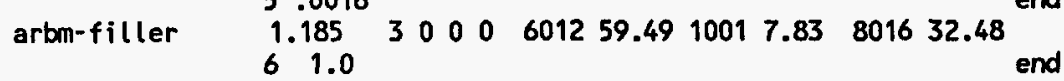
end comp end symmslabcell 17.6714 .921315 .242 end 38 cans of damp uranium oxide, $U_{3} \mathrm{O}_{8}$, separated by $2.43 \mathrm{~cm}$ of plexiglas, - Plexiglas reflected

read parm run=yes npg $=600$ gen=405 nsk=5 nub=yes end parm

read geom

unit $\mathbf{5 1}$

end


con=' fuel box with moderator $+x_{1}+y_{0}+z$

cuboid $116 \mathrm{p} 7.46$

cuboid $216 \mathrm{p} 7.62$

reflector 311.2150 .01 .2150 .01 .2150 .0 unit 52

com=' fuel box with moderator $-x,+y,+z$

cuboid $116 p 7.46$

cuboid $216 p 7.62$

reflector $310.0 \quad 1.215 \quad 1.215 \quad 0.01 .2150 .01$ unit 53

com $=$ ' fuel box with moderator $+x_{1}+y_{1}-y_{1}+z$ '

cuboid $116 p 7.46$

cuboid $216 \mathrm{p} 7.62$

reflector $311.215 \quad 0.0 \quad 2 r 1.215 \quad 1.2150 .0$

unit 54

com=' fuel box with moderator $-x_{1}+y_{1}-y_{0}+2$

cuboid $116 \mathrm{p} 7.46$

cuboid $2,607.62$

reflector $310.0 \quad 1.215 \quad 2 r 1.215 \quad 1.2150 .01$

unit 55

com=' fuel box with moderator $+x,-y_{1}+z$ '

cuboid $116 p 7.46$

cuboid $216 p 7.62$

reflector $311.215 \quad 0.0 \quad 0.0 \quad 1.215 \quad 1.215 \quad 0.01$

unit 56

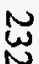

com=' fuel box with moderator $-x,-y,+z$ '

cuboid $116 \mathrm{pp} 7.46$

cuboid $216 \mathrm{p} 7.62$

reflector $3 \quad 1 \quad 0.0 \quad 1.215 \quad 0.0 \quad 1.215 \quad 1.215 \quad 0.01$

unit 57

com $=$ ' fuel box with moderator $+x,+y,+z,-z$ '

cuboid $116 p 7.46$

cuboid 21607.62

reflector $3 \uparrow 1.215 \quad 0.0 \quad 1.215 \quad 0.0 \quad 2 r 1.2151$

unit 58

com=' fuel box with moderator $-x,+y,+z,-z$ '

cuboid $116 \mathrm{p} 7.46$

cuboid $216 p 7.62$

reflector $310.0 \quad 1.215 \quad 1.215 \quad 0.0 \quad 2 r 1.215$ unit 59

com=' fuel box with moderator $+x,+y,-y,+z,-z$ '

cuboid 11 6p7.46

cuboid $216 \mathrm{pp} 7.62$

reflector $311.2150 .04 r 1.215$

unit 60

com=' fuel box with moderator $-x,+y,-y,+z,-z^{\prime}$

cuboid $116 p 7.46$

cuboid 21607.62

reflector $310.05 r 1.215$

unit 61

com $=$ 'fuel box with moderator $+x,-y,+z,-z$ ' cuboid $196 \mathrm{p} 7,46$

cuboid $216 \mathrm{p} 7.62$

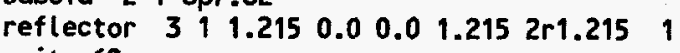
unit 62

com=' fuel box with moderator $-x,-y,+z,-z^{\prime}$

cuboid 11 6p7.46

cuboid $216 \mathrm{pp} 7.62$

reflector $310.0 \quad 1.2150 .0 \quad 1.215 \quad 211.215 \quad 1$ unit 63

com=' fuel box with moderator $-x_{1}+y,-z$ '

cuboid $116 \mathrm{kp} 7.46$

cuboid 21607.62

reflector $310.0 \quad 1.215 \quad 1.215 \quad 0.0 \quad 0.0 \quad 1.215 \quad 1$

unit 64

com $=$ ' fuel box with moderator $+x_{1}+y_{1}-y_{1}-z^{\prime}$

cuboid $116 p 7.46$

cuboid $216 \mathrm{p7.62}$

reflector $311.2150 .02 r 1.215 \quad 0.01 .215 \quad 1$

unit 65

com=' fuel box with moderator $-x,+y,-y,-z$ '

cuboid $116 p 7.46$

cuboid $216 \mathrm{p} 7.62$

reflector $310.0 \quad 1.215 \quad 2 r 1.215 \quad 0.0 \quad 1.215 \quad 1$

unit 66

com=' fuel box with moderator $-x,-y,-z$ '

cuboid $116 \mathrm{p} 7.46$

cuboid $216 p 7.62$

reflector $310.0 \quad 1.215 \quad 0.0 \quad 1.215 \quad 0.01 .215 \quad 1$ unit 67

com=' fuel box with moderator $+x,+y,-z$ '

cuboid $116 \mathrm{p} 7.46$

cuboid $216 \mathrm{p} 7.62$

reflector $\begin{array}{lllllllll} & 1 & 1.215 & 0.0 & 1.215 & 0.0 & 0.0 & 1.215 & 1\end{array}$

unit 68

com $=$ ' fuel box with moderator $+x,-y,-z$ '

cuboid $1116 \mathrm{p} 7.46$

cuboid $216 p 7.62$

$\begin{array}{llllllllll}\text { reflector } & 3 & 1 & 1.215 & 0.0 & 0.0 & 1.215 & 0.0 & 1.215 & 1\end{array}$

unit 69

com=' empty fuel location edge'

cuboid 0 i 6 p8.2275

unit 70

com='empty fuel location middle'

cuboid $012 p 8.22752 p 8.8352 p 8.2275$

unit 11

com='north split table core'

array $13 * 0.0$

cuboid $0132.91 \cdot .5968 .25 \quad 0.0 \quad 50.58 \quad 0.0$

reflector $6 \begin{array}{llllllll}1 & 0 & 4.9 & 8.5 & 0.0 & 7.3 & 26.1 & 1\end{array}$

reflector $01100.6 \quad .75 \quad 3 * 01$

unit 12 
comlso'south split table core' array $23 * 0.0$

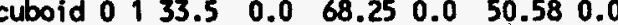
reflector $6,12.2 \quad 0 \quad 8.5 \quad 0.07 .326 .1$, reflector $0111.3 \quad 0.753 * 01$ unit 21

comine'north bottom reflector '

$\begin{array}{lll}\text { com='north bottom reflector ' } \\ \text { cuboid } 3,7.4 & 0.02 p 64.05 & 2 p 12.60\end{array}$ cuboid $5139.0 \quad 0.02 p 64.05 \quad 2 p 12.60$ unit 22

com='north top reflector

cuboid $3,7.4 \quad 0.02064 .052012 .00$

cuboid $5,39.0 \quad 0.02064 .052 p 12.00$

unit 23

comlnorth side reflector '

cuboid $317.4 \quad 0.02 p 12.65 \quad 57.88 \quad-26.1$ cuboid $5139.0 \quad 0.02 p 12.6557 .88 \quad-26.1$ unit 24

come'south bottom reflector with tris' conf south bottom reflector with $.55 \quad 2 p 12.60$ cuboid $5147.0 \quad 0.02 p 64.05 \quad 2 p 12.60$ unit 25

com='south top reflector with tris' cuboid $0110.2 \quad 0.0 .2 \mathrm{p} 2.55 \quad 2 \mathrm{p} 12.00$ cuboid $5147.0 \quad 0.02 p 64.052 p 12.00$ unit 26

com=' south side reflector with tris' cuboid 5 i $47.0 \quad 0.02 p 12.65 \quad 57.88 \quad-26.1$ unit 31

com= ' north array coretside array $33 \star 0.0$

unit 32

con='south array coretside'

array $43 * 0.0$

unit 33

com='north array'

array $53 * 0.0$

reflector $41025.24 \mathrm{ro} 0.0$

reflector $311.2304 r 0.0$

unit 34

com=' south array'

array $63 * 0.0$

reflector $5126.5 \quad 5 r 0.0$

reflector 31101.234 ro.0

reflector $0 \begin{array}{lllllll}1 & 0 & .31 & 4 \mathrm{ro} & 0.0\end{array}$

global

unit 35

unit 35

com= total

end geometry read array

ara $=1$ nux $=2$ nuy $=4$ nuz $=3$

com='north split table core'

fill 51525354 lq2 $5556 \quad 57585960 \quad 1 q 2 \quad 61626963 \quad 64$ 651926966

end fill

ara $=2$ nux $=2$ nuy $=4$ nuz $=3$

com=' south split table core'

fill $515253 \quad 54$ 1q2 $5556 \quad 57585960 \quad 1 q 2 \quad 6162 \quad 67 \quad 6964$ 701926869

ara=3 nux=1 nuy=3 nuz=1

com='north core with side wall'

fill 231123

end fill

ara $=4$ nux $=1$ nuy $=3$ nuz $=1$

com=' south core with side wall'

fill 261226

end fill

fill nux $=1$ nuz $=3$

com='north assembly'

ar $a=6$ nux $=1$ nuy $=1$ nuz $=3$

comp' south assembly'

fill 243225

ara $=7$ nux $=2$ nuy=1 nuz $=1$

com=' total '

fill 3334

end array

end fill

end data

end

cr1653as

$=\operatorname{csas} 25$

cr1653as

44group latticecell

u308 1 den=4.54 1293.0922340 .03922354 .4892236

den $=4.541293 .0$

end

h20

arbm-baggie 1 den $=.16060$ 140000.12

arbm-tape(vinyl) $1.374-27000 \quad 601245.9110015 .928016 \quad 10.82$ 1700025.73200006 .9220001 .6820001 .12

end $f i l l$

end fill

arbm-tape(mylar) $1.832-23$

arbm-moderator $1.1853000061259 .4910017 .83 \quad 801632.483$ end arbm-plex(reg) $\quad 1.1853000601259 .5910017 .848016$

32.234 .9935

arbm-plex(paper) $0.7663000601242 .1710016 .48 \quad 8016$

49.54 .0049

0.7283000601286 .29100111 .678016

arbm-plex(glue)

end

end

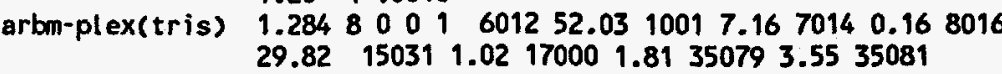


3.555 .9935

$0.7663000 \quad 601242.1710016 .48 \quad 8016 \quad 49.5$ end

5.0049

eno

arbm-plex(glue) $\quad 0.7283000061286 .291001 \quad 11.678016 \quad 1.20$ 5.0016

arbm-filler

$$
\begin{aligned}
& 1.1853 \\
& 61.0
\end{aligned}
$$

end comp

symmslabcell 17.6714 .921315 .242

$001259.4910017 .83 \quad 8016 \quad 32.48$

end

end

38 cans of damp uranium oxide, $U_{3} \mathrm{O}_{8}$, separated by $2.43 \mathrm{~cm}$ of plexiglas

"Plexiglas reflected

read parm run=yes npg $=600$ gen $=405$ nsk $=5$ nub=yes end parm

read geom

unit 1

com=' fuel box $15.24 \mathrm{~cm}$ on a side with $.16 \mathrm{~cm}$ walls !

cuboid $1116 \mathrm{p} 7.46$

cuboid 21607.62

unit 2

com=' $x$-face interstitial moderator'

cuboid $312 p 1.215$ 4p7.62

unit 3

com=' $y$-face interstitial moderator

cuboid $312 \mathrm{p} 7.62$ 2p1.215 2p7.62

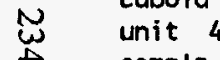

com='z-face interstitial moderator'

cuboid $314 \mathrm{p} 7.622 \mathrm{pl} .215$

unit 5

com='more $x$-face moderator'

cuboid $314 \mathrm{pl} 1.2152 \mathrm{p} 7.62$

unit 6

com=' 'more $y$-face moderator'

cuboid $312 p 7.62 \quad 4 p 1.215$

unit 7

com='more $z$-face moderator'

cuboid $312 p 1.2152 p 7.62 \quad 2 p 1.215$

unit 8

com=' last of interstitial moderator"

cuboid $316 p 1.215$

unit 51

com='empty fuel location

cuboid $016 \mathrm{p} 7.620$

unit 52

com $=1 x-$ face moderator void'

cuboid $012 p 1.2154 p 7.62$

unit 53

com=' $y$-face moderator void'

cuboid $012 p 7.622 p 1.2152 p 7.62$

unit 11

com='north split table core' array $13 * 0.0$

cuboid $0132.91 \cdot-5969.0 \quad 0.0 \quad 50.58 \quad 0.0$

reflector $610 \quad 4.98 .5 \quad 0.07 .326 .11$

reflector $\begin{array}{llllllll}0 & 1 & 0 & 0.6 & 4 * 0 & 1\end{array}$

unit 12

com='south split table core'

array $23 * 0.0$

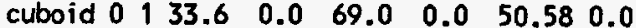

reflector $\begin{array}{lllllllll}6 & 1 & 12.2 & 0 & 8.5 & 0.0 & 7.3 & 26.1 & 1\end{array}$

reflector $0 \begin{array}{lllll}0 & 1.3 & 0 & 4 * 0 & 1\end{array}$

unit 21

com=' north bottom reflector '

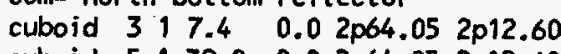

cuboid $5139.0 \quad 0.02 p 64.05 \quad 2 p 12.60$

unit 22

com=' north top reflector :

cuboid $317.4 \quad 0.02 p 64.05 \quad 2 p 12.00$

cuboid $5,39.0 \quad 0.02064 .05 \quad 2 p 12.00$

unit 23

com='north side reflector '

$\begin{array}{lllllll}\text { cuboid } 3 & 1 & 7.4 & 0.0 & 2 \mathrm{p} 12.65 & 57.88 & -26.1\end{array}$

$\begin{array}{lllllll}\text { cuboid } 5139.0 & 0.0 & 2 p 12.65 & 57.88 & -26.1\end{array}$

unit 24

com=' south bottom reflector with tris'

cuboid $01110.2 \quad 0.02 p 2.55 \quad 2 p 12.60$

cuboid $5147.1 \quad 0.02 p 64.05 \quad 2 p 12.60$

unit 25

com=' south top reflector with tris'

$\begin{array}{llllll}\text { cuboid } 0 & 1 & 10.2 & 0.0 & 2 \mathrm{p} 2.55 & 2 \mathrm{p} 12.00\end{array}$

cuboid $5147.1 \quad 0.02 p 64.05 \quad 2 p 12.00$

unit 26

com='south side reflector with tris'

$\begin{array}{llllllll}\text { cuboid } 5 & 1 & 47.1 & 0.0 & 2 p 12.65 & 57.88 & -26.1\end{array}$

unit 31

com=' nor th array coretside'

array $3 * 0.0$

unit 32

com=' south array coretside'

array $43 * 0.0$

unit 33

com='north array'

array $53 * 0.0$

reflector $41025.24 r 0.01$

reflector $311.2304 r 0.01$

unit 34

com='south array'

array $63 * 0.0$

reflector $010.5 \quad 5 r 0.01$

reflector $5126.5 \quad 5 r 0.01$

reflector $311001.23 \quad 4 r 0.0$ ?

reflector $01101.26 \quad 4 \mathrm{r} 0.01$ 
global

unit 35

com $=$ ' total $'$

array $73 * 0.0$

end geometry

read array

ar $a=1$ nux $=3$ nuy $=7$ nuz $=5$

com='north split table core'

fill $1213532 q 6121$

$4746862 q 6474$

1942

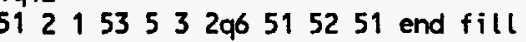

ara $=2$ nux $=3$ nuy $=7$ nuz $=5$

com=' south spl it table core'

fill $1213532 q 6121$

474686296474

1942

$125135532 q 6515251$ end fill

ara $=3$ nux $=1$ nuy $=3$ nuz $=1$

com='north core with side wall'

fill 231123

ara $=4$ nux $=1$ nuy $=3$ nuz $=1$

N fill 261226

ara $=5$ nux $=1$ nuy $=1$ nuz $=3$

com $=$ ' nor th assembly'

fill 213122

ara $=6$ nux $=1$ nuy $=1$ nuz $=3$

com=' south assembly'

fill 243225

ara $=7$ nux $=2$ nuy $=1$ nuz $=$

com=' total'

fill 3334

end array

end $f i l l$

end $f i l l$

end $\mathrm{fill}$

end data

end

\section{cr2500s}

$=\operatorname{csas} 25$

cr2500s

44 group

u308

latticecell

1 den=4.54 1293.0922340 .03922354 .4892236

0.099223895 .4

end

h20

1 den $=.27248$

end

arbm-baggie

$.596-23000 \quad 601284.9100114 .0180161 .201$ end

arbm-al1100

140000.12

end
$1700025.73200006 .9220001 .6 \quad 820001.12 \quad$ end arbm-tape(mylar) $2.748-23000601265.501001 \quad 6.838016 \quad 27.02$ 2

end arbm-moderator $1.1853000 \quad 601259.4910017 .83 \quad 801632.483$ end arbm-plex(reg) $\quad 1.1853000 \quad 601259.5910017 .84 \quad 801632.234$ .9935

$0 \quad 601242.1710016 .48 \quad 801649.5$

and

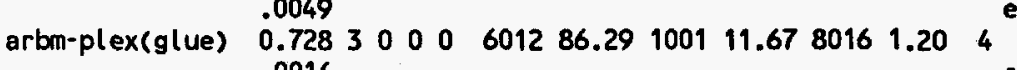
0016

end

arbm-plex(tris) $\quad \begin{array}{lllllll}1.284 & 8 & 0 & 0 & 1 & 601252.0310017 .16 \quad 7014 & 0.16\end{array}$ 801629.82150311 .02170001 .81350793 .5535081 3.555 .9935

arbm-plex(paper) $0.76630000601242 .1710016 .48 \quad 8016 \quad 49.5$

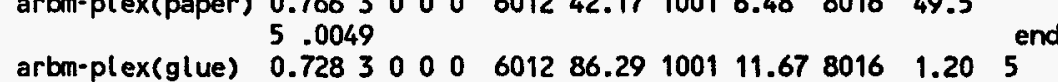

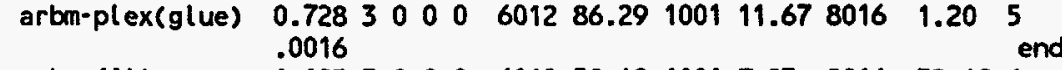
$\begin{array}{lllllll}\text { arbm-filler } & 1.1853000601259 .4910017 .838016 & 32.486 & \\ & 1.0\end{array}$

end comp

symmslabcell 17.6714 .921315 .242 end 30 cans of damp uranium oxide, $\mathrm{U}_{3} \mathrm{O}_{8}$, separated by $2.43 \mathrm{~cm}$ of Plexiglas, - $P$ lexiglas reflected

read parm run=yes npg=600 gen=405 nsk $=5$ nub=yes end parm

read geom

unit 1

com=' fuel box $15.24 \mathrm{~cm}$ on a side with $.16 \mathrm{~cm}$ walls '

cuboid $116 p 7.46$

cuboid $216 \mathrm{p} 7.62$

unit 2

com='x-face interstitial moderator'

cuboid $312 p 1.2154 p 7.62$

unit 3

com=' $y$-face interstitial moderator'

cuboid $312 \mathrm{p} 7.622 \mathrm{p} 1.2152 \mathrm{p} 7.62$

unit 4

com=' $z$-face interstitial moderator'

cuboid $314 p 7.62$ 2p1.215

unit 5

con $=$ 'more $x$-face moderator'

cuboid $314 \mathrm{p} 1.2152 \mathrm{p} 7.62$

unit 6

com=' more $y$-face moderator'

cuboid 31 2p7.62 4p1.215

unit 7

com=' 'more $z$-face moderator'

cuboid $312 \mathrm{p} 1.215$ 2p7.62 2p1.215

unit 8

com='last of interstitial moderator'

cuboid $316 \mathrm{p} 1.215$ 
unit 51

com=' empty fuel location'

cuboid $016 p 7.620$

unit 53

com='y-face moderator void'

cuboid $012 p 7.62$ 2p1.215 2p7.62

unit 11

com='north split table core'

array $13 * 0.0$

cuboid $0132.91-.59 \quad 77.50 \quad 0.0 \quad 50.58 \quad 0.0$

reflector $614 * 02.4230 .01$

reflector 011000000.301

unit 12

com='south split table core'

array $23 * 0.0$

$\begin{array}{llllllll}\text { cuboid } 0 & 1 & 33.5 & 0.0 & 77.50 & 0.0 & 50.58 & 0.0\end{array}$

reflector $6113.5 \quad 3 * 0 \quad 2.42 \quad 30.01$

reflector 01100000.301

unit - 21

com='north bottom reflector.

cuboid $311.90 \quad 0.02 p 64.05 \quad 2 p 12.60$

cuboid $5133.500 .02 p 64.052 p 12.60$

unit 22

com=' nor th top reflector '

cuboid $311.90 \quad 0.02 p 64.052 p 12.00$

cuboid $5133.500 .02 p 64.052 p 12.00$

unit 23

com='north side reflector '

cuboid $311.90 \quad 0.0 \quad 2 p 12.65 \quad 53.3 \quad-30.0$

cuboid $5133.50 \quad 0.0 \quad 2 p 12.65 \quad 53.3 \quad-30.0$

unit 24

com=' south bottom reflector with tris'

cuboid $0110.20 .02 p 2.55 \quad 2 p 12.60$

cuboid $5147.0 \quad 0.02064 .052012 .60$

unit 25

com='south top reflector with tris'

cuboid $0110.2 \quad 0.02 \mathrm{p} 2.55 \quad 2 \mathrm{p} 12.00$ cuboid $5147.0 \quad 0.02 p 64.052 p 12.00$

unit 26

com='south side reflector with tris'

cuboid $5147.0 \quad 0.0 \quad 2 p 12.65 \quad 53.3 \quad-30.0$

unit 31

com= 'north array coretside'

array $33^{\star} 0.0$

unit 32

com=' south array coretside'

array $433^{*} 0.0$

unit 33

con=' north array'

array $53 * 0.0$

reflector 41025.24 ro.0 reflector 311.2304 ro.0 1

unit 34

conl =' south array

array $63 * 0.0$

reflector $5126.5 \quad 5 r 0.0$

reflector $3101.234 \mathrm{ro} 0.0$

reflector $0110.57 \quad 4$ ro.0 1

global

unit 35

com= 1 total 1

array $73 * 0.0$

end geometry

read array

ara $=1$ nux $=3$ nuy $=5$ nuz $=5$

com='north split table core'

fill 121353196121

474686196474

1930

512153531965121 end fill

ara $=2$ nux $=3$ nuy $=5$ nuz $=5$

com='south split table core'

fill 121353196121

474686196474

1930

$125135531 q 61251$ end fill

ara $=3$ nux $=1$ nuy $=3$ nuz $=1$

conl =' north core with side wall'

fill 231123

ara $=4$ nux $=1$ nuy $=3$ nuz $=1$

end fill

com=1 south core with side wall'

fill 261226

ara $=5$ nux $=1$ nuy $=1$ nuz $=3$

com=' north assembl $y$ '

fill 213122

ara $=6$ nux $=1$ nuy $=1$ nuz $=3$

com= 'south assembly'

fill 243225

com $=$ ' total 1

fill 3334

end array

end fill

end $f i l l$

end data

end

dsn399-1

$=\operatorname{csas} 25$

dsn399-1

44group latticecel

uo2 $\quad 10.947293922354 .7429223895 .258$ 
$\begin{array}{lll}\text { al } & 2 & 1.0 \\ \text { h2o } & 3 & 1.0\end{array}$

arbmss 7.9030012430419 .172630471 .40283049 .374 hf 5 den $=13.29$ uo2 60.947293922354 .7429223895 .258 end

\section{end comp}

squarepitch 1.350 .79130 .9420 .820

more data res $=6$ cylinder .395 dan $(6)=.989923$ end more

$4-18 \times 18$ assemblies separated by $0.1046-\mathrm{cm}$-thick hafnium plates and

$-1.6954 \mathrm{~cm}$ of water, plate-to-assembly distance of $0.9 \mathrm{~cm}, 37.66 \mathrm{~cm}$

water height

read parm run=yes gen=405 npg=600 nsk=5 nub=yes end parm

\section{read geom}

unit 1

cyl inder 110.395

cylinder 010.410

cylinder

cylinder 210.470

$37.66 \quad 0.0$

37.660 .0

37.660 .0

cuboid $314 \mathrm{p0} .675$

37.660 .0

cyl inder 210.470

$0.25 \quad 0.0$

0.250 .0

$\begin{array}{llll}\text { cuboid } 4,14 \mathrm{p} 0.675 & 0.25 & 0.0\end{array}$

unit 3

$2.75 \quad 1.75$

$\begin{array}{llllll}\text { cuboid } & 3 & 1 & 4 \mathrm{p} 0.675 & 2.75 & 1.2 \\ \text { cuboid } & 4 & 1 & 4 \mathrm{p} 0.675 & 2.75 & 0.0\end{array}$

unit 4

array $13 * 0.0$

reflector $31024.8 \quad 4$ ro 1

unit 5

cuboid $\quad 512 \mathrm{2p} .0523 \quad 24.30040 .66 \quad 0.8$

$\begin{array}{llllll}\text { cuboid } 3 & 12 \mathrm{p} .9 & 24.30 & 40.66 & 0.8\end{array}$

$\begin{array}{lllll}\text { cuboid } \quad 412 \mathrm{p} .9 & 24.30 & 40.66 & 0.0\end{array}$

unit 6

array $13 * 0.0$

reflector 3124.804 ro 1

unit 7

array $23 * 0.0$

unit 8

$\begin{array}{llllllll}\text { cuboid } \quad 3149.9477 & 0 & 24.8 & 0 & 40.66 & 0.8\end{array}$

$\begin{array}{lllllll}\text { cuboid } \quad 5 & 150.0523 & 0 & 24.8 & 0 & 40.66 & 0.8\end{array}$

$\begin{array}{llllllll}\text { cuboid } \quad 31100 . & 0 & 24.80 & 40.66 & 0.8\end{array}$

cuboid $\quad 41100 . \quad 024.80 \quad 40.660 .0$

unit 9

cuboid $3149.947700 .8477040 .66 \quad 0.8$

cuboid $5,50.052300 .8477040 .660 .8$

$\begin{array}{llllllll}\text { cuboid } \quad 31 & 3100 & 0 & 0.8477 & 0 & 40.66 & 0.8\end{array}$

cuboid $51100 \quad 00.9523040 .66 \quad 0.8$

cuboid $41100 \quad 00.9523040 .660 .0$

end

end

end

end

\author{
cuboid $\quad 3149.947700 .8477040 .66 \quad 0.8$ \\ $\begin{array}{lllllllllll}\text { cuboid } \quad 5 & 1 & 50.0523 & 0 & 0.8477 & 0 & 40.66 & 0.8\end{array}$ \\ $\begin{array}{lllllllll}\text { cuboid } \quad 31 & 3100 & 0 & 0.8477 & 0 & 40.66 & 0.8\end{array}$ \\ cuboid $41100 \quad 00.8477040 .660 .0$ \\ unit 11 \\ array 3 3*0.0 \\ reflector 314 r20.0 0.020 .01 \\ unit 12

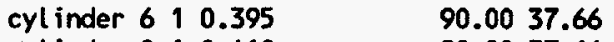 \\ cylinder $0110.410 \quad 90.0037 .66$ \\ cyl inder $2110.470 \quad 90.0037 .66$

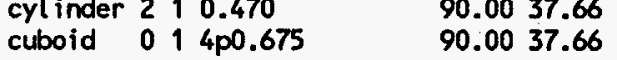 \\ unit 13 \\ array $43 * 0.0$ \\ reflector $01024.8 \quad 2 r 07.80 .0$ \\ unit 14 \\ cuboid $\quad 512 p .052324 .3 \quad 0 \quad 97.8037 .66$

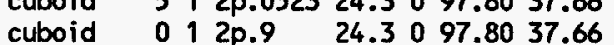 \\ cuboid \\ array $43 * 0.0$ \\ reflector $0 \quad 124.8 \quad 02$ r $0 \quad 7.8 \quad 0.0$ \\ unit 16 \\ array $53 * 0.0$ \\ unit 17 \\ cuboid 0149.9477024 .8097 .8037 .66 \\ cuboid $\quad 5149.94230024 .8 \quad 097.8037 .66$

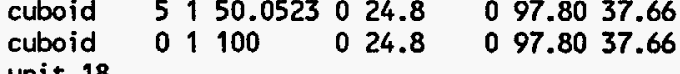 \\ cuboid 0149.9477000 .8477097 .8037 .66 \\ cuboid 5150.0523000 .8477097 .8037 .66 \\ cuboid $\quad 01100 \quad 0 \quad 0.8477097 .8037 .66$ \\ cuboid $\quad 51100 \quad 0 \quad 0.9523 \quad 0 \quad 97.8037 .66$ \\ unit 19 \\ cuboid $0149.9477 \quad 0 \quad 0.8477097 .8037 .66$ \\ cuboid 5150.0523000 .8477097 .8037 .66

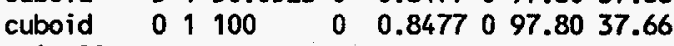 \\ unit 20 \\ array $63 * 0.0$ \\ reflector $014 \mathrm{r} 20.02 \mathrm{r} 0.01$ \\ global \\ unit 21 \\ array $73 * 0.0$ \\ end geom
}

read array

ara=1 nux=18 nuy=18 nuz=3 fill $324 r 3 \quad 324 r 2324 r 1$ end fill

ara $=2$ nux $=3$ nuy=1 nuz=1 fill $456 \quad$ end fill

end fill

ara $=3$ nux $=1$ nuy $=6$ nuz $=1$ fill 879 ill 3078

ara $=4$ nux=18 nuy=18 nuz=1 fill $324 r 12 \quad$ end fill

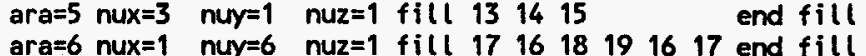


44 group latticecell

uo2 10.947293922354 .7429223895 .258 end

$\begin{array}{lll}\text { al } & 2 & 1.0 \\ \text { h20 } & 3 & 1.0\end{array}$

arbmss 7.90300012430419 .172630471 .40283049 .374 hf 5 den $=13.29$

uo2 $6 \quad 0.947293922354 .7429223895 .258$ end comp

squarepitch 1.350 .79130 .9420 .820

4-18 $\times 18$ assemblies separated by $0.1046-\mathrm{cm}$-thick hafnium plates and $-5.6954 \mathrm{~cm}$ of water, plate-to-assembly distance of $2.9 \mathrm{~cm}, 53.40 \mathrm{~cm}$ 'water height

read parm run=yes gen=405 npg=600 nsk=5 nub=yes end parm

\section{read geom}

unit 1

cylinder 0110.410

cyl inder $2,0.470$

cuboid $314 \mathrm{p} 0.675$

$53.40 \quad 0.0$

53.400 .0

53.400 .0

53.400 .0

$\begin{array}{ll}0.25 & 0.0 \\ 0.25 & 0.0\end{array}$

cylinder 310.500

$0.25 \quad 0.0$

cuboid

cylinder 210.470

$2.75 \quad 1.75$

$2.75 \quad 1.2$

cuboid $314 \mathrm{p0} 0.67$

$2.75 \quad 0.0$

unit 4

array $13 * 0.0$

reflector 31022.84 ro 1

unit 5

$\begin{array}{lllllllll}\text { cuboid } & 5 & 1 & 2 p .0523 & 24.3 & 0 & 56.40 & 0.8 \\ \text { cuboid } & 3 & 1 & 2 p 2.9 & 24.3 & 0 & 56.40 & 0.8\end{array}$

$\begin{array}{llllll}\text { cuboid } \quad 412 \mathrm{p2.9} & 24.3 & 0 & 56.40 & 0.0\end{array}$

unit 6

array $13 * 0.0$

reflector 3122.804 ro

unit 7

array $23 * 0.0$

unit 8

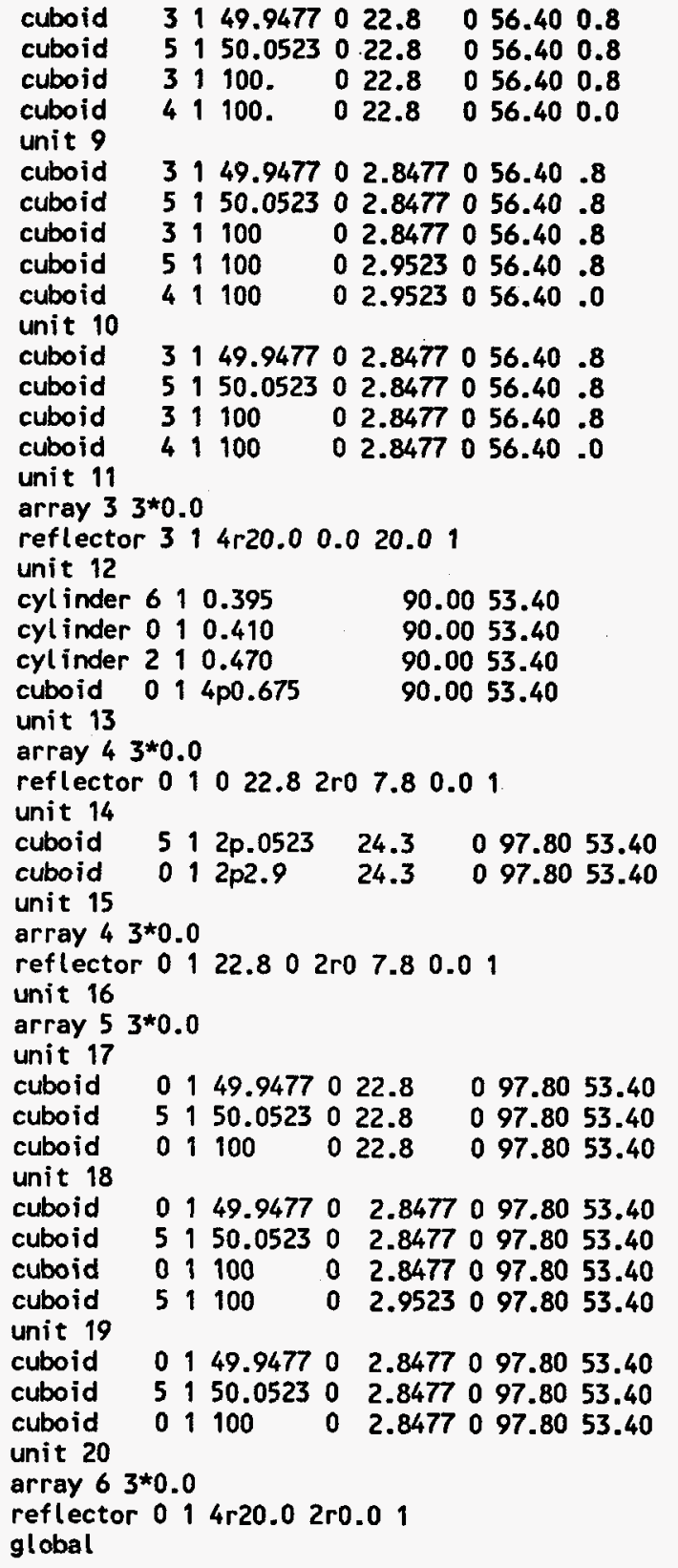


unit 21

array $73 * 0.0$

end geom

read array

ara $=1$ nux=18 nuy=18 nuz=3 fill $324 r 3324 r 2324 r 1$ end fill ara $=2$ nux $=3$ nuy=1 nuz=1 fill $456 \quad$ end fill ara $=3$ nux $=1$ nuy $=6$ nuz=1 fill 8791078 end fill ara $=4$ nux $=18$ nuy=18 nuz=1 fill $324 r 12$ end fill ara=5 nux=3 nuy=1 nuz=1 fill $13 \quad 14 \quad 15$ end fill ara $=6$ nux $=1$ nuy $=6$ nuz=1 fill $17 \quad 16 \quad 18 \quad 1916 \quad 17$ end fill $\begin{array}{llllllll}a r a=6 & \text { nux }=1 & \text { nuy } a=7 \\ \text { nux }=1 & \text { nuy=1 nuz=2 fill } & 11 & 20 & & \text { end fill }\end{array}$ end array

end data

end

dsn399-3

$=\operatorname{csas} 25$

dsn399-3

44group latticecell

uo2 10.947293922354 .7429223895 .258

al 21.0

arbmss 7.9030012430419 .172630471 .40283049 .374 end hf 5 den=13.29 end uo2 60.947293922354 .7429223895 .258 end end comp

squarepitch 1.600 .79130 .9420 .820

more data res $=6$ cyl inder .395 dan $(6)=.991569$ end more

1-21x21 assembly surrounded by $0.1046-\mathrm{cm}-$ thick hafnium plates and 0.8 $\mathrm{cm}$ of water, plate-to-assembly distance of $0.8 \mathrm{~cm}, 49.99 \mathrm{~cm}$ water height

read parm run=yes gen $=405 \mathrm{npg}=600$ nsk $=5$ nub=yes end parm

\section{read geom}

unit 1

cyl inder $110.395 \quad 49.990 .0$

cyl inder $0110.410 \quad 49.990 .0$

cyl inder $210.470 \quad 49.990 .0$

cuboid 314 p0.8 49.990 .0

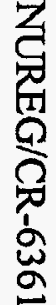

cyl inder $210.470 \quad 0.250 .0$

cylinder $310.500 \quad 0.250 .0$

cuboid $414 \mathrm{p0} .8 \quad 0.250 .0$

unit 3

$2.35 \quad 1.35$

cyl inder $210.470 \quad 2.351 .35$

$\begin{array}{llllll}\text { cuboid } & 3 & 1 & 4 \mathrm{p} 0.8 & 2.35 & 0.8 \\ \text { cuboid } & 4 & 1 & 4 \mathrm{p} 0.8 & 2.35 & 0.0\end{array}$

unit 4

cuboid $3116.2954 \quad 0 \quad 16.2954 \quad 0$

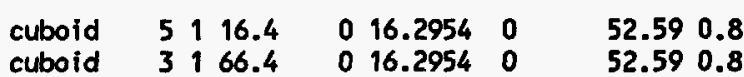

cuboid $4166.4 \quad 016.29540 \quad 52.590 .0$

cuboid

unit 5

cuboid $\quad 5116.295400 .0 \quad-0.104652 .590 .8$

cuboid $\quad 3116.2954 \quad 0 \quad 33.7046-0.1046 \quad 52.590 .8$

cuboid $\quad 4116.2954033 .7046-0.104652 .590 .0$

unit 6

array $13 * 0.0$

reflector 514 r0.1046 2r0.0

unit 7

cuboid $\quad 3116.2954033 .7046 \quad 0.0 \quad 52.590 .8$

cuboid 51 16.2954 $033.7046 \cdot .1046 \quad 52.590 .8$

cuboid $4116.2954033 .7046 \cdots .1046 \quad 52.590 .0$

unit 8

array $23 * 0.0$

unit 9

$\begin{array}{llllll}3150 . & 0 & 16.29540 & 52.59 & 0.8\end{array}$

cuboid $3166.4 \quad 016.29540 \quad 52.590 .8$

$\begin{array}{lllll}\text { cuboid } 4166.4 & 0 & 16.29540 & 52.590 .0\end{array}$

unit 10

array $3 * 0.0$

reflector 314 r20.0 0.020 .01

unit 11

cyl inder $6 \quad 1 \quad 0.395$

cyl inder 0110.410

cylinder 210.470

cuboid $014 \mathrm{p} 0.8$

unit 12

$\begin{array}{lllllll}0 & 1 & 16.2954 & 0 & 16.29540 & 98.20 & 49.99\end{array}$

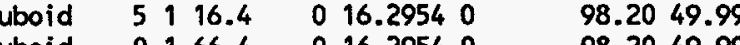

cuboid $\quad 0166.4 \quad 016.29540 \quad 98.2049 .99$

$\begin{array}{llllllll}\text { unit 13 } & 5116.2954 & 0 & 0.0 & -0.104698 .20 & 49.99\end{array}$

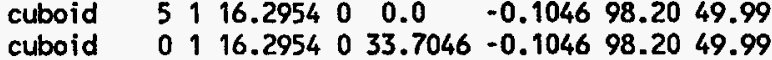

cubitid

array $43 * 0.0$

reflector $514 \mathrm{r} 0.10462 \mathrm{r} 0.01$

reflector $014 \mathrm{ro.0} \quad 8.201$

unit 15

cuboid $0116.2954 \quad 0 \quad 33.7046 \quad 0.0 \quad 98.2049 .99$

cuboid $5116.2954033 .7046 \quad-.1046 \quad 98.2049 .99$

unit 16

array $53 * 0.0$

unit 17

cuboid $\quad 5150.1046016 .29540 \quad 98.2049 .99$

cuboid $0166.4 \quad 016.29540 \quad 98.2049 .99$

unit 18

array $63 * 0.0$ 
end geom

read array

ara=1 nux=21 nuy=21 nuz=3 fill $441 r^{3} \quad 441 r^{2} \quad 441 r 1$ end fill ara $=2$ nux $=3$ nuy $=21$ nuz=3 fill $441 r 3 \quad 441 r 2 \quad 441 r 1$ end fill
nuy=1 nuz=1 fill 567 $z=1$ fill 567

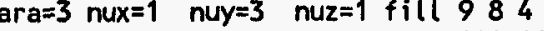

ara $a=4$ nux $=21$ nuy $=21$ nuz=1 fill $441 r 11$

ara $=5$ nux $=3$ nuy $=1$ nuz $=1$ fill $13 \quad 14 \quad 15$

end fill

ara $=5$ nux $=3$ nuy $=1$ nuz $=1$ fill 13 14 15

end fill

end array

end data

end

dsn399-4

$=\operatorname{csas} 25$

dsn399-4

44group latticecel

402 $\quad 10.947293922354 .7429223895 .258$

at 21.0

$\stackrel{N}{t}$ h2o 31.0

arbmss 7.9030012430419 .172630471 .40283049 .374 end

hf 5 den $=13.29$ end

uo2 $60.947293922354 .7429223895 .258 \quad$ end end comp

squarepitch 1.600 .79130 .9420 .820

more data res $=6$ cyl inder .395 dan $(6)=.991569$ end more

$9-20 \times 20$ assembly surrounded by $0.1046-\mathrm{cm}-$ thick hafnium plates and 2.4

cm of water, plate-to-assembly distance of $2.4 \mathrm{~cm}, 52.83 \mathrm{~cm}$ water

height

read parm run=yes gen $=405$ npg $=600$ nsk $=5$ nub=yes end parm

read geon

unit 1

cyl inder 110.395

cylinder 0110.410

cylinder 210.470

cuboid $3 \quad 14 \mathrm{p0} .8$

unit 2

cyl inder 290.470

cyl inder 310.500

cuboid $414 \mathrm{p0} .8$

unit 3

cyl inder 210.470

cuboid $3,14 \mathrm{p0} .8$

unit 4

$\begin{array}{ll}52.83 & 0.0 \\ 52.83 & 0.0 \\ 52.83 & 0.0 \\ 52.83 & 0.0 \\ & \\ 0.25 & 0.0 \\ 0.25 & 0.0 \\ 0.25 & 0.0 \\ & \\ 2.35 & 1.35 \\ 2.35 & 0.8\end{array}$

$\begin{array}{lllllllll}\text { cuboid } \quad 31 & 16.2954 & 0 & 16.2954 & 0 & 55.43 & 0.8\end{array}$

$\begin{array}{llllllll}\text { cuboid } \quad 5 & 1 & 16.4 & 0 & 16.2954 & 0 & 55.43 & 0.8\end{array}$

cuboid $\quad 3166.4 \quad 016.2954055 .43 \quad 0.8$

unit 5

cuboid $\quad 5116.2954 \quad 0 \quad 0.1046 \quad 0 \quad 55.43 \quad 0.8$

$\begin{array}{lllllllll}\text { cuboid } & 3 & 1 & 16.2954 & 0 & 33.8092 & 0 & 55.43 & 0.8\end{array}$

unit 6

array $13 * 0.0$

reflector $391.601 .602 r 0.0$

reflector $514 \mathrm{r0} .10462 \mathrm{r0.0}$

unit 7

cuboid $\quad 3116.2954 \quad 0 \quad 33.7046 \quad 0 \quad 55.43 \quad 0.8$

$\begin{array}{lllllllll}\text { cuboid } \quad 51 & 16.2954 & 0 & 33.8092 & 0 & 55.43 & 0.8\end{array}$

unit 8

array $23 * 0.0$

unit 9

$\begin{array}{llllllll}\text { cuboid } & 3150 . & 0 & 16.2954 & 0 & 55.43 & 0.8\end{array}$

$\begin{array}{lllllllll}\text { cuboid } 5 & 5 & 50.1046 & 0 & 16.2954 & 0 & 55.43 & 0.8\end{array}$

$\begin{array}{llllllll}\text { cuboid } & 31 & 166.4 & 0 & 16.2954 & 0 & 55.43 & 0.8\end{array}$

unit 10

array $33 * 0.0$

reflector $41500.0 \quad 0.8$

reflector $314 r 20.00 .020 .01$

unit 11

cyt inder 610.395

cyl inder 0190.410

cyl inder 210.470

cuboid $014 \mathrm{po} .8$

90.0052 .83

90.0052 .83

90.0052 .83

cuboid

unit 12

cuboid 5116.4

cuboid $0166.4 \quad 0 \quad 016.2954098 .2052 .83$

cuboid $5,16.2954 \quad 0 \quad 0.1046098 .2052 .83$

cuboid

$033.8092 \quad 0 \quad 98.2052 .83$

unit 14

array $43 * 0.0$

reflector 011.601 .608 .20

reflector 514 ro.1046 2ro.0 1

unit 15

cuboid $\quad 0116.2954 \quad 0 \quad 33.7046 \quad 0 \quad 98.2052 .83$

cuboid $5116.2954 \quad 033.8092098 .2052 .83$

unit 16

$\star 0.0$

array 5

unit 17

cuboid $0150 . \quad 0 \quad 16.2954098 .2052 .83$

cuboid $5150.1046 \quad 0 \quad 16.29540 \quad 98.2052 .83$

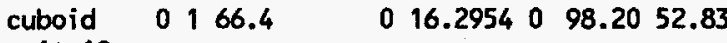

unit 18

array $63 * 0.0$

reflector $014 \mathrm{r} 20.02 \mathrm{r} 0.0$ 
global

unit 19

array $73 * 0.0$

end geom

read array

ara=1 nux=20 nuy=20 nuz=3 fill $400 r 3400 r 2400 r 1$ end fill

ara $=2$ nux $=3$ nuy $=1 \quad n u z=1$ fill 5 6 67

$\operatorname{ara}=3$ nux $=1$

nuy $=3$ nuz=1 fill 9884

end fill

end fill

end fill

ara $=6$ nux $=1$ nuy $=3 \quad$ nuz=1 fill $17 \quad 16 \quad 12$

end fill

ara $=7$ nux $=1$ nuy=1 nuz=2 fill 1018

end $f i l l$

end array

end data

end

epru65

\#csas25

epru65

44 group

$\begin{array}{lllll} & & \end{array}$ 0.01719223897 .62

$N \quad$ al 31.0293

h20 $4 \begin{array}{llll}1.0 & 293\end{array}$

pb $5 \quad 1.0 .293$

end comp

squarepitch $1.5621 \quad 1.117614 \quad 1.27003 \quad$ end

Core of 708 fuel rods, $111.76 \mathrm{~cm}$ water height with $0.9 \mathrm{ppm}$ boron

read parm gen $=405$ npg $=600$ nsk $=5$ nub=yes run=yes end parm

read geom

unit 1

cylinder $\quad 31.6352 .5402 .2225$

cuboid $\quad 41 \quad .78105-.78105 .78105 \cdot .781052 .540$

cuboid $\quad 31 \quad .78105-.78105 .78105-.781052 .540$

unit 2

cylinder 31.6355 .0802 .540

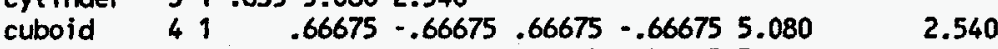

$\begin{array}{llllllll}\text { cuboid } & 31 & .78105 & -.78105 & .78105 & -.78105 & 5.080 & 2.540\end{array}$

cylinder $\quad 31.55887 .3025$

cylinder 11.558895 .885

cylinder 31.63595 .885

5.080

cylinder

5.080

unit 4

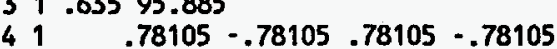

$95.885 \quad 5.080$

cylinder

$\begin{array}{llll}3 & 1 & .635 & 98.4250\end{array}$

95.885

95.885

\begin{tabular}{l}
$98.425041 .74041 \cdot .7404198 .4250 \quad 95.885$ \\
\hline
\end{tabular} unit 5

cylinder 11.558898 .742598 .4250

cyl inder 31.635100 .012598 .4250

cuboid $\quad 41.78105-.78105 .78105-.78105100 .012598 .4250$

cuboid $\quad 51.78105-.78105 .78105-.78105 \quad 100.965 \quad 98.4250$

unit 6

cuboid $\quad 4 \quad 1.78105-.78105 .78105-.781052 .540 \quad 2.2225$

cuboid $\quad 31.78105-.78105 .78105-.781052 .540 \quad 0$

unit 7

cuboid

cuboid

unit 8

cuboid

unit 9

cuboid

cuboid

unit 10

cuboid

$41.66675-.66675 .66675-.666755 .080$

2.540

$. .78105 .78105-.781055 .080$

2.540

$41.78105-.78105 .78105-.78105$

$95.885 \quad 5.080$

cuboid

global

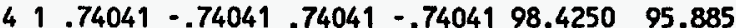

$31.78105-.78105 .78105-.7810598 .4250 \quad 95.885$

100

reflector $4130303030 \quad 15.2430 \quad 1$

end geom

read array

ara $=1$ nux $=31$ nuy=31 nuz=5

fill

$13 r 6$ 5r1 23r6 11r1 18r6 15r1 15r6 17r1 13r6 19r1 11r6 21r1 9r6 $23 r 1 \quad 7 r 6 \quad 25 r 1 \quad 5 r 6 \quad 27 r 1 \quad 2 r 6 \quad 1931$ 1r6 $29 r 1$ 1r6 2931

$154 r 1 \quad 1 r 6 \quad 155 b 403$

$\begin{array}{lllllllllllllll}13 r 7 & 5 r 2 & 23 r 7 & 11 r 2 & 18 r 7 & 15 r 2 & 15 r 7 & 17 r 2 & 13 r 7 & 19 r 2 & 11 r 7 & 21 r 2 & 9 r 7\end{array}$ $\begin{array}{lllllllllll}23 r 2 & 7 r 7 & 25 r 2 & 5 r 7 & 27 r 2 & 2 r 7 & 1931 & 1 r 7 & 29 r 2 & 1 r 7 & 2931\end{array}$ $154 r 21 r 7155 b 403$

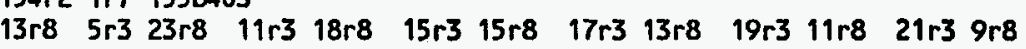
$\begin{array}{lllllllllll}23 r 3 & 7 r 8 & 25 r 3 & 5 r 8 & 27 r 3 & 2 r 8 & 1 q 31 & 1 r 8 & 29 r 3 & 1 r 8 & 2 q 31\end{array}$

$154 r 31 r 8155 b 403$

$13 r 9$ 5r4 23r9 11r4 18r9 15r4 15r9 17r4 13r9 19r4 11r9 21r4 9r9 $\begin{array}{lllllllllll}23 r 4 & 7 r 9 & 25 r 4 & 5 r 9 & 27 r 4 & 2 r 9 & 1031 & 1 r 9 & 29 r 4 & 1 r 9 & 2931\end{array}$

$154 r 41 r 9155 b 403$

$13 r 10$ 5r5 23r10 11r5 18r10 15r5 15r10 17r5 13r10 19r5 11r10 21r5 9r10 $23 r 5$ 7r10 25r5 5r10 27r5 2r10 1q31 1r10 29r5 1r10 2q31

$154 r 5 \quad 1 \times 10 \quad 1556403$

end fill

end array

end data

end

espru65b

=csas 25

epru65 
44group

1 den=9.20 $1.029392234 \quad 0.0137922352 .35 \quad 92236$ 0.01719223897 .62

al $\quad 3 \quad 1.0293$

h2o $4 \quad 1.0293$

boron 4 den=463.8-6

squarepitch $1.56211 .117614 \quad 1.27003$ end

Core of 1201 fuel rods, $111.76 \mathrm{~cm}$ water height with $463.8 \mathrm{ppm}$ boron read parm gen $=405 \mathrm{npg}=600 \mathrm{nsk}=5$ nub=yes run=yes end parm

read geom

unit 1

cylinder $\quad 31.6352 .5402 .2225$

$\begin{array}{llllllll}\text { cuboid } & 41 & .78105 & -.78105 & .78105 & -.78105 & 2.540 & 2.2225\end{array}$

cuboid $\quad 31 \quad .78105 \cdot .78105 .78105 \cdot .781052 .5400$

unit 2

cyl inde

31.6355 .0802 .540

cuboid $41 \quad .66675 \cdot .66675 .66675 \cdot .666755 .0802 .540$

unit

unit 3

cylinder

cylinder

cuboid

unit 4

cyl inder

cuboid

cuboid

unit 5

cyl inder

cyl inder

cuboid

unit 6

cuboid

cuboid

unit 7

cuboid $\quad 41 \quad .66675 \cdot .66675 .66675-.666755 .080$

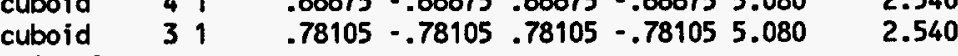

unit 8

cuboid $\quad 41 \quad .78105-.78105 .78105-.7810595 .885 \quad 5.080$

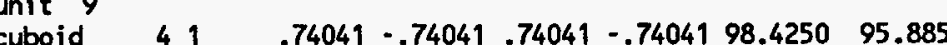

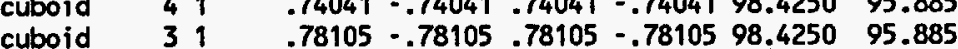
cuboid $31 \quad .78105 \cdot .78105 .78105 \cdot .7810598 .425095 .885$ $\begin{array}{llllllll}\text { cuboid } 41 & .78105 & -.78105 & .78105 & -.78105 & 100.0125 & 98.4250\end{array}$ global unit 11

array 1000

reflector 413030303015.2430 ,

end geom read array

ara $=1$ nux $=39$ nuyy $=39$ nuz=5

fill

$15 r 6$ 9r1 27r6 15r1 22r6 19r1 18r6 23r1 15r6 25r1 13r6

$27 r 111 r 6$

$\begin{array}{lllllllllll}29 r 1 & 9 r 6 & 31 r 1 & 7 r 6 & 33 r 1 & 3 r 6 & 1939 & 2 r 6 & 35 r 1 & 2 r 6 & 1 q 39\end{array}$

$1 r 637 r 1 \quad 1 r 6 \quad 2 q 39351 r 13516585$

$\begin{array}{llllllllllllllllll}15 r 7 & 9 r 2 & 27 r 7 & 15 r 2 & 22 r 7 & 19 r 2 & 18 r 7 & 23 r 2 & 15 r 7 & 25 r 2 & 13 r 7\end{array}$

$27 r^{2} 11 r 7$

$\begin{array}{lllllllllll}29 r 2 & 9 r 7 & 31 r 2 & 7 r 7 & 33 r 2 & 3 r 7 & 1039 & 2 r 7 & 35 r 2 & 2 r 7 & 1039\end{array}$ $1 r 737 r 2 \quad 1 r 72 q 39351 r 23516585$

$\begin{array}{llllllllllllllll}15 r 8 & 9 r 3 & 27 r 8 & 15 r 3 & 22 r 8 & 19 r 3 & 18 r 8 & 23 r 3 & 15 r 8 & 25 r 3 & 13 r 8 & 27 r 3 & 11 r 8\end{array}$ $\begin{array}{lllllllllll}29 r 3 & 9 r 8 & 31 r 3 & 7 r 8 & 33 r 3 & 3 r 8 & 1939 & 2 r 8 & 35 r 3 & 2 r 8 & 1939\end{array}$ $1 r 837 r 3 \quad 1 r 8 \quad 2 q 39351 r 3 \quad 351 b 585$

$15 r 9$ 9r4 27r9 $15 r 4$ 22r9 $19 r 4$ 18r9 $23 r 4$ 15r9 $25 r 4$ 13r9 $27 r 4$ 11r9 $\begin{array}{lllllllllll}29 r 4 & 9 r 9 & 31 r 4 & 7 r 9 & 33 r 4 & 3 r 9 & 1939 & 2 r 9 & 35 r 4 & 2 r 9 & 1939\end{array}$

$1 r^{2} 37 r_{4} \quad 1 r^{2} 2 q^{3} 3351 r^{4} 3516585$

$15 r 10$ 9r5 27r10 15r5 22r10 19r5 18r10 23r5 15r10 25r5 13r10 27r5 1r10 $29 r 5$ 9r10 31r5 $7 r 1033 r 5 \quad 3 r 10 \quad 1939$ 2r10 $35 r 5 \quad 2 r 101939$ end fill $1 r 1037 r 5 \cdot 1 r 102 q 39351 r 5351 b 585$

end array

end data

end

epruT5

\#csas25

epru75

44group latticecell

uo2 1 den=9.20 $1.029392234 \quad 0.0137922352 .3592236$ 0.01719223897 .62

al $3 \quad 1.0293$

h20 $4 \quad 1.0293$

end comp

squarepitch $1.905 \quad 1.117614 \quad 1.27003$

cors $112.08 \mathrm{~cm}$ water height with $0.5 \mathrm{ppm}$ boron

read parm gen $=405$ npg=600 nsk $=5$ nub=yes run=yes end parm

read geom

unit 1

cylinder 31.6355 .0803 .175

$\begin{array}{llllllll}\text { cuboid } & 41 & 0.9525 & -0.9525 & 0.9525 & -0.9525 & 5.080 & 3.175\end{array}$

$\begin{array}{lllllllllll}\text { cuboid } & 31 & 0.9525 & -0.9525 & 0.9525 & -0.9525 & 5.080 & 0\end{array}$

unit 2

cylinder $\quad 31.635 \quad 6.9855 .080$

cyl inder 41.744226 .9855 .080

$\begin{array}{lllllllll}\text { cuboid } & 3 & 1 & 0.9525 & -0.9525 & 0.9525 & -0.9525 & 6.985 & 5.080\end{array}$

unit 3

cylinder 111.558893 .986 .985

cylinder 31.63593 .986 .985 


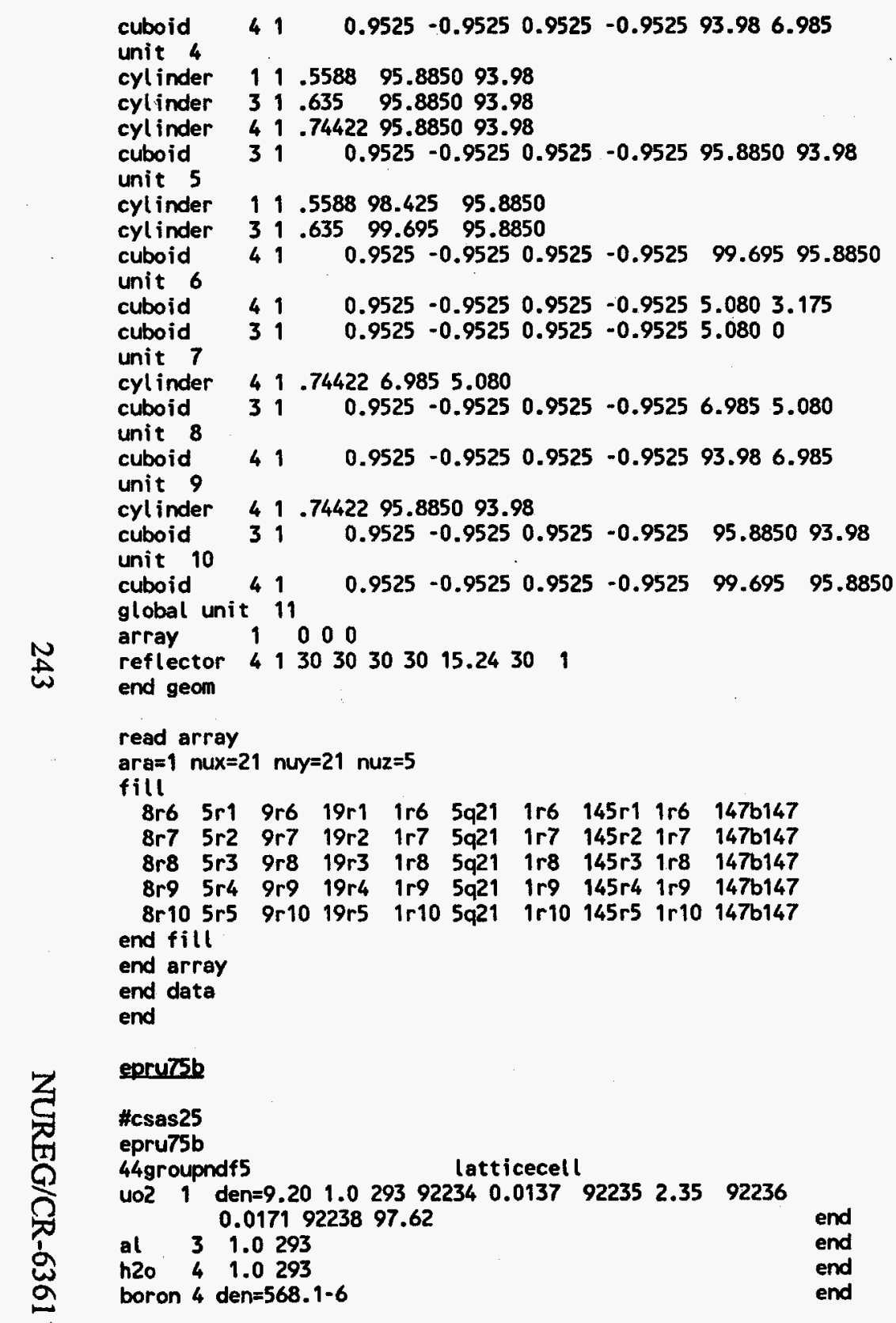

end comp

squarepitch $1.905 \quad 1.1176 \quad 14 \quad 1.27003$

3 end

Core of 1201 fuel rods, $112.08 \mathrm{~cm}$ water height with $568 \mathrm{ppm}$ boron read parm gen $=405$ npg $=600$ nsk $=5$ nub=yes run=yes end parm

\section{read geom}

unit 1

cyl inder 31.6355 .0803 .175

$\begin{array}{llllllll}\text { cuboid } & 4 & 0.9525 & -0.9525 & 0.9525 & -0.9525 & 5.080 & 3.175\end{array}$

$\begin{array}{llllllllll}\text { cuboid } & 3 & 1 & 0.9525 & -0.9525 & 0.9525 & -0.9525 & 5.080 & 0\end{array}$

unit 2

cylinder $31.635 \quad 6.9855 .080$

cyl inder 41.744226 .9855 .080

$\begin{array}{llllllll}\text { cuboid } \quad 31 & 0.9525 & -0.9525 & 0.9525 & -0.9525 & 6.985 & 5.080\end{array}$

unit 3

cyl inder $\quad 11.558893 .986 .985$

cylinder 11.53593 .986 .985

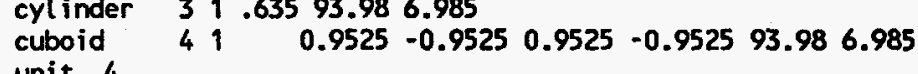

unit 4

cylinder $\quad 11.5588 \quad 95.885093 .98$

cylinder $31.635 \quad 95.885093 .98$

cylinder $\quad 41.7442295 .885093 .98$

$\begin{array}{llllllll}\text { cuboid } & 3 & 0.9525 & -0.9525 & 0.9525 & -0.9525 & 95.8850 & 93.98\end{array}$

unit

cyl inder $11.558898 .425 \quad 95.8850$

cylinder $\quad 3 \quad 1.635 \quad 99.695 \quad 95.8850$

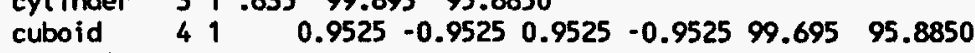

unit 6

$\begin{array}{lllllllll}\text { cuboid } \quad 41 & 0.9525 & -0.9525 & 0.9525 & -0.9525 & 5.080 & 3.175\end{array}$

$\begin{array}{llllllllllll}\text { cuboid } & 31 & 0.9525 & -0.9525 & 0.9525 & -0.9525 & 5.080 & 0\end{array}$

unit 7

cylinder $\quad 41.744226 .985 \mathbf{5 . 0 8 0}$

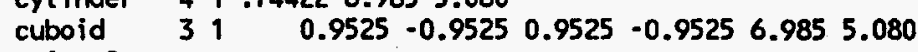

unit 8

$\begin{array}{lllllllll}\text { cuboid } \quad 41 & 0.9525 & -0.9525 & 0.9525 & -0.9525 & 93.98 & 6.985\end{array}$

unit 9

cylinder $\quad 41.7442295 .885093 .98$

$\begin{array}{lllllllll}\text { cuboid } & 3 & 0 & 0.9525 & -0.9525 & 0.9525 & -0.9525 & 95.8850 & 93.98\end{array}$

$\begin{array}{lllllllll}\text { unit } 10 & & & & & & & & \\ \text { cuboid } & 4 & 1 & 0.9525 & -0.9525 & 0.9525 & -0.9525 & 99.695 & 95.8850\end{array}$

global unit 11

array 1000

reflector $413030303015.2430 \quad 1$

end geom

read array

ara $=1$ nux $=39$ nuy $=39$ nuz $=5$

fill

$15 r 6$ 9r1 $27 r 6 \quad 15 r 122 r 6 \quad 19 r 1$ 18r6 23r1 15r6 $25 r 1$ 13r6 27r1 11r6 $29 r 1$ 9r6 31r1 7r6 33r1 3r6 1q39 2r6 35r1 2r6 1939 $1 r 637 r 1 \quad 1 r 62 q 39351 r 13516585$ 


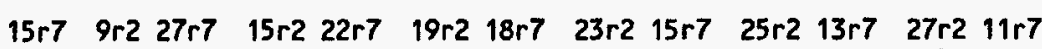
$\begin{array}{lllllllllll}29 r 2 & 9 r 7 & 31 r 2 & 7 r 7 & 33 r 2 & 3 r 7 & 1 q 39 & 2 r 7 & 35 r 2 & 2 r 7 & 1 q 39\end{array}$ $1 r 737 r^{2} \quad 1 r 72939351 r^{2} 351 b 585$

$\begin{array}{lllllllllllllllll}15 r 8 & 9 r 3 & 27 r 8 & 15 r 3 & 22 r 8 & 19 r 3 & 18 r 8 & 23 r 3 & 15 r 8 & 25 r 3 & 13 r 8 & 27 r 3 & 11 r 8\end{array}$ $\begin{array}{lllllllllll}29 r 3 & 9 r 8 & 31 r 3 & 7 r 8 & 33 r 3 & 3 r 8 & 1939 & 2 r 8 & 35 r 3 & 2 r 8 & 1939\end{array}$ $1 r 8 \quad 37 r 3 \quad 1 r 8 \quad 2 q 39351 r 3 \quad 351 b 585$

$15 r 9$ 9r4 27r9 $15 r 422 r 9$ 19r4 $18 r 923 r 4 \quad 15 r 9 \quad 25 r 4 \quad 13 r 9 \quad 27 r 4 \quad 11 r 9$

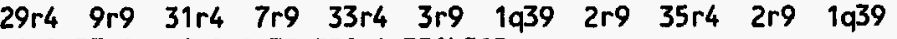
$1 r 937 r 4 \quad 1 r 92939351 r 4351 b 585$

$15 r 109 r 5 \quad 27 r 10 \quad 15 r 5 \quad 22 r 10 \quad 19 r 5 \quad 18 r 10 \quad 23 r 5 \quad 15 r 10 \quad 25 r 5 \quad 13 r 10 \quad 27 r 5 \quad 1 r 10$

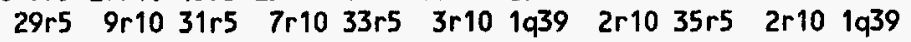
end $f i l l$ 1 ir10 $37 r 5$ ir10 $2939351 r 53516585$

\section{end array}

end data

end

epru87

\#csas 25

epru87

44 group

no2 1 den=9.20 $1.0 \quad 293 \quad 92234 \quad 0.0137 \quad 92235 \quad 2.35 \quad 92236$ 0.01719223897 .62

$\begin{array}{llll}\text { al } & 3 & 1.0 & 293 \\ \text { h20 } & 4 & 1.0 & 293\end{array}$

pb $5 \quad 1.0293$

end comp

squarepitch $2.20981 .117614 \quad 1.27003$

end

end

Core of 342 fuel rods, $111.76 \mathrm{~cm}$ water height with $0.9 \mathrm{ppm}$ boron

read parm gen $=405 \mathrm{npg}=600 \mathrm{nsk}=5$ nub=yes run=yes end parm

read geom

unit 1

cylinder 31.6352 .5402 .225

cuboid $\quad 41 \quad .78105-.78105 .78105-.781052 .5402 .225$

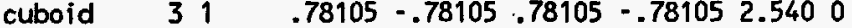

unit 2

cyl inder $\quad 31.6355 .0802 .540$

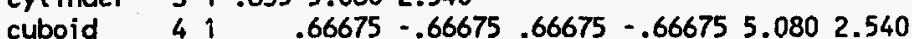

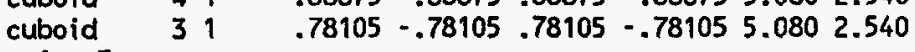

unit 3

cylinder 31.55887 .30255 .080

cylinder $11.558895 .885 \mathbf{5 . 0 8 0}$

cylinder 31.63595 .8855 .080

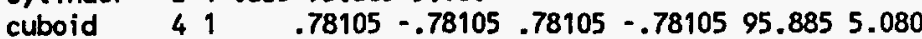

unit 4

cylinder 11.558898 .425095 .885

cylinder 31.63598 .425095 .885

cuboid $41.74041-.74041 .74041-.7404198 .425095 .885$

cuboid $\quad 31 \quad .78105-.78105 .78105-.7810598 .425095 .885$ unit 5

cylinder 11.558898 .742598 .4250

cylinder 31.635100 .012598 .4250

cuboid $41 \quad .78105-.78105 .78105-.78105100 .012598 .4250$

$\begin{array}{llllllll}\text { cuboid } & 51 & .78105 & -.78105 & .78105 & -.78105 & 100.965 & 98.4250\end{array}$

unit 6

cuboid

$41 \quad .78105-.78105 .78105-.781052 .5402 .225$

cuboid

Unit 7

cuboid

cuboid

unit 8

cuboid

unit 9

$\begin{array}{llllll} & & & \\ \text { 4 } & .74041 & -.74041 .74041 & -.7404198 .425095 .885\end{array}$

unit 10

cuboid $\quad 41 \quad .78105-.78105 .78105 \cdot .78105 \quad 100.012598 .4250$

cuboid $\quad 51 \quad .78105-.78105 .78105 \quad-.78105 \quad 100.965 \quad 98.4250$

global unit 11

array

10000

end geom

read array

ara $=1$ nux $=29$ nuy=29 nuz=5

fill

$10 r 6164 q 29 r 69 r 6166 q 2$ 6r6 $6 r 6168 q 25 r 6 \quad 5 r 6169 q 24 r 6$

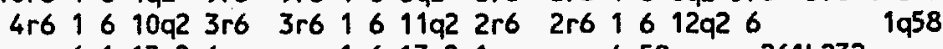

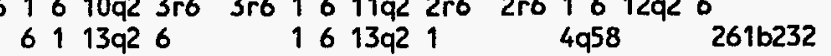

$9 r 6165 q 2 \quad 8 r 6$ 10r6 $164 q 29 r 6$

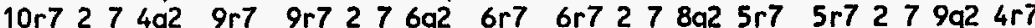

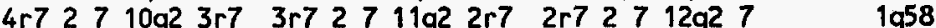

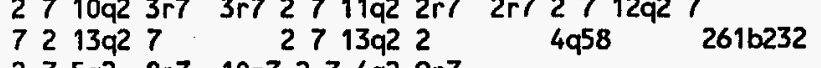

$9 r 72 \begin{array}{llllll}9 q^{2} & 8 r 7 & 10 r 7 & 27 & 4 q 2 & 9 r 7\end{array}$

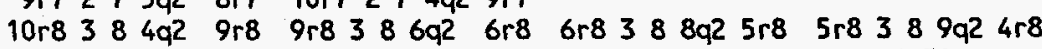

$\begin{array}{llllllllllllllll}4 r 83 & 3 & 10 q 2 & 3 r 8 & 3 r 8 & 3 & 8 & 11 q 2 & 2 r 8 & 2 r 8 & 3 & 8 & 12 q 2 & 8 & 1 q 58\end{array}$ $\begin{array}{llllll}8 & 313 q 2 & 8 & 38 & 13 q 2 & 3\end{array}$

$9 r_{8} 38 \quad 5 q 2 \quad 8 r 8 \quad 10 r 838 \quad 4 q 2 \quad 9 r 8$

$10 r 9494 q^{2} 9 r 9$ 9r9 $496 q^{2}$ 6r9 6r9 49 8q2 $5 r^{\circ} \quad 5 r 9499 q^{2} 4 r^{\circ}$

$4 r 949$ 10q2 3r9 $3 r 949$ 11q2 $2 r 9$ 2r9 49 12q2 9 1q58

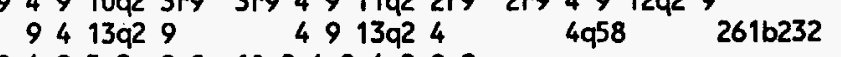

$9 r 9495 q^{2} \quad 8 r 9$ 10r9 $494 q_{2} 9 r 9$

$10 r 105104 q 2$ 9r10 9r10 5 10 6q2 6r10 6r10 5 10 $8 q 25 r 105 r 10510$ $9 q 24 r 10$

$\begin{array}{llllllllllllllll}4 r 10 & 5 & 10 & 10 q 2 & 3 r 10 & 3 r 10 & 5 & 10 & 11 q 2 & 2 r 10 & 2 r 10 & 5 & 10 & 12 q 2 & 10 & 1 q 58\end{array}$

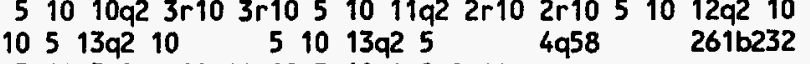

9r10 5 10 $5 q^{2} 8 r_{10} 10 r 105104 q 29 r 10$

end fill

end array 
end data

end

\section{eprus7b}

\#csas25

epru87b

44 group

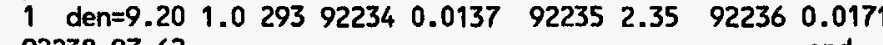

al 31.0293

h2o $4 \quad 1.0293$

boron 4 den $=285.8-6$

end comp

squarepitch $2.2098 \quad 1.117614 \quad 1.27003$

Core of 885 fuel rods, $111.76 \mathrm{~cm}$ water height with $285.8 \mathrm{ppm}$ boron

read parm gen $=405 \mathrm{npg}=600 \mathrm{nsk}=5$ nub=yes run=yes end parm

read geom

unit 1

cylinder $3 \quad 1.6352 .5402 .2225$

cuboid $\quad 41.1 .1049-1.10491 .1049-1.10492 .5402 .2225$

cuboid

unit 2

unit 2

cylinder 31.6355 .0802 .540

cuboid

cuboid

cylinder

$\begin{array}{lll}41 & 99060-.99060 .99060-.990605 .0802 .540\end{array}$

cylinder

31

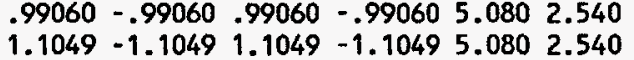

cylinder 31.63595 .8855 .080

cuboid

$41 \quad 1.1049-1.10491 .1049-1.104995 .8855 .080$

cyl inder

cuboid $\quad 4 T^{1.06426}-1.064261 .06426-1.0642698 .425095 .885$

cuboid $31 \quad 1.1049-1.10491 .1049-1.104998 .425095 .885$

unit 5 cylinder 11.558898 .742598 .4250

cylinder 19.558898 .742598 .4250

$\begin{array}{llllllll}\text { cylinder } & 3 & 1 & .635 & 100.0125 & 98.4250 & & \\ \text { cuboid } & 4 & 1 & 1.1049 & -1.1049 & 1.1049 & -1.1049 & 100.012598 .4250\end{array}$

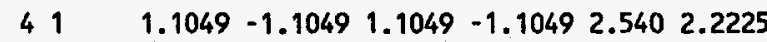

cuboid $\quad 31 \quad 1.1049-1.10491 .1049-1.10492 .5400$

unit 7

cuboid

$41 \quad .99060-.99060 .99060-.990605 .0802 .540$

$1.1049-1.10491 .1049-1.10495 .0802 .540$

unit 8

cuboid 3

unit 9

$41 \quad 1.1049-1.10491 .1049-1.104995 .8855 .080$

cuboid

$1.06426-1.06426 \quad 1.06426-1.0642698 .425095 .885$

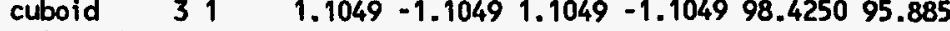

unit 10

cuboid

41

$1.1049-1.10491 .1049-1.1049100 .012598 .4250$

global

1000

reflector $\begin{array}{lllllllll}4 & 1 & 30 & 30 & 30 & 30 & 15.24 & 30 & 1\end{array}$

end geom

read array

ara $=1$ nux $=33$ nuy $=33$ nuz=5

fill

$11 r 6$ 11r1 20r6 15r1 16r6 19r1 13r6 21r1 11r6 23r1 9r6 25r1 7r6

$27 r 1$ 5r6 29r1 2r6 1q33 1r6 31r1 1r6 1q33 363r1 $363 \mathrm{b3} 363$

$\begin{array}{llllllllllllll}11 r 7 & 11 r 2 & 20 r 7 & 15 r 2 & 16 r 7 & 19 r 2 & 13 r 7 & 21 r 2 & 11 r 7 & 23 r 2 & 9 r 7 & 25 r 2 & 7 r 7\end{array}$

$27 r 2 \quad 5 r 729 r 2 \quad 2 r 71933 \quad 1 r 731 r 2 \quad 1 r 7$ 1q33 $363 r 2 \quad 363 b 363$

$11 r 811 r 3 \quad 20 r 8 \quad 15 r 3 \quad 16 r 8$ 19r3 $13 r 8$ 21r3 11 r8 $23 r 3$ 9r8 $25 r 3 \quad 7 r 8$

$\begin{array}{lllllllllll}27 r 3 & 5 r 8 & 29 r 3 & 2 r 8 & 1933 & 1 r 8 & 31 r 3 & 1 r 8 & 1933 & 363 r 3 & 363 b 363\end{array}$

$11 r 9$ 11r4 $20 r 9$ 15r4 16r9 19r4 13ro 21r4 11r9 23r4 9ro 25r4 7ro

$27 r 4$ 5r9 29r4 2r9 1033 1r9 31r4 1r9 1a33 $363 r 4363 b 363$

$11 r 1011 r 5 \quad 20 r 10 \quad 15 r 5 \quad 16 r 10$ 19r5 $13 r 10 \quad 21 r 5$ 11r10 23r5 9r10 25r5 7r10

$27 r 5 \quad 5 r 1029 r 5 \quad 2 r 10 \quad 1933$ 1r10 $31 r 5$ 1r10 1q33 $363 r 5 \quad 363 b 363$

end $f i l l$

end array

end data

end

nse71h1

\#csas25

nse71h1

44 group latticecell

uo2 $\quad 1$ den=10.38 1.0 293922354.7429223895 .258

$12000 \quad 0.47 \quad 14000 \quad 0.43$

260000.22

h2o $\quad 31.0$

al $\quad 50.437 \quad 72630471.402430419 .16283049 .4441$ end

arbmstl 7.9030012630471 .402430419 .16283049 .445 .505 end

h20 $\quad 50.058$ and

10.062

at 60.62

uo2 70.946293922354 .7429223895 .258 end

end comp

$\begin{array}{lllllllll}\text { triangpitch } & 1.35 & 0.79 & 1 & 0.94 & 2 & 0.82 & 0\end{array}$

more data res $=7$ cyl 0.395 dan $(7)=.98636$

end

Core of 547 fuel rods in a hexagonal lattice, $60.93 \mathrm{~cm}$ water height

read parm run=yes gen $=405 \mathrm{npg}=600 \mathrm{nsk}=5$ nub=yes end parm

read geom

unit 2

zhemicyl+y $11.395 \quad 60.93 \quad 0.0$ 


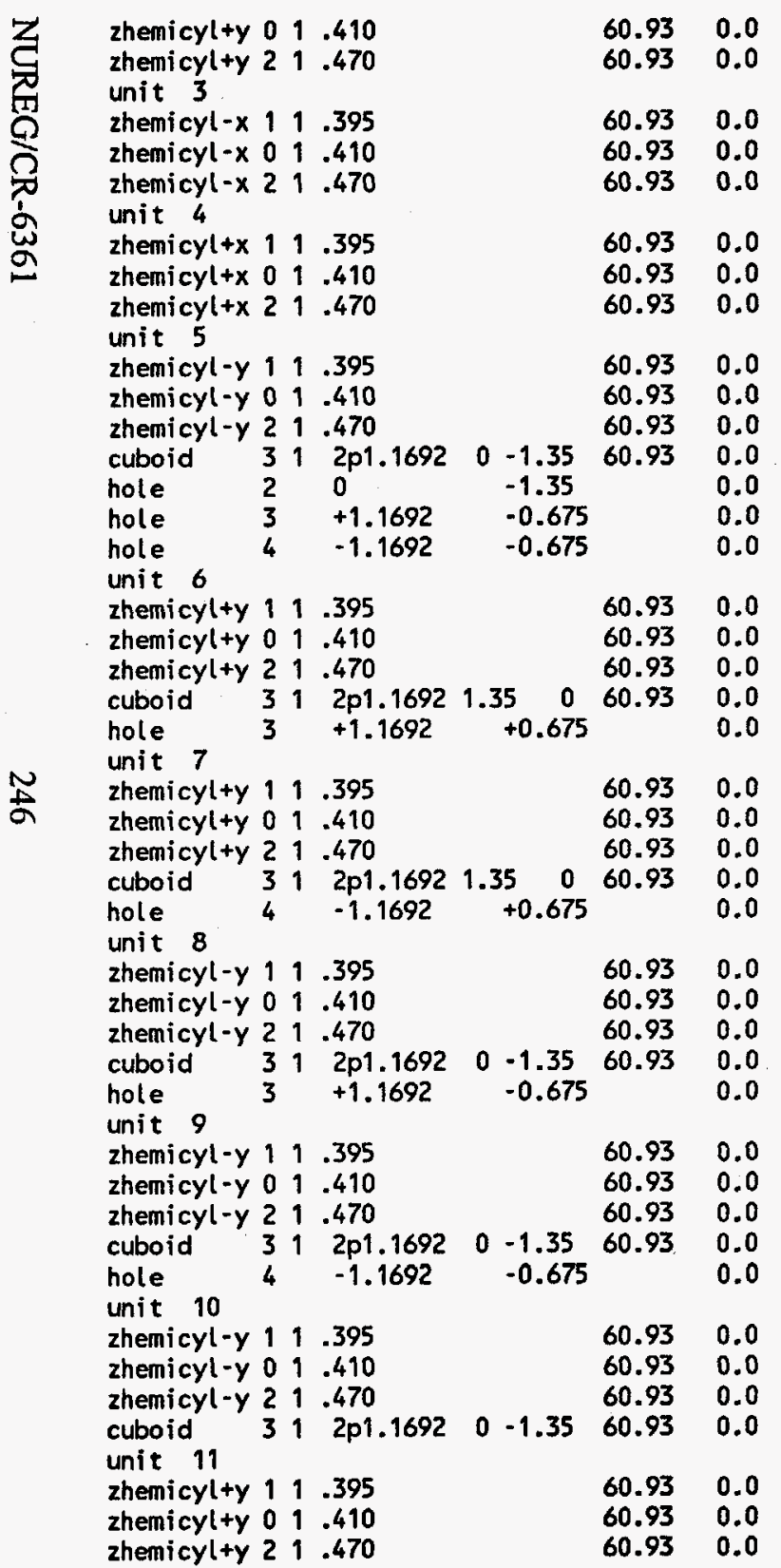

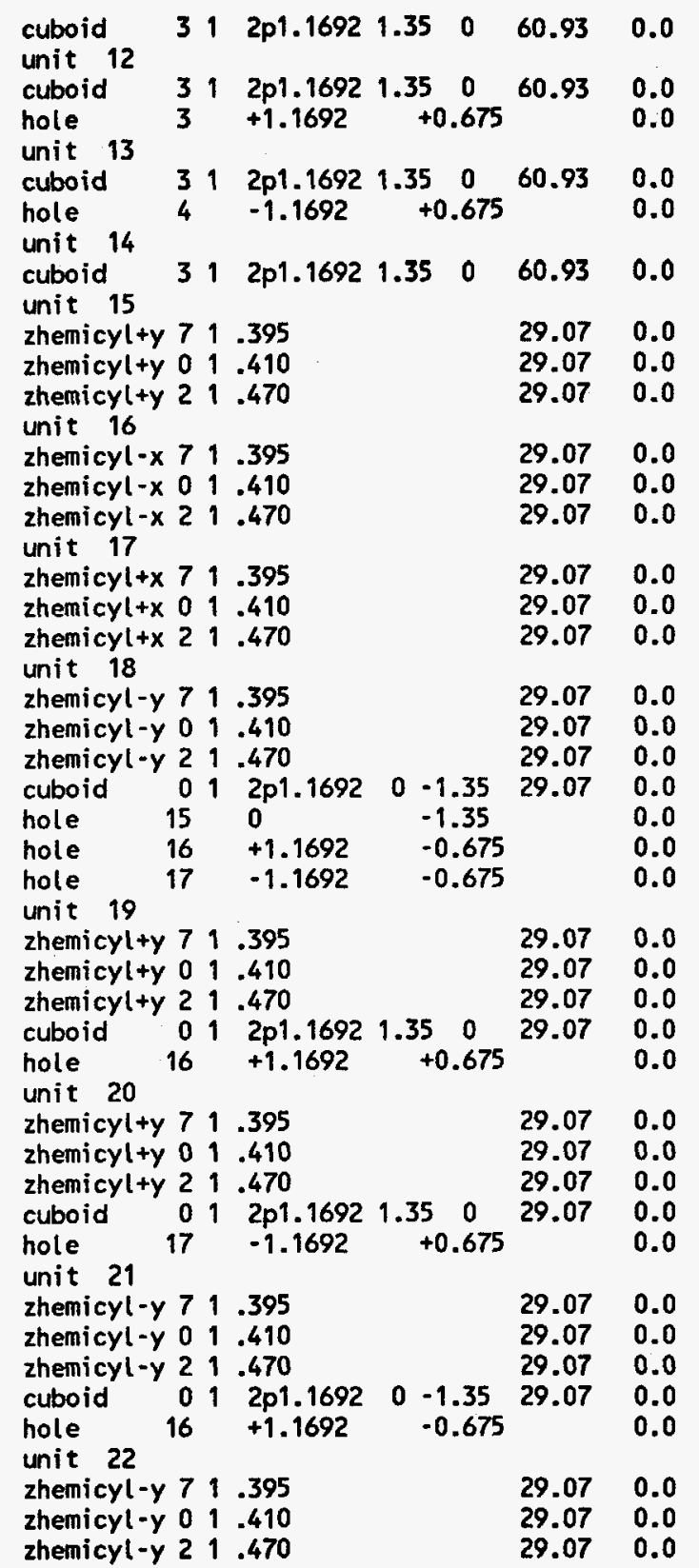




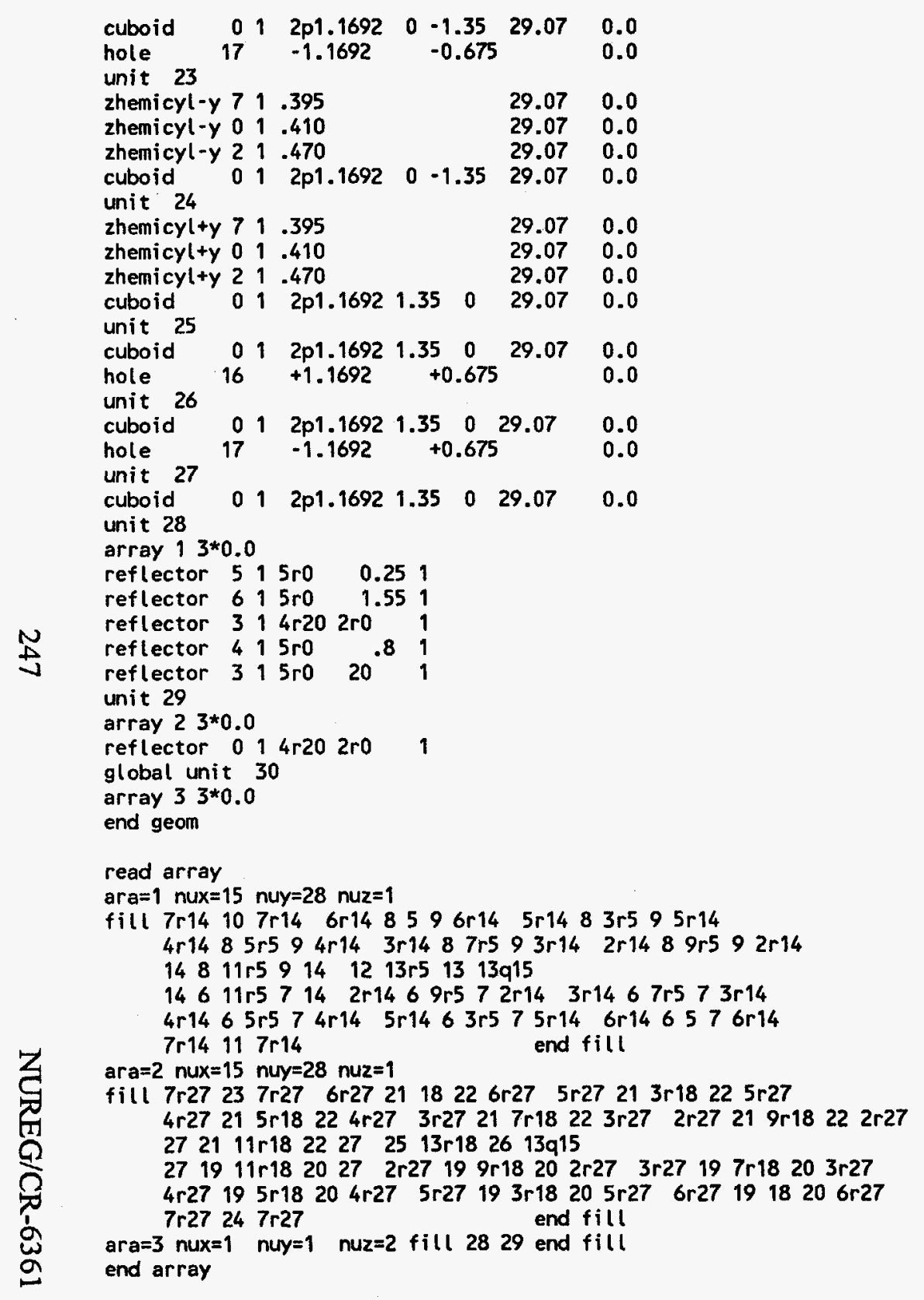

end data

end

\section{nse71h?}

\#csas25

nse71h2

44group latticecel

uo2 1 den=10.38 1.0293922354 .7429223895 .258

arbmag5 $2.704 \quad 0 \quad 0 \quad 1 \quad 1302798.8512000 \quad 0.47 \quad 14000 \quad 0.43$ 260000.2221293

h20 $\quad 31.0$

arbmstl 7.9030012630471 .402430419 .16283049 .4441 end

al 50.437 and

arbmstl 7.9030012630471 .402430419 .16283049 .445 .505 end

h20 $50.058 \quad 50.62$

60.62

at 60.62

end

uo2 70.946293922354 .7429223895 .258 end

$\begin{array}{llllllllll}\text { triangpitch } & 1.72 & 0.79 & 1 & 3 & 0.94 & 2 & 0.82 & 0\end{array}$ end

more data res $=7$ cyl 0.395 dan $(7)=.98899$ end

Core of 271 fuel rods in a hexagonal lattice, $68.06 \mathrm{~cm}$ water height

read parm run=yes gen $=405$ npg $=600$ nsk $=5$ nub=yes end parm

\section{read geom}

unit 2

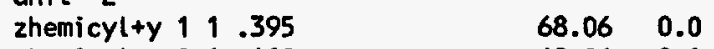

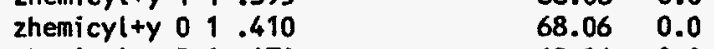

$\begin{array}{lllll}\text { zhemicylty } 2 & 1.470 & 68.06 & 0.0\end{array}$

unit 3

$\begin{array}{lllll}\text { zhemicyl-x } & 1 & 1.395 & 68.06 & 0.0\end{array}$

zhemicyl-x $01 . .410 \quad 68.06 \quad 0.0$

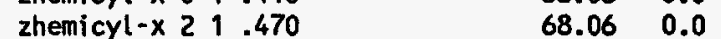

unit 4

zhemicyl+x $11.395 \quad 68.06 \quad 0.0$

$\begin{array}{llllll}\text { zhemicyl+x } & 0 & 1 & .410 & 68.06 & 0.0\end{array}$

$\begin{array}{lllll}\text { zhemicyl+x } 2 & 1.470 & 68.06 & 0.0\end{array}$

unit 5

zhemicyl-y $11.395 \quad 68.06 \quad 0.0$

zhemicyl-y $011.410 \quad 68.06 \quad 0.0$

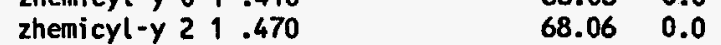

$\begin{array}{llllllll}\text { cuboid } & 3 & 1 & 2 p 1.4896 & 0 & -1.72 & 68.06 & 0.0\end{array}$

$\begin{array}{lllll}\text { hole } & 2 & 0 & -1.72 & 0.0\end{array}$

$\begin{array}{lllll}\text { hole } & 3 & +1.4896 & -0.860 & 0.0\end{array}$

hole $\quad 4 \quad-1.4896 \quad-0.860$

unit 6

$68.06 \quad 0.0$

zhemicylty 111.395

zhemicylty 01.410

zhemicylty 21.470

$\begin{array}{ll}68.06 & 0.0 \\ 68.06 & 0.0\end{array}$

(n)

(n)

(n)

$\begin{array}{lllllllllllllll}\text { cuboid } & 3 & 1 & 2 p 1.4896 & 1.72 & 0 & 68.06 & 0.0\end{array}$


reflector $6115 \mathrm{ro} \quad 1.551$

$\begin{array}{lllllll}\text { reflector } & 3 & 1 & 4 \mathrm{r} 20 & 2 \mathrm{ro} & & 1 \\ \text { reflector } & 4 & 1 & 5 \mathrm{ro} & .8 & 1\end{array}$

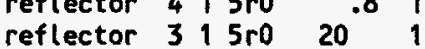

unit 29

unit 29

reflector 0 i $4 \mathrm{r} 202 \mathrm{2r} 0 \quad 1$

globat unit 30

array $33 * 0.0$

end geom

read array

ara $=1$ nux $=11$ nuy $=20$ nuz $=1$

fill $5 r 1410 \quad 5 r 14 \quad 4 r 148594 r 14 \quad 3 r 14 \quad 8 \quad 3 r 593 r 14$

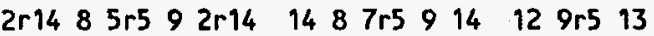
$9 q 11$

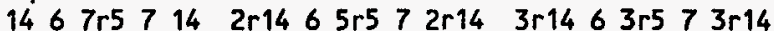
$4 r 146574 r 14 \quad 5 r 14115 r 14$ end fill ara $=2$ nux $=11$ nuy $=20$ nuz=1

fill $5 r 27 \quad 23 \quad 5 r 27 \quad 4 r 2721 \quad 1822 \quad 4 r 27 \quad 3 r 27 \quad 21 \quad 3 r 18 \quad 22 \quad 3 r 27$

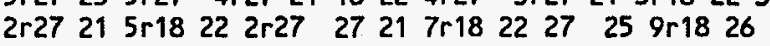
$9 q 11$

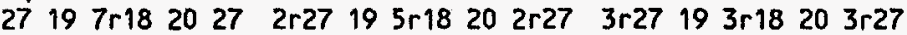
$4 r 27 \quad 19 \quad 18204 r 27 \quad 5 r 27 \quad 245 r 27$ end fill

ara $=3$ nux $=1$ nuy=1 nuz=2 fill 2829 and fill

end array

end data

end

nse71h3

\#csas 25

nse71h3

44 group latticecell

102 1 den=10.38 1.0293922354 .7429223895 .258 $2.7040011302798 .85120000 .47 \quad 14000 \quad 0.43$

arbmags 260000.2221293

h20 31.0

arbmstl $\quad 7.9030012630471 .4024304 \quad 19.16283049 .44 \quad 41$ 50.437

arbmstl $7.9030012630471 .402430419 .16283049 .445 \quad .505$ h2o $\quad 50.058$

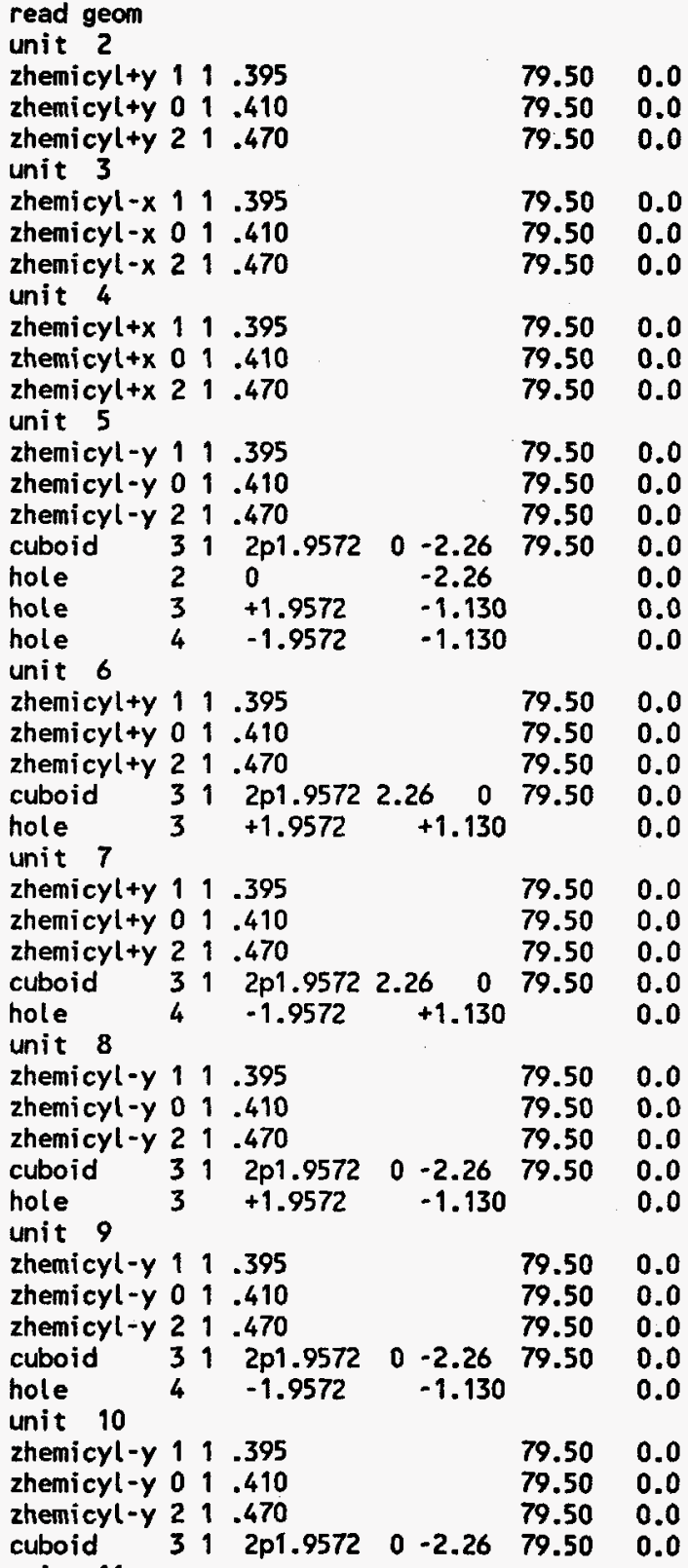




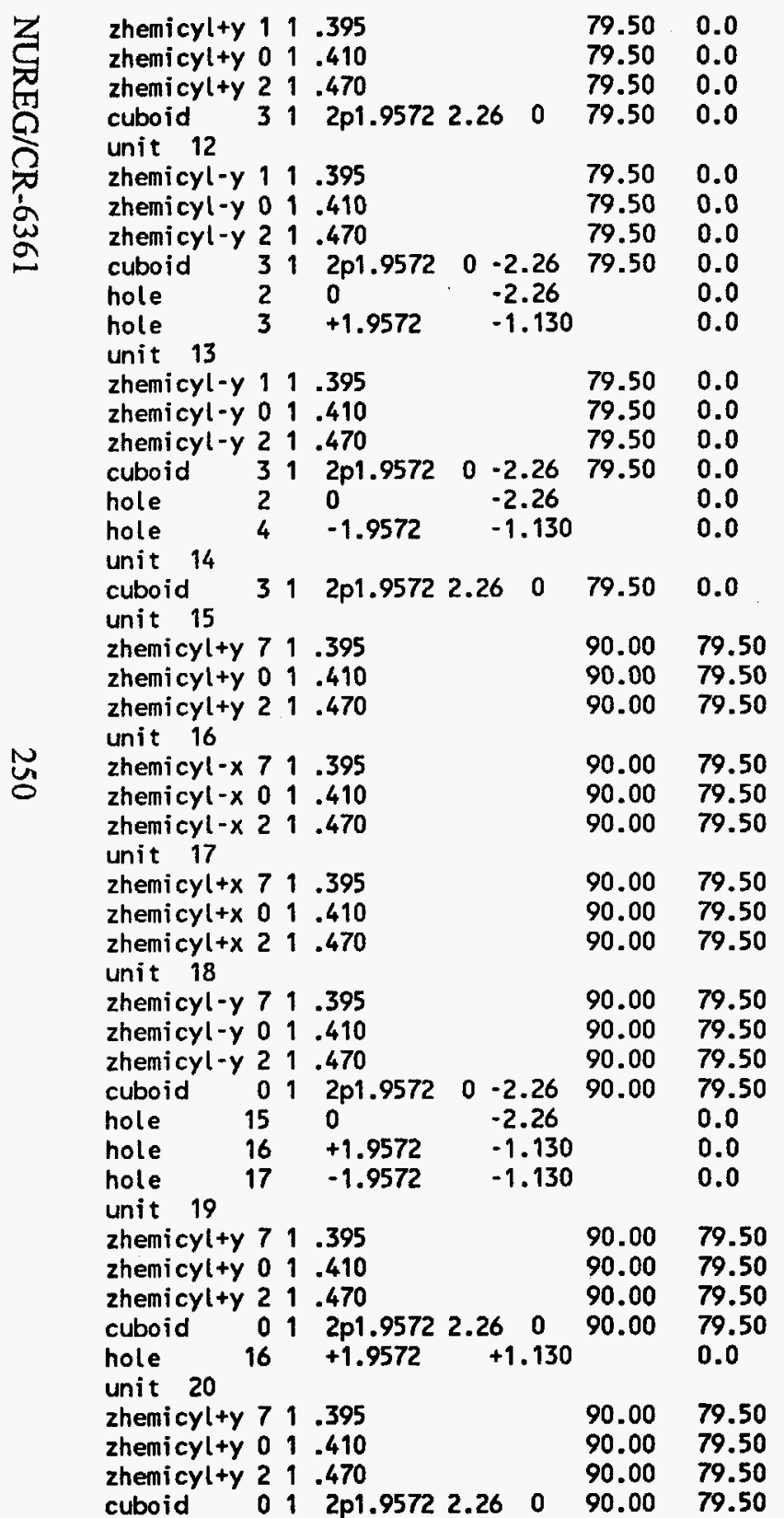

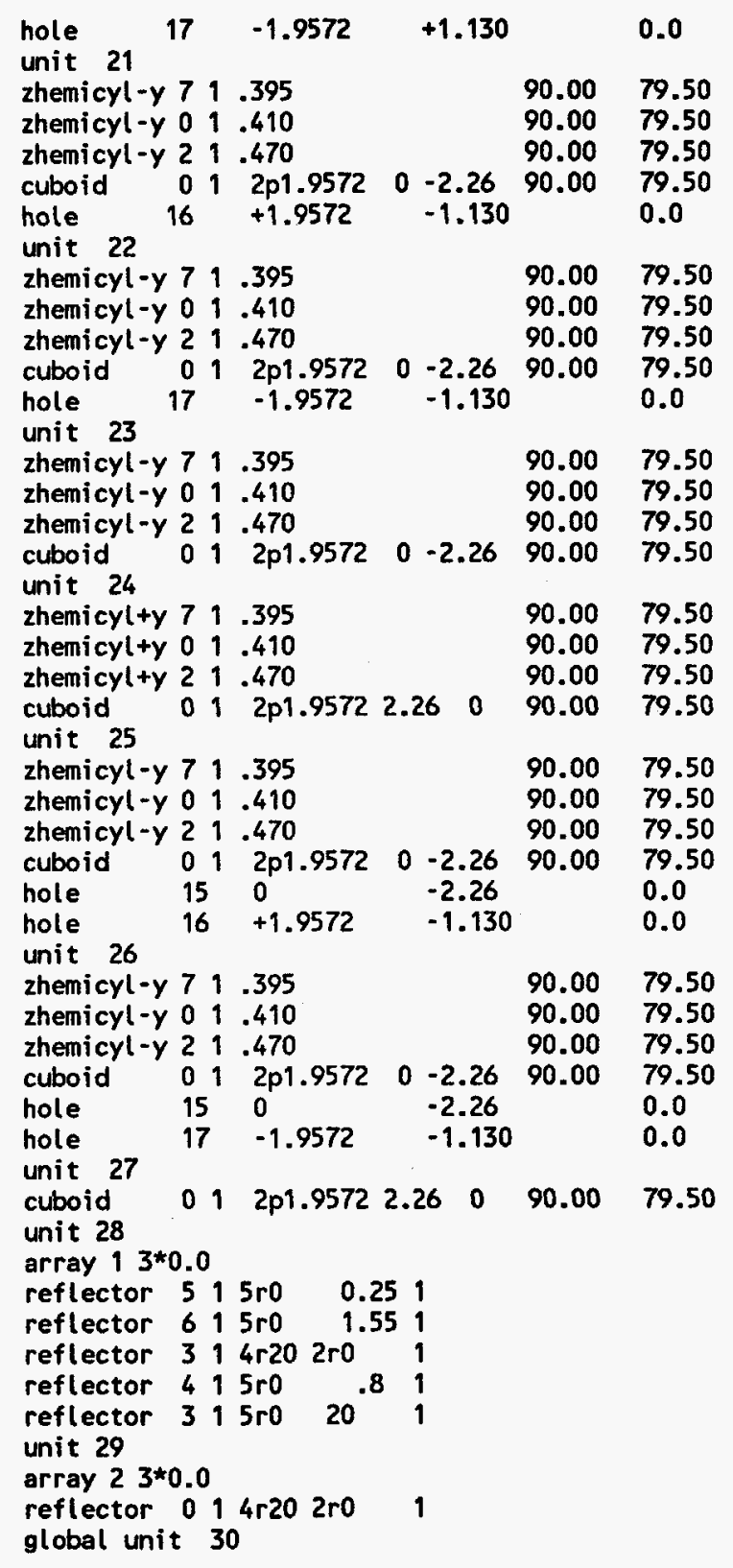


array $33 * 0.0$

end geom

read array

ara $=1$ nux $=9$ nuyy $=18$ nuz $=1$

fill $4 r 14 \quad 104 r 14 \quad 3 r 148593 r 14 \quad 2 r 1483 r 592 r 14$

$\begin{array}{lllllllllll}14 & 8 & 5 r 5 & 9 & 14 & 8 & 7 r 5 & 9 & 12 & 7 r 5 & 13\end{array}$

$$
799
$$

$67 r 57 \quad 1465 r 5714 \quad 2 r 1463 r 572 r 14$

$3 r 146573 r_{14} 4 r 14114 r 14$ end fill

ara $=2$ nux $=9$ nuy=18 nuz $=1$

$\begin{array}{lllllllllllllllll}\text { fill } & 4 r 27 & 23 & 4 r 27 & 3 r 27 & 21 & 18 & 22 & 3 r 27 & 2 r 27 & 21 & 3 r 18 & 22 & 2 r 27\end{array}$

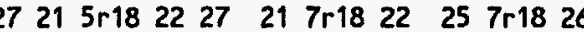

799

$\begin{array}{lllllllllllllll}19 & 7 r 18 & 20 & 27 & 19 & 5 r 18 & 20 & 27 & 2 r 27 & 19 & 3 r 18 & 20 & 2 r 27\end{array}$

$3 r 27191820 \quad 3 r 27 \quad 4 r 2724 \quad 4 r 27$ end $f i l l$

ara $=3$ nux $=1$ nuy $=1$ nuz $=2$ fill 2829 end fill

end array

end data

end

\section{nse71sa}

$=\operatorname{csas} 25$

nse71sq

44group latticecell

0.947293922354 .7429223895 .258

arbmag5 $2.7040011302798 .8512000 \quad 0.4714000 \quad 0.43$ 260000.2221293

h20 $\quad 31.0$

arbmss $\quad 7.9030001 .24304 \quad 19.172630471 .40283049 .374$ 51.0

end comp

squarepitch $1.26 \quad 0.79130 .9420 .820$

Core of $22 \times 22$ fuel rods, $90.69 \mathrm{~cm}$ water height

read parm run=yes gen $=405$ npg $=600$ nsk $=5$ nub=yes end parm

read geom

unit 1

cylinder 110.395

cylinder 010.410

cylinder 210.470

cuboid $314 \mathrm{p} 0.63$

unit

cyl inder 5 1 0.470

cyl inder 310.500

cuboid $414 \mathrm{p} 0.63$

unit 3

cylinder 510.470

cuboid $314 \mathrm{p0} .63$

cuboid $414 \mathrm{p0} .63$
$90.00 \quad 0.0$

$90.00 \quad 0.0$

$90.00 \quad 0.0$

90.690 .0

$\begin{array}{ll}0.25 & 0.0\end{array}$

$0.25 \quad 0.0$

0.250 .0

$2.35 \quad 0.8$

$2.35 \quad 0.8$ unit 4

array $13 * 0.0$

global

unit 5

array $23 * 0.0$

reflector 314 r20.0 0.020 .01

end geom

read array

ara=1 nux= 1 nuy=1 nuz=3 fill 3221 end fill

ara $=2$ nux $=22$ nuy $=22$ nuz $=1$ fill $f 4$ end fill

end array

end data

nse71u1

$=\operatorname{csas} 25$

nse71w1

44 group latticecell

uo2 10.947293922354 .7429223895 .258

arbmag5 $2.7040011302798 .85120000 .4714000 \quad 0.43$ 260000.2221293

h20 31.0 arbmss 7.9030012400019 .172600071 .40280009 .374 end al 51.0

squarepitch 1.260 .79130 .9420 .820

Core of $22 \times 22$ positions: 459 fuel rods, 25 water holes, $81.36 \mathrm{~cm}$ water

"height

read parm run=yes gen $=405$ npg $=600$ nsk $=5$ nub=yes end parm

read geom

unit 1

cyl inder $110.395 \quad 81.360 .0$

cylinder $010.410 \quad 81.360 .0$

cylinder $210.470 \quad 81.360 .0$

cuboid 314 po.63 81.360 .0

unit 2

cylinder 510.470

cylinder 310.500

81.360 .0

cuboid $414 \mathrm{po} .63$

$\begin{array}{ll}0.25 & 0.0 \\ 0.25 & 0.0\end{array}$

$0.25 \quad 0.0$

unit 3

cylinder $5,0.470$

$\begin{array}{lllll}\text { cuboid } 4 & 1 & 4 \mathrm{p0} .63 & 2.35 & 0.8 \\ & & \end{array}$

unit 4

array $13 * 0.0$

unit 5

cylinder $310.500 \quad 0.00 \quad-0.25$

$\begin{array}{lllll}\text { cuboid } 414 \mathrm{p} 0.63 & 0.00 & -0.25\end{array}$ 
read array

ara=1 nux=1 nuy=1 nuz=3 fill 321 end fill ara=2 nux=22 nuy=22 nuz=1 fill $5 \quad 4 r 4 \quad 3 q 5 \quad 5 \quad 4 \quad 88 r 4 \quad 3 q 110 \quad 5 \quad 4 r 4$

$$
3 q 55422 r 4
$$

\section{end array}

end data

end

\section{nse71w?}

\section{$=\operatorname{csas} 25$}

nse71w2

44group latticecel

uo2 $10.947293922354 .7429223895 .258 \quad$ end

arbmag5 $2.70400111302798 .8512000 \quad 0.47 \quad 14000 \quad 0.43$ 260000.2221293

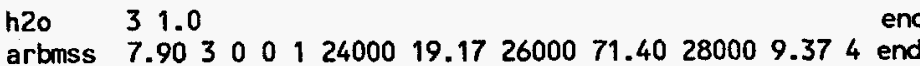

end al 51.0

end

end comp

squarepitch $1.26 \quad 0.79130 .9420 .820$ end

Core of $22 \times 22$ positions: 420 fuel rods, 64 water holes, $73.05 \mathrm{~cm}$ water height

read parm run=yes gen=405 npg=600 nsk=5 nub=yes end parm

\section{read geom}

unit

cylinder 010.410

cyl inder 210.470

73.050 .0

cuboid $314 \mathrm{p0} .63$

73.050 .0

unit 2

73.050 .0

$73.05 \quad 0.0$

cylinder 510.470

$0.25 \quad 0.0$

cylinder 310.500

cuboid $414 \mathrm{p0} .63$

unit 3

$0.25 \quad 0.0$

cylinder 5 1 $0.470 \quad 2.35 \quad 0.8$

$\begin{array}{llll}\text { cuboid } 314 \mathrm{p0} 0.63 & 2.35 & 0.8\end{array}$

$\begin{array}{llll}\text { cuboid } 414 \mathrm{p0} 0.63 & 2.35 & 0.0\end{array}$

unit 4

2.350 .0

array $13 * 0.0$

unit 5

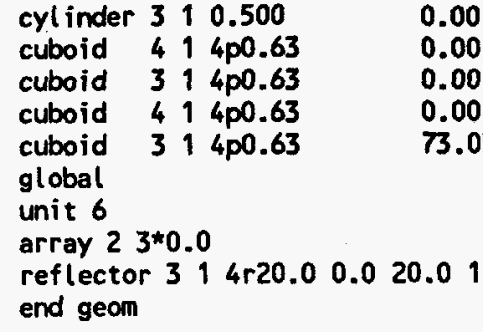

249-194

\section{$=\operatorname{csas} 25$ parm=size $=300000$} p49-194 44group uo2

c

c

3 den=1.321 221293

s $\quad 3$ den=1.321 .017 293

ca 3 den=1.321 .114 293

si 3 den=1.321.003 293

$\begin{array}{lllll}\text { h20 } & 4 & 1.0 & & 293 \\ \text { h } & 5 & d e n=1.185 & .08 & 293\end{array}$

h $\quad 5$ den=1.185 $.08 \quad 293$

c 5 den $=1.185 .60293$

poly(h20) 6 den=0.905 $1.0 \quad 293$

end comp

triangpitch $1.598 \quad 1.26514 \quad 4 \quad 1.415 \quad 2 \quad 1.2830$

Core of 1185 fuel rods in a hexagonal lattice

read parm run=yes gen $=405$ npg $=600$ nsk $=5$ nub=yes end parm

\section{read geom}

unit 1

com=! 0.0 to $2.54 \mathrm{~cm} 01$

zhemicylt+x 31 . $14415 \quad 2.54 \quad 0.0$

zhemicyltx 2 1 1.70752 .540 .0

$\begin{array}{llllll}\text { zhemicyl+x } & 4 & 1 & .714 & 2.54 & 0.0\end{array}$

unit 2

com=! 0.0 to $2.54 \mathrm{~cm} 01$ ! end $f i l l$ 


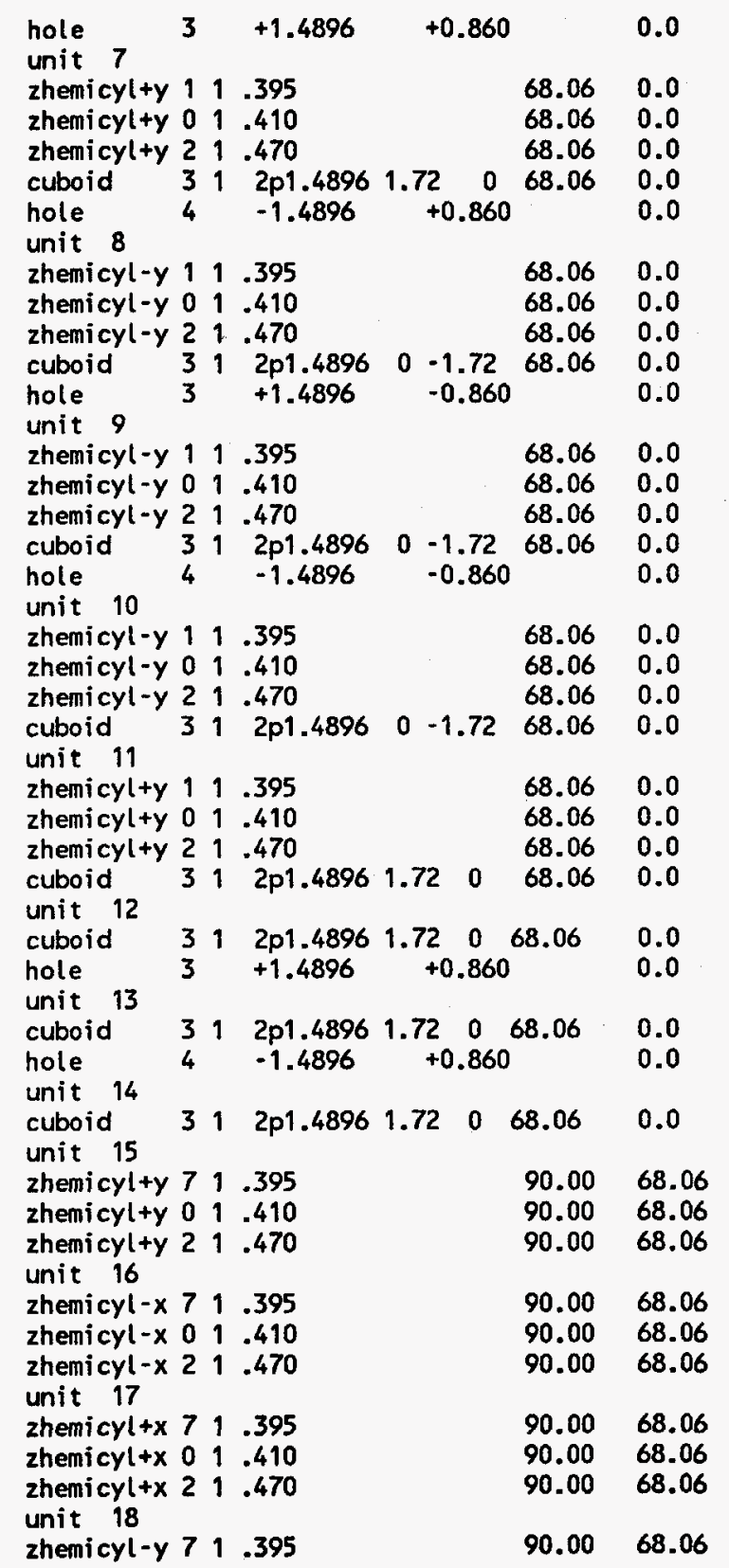

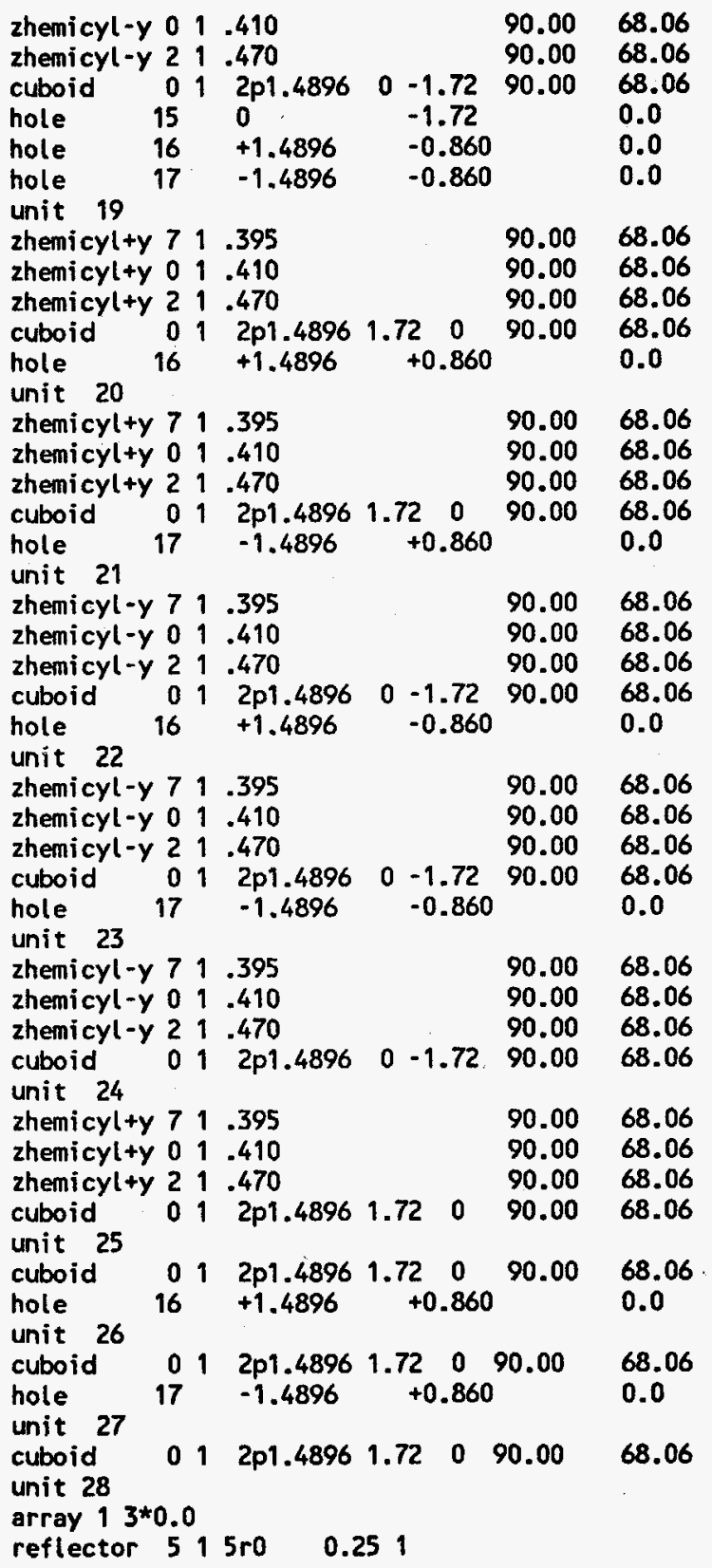


com=! 0.0 to $1.35 \mathrm{~cm} 01$ ! zhemicyl+x $3 \quad 1 \quad .6415 \quad 1.35 \quad 0.0$ zhemicyl+x $2 \quad 1 \quad .7075 \quad 1.350 .0$ $\begin{array}{llllll}\text { zhemicyl+x } & 4 & 1 & .714 & 1.35 & 0.0\end{array}$ unit 26

com=! 0.0 to $1.35 \mathrm{~cm} 01$ ! zhemicyl-y $3 \quad 1 \quad 64151.350 .0$ zhemicyl-y 21.70751 .350 .0

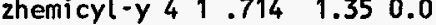
unit 27

com=! $\quad 0.0$ to $\quad 1.35 \mathrm{~cm} 01$ zhemicyl-x $31.6415 \quad 1.350 .0$ zhemicyl-x $21.7075 \quad 1.350 .0$

$\begin{array}{lllllll}N & \text { zhemicyl-x } & 4 & 1 & .714 & 1.35 & 0.0\end{array}$ unit 28

com=! 0.0 to $1.35 \mathrm{~cm} 01$ ! zhemicylty $3 \quad 1 \quad 6415 \quad 1.350 .0$ zhemicylty $21.7075 \quad 1.35 \quad 0.0$

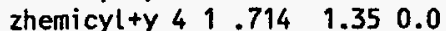
unit 31

com=! 0.0 to $0.381 \mathrm{~cm} 01$ zhemicylt+x $3 \quad 1 \quad .6415 \quad 0.3810 .0$ zhemicyl+x $21.7075 \quad 0.3810 .0$ $\begin{array}{llllll}\text { zhemicyl+x } & 4 & 1 & .714 & 0.381 & 0.0\end{array}$ unit 32

com $=$ ! 0.0 to $0.381 \mathrm{~cm} 01$ zhemicyl-y $3 \quad 1 \quad .6415 \quad 0.3810 .0$ zhemicyl-y 21.70750 .3810 .0 $\begin{array}{lllllll}\text { zhemicyl-y } & 4 & 1 & .714 & 0.381 & 0.0\end{array}$ unit 33

com=! $\quad 0.0$ to $0.381 \mathrm{~cm} 01$. zhemicyl- $x \quad 3 \quad 1 \quad 6415 \quad 0.3810 .0$ zhemicyl-x 21.70750 .3810 .0 themicyl-x 21.70750 .3810 .0

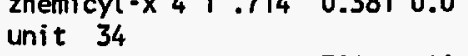

com=! $\quad 0.0$ to $0.381 \mathrm{~cm} 01$ zhemicylty $3 \quad 1.6415 \quad 0.3810 .0$ zhemicyl+y 21.70750 .3810 .0 zhemicylty $4 \begin{array}{lllll}1 & .714 & 0.381 & 0.0\end{array}$

unit 101

com $=1 \quad 0.0$ to $1.35 \mathrm{~cm} 01$ ।

zhemicyl+x $31.6415 \quad 1.35 \quad 0.0$ zhemicyl+x $29.7075 \quad 1.35 \quad 0.0$

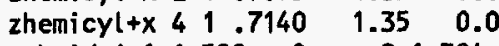
$\begin{array}{lllllll}\text { cuboid } 6 & 1 & 1.598 & 0 & 2 p 1.384 & 1.35 & 0.0\end{array}$

$\begin{array}{lllll}\text { hole } & 26 & .799 & 1.384 & 0.0\end{array}$

$\begin{array}{lllll}\text { hole } & 27 & 1.598 & 0 & 0.0\end{array}$

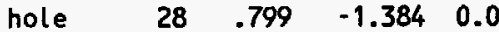

unit 102

com $=$ ! 0.0 to $1.35 \mathrm{~cm} 02$ !

$\begin{array}{llllll}\text { zhemicyl+x } & 3 & 1 & .6415 & 1.35 & 0.0\end{array}$

zhemicyl+x $211.7075 \quad 1.350 .0$

zhemicyl+x $\begin{array}{lllll}4 & 1 & .7140 & 1.35 & 0.0\end{array}$

cuboid $611.598 \quad 0 \quad 2 p 1.384 \quad 1.35 \quad 0.0$

$\begin{array}{lllll}\text { hole } & 26 & .799 & 1.384 & 0.0\end{array}$

hole $27 \quad 7.598 \quad 0$

unit 103

com=! 0.0 to $1.35 \mathrm{~cm} 03$ !

zhemicyl+x $3 \begin{array}{lllll} & 1 & .6415 & 1.35 & 0.0\end{array}$

zhemicyl+x $21.7075 \quad 1.35 \quad 0.0$

zhemicyl+x $4 \begin{array}{lllll} & 1 & .7140 & 1.35 & 0.0\end{array}$

$\begin{array}{lllllll}\text { cuboid } 6 & 1 & 1.598 & 0 & 2 p 1.384 & 1.35 & 0.0\end{array}$

$\begin{array}{lllll}\text { hole } & 26 & .799 & 1.384 & 0.0\end{array}$

$\begin{array}{lllrl}\text { hole } & 26 & .799 & 1.384 & 0.0 \\ \text { hole } & 28 & .799 & -1.384 & 0.0\end{array}$

unit 104

com=! 0.0 to $1.35 \mathrm{~cm} 04$ !

zhemicyl+x 31 1.6415 $1.35 \quad 0$

zhemicyl+x $21.7075 \quad 1.35$

zhemicyl+x $4 \begin{array}{lllll} & 1 & .7140 & 1.35 & 0\end{array}$

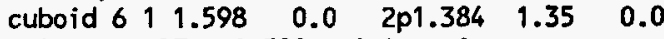

$\begin{array}{lllll}\text { hole } & 27 & 1.598 & 0.0 & 0\end{array}$

$\begin{array}{lllll}\text { hole } & 28 & .799 & -1.384 & 0\end{array}$

unit 105

com=! 0.0 to $1.35 \mathrm{~cm} 05$ !

zhemicyl+x $31.6415 \quad 1.35$

zhemicyl+x $211.7075 \quad 1.35 \quad 0$

zhemicyl $+x \quad 4 \quad 1.7140 \quad 1.35 \quad 0$

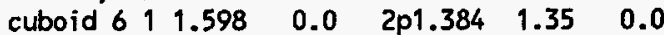

hole

$\begin{array}{llll}26 & .799 & 1.384 & 0\end{array}$

unit 106

com=! 0.0 to $1.35 \mathrm{~cm} 06$ !

zhemicyl+x $31.6415 \quad 1.35$

zhemicyltx $21.7075 \quad 1.35 \quad 0$

zhemicyl $+x \quad 4 \quad 1.7140 \quad 1.35 \quad 0$

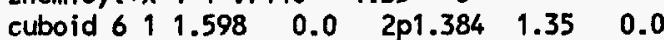

hole

unit 107

com=! 0.0 to $\quad 1.35 \mathrm{~cm} 07$

zhemicyl+x $3 \quad 1 \quad 6415 \quad 1.35 \quad 0$

zhemicyl+x $21.7075 \quad 1.35 \quad 0$ 
zhemicyl+x $4 \begin{array}{lllll}1 & .7140 & 1.35 & 0\end{array}$

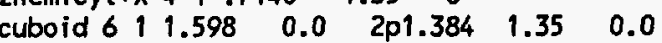
hole

unit 108 comel 0.03 to 3415 zhemicylt+x $21.7075 \quad 1.350$ zhemicyl+x $41.7140 \quad 1.35 \quad 0$

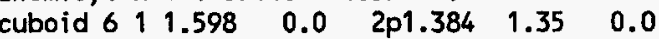
unit 109

com=! 0.0 to $1.35 \mathrm{~cm} 09$ !

zhemicyl-y $3 \quad 1.6415 \quad 1.35 \quad 0$ zhemicyl-y $21.7075 \quad 1.35 \quad 0$ zhemicyl-y 4 1 $1.7140 \quad 1.35 \quad 0$ cuboid $612 \mathrm{p} 0.799 \quad 0.0-2.768$ hole $27 \quad 0.799-1.384 \quad 0$ $\begin{array}{lllll}\text { hole } & 28 & 0.0 & -2.768 & 0\end{array}$ unit 110

com=! 0.0 to $\quad 1.35 \mathrm{~cm} 10$ ! zhemicyl-y $3 \quad 1.6415 \quad 1.35 \quad 0$ zhemicyl-y $2 \quad 1.7075 \quad 1.35 \quad 0$ zhemicyl-y $4 \begin{array}{lllll} & 1.7140 & 1.35 & 0\end{array}$ cuboid $612 \mathrm{p} 0.799 \quad 0.0-2.768$ hole $\quad 27 \quad 0.799-1.384 \quad 0$

com=! 0.0 to $1.35 \mathrm{~cm} 11$ ! zhemicyl-y $3 \quad 1.6415 \quad 1.35 \quad 0$ zhemicyl-y $2 \quad 1.7075 \quad 1.35 \quad 0$ zhemicyl-y $4 \quad 1 \quad .7140 \quad 1.35 \quad 0$ cuboid 6 i $2 \mathrm{p} 0.799 \quad 0.0-2.768$ $\begin{array}{lllll}\text { hole } & 28 & 0.0 & -2.768 & 0\end{array}$ unit 112

com=! 0.0 to $1.35 \mathrm{~cm} 12$ ! zhemicyl-y $3 \quad 1 \quad .6415 \quad 1.35 \quad 0$ zhemicyl-y $2 \quad 1.7075 \quad 1.35 \quad 0$ zhemicyl-y $4 \begin{array}{lllll} & 1 & .7140 & 1.35 & 0\end{array}$ cuboid 61 2p0.799 $0 \quad-2.768$

com=! 0.0 to $1.35 \mathrm{~cm} 13$ !

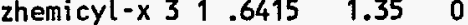
zhemicyl-x $2 \quad 1.7075 \quad 1.35 \quad 0$ zhemicyl-x $4 \quad 1 \quad 7140 \quad 1.35$

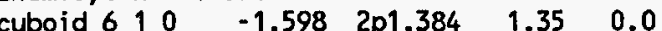
hole 0

unit 114

com=! 0.0 to $1.35 \mathrm{~cm} 14$ ! zhemicyl-x $3 \quad 1 \quad .6415 \quad 1.35 \quad 0$ zhemicyl-x $2 \quad 1.7075 \quad 1.35 \quad 0$ $\begin{array}{llllll}\text { zhemicyl-x } & 4 & 1 & .7140 & 1.35 & 0\end{array}$ cuboid $610 \quad-1.598 \quad 2 p 1.384 \quad 1.35 \quad 0.0$ cuboid 115

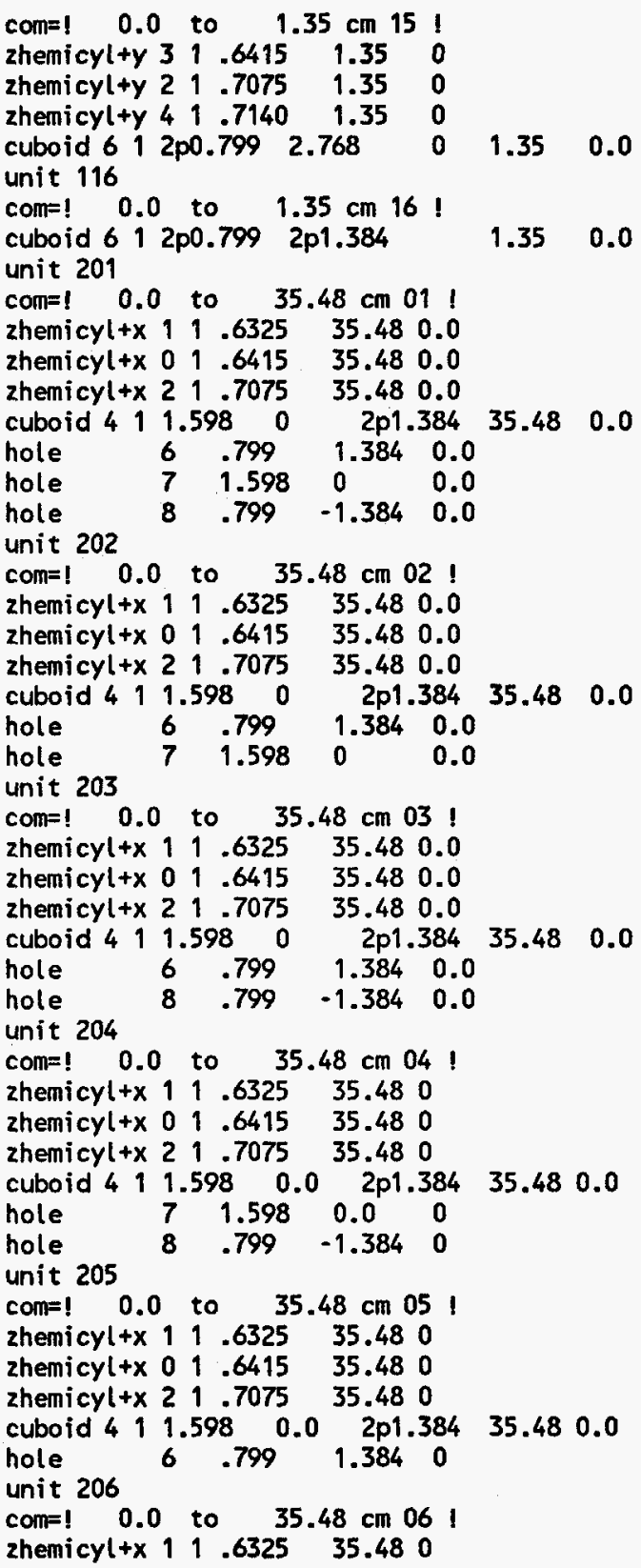


zhemicyl+x $01.6415 \quad 35.480$

zhemicyl+x $21.7075 \quad 35.480$

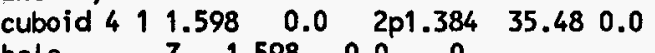

hole

unit 207

com=! 0.0 to $\quad 35.48 \mathrm{~cm} 07$ !

2hemicyl+x $11.6325 \quad 35.48$

2hemicyltx o $1.6415 \quad 35.480$

zhemicylt $x 21.7075 \quad 35.480$

$\begin{array}{lllllll}\text { cuboid } 411.598 \quad 0.0 & 2 p 1.384 & 35.48 & 0.0\end{array}$

hole

$\begin{array}{llll}8 & .799 & -1.384 & 0\end{array}$

unit 208

com $=$ ! 0.0 to $\quad 35.48 \mathrm{~cm} 08$

zhemicyl+x $11.6325 \quad 35.480$

zhemicyl+x $0 \quad 1 \quad .6415 \quad 35.480$

zhemicyl+x $21.7075 \quad 35.480$

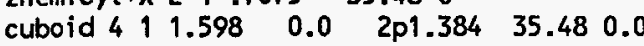

unit 209

com=! 0.0 to $35.48 \mathrm{~cm} 09$ !

zhemicyl-y $11.6325 \quad 35.480$

zhemicyl-y $01.6415 \quad 35.480$

zhemicyl-y 01.6415350758

zhemicyl-y 21.70750 .35 .480

0.75 .480 .0

hole 7

hole $\quad \begin{array}{lllll} & 8 & 0.0 & -2.768 & 0\end{array}$

N unit 210

com $=$ ! 0.0 to $\quad 35.48 \mathrm{~cm} 10$

zhemicyl-y $11.6325 \quad 35.480$

zhemicyl-y $01.6415 \quad 35.480$

2hemicyl-y o i. 647535.480

chemicyl $120.7990 .0-2.768$

$\begin{array}{lllll}\text { cuboid } 4 & 1 & \text { 2p0.799 } 0.0 & -2.768 \\ \text { hole } & 7 & 0.799 & -1.384 & 0\end{array}$

$35.48 \quad 0.0$

com=! $\quad 0.0$ to $35.48 \mathrm{~cm} 11$ !

zhemicyl-y $111.6325 \quad 35.480$

zhemicyl-y $01.6415 \quad 35.480$

zhemicyl-y $21.7075 \quad 35.480$

$\begin{array}{llllll}\text { cuboid } 412 \mathrm{p} 0.799 & 0.0 & -2.768 & 35.48 & 0.0\end{array}$

$\begin{array}{lllll}\text { hole } & 8 & 0.0 & -2.768 & 0\end{array}$

unit 212

com=! 0.0 to $35.48 \mathrm{~cm} 12$ !

zhemicyl-y $11.6325 \quad 35.480$

zhemicyl-y $01.6415 \quad 35.480$

zhemicyl-y $21.7075 \quad 35.480$

cuboid 4

unit 213

$35.48 \quad 0.0$

com $=$ ! $\quad 0.0$ to $\quad 35.48 \mathrm{~cm} 13$ !

zhemicyl-x $11.6325 \quad 35.480$

zhemicyl-x $0101.6415 \quad 35.48 \quad 0$

zhemicyl-x $21.7075 \quad 35.480$

cuboid $410 \quad-1.598 \quad 2 p 1.384$ hole

unit 214

com=1 0.0 to $35.48 \mathrm{~cm} 14$

zhemicyl-x $11.6325 \quad 35.480$

zhemicyl-x $01.6415 \quad 35.480$

zhemicyl-x $21.7075 \quad 35.480$

cuboid $410 \quad-1.598 \quad 2 p 1.384$

35.480 .0

unit 215

com=! 0.0 to $35.48 \mathrm{~cm} 15$ ।

zhemicylty $11.6325 \quad 35.480$

zhemicylty $001.6415 \quad 35.480$

zhemicyl+y $21.7075 \quad 35.480$

cuboid $412 \mathrm{2p0} .799 \quad 2.768 \quad 0$.

unit 216

35.480 .0

conl $=10.0$ to $35.48 \mathrm{~cm} 16$ !

cuboid $41200.799 \quad 201.384$

unit 301

com=! 0.0 to $1.35 \mathrm{~cm} 01$ !

zhemicyl+x 111 . $6325 \quad 1.35 \quad 0.0$

zhemicyl+x $0 \begin{array}{lllll} & 1 & .6415 & 1.35 & 0.0\end{array}$

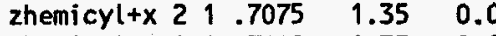

zhemicyl+x $4 \quad 1.7140 \quad 1.35 \quad 0.0$

$\begin{array}{lllllll}\text { cuboid } 61 & 1.598 & 0 & 2 p 1.384 & 1.35 & 0.0\end{array}$

$\begin{array}{lllll}\text { hole } & 10 & .799 & 1.384 & 0.0\end{array}$

$\begin{array}{lllll}\text { hole } & 10 & .799 & 1.384 & 0.0 \\ \text { hole } & 11 & 1.598 & 0 & 0.0\end{array}$

$\begin{array}{lllll}\text { hole } & 12 & .799 & -1.384 & 0.0\end{array}$

unit 302

com=! 0.0 to $1.35 \mathrm{~cm} \quad 02$ !

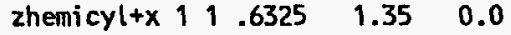

zhemicyl+x $0101.6415 \quad 1.35 \quad 0.0$

zhemicyl+x $2 \quad 1.7075 \quad 1.35 \cdot 0.0$

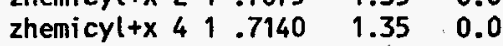

$\begin{array}{lllllll}\text { cuboid } 6 & 1 & 1.598 & 0 & 2 p 1.384 & 1.35 & 0.0\end{array}$

$\begin{array}{lllll}\text { hole } & 10 & .799 & 1.384 & 0.0\end{array}$

$\begin{array}{lllll}\text { hole } & 11 & 1.598 & 0 & 0.0\end{array}$

unit 303

com $=$ ! 0.0 to $1.35 \mathrm{~cm} 03$ !

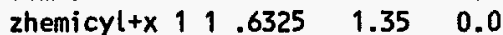

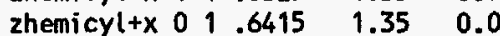

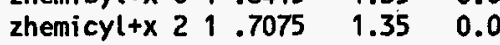

zhemicyl+x $4 \quad 1.7140 \quad 1.35 \quad 0.0$

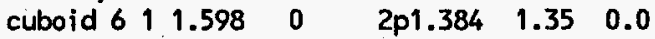

$\begin{array}{lllll}\text { hole } & 10 & .799 & 1.384 & 0.0\end{array}$

$\begin{array}{lllll}\text { hole } & 12 & .799 & -1.384 & 0.0\end{array}$

unit 304

com $=$ ! 0.0 to $1.35 \mathrm{~cm} 04$ !

zhemicyl+x $119.6325 \quad 1.35 \quad 0$

zhemicyl+x 001 . $1.6415 \quad 1.35 \quad 0$

zhemicyl+x $21.7075 \quad 1.350$

zhemicyt+x $4 \quad 1 \quad .7140 \quad 1.35 \quad 0$

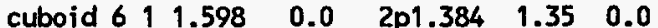



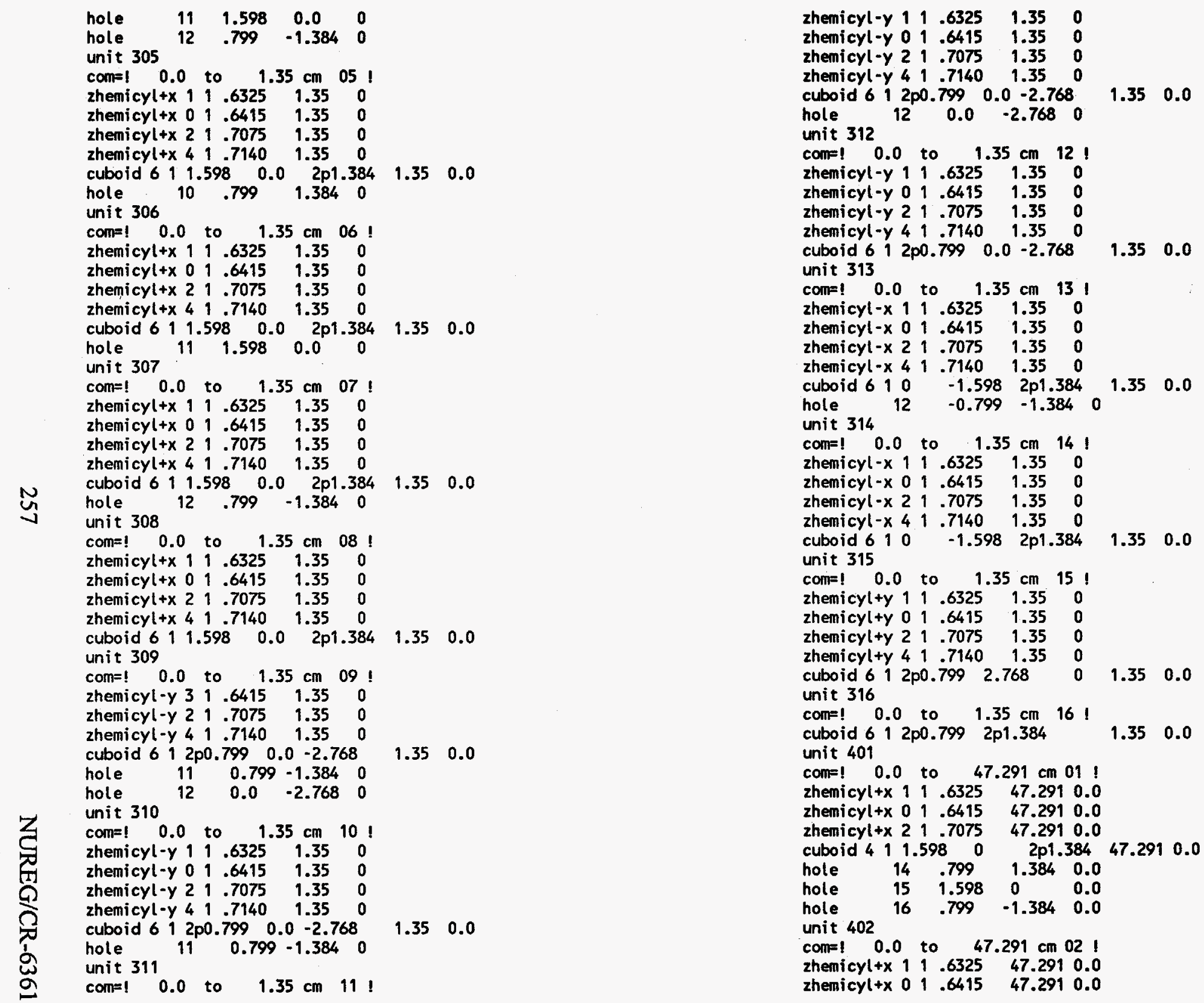
unit 403

com=! 0.0 to $47.291 \mathrm{~cm} 03$ ! zhemicyl+x 11 1.6325 47.2910 .0 zhemicyl+x $01.6415 \quad 47.2910 .0$ zhemicylt+x 21.707547 .2910 .0 $\begin{array}{cccccc}\text { zhemicyl+x } & 2 & 1 & .7075 & 47.291 & 0.0 \\ \text { cuboid } 4 & 1.598 & 0 & 2 p 1.384 & 47.291 & 0.0\end{array}$ $\begin{array}{llllll}\text { cuboid } 4 & 1 & 1.598 & 0 & 2 p 1.384 \\ \text { hole } & 14 & .799 & 1.384 & 0.0\end{array}$ $\begin{array}{lllrl}\text { hole } & 14 & .799 & 1.384 & 0.0 \\ \text { hole } & 16 & .799 & -1.384 & 0.0\end{array}$ unit 404

com=! 0.0 to $\quad 47.291 \mathrm{~cm} 04$ zhemicyl+x $11.6325 \quad 47.2910$ zhemicyltx $01.6415 \quad 47.2910$ zhemicyltx $01.6415 \quad 47.2910$ $\begin{array}{llllllll}\text { zhemicyltx } & .7075 & 47.291 & & & & \end{array}$

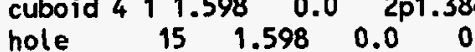
hole $\quad \begin{array}{llll}16 & .799 & -1.384 & 0\end{array}$

unit $\mathbf{4 0 5}$

com=! 0.0 to $\quad 47.291 \mathrm{~cm} 05$ ! zhemicyl+x 11 . $6325 \quad 47.2910$ zhemicyl+x $0 \begin{array}{lllll} & 1 & .6415 & 47.2910\end{array}$ zhemicyl+x $21.7075 \quad 47.2910$

unit 406

com $=! \quad 0.0$ to $\quad 47.291 \mathrm{~cm} 06$

zhemicyl $+x \quad 1 \quad 1.6325 \quad 47.2910$ zhemicyl+x $0 \quad 1 \quad .6415 \quad 47.2910$ zhemicyl+x $21.7075 \quad 47.2910$

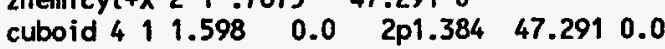
hole $\begin{array}{llll}15 & 1.598 & 0.0 & 0\end{array}$

unit 407

com=! 0.0 to $47.291 \mathrm{~cm} 07$ zhemicyl+x 11 1 .6325 47.2910 zhemicyl+x $01.6415 \quad 47.2910$ chemicyl tx $21.7075 \quad 47.2910$ $\begin{array}{lllllll}\text { cuboid } 4 & 1 & 1.598 & 0.0 & 2 p 1.384 & 47.291 & 0.0\end{array}$ hole $\quad 16 \quad .799 \quad-1.384 \quad 0$ unit 408

com=! 0.0 to $\quad 47.291 \mathrm{~cm} 08$ zhemicyl+x $11.6325 \quad 47.2910$ zhemicyl+x $01.6415 \quad 47.2910$ zhemicyltx $21.7075 \quad 47.2910$ $\begin{array}{llllll}\text { zhemicyltx } & 1 & .7075 & & \\ \text { cuboid } 41 & 1.598 & 0.0 & 2 p 1.384 & 47.291 & 0.0\end{array}$

cuboid 4
unit 409

com=! 0.0 to $\quad 47.291 \mathrm{~cm} 09$ ! zhemicyl-y $11.6325 \quad 47.2910$ zhemicyl-y $01.6415 \quad 47.2910$

zhemicyl-y $21.7075 \quad 47.2910$

cuboid 412 2p0.799 $0.0 \quad-2.768$ hole $\quad 15 \quad 0.799 \cdot 1.384 \quad 0$ hole $\quad \begin{array}{llll}16 & 0.0 & -2.768 & 0\end{array}$

unit 410

com=! 0.0 to $47.291 \mathrm{~cm} 10$ ! zhemicyl-y 11 1 .6325 47.2910 zhemicyl-y $01.6415 \quad 47.2910$ zhemicyl-y $21.7075 \quad 47.2910$ cuboid $412 p 0.799 \quad 0.0-2.768 \quad 47.2910 .0$ hole $15 \quad 0.799-1.384 \quad 0$ hole 411

com=! 0.0 to $47.291 \mathrm{~cm} 11$ zhemicyl-y $111.6325 \quad 47.2910$ zhemicyl-y $011.6415 \quad 47.2910$ zhemicyl-y $21.7075 \quad 47.2910$ cuboid $412 \mathrm{p} 0.799 \quad 0.0-2.768 \quad 47.291 \quad 0.0$ cuboid 4 16 2 o.799 $0.0-2.768$ unit 412

com=! 0.0 to $47.291 \mathrm{~cm} 12$ zhemicyl-y 11 1 .6325 47.2910 zhemicyl-y $0101.6415 \quad 47.2910$ zhemicyl-y $21.7075 \quad 47.2910$ cuboid $412 \mathrm{p} 0.799 \quad 0.0-2.768$ unit 413

com $=$ ! 0.0 to $47.291 \mathrm{~cm} 13$ zhemicyl-x 11 . $1325 \quad 47.2910$ zhemicyl-x $001.6415 \quad 47.2910$ zhemicyl-x $21.7075 \quad 47.2910$ $\begin{array}{lllll}\text { cuboid } 410 & -1.598 & 2 p 1.384 & 47.291 & 0.0\end{array}$ hole

unit 414

confl 0.0 to $47.291 \mathrm{~cm} 14$

zhemicyl-x $19.6325 \quad 47.2910$ zhemicyl-x $01.6415 \quad 47.2910$ zhemicyl-x $21.7075 \quad 47.2910$

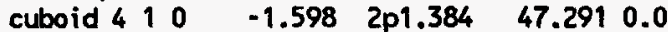
unit 415

com=! 0.0 to $47.291 \mathrm{~cm} 15$ conficy 0.0 to 1 1 6325.291 .632547 .2910 zhemicylty $01.6415 \quad 47.2910$ zhemicylty $21.7075 \quad 47.2910$ cuboid 412 2po.799 2.768 unit 416

com=! 0.0 to $47.291 \mathrm{~cm} 16$ cuboid $412 \mathrm{p0} .799201 .384 \quad 47.2910 .0$ unit 501

com=! 0.0 to $5.969 \mathrm{~cm} 01$ ! zhemicyl+x $11.6325 \quad 5.9690 .0$ zhemicyl+x $01.6415 \quad 5.9690 .0$ zhemicyltx $2, .7075 \quad 5.9690 .0$ 


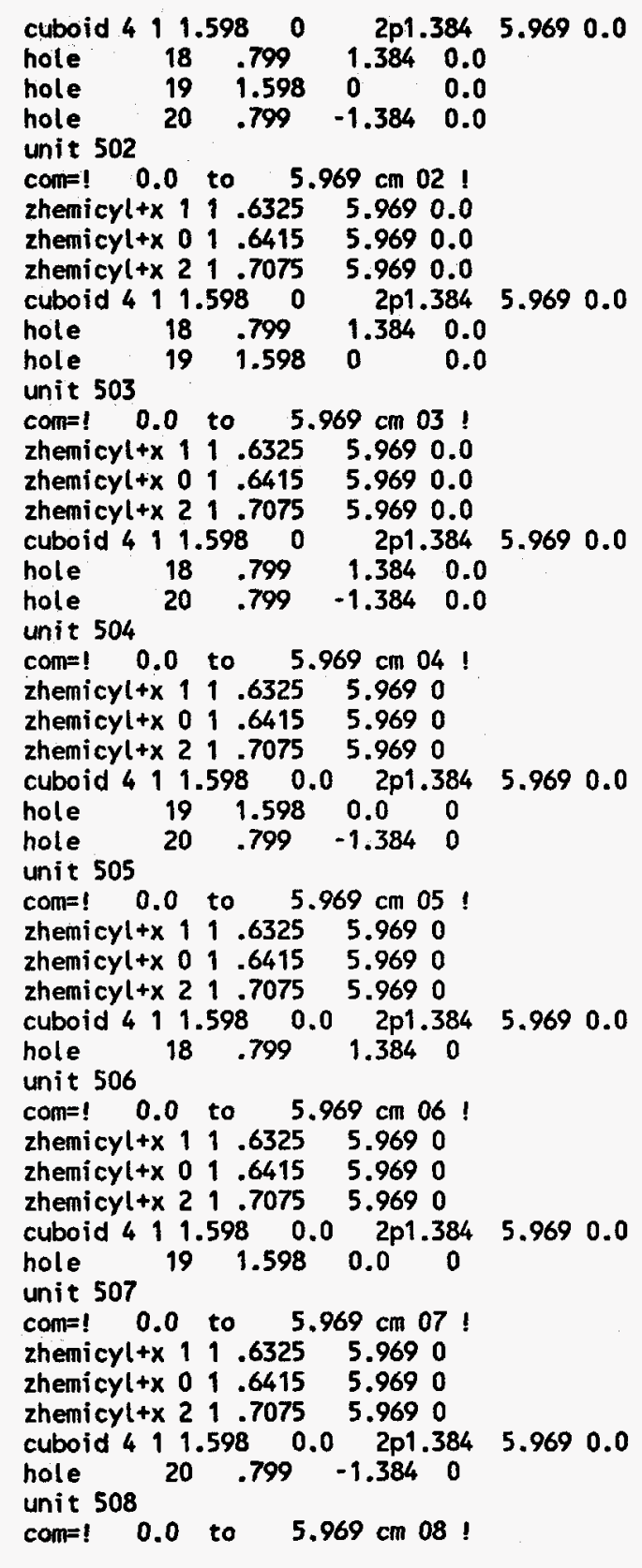

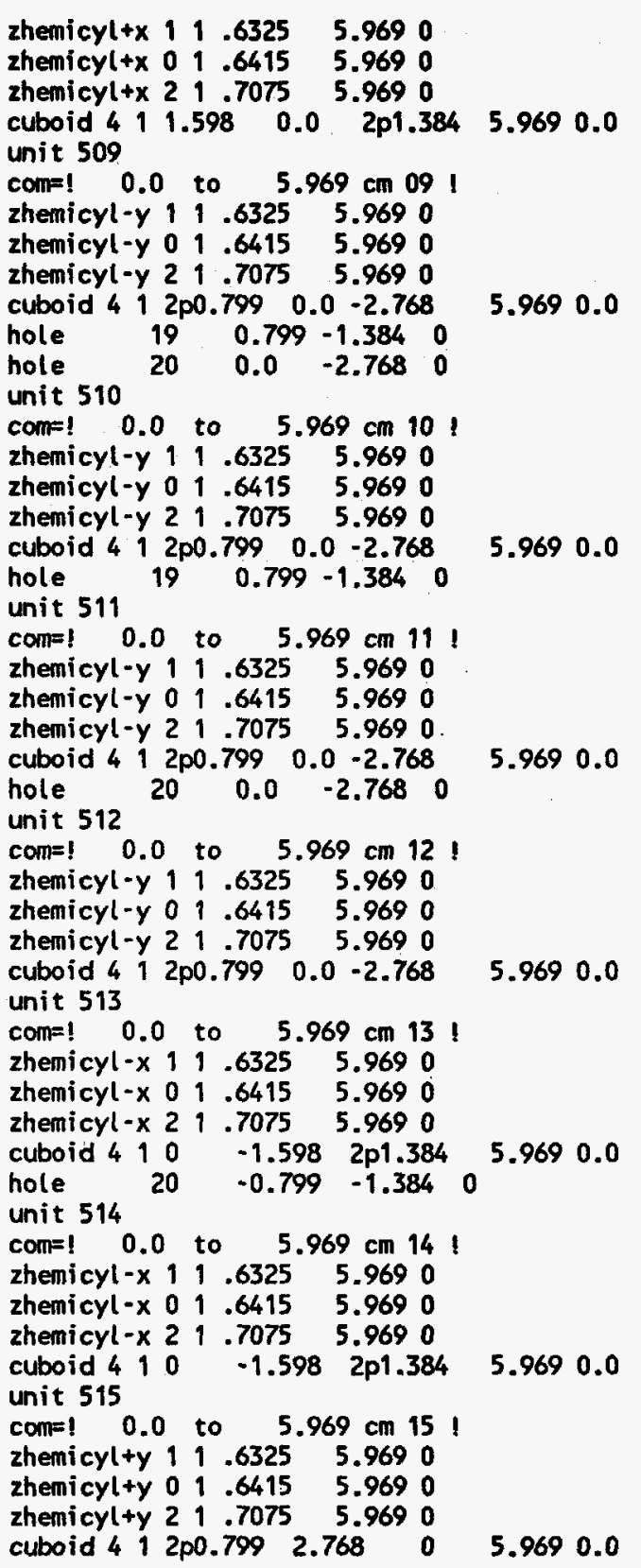



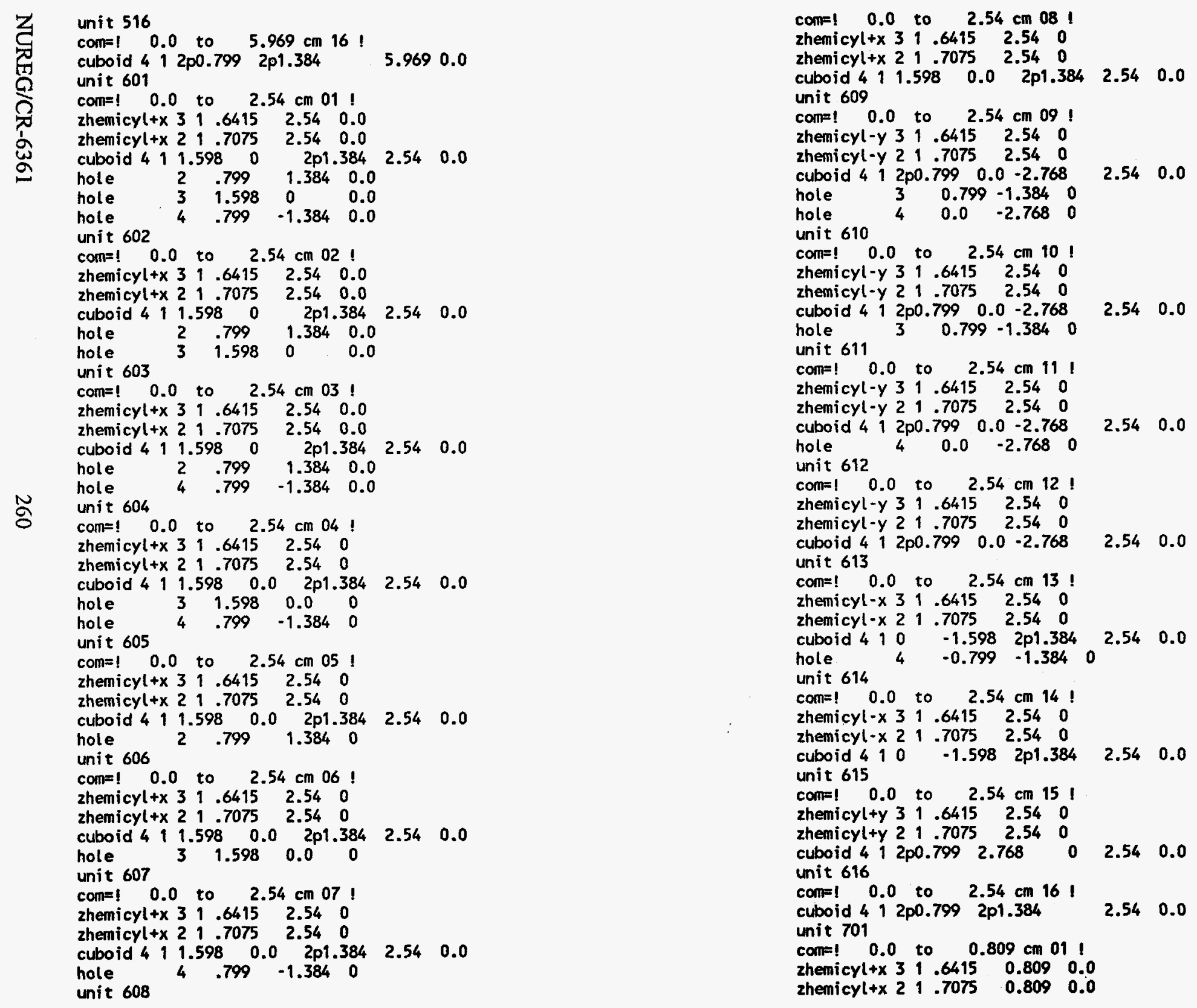


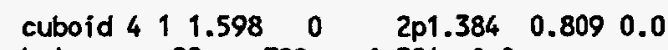

$\begin{array}{lllll}\text { hole } & 22 & .799 & 1.384 & 0.0\end{array}$

$\begin{array}{lllll}\text { hole } & 23 & 1.598 & 0 & 0.0\end{array}$

$\begin{array}{lllll}\text { hole } & 24 & .799 & -1.384 & 0.0\end{array}$

unit 702

com=! 0.0 to $0.809 \mathrm{~cm} 02$ !

zhemicyl+x $3 \quad 1 \quad 6415 \quad 0.809 \quad 0.0$

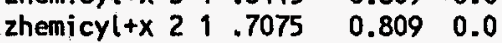

$\begin{array}{llllll}\text { cuboid } 411.598 & 0 & 2 p 1.384 & 0.809 & 0.0\end{array}$

$\begin{array}{llllll}\text { hole } & 22 & .799 & 1.384 & 0.0\end{array}$

hole

$\begin{array}{llll}23 & 1.598 & 0 & 0.0\end{array}$

unit 703

com=! $\quad 0.0$ to $0.809 \mathrm{~cm} 03$ !

$\begin{array}{llllll}\text { zhemicyl+x } 3 & 1 & .6415 & 0.809 & 0.0\end{array}$

themicyltx 21.70750 .8090 .0

$\begin{array}{llllll}\text { cuboid } 41 & 1.598 & 0 & 2 p 1.384 & 0.809 & 0.0\end{array}$

$\begin{array}{llllll}\text { hole } & 22 & .799 & 1.384 & 0.0\end{array}$

$\begin{array}{lllll}\text { hole } & 24 & .799 & -1.384 & 0.0\end{array}$

unit 704

com=! 0.0 to $0.809 \mathrm{~cm} 04$ !

chemicyl+x $3,6415 \quad 0.8090$

zhemicyl $+x \quad 2 \quad 1 \quad .7075 \quad 0.809 \quad 0$

$\begin{array}{llllll} & \end{array}$

$\begin{array}{lllll}\text { hole } & 23 & 1.598 & 0.0 & 0\end{array}$

$\begin{array}{lllll}\text { hole } & 24 & .799 & -1.384 & 0\end{array}$

O unit 705

com=! 0.0 to $0.809 \mathrm{~cm} \mathrm{05}$

zhemicyl+x $3 \quad 1 \quad .6415 \quad 0.809 \quad 0$

themicyl+x $21.7075 \quad 0.8090$

$\begin{array}{lllllll}\text { cuboid } 4 & 1 & 1.598 & 0.0 & 2 \mathrm{p} 1.384 & 0.809 & 0.0\end{array}$

hole

unit 706

0.0 to $0.809 \mathrm{~cm} 06$

zhemicyl+x $3 \quad 1 \quad .6415 \quad 0.809 \quad 0$

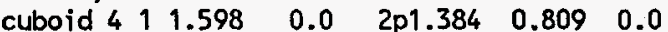

hole $231.598 \quad 0.0 \quad 0$

unit 707

com=! 0.0 to $0.809 \mathrm{~cm} 07$ !

$\begin{array}{llllll}\text { zhemicyl+x } & 3 & 1 & .6415 & 0.809 & 0\end{array}$

zhemicyl $+x \quad 2 \quad 1 \quad .7075 \quad 0.809 \quad 0$

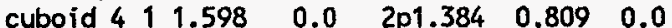

hole

unit 708

com $=$ ! 0.0 to $0.809 \mathrm{~cm} 08$ !

$\begin{array}{lllll}\text { zhemicyltx } 3 & 1.6415 & 0.809 & 0\end{array}$

zhemicyl+x $2 \quad 1 \quad .7075 \quad 0.809 \quad 0$

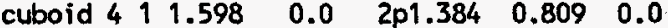

unit 709

com $=1 \quad 0.0$ to $0.809 \mathrm{~cm} 09$

zhemicyl-y 31.64150 .8090 zhemicyl-y $2 \begin{array}{lllll}2 & 1 & .7075 & 0.809 & 0\end{array}$

cuboid 41 2p0.799 $0.0-2.768$

hole $23 \quad 0.799-1.3840$

hole $\quad \begin{array}{lllll}24 & 0.0 & -2.768 & 0\end{array}$

unit 710

com=! $\quad 0.0$ to $0.809 \mathrm{~cm} 10$

zhemicyl-y $3 \quad 1 \quad 6415 \quad 0.809 \quad 0$

$\begin{array}{lllll}\text { zhemicyl-y } 2 & 1 & .7075 & 0.809 & 0\end{array}$

cuboid $412 \mathrm{p} 0.799 \quad 0.0-2.768$

$\begin{array}{llllll}\text { hole } & 23 & 0.799 & -1.384 & 0\end{array}$

unit 711

com=! 0.0 to $0.809 \mathrm{~cm} 11$

$\begin{array}{lllll}0.0 & & 0.809 & \\ & 0.809 & 0\end{array}$

zhemicyl-y $21.7075 \quad 0.809 \quad 0$

cuboid $412 \mathrm{2p0} .799 \quad 0.0-2.768$

hole $24 \quad 0.0 \quad-2.768 \quad 0$

unit 712

comle! 0.0 to $0.809 \mathrm{~cm} 12$

zhemicyl-y $31.6415 \quad 0.809 \quad 0$

zhemicyl-y $21.7075 \quad 0.809 \quad 0$

cuboid $41200.799 \quad 0.0-2.768$

unit 713

com=! 0.0 to $0.809 \mathrm{~cm} 13$ !

zhemicyl-x $3 \quad 1 \quad 64150.8090$

zhemicyl-x 2 \begin{tabular}{lllll}
\hline & 1.7075 & 0.809 & 0
\end{tabular}

$\begin{array}{llllll}\text { cuboid } 410 & -1.598 & 2 p 1.384 & 0.809 & 0.0\end{array}$

hole $\quad \begin{array}{llll}24 & -0.799 & -1.384 & 0\end{array}$

unit 714

com $=1 \quad 0.0$ to $0.809 \mathrm{~cm} 14$

zhemicyl-x 31.64150 .8090

zhemicyl-x $21.7075 \quad 0.809 \quad 0$

$\begin{array}{llllll}\text { cuboid } 410 & -1.598 & 2 p 1.384 & 0.809 & 0.0\end{array}$

unit 715

com=! $\quad 0.0$ to $0.809 \mathrm{~cm} 15$

zhemicylty $31.6415 \quad 0.8090$

zhemicylty $21.7075 \quad 0.809 \quad 0$

cuboid 41200.7992 .768

unit 716

com=! 0.0 to $0.809 \mathrm{~cm} 16$

cuboid $412 \mathrm{po} 0.799 \quad 2 \mathrm{p} 1.384$

$0.809 \quad 0.0$

unit 801

com=! 0.0 to $0.381 \mathrm{~cm} 01$ !

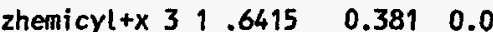

$\begin{array}{lllll}\text { zhemi cyl+x } 2 & 1 & .7075 & 0.381 & 0.0\end{array}$

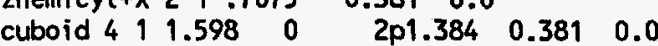

hole $\quad \begin{array}{llll}32 & .799 & 1.384 & 0.0\end{array}$

$\begin{array}{lllll}\text { hole } & 33 & 1.598 & 0 & 0.0\end{array}$

$\begin{array}{lllll}\text { hole } & 34 & .799 & -1.384 & 0.0\end{array}$

unit 802

com=! 0.0 to $0.381 \mathrm{~cm} 02$ !

zhemicyltx 31.64150 .3810 .0 
zhemicyl+x $21.7075 \quad 0.381 \quad 0.0$

$\begin{array}{lllllll}\text { cuboid } 4 & 1 & 1.598 & 0 & 2 p 1.384 & 0.381 & 0.0\end{array}$

$\begin{array}{llllll}\text { hole } & 32 & .799 & 1.384 & 0.0\end{array}$

hole $\quad \begin{array}{llll}33 & 1.598 & 0 & 0.0\end{array}$

unit 803

com $=10.0$ to $0.381 \mathrm{~cm} 03$ !

$\begin{array}{llllll}\text { zhemicylt+x } & 3 & 1 & .6415 & 0.381 & 0.0\end{array}$

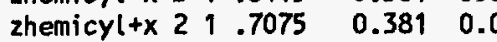

$\begin{array}{lllllll}\text { cuboid } 4 & 1 & 1.598 & 0 & 2 p 1.384 & 0.381 & 0.0\end{array}$

$\begin{array}{llllll}\text { hole } & 32 & .799 & 1.384 & 0.0\end{array}$

$\begin{array}{lllll}\text { hole } & 34 & .799 & -1.384 & 0.0\end{array}$

unit 804

com=! 0.0 to $0.381 \mathrm{~cm} 04$ !

$\begin{array}{llllll}\text { zhemicyl+x } & 3 & 1 & .6415 & 0.381 & 0\end{array}$

zhemicyltx 21 . 10750.3810

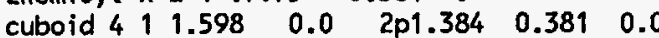

hole $\quad \begin{array}{llll}33 & 1.598 & 0.0 & 0\end{array}$

$\begin{array}{lllll}\text { hole } & 34 & .799 & -1.384 & 0\end{array}$

unit 805

com=! 0.0 to $0.381 \mathrm{~cm} 05$ !

zhemicyltx $\begin{array}{llllll}3 & 1 & .6415 & 0.381 & 0\end{array}$

zhemicyltx $21.7075 \quad 0.3810$

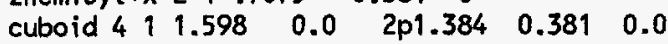

hole

unit 806

今 com=! 0.0 to $0.381 \mathrm{~cm} 06$ !

zhemicyltx $3 \quad \begin{array}{lllll}1 & .6415 & 0.381 & 0\end{array}$

zhemicyl+x $2 \quad 1 \quad .7075 \quad 0.3810$

$\begin{array}{lllllll}\text { cuboid } 4 & 1 & 1.598 & 0.0 & 2 \mathrm{p} 1.384 & 0.381 & 0.0\end{array}$

hole

unit 807

com=! 0.0 to $0.381 \mathrm{~cm} 07$ !

zhemicyl+x $\begin{array}{llllll}3 & 1 & .6415 & 0.381 & 0\end{array}$

zhemicyl+x $2 \quad 1 \quad .7075 \quad 0.3810$

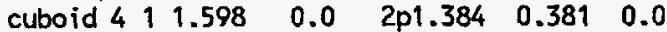

$\begin{array}{lllll}\text { hole } & 34 & .799 & -1.384 & 0\end{array}$

unit 808

com $=$ ! 0.0 to $0.381 \mathrm{~cm} 08$ !

zhemicyltx $\begin{array}{lllll}3 & 1 & .6415 & 0.381 & 0\end{array}$

zhemicyl+x $2 \quad 1 \quad .7075 \quad 0.381 \quad 0$

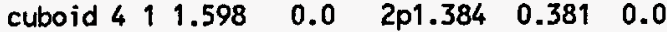

unit 809

com $=$ ! 0.0 to $0.381 \mathrm{~cm} 09$ !

zhemicyl-y $31.6415 \quad 0.3810$

zhemicyl-y $21.7075 \quad 0.381$

cuboid 4 I 2 p0.799 $0.0-2.768$

hole $33 \quad 0.799-1.384$

hole $\quad \begin{array}{lllll}34 & 0.0 & -2.768 & 0\end{array}$

unit 810

com=! 0.0 to $0.381 \mathrm{~cm} 10$ !

zhemicyl-y 31.64150 .3810 zhemicyl-y $21.7075 \quad 0.3810$

cuboid $412 \mathrm{p} 0.799 \quad 0.0-2.768$

hole

$0.381 \quad 0.0$

unit 811

com=! 0.0 to $0.381 \mathrm{~cm} 11$ !

zhemicyl-y $3 \quad 1.6415 \quad 0.3810$

zhemicyl-y $21.7075 \quad 0.381$

cuboid 4 1 2 p0.790 $0.0-2.768$

$\begin{array}{lllll}\text { hole } & 34 & 0.0 & -2.768 & 0\end{array}$

hole

unit 812

com=! 0.0 to $0.381 \mathrm{~cm} 12$ !

zhenicyl-y $31.6415 \quad 0.3810$

zhemicyl-y $21.7075 \quad 0.381$

$\begin{array}{lllll}\text { cuboid } 412 p 0.799 & 0.0 & -2.768\end{array}$

unit 813

com=! 0.0 to $0.381 \mathrm{~cm} 13$ !

zhemicyl-x $3 \quad 1$. 64150.3810

zhemicyl- $x \quad 21.7075 \quad 0.381 \quad 0$

$\begin{array}{llllll}\text { cuboid } 410 & -1.598 & 2 \mathrm{p} 1.384 & 0.381 & 0.0\end{array}$

$\begin{array}{lllll}\text { hole } & 34 & -0.799 & -1.384 & 0\end{array}$

unit 814

con $=$ ! 0.0 to $0.381 \mathrm{~cm} 14$ !

zhemicyl-x $3 \quad 1.6415 \quad 0.3810$

zhemicyl-x $21.7075 \quad 0.381$

$\begin{array}{llll}\text { cuboid } 410 & -1.598 & 2 p 1.384\end{array}$

unit 815

com=! 0.0 to $0.381 \mathrm{~cm} 15$ !

zhemicylty $31.6415 \quad 0.3810$

zhemicylty $21.7075 \quad 0.381$

unit 816

com=! 0.0 to $0.381 \mathrm{~cm} 16$ !

cuboid $412 \mathrm{p} 0.799 \quad 2 \mathrm{p} 1.384$

global unit 900

com=! reflector region!

$\begin{array}{lllll}\text { array } 1 & -30.362 & -29.064 & 0.0\end{array}$

reflector $51100000002.54 \quad 1$

zcylinder $4176.0 \quad 109.14-22.86$

end geom

read array

ara $=1$ nux $=38$ nuy $=21$ nuz=9

com=! full array of fuel pins!

\begin{tabular}{|c|c|c|c|c|c|c|c|c|}
\hline $\begin{array}{r}11 r 716 \\
8 r 716 \\
6 r 716 \\
5 r 716 \\
4 r 716 \\
3 r 716 \\
2 r 716\end{array}$ & $\begin{array}{r}3 r 712 \\
712 \\
712 \\
710 \\
710 \\
710 \\
710\end{array}$ & $\begin{array}{l}710 \\
710 \\
710\end{array}$ & 702 & $\begin{array}{r}9 r 702 \\
16 r 701 \\
22 r 701 \\
25 r 701 \\
28 r 701 \\
30 r 701 \\
32 r 701\end{array}$ & $\begin{array}{l}705 \\
702 \\
705 \\
702 \\
705 \\
705 \\
705\end{array}$ & $\begin{array}{r}2 r 712 \\
705 \\
7 r 716 \\
705 \\
4 r 716 \\
3 r 716 \\
2 r 716\end{array}$ & $\begin{array}{r}11 r 716 \\
712 \\
5 r 716\end{array}$ & $8 r 716$ \\
\hline
\end{tabular}




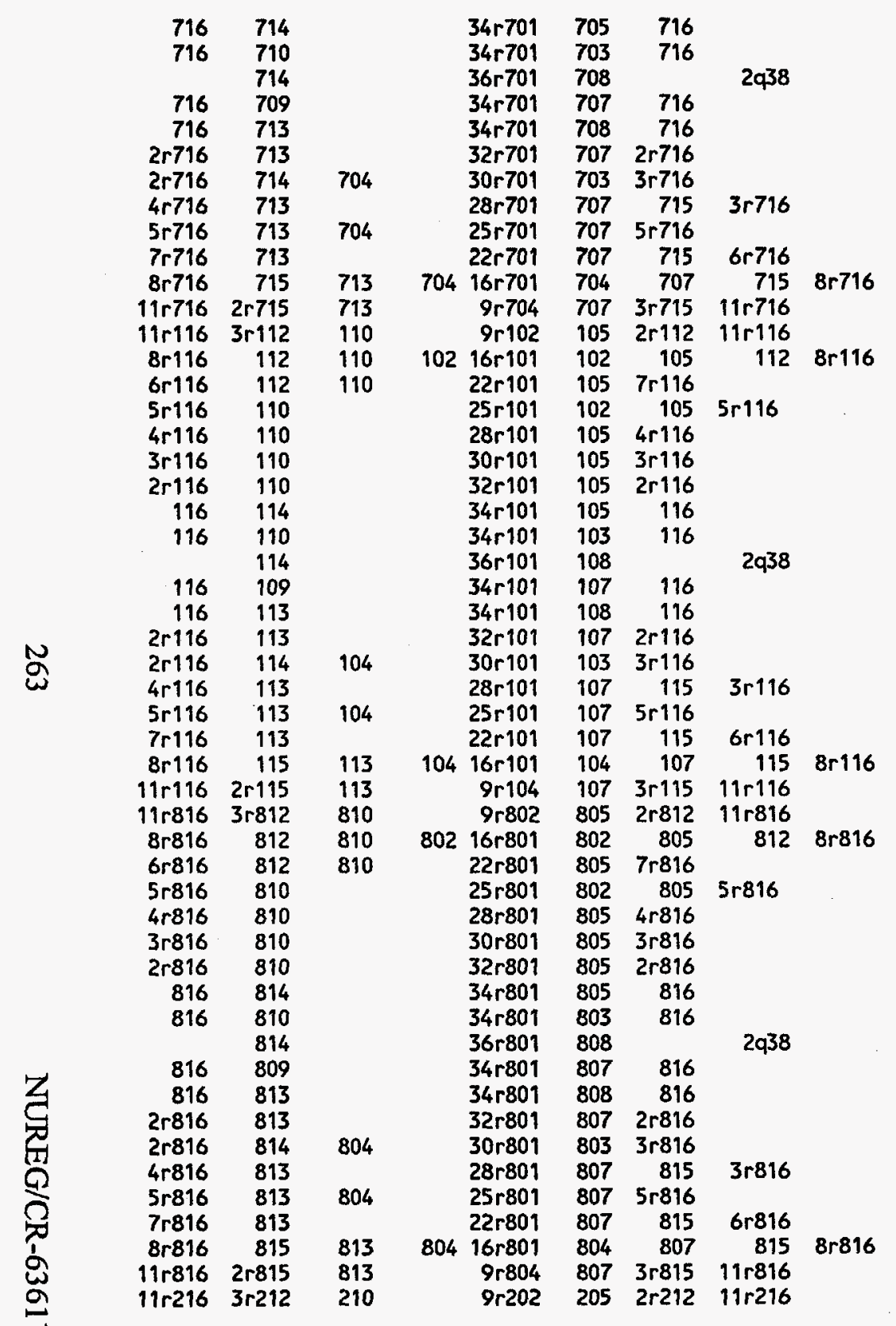

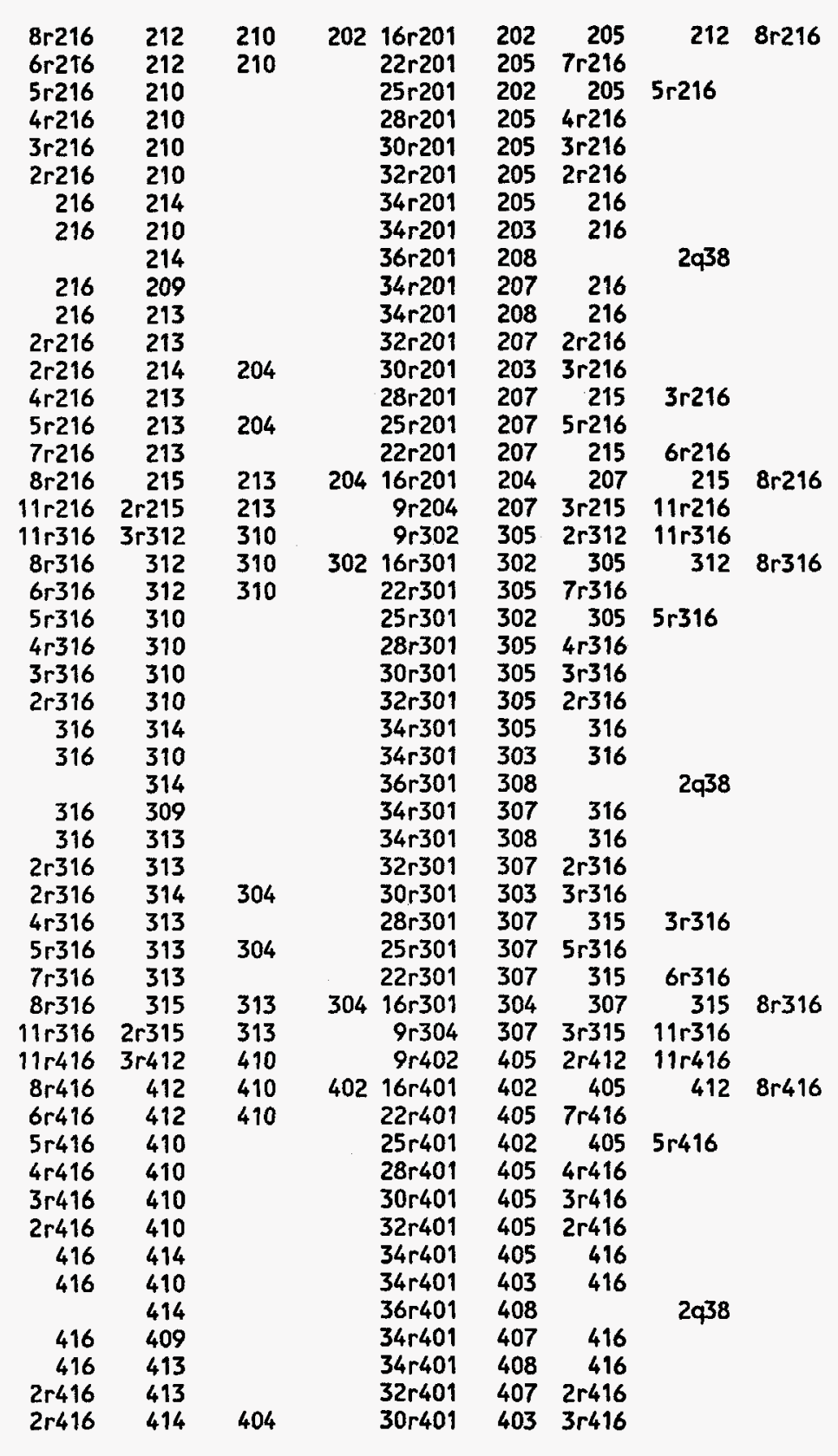




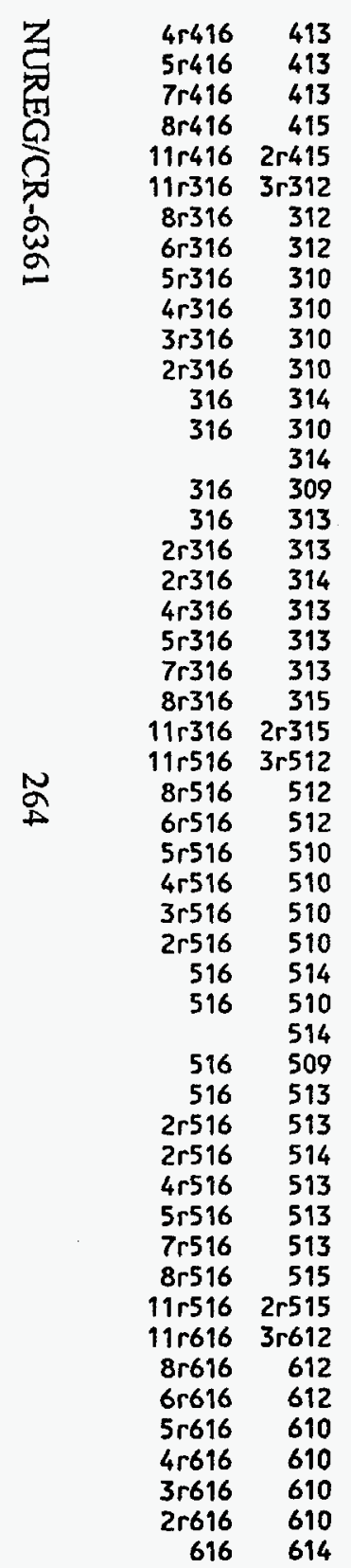

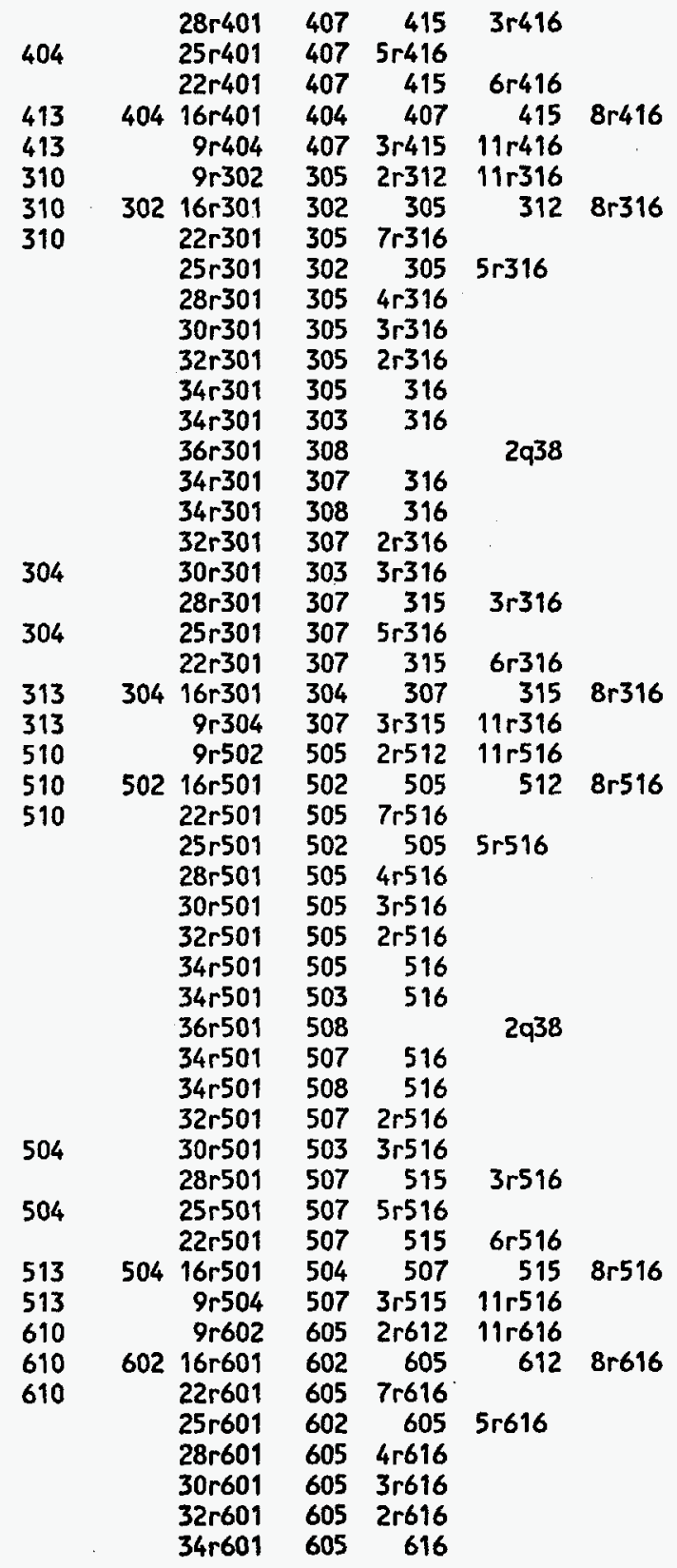

\begin{tabular}{|c|c|c|c|c|c|c|c|}
\hline $\begin{array}{r}616 \\
616 \\
616 \\
2 r 616 \\
2 r 616 \\
4 r 616 \\
5 r 616 \\
7 r 616 \\
8 r 616 \\
11 r 616\end{array}$ & $\begin{array}{r}610 \\
614 \\
609 \\
613 \\
613 \\
614 \\
613 \\
613 \\
613 \\
615 \\
2 r 615\end{array}$ & $\begin{array}{l}604 \\
604 \\
613 \\
613\end{array}$ & 604 & $\begin{array}{r}34 r 601 \\
36 r 601 \\
34 r 601 \\
34 r 601 \\
32 r 601 \\
30 r 601 \\
28 r 601 \\
25 r 601 \\
22 r 601 \\
16 r 601 \\
9 r 604\end{array}$ & $\begin{array}{l}603 \\
608 \\
607 \\
608 \\
607 \\
603 \\
607 \\
607 \\
607 \\
604 \\
607\end{array}$ & $\begin{array}{r}616 \\
\\
616 \\
616 \\
2 r 616 \\
3 r 616 \\
615 \\
5 r 616 \\
615 \\
607 \\
3 r 615\end{array}$ & $\begin{array}{r}3 r 616 \\
6 r 616 \\
615 \\
11 r 616\end{array}$ \\
\hline
\end{tabular}

22438 al

\section{$=\operatorname{csas} 25$}

p2438al

44group latticecell

uo2 10.84293922352 .359223897 .65 end

at 1.0

h20 31.0

plexiglas 41.0

arbmal $\quad 2.692800011302797 .1524000 .2129000 .12$ $26000.8225055 .21 \quad 14000 \quad 8216000 \quad .0622000$

.615 end

end comp

squarepitch 2.0321 .1176131 .272 end

3-20x16 assemblies separated by $0.625-\mathrm{cm}$-thick aluminum plates and $-8.045 \mathrm{~cm}$ of water, plate-to-assembly distance of $0.645 \mathrm{~cm}$ read parm run=yes gen $=405 \mathrm{npg}=600 \mathrm{nsk}=5$ nub=yes end parm

read geom

unit 1

cylinder 110.5588

$\begin{array}{lll}2 & 96.52 & -1.27\end{array}$

$\begin{array}{llll}\text { cuboid } 314 \mathrm{p} 1.016 & 96.52 & -1.27\end{array}$

unit 2

array $13 * 0.0$

reflector 312 r0.0 2r1.544 2r0.0 1

unit 3

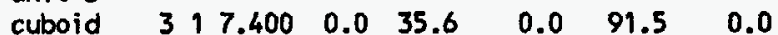

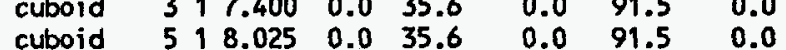

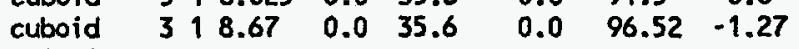

unit 4

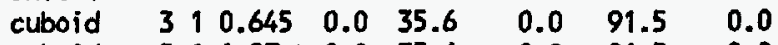

$\begin{array}{llllllll}\text { cuboid } 5 & 1 & 1.27 & 0.0 & 35.6 & 0.0 & 91.5 & 0.0 \\ & 3 & 1.67 & 0.0 & 35.6 & 0.0 & 96.52 & -1.27\end{array}$

global unit 5 
array $23 * 0.0$

reflector $4,5 r_{0} 2.54$,

reflector 312 r30.5 2r28.96 2r15.31

end geometry

\section{read array}

ara $=1$ nux $=20$ nuy $=16$ nuz=1 fill $f 1$ and fill ara=2 nux $=5$ nuy $=1$ nuz=1 fill 232242 end fill end array

end data

end

22438 ba

$=$ csas 25

p2438ba

44group latticecell

uo2 $\quad 10.84293922352 .359223897 .65$

al 21.0

h20 $\quad 31.0$

plexiglas 41.0

$2.49 \quad 510$
arbmboral

$60127.9726000 .3314000 .251293 \quad$ end

end comp

1.1176131 .272

$2-22 \times 16$ and $1-20 \times 16$ (center) assemblies separated by $0.713-\mathrm{cm}$-thick Boral-A plates and $4.337 \mathrm{~cm}$ of water, plate-to-assembly distance of $0.645 \mathrm{~cm}$

read parm run=yes gen $=405 \mathrm{npg}=600 \mathrm{nsk}=5$ nub=yes end parm

read geom

unit 1

cyl inder 1110.5588

cylinder 210.635

cuboid $314 \mathrm{pl} 1.016$

$91.44 \quad 0.0$

$96.52-1.27$

unit 2

$96.52-1.27$

array $13 * 0.0$

reflector 312 r0.0 $2 r 1.9942$ 2r0.0 1

unit 3

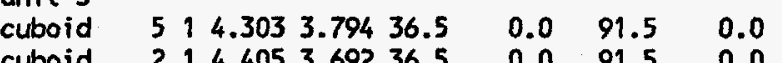

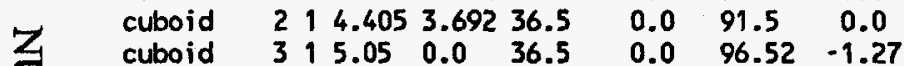

cuboid

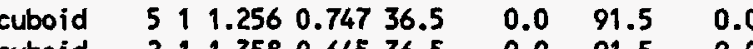

$\begin{array}{llll}36.5 & 0.0 & 91.5 & 0.0\end{array}$

cuboid

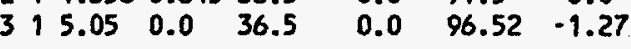

tefray 2 3*0.0

reflector $312 r 0.02 r 1.9942 r 0.0$ 1

global unit 6

array $3 \quad 3 * 0.0$

reflector $415 r 0 \quad 2.54 \quad 1$

reflector $312 r 30.5 \quad 2 r 26.512 r 15.31$

end geometry

read array

ara=1 nux=22 nuy=16 nuz=1 fill $f 1$ end fill

ara $=2$ nux $=20$ nuy $=16$ nuz=1 fill fi 1 end fill

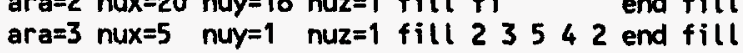

end array

end data

end

\section{$2438 c 4$}

=csas 25

p2438cu

44 group latticecel

uo2 10.84293922352 .359223897 .65 end

at 21.0

h20 $\quad 31.0$

plexíglas 41.0

cu 5 den $=8.913$

end comp

squarepitch 2.0321 .1176131 .272

$5.974 \mathrm{~cm}$ of water plate-to-assembly distance copper plates and

read geom

unit 1

$\begin{array}{llllll}\text { cylinder } & 1 & 1 & 0.5588 & 91.44 & 0.0 \\ \text { cylinder } & 2 & 1 & 0.635 & 96.52 & -1.27\end{array}$

$\begin{array}{llllll}\text { cylinder } & 2 & 1 & 0.635 & 96.52 & -1.27 \\ \text { cuboid } & 3 & 1 & 4 p 1.016 & 96.52 & -1.27\end{array}$

unit 2

array $13 * 0.0$

reflector $312 r 0.02 r 1.5442 r 0.01$

unit 3

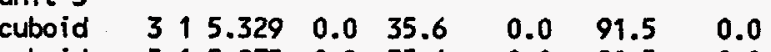

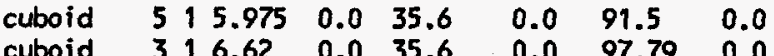

cuboid 316.62 o.0

unit 4

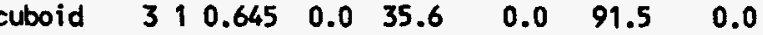

$\begin{array}{llllllll}\text { cuboid } 5 & 51 & 1.291 & 0.0 & 35.6 & 0.0 & 91.5 & 0.0\end{array}$

$\begin{array}{llllllll}\text { cuboid } 3 & 1 & 6.62 & 0.0 & 35.6 & 0.0 & 97.79 & 0.0\end{array}$

global unit 5

array $23 * 0.0$

reflector $415 r 02.54 \quad 1$

reflector 312 r30.5 2r28.96 2r15.3

end geometry 
end comp

7.9360012430418 .56290000 .2726304

squarepitch $2.032 \quad 1.1176 \quad 131.272$

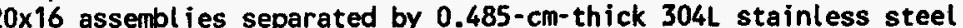

plates and $6.395 \mathrm{~cm}$ of water, plate-to-assembly distance of $0.645 \mathrm{~cm}$

\section{p2438sig}

$=\operatorname{csas} 25$

p2438sig

44 group latticecell

uo2 10.84293922352 .359223897 .65 end

at 1.02

h20 31.0

plexiglas 41.0

end comp

squarepitch 2.0321 .1176131 .272

$3-20 \times 16$ assembl ies separated by $8.39 \mathrm{~cm}$ of water

read parm run $=y e s$ gen $=405 \mathrm{npg}=600 \mathrm{nsk}=5$ nub=yes end parm

read geom

unit 1

cyl inder $\begin{array}{lllll}1 & 1 & 0.5588 & 91.44 & 0.0\end{array}$

$\begin{array}{llllll}\text { cyl inder } 2 & 1 & 0.635 & 96.52 & -1.27\end{array}$

cuboid $314 \mathrm{p} 1.016$

$96.52-1.27$

unit 2

array $13 * 0.0$

unit 3

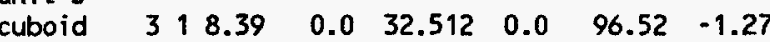

global unit 4

array $23 * 0.0$

reflector $\quad 415 r 02.54$

reflector $314 \mathrm{r} 30.52 \mathrm{2r} 15.3$

end geometry

read array

ara $=1$ nux $=20$ nuy $=16$ nuz $=1$ fill $f 1$ end fill

ara=2 nux=5 nuy $=1$ nuz=1 fill 233232 end fill

end array

end data

\section{p2438ss}

$=\operatorname{csas} 25$

p2438ss

44 group latticecell

uo2 10.84293922352 .359223897 .65 end

al 21.0

read geon

unit 1

cylinder $1110.5588 \quad 92.72 \quad 1.27$

$\begin{array}{lllllll}\text { cylinder } & 2 & 1 & 0.635 & 97.79 & 0.00 \\ \text { cuboid } & 3 & 1 & 401.016 & 97.79 & 0.00\end{array}$

cuboid $314 \mathrm{p} 1.016$

unit

array $13 * 0.0$

$\begin{array}{lllllll}\text { cuboid } 3140.64 & 0.0 & 34.056 & -1.544 & 97.79 & 0.0\end{array}$

unit 3

$\begin{array}{llllllll}\text { cuboid } 3 & 3 & 5.750 & 0.0 & 35.6 & 0.0 & 91.5 & 0.0\end{array}$

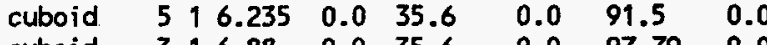

$\begin{array}{lllllll}\text { cuboid } 316.88 & 0.0 & 35.6 & 0.0 & 97.79 & 0.0\end{array}$

unit $4 \quad 3 \quad 10.645 \quad 0.0 \quad 35.6 \quad 0.0 \quad 91.5 \quad 0.0$

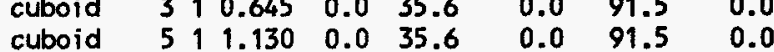

$\begin{array}{lllllllll}\text { cuboid } & 5 & 1 & 1.130 & 0.0 & 35.6 & 0.0 & 91.5 & 0.0 \\ \text { cuboid } & 3 & 1 & 6.88 & 0.0 & 35.6 & 0.0 & 97.79 & 0.0\end{array}$

global unit 5

array $2 \quad 3 * 0.0$

reflector $415 \mathrm{ro} \quad 2.541$

reflector $314 r 30.515 .215 .3$

end geometry

read array

ara $=1$ nux $=20$ nuy=16 nuz=1 fill $f 1$ end fill ara=2 nux=5 nuy=1 nuz=1 fill 232242 end fill

end array

end data

end

2243825

$=\operatorname{csas} 25$

p2438zr

44group latticecell

4ho? 10.84293922352 .359223897 .65

al 21.0

h20 $\quad 31.0$

plexiglas 41.0

24000.1326000 .215 .16500001 .5

squarepitch 2.0321 .1176131 .272 
3-20×16 assemblies separated by $0.652-\mathrm{cm}-$ thick Zircaloy-4 plates and $-8.138 \mathrm{~cm}$ of water, plate-to-assembly distance of $0.645 \mathrm{~cm}$ read parm run=yes gen $=405 \mathrm{npg}=600$ nsk $=5$ nub=yes end parm

\section{read geom}

unit 1

cyl inder 110.5588

cylinder 210.635

cuboid 314 p1.016

$91.44 \quad 0.0$

$96.52-1.27$

unit 2

array $13 * 0.0$

reflector $312 r 0.0 \quad 2 r 1.5442 r 0.01$

unit 3

$\begin{array}{llllllll}\text { cuboid } 3 & 3 & 17.493 & 0.0 & 35.6 & 0.0 & 91.5 & 0.0\end{array}$

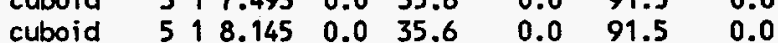

$\begin{array}{llllllll}\text { cuboid } 3 & 1 & 8.79 & 0.0 & 35.6 & 0.0 & 97.79 & 0.0\end{array}$

unit 4

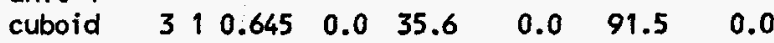

$\begin{array}{llllllll}\text { cuboid } \quad 5 & 1 & 1.297 & 0.0 & 35.6 & 0.0 & 91.5 & 0.0\end{array}$

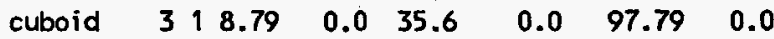

global unit 5

array $2 \quad 3 * 0.0$

reflector 415 ro 2.54

reflector 312 r30.5 2 r28.96 2r15.3 1

end geometry

read array

ara $=1$ nux $=20$ nuy=16 nuz $=1$ fill $f 1$ end fill

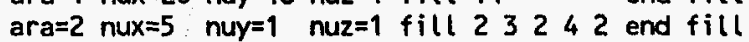

end array

end data

end

p2615al

$=\operatorname{csas} 25$

p2615al

44 group latticecell

uo2 10.949293922354 .319223895 .69 end

at 21.0 end

h2o 31.0

$\begin{array}{lllllll}\text { arbmal } & 2.692800 & 0 & 1302797.1524000 .2129000 .12\end{array}$ 26000.8225055 .2114000 .8216000 .0622000
.615

arbmrubber 1.32160000601258 .10016 .52000011 .4 160001.7801622 .1140000 .36

end comp

2.5401 .2649131 .414721 .28270

end

$3-15 \times 8$ assembl ies separated by $0.625-\mathrm{cm}-$ thick aluminum plates and
$-10.095 \mathrm{~cm}$ of water, plate-to-assembly distance of $0.105 \mathrm{~cm}$ read parm run=yes gen $=405$ npg $=600$ nsk $=5$ nub=yes end parm

read geom

unit 1

cylinder 110.63245

cyl inder 010.64135

cylinder 610.64135

cyl inder 210.70735

cuboid $314 \mathrm{p} 1.270$

$91.44 \quad 0.0$

$93.98 \quad-2.54$

$93.98-2.54$

$93.98-2.54$

unit 2

reflector 312 ro $2 r 7.642 r 0 \quad 1$

unit 3

$\begin{array}{llllll}\text { cuboid } & 3 & 1 & .105 & -10.615 & 2 \mathrm{p} 17.8\end{array}$

$88.96-2.54$

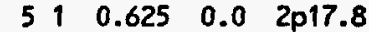

$93.98 \quad-2.54$

global unit 5

array $2 \quad-67.87 \quad-17.8 \quad 0$.

reflector $415 r 0.0 \quad 2.541$

reflector $312 r 30.5 \quad 2 r 22.86 \quad 2 r 15.31$

end geometry

read array

ara $=1$ nux $=15$ nuy $=8$ nuz $=1$ fill $f 1$ end fill

ara $=2$ nux $=5$ nuy=1 nuz=1 fill 2332442 end fill

end array

end data

end

p2615ba

$=\operatorname{csas} 25$

p2615ba

44group latticecell

uo2 10.949293922354 .319223895 .69 end

al 21.0

h2o $\quad 31.0$

plexiglas 41.0

arbmboral $2.49 \quad 4101500028.71302763 .0$

$60127.97260000 .33 \quad 5 \quad 1.293$

end

end

arbmrubber 1.32160000601258 .10016 .52000011 .4 160001.7801622 .1140000 .36

end comp

squarepitch 2.5401 .2649131 .414721 .28270

end

3-15x8 assemblies separated by $0.713-\mathrm{cm}$-thick Boral-A plates and $-6.007 \mathrm{~cm}$ of water, plate-to-assembly distance of $3.277 \mathrm{~cm}$

read parm run=yes gen $=405$ npg $=600$ nsk $=5$ nub=yes end parm 
read geom

unit 1

cylinder 110.63245

cylinder 0110.64135

cylinder 610.64135

cylinder 210.70735

cuboid $314 \mathrm{p} 1.270$

unit 2

array $13 * 0.0$

$\begin{array}{llllll}\text { reflector } & 31 & 2 r 0 & 2 r 8.09 & 2 r 0 & 1\end{array}$

unit 3

cuboid $59-0.102-0.6112 p 18.25$

$\begin{array}{llllll}\text { cuboid } \quad 2 & 1 & 0.0 & -0.713 & 2 p 18.25\end{array}$

$\begin{array}{llllll}\text { cuboid } \quad 313.277 & -3.443 & 2 p 18.25\end{array}$

$88.96 \quad-2.54$

$88.96-2.54$

cuboid

$93.98 \quad-2.54$

unit 4

$0.6110 .102 \quad 2 p 18.25$

$88.96-2.54$

$\begin{array}{ll}88.96 & -2.54 \\ 93.98 & -2.54\end{array}$

$\begin{array}{llllll}\text { cuboid } & 2 & 1 & 0.713 & 0.0 & 2 p 18.25\end{array}$

cuboid $31 \quad 3.443-3.2772 p 18.25$

global unit 5

array $2 \quad-63.87 \quad-18.250$.

reflector $\quad 415 r 0.0 \quad 2.541$

reflector $312 r 30.52 r 22.412 r 15.3$,

end geometry



read array

ara=1 nux=15 nuy=8 nuz=1 fill $f 1$ end fill

ara=2 nux=5 nuy=1 nuz=1 fill $23 \quad 3 \quad 2 \quad 4 \quad 2$ end fill

end array

end data

end

p2615cd1

\section{$=\operatorname{csas} 25$}

p2615cd1

44group latticecell

uo2 10.949293922354 .319223895 .69

al 21.0

plexiglas 41.0

cd 50.997

arbmrubber 1.3216000601258 .10016 .52000011 .4

160001.7801622 .1140000 .36

\section{end comp}

squarepitch 25401.2649131 .414721 .28270

3-15x8 assemblies separated by $0.2006-\mathrm{cm}$-thick cadmium

$7.0794 \mathrm{~cm}$ of water, plate-to-assembly distance of $3.277 \mathrm{~cm}$

read parm run=yes gen $=405 \mathrm{npg}=600 \mathrm{nsk}=5$ nub=yes end parm

read geom

unit 1 cylinder 110.63245

cylinder 010.64135

cyl inder 610.64135

cyl inder 210.70735

cuboid $314 p 1.270$

unit 2

$1.44 \quad 0.0$

$93.98-2.54$

$93.98-2.54$

$93.98-2.54$

array $13 * 0.0$

reflector $\quad 3 \quad 12 \mathrm{r} 0 \quad 2 \mathrm{r} 7.642 \mathrm{rO} \quad 1$

unit 3

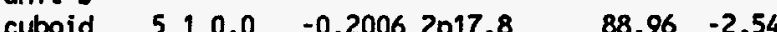

$\begin{array}{lllllll}\text { cuboid } 3 & 1 & 3.277 & -4.003 & 2 p 17.8 & 93.98 & -2.54\end{array}$

unit 4

$\begin{array}{lllllll}\text { cuboid } 5 & 5 & 0.2006 & 0.0 & 2 p 17.8 & 88.96 & -2.54\end{array}$

$\begin{array}{lllllll}\text { cuboid } 314.003 & -3.277 & 2 p 17.8 & 93.98 & -2.54\end{array}$

global unit 5

$\begin{array}{llll}\text { array } 2 & -64.43 & -17.8 & 0 .\end{array}$

reflector $495 \mathrm{ro} .0 \quad 2.541$

reflector $312 r 30.52 r 22.862 r 15.31$

end geometry

read array

ar $a=1$ nux $=15$ nuy $=8$ nuz $=1$ fill $f 1$ end fill

ara $=2$ nux $=5$ nuy=1 nuz=1 fill $232 \quad 2 \quad 4 \quad 2$ end fill

end array

end data

end

\section{$22615 \mathrm{~cd} 2$}

$=\operatorname{csas} 25$

p2615cd2

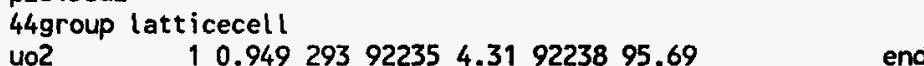

at 21.0

h20 31.0

plexiglas 41.0

arbmrubber 1.32160000601258 .10016 .52000011 .4 $160001.7801622 .1140000 .36 \quad$ end

end comp

squarepitch 2.5401 .2649131 .414721 .28270 end

3-15x8 assemblies separated by $0.2006-\mathrm{cm}-$ thick cadmium plates and $3.674 \mathrm{~cm}$ of water, plate-to-assembly distance of $0.529 \mathrm{~cm}$ read parm run=yes $g e n=405 \mathrm{npg}=600$ nsk $=5$ nub=yes end parm

read geom

unit 1

cyl inder 110.63245

cylinder 010.64135

cyt inder 610.64135

cylinder 210.70735

cuboid $314 \mathrm{p} 1.270$

$\begin{array}{ll}93.98 & -2.54 \\ 93.98 & -2.54\end{array}$


unit 2

array $13 * 0.0$

$\begin{array}{lllllll}\text { reflector } & 3 & 1 & 2 r 0 & 2 r 7.64 & 2 r 0 & 1\end{array}$

unit 3

cuboid $\quad \begin{array}{lllllll}5 & 1 & 0.0 & -0.2006 & 2 p 17.8 & 88.96 & -2.54\end{array}$

$\begin{array}{llllllll}\text { cuboid } & 3 & 1 & 0.529 & -5.151 & 2 p 17.8 & 93.98 & -2.54\end{array}$

unit 4

8.98 .9 .94

$\begin{array}{lllllllll}\text { cuboid } & 5 & 1 & 0.2006 & 0.0 & 2 p 17.8 & 88.96 & -2.54\end{array}$

$\begin{array}{llllllll}\text { cuboid } 3 & 1 & 5.151 & -0.529 & 2 p 17.8 & 93.98 & -2.54\end{array}$

global unit 5

$\begin{array}{llll}\text { array } 2 & -62.83 & -17.8,0 . & 0 .\end{array}$

reflector 312 r30.5 2 r22.86 2r15.31

end geometry

\section{read array}

ara $=1$ nux $=15$ nuy=8 nuz=1 fill $f 1$ end fill

ara=2 nux=5 nuy=1 nuz=1 fill $2 \quad 3 \quad 2 \quad 4 \quad 2$ end fill

end array

end data

end

\section{p2615cu}

$=\operatorname{csas} 25$

p2615cu

44 group latticecell

uo2 10.949293922354 .319223895 .69

al

h20 31.0

plexiglas 41.0

end

end

cu 5 den $=8.913$

arbmrubber 1.32160000601258 .10016 .52000011 .4

160001.7801622 .1140000 .36

\section{end comp}

end

squarepitch 2.5401 .2649131 .414721 .28270 end

3-15x8 assembl jes separated by $0.646-\mathrm{cm}$-thick copper plates and 7.504 $\mathrm{cm}$ of water, plate-to-assembly distance of $0.084 \mathrm{~cm}$ read parm run=yes gen $=405$ npg $=600$ nsk $=5$ nub=yes end parm

\section{read geom}

unit 1

cyl inder 110.63245

cylinder 0010.64135

$\begin{array}{llll}\text { cylinder } & 0 & 1 & 0.64135 \\ \text { cylinder } & 6 & 1 & 0.64135\end{array}$

cylinder 2.10 .70735

cuboid $314 \mathrm{p} 1.270$

unit 2

array $13 * 0.0$

reflector 312 ro $2 r 7.642$ ro 1

unit 3

$91.44 \quad 0.0$

$91.44 \quad 0.0$

$93.98-2.54$

$93.98 \quad-2.54$

$93.98-2.54$

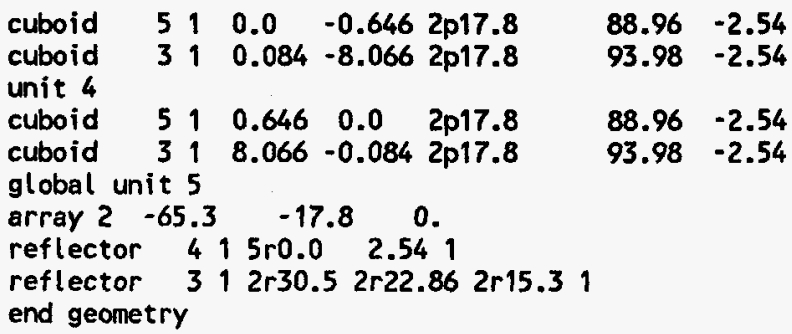

$\begin{array}{llllllll}\text { cuboid } \quad 5 & 1 & 0.0 & -0.646 & 2 \mathrm{p} 17.8 & 88.96 & -2.54\end{array}$

$\begin{array}{lllllll}\text { cuboid } 31 & 0.084 & -8.066 & 2 p 17.8 & 93.98 & -2.54\end{array}$

unit 4

$\begin{array}{llllllll}\text { cuboid } & 5 & 1 & 0.646 & 0.0 & 2 p 17.8 & 88.96 & -2.54\end{array}$

$\begin{array}{llllllll}\text { cuboid } \quad 3 & 1 & 8.066 & -0.084 & 2 p 17.8 & 93.98 & -2.54\end{array}$

global unit 5

$\begin{array}{llll}\text { array } 2 & -65.3 & -17.8 & 0 .\end{array}$

reflector $415 r 0.0 \quad 2.541$

reflector 312 r $30.52 r 22.862 r 15.31$

end geometry

read array

ara $=1$ nux $=15$ nuy $=8$ nuz $=1$ fill $f 1$ end $f i l l$

ara=2 nux=5 nuy=1 nuz=1 fill $2 \quad 3 \quad 2 \quad 4 \quad 2$ end fill

end array

end data

end

p2615ss

$=\operatorname{csas} 25$

p2615ss

44 group latticecell

uo2 10.949293922354 .319223895 .69

al 21.0

h20 31.0

plexiglas 41.0

s. 504.0

arbmrubber 1.32160000601258 .10016 .52000011 .4 160001.7801622 .1140000 .36

end

end comp

3-15x8 assemblies separated by $0.485-\mathrm{cm}-$ thick $304 \mathrm{~L}$ stainless steel

plates and $8.095 \mathrm{~cm}$ of water, plate-to-assembly distance of $0.245 \mathrm{~cm}$ read parm run=yes gen $=405 \mathrm{npg}=600$ nsk $=5$ nub=yes end parm

read geom

unit 1

cylinder 110.63245

cylinder 0110.64135

cylinder 610.64135

cylinder 210.70735

cuboid $314 \mathrm{p} 1.270$

unit 2

array $13 * 0.0$

reflector 31 2ro $2 r 7.642$ ro

unit 3

cuboid $51 \quad 0.0 \quad-0.4852017 .8$

$\begin{array}{llllll}\text { cuboid } & 31 & 0.245 & -8.335 & 2 p 17.8\end{array}$

$88.96-2.54$

Unit 4

$\begin{array}{lllll}5 & 1 & 0.485 & 0.0 & 2 p 17.8\end{array}$

$88.96-2.54$ 
$\begin{array}{llllllll}\text { cuboid } \quad 3 & 1 & 8.335 & -0.245 & 2 p 17.8 & 93.98 & -2.54\end{array}$

global unit 5

$\begin{array}{llll}\text { array } 2 & -65.73 & -17.8 & 0 .\end{array}$

reflector $415 r 0.0 \quad 2.54$

reflector 312 r30.5 2r22.86 2r15.3

end geometry

read array

ar $a=1$ nux $=15$ nuy $=8$ nuz $=1$ fill $f \mathfrak{i}$ end fill

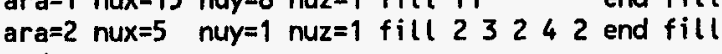

end array

end data

end

\section{p2615zr}

$=c \operatorname{sas} 25$

p2615zr

44group latticecel

uo2 10.949293922354 .319223895 .69 end

at $\quad 21.0$

h20 31.0

plexiglas 41.0

$\begin{array}{llllllllll} & & & \end{array}$

6.324001

$\stackrel{N}{O}$

arbmrubber $1.32160000012 \quad 58.01001 \quad 6.5 \quad 2000011.4$ 160001.7801622 .1140000 .36 end

end comp

squarepitch $2.5401 .264913 \quad 1.414721 .2827 \quad 0$ end

3-15 88 ass

$-10.268 \mathrm{~cm}$ of water, plate-to-assembly distance of $0.078 \mathrm{~cm}$

read parm run=yes gen $=405 \mathrm{npg}=600 \mathrm{nsk}=5$ nub=yes end parm

read geom

unit 1

cylinder 110.63245

cylinder 010.64135

cylinder 610.64135

cylinder $2 \begin{array}{lll}2 & 1 & 0.70735\end{array}$

cuboid 3 1 4 p1.270

unit 3

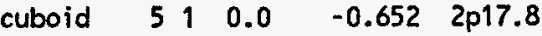

cuboid

$\begin{array}{lllll}3 & 1 & 0.078 & -10.842 & 2 p 17.8\end{array}$

$93.98-2.54$

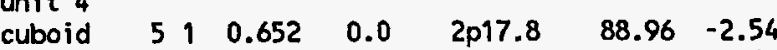

$\begin{array}{llllllll}\text { cuboid } & 3 & 1 & 10.842 & -0.078 & 2 p 17.8 & 93.98 & -2.54\end{array}$

global unit 5

$\begin{array}{llll}\text { array } 2 & -68.07 & -17.8 & 0 .\end{array}$
$88.96-2.54$

$93.98-2.54$

$\begin{array}{ll}3.98 & -2.54\end{array}$ reflector $\quad 415 r 0.0 \quad 2.541$

reflector 312 r30.5 2 r22.86 2r15.31

end geometry

read array

ara=1 nux $=15$ nuy $=8$ nuz $=1$ fill $f 1$ end fill

ara $=2$ nux $=5$ nuy $=1$ nuz=1 fill $2 \quad 3 \quad 2 \quad 4 \quad 2$ end fill

end array

end data

end

0282711

$=\operatorname{csas} 25$

p2827। 1

44group latticecell

$\begin{array}{lrl}\text { 4.2 } & 10.8429392235 \quad 2.359223897 .65\end{array}$

uor $\quad 21.0$

h20 $\quad 31.0$

plexiglas 41.0

h20 51.0

pb $\quad 6.9758$

end comp

$2.0321 .1176 \quad 131.272$

3-19x16 assemblies separated by $13.27 \mathrm{~cm}$ of water, assemb

'separated from lead reflecting walls by $0.66 \mathrm{~cm}$ of water

read parm run=yes gen $=405 \mathrm{npg}=600$ nsk $=5$ nub=yes end parm

read geom

unit 1

cylinder 190.5588

cylinder 210.635

$\begin{array}{llll}\text { cylinder } & 2 & 1 & 0.635 \\ \text { cuboid } & 3 & 1 & 4 \mathrm{p} 1.016\end{array}$

$91.44 \quad 0.0$

$96.52 \quad-1.27$

unit 2

array $13 * 0.0$

unit 3

$\begin{array}{lllllllll}\text { cuboid } & 5 & 1 & 12.958 & 0.0 & 32.512 & 0.0 & 96.52 & -1.27\end{array}$

global unit 4

global $2-70.87-16.256$

$\begin{array}{llllll}\text { reflector } & 4 & 1 & 5 \mathrm{r} 0.0 & 2.54 & 1\end{array}$

$\begin{array}{lllllll}\text { cuboid } \quad 51 & 2 \mathrm{p} 82.0 & \text { 2p16.916 } & 104.29 & -19.11\end{array}$

$\begin{array}{lllllll}\text { reflector } & 6 & 1 & 2 r 0.0 & 2 r 10.2 & 2 r 0.0 & 1 \\ \text { reflector } & 5 & 1 & 2 r 30.5 & 2 r 19.64 & 7.430 & 1\end{array}$

end geometry

read array

ara $=1$ nux $=19$ nuy=16 nuz=1 fill $f 1$ end fill

ara=2 nux=5 nuy=1 nuz=1 fill 23232 end fill

end array

end data

end end

end

nd

end

end

end 
0282712

\section{$=$ csas 25}

p282712

44group latticecell

uo2 $\quad 10.84293922352 .359223897 .65$

21.0

$\begin{array}{lll}2 & 2 & 1.0 \\ \text { h } & 3 & 1.0\end{array}$

plexiglas 41.0

h2o 51.0

$\mathrm{pb} \quad 6.9758$

end comp

squarepitch 2.0321 .1176131 .272

$3-19 \times 16$ assenblies separated by

'separated from lead reflecting walls by $2.616 \mathrm{~cm}$ of water read parm run=yes gen $=405 \mathrm{npg}=600 \mathrm{nsk}=5$ nub=yes end parm

read geom

unit 1

cyl inder 110.5588

cylinder 210.635

$91.44 \quad 0.0$

$96.52-1.27$

unit 2

$96.52-1.27$

array $13 * 0.0$

$N$ unit 3

$\begin{array}{lllllllll} & 5 & 1 & 10.488 & 0.0 & 32.512 & 0.0 & 96.52 & -1.27\end{array}$

global unit 4

$\begin{array}{llll}\text { array } 2 & -68.4 & -16.256 & 0\end{array}$

$\begin{array}{llll}\text { reflector } 4 & 4 & 5 \mathrm{r} 0.0 & 2.54\end{array}$

$\begin{array}{lllllll}\text { cuboid } & 5 & 1 & 2 p 82.0 & 2 p 18.872 & 104.29 & -19.11^{1}\end{array}$

$\begin{array}{lllllll}\text { reflector } & 6 & 1 & 2 \mathrm{r} 0.0 & 2 \mathrm{r} 10.2 & 2 \mathrm{r} 0.0 & 1\end{array}$

reflector $51 \quad 2 r 30.5 \quad 2 r 17.6847 .43$

end geometry

read array

ara $=1$ nux $=19$ nuy=16 nuz=1 fill $f 1$ end fill

ara $=2$ nux $=5$ nuy=1 nuz=1 fill $2 \quad 3 \quad 2 \quad 3 \quad 2$ end fill

end array

end data

end

Z

p282713

$=\operatorname{csas} 25$

p282713

44group latticecelt

uo2 $\quad 10.949293922354 .319223895 .69$

al

21.0

h2o 31.0

plexiglas 41.0

$\mathrm{pb} \quad 5 \quad .9758$ arbmrubber 1.3216000601258 .10016 .52000011 .4 $160001.7801622 .114000 \quad 0.37$

end comp

squarepitch 2.5401 .2649131 .414721 .28270

end

$3-13 \times 8$ assemblies separated by $20.78 \mathrm{~cm}$ of water, assemblies separated

from lead reflecting walls by $0.66 \mathrm{~cm}$ of water

read parm run=yes gen $=405 \mathrm{npg}=600 \mathrm{nsk}=5$ nub=yes end parm

read geom

unit 1

cylinder 110.63245

cyl inder 010.64135

cylinder 710.64135

cylinder 210.70735

cuboid $314 \mathrm{p} 1.270$

$93.98-2.54$

$93.98-2.54$

unit 2

array $13 * 0.0$

unit 3

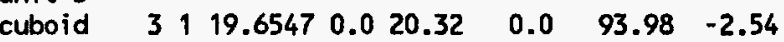

global unit 4

array $2-69.1847 \cdot 10.16 \quad 0$

reflector $415 r 0.0 \quad 2.54$

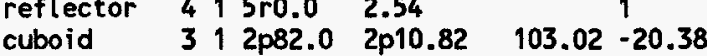

reflector $5 \begin{array}{llllll}5 & 1 & 2 \mathrm{r} 0.0 & 2 \mathrm{r} 10.2 & 2 \mathrm{r} 0.0 & 1\end{array}$

reflector $312 r 30.5 \quad 2 r 19.64: 6.160$

end geometry

read array

ara $=1$ nux $=13$ nuy $=8$ nuz $=1$ fill $f 1$ end $f i l l$

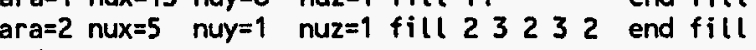

end array

end data

end

\section{p282714}

$=\operatorname{csas} 25$

p282714

44 group latticecell

uo2 10.949293922354 .319223895 .69

al 21.0

h20 $\quad 31.0$

plexiglas 41.0

h20 51.0

pb $\quad 6.9758$

arbmrubber 1.32160000601258 .10016 .52000011 .4 $160001.7801622 .114000 \quad 0.37$

end comp

squarepitch $2.5401 .2649131 .41472 \quad 1.28270$

3-13×8 assembl ies separated by $19.04 \mathrm{~cm}$ of water, assemblies separated

from lead reflecting walls by $1.321 \mathrm{~cm}$ of water,

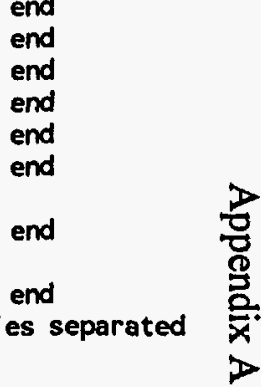


read array

ara $=1$ nux=13 nuy $=8$ nuz=1 fill $f 1$ and $f i l l$ ara $=2$ nux $=5$ nuy $=1$ nuz $=1$ fill $\begin{array}{lllllllll}2 & 3 & 3 & 2 & \text { end fill }\end{array}$

$N$ end array

N end data

end

\section{p2827slg}

\section{$=\operatorname{csas} 25$}

p2827sig

44 group latticecell

uo2 10.84293922352 .359223897 .65

al 21.0

h20 31.0

plexiglas 41.0

41.0

end comp

squarepitch 2.0321 .1176131 .272

3-19×16 assemblies separated by $8.31 \mathrm{~cm}$ of water

read parm run=yes gen $=405$ npg $=600$ nsk $=5$ nub=yes end parm

\section{read geom}

unit

cyl inder 110.5588

cyl inder 210.635

cuboid $314 \mathrm{p1.016}$

unit 2

array $13 * 0.0$

unit 3

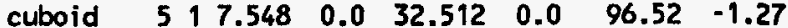

global unit 4

array $2-65.460-16.2560$.

reflector $415 r 0.0 \quad 2.54$

reflector 514 r30.5 15.215 .3

end geometry

read array

ara=1 nux=19 nuy=16 nuz=1 fill $f 1$ end fill

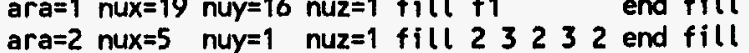

end array

end data

end

22827u1

$=\operatorname{csas} 25$

p2827ut

44 group latticecell

uo2 10.84293922352 .359223897 .65 end

at $\quad 21.0$

h2o 31.0

plexiglas 41.0

h20 $\quad 51.0$

end comp

6.9816329392235 .1999223899 .801

squarepitch $2.0321 .1176 \quad 131.272$

3-19×16 assemblies separated by $11.83 \mathrm{~cm}$ of water, assemblies separated from depleted uranium reflecting walls by $0.0 \mathrm{~cm}$ of water read parm run=yes gen $=405 \mathrm{npg}=600 \mathrm{nsk}=5$ nub=yes end parm

read geom

unit 1

$\begin{array}{llllll}\text { cylinder } & 1 & 1 & 0.5588 & 91.44 & 0.0\end{array}$

cylinder $210.635 \quad 96.52 \quad-1.27$

cuboid $314 \mathrm{p} 1.016 \quad 96.52-1.27$

unit 2

array $13 * 0.0$

unit 3

cuboid $\quad \begin{array}{lllllllll}5 & 1 & 11.068 & 0.0 & 32.512 & 0.0 & 96.52 & -1.27\end{array}$

global unit 4

array $2 \quad-68.98 \quad-16.256 \quad 0$.

reflector $\quad 415 r 0.0 \quad 2.54$

$\begin{array}{llllll}\text { cuboid } \quad 5 & 1 & 2 p 76.15 & 2 p 16.256 & 102.79 & -19.11\end{array}$

$\begin{array}{lllllll}\text { reflector } & 6 & 1 & 2 \mathrm{r} 0.0 & 2 \mathrm{r} 7.65 & 2 \mathrm{r} 0.0 & 1\end{array}$

$\begin{array}{llllll}\text { reflector } & 5 & 1 & 2 r 30.5 & 2 r 22.85 & 8.930\end{array}$

end geometry

read array

ara $=1$ nux $=19$ nuy=16 nuz=1 fill fi fill 
ara=2 nux=5 nuy=1 nuz=1 fill 2332332 end fill

end array

end data

end

\section{p2827ur}

$=\operatorname{csas} 25$

p2827u2

44group latticecel

al 21.0

h20 31.0

plexiglas 41.0

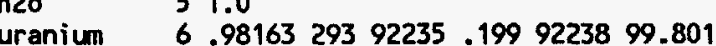

uranium

end

end

squarepitch 2.0321 .1176131 .272

from separated by $14.11 \mathrm{~cm}$ of water, assembl ies

'separated from depleted uranium reflecting walls by $1.956 \mathrm{~cm}$ of water

read parm run=yes gen $=405 \mathrm{npg}=600 \mathrm{nsk}=5$ nub $=y e s$ end parm

read geom

unit

cylinder $110.5588 \quad 91.44 \quad 0.0$

N cylinder 210.635

unit 2

array $13 * 0.0$

unit 3

$\begin{array}{ll}96.52 & -1.27 \\ 96.52 & -1.27\end{array}$

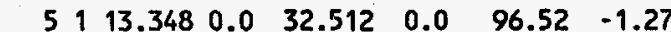

global unit 4

array $2 \quad-71.26 \quad-16.256 \quad 0$.

reflector $415 r 0.0 \quad 2.54$

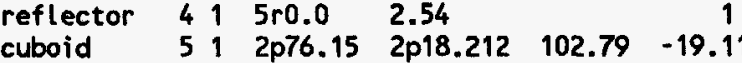

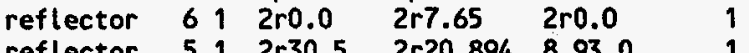

reflector $51 \quad 2 r 30.5 \quad 2 r 20.894 \quad 8.93$

end geometry

read array

ara=1 nux=19 nuy=16 nuz=1 fill $f 1$ end fill

ara $=2$ nux $=5$ nuy=1 nuz=1 fill 233232 end fill

end array

end data

end

2282743

$=\operatorname{csas} 25$

p2827u3

44group latticecell

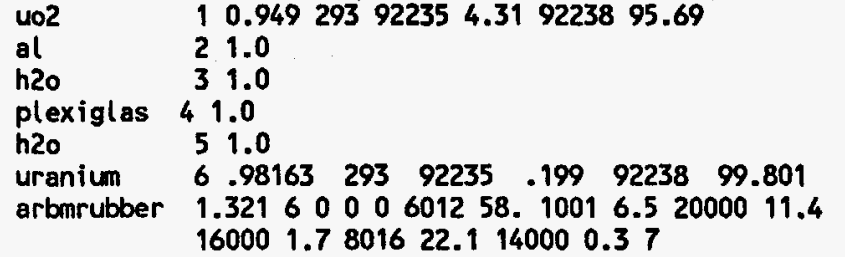

end

end

end

end

end

end

end comp

squarepitch $2.540 \quad 1.2649 \quad 13 \quad 1.41472 \quad 1.28270$

end

3-13×8 assemblies separated by $15.38 \mathrm{~cm}$ of water, assemblies separated

'from depleted uranium reflecting walls by $0.0 \mathrm{~cm}$ of water

read parm run=yes gen $=405 \mathrm{npg}=600$ nsk $=5$ nub=yes end parm

read geom

unit 1

cylinder $110.63245 \quad 91.44 \quad 0.0$

cylinder $010.64135 \quad 91.44 \quad 0.0$

cylinder $710.64135 \quad 93.98-2.54$

cylinder $210.70735 \quad 93.98 \quad-2.54$

cuboid $314 \mathrm{p} 1.270 \quad 93.98 \quad-2.54$

unit 2

array $13 * 0.0$

unit 3

$\begin{array}{lllllllll} & 5 & 1 & 14.2547 & 0.0 & 20.32 & 0.0 & 93.98 & -2.54\end{array}$

global unit 4

array $2-63.7847-10.16 \quad 0$

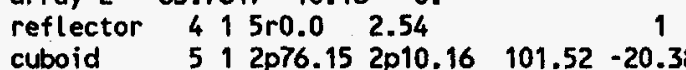

reflector $612 \mathrm{r} 0.0 \quad 2 r 7.65 \quad 2 \mathrm{r0} .0$

reflector $512 r 30.52 r 22.857 .660$

end geometry

read array

ara $=1$ nux $=13$ nuy $=8$ nuz $=1$ fill $f 1$ end $f i l l$

ara=2 nux=5 nuy=1 nuz=1 fill 232232 end fill

end array

end data

end

22827u4

$=\operatorname{csas} 25$

p2827u

44group latticecell

402 10.949293922354 .319223895 .69

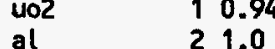

al $\quad 21.0$

plexiglas 41.0

hro 51.0 
uranium $\quad 6.98163 \quad 293 \quad 92235 \quad .199 \quad 92238 \quad 99.801 \quad$ end arbmrubber 1.3216000601258 .10016 .52000011 .4 $160001.7801622 .114000 \quad 0.37$

end comp

squarepitch 2.5401 .2649131 .414721 .28270 end

squarepitch 2.5401 .2649131 .414721 .28270 end

'separated from depleted uranium reflecting walls by $1.956 \mathrm{~cm}$ of water

read parm run=yes gen $=405$ npg $=600$ nsk $=5$ nub=yes end parm

read geom

unit 1

cylinder 110.63245

cylinder 010.64135

cylinder 710.64135

cylinder 210.70735

$93.98-2.54$

unit 2

$\begin{array}{lllllll}\text { unit } 3 & 5 & 14.1947 & 0.020 .32 & 0.0 & 93.98 & -2.54\end{array}$

global unit 4

global unit $4.9147-10.16$

reflector $495 \mathrm{r} 0.0 \quad 2.54$

$\begin{array}{llllll}\text { cuboid } \quad 5 & 1 & 2 p 76.15 & 2 p 12.116 & 101.52 & -20.38\end{array}$

reflector $612 r 0.0 \quad 2 r 7.65 \quad 2 r 0.0$

$\begin{array}{lllll}N & \text { reflector } 512 r 30.5 & 2 r 20.894 & 7.660\end{array}$

end geometry

read array

ar $a=1$ nux=12 nuy=8 nuz=1 fill $f 1$ end $f i l l$

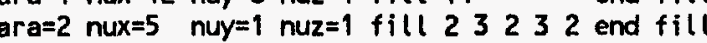

end array

end data

end

\section{p3314al}

$=$ csas 25

p3314al

44 group latticecell

uo2 10.949293922354 .319223895 .69

al 21.0

h20 31.0

plexiglas 41.0

arbmrubber 1.3216000601258 .10016 .52000011 .4 $160001.7801622 .1 \quad 14000 \quad 0.35$

al 6 den $=2.692 \quad .9715$

cr 6 den=2.692 .0021

cu 6 den $=2.692 .0012$

cu 6 den $=2.692 .0012$

m 6 den=2.692 .0021 si 6 den $=2.692 .0082$

s 6 den $=2.692 .0006$

ti 6 den=2.692 .0061

end comp

squarepitch 1.8921 .2649131 .414721 .28270 end $2-9 \times 12$ and $2-9 \times 1$ assembl ies separated by $0.625-\mathrm{cm}$-thick aluminum plates and $2.205-$ and $8.415-\mathrm{cm}$ of water

read parm run=yes gen $=405 \mathrm{npg}=600 \mathrm{nsk}=5$ nub=yes end parm

read geom

unit 1

cyl inder $110.63245 \quad 91.44 \quad 0.0$

cylinder $010.64135 \quad 91.44 \quad 0.0$

cylinder $510.64135 \quad 93.98-2.54$

cyl inder $210.70735 \quad 93.98 \quad-2.54$

cuboid 314 p.9460 $\quad 93.98-2.54$

unit 2

$\begin{array}{lllllllll}\text { cuboid } & 3 & 1 & 17.957 & 0.0 & 22.704 & 0.0 & 93.98 & -2.54\end{array}$

unit 3

unit 4

$\begin{array}{lllllllll}6 & 1 & 2.83 & 2.205 & 22.704 & 0.0 & 88.96 & -2.54\end{array}$

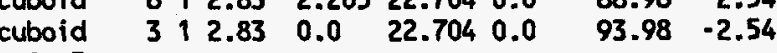

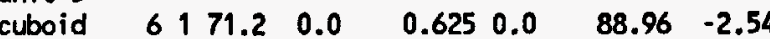

$\begin{array}{lllllll}\text { cuboid } & 3171.2 & 0.0 & 0.625 & 0.0 & 93.98 & -2.54\end{array}$

$\begin{array}{lllllllll}\text { cuboid } & 3 & 1 & 36.39 & 0.0 & 8.415 & 0.0 & 88.96 & -2.54\end{array}$

$\begin{array}{llllllll} & & & \end{array}$

$\begin{array}{llllllll}\text { cuboid } & 3 & 171.2 & 0.0 & 8.415 & 0.0 & 93.98 & -2.54\end{array}$

unit 7

cuboid

unit 8

cuboid

$\begin{array}{llllllll}3 & 1 & 17.157 & 0.0 & 1.892 & 0.0 & 93.98 & -2.54\end{array}$

cuboid

$\begin{array}{llllllll}6 & 1 & 2.83 & 2.205 & 1.892 & 0.0 & 88.96 & -2.54\end{array}$

array $23 * 0.0$

unit 10

$\begin{array}{llllllllll}\text { cuboid } & 3 & 1 & 36.39 & 0.0 & 37.564 & 0.0 & 88.96 & -2.54\end{array}$

cuboid $\quad \begin{array}{lllllllll}6 & 1 & 37.015 & 0.0 & 37.564 & 0.0 & 88.96 & -2.54\end{array}$

$\begin{array}{llllllll}\text { cuboid } & 3171.2 & 0.0 & 37.564 & 0.0 & 93.98 & -2.54\end{array}$

unit 11

array $3 * 0.0$

unit 12

array $43 * 0.0$

global unit 13

array $53 * 0.0$

reflector $415 r 0.0 \quad 2.54 \quad 1$

reflector $314 r_{30.0} 2 r 15.0$

end geometry 
read array

ara $=1$ nux=9 nuy=12 nuz=1 fill $f 1$ end fill

ara $=2$ nux $=9$ nuy=1 nuz=1 fill fl end $f i l l$

ara $=3$ nux $=5$ nuy=1 nuz=1 fill $2 \quad 3 \quad 4 \quad 3 \quad 2$ end fill

ara $=4$ nux $=5$ nuy $=1$ nuz $=1$ fill 79897 end fill

ara $=5$ nux=1 nuy=5 nuz=1 fill $1156 \quad 12 \quad 10$ end fill

end array

end data

end

\section{4ba}

$=\operatorname{csas} 25$

p3314ba

44group latticecell

uo2 $\quad 10.949293922354 .319223895 .69$

al

21.0

31.0

arbmrubber 1.3216000601258 .10016 .52000011 .4

$160001.7801622 .1 \quad 14000 \quad 0.35$

6 den $=2.49 \quad .287$

6 den=2.49 $\quad .0797$

6 den=2.49 $\quad .6249$

6 den=2.49 $\quad .0005$

6 den $=2.49 \quad .0009$

$\begin{array}{lll}6 \text { den }=2.49 & .0009 \\ 6 \text { den } & .49 & .0033\end{array}$

6 den=2.49 $\quad .0005$

6 den $=2.49 \quad .0005$

6 den $=2.49 \quad .0002$

6 den $=2.49 \quad .0002$

6 den $=2.49 \quad .0020$

6 den=2.49 $\quad .0003$

end

end

end

end

end

end

end

end

end

end

end

end

end

end

end

end

end

end comp

squarepitch 1.8921 .2649 1 31.414721 .28270 end

$2-11 \times 14$ and $2-11 \times 16$ assemblies separated by $0.713-\mathrm{cm}-$ thick Boral-A plates and 2.117- and $4.087-\mathrm{cm}$ of water

read parm run=yes gen $=405 \mathrm{npg}=600 \mathrm{nsk}=5$ nub=yes end parm

read geom

unit 1

cyl inder 110.63245

cyl inder 010.64135

cyl inder 510.6413

cylinder 210.70735

cuboid $314 \mathrm{p} .9460$

unit 2

$\begin{array}{lllll}1 & 13.373 & 0.0 & 26.488 & 0.0\end{array}$

cuboid

array $13 * 0.0$ unit 4

cuboid

$\begin{array}{llllllll}6 & 1 & 2.728 & 2.219 & 26.488 & 0.0 & 88.96 & -2.54\end{array}$

$\begin{array}{llllllll}2 & 1 & 2.83 & 2.117 & 26.488 & 0.0 & 88.96 & -2.54\end{array}$

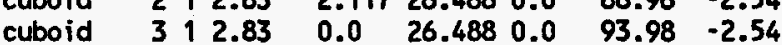

unit 5

unit

cuboid

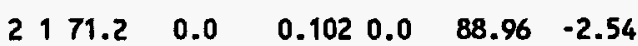

$\begin{array}{llllllll}6 & 1 & 71.2 & 0.0 & 0.611 & 0.0 & 88.96 & -2.54\end{array}$

$\begin{array}{llllllll}2 & 1 & 71.2 & 0.0 & 0.713 & 0.0 & 88.96 & -2.54\end{array}$

$\begin{array}{llllllll}\text { cuboid } & 3 & 171.2 & 0.0 & 0.713 & 0.0 & 93.98 & -2.54\end{array}$

unit 6

cuboid

cuboid

cuboid

3136.3020 .0

$\begin{array}{llllllll}2 & 1 & 36.404 & 0.0 & 4.087 & 0.0 & 88.96 & -2.54\end{array}$

$\begin{array}{llllllll}6 & 1 & 36.913 & 0.0 & 4.087 & 0.0 & 88.96 & -2.54\end{array}$

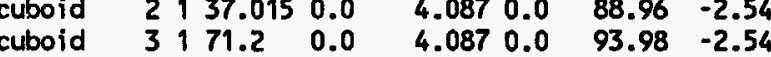

unit 7

cuboid

unit 8

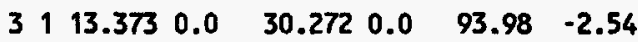

$\begin{array}{lllllll}6 & 12.728 & 2.219 & 30.272 & 0.0 & 88.96 & -2.54\end{array}$

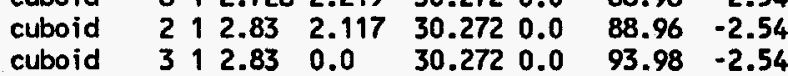

unit 9

unit 10

cuboid $\quad 3136.302 \quad 0.0 \quad 9.64 \quad 0.0 \quad 88.96 \quad-2.54$

$\begin{array}{llllllll}\text { cuboid } 2 & 2 & 36.404 & 0.0 & 9.64 & 0.0 & 88.96 & -2.54\end{array}$

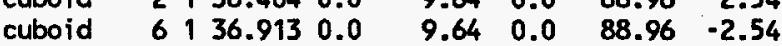

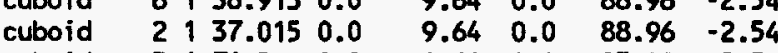

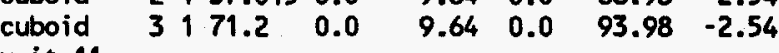

unit 11

array $3 * 0.0$

unit 12

array $43 * 0.0$

global unit 13

array $5 \quad 3 * 0.0$

reflector $415 r 0.0 \quad 2.54$

reflector $314 r 30.0 \quad 2 r 15.0$

end geometry

read array

ara=1 nux=11 nuy=14 nuz=1 fill f 1 end fill

ar $a=2$ nux $=11$ nuy=16 nuz=1 fill f1

ara $=3$ end fill

ara=3 nux=5 nuy=1 nuz=1 fill $25343 \quad 3 \quad$ end fill ara $=4$ nux $=5$ nuy=1 nuz=1 fill 79897 end fill ara=5 nux=1 nuy=5 nuz=1 fill 11561210 end fill

end array

end data

end 
44group latticecell

$402 \quad 10.949$

0.949293922354 .319223895 .69

21.0

$20 \quad 31.0$

plexiglas 41.0

arbmrubber 1.3216000601258 .10016 .52000011 .4 160001.7801622 .1140000 .35

b $\quad 6$ den=2.47 $\quad .3188$

c $\quad 6$ den $=2.47 \quad .0886$

al $\quad 6$ den=2.47 $\quad .5926$

fe $\quad 6$ den=2.47 .0005

mg 6 den=2.47 .0001

na 6 den $=2.47 \quad .0002$

end comp

6 den $=2.47 \quad .0006$

squarepitch 1.8921 .2649131 .414721 .28270

$2-11 \times 14$ and $2-11 \times 14$ assemblies separated by $0.231-\mathrm{cm}$ - thick Boral-c plates and 2.599- and 3.299- $\mathrm{cm}$ of water

read parm run=yes gen $=405$ npg $=600$ nsk $=5$ nub=yes end parm

read geom

unit 1

cylinder 110.63245

cylinder 010.64135

cylinder 510.64135

cylinder 2110.70735

cuboid $314 \mathrm{p} .9460$

end

end

end

end

end

end

end

end

end

unit 2

$\begin{array}{llllllll}3 & 1 & 13.373 & 0.0 & 26.488 & 0.0 & 93.98 & -2.54\end{array}$

unit 3

array $13 * 0.0$

unit 4

cuboid $\quad 612.805 \quad 2.62426 .488 \quad 0.0 \quad 88.96 \quad-2.54$

$\begin{array}{lllllllll}\text { cuboid } 2 & 1 & 2.83 & 2.599 & 26.488 & 0.0 & 88.96 & -2.54\end{array}$

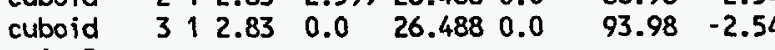

unit 5

cuboid $\quad \begin{array}{llllllll}6 & 1 & 71.2 & 0.0 & 0.206 & 0.025 & 88.96 & -2.54\end{array}$

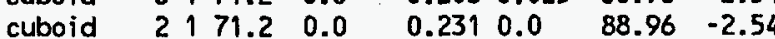

$\begin{array}{lllllllll}\text { cuboid } & 3 & 1 & 71.2 & 0.0 & 0.231 & 0.0 & 93.98 & -2.54\end{array}$

unit 6

$\begin{array}{lllllllll}\text { cuboid } \quad 3 & 1 & 36.784 & 0.0 & 3.299 & 0.0 & 88.96 & -2.54\end{array}$

$\begin{array}{lllllllll}\text { cuboid } \quad 2 & 1 & 36.809 & 0.0 & 3.299 & 0.0 & 88.96 & -2.54\end{array}$

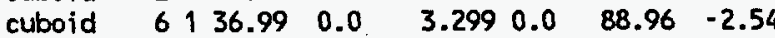

cuboid $2137.0150 .0 \quad 3.299 \quad 0.0 \quad 88.96 \quad-2.54$

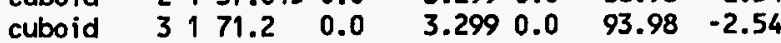

unit 7

array $23 * 0.0$ unit 8
cuboid

$\begin{array}{lllll} & 0.98 & -2.54\end{array}$

$\begin{array}{lllllllll}\text { cuboid } 6 & 1 & 36.99 & 0.0 & 14.694 & 0.0 & 88.96 & -2.54\end{array}$

$\begin{array}{lllllllll}\text { cuboid } \quad 2 & 137.015 & 0.0 & 14.694 & 0.0 & 88.96 & -2.54\end{array}$

$\begin{array}{lllllllll}\text { cuboid } \quad 3171.2 & 0.0 & 14.694 & 0.0 & 93.98 & -2.54\end{array}$

global unit 9

array $3 \quad 3 * 0.0$

reflector $415 r 0.0 \quad 2.54 \quad 1$

reflector $314 r 30.0 \quad 2 r 15.01$

end geometry

read array

ara $=1$ nux $=11$ nuy $=14$ nuz $=1$ fill $f 1$

end fill

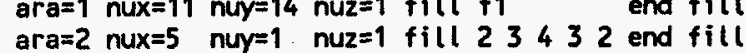

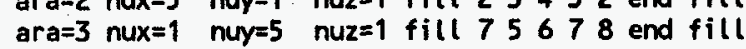
end array

end data

end

\section{p3314bf1}

$=\operatorname{csas} 25$

p3314bf1

44 group latticecell

vo2 10.949293922354 .319223895 .69 end

21.0

h2o 31 .0

plexiglas 41.0

arbmrubber 1.3216000601258 .10016 .52000011 .4

160001.7801622 .1140000 .3

boron 6 den=1.731.3274

c $\quad 6$ den=1.731 .2113

cr $\quad 6$ den $=1.731 .0265$

fe $\quad 6$ den $=1.731 .0005$

6 den=1.731.2101

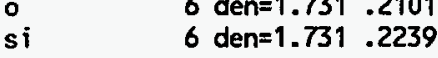

end comp

$2-11 \times 14$ and $2-11 \times 14$ assemblies separated by $0.546-\mathrm{cm}-$ thick Boroflex plates and 2.284- and $3.054-\mathrm{cm}$ of water

read parm run=yes gen $=405$ npg $=600$ nsk $=5$ nub=yes end parm

read geom

unit 1

cylinder 1110.63245

cylinder 0110.64135

cylinder 510.64135

cylinder 210.70735

cuboid $314 \mathrm{p} .9460$ 
unit 2

array $13 * 0.0$

unit 3

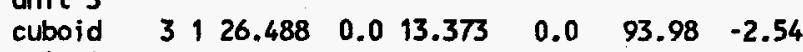

unit 4

$\begin{array}{lllllllll}\text { cuboid } & 6 & 1 & 26.488 & 0.0 & 2.67 & 2.444 & 88.96 & -2.54\end{array}$ $\begin{array}{lllllllll}\text { cuboid } & 4 & 1 & 26.488 & 0.0 & 2.83 & 2.284 & 88.96 & -2.54\end{array}$

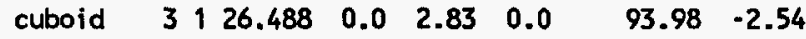

unit 5

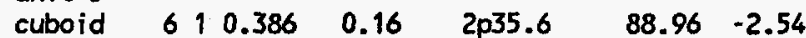
$\begin{array}{lllllll}\text { cuboid } 4 & 4 & 0.546 & 0.0 & 2 p 35.6 & 88.96 & -2.54\end{array}$

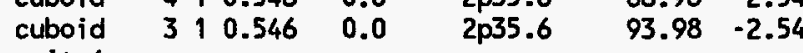

unit 6

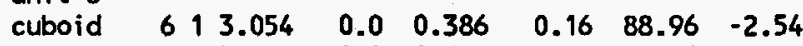

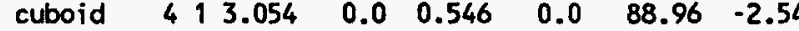
$\begin{array}{lllllllll}\text { cuboid } & 3 & 1 & 3.054 & 0.0 & 34.731 & -36.469 & 93.98 & -2.54\end{array}$

unit 7

cuboid $\quad \begin{array}{llllllll}6 & 1 & 14.624 & 0.0 & 0.386 & 0.16 & 88.96 & -2.54\end{array}$ $\begin{array}{llllllll}\text { cuboid } \quad 4 & 1 & 14.624 & 0.0 & 0.546 & 0.0 & 88.96 & -2.54\end{array}$ $\begin{array}{llllllll}\text { cuboid } 31 & 314.624 & 0.0 & 34.731 & -36.469 & 93.98 & -2.54\end{array}$

array $23 * 0.0$

global unit 9

array $3 \quad 3 * 0.0$

ป

reflector $495 r 0.0 \quad 2.54$,

reflector $314 \mathrm{r30.0} 2 \mathrm{r} 15.01$

end geometry

\section{read array}

ara=1 nux=14 nuy=11 nuz=1 fill fl end fill

ar $a=2$ nux $=1$ nuy $=5$ nuz=1 fill $3 \quad 2 \quad 4 \quad 2 \quad 3$ end fill

ara $=3$ nux $=5$ nuy $=1$ nuz=1 fill $8 \quad 5 \quad 6 \quad 8 \quad 7$ end fill

end array

end data

end

\section{p3314bf2}

\section{$=\operatorname{csas} 25$}

\section{p3314bf2}

44 group latticecel 1

10.949293922354 .319223895 .69

h2o 31.0

$\begin{array}{lllll} & \end{array}$

$2-11 \times 14$ and $2-11 \times 16$ assemblies separated by $0.772-\mathrm{cm}-$ thick Boroflex plates and 2.058- and $4.168 \mathrm{-cm}$ of water

read parm run=yes gen $=405$ npg $=600$ nsk $=5$ nub=yes end parm

\section{read geom}

unit 1

cyl inder 110.63245

cylinder 010.64135

cylinder 510.64135

cylinder 210.70735

cuboid $3 \uparrow 4 \mathrm{p} .9460$

unit

array $13 * 0.0$

unit 3

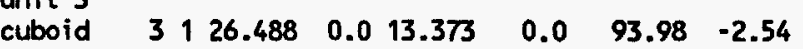

unit 4

cuboid $\quad \begin{array}{llllllll}6 & 1 & 26.488 & 0.0 & 2.67 & 2.218 & 88.96 & -2.54\end{array}$

$\begin{array}{lllllllll}\text { cuboid } \quad 4 & 1 & 26.488 & 0.0 & 2.83 & 2.058 & 88.96 & -2.54\end{array}$

$\begin{array}{llllllll}\text { cuboid } 3 & 3 & 26.488 & 0.0 & 2.83 & 0.0 & 93.98 & -2.54\end{array}$

unit 5

array $23 * 0.0$

unit 6

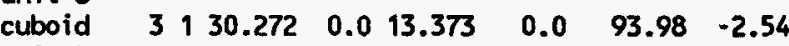

unit 7

cuboid $\quad \begin{array}{llllllll}6 & 1 & 30.272 & 0.0 & 2.67 & 2.218 & 88.96 & -2.54\end{array}$

$\begin{array}{llllllll}\text { cuboid } \quad 4 & 4 & 30.272 & 0.0 & 2.83 & 2.058 & 88.96 & -2.54\end{array}$

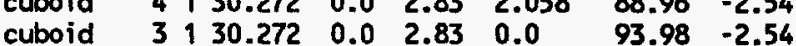

unit 8

$93.98-2.54$

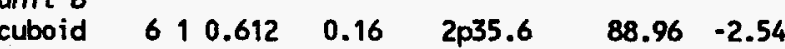

cuboid $\begin{array}{lllllll}4 & 1 & 0.772 & 0.0 & 2 p 35.6 & 88.96 & -2.54\end{array}$

$\begin{array}{llllllll}\text { cuboid } & 3 & 1 & 0.772 & 0.0 & 2 p 35.6 & 93.98 & -2.54\end{array}$

unit 9

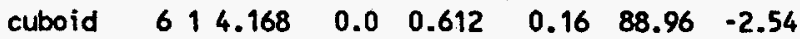

$\begin{array}{llllllll}\text { cuboid } 4 & 4 & 4.168 & 0.0 & 0.772 & 0.0 & 88.96 & -2.54\end{array}$

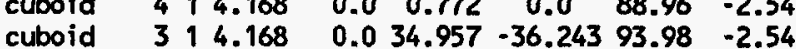

$\begin{array}{lllllllll}\text { cuboid } & 3 & 1 & 4.168 & 0.0 & 34.957 & -36.243 & 93.98 & -2.54\end{array}$

$\begin{array}{llllllll}\text { cuboid } 6 & 19.500 & 0.0 & 0.612 & 0.16 & 88.96 & -2.54\end{array}$

$\begin{array}{llllllll}\text { cuboid } \quad 4 & 4 & 9.500 & 0.0 & 0.772 & 0.0 & 88.96 & -2.54\end{array}$

$\begin{array}{llllllll}\text { cuboid } \quad 319.500 & 0.0 & 34.957 & -36.243 & 93.98 & -2.54\end{array}$

unit 11

array $33 * 0.0$

unit 12

array $43^{*} 0.0$

global unit 13

array $5 \quad 3 * 0.0$

reflector $\quad 415 r 0.0 \quad 2.54 \quad 1$ 


\section{reflector $\quad 314 r 30.0 \quad 2 r 15.01$} end geometry

\section{read array}

$\begin{array}{ll}\text { ara }=1 \text { nux }=14 \text { nuy=11 nuz=1 fill f } 1 & \text { end fill } \\ \text { ara } a=2 \text { nux }=16 \text { nuy=11 nuz=1 fill f1 } & \text { end fill }\end{array}$ ara $=2$ nux $=16$ nuy=11 nuz=1 fill fi

ara=3 nux=1 nuy $=5$ nuz=1 fill $3 \begin{array}{lllllll}3 & 2 & 4 & 2 & 3\end{array}$ end fill ara $=4$ nux=1 nuy=5 nuz=1 fill 65756 and fill ara $=5$ nux $=5$ nuy=1 nuz=1 fill 11891210 end fill end array

end data

end

\section{p3314bs1}

\section{=csas 25}

p3314bs 1

44group latticecel

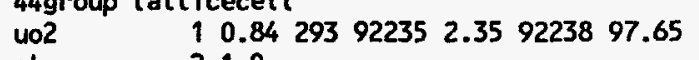

al $\quad 21.0$

h20 $\quad 31.0$

plexiglas 41.0

cr $\quad 5$ den=7.90 .1903

b $\quad 5$ den=7.90 .0105

$\underset{\infty}{\infty}$

ni $\quad 5$ den=7.90 .0953

m $\quad 5$ den=7.90 .0158

cu $\quad 5$ den $=7.90 .0028$

mo

5 den $=7.90 \quad .0049$

end comp

squarepitch 1.6841 .1176131 .272 end geometry

$2-17 \times 20$ and $1-25 \times 20$ (center) assembl ies separated by $0.298-\mathrm{cm}-\mathrm{thick}$

$-1.1 \mathrm{wt} \% \mathrm{~B}$ stainless steel plates and $3.562 \mathrm{~cm}$ of water

read parm run=yes gen $=405 \mathrm{npg}=600 \mathrm{nsk}=5$ nub=yes end parm

\section{read geom}

unit 1

cylinder 1110.5588

cylinder 210.635

cuboid $314 \mathrm{p0} 0.842$

$91.44 \quad 0.0$

$96.52-1.27$

unit 2

array $13 * 0.0$

reflector $312 \mathrm{ro} \quad 1.920 .02 \mathrm{ro} \quad 1$

unit 3

array $23 * 0.0$

reflector $312 r 0 \quad 1.92 \quad 0.02 r 0 \quad 1$

unit 4

cuboid $51 \quad 0.0 \quad-0.298 \quad 35.6 \quad 0.0$

$\begin{array}{lllllllll}\text { cuboid } & 5 & 1 & 0.0 & -0.298 & 35.6 & 0.0 & 90.23 & -1.27 \\ \text { cuboid } & 3 & 1 & 0.0 & -3.86 & 35.6 & 0.0 & 96.52 & -1.27\end{array}$

unit 5

unit 5

$\begin{array}{llllll}51 & 0.298 & 0.0 & 35.6 & 0.0\end{array}$

$90.23-1.27$

$\begin{array}{lllllllll}\text { cuboid } & 3 & 1 & 3.86 & 0.0 & 35.6 & 0.0 & 96.52 & -1.27\end{array}$

global unit 9

$\begin{array}{llll}\text { array } 3 & -53.538 & -17.8 & 0 .\end{array}$

reflector $415 \mathrm{r} 0.0 \quad 2.54 \quad 1$

reflector $314 r 30.0 \quad 2 r 15.01$

end geometry

\section{read array}

ara $=1$ nux $=25$ nuy $=20$ nuz $=1$ fill $f 1$ end fill

ara $=2$ nux $=17$ nuy $=20$ nuz $=1$ fill $f 1 \quad$ end fill

ara $=3$ nux $=5$ nuy=1 nuz=1 fill 344253 end fill

end array

end data

end

\section{p3314bs2}

$=\operatorname{csas} 25$

p3314bs2

44group latticecell

uo2 $10.84293922352 .359223897 .65 \quad$

al 21.0

h20 31.0

plexiglas 41.0

cr $\quad 5$ den $=7.77 .1960$

b $\quad 5$ den=7.77.0162

fe $\quad 5$ den=7.77 .6640

ni $\quad 5$ den=7.77 .1012

mn $\quad 5$ den=7.77.0169

cu $\quad 5$ den $=7.77 .0026$

mo $\quad 5$ den=7.77 .0031

end comp

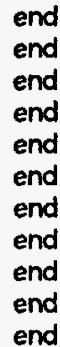

squarepitch 1.6841 .1176131 .272 end geometry

$2-17 \times 20$ and $1-25 \times 20$ (center) assemblies separated by $0.298-\mathrm{cm}-$ thick $1.1 \mathrm{wt \%}$ B stainless steel plates and $3.162 \mathrm{~cm}$ of water

read parm run=yes gen $=405$ npg $=600$ nsk $=5$ nub=yes end parm

read geom

unit 1

cyl inder 1110.5588

cyl inder 210.635

cuboid $314 \mathrm{p0} .842$

unit 2

array $13 * 0.0$

reflector $312 \mathrm{ro} \quad 1.92 \quad 0.0 \quad 2 \mathrm{r} 0 \quad 1$

unit 3

array $23 * 0.0$

reflector $312 \mathrm{r} 0 \quad 1.920 .02 \mathrm{r} 0 \quad 1$

unit 4

$\begin{array}{lllllllll}\text { cuboid } & 5 & 1 & 0.0 & -0.298 & 35.6 & 0.0 & 90.23 & -1.27\end{array}$ 
unit 5

cuboid $\quad \begin{array}{lllllllll}5 & 1 & 0.298 & 0.0 & 35.6 & 0.0 & 90.23 & -1.27\end{array}$

$\begin{array}{lllllllll}\text { cuboid } & 3 & 1 & 3.46 & 0.0 & 35.6 & 0.0 & 96.52 & -1.27\end{array}$

global unit 9

$\begin{array}{llll}\text { array } 3 & -53.138 & -17.8 & 0 .\end{array}$

reflector $\quad 415 r 0.0 \quad 2.54 \quad 1$

reflector $314 \mathrm{r30.0} 2 \mathrm{r} 15.01$

end geometry

\section{read array}

ara $=1$ nux $=25$ nuy=20 nuz=1 fill $f 1$ end fill ara=2 nux=17 nuy=20 nuz=1 fill f 1 end fill ara=3 nux=5 nuy=1 nuz=1 fill 342553 end fill end array

end data

end

\section{D3314bs3}

$=\operatorname{csas} 25$

p3314bs 3

44 group latticecel

uo2 10.949293922354 .319223895 .69 end

al 21.0

h20 31.0

plexiglas 41.0

cr 5 den $=7.90 .1903$

b $\quad 5$ den=7.90.0105

fe $\quad 5$ den=7.90.6804

ni $\quad 5$ den $=7.90 .0953$

$\mathrm{mn} \quad 5$ den=7.90 .0158

$\mathrm{cu} \quad 5$ den=7.90.0028

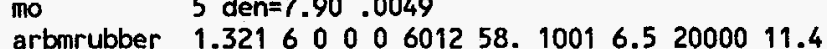

1.3216000601258

end comp

160001.7801622 .1140000 .36

squarepitch $1.892 \quad 1.2649 \quad 131.41472 \quad 1.28270$ end 3-12x16 assemblies separated by $0.298-\mathrm{cm}-$ thick $1.1 \mathrm{wt} \%$ B stainless steel plates and $6.932 \mathrm{~cm}$ of water

read parm run=yes gen $=405$ npg $=600$ nsk $=5$ nub=yes end parm

\section{read geom}

\section{unit 1}

cylinder 1110.63245

cylinder 0110.64135

cylinder 610.64135

cylinder 210.70735

cylinder 210.70735

cuboid

array $13 * 0$.

$\begin{array}{llllll}\text { reflector } & 3 & 2 \mathrm{rO} & 2 \mathrm{r} 2.664 & 2 \mathrm{rO} & 1\end{array}$ unit 3

cuboid

$\begin{array}{lllll}5 & 1 & 0.0 & -0.298 & 2 p 17.8\end{array}$

$88.96-2.54$

cuboid

unit 4

$93.98-2.54$

$\begin{array}{llllllll}5 & 1 & 0.298 & 0.0 & 2 p 17.8 & 88.96 & -2.54\end{array}$

$\begin{array}{lllllll}\text { cuboid } 3 & 1 & 07.23 & 0.0 & 2 p 17.8 & 93.98 & -2.54\end{array}$

global unit 5

$\begin{array}{llll}\text { array } 2 & -41.286 & -17.8 & 0 .\end{array}$

reflector $\quad 415 \mathrm{r} 0.0 \quad 2.54 \quad 1$

reflector $314 \mathrm{r} 30.0 \quad 2 \mathrm{r} 15.0$

end geometry

read array

ara $a=1$ nux $=12$ nuy=16 nuz $=1$ fill $f 1$ end $f i l l$

ara $=2$ nux $=5$ nuy $=1$ nuz=1 fill $23 \quad 3 \quad 242$ end fill

end array

end data

end

\section{p3314bs4}

\section{$=\operatorname{csas} 25$}

p3314bs4

44group latticecell

uo2 10.949293922354 .319223895 .69

al 21.0

h2o $\quad 31.0$

plexiglas 41.0

cr 5 den $=7.77 .1960$

b $\quad 5$ den=7.77.0162

fe $\quad 5$ den $=7.77 .6640$

ni $\quad 5$ den=7.77 .1012

ni 5 den=7.77.0169

min $\quad 5$ den=7.77.0169

mo $\quad 5$ den=7.77.0031

arbmrubber 1.32160000601258 .10016 .52000011 .4 160001.7801622 .1140000 .36

end comp

squarepitch $1.892 \quad 1.2649131 .414721 .28270$

3-12×16 assemblies separated by $0.298-\mathrm{cm}-$ thick $1.6 \mathrm{wt} \%$ B stainless steel plates and $6.332 \mathrm{~cm}$ of water

read parm run=yes gen $=405$ npg $=600$ nsk $=5$ nub=yes end parm

\section{read geom}

unit 1

$\begin{array}{llllll}\text { cyl inder } & 1 & 1 & 0.63245 & 91.44 & 0.0\end{array}$

cylinder $010.64135 \quad 91.44 \quad 0.0$

cylinder $610.64135 \quad 93.98 \quad-2.54$

cylinder $210.70735 \quad 93.98-2.54$

$\begin{array}{llll}\text { cuboid } 314 \mathrm{p0.946} & 93.98 & -2.54\end{array}$

$\begin{array}{ll}93.98 & -2.54 \\ 93.98 & -2.54\end{array}$

unit 2 
$\begin{array}{llll}\text { array } 2 & -40.686 & -17.8 & 0 .\end{array}$

$\begin{array}{llllll}\text { reflector } & 4 & 9 & 5 r 0.0 & 2.54 & 1 \\ \text { reflector } & 3 & 1 & 4 \mathrm{r} 30.0 & 2.15 .0 & 1\end{array}$

end geometry

read array

ara $=1$ nux $=12$ nuy $=16$ nuz $=1$ fill $f 1$ end $f i l l$

ara $=2$ nux $=5$ nuy $=1$ nuz=1 fill $2 \quad 3 \quad 2 \quad 4 \quad 2$ end fill

end array

end data

end

\section{p3314cd1}

$=\operatorname{csas} 25$

p3314cd1

44 group latticecell

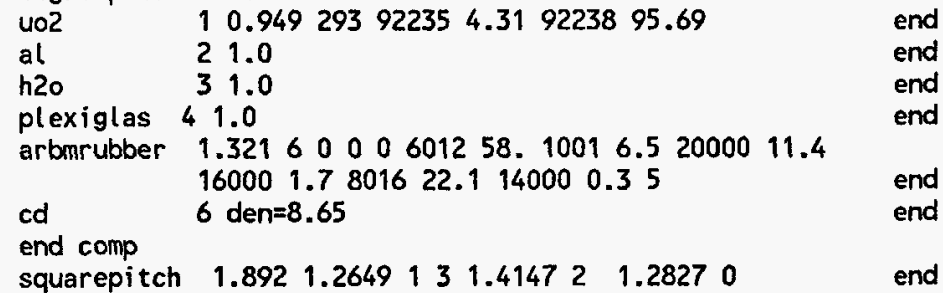

end

end

end

end

end

$2-11 \times 14$ and $2.11 \times 13$ assemblies separated by $0.061546-\mathrm{cm}-$ thick cadmium plates and $2.769-$ and $5.239-\mathrm{cm}$ of water

read parm run=yes gen $=405 \mathrm{npg}=600 \mathrm{nsk}=5$ nub=yes end parm

read geom

unit 1

cylinder 110.63245

cylinder 010.64135

cylinder 510.64135

cylinder 210.70735

cuboid $314 \mathrm{p} .9460$

unit 2

$\begin{array}{llllllll}3 & 1 & 13.373 & 0.0 & 26.488 & 0.0 & 93.98 & -2.54\end{array}$

unit 3

array $13 * 0.0$

unit 4

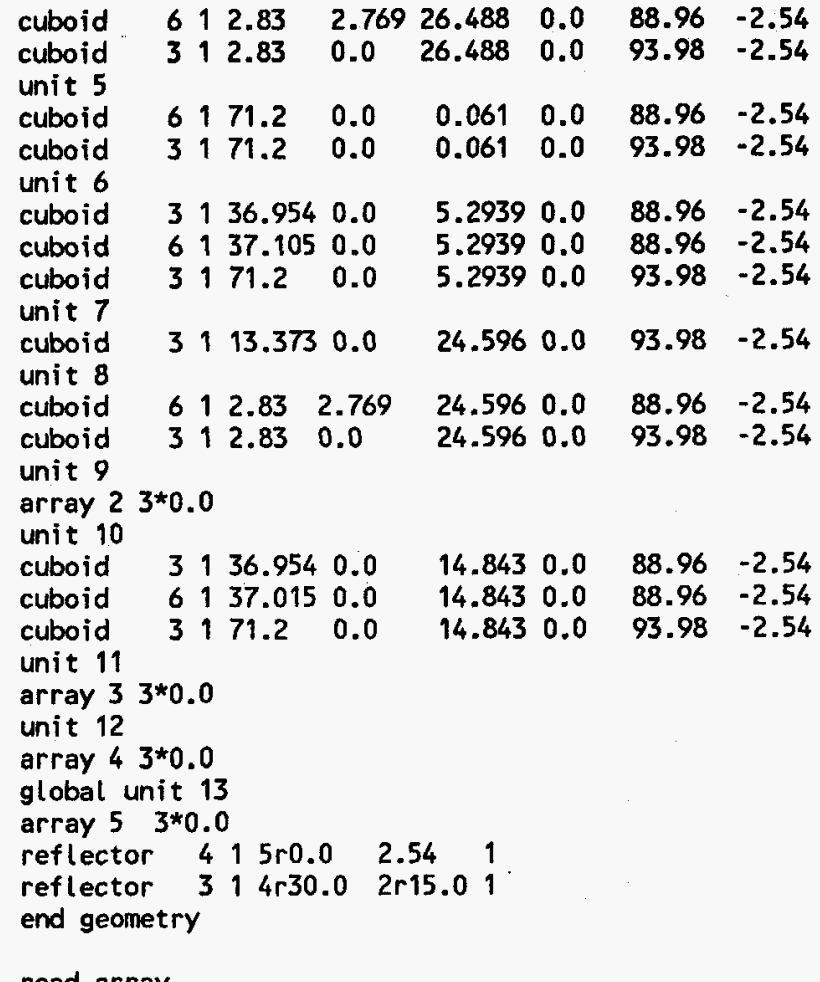

read array

ara $=1$ nux $=11$ nuy=14 nuz=1 fill $f 1$ end fill ara $=2$ nux $=11$ nuy $=13$ nuz=1 fill $f 1$ end fill

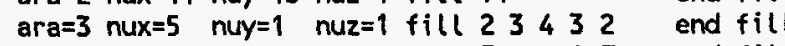
ara $=4$ nux $=5$ nuy $=1$ nuz=1 fill 79897 end fill ara $=5$ nux=1 nuy=5 nuz=1 fill $11 \quad 5 \quad 6 \quad 12 \quad 10$ end fill end array

end data

end

\section{p3314cd?}

$=\operatorname{csas} 25$

p3314cd2

44 group latticecell

$\begin{array}{lllll}\text { 44group latticecell } & & & \\ \text { uo2 } & 0.84 & 29392235 & 2.359223897 .65\end{array}$

$\begin{array}{lll}\text { uor } & 1 & 0.84 \\ \text { al } & 2 & 1.0\end{array}$

$\begin{array}{lll}\text { al } & 2 & 1.0 \\ \text { h20 } & 3 & 1.0\end{array}$

plexiglas 41.0

cd $\quad 5$ den $=8.65$

end comp end
end
end
end
end 
squarepitch $1.6841 .1176,31.272$ end geometry

$2-17 \times 20$ and $1-25 \times 20$ (center) assemblies separated by $0.061-\mathrm{cm}-$ thick cadmium plates and $2.979 \mathrm{~cm}$ of water

read parm run=yes gen=405 npg=600 nsk=5 nub=yes end parm

\section{read geom}

unit

cyl inder 110.5588

cyl inder 210.635

cuboid $314 \mathrm{p} 0.842$

$91.44 \quad 0.0$

$96.52-1.27$

unit 2

$96.52-1.27$

array $13 * 0.0$

reflector 312 ro 1.920 .02 ro 1

unit 3

array $23 * 0.0$

reflector 312 ro 1.920 .02 ro 1

unit 4

cuboid $\quad \begin{array}{lllllll}5 & 1 & 0.0 & -0.061 & 35.6 & 0.0\end{array}$

$\begin{array}{llllllll}\text { cuboid } & 3 & 1 & 0.0 & -3.04 & 35.6 & 0.0\end{array}$

unit 5

$\begin{array}{llllll}3 & 1 & 0.0 & -3.04 & 35.6 & 0.0\end{array}$

$90.23-1.27$

$96.52-1.27$

$\begin{array}{lllllllll}\text { cuboid } & 5 & 1 & 0.061 & 0.0 & 35.6 & 0.0 & 90.23 & -1.27\end{array}$

$\begin{array}{lllllllll}\text { cuboid } & 3 & 1 & 3.04 & 0.0 & 35.6 & 0.0 & 96.52 & -1.27\end{array}$

global unit 9

$\begin{array}{llll}\text { array } 3 & -52.718 & -17.8 & 0 .\end{array}$

reflector $\quad 415 \mathrm{r}_{0.0} \quad 2.54 \quad 1$

reflector $314 \mathrm{r} 30.0$ 2r15.01

end geometry

read array

ara=1 nux=25 nuy=20 nuz=1 fill $f 1$ end fill

ara=2 nux=17 nuy=20 nuz=1 fill $f 1$ end fill

ara=3 nux $=5$ nuy=1 nuz=1 fill 342253 end fill

end array

end data

end

\section{p3314cu1}

$=\operatorname{csas} 25$

p3314cu1

44group latticecell
10.949293922354 .319223895 .69

21.0

h2o

31.0

artmrubber $1.32160000601258,10016.520000 \quad 11.4$

$160001.7801622 .114000 \quad 0.35$

6 den $=8.913$

end comp

squarepitch 1.8921 .2649131 .414721 .28270 plates and 2.493- and 5.603-cm of water

read parm run=yes gen $=405 \mathrm{npg}=600$ nsk $=5$ nub=yes end parm

\section{read geom}

unit 1

cylinder 1110.63245

cylinder 010.6413

cylinder 510.64135

$\begin{array}{llll}\text { cylinder } & 2 & 1 & 0.70735 \\ \text { cuboid } & 3 & 1 & 4 p .9460\end{array}$

$\begin{array}{lll}93.98 & -2.54\end{array}$

$\begin{array}{llllllll}\text { cuboid } & 3,12.157 & 0.0 & 22.704 & 0.0 & 93.98 & -2.54\end{array}$

unit 3

unit 4

$\begin{array}{lllllllll}6 & 1 & 2.83 & 2.493 & 22.704 & 0.0 & 88.96 & -2.54\end{array}$

cuboid

$-2.54$

$\begin{array}{llllllll} & & \end{array}$

$\begin{array}{lllllllll}\text { cuboid } & 3 & 1 & 61.2 & 0.0 & 0.337 & 0.0 & 93.98 & -2.54\end{array}$

$\begin{array}{lllllllll}\text { cuboid } & 3 & 1 & 31.678 & 0.0 & 5.603 & 0.0 & 88.96 & -2.54\end{array}$

$\begin{array}{lllllllll}\text { cuboid } & 6 & 1 & 32.015 & 0.0 & 5.603 & 0.0 & 88.96 & -2.54\end{array}$

$\begin{array}{llllllll}\text { cuboid } 3161.2 & 0.0 & 5.603 & 0.0 & 93.98 & -2.54\end{array}$

$\begin{array}{llllllllll}3 & 1 & 12.157 & 0.0 & 13.244 & 0.0 & 93.98 & -2.54\end{array}$

$\begin{array}{llllllllll}\text { cuboid } & 6 & 1 & 2.83 & 2.493 & 13.244 & 0.0 & 88.96 & -2.54\end{array}$

$\begin{array}{lllllllll}\text { cuboid } & 3 & 1 & 2.83 & 0.0 & 13.244 & 0.0 & 93.98 & -2.54\end{array}$

unit 9

array $23 * 0.0$

$\begin{array}{llllllllll}\text { unit } 10 & & & & & & & & & \end{array}$

$\begin{array}{lllllllll}\text { cuboid } & 3 & 1 & 31.678 & 0.0 & 19.312 & 0.0 & 88.96 & -2.54 \\ \text { cuboid } & 6 & 1 & 32.015 & 0.0 & 19.312 & 0.0 & 88.96 & -2.54\end{array}$

$\begin{array}{lllllllll}\text { cuboid } & 6 & 1 & 32.015 & 0.0 & 19.312 & 0.0 & 88.96 & -2.54 \\ \text { cuboid } & 3 & 1 & 61.2 & 0.0 & 19.312 & 0.0 & 93.98 & -2.54\end{array}$

unit 11

array $33^{*} 0.0$

unit 12

array $43 * 0.0$

glabal unit 13

global unit 13

reflector $\quad 415 r 0.0 \quad 2.54$

reflector 314 r30.0 2 r15.0

end geometry

read array

ara=1 nux $=9$ nuy=12 nuz=1 fill $f 1$ end $f i l l$

ara $=2$ nux=9 nuy=7 nuz=1 fill $f 1 \quad$ end fill

ar $a=3$ nux $=5$ nuy=1 nuz=1 fill $2 \quad 34332$ end fill

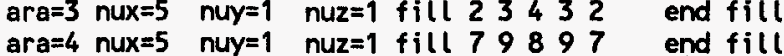

ara=5 nux=1 nuy=5 nuz=1 fill $115 \quad 5 \quad 12 \quad 10$ end fill 


\section{p3314cu2}

$=\operatorname{csas} 25$

$3314 \mathrm{cu} 2$

44 group latticecell

uo2 10.949293922354 .319223895 .69 end

al 21.0

h2o

$$
31.0
$$

plexiglas 41.0

arbmrubber $1.32160000601258,10016.52000011 .4$ 160001.7801622 .1140000 .35 end

end comp

$$
6 \text { den }=8.913
$$

ditch 1.8921 .2649131 .414721 .28270

$2-9 \times 12$ and $2-9 \times 5$ assemblies separated by $0.646-\mathrm{cm}-$ thick copper

"plates and 2.184- and 2.024- $\mathrm{cm}$ of water

read parm run=yes gen $=405 \mathrm{npg}=600 \mathrm{nsk}=5$ nub=yes end parm

read geom

unit 1

cylinder 110.6324

cylinder 010.64135

cylinder 510.64135

cylinder 210.70735

cuboid $314 \mathrm{p} .9460$

unit 2

314 p. 9460

cuboid

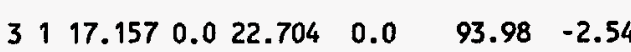

arit 3

unit 4

$\begin{array}{lllllllll} & 6 & 12.83 & 2.184 & 22.704 & 0.0 & 88.96 & -2.54\end{array}$

$\begin{array}{lllllllll}\text { cuboid } & 3 & 1 & 2.83 & 0.0 & 22.704 & 0.0 & 93.98 & -2.54\end{array}$

$\begin{array}{llll}6171.2 & 0.0 & 0.646 & 0.0\end{array}$

$88.96-2.54$

cuboid

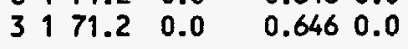

$93.98-2.54$

unit 6

$\begin{array}{llllllll}6 & 1 & 37.015 & 0.0 & 2.024 & 0.0 & 88.96 & -2.54\end{array}$

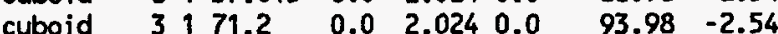

unit 7

cuboid

$\begin{array}{llllll}3 & 1 & 17.157 & 0.0 & 9.46 & 0.0\end{array}$

$93.98-2.54$

cuboid $\quad \begin{array}{llllllll}6 & 1 & 2.83 & 2.184 & 9.46 & 0.0 & 88.96 & -2.54\end{array}$

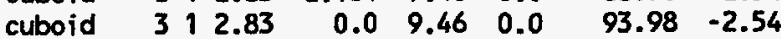

unit 9

array $23 * 0.0$

unit 10 cuboi

array 3 3*0.0

unit 12

array $43 * 0.0$

global unit 13

array $5 \quad 3 * 0.0$

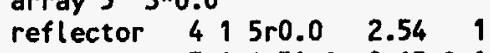

reflector $314 \mathrm{r} 30.0 \quad 2 \mathrm{r} 15.01$

end geometry

read array

ara $=1$ nux $=9$ nuy=12 nuz=1 fill $f 1$ end fill

ara $=2$ nux $=9$ nuy $=5$ nuz=1 fill $f 1$ end fill

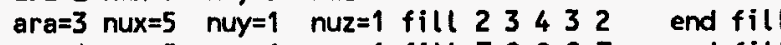
ara $=4$ nux $=5$ nuy $=1$ nuz $=1$ fill 79897 end fill ara $=5$ nux $=1$ nuy=5 nuz=1 fill $1156 \quad 12 \quad 10$ end fill

end array

end data

end

\section{4cu3}

$=\operatorname{csas} 25$

p3314cu3

44group latticecell

al 21.0

h20 $\quad 31.0$

plexiglas 41.0

5 den $=8.913$

arbmrubber 1.3216000601258 .10016 .52000011 .4

160001.7801622 .1140000 .36

end comp

squarepitch 1.8921 .2649131 .414721 .28270 end

$3-12 \times 16$ assemblies separated by $0.337-\mathrm{cm}-\mathrm{thick}$ copper plates and $-10.023 \mathrm{~cm}$ of water

read parm run=yes gen $=405$ npg $=600$ nsk $=5$ nub=yes end parm

read geom

unit 1

cylinder 110.63245

cylinder 010.64135

cyl inder 610.64135

cyl inder 210.70735

cuboid $314 \mathrm{p} .946$

$91.44 \quad 0.0$

$91.44 \quad 0.0$

$93.98-2.54$

$93.98-2.54$

unit 2

$93.98 \quad-2.54$

array $13 * 0.0$

reflector 312 ro 2ro.164 2ro

unit 3 


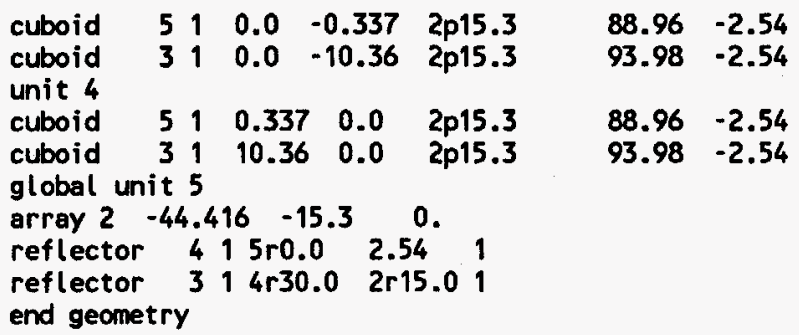

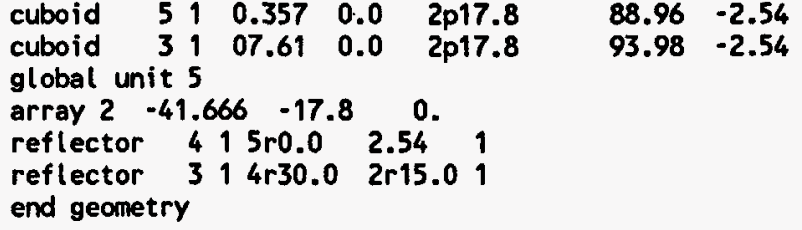

p3314cus

$=\operatorname{csas} 25$

p3314cu5

44 group latticecelt

$\begin{array}{llllll}\text { uo2 } & 1 & 0.84 & 29392235 & 2.359223897 .65\end{array}$

al 21.0

h20 $\quad 31.0$

plexiglas 41.0

cu 5 den $=8.913$

end comp

squarepitch 1.6841 .1176131 .272 end geometry

$2-20 \times 18$ and $1-25 \times 18$ (center) assemblies separated by $0.337-\mathrm{cm}-$ thick copper plates and $4.903 \mathrm{~cm}$ of water

read parm run=yes gen $=405$ npg $=600$ nsk $=5$ nub=yes end parm

read geom

unit 1

cyl inder 1110.5588

cyl inder 210.635

cuboid $314 \mathrm{p0} .842$

unit 2

0.0

$96.52-1.27$

array $13 * 0.0$

$\begin{array}{llllllll} & & & & & \end{array}$

unit 3

array $23 * 0.0$

reflector $\quad 312$ ro $\quad 0.288 \quad 0.02$ ro $\quad 1$

unit 4

$\begin{array}{lllllllll}\text { cuboid } & 5 & 1 & 0.0 & -0.337 & 30.6 & 0.0 & 90.23 & -1.27\end{array}$

$\begin{array}{llllllllll}\text { cuboid } & 3 & 1 & 0.0 & -5.24 & 30.6 & 0.0 & 96.52 & -1.27\end{array}$

unit 5

$\begin{array}{lllllllll}\text { cuboid } & 5 & 1 & 0.337 & 0.0 & 30.6 & 0.0 & 90.23 & -1.27\end{array}$

$\begin{array}{lllllllll}\text { cuboid } & 3 & 1 & 5.24 & 0.0 & 30.6 & 0.0 & 96.52 & -1.27\end{array}$

global unit 9

$\begin{array}{llll}\text { array } 3 & -59.97 & -15.3 & 0 .\end{array}$ 
reflector $314 r 30.0 \quad 2 r 15.01$

end geometry

read array

ar $a=1$ nux $=25$ nuy=18 nuz=1 fill $f 1$ end fill

ara=2 nux=20 nuy=18 nuz=1 fill $f 1$ end fill

ara=3 nux=5 nuy=1 nuz=1 fill 342553 end fill

end array

end data

end

\section{D3314cus}

$=\operatorname{csas} 25$

p3314cu6

44 group latticecell

$\begin{array}{llllll}\text { uo2 } & 0.84 & 29392235 & 2.3592238 & 97.65\end{array}$

end

al 21.0

h20 31.0

plexiglas 41.0

cu $\quad 5$ den $=8.91 .99$

$\begin{array}{ll}\mathrm{cu} & 5 \text { den }=8.91 \\ \mathrm{~cd} & 5 \text { den }=8.91 .01\end{array}$

end comp

squarepitch 1.6841 .1176131 .272 end geometry

$2-20 \times 18$ and $1-25 \times 18$ (center) assemblies separated by $0.357-\mathrm{cm}-$ thick

-1 wt\% cadmium copper plates and $2.243 \mathrm{~cm}$ of water

$\infty$ read parm run=yes gen $=405 \mathrm{npg}=600 \mathrm{nsk}=5$ nub=yes end parm

read geom

unit 1

cyl inder 110.5588

cyl inder 210.635

cuboid $314 \mathrm{p} 0.842$

unit 2

array $13 * 0,0$

reflector $312 \mathrm{r} 0 \quad 5.288 \quad 0.02 \mathrm{ro} \quad 1$

unit 3

array $23 * 0.0$

reflector 312 ro $\quad 5.288 \quad 0.0 \quad 2$ ro $\quad 1$

unit 4

cuboid $\quad \begin{array}{lllllll}5 & 1 & 0.0 & -0.357 & 35.6 & 0.0\end{array}$

cuboid

$\begin{array}{llllll}3 & 1 & 0.0 & -2.60 & 35.6 & 0.0\end{array}$

$90.23 \quad-1.27$

unit 5

$96.52-1.27$

$\begin{array}{llllllllll} & & & & & \end{array}$

$\begin{array}{llllllll} & \\ \text { cuboid } 3 & 1 & 2.60 & 0.0 & 35.6 & 0.0 & 96.52 & -1.27\end{array}$

global unit 9

array $3 \quad-57.33 \quad-17.8 \quad 0$.

reflector $415 r 0.0 \quad 2.54 \quad 1$

reflector $3,4 r 30.0 \quad 2 r 15.01$

end geometry read array

ara $=1$ nux $=25$ nuy=18 nuz=1 fill $f 1$ end fill

ara $=2$ nux=20 nuy=18 nuz=1 fill $f 1$ end fill

ara=3 nux=5 nuy=1 nuz=1 fill 34253 end fill

end array

end data

end

\section{p3314sig}

$=\operatorname{csas} 25$

p3314slg

44 group latticecell

402 $\quad 10.949293922354 .319223895 .69$

$\begin{array}{llllll}\text { uo2 } & 1 & 0.949293922354 .319223895 .69 & \text { end } \\ \text { al } & 2 & 1.0 & & \end{array}$

plexiglas 41.0

arbmrubber 1.3216000601258 .10016 .52000011 .4 160001.7801622 .1140000 .35

end

end

end comp

1.8921 .2649131 .414721 .28270

end

squarepitch 1.8921 .2649131 .414721 .28270 end

$2-9 \times 12$ and $2-9 \times 1$ assembl ies separated by 2.83 - and $10.86-\mathrm{cm}$ of water read parm run=yes gen $=405$ npg $=600$ nsk $=5$ nub=yes end parm

read geom

unit 1

cylinder $110.63245 \quad 91.44 \quad 0.0$

cylinder $010.64135 \quad 91.44 \quad 0.0$

cylinder $510.64135 \quad 93.98-2.54$

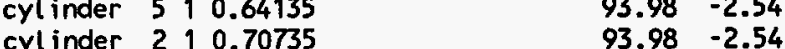

cuboid 314 p.9460 $\quad 93.98 \quad-2.54$

unit 2

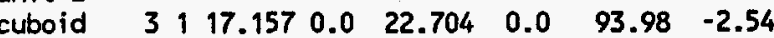

unit 3

array $13 * 0.0$

unit 4

cuboid $\quad \begin{array}{llllllll}3 & 1 & 2.83 & 0.0 & 22.704 & 0.0 & 93.98 & -2.54\end{array}$

$\begin{array}{llllllll}\text { unit } 5 & & & & & & \\ \text { cuboid } & 3171.2 & 0.0 & 10.86 & 0.0 & 93.98 & -2.54\end{array}$

unit 6

$\begin{array}{lllllllll}\text { cuboid } & 3 & 1 & 17.157 & 0.0 & 1.892 & 0.0 & 93.98 & -2.54\end{array}$

$\begin{array}{llllllll}\text { unit } 7 & 312.83 & 0.0 & 1.892 & 0.0 & 93.98 & -2.54\end{array}$

cuboic

array $23 * 0.0$

unit 9

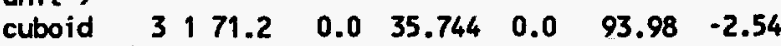

unit 10

array $3 * 0.0$

unit 11

array $43^{* 0.0}$ 
global unit 12

array $5 \quad 3 * 0.0$

reflector $\quad 415 \mathrm{r} 0.0 \quad 2.54 \quad 1$

reflector $314 \mathrm{r} 30.0 \quad 2 \mathrm{r} 15.01$

end geometry

\section{read array}

ara $=1$ nux $=9$ nuy=12 nuz=1 $f i l l$ f 1 end $f i l l$

ara $=2$ nux=9 nuy=1 nuz=1 fill $f 1$ end fill

ara $=3$ nux $=5$ nuy=1 nuz=1 fill $2 \quad 3 \quad 4 \quad 3 \quad 2$ end fill

ara $=4$ nux $=5$ nuy=1 nuz=1 fill $6 \quad 87886$ end fill

ara $=5$ nux $=1$ nuy $=4$ nuz $=1$ fill 105119 end fill

end array

end data

end

\section{p3314ss1}

\section{$=\operatorname{csas} 25$}

4ss 1

44 group latticecell

uo2 10.949293922354 .319223895 .69

al 21.0

h20 $\quad 31.0$

plexiglas 41.0

arbmrubber $1.3216000601258,10016.52000011 .4$

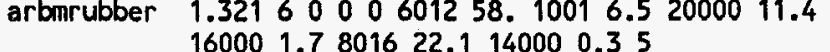

cr $\quad 6$ den $=7.93 .1856$

$\begin{array}{lll}\text { cu } & 6 \text { den=7.93 } & .0027 \\ \text { fe } & 6 \text { den=7.93 } & .6824\end{array}$

$\mathrm{mn} \quad 6$ den $=7.93 .0158$

mo 6 den=7.93 .0026

ni $\quad 6$ den=7.93 .1109

end comp

squarepitch 1.8921 .2649131 .414721 .28270

$2-9 \times 12$ and $2-9 \times 2$ assemblies separated by $0.302-\mathrm{cm}-\mathrm{thick} 0.0$ wt\% 8

'stainless steel plates and 2.528 - and $3.078-\mathrm{cm}$ of water

read parm run=yes gen $=405 \mathrm{npg}=600 \mathrm{nsk}=5$ nub=yes end parm

read geom

unit 1

cylinder 110.63245

cylinder 010.64135

cylinder 510.64135

cylinder 210.70735

cuboid $314 p .9460$

unit 2

117.1570 .0

cuboid

3117.1570 .0

$22.704 \quad 0.0$

array $13 * 0.0$

unit 4

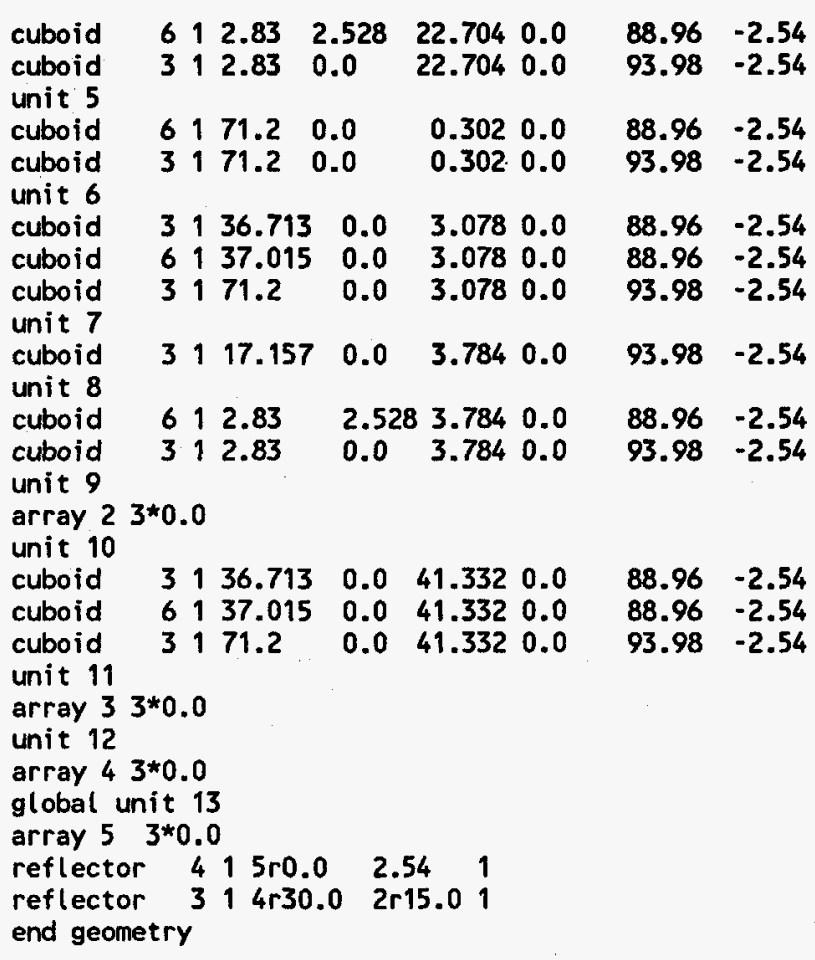

read array

ara=1 nux=9 nuy=12 nuz=1 fill $f 1$ end fill

ara $=2$ nux $=9$ nuy=2 nuz $=1$ fill $f 1 \quad$ end fill

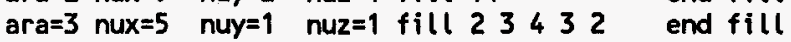
ara $=4$ nux $=5$ nuy=1 nuz=1 fill 798997 end fill ara $=5$ nux=1 nuy=5 nuz=1 fill $1156 \quad 12 \quad 10$ end fill end array

end data

end

p3314ss?

$=\operatorname{csas} 25$

p3314ss2

44group latticecell

uo2 $\quad 10.949293922354 .319223895 .69$

$\begin{array}{lll}1 & 0.949 \\ 2 & 1.0\end{array}$

h2o 31.0

plexiglas 41.0

arbmrubber 1.3216000601258 .10016 .52000011 .4 end
end
end
end 
read geom

unit 1

cylinder 110.63245

cyl inder 010.64135

cylinder 5110.64135

cylinder 2110.70735

cuboid $3 \begin{array}{llll}4 p .9460 \\ \text { cunit }\end{array}$

unit 2

cuboid

$\begin{array}{lllllllll}3 & 1 & 17.157 & 0.0 & 22.704 & 0.0 & 93.98 & -2.54\end{array}$

unit 3

array $13 * 0.0$

\begin{tabular}{|c|c|c|c|c|c|c|}
\hline \\
\hline & $\begin{array}{lll}6 & 1 & 71.2 \\
3 & 1 & 71.2\end{array}$ & $\begin{array}{l}0.0 \\
0.0\end{array}$ & $\begin{array}{l}0.302 \\
0.302\end{array}$ & $\begin{array}{l}0.0 \\
0.0\end{array}$ & $\begin{array}{l}88.96 \\
93.98\end{array}$ & $\begin{array}{l}-2.54 \\
-2.54\end{array}$ \\
\hline $\begin{array}{l}\text { cuboid } \\
\text { cuboid } \\
\text { cuboid }\end{array}$ & $\begin{array}{lll}3 & 1 & 36.713 \\
6 & 1 & 37.015 \\
3 & 1 & 71.2\end{array}$ & $\begin{array}{l}0.0 \\
0.0 \\
0.0\end{array}$ & $\begin{array}{l}11.248 \\
11.248 \\
11.248\end{array}$ & $\begin{array}{l}0.0 \\
0.0 \\
0.0\end{array}$ & $\begin{array}{l}88.96 \\
88.96 \\
93.98\end{array}$ & $\begin{array}{l}-2.54 \\
-2.54 \\
-2.54\end{array}$ \\
\hline cuboid & 3117.157 & 0.0 & 24.596 & 0.0 & 93.98 & -2.54 \\
\hline $\begin{array}{l}\text { cuboid } \\
\text { cuboid } \\
\text { unit } 9 \\
\text { array } 2 \\
\text { unit } 10\end{array}$ & $\begin{array}{rrr}6 & 1 & 2.83 \\
3 & 1 & 2.83 \\
& \\
* 0 & .0\end{array}$ & $\begin{array}{l}2.528 \\
0.0\end{array}$ & $\begin{array}{l}24.596 \\
24.596\end{array}$ & $\begin{array}{l}0.0 \\
0.0\end{array}$ & $\begin{array}{l}88.96 \\
93.98\end{array}$ & $\begin{array}{l}-2.54 \\
-2.54\end{array}$ \\
\hline $\begin{array}{l}\text { cuboid } \\
\text { cuboid } \\
\text { cuboid } \\
\text { unit } 11 \\
\text { array } 3 \\
\text { unit } 12 \\
\text { array } 4\end{array}$ & $\begin{array}{lll}3 & 1 & 36.713 \\
6 & 1 & 37.015 \\
3 & 1 & 71.2 \\
& & \\
& 0.0\end{array}$ & $\begin{array}{l}0.0 \\
0.0 \\
0.0\end{array}$ & $\begin{array}{l}12.35 \\
12.35 \\
12.35\end{array}$ & $\begin{array}{l}0.0 \\
0.0 \\
0.0\end{array}$ & $\begin{array}{l}88.96 \\
88.96 \\
93.98\end{array}$ & $\begin{array}{l}-2.54 \\
-2.54 \\
-2.54\end{array}$ \\
\hline
\end{tabular}

$\begin{array}{llllll}\text { reflector } & 4 & 1 & 5 \mathrm{r} 0.0 & 2.54 & 1 \\ \text { reflector } & 3 & 1 & 4 \mathrm{r} 30.0 & 2 \mathrm{r} 15.0 & 1\end{array}$

end geometry

read array

ara $=1$ nux $=9$ nuy=12 nuz=1 fill $f 1$ end fill

ara $=2$ nux $=9$ nuy=13 nuz=1 fill $f 1$ end fill

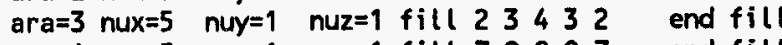

ara $=4$ nux=5 nuy=1 nuz=1 fill 79897 end fill

ara $=5$ nux $=1$ nuy=5 nuz=1 fill $1156 \quad 12 \quad 10$ end fill

end array

end data

end

\section{p3314ss3}

$=\operatorname{csas} 25$

p3314ss3

44group latticecell

10.949293922354 .319223895 .69

al 21.0

h20 $\quad 31.0$

plexiglas 41.0

end

arbmrubber 1.3216000601258 .10016 .52000011 .4

160001.7801622 .1140000 .35 end

cr 6 den $=7.93 .1856 \quad 6$ end

cu 6 den=7.93 .0027 end

$\begin{array}{llll}\text { fe } & 6 \text { den=7.93 } & .6824 & \text { end } \\ \mathrm{mn} & 6 \text { den=7.93 } & .0158 & \text { end }\end{array}$

mo 6 den=7.93.0026 end

ni 6 den=7.93.1109 end

end comp

squarepitch 1.8921 .2649131 .414721 .28270 end $2-9 \times 12$ and $2-9 \times 5$ assembl ies separated by $0.485-\mathrm{cm}-$ thick $0.0 \mathrm{wt} \% \mathrm{~B}$ stainless steel plates and $2.83-$ and $4.47-\mathrm{cm}$ of water

read parm run=yes gen $=405 \mathrm{npg}=600 \mathrm{nsk}=5$ nub=yes end parm

read geom

unit 1

cylinder $1110.63245 \quad 91.44 \quad 0.0$

cylinder $010.64135 \quad 91.44 \quad 0.0$

cyl inder $510.64135 \quad 93.98-2.54$

$\begin{array}{llllll}\text { cyl inder } & 5 & 1 & 0.64135 & 93.98 & -2.54 \\ \text { cyl inder } 2 & 1 & 0.70735 & 93.98 & -2.54\end{array}$

$\begin{array}{llllll}\text { cyl inder } & 2 & 1 & 0.70735 & 93.98 & -2.54 \\ \text { cuboid } & 3 & 1 & 4 p .9460 & 93.98 & -2.54\end{array}$

unit 2

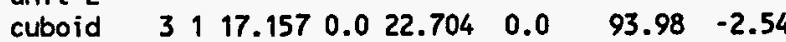

unit 3

array $13 * 0.0$

unit 4

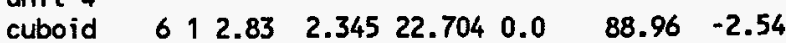

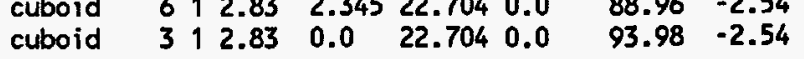




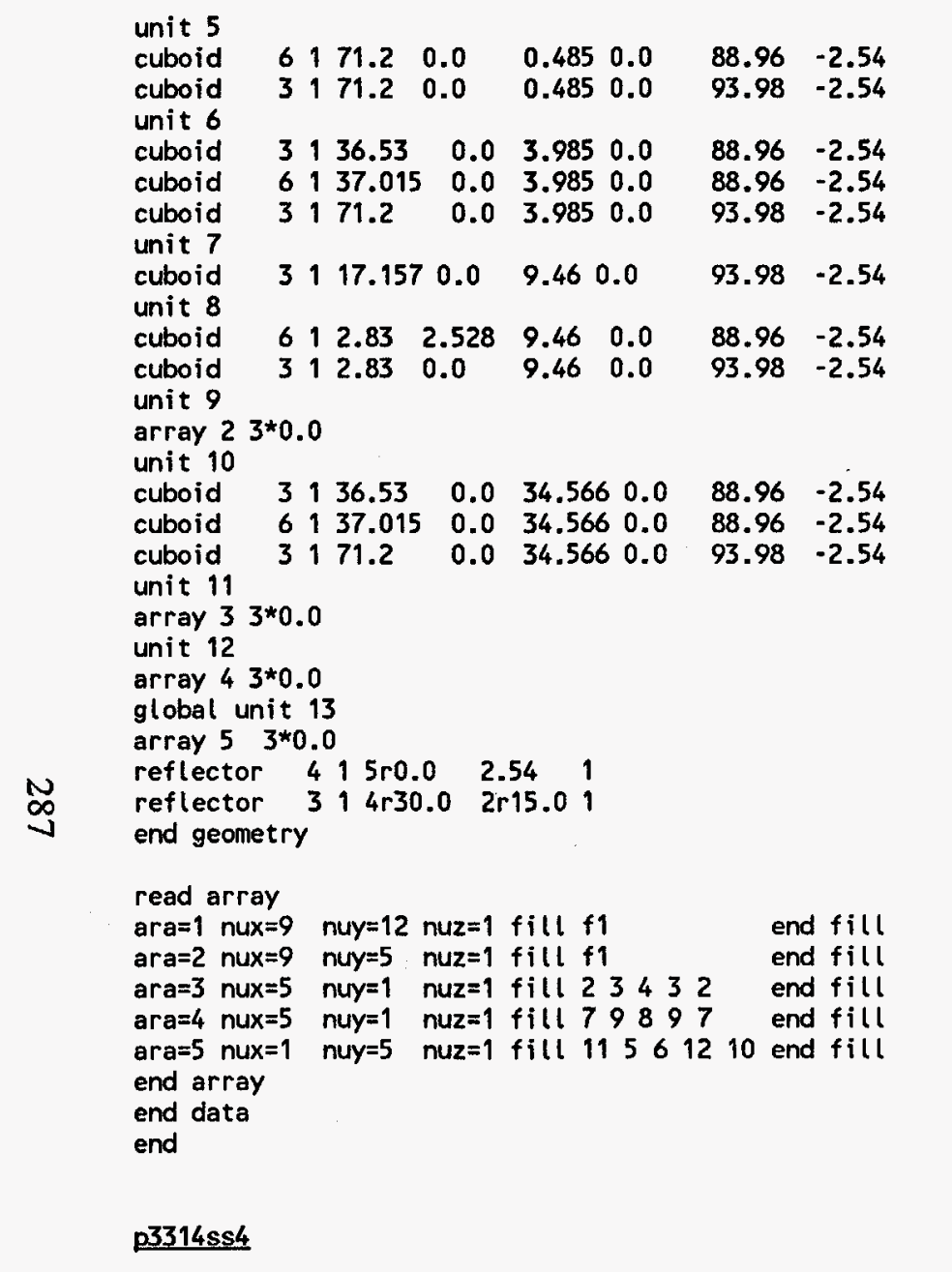

$=\operatorname{csas} 25$
p3314ss

44 group latticecell

uo2 10.949293922354 .319223895 .69 21.0

31.0

arbmrubber 1.32160000601258 .10016 .52000011 .4 160001.7801622 .1140000 .35

cr 160001.7801622 .1
6 den $=7.93 .1856$

$\begin{array}{llll}\mathrm{cu} & 6 \text { den=7.93 } .0027 & \text { end } \\ \mathrm{fe} & 6 \text { den=7.93 } & .6824 & \text { end } \\ \mathrm{mn} & 6 \text { den=7.93 } .0158 & \text { end } \\ \mathrm{mo} & 6 \text { den=7.93 } .0026 & \text { end } \\ \mathrm{ni} & 6 \text { den=7.93 } .1109 & \text { end } \\ \text { end comp } & & \end{array}$

squarepitch 1.8921 .2649131 .414721 .28270 end $2-9 \times 12$ and $2-9 \times 12$ assembl ies separated by $0.485-\mathrm{cm}-$ thick 0.0 wt\% B stainless steel plates and $2.83-$ and $8.36-\mathrm{cm}$ of water read parm run=yes gen $=405$ npg=600 nsk=5 nub=yes end parm

read geom

unit

cylinder $110.63245 \quad 91.44 \quad 0.0$

cylinder $0110.64135 \quad 91.44 \quad 0.0$

cylinder 5 1 $0.64135 \quad 93.98 \quad-2.54$

cyl inder $210.70735 \quad 93.98 \quad-2.54$

$\begin{array}{lllll}\text { cuboid } 314 \mathrm{p} .9460 & 93.98 & -2.54\end{array}$

unit 2

$93.98-2.54$

cuboid

$3117.157 \quad 0.022 .704 \quad 0.0$

$-2.54$

unit 3

array $13 * 0.0$

unit 4

cuboid $\begin{array}{llllllll}6 & 1 & 2.83 & 2.345 & 22.704 & 0.0 & 88.96 & -2.54\end{array}$

$\begin{array}{lllllllll}\text { cuboid } & 3 & 1 & 2.83 & 0.0 & 22.704 & 0.0 & 93.98 & -2.54\end{array}$

unit 5

$\begin{array}{llllllll}\text { cuboid } \quad 6 & 171.2 & 0.0 & 0.485 & 0.0 & 88.96 & -2.54\end{array}$

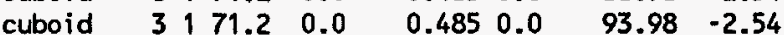

unit 6

$\begin{array}{lllllllll}\text { cuboid } & 3 & 1 & 36.53 & 0.0 & 7.875 & 0.0 & 88.96 & -2.54\end{array}$

$\begin{array}{llllllll}\text { cuboid } \quad 6 & 1 & 37.015 & 0.0 & 7.875 & 0.0 & 88.96 & -2.54\end{array}$

$\begin{array}{lllllll}\text { cuboid } 3171.2 & 0.0 & 7.875 & 0.0 & 93.98 & -2.54\end{array}$

unit 7

cuboid

$\begin{array}{llllllll}3 & 1 & 17.157 & 0.0 & 22.704 & 0.0 & 93.98 & -2.54\end{array}$

unit 8

cuboid

cuboid

$\begin{array}{lllllll}6 & 1 & 2.83 & 2.345 & 22.704 & 0.0\end{array}$

$88.96-2.54$

unit 9

array $23 * 0.0$

unit 10

$\begin{array}{lllllllll}\text { cuboid } & 3 & 1 & 36.53 & 0.0 & 17.432 & 0.0 & 88.96 & -2.54\end{array}$

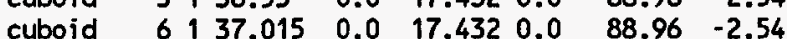

$\begin{array}{llllllll}\text { cuboid } 3171.2 & 0.0 & 17.432 & 0.0 & 93.98 & -2.54\end{array}$

unit 11

array $3 * 0.0$

unit 12

array $43 * 0.0$

global unit 13

array $5 \quad 3 * 0.0$

reflector $415 r 0.0 \quad 2.54$ 
reflector $314 r 30.0 \quad 2 r 15.01$

end geometry

read array

ara $=1$ nux=9 nuy=12 nuz=1 fill $f 1$

end $f i l l$

ara $=2$ nux=9 nuy=12 nuz=1 fill $f 1$

end fill

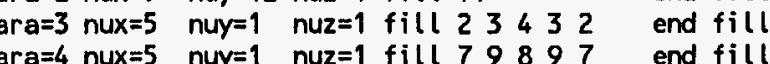

ara $=5$ nux=1 nuy=5 nuz=1 fill $115 \quad 6 \quad 12 \quad 10$ end fill

end array

end data

end

\section{p3314ss5}

\section{$=\operatorname{csas} 25$}

p3314ss5

44 group latticecell

uo2 10.84293922352 .359223897 .65

al $\quad 21.0$

h20 31.0

plexiglas 41.0

cr $\quad 5$ den=7.93 .1856

cu 5 den=7.93 .0027

fe $\quad 5$ den=7.93 .6824

$\sim \quad \mathrm{mn} \quad 5$ den=7.93 .0158

mo $\quad 5$ den=7.93 .0026

ni 5 den=7.93 .1109

squarepitch 1.6841 .1176131 .272 end geometry

$2-17 \times 10$ and $1-25 \times 20$ (center) assemblies separated by $0.302-\mathrm{cm}$-thick

10.0 wt \% B stainless steel plates and $7.80 \mathrm{~cm}$ of water

read parm run=yes gen $=405 \mathrm{npg}=600 \mathrm{nsk}=5$ nub=yes end parm

read geom

unit 1

cyl inder 110.5588

cyl inder 210.635

cylinger

unit

$\begin{array}{ll}91.44 & 0.0\end{array}$

unit

$96.52-1.27$

array $13 * 0.0$

reflector $312 \mathrm{ro} \quad 1.920 .02 \mathrm{ro} \quad 1$

unit 3

array $23 * 0.0$

reflector $312 \mathrm{ro} \quad 1.920 .02 \mathrm{r} 0 \quad 1$

unit 4

$\begin{array}{lllllllll}5 & 1 & 0.0 & -0.302 & 35.6 & 0.0 & 90.23 & -1.27\end{array}$

$\begin{array}{lllllllll}\text { cuboid } & 3 & 1 & 0.0 & -7.80 & 35.6 & 0.0 & 96.52 & -1.27\end{array}$

$90.23-1.27$

$\begin{array}{lrrrrrrrr}\text { cuboid } & 5 & 1 & 0.302 & 0.0 & 35.6 & 0.0 & 90.23 & -1.27 \\ \text { cuboid } & 3 & 1 & 7.80 & 0.0 & 35.6 & 0.0 & 96.52 & -1.27\end{array}$

global unit 9 array $3 \quad-57.478-17.8$. 0.

reflector $415 \mathrm{r} 0.0 \quad 2.54 \quad 1$

reflector $3 \uparrow 4 r 30.0 \quad 2 r 15.01$

end geometry

read array

ara $=1$ nux $=25$ nuy $=20$ nuz= 1 fill $f i$ end fill

ara=2 nux=17 nuy $=20$ nuz=1 fill $f 1$ end fill

ara=3 nux=5 nuy=1 nuz=1 fill $3 \quad 4 \quad 2 \quad 5 \quad 3$ end fill

end array

end data

end

\section{D33145s6}

$=\operatorname{csas} 25$

p3314ss 6

44 group latticecell

uo2 $10.949293922354 .319223895 .69 \quad$ end

al 21.0

h20 31.0

plexiglas 41.0

cr $\quad 5$ den=7.93 .1856

cu 5 den $=7.93 .0027$

fe $\quad 5$ den=7.93 .6824

den 7.93 .0

den $=7.93$.015

$\begin{array}{llll}\text { mo } & 5 \text { den=7.93 } & .0026 & \text { end } \\ \text { ni } & 5 \text { den=7.93 } & .1109 & \text { end }\end{array}$

arbmrubber 1.3216000601258 .10016 .52000011 .4 160001.7801622 .1140000 .36

end comp

squarepitch 1.8921 .2649131 .414721 .28270 end

$3-12 \times 16$ assemblies separated by $0.302-\mathrm{cm}$-thick $0.0 \mathrm{wt} \%$ B stainless steel plates and $10.218 \mathrm{~cm}$ of water

read parm run=yes gen $=405 \mathrm{npg}=600 \mathrm{nsk}=5$ nub=yes end parm

read geom

unit 1

cylinder 110.63245

cylinder 010.64135

cylinder $6 \quad 10.64135$

cylinder 210.70735

cuboid $314 \mathrm{p0} 0.946$

$91.44 \quad 0.0$

$93.98 \quad-2.54$

$93.98-2.54$

unit 2

array $13 * 0.0$

reflector $312 \mathrm{r} 0 \quad 2 \mathrm{r} 2.6642 \mathrm{r} 0$

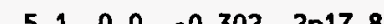

$\begin{array}{llllll}\text { cuboid } & 3 & 1 & 0.0 & -10.52 & 2 p 17.8\end{array}$

unit 4

cuboid

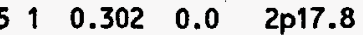

cuboid

$\begin{array}{lllll}3 & 1 & 10.52 & 0.0 & 2 p 17.8\end{array}$

$88.96-2.54$

$93.98 \quad-2.54$ 
global unit 5

array $2-44.576-17.8 \quad 0$.

reflector $\quad \begin{array}{lllll}1 & 1 & 5 \mathrm{r} 0.0 & 2.54 & 1\end{array}$

end geometry

read array

ara $=1$ nux=12 nuy=16 nuz=1 fill $f 1$ end $f i l l$ ara=2 nux=5 nuy=1 nuz=1 fill $2 \quad 3 \quad 2 \quad 4 \quad 2$ end fill end array

end data

end

\section{$23314 \times 1$}

$=\operatorname{csas} 25$

p3314w1

44 group latticecel

uo2 $\quad 10.949293922354 .319223895 .69$

1.0

$\begin{array}{ll}2 & 1.0 \\ 3 & 1.0\end{array}$

plexiglas 41.0

arbmrubber 1.32160000601258 .10016 .52000011 .4 160001.7801622 .1140000 .35

end comp

$N \quad$ squarepitch 1.8921 .2649131 .414721 .28270 end geometry Core of $14 \times 14$ positions: 168 fuel rods and 25 water holes read parm run=yes gen $=405 \mathrm{npg}=600 \mathrm{nsk}=5$ nub $=$ yes end parm

read geom

unit

cylinder 1110.63245

cylinder 0110.64135

cylinder 510.64135

cyl inder 210.70735

cuboid $314 \mathrm{p} .9460$

unit 2

314 p. 9460

unit 3

array $13 * 0.0$

unit 4

array $23 * 0.0$

unit 5

array $3 * 0.0$

global unit 6

array $4 \quad 3 * 0.0$

reflector $\quad 415 \mathrm{ro.0} \quad 2.541$

reflector $314 \mathrm{r} 30,15.215 .31$

end geometry read array

ara=1 nux=14 nuy=1 nuz=1 fill $f 1$ end fill ara=2 nux=14 nuy=1 nuz=1 fill $2 r 1124 \mathrm{q} 2 \quad 2 r 1$ end fill ara $=3$ nux $=14$ nuy $=1$ nuz $=1$ fill $11 r 13 r 2$ end fill ara $=4$ nux=1 nuy=14 nuz=1 fill $2 r 3434$ q2 35 end fill end array

end data

end

\section{2}

$=\operatorname{csas} 25$

p3314W2

44group latticecell

uo2 10.840293922352 .359223897 .65

at 21.0

h20 31.0

plexiglas 41.0

end comp

squarepitch 1.6841 .1176131 .272 end geometry

Core of $23 \times 23$ positions: 486 fuel rods and 25 water holes

read parm run=yes gen $=405$ npg $=600$ nsk $=5$ nub=yes end parm

read geom

unit 1

cylinder 110.5588

cyl inder 210.635

$\begin{array}{rr}91.44 & 0.0 \\ 96.52 & -1.27\end{array}$

$\begin{array}{llllll}\text { cuboid } & 3 & 1 & 400.842 & 96.52 & -1.27\end{array}$

unit 2

cuboid $314 \mathrm{p} .842$

$96.52-1.27$

unit 4

array $23 * 0.0$

unit 5

array $3 * 0.0$

global unit 6

array $4 \quad 3 * 0.0$

reflector $415 \mathrm{r} 0.0 \quad 2.541$

reflector $314 \mathrm{r30}, 15.215 .3$

end geometry

read array

ara $=1$ nux=23 nuy=1 nuz=1 fill fi fill ara=2 nux=23 nuy=1 nuz=1 fill $5 r 1 \quad 1 \quad 2 \quad 4 q 2 \quad 8 r 1$ end fill ara $=3$ nux $=23$ nuy=1 nuz $=1$ fill $5 r 1$ 18r2 end fill ara $=4$ nux=1 nuy=23 nuz=1 fill $7 r 3434 q 25 r 35$ end fill

end array

end data

enc 
h2o

artmrubber 1.3216000601258 .10016 .52000011 .4 160001.7801622 .1140000 .36

end comp

squarepitch 1.8921 .2649131 .414721 .28270 - thick 2 ircaloy- 4 " plates and 2.178- and $10.388-\mathrm{cm}$ of water

read parm run=yes gen $=405$ npg $=600$ nsk $=5$ nub=yes end parm

\section{read geom}

unit 1

cylinder 110.63245

cylinder 010.64135

unit 3

array $13 * 0.0$

array

unit 4

$\begin{array}{llllllll}7 & 1 & 2.83 & 2.178 & 22.704 & 0.0 & 88.96 & -2.54\end{array}$

$\begin{array}{llllllll}\text { cuboid } 5 & 1 & 2.83 & 0.0 & 22.704 & 0.0 & 93.98 & -2.54\end{array}$

unit 5

cuboid

unit 6

unit 6

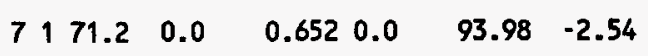

cuboid

unit 7

cuboid

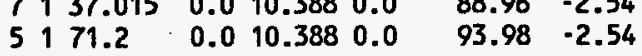

$88.96-2.54$

unit 8

$\begin{array}{llllll}5 & 1 & 17.157 & 0.0 & 3.784 & 0.0\end{array}$

cuboid

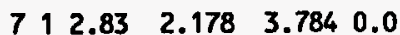

cuboid

$88.96-2.54$

unit 9

array $23 * 0.0$

unit 10

$\begin{array}{lllllllll}\text { cuboid } \quad 5 & 1 & 36.363 & 0.0 & 33.672 & 0.0 & 88.96 & -2.54\end{array}$

$\begin{array}{lllllllll}\text { cuboid } \quad 7137.015 & 0.0 & 33.672 & 0.0 & 88.96 & -2.54\end{array}$

$\begin{array}{llllllll}\text { cuboid } \quad 5171.2 & 0.0 & 33.672 & 0.0 & 93.98 & -2.54\end{array}$

array $33 * 0.0$

unit 12

array $43 * 0.0$

global unit 13

array $5 \quad 3 * 0.0$

reflector $\quad 415 r 0.0 \quad 2.54 \quad 1$

reflector 514 r30.0 2 r 15.01

end geometry

\section{read array}

ara=1 nux=9 nuy=12 nuz=1 fill $f 1$ end fill ara $=2$ nux $=9$ nuy $=2$ nuz $=1$ fill $f 1$ end fill

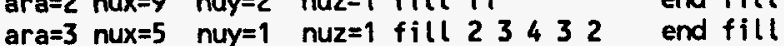
ara=4 nux=5 nuy=1 nuz=1 fill 79897 end fill ara $=5$ nux $=1$ nuy=5 nuz=1 fill $1156 \quad 12 \quad 10$ end fill end array

end data

end

p3602bb

$=\operatorname{csas} 25$

p3602bb

44group latticecell

402 10.949293922354 .319223895 .69

li

h20 31.0

plexiglas 41.0

end $\begin{array}{llllllll}1.28 & 14000.22 & 28304 & .79 & 42000 & .49 & 24304 & .12\end{array}$ $29000.13 \quad 5$

arbmrubber $1.321600001258,10016.52000011 .4$

160001.7801622 .1140000 .36

al $\quad 7$ den $=2.50 .612$

boron 7 den=2.50 .3036

c 7 den $=2.50 \quad .0843$ 3-12×16 assembl ies separated by $0.292-\mathrm{cm}$-thick Boral $-\mathrm{B}$ plates and $-8.008 \mathrm{~cm}$ of water, assembl ies separated from stainless steel reflecting walls by $1.956 \mathrm{~cm}$ of water

read parm run=yes gen $=405$ npg $=600$ nsk $=5$ nub=yes end parm

read geom

unit 1

cylinder $110.63245 \quad 91.44 \quad 0.0$

cylinder $00110.64135 \quad 91.44 \quad 0.0$

cylinder $610.64135 \quad 93.98-2.54$

cylinder $210.70735 \quad 93.98$-2.54

cuboid $314 p .9460 \quad 93.98 \quad-2.54$ 
unit 2

array $13 * 0.0$

unit 3

$\begin{array}{lllllllll}\text { cuboid } & 3 & 1 & 7.5307 & 0.0 & 30.272 & 0.0 & 93.98 & -2.54\end{array}$

unit

$\begin{array}{llllllll}\text { cuboid } \quad 71 & 0.292 & 0.0 & 2 p 15.1 & 88.96 & -2.54\end{array}$

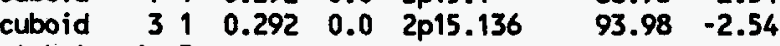

global unit 5

array $2-41.8787-15.136 \quad 0$.

reflector $415 \mathrm{r0.0} \quad 2.54 \quad 1$

$\begin{array}{llllllll}\text { cuboid } \quad 312 p 73.65 & 2 p 17.0924 & 101.52 & -20.38\end{array}$

reflector $\quad 512 r 0.0 \quad 2 r 17.85 \quad 2 r 0.0$

reflector $312 r 30.5 \quad 2 \times 10.694 \quad 7.5401$

end geometry

read array

ara $=1$ nux $=12$ nuy=16 nuz=1 fill $f 1$ end fill

ara=2 nux=7 nuy=1 nuz=1 fill 2342432 end fill

end array

end data

end

23602bs1

$=\operatorname{csas} 25$

No p3602bs 1

$\begin{array}{llllll}\text { 44group latticecell } & & & & & \\ \text { uo2 } & 10.84 & 09392235 & 2.359223897 .65\end{array}$

uo2 $\quad 10.84293922352 .359223897 .65$

at 21.0

h20 31.0

plexiglas 41.0

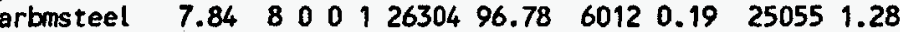

end

boron 6 den $=7.9 .0105$

crss 6 den=7.9.1903

$\begin{array}{lll}\text { crss } & 6 \text { den=7.9. } & .1903 \\ \text { cu } & 6 \text { den=7.9.0028 }\end{array}$

fess $\quad 6$ den=7.9.

mn $\quad 6$ den=7.9.0158

mo $\quad 6$ den=7.9 .0049

niss $\quad 6$ den=7.9.0953

end comp

squarepitch $1.6841 .1176+31.27$

2-18x20 and $1-25 \times 18$ (center) assemblies

1.1 wt\% B $304 \mathrm{~L}$ stainless steel plates and $4.502 \mathrm{~cm}$ of water,

assemblies separated from stainless steel reflecting walls by 1.321

cm of water

read parm run=yes gen $=405$ npg $=600$ nsk $=5$ nub=yes end parm

read geom

unit

cyl inder 110.5588

91.44

0. cylinder 210.635

cuboid $314 \mathrm{p0} 0.842$

$96.52-1.27$

unit 2

$96.52 \quad-1.27$

array $13 \star 0.0$

$\begin{array}{lllllllllll}\text { cuboid } & 3 & 1 & 4.088 & 0.0 & 30.312 & 0.0 & 96.52 & -1.27\end{array}$

cuboid

cuboid

unit 5

array $23 * 0.0$

global unit 6

array $3 \quad-59.116 \quad-15.156 \quad 0$

$\begin{array}{llllll}\text { reflector } \quad 4 & 15 \mathrm{5r0.0} & 2.541\end{array}$

cuboid $\quad 312 p 73.65$ 2p16.477 102.79-19.11

$\begin{array}{lllllll}\text { reflector } & 5 & 1 & 2 r 0.0 & 2 r 17.85 & 2 r 0.0 & 1 \\ \text { reflector } & 3 & 1 & 2 r 30.5 & 2 r 11.329 & 8.93 & 0\end{array}$

end geometry

read array

ara $=1$ nux $=20$ nuy $=18$ nuz $=1$ fill $f 1$

ara $=2$ nux $=25$ nuy $=18$ nuz $=1$ fill $f 1$

$\begin{array}{llllll}1 & 0.298 & 0.0 & 2 p 15.1 & 90.23 & -1.27 \\ 1 & 0.298 & 0.0 & 2 p 15.156 & 96.52 & -1.27\end{array}$

ara $=3$ nux $=7$

end data

p3602bs?

$=c s a s 25$

p3602bs2

44group latticecell

uo2 $\quad 10.949293922354 .319223895 .69$

al 21.0

h2o $\quad 31.0$

plexiglas 41.0

arbmsteel $\quad 7.84 \quad 8 \quad 0 \quad 0 \quad 1 \quad 26304 \quad 96.78 \quad 6012 \quad 0.19 \quad 25055 \quad 1.28$

arbmrubber 1.3216000601258 .10016 .52000011 .4

160001.7801622 .1140000 .36

boron $\quad 7$ den=7.9.0105

crss $\quad 7$ den $=7.9 .1903$

cu 7 den=7.9.0028

fess $\quad 7$ den $=7.9 .6804$

mn $\quad 7$ den=7.9.0158

mo $\quad 7$ den $=7.9 .0049$

niss $\quad 7$ den=7.9.0953

end comp

end fill

end fill

squarepitch

$2-20 \times 18$ and $1-25 \times 18$ (center) assemblies separated by $0.061-\mathrm{cm}-$ thick

cadmium plates and $3.799 \mathrm{~cm}$ of water, assemblies separated from end

end

end

end

end

end

end

end

end 
'stainless steel reflecting walls by $1.321 \mathrm{~cm}$ of water

read parm run=yes gen $=405$ npg $=600$ nsk $=5$ nub=yes end parm

read geom

unit 1

cylinder 110.63245

cylinder 0110.64135

cyl inder 610.64135

cylinder 210.70735

cuboid 3 i 3 p.9460

unit 2

array $13 * 0.0$

unit 3

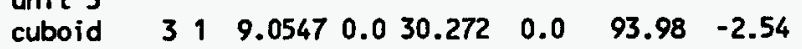

$\begin{array}{llllll}7 & 0.0587 & 0.030 .272 & 0.0 & 93.98 & -2.54\end{array}$

$\begin{array}{llllllll}\text { cuboid } & 7 & 1 & 0.298 & 0.0 & 2 p 15.1 & 88.96 & -2.54 \\ \text { cuboid } & 3 & 1 & 0.298 & 0.0 & 2 p 15.136 & 93.98 & -2.54\end{array}$

global unit 5

array $2-43.4087-15.1360$.

$\begin{array}{llll}\text { reflector } 495 r 0.0 & 2.54 \quad 1\end{array}$

cuboid $\quad 31$ 2p73.65 2p17.092 101.52 -20.38

$\begin{array}{lllllll}\text { reflector } 5 & 1 & 2 \mathrm{r} 0.0 & 2 \mathrm{r} 17.85 & 2 \mathrm{r} 0.0 & 1\end{array}$

reflector $312 r 30.5 \quad 2 r 10.69475401$

end geometry

read array

ara $=1$ nux $=12$ nuy=16 nuz=1 fill $\mathrm{fl}$

$91.44 \quad 0.0$

$91.44 \quad 0.0$

$93.98-2.54$

$93.98-2.54$

ara $=2$ nux $=7$

end

\section{$03602 \mathrm{cd1}$}

\section{$=\operatorname{csas} 25$}

p3602cd1

44group latticecell

$\begin{array}{lllll}\text { 44 } & 1 & 0.8429392235 & 2.3592238 & 97.65\end{array}$

uor 20.8

al $\quad 21.0$

plexiglas 41.0

$\begin{array}{llllllllllll}\text { arbmsteel } & 7.84 & 8 & 0 & 0 & 1 & 26304 & 96.78 & 6012 & 0.19 & 25055 & 1.28\end{array}$

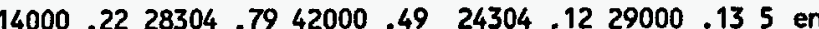

cd

$$
6 \text { den }=8.65
$$

end comp

guarepitch 1.6841 .1176131 .272

$2-20 \times 18$ and 1-25x18(center) assemblies separated by $0.061-\mathrm{cm}$-thick

cadmium plates and $3.799 \mathrm{~cm}$ of water, assembl ies separated from

'stainless steel reflecting walls by $1.321 \mathrm{~cm}$ of water

read parm run=yes gen $=405 \mathrm{npg}=600$ nsk $=5$ nub=yes end parm read geom

unit 1

cylinder 110.5588

cylinder 210.635

$\begin{array}{llllll}\text { cylinder } & 2 & 1 & 0.635 & 96.52 & -1.27 \\ \text { cuboid } & 3 & 1 & 4 \mathrm{p0} .842 & 96.52 & -1.27\end{array}$

unit 2

array $13 * 0.0$

unit 3

$\begin{array}{lllllllll}\text { cuboid } & 3 & 1 & 3.385 & 0.0 & 30.312 & 0.0 & 96.52 & -1.27\end{array}$

cuboid $610.0610 .02 p 15.1 \quad 90.23 \quad-1.27$

cuboid $310.061 \quad 0.02 p 15.156 \quad 96.52 \quad-1.27$

unit 5

array $23 * 0.0$

global unit 6

array $3-58.115-15.156 \quad 0$.

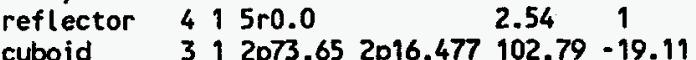

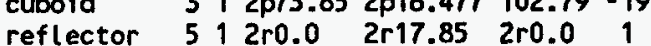

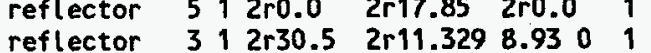

reflector 3

read array

ara $=1$ nux $=20$ nuy=18 nuz=1 fill $f 1$ end fill ara $=2$ nux $=25$ nuy $=18$ nuz $=1$ fill $f 1 \quad$ end fill ara $=3$ nux $=7$ nuy $=1$ nuz=1 fill 23445432 end fill end array

end data

end

$3602 \mathrm{~cd} 2$

$=\operatorname{csas} 25$

p3602cd2

44 group latticecel

$002 \quad 10.949293922354 .319223895 .69$

al 21.0

h20 31.0

25055 end

$\begin{array}{llllllllll}7.84 & 8 & 0 & 0 & 1 & 26304 & 96.78 & 6012 & 0.19 & 25055\end{array}$ $\begin{array}{lllllllll}1.28 & 14000 & .22 & 28304 & .79 & 42000 & .49 & 24304.12 & \\ 29000.13 & 5 & & \end{array}$

arbmrubber 1.32160000601258 .10016 .52000011 .4 160001.7801622 .1140000 .36

cd

7 den $=8.65$

end

$\begin{array}{lllllll}\text { end comp } & & & & \end{array}$

3-12x16 assemblies separated by $0.061-\mathrm{cm}$-thick cadmium plates and $\mathbf{8 . 7 9 9} \mathrm{cm}$ of water, assembl ies separated from stainless steel

'reflecting walls by $1.956 \mathrm{~cm}$ of water

read parm run=yes gen $=405 \mathrm{npg}=600$ nsk $=5$ nub=yes end parm 


\section{read geom}

unit 1

cylinder 1110.63245

cyl inder 010.64135

cylinder 610.64135

cyl inder 210.7073

$91.44 \quad 0.0$

$314 p .9460$

$93.98-2.54$

array $13 * 0.0$

unit 3

$\begin{array}{llllllll}\text { cuboid } & 31 & 8.4017 & 0.0 & 30.272 & 0.0 & 93.98 & -2.54\end{array}$

$\begin{array}{llllllll}\text { cuboid } & 71 & 0.061 & 0.0 & 2 p 15.1 & 88.96 & -2.54\end{array}$

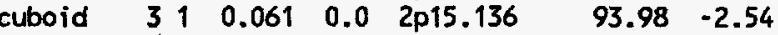

global unit 5

array $2-42.5187-15.136 \quad 0$

reflector $415 \mathrm{r0.0} \quad 2.54$,

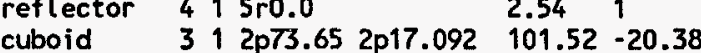

$\begin{array}{lllllll}\text { cuboid } & 3 & 1 & 2 \mathrm{p} 73.65 & 2 \mathrm{p} 17.092 & 101.52 & -20.38 \\ \text { reflector } & 5 & 1 & 2 \mathrm{r} 0.0 & 2 \mathrm{r} 17.85 & 2 \mathrm{r0.0} & 1\end{array}$

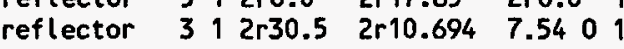

end geometry

read array

ara=1 nux=12 nuy=16 nuz=1 fill $f 1 \quad$ end $f i l l$

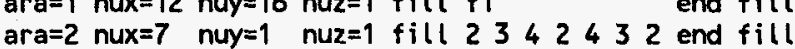

end array

end data

end

23602cu1

$=\operatorname{csas} 25$

p3602cu1

44 group latticecell

no2 $\quad 10.84293922352 .359223897 .65$

at $\quad 21.0$

h20 31.0

$\begin{array}{rrrrrrrrr} & & & & & & & & \end{array}$

cu

$14000.22 \quad 28304 \quad 79 \quad 42000 \quad 49 \quad 24304 \quad 1229000.135$ end 6 den $=8.913$

squarepitch 1.6841 .1176131 .272

$2-20 \times 18$ and $1.25 \times 18$ (center) assemblies separated by $0.337-\mathrm{cm}-$ thick

copper plates and $7.453 \mathrm{~cm}$ of water assemblies separated from

stainless steel reflecting walls by $1.321 \mathrm{~cm}$ of water

read parm run=yes gen $=405 \mathrm{npg}=600$ nsk $=5$ nub=yes end parm

read geom

unit 1

cyl inder 110.5588

91.440 .0

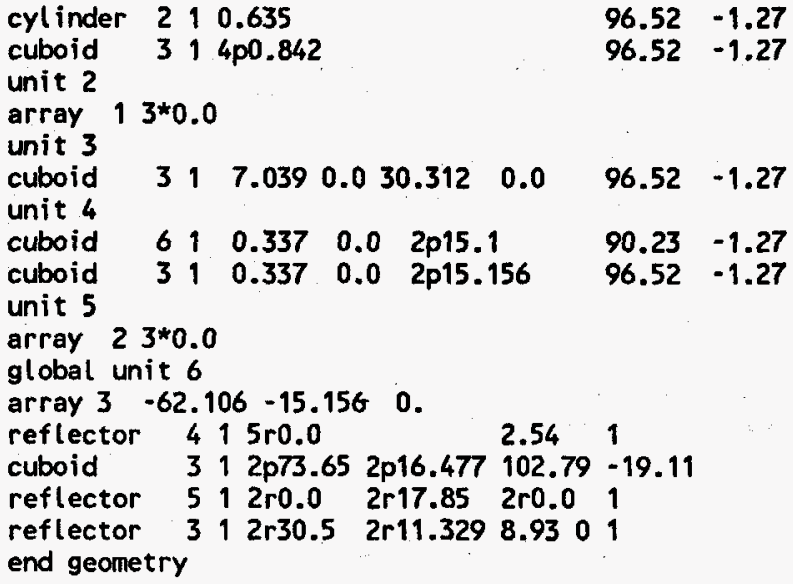

14000.2228304 .7942000 .4924304 .1229000 .135 end cu 6 den=8.910.99 end cd den=8.910.01

end comp

squarepitch 1.6841 .1176131 .272 wt\% cadmiun copper plates and $5.073 \mathrm{~cm}$ of water, assembl ies separated - from stainless steel reflecting walls by $1.321 \mathrm{~cm}$ of water

read parm run=yes gen $=405 \mathrm{npg}=600 \mathrm{nsk}=5$ nub=yes end parm

read geom

unit 1

cylinder $110.5588 \quad 91.44 \quad 0.0$

cylinder 210.635 


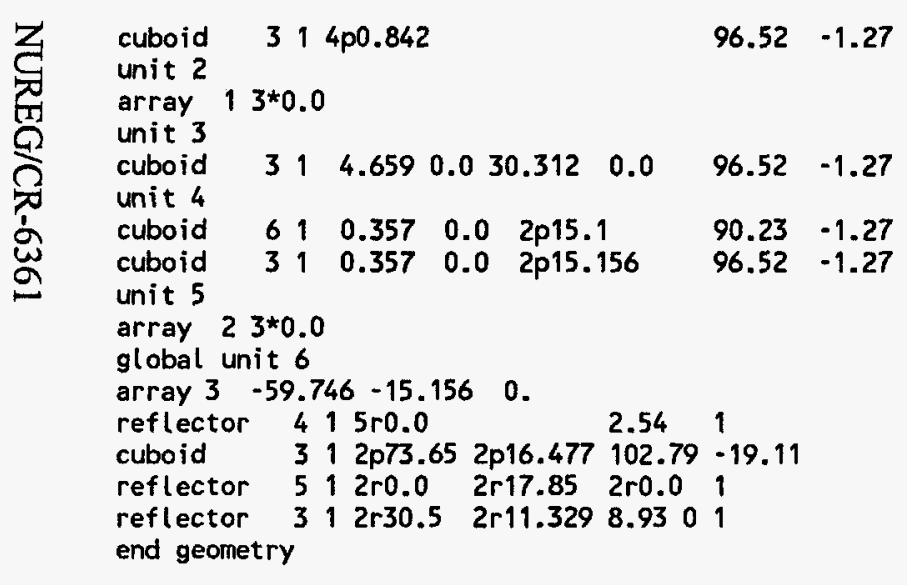

\section{read array}

ara $=1$ nux $=20$ nuy $=18$ nuz $=1$ fill $f 1$

end $f i l l$

ara $=2$ nux $=25$ nuy $=18$ nuz $=1$ fill $f 1$

end fill

ara $=3$ nux=7 nuy=1 nuz=1 fill $2 \quad 3 \quad 4 \quad 5 \quad 4 \quad 3 \quad 2$ end fill

end array

end data

end

요

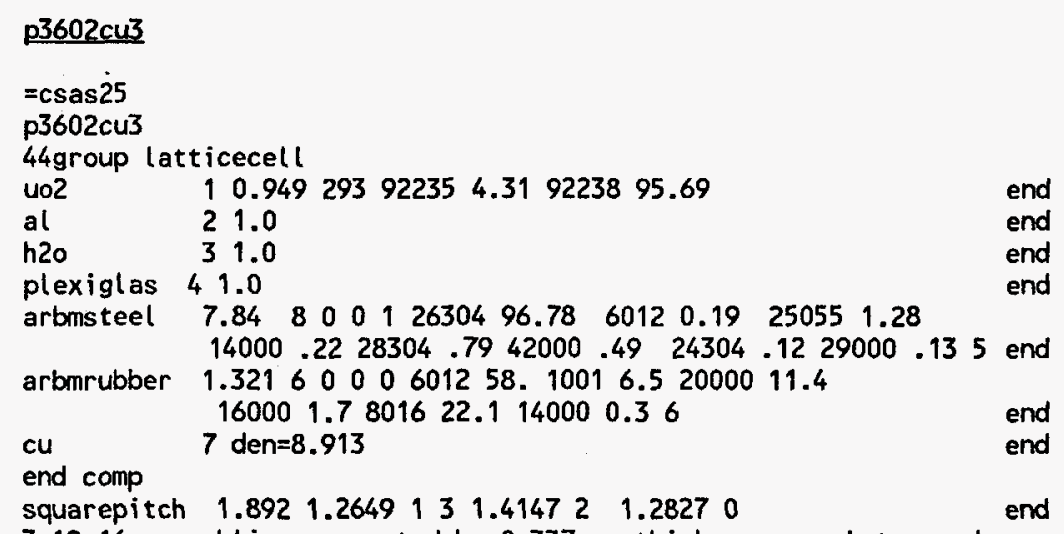

3-12×16 assemblies separated by $0.337-\mathrm{cm}-$ thick copper plates and

$13.133 \mathrm{~cm}$ of water, assemblies separated from stainless steel

reflecting walls by $1.956 \mathrm{~cm}$ of water

read parm run=yes gen $=405 \mathrm{npg}=600 \mathrm{nsk}=5$ nub=yes end parm

\section{read geom}

unit 1

cyl inder 110.63245

cylinder 010.64135 $\begin{array}{ll}91.44 & 0.0 \\ 91.44 & 0.0\end{array}$

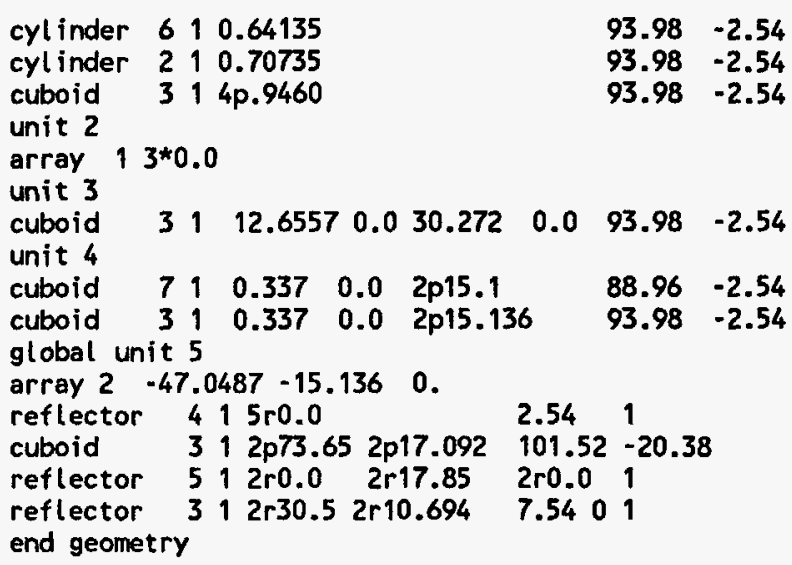




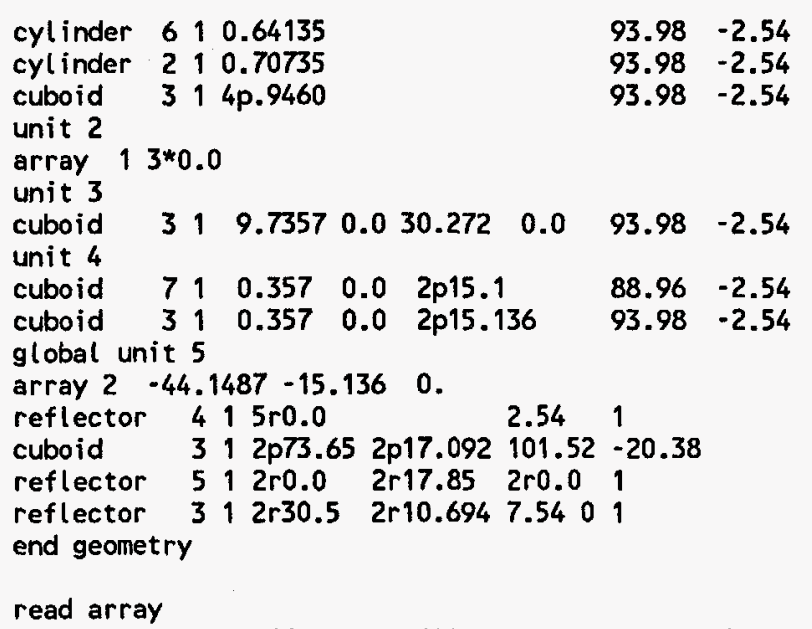

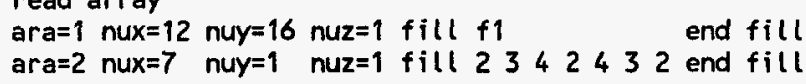

end array

end data

end

\section{p3602n11}

$=\operatorname{csas} 25$

p3602n11

44 group latticecell

uo2 14 group latticecell 0.84293922352 .359223897 .65

al 21.0

plexiglas 41.0

h20 5 i.0

end

end

end

end

$\begin{array}{llllllllllll}\text { arbmsteel } \quad 7.84 & 8 & 0 & 0 & 1 & 26304 & 96.78 & 6012 & 0.19 & 25055 & 1.28\end{array}$

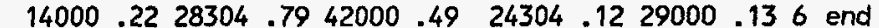

end comp

squarepitch 1.6841 .1176131 .272

end

$2-20 \times 18$ and $1-25 \times 18$ (center) assembl ies separated by $8.98 \mathrm{~cm}$ of water,

read parm run=yes gen $=405 \mathrm{npg}=600 \mathrm{nsk}=5$ nub=yes end parm

read geom

unit 1

cylinder 1110.5588

cylinder 210.635

cuboid 3140.842

$91.44 \quad 0.0$

$96.52-1.27$

unit 2

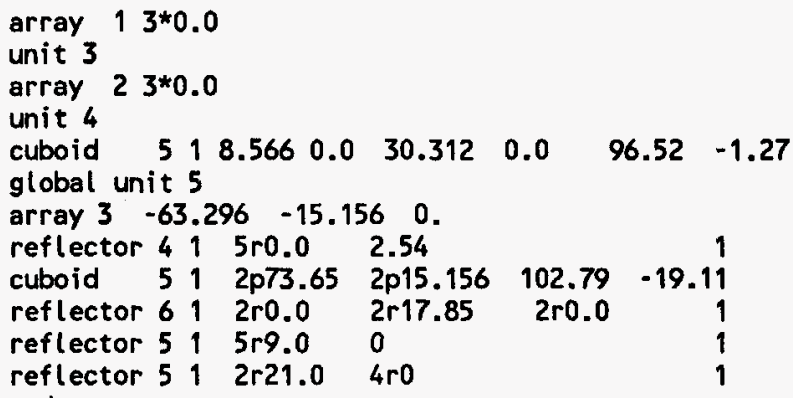

p3602n12

$=c s a s 25$

p3602n12

44 group latticecell

uo2 10.84293922352 .359223897 .65 end

al 21.0 end

20

h20 $\quad 51.0 \quad 7.84 \quad 800,12630496.78 \quad 6012 \quad 0.19 \quad 250551.28$

$\begin{array}{llllllllllll}\text { arbmsteel } & 7.84 & 8 & 0 & 0 & 1 & 26304 & 96.78 & 6012 & 0.19 & 25055 & 1.28 \\ & 14000 & .22 & 28304 & .79 & 42000 & .49 & 24304 & .12 & 29000 & .13 & 6\end{array}$ end

end comp

squarepitch 1.6841 .1176131 .272 end

ies separated by $9.58 \mathrm{~cm}$ of water. assemblies separated from stainless steel reflecting walls by $0.66 \mathrm{~cm}$ of water

read parm run=yes gen $=405 \mathrm{npg}=600 \mathrm{nsk}=5$ nub=yes end parm

read geom

unit 1

cyl inder 110.5588

cylinder 290.635

$\begin{array}{lllll}\text { cuboid } & 3 & 1 & 4 p .842\end{array}$

unit 2

$96.52-1.27$

array $13 * 0.0$

unit 3

array $23 * 0.0$ 
unit 4

$\begin{array}{lllllllll}\text { cuboid } \quad 5 & 1 & 9.166 & 0.0 & 30.312 & 0.0 & 96.52 & -1.27\end{array}$

global unit 5

array $3-63.896-15.156 \quad 0$.

reflector $415 \mathrm{ro} 0$ (2.54

$\begin{array}{lllllll}\text { cuboid } 5 & 1 & 2 p 73.65 & 2 p 15.816 & 102.79 & -19.11\end{array}$

$\begin{array}{lllll}\text { reflector } 6 & 1 & 2 \mathrm{r} 0.0 & 2 \mathrm{r} 17.85 & 2 \mathrm{r} 0.0\end{array}$

reflector 51 1 $5 r 9.0 \quad 0$

reflector 51 2r21.0 4 ro

end geometry

read array

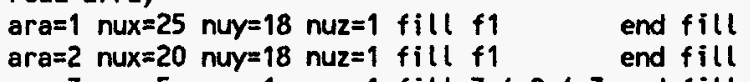

ara $=3$ nux $=5$ nuy=1 nuz=1 fill 342443 end fill

end array

end data

\section{D3602n13}

$=\operatorname{csas} 25$

p3602n13

44group latticecell

10.84293922352 .359223897 .65

21.0

h2o 31.0

plexiglas $4 \begin{aligned} & 4 \\ & \text { h2o }\end{aligned}$

$\begin{array}{llllllllllll}\text { arbmsteel } & 7.84 & 8 & 0 & 0 & 1 & 26304 & 96.78 & 6012 & 0.19 & 25055 & 1.28\end{array}$

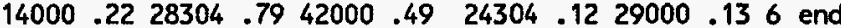

end comp

squarepitch 1.6841 .1176131 .272 end

$2-20 \times 18$ and $1-25 \times 18$ (center) assemblies separated by $9.66 \mathrm{~cm}$ of water

assemblies separated from stainless steel reflecting walls by 1.684

' $\mathrm{cm}$ of water

read parm run=yes gen $=405 \mathrm{npg}=600 \mathrm{nsk}=5$ nub=yes end parm

read geom

unit 1

cylinder $1 \uparrow 0.5588$

cylinder 210.635

cuboid $314 \mathrm{p} .842$

$91.44 \quad 0.0$

unit 2

$96.52-1.27$

array $13 * 0.0$

unit 3

array $23 * 0.0$

unit 4

$\begin{array}{llllllll}\text { cuboid } 5 & 5 & 9.246 & 0.0 & 30.312 & 0.0 & 96.52 & -1.27\end{array}$

global unit 5

array $3 \quad-63.976-15.1560$. $\begin{array}{lllllll}\text { reflector } & 4 & 1 & 5 r 0.0 & 2.54 & & 1 \\ \text { cuboid } & 5 & 1 & 2 p 73.65 & 2 p 16.84 & 102.79 & -19.11\end{array}$

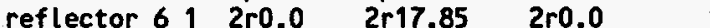

reflector 51 5r9.0

reflector $512 \mathrm{r} 21.0 \quad 4 \mathrm{ro}$

end geometry

read array

ara $=1$ nux $=25$ nuy=18 nuz=1 fill $f 1$ end fill

ara=2 nux=20 nuy=18 nuz=1 fill $f 1$ end fill

ara $=3$ nux $=5$ nuy=1 nuz=1 fill 342443 end fill

end array

end data

end

p3602n14

$=\operatorname{csas} 25$

$03602 n 14$

44 roup latticecell

uo2 1.0 .84293922352 .359223897 .65

al 21.0

h2o $\quad 31.0$

plexiglas 41.0

$\begin{array}{llllllllllllllll}\text { arbmsteel } & 7.84 & 8 & 0 & 0 & 1 & 26304 & 96.78 & 6012 & 0.19 & 25055 & 1.28 & 14000 & .22\end{array}$

$28304.7942000 .49 \quad 24304.12 \quad 29000.13 \quad 6$ end

end comp

squarepitch 1.6841 .1176131 .272

$2-20 \times 18$ and $1-25 \times 18$ (center) assembl ies separated by $8.54 \mathrm{~cm}$ of water, assemblies separated from stainless steel reflecting walls by 3.912 $\mathrm{cm}$ of water

read parm run=yes gen $=405$ npg $=600$ nsk $=5$ nub=yes end parm

read geom

unit 1

cylinder 110.5588

cylinder 210.635

cuboid $314 \mathrm{p} .842$

$91.44 \quad 0.0$

unit 2

$8.52-1.27$

array $13 * 0.0$

unit 3

array $23 * 0.0$

unit 4

$\begin{array}{lllllllll}\text { cuboid } \quad 5 & 1 & 8.126 & 0.0 & 30.312 & 0.0 & 96.52 & -1.27\end{array}$

global unit 5

array $3-62.856-15.156 \quad 0$.

reflector $415 r 0.002 .54$

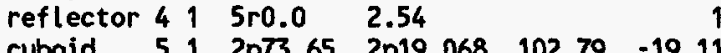

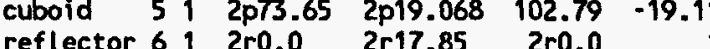

$\begin{array}{llllll}\text { reflector } 6 & 1 & 2 r 0.0 & 2 r 17.85 & 2 r 0.0 & 1 \\ \text { reflector } 5 & 1 & 5 r 9.0 & 0 & & 1\end{array}$ 
reflector 512 21.0 4 ro

end geometry

read array

ara $=1$ nux $=25$ nuy=18 nuz=1 fill $f 1$ end $f i l l$

$a r a=2$ nux $=20$ nuy=18 nuz=1 fill $f 1$ end fill

ara=3 nux $=5$ nuy $=1$ nuz $=1$ fill 344243 end fill

end array

end data

end

23602n21

$=\operatorname{csas} 25$

p3602n21

44 group latticecell

uo2 $10.84293922352 .359223897 .65 \quad$ end

al 21.0

h20 31.0

plexiglas 41.0

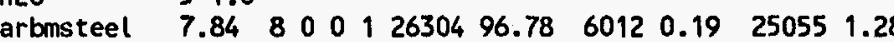

14000.2228304 .7942000 .4924304 .1229000 .136 end

end comp

squarepitch 2.0321 .1176131 .272

No 3-19×16 assemblies separated by $11.20 \mathrm{~cm}$ of water, assemblies

separated from stainless steel reflecting walls by $0.66 \mathrm{~cm}$ of water

read parm run=yes gen $=405$ npg $=600$ nsk $=5$ nub=yes end parm

read geom

unit

cylinder 110.5588

cylinder 210.635

cuboid $314 \mathrm{p} 1.016$

$96.52-1.27$

unit 2

$96.52-1.27$

array $13 * 0.0$

unit 3

cuboid $519.598 \quad 0.0 \quad 32.512 \quad 0.0 \quad 96.52 \quad-1.27$

global unit

array $2-67.51 \quad-16.256 \quad 0$.

reflector $4125 \mathrm{r} 0.0 \quad 2.54$

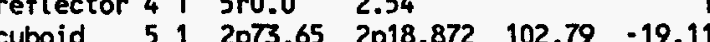

$\begin{array}{lllll}\text { reflector } 61 & 1 & 20.0 & 2 r 17.85 & 2 \times 0.0\end{array}$

reflector 61125.0

reflector 51 2r21.0 4 ro

$4 \mathrm{ro}$

end geometry

read array

ara=1 nux=19 nuy=16 nuz=1 fill $f 1 \quad$ end fill

ara $=2$ nux $=5$ nuy=1 nuz=1 fill $2 \quad 3 \quad 2 \quad 3 \quad 2$ end fill

end array end data

end

103602n22

$=\operatorname{csas} 25$

$03602 n 22$

44 roup latticecell

Uo2 $\quad 10.84293922352 .359223897 .65$

al 21.0

h2o 31.0

plexiglas 41.0

arbmsteel $\quad \begin{array}{llllllllllll}7.84 & 8 & 0 & 0 & 1 & 26304 & 96.78 & 6012 & 0.19 & 25055 & 1.28\end{array}$ $14000 \quad 22 \quad 28304 \quad 79 \quad 42000.49 \quad 24304.1229000 .136$ end

end comp

squarepitch 2.0321 .1176131 .272 read parm run=yes gen $=405$ npg $=600$ nsk $=5$ nub $=y e s$ end parm

read geom

unit 1

cylinder 110.5588

cylinder 210.635

cuboid $314 \mathrm{pl} .016$

unit 2

$96.52-1.27$

array $13 * 0.0$

unit 3

$\begin{array}{lllllllll}\text { cuboid } \quad 5 & 1 & 10.438 & 0.0 & 32.512 & 0.0 & 96.52 & -1.27\end{array}$

global unit 4

array $2-68.35 \quad-16.256 \quad 0$

reflector 41 5ro.0 2.54

$\begin{array}{lllllll}\text { cuboid } 5 & 1 & 2 p 73.65 & 2 p 16.916 & 102.79 & -19.11\end{array}$

reflector $612 \mathrm{r} 0.0 \quad 2 \mathrm{r} 17.85 \quad 2 \mathrm{r0.0}$

reflector $5115 \mathrm{rO}^{\circ} \mathrm{O}$

reflector 512 r21.0 $4 \mathrm{r}^{\circ}$

end geometry

read array

ara $=1$ nux=19 nuy=16 nuz=1 fill $f 1$ end fill

ara=2 nux=5 nuy=1 nuz=1 fill 232232 end fill

end array

end data

end

$123002 n 31$

$=c s a s 25$

p3602n31

44group latticecell

end
end
end
end
end 
arbmsteel $7.84 \quad 8 \quad 0 \quad 0 \quad 12630496.78 \quad 6012 \quad 0.19 \quad 25055 \quad 1.28 \quad$ end $\begin{array}{lllllllllll}14000 & .22 & 28304 & .79 & 42000 & .49 & 24304 & .12 & 29000 & .13 & 6\end{array}$ end end comp

squarepitch $1.892 \quad 1.2649131 .41472 \quad 1.28270$

end

$3-12 \times 16$ assembl ies separated by $14.87 \mathrm{~cm}$ of water, assemblies

separated from stainless steel reflecting walls by $0.0 \mathrm{~cm}$ of water read parm run=yes gen $=405$ npg $=600$ nsk $=5$ nub=yes end parm

\section{read geom}

unit 1

cyl inder 110.63245

$91.44 \quad 0.0$

cyl inder 010.64135

$91.44 \quad 0.0$

cyl inder 710.64135

$93.98-2.54$

cyl inder 210.70735

$93.98-2.54$

unit 2

$93.98-2.54$

array $13 * 0.0$

unit 3

$\begin{array}{lllllllll} & 51 & 14.3927 & 0.0 & 30.272 & 0.0 & 93.98 & -2.54\end{array}$

global unit 5

array $2-48.4487-15.1360$.

reflector $\quad 415 r 0.0 \quad 2.541$

$\begin{array}{lllllll}\text { cuboid } \quad 512 p 73.65 & 2 p 15.136 & 101.52 & -20.38\end{array}$

reflector 612 ro.0 2 r17.85 2 r0.0 1

reflector $515 \mathrm{r8.0} \quad 0 \quad 1$

reflector $514 r 3.0$ 2ro 1

reflector $512 \mathrm{r} 20.0 \quad 4 \mathrm{ro} 1$

end geometry

read array

ara $=1$ nux=12 nuy=16 nuz=1 fill $f 1$ end fill

ara $=2$ nux=5 nuy=1 nuz=1 fill $2323 \quad 2$ end fill

end array

end data

\section{$103602 n 32$}

$=\operatorname{csas} 25$

p3602n32

44group latticecell

402 $\quad 10.949293922354 .319223895 .69$

$\begin{array}{lll}\text { al } & 2 & 1.0 \\ \text { h20 } & 3 & 1.0\end{array}$

end

end

$$
\text { nd }
$$

r

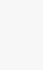


arbmsteel $\quad \begin{array}{lllllllllll}7.84 & 8 & 0 & 0 & 1 & 26304 & 96.78 & 6012 & 0.19 & 25055 & 1.28\end{array}$ $14000.22 \quad 28304.79 \quad 42000.49 \quad 24304.12 \quad 29000.136$ end arbmrubber

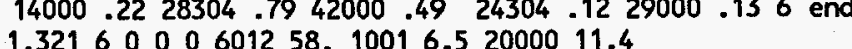
$160001.7801622 .1 \quad 14000 \quad 0.37$

end comp

squarepitch 1.8921 .2649131 .414721 .28270 end

$3-12 \times 16$ assemblies separated by $15.87 \mathrm{~cm}$ of water, assemblies - separated from stainless steel reflecting walls by $1.321 \mathrm{~cm}$ of water read parm run=yes gen $=405$ npg $=600$ nsk $=5$ nub=yes end parm

\section{read geom}

unit

cylinder 1110.63245

cylinder 010.64135

cylinder $7,0.64135$

cylinder 210.70735

cuboid $314 \mathrm{p} .9460$

$91.44 \quad 0.0$

$93.98-2.54$

unit 2

$93.98-2.54$

array $13 * 0.0$

unit 3

$\begin{array}{llllllll}\text { cuboid } \quad 51 & 515.3927 & 0.0 & 30.272 & 0.0 & 93.98 & -2.54\end{array}$

global unit 5

array $2 \quad-49.4487-15.136 \quad 0$.

reflector $\quad 415 r 0.0 \quad 2.541$

$\begin{array}{llllllll} & \text { cuboid } & 5 & 1 & 2 \mathrm{p} 73.65 & 2 \mathrm{p} 16.457 & 101.52 & -20.38\end{array}$

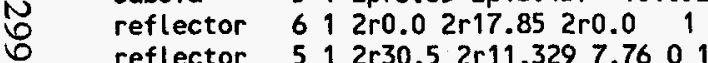

end geometry

read array

ara $=1$ nux $=12$ nuy=16 nuz $=1$ fill $f 1$ end $f i l l$

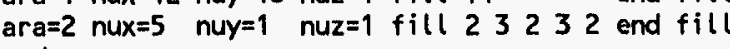

end array

end data

end

\section{p3602n34}

$=\operatorname{csas} 25$

p3602n34

44group latticecell

44 group lat

al $\quad 21.0$

h2o 31.0

plexiglas 41.0

h2o 51.0

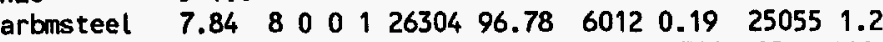

arbmrubber $1.3216000601258 .10016 .520000 \quad 11.4$

160001.7801622 .1140000 .37 end comp

squarepitch $1.892 \quad 1.2649131 .414721 .28270$

$15.84 \mathrm{~cm}$ of water, assemblies

separated from stainless steel reflecting walls by $1.956 \mathrm{~cm}$ of water read parm run=yes gen $=405 \mathrm{npg}=600 \mathrm{nsk}=5$ nub=yes end parm

read geom

unit 1

cylinder 110.63245

cyl inder 0 i 0.64135

cylinder $7,10.64135$

cylinder $\begin{array}{llll}7 & 1 & 0.64135 \\ \text { cylinder } & 2 & 1 & 0.70735\end{array}$

$\begin{array}{llll}\text { cyl inder } & 2 & 1 & 0.70735 \\ \text { cuboid } & 3 & 1 & 40.9460\end{array}$

$91.44 \quad 0.0$

$93.98-2.54$

$93.98-2.54$

unit 2

$93.98-2.54$

array $13 * 0.0$

unit 3

$\begin{array}{lllllllll}\text { cuboid } & 5 & 1 & 15.3627 & 0.0 & 30.272 & 0.0 & 93.98 & -2.54\end{array}$

global unit 5

array $2-49.4187-15.1360$.

reflector $415 r 0.0 \quad 2.541$

$\begin{array}{llllll}\text { cuboid } \quad 512 p 73.65 & 2 p 17.092 \quad 101.52 & -20.38\end{array}$

reflector $612 \mathrm{2r0} .02 \mathrm{2r} 17.852 \mathrm{2r0} .0$

reflector $515 r 8.0 \quad 01$

reflector $514 \mathrm{r} 3.0 \quad 2 \mathrm{r} 01$

reflector $512 \mathrm{r} 20.0 \quad 4 \mathrm{ro} \quad 1$

end geometry

read array

ara $=1$ nux $=12$ nuy $=16$ nuz $=1$ fill $f 1$ end $f i l l$

ara $=2$ nux=5 nuy=1 nuz=1 fill $2 \quad 3 \quad 2 \quad 3 \quad 2$ end fill

end array

end data

end

p3602n35

$=\operatorname{csas} 25$

$03602 n 35$

44 group latticecell

uo2 10.949293922354 .319223895 .69

al 21.0

h20 $\quad 31.0$

plexiglas 41.0

51.0

$\begin{array}{lllllllllllllll}\text { arbmsteel } & 7.84 & 8 & 0 & 0 & 1 & 26304 & 96.78 & 6012 & 0.19 & 25055 & 1.28 & 14000 & 22\end{array}$

$\begin{array}{lllllllllll}28304 & 79 & 42000 & 49 & 24304 & .12 & 29000 & .13 & 6\end{array}$ end

arbmrubber 1.32160000601258 .10016 .52000011 .4 160001.7801622 .1140000 .37

end comp

squarepitch $1.8921 .2649131 .41472 \quad 1.28270$

end

$3-12 \times 16$ assemblies separated by $15.45 \mathrm{~cm}$ of nater, assemblies 
separated from stainless steel reflecting walls by $2.616 \mathrm{~cm}$ of water read parm run=yes gen $=405$ npg $=600$ nsk $=5$ nub=yes end parm

\section{read geom}

cylinder 110.63245

cylinder 010.64135

cylinder 710.64135

cylinder 210.70735

cuboid $314 \mathrm{p} .9460$

unit 2

$91.44 \quad 0.0$

$\begin{array}{ll}91.44 & 0.0 \\ 93.98 & -2.54\end{array}$

$93.98-2.54$

$93.98-2.54$

array $13 * 0.0$

unit 3

$\begin{array}{llllllllll}\text { cuboid } & 5 & 1 & 14.9727 & 0.0 & 30.272 & 0.0 & 93.98 & -2.54\end{array}$

global unit 5

array $2 \quad-49.0287-15.136 \quad 0$.

reflector $415 \mathrm{r} 0.0 \quad 2.541$

$\begin{array}{llllllll} & 51 & 2 p 73.65 & 2 p 17.752 & 101.52 & -20.38\end{array}$

reflector $612 \mathrm{ro} .02 \mathrm{r} 17.852 \mathrm{r0} .0$

reflector $515 r 8.0 \quad 01$

reflector $514 \mathrm{r} 3.0 \quad 2 \mathrm{r} 0 \quad 1$

reflector 512 r20.0 4 ro 1

end geometry

read array

ara=1 nux=12 nuy=16 nuz=1 $f i l l$ fl end $f i l l$

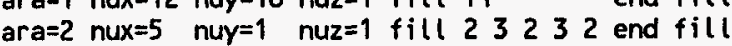

end array

end data

enc

D3602n36

$=c s a s 25$

p3602n 36

4 group latticecel

uo2 10.949293922354 .319223895 .69 end

at 21.0

h2o $\quad 31.0$

plexiglas 41.0

h20 51.0

arbmsteel $\quad \begin{array}{lllllllllll}7.84 & 8 & 0 & 0 & 1 & 26304 & 96.78 & 6012 & 0.19 & 25055 & 1.28\end{array}$

$14000.2228304 .7942000 .49 \quad 24304.1229000 .136$ end

arbmrubber 1.3216000601258 .10016 .52000011 .4

160001.7801622 .1140000 .37

end comp

end

squarepitch $1.892 \quad 1.2649131 .41472 \quad 1.28270$

$3-12 \times 16$ assemblies separated by $13.82 \mathrm{~cm}$ of water, assembl ies

separated from stainless steel reflecting walls by $5.405 \mathrm{~cm}$ of water read parm run=yes gen $=405$ npg $=600$ nsk $=5$ nub=yes end parm read geom

unit 1

cylinder $110.63245 \quad 91.44 \quad 0.0$

cylinder 0110.64135

$91.44 \quad 0.0$

$93.98-2.54$

$\begin{array}{llllll}\text { cyl inder } & 7 & 1 & 0.64135 & 93.98 & -2.54 \\ \text { cyl inder } 2 & 1 & 0.70735 & 93.98 & -2.54 \\ \text { cuboid } & 3 & 1 & 4 p .9460 & 93.98 & -2.54\end{array}$

cuboid $314 \mathrm{p} .9460$

$93.98-2.54$

array $13 * 0.0$

unit 3

$\begin{array}{llllllllll}\text { cuboid } 5 & 5 & 1 & 13.3427 & 0.0 & 30.272 & 0.0 & 93.98 & -2.54\end{array}$

global unit 5

array $2-47.3987-15.1360$.

reflector $415 \mathrm{r} 0.0 \quad 2.541$

$\begin{array}{llllllll}\text { cuboid } \quad 5 & 1 & 2 \mathrm{p} 73.65 & 2 \mathrm{p} 20.541 & 101.52 & -20.38\end{array}$

reflector $\quad 612$ r0.0 2r17.85 2r0.0 1

reflector $51958.0 \quad 0 \quad 1$

reflector $514 \mathrm{r} 3.0 \quad 2 r 0$

reflector $512 \mathrm{r} 20.0 \quad 4 \mathrm{rO}$

end geometry

read array

ara $=1$ nux $=12$ nuy=16 nuz=1 fill $f 1$ end $\mathrm{fill}$ ara $=2$ nux=5 nuy=1 nuz=1 fill $2 \quad 3 \quad 2 \quad 3 \quad 2$ end fill

end array

end data

end

$3602 n 41$

$=\operatorname{csas} 25$

p3602n41

44group latticecell

402 10.949293922354319223895 .69

al

h20 $\quad 31.0$

plexiglas 41.0

$\begin{array}{lllllllllllll}\text { arbmsteel } & 7.84 & 8 & 0 & 0 & 1 & 26304 & 96.78 & 6012 & 0.19 & 25055 & 1.28\end{array}$

$\begin{array}{lllllllllll}14000 & .22 & 28304 & .79 & 42000 & 49 & 24304 & .1229000 & 136 & \text { end }\end{array}$

arbmrubber 1.3216000601258 .10016 .52000011 .4 160001.7801622 .1140000 .37

end

end comp

squarepitch 2.5401 .2649131 .414721 .28270

3-13×8 assemblies separated by $12.89 \mathrm{~cm}$ of water, assemblies

separated from stainless steel reflecting walls by $0.0 \mathrm{~cm}$ of water

read parm run=yes gen $=405 \mathrm{npg}=600$ nsk $=5$ nub=yes end parm

read geom

unit 1

cyl inder 110.63245

$91.44 \quad 0.0$ 


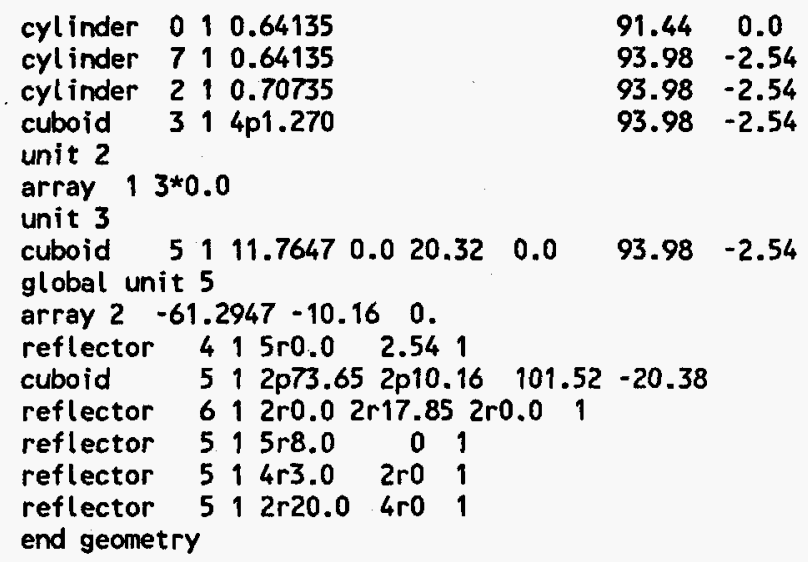

\section{$3602 n 42$}

$=\operatorname{csas} 25$

p3602n42

44group latticecell

uo2 10.949293922354 .319223895 .69 end

at 21.0

h20 31.0

plexiglas 41.0

h20 51.0

arbmsteel $\quad 7.84800012630496 .7860120 .1925055 \quad 1.28 \quad 14000$ $.22 \quad 28304.7942000 \quad .49 \quad 24304.12 \quad 29000.13 \quad 6$

arbmrubber 1.3216000601258 .10016 .52000011 .4

$160001.7801622 .114000 \quad 0.37$

end comp

gquarepitch 2.5401 .2649131 .414721 .28270

$3-13 \times 8$ assemblies separated by $14.12 \mathrm{~cm}$ of water, assemblies read parm run=yes gen $=405 \mathrm{npg}=600 \mathrm{nsk}=5$ nub $=y e s$ end parm

\section{read geom}

unit 1

cylinder 110.63245

cylinder 0110.64135

cylinder 710.64135

cylinder 210.70735

$\begin{array}{cc}91.44 & 0.0 \\ 91.44 & 0.0 \\ 93.98 & -2.54 \\ 93.98 & -2.54\end{array}$

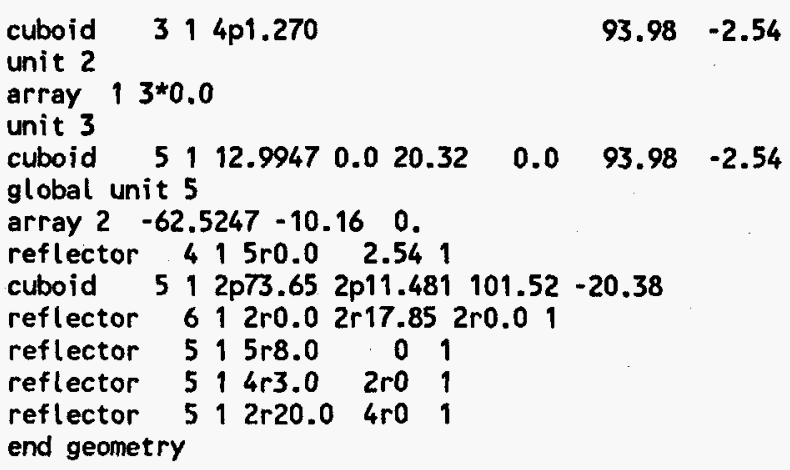

read array

ara=1 nux=13 nuy=8 nuz $=1$ fill $f 1$ end fill

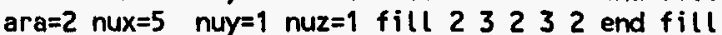

end array

end data

end

\section{p3602n43}

$=\operatorname{csas} 25$

$3602 n 43$

44 group latticecell

uo2 10.949293922354 .319223895 .69 end

al 21.0

h2o $\quad 31.0$

plexiglas 41.0

h20 51.0

$\begin{array}{lllllllllllll} & 7.84 & 8 & 0 & 0 & 1 & 26304 & 96.78 & 6012 & 0.19 & 25055 & 1.28\end{array}$ arbmrubber 1.3216000601258 .10016 .52000011 .4

end comp 160001.7801622 .1140000 .37

squarepitch 2.5401 .2649131 .414721 .28270

end

3-13x8 assemblies separated by $12.44 \mathrm{~cm}$ of water, assembl ies

separated from stainless steel reflecting walls by $2.616 \mathrm{~cm}$ of water read parm run=yes gen $=405$ npg $=600$ nsk $=5$ nub=yes end parm

read geon

unit 1

cylinder 110.63245

cylinder 0110.64135

cylinder 710.64135

cylinder 210.70735

cylinder 210.70735

cuboid $314 \mathrm{pl} .270$

array $13 * 0.0$
$91.44 \quad 0.0$

$93.98-2.54$

$93.98 \quad-2.54$

$\begin{array}{lll}93.98 & -2.54\end{array}$ 


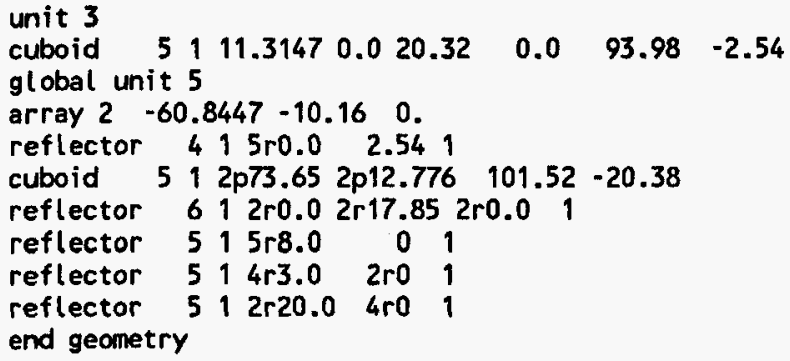

p3602ss1

\section{csas 25}

p3602ss

44 group latticecell

(a)

31.0

$\begin{array}{llllllllllll}\text { arbmsteel } & 7.84 & 8 & 0 & 0 & 1 & 26304 & 96.78 & 6012 & 0.19 & 25055 & 1.28\end{array}$

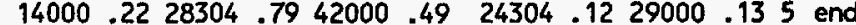

crss $\quad 6$ den=7.93.1856 end

cu 6 den=7.93 .0027 end

fess 6 den=7.93 .6824 end

mn 6 den=7.93 .0158 end

$\begin{array}{llll}\text { mo } & 6 \text { den=7.93 } .0026 & \text { end } \\ \text { niss } & 6 \text { den=7.93 } & & \end{array}$

end comp

squarepitch 1.6841 .1176131 .27

$2-20 \times 18$ and $1-25 \times 18$ (center) assemblies separated by $0.302-c m$ thick

$304 \mathrm{~L}$ stainless steel plates and $7.978 \mathrm{~cm}$ of water, assemblies

separated from stainless steel reflecting walls by $1.321 \mathrm{~cm}$ of water read parm run=yes gen $=405 \mathrm{npg}=600 \mathrm{nsk}=5$ nub=yes end parm

read geom

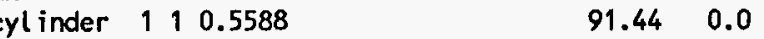

cylinder $210.635 \quad 96.52-1.27$

$\begin{array}{lllllllll}\text { cuboid } \quad 3 & 1 & 0.842 & -0.842 & 0.842 & -0.842 & 96.52 & -1.27\end{array}$

unit 2

array $13 * 0.0$

unit 3

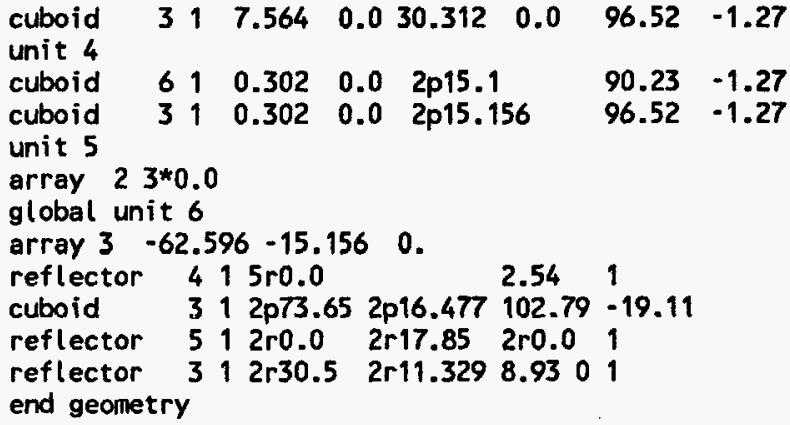

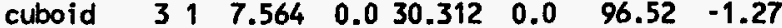

unit 4

cuboid

$\begin{array}{lllll}6 & 1 & 0.302 & 0.0 & 2 p 15.1\end{array}$

$90.23-1.27$

cuboid

unit 5

array $23 * 0.0$

global unit 6

array $3-62.596-15.156 \quad 0$

reflector $\quad 415 \mathrm{ro.0} \quad 2.54$,

$\begin{array}{lllllll}\text { cuboid } & 3 & 1 & 2 \mathrm{p} 73.65 & 2 \mathrm{p} 16.477 & 102.79 & -19.11\end{array}$

reflector 51 2r0.0 2 r17.85 2 ro.0 1

reflector $312 r 30.5 \quad 2 r 11.3298 .9301$

end geometry

read array

ara=1 $n u x=20$ nuy=18 nuz=1 fill $f$

ara $=3$ nux $=7$ nuy=1 nuz=1 fill 2345432 end fill

end array

end data

end

\section{p3602ss?}

$=\operatorname{csas} 25$

p3602ss2

44group latticecel

uo2 10.949293922354 .319223895 .69 end

at 21.0

h20 31.0

plexiglas 41.0

$\begin{array}{lrllllllllll}\text { arbmsteel } & 7.84 & 8 & 0 & 0 & 1 & 26304 & 96.78 & 6012 & 0.19 & 25055 & 1.28\end{array}$ 14000.2228304 .7942000 .4924304 .1229000 .135 end

arbmrubber 1.3216000601258 .10016 .52000011 .4 160001.7801622 .1140000 .36

crss $\quad 7$ den $=7.93 .1856$

cu $\quad 7$ den=7.93 .0027

fess $\quad 7$ den=7.93 .6824

mn $\quad 7$ den=7.93 .0158

mo 7 den=7.93.0026

niss

7 den=7.93 .1109

end comp

squarepitch 1.8921 .2649131 .414721 .28270

$3-12 \times 16$ assemblies separated by $0.302-c m$-thick $304 \mathrm{~L}$ stainless steel -plates and $13.448 \mathrm{~cm}$ of water, assembl ies separated from stainless 'steel reflecting walls by $1.956 \mathrm{~cm}$ of water

read parm run=yes gen $=405 \mathrm{npg}=600 \mathrm{nsk}=5$ nub=yes end parm

read geom

unit 1

cylinder $110.63245 \quad 91.44 \quad 0.0$ 


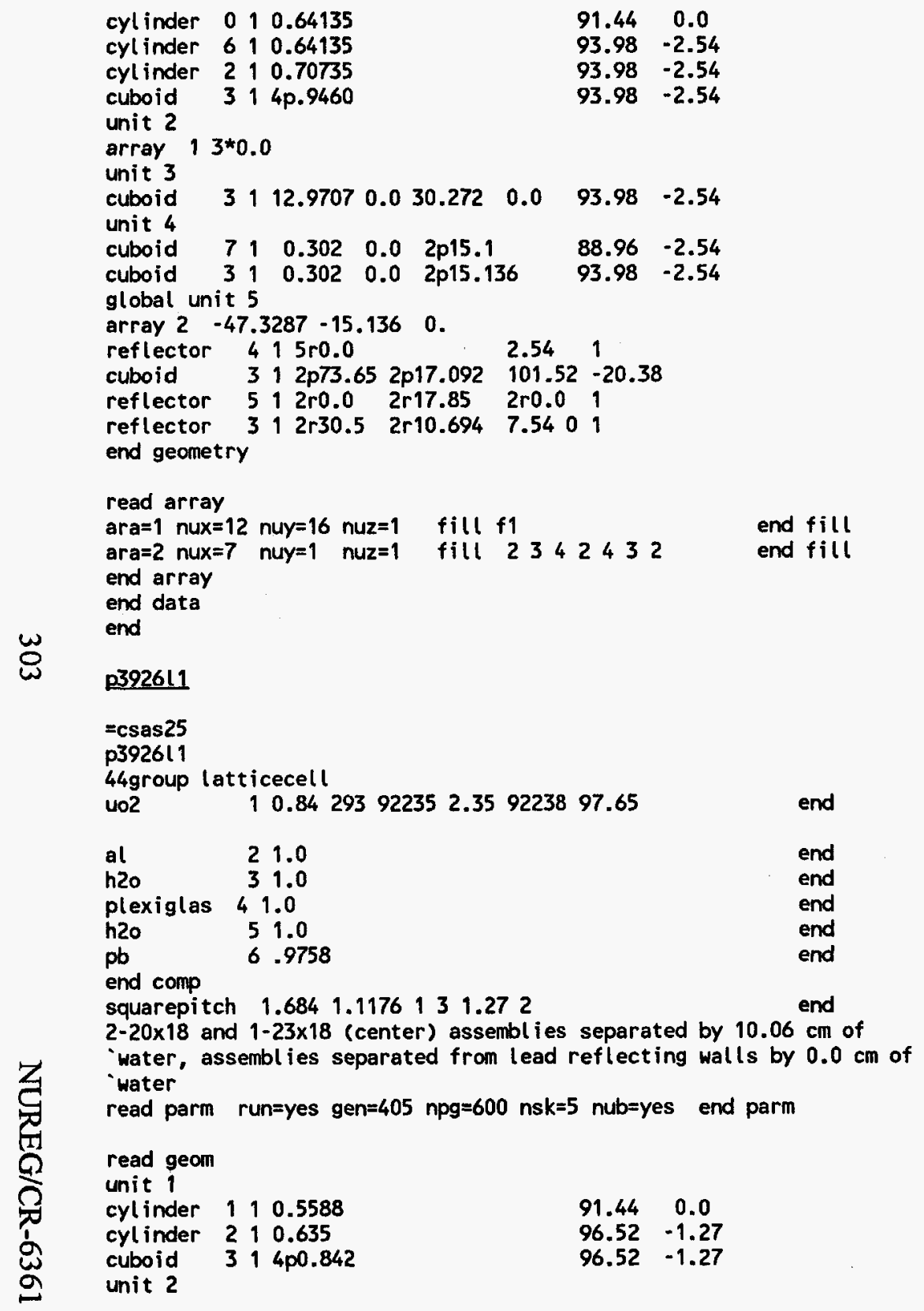

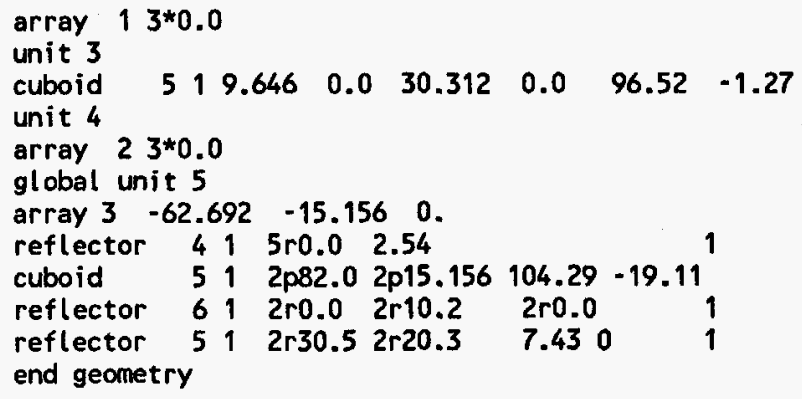$$
\text { global unit } 5
$$ 
array $3 \quad-62.742 \quad-15.156 \quad 0$.

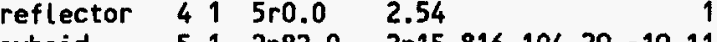

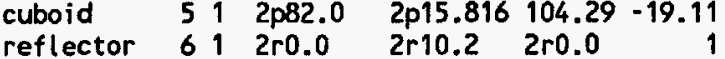

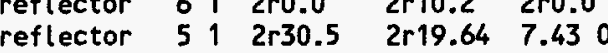

end geometry

read array

ara $=1$ nux $=23$ nuy=18 nuz $=1$ fill $f 1$ end $f i l l$

ara $=2$ nux $=20$ nuy $=18$ nuz=1 fill $f 1$ end fill

ara=3 nux=5 nuy=1 nuz=1 fill $4 \begin{array}{llllll}3 & 2 & 3 & 4 & \text { end fill }\end{array}$

end array

end data

end

\section{D392613}

$=\operatorname{csas} 25$

p392613

44 group latticecel

uo2 10.84293922352 .359223897 .65 end

at 21.0

h20 31.0

plexiglas $4 \quad 1.0$

h20 51.0

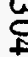

$\begin{array}{ll}5 & 1.0 \\ 6 & .9758\end{array}$

end

end

end

end

end

end comp

squarepitch $1.6841 .117613 \quad 1.272$ 2 water, assemblies separated from lead reflecting walls by $3.276 \mathrm{~cm}$ of water

read parm run=yes gen $=405$ npg=600 nsk=5 nub=yes end parm

read geom

unit 1

cyl inder 1110.5588

cylinder 210.635

cuboid $314 \mathrm{p0} .842$

$91.44 \quad 0.0$

$96.52-1.27$

unit 2

array $13 * 0.0$

unit 3

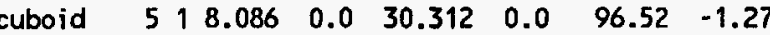

unit 4

array $23 * 0.0$

global unit 5

array $3 \quad-61.132 \quad-15.156 \quad 0$.

reflector 41 5ro.0 2.54

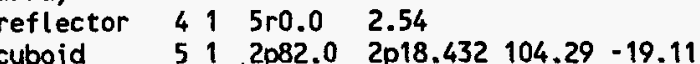

$2 p 18.432104 .29-19.11$

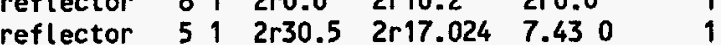

end geometry read array

ara=1 nux=23 nuy=18 nuz=1 fill $f 1$ end fill

ara $=2$ nux $=20$ nuy $=18$ nuz=1 fill $f 1$ end fill

ara $=3$ nux $=5$ nuy $=1$ nuz $=1$ fill $4 \begin{array}{lllllll}3 & 2 & 3 & 4 & \text { end } f i l l\end{array}$

end array

end data

end

2392614

$=\operatorname{csas} 25$

p392614

44 group latt icecel

uo2 10.949293922354 .319223895 .69 end

al 21.0

h20 31.0

plexiglas 41.0

h20 51.0

pb 6.9758

arbrirubber 1.3216000601258 .10016 .52000011 .4 160001.7801622 .1140000 .37

end comp

squarepitch $1.892 \quad 1.2649131 .414721 .28270$ end

3-12x16 assemblies separated by $17.74 \mathrm{~cm}$ of water, assemblies

separated from lead reflecting walls by $0.0 \mathrm{~cm}$ of water

read parm run=yes gen $=405 \mathrm{npg}=600$ nsk $=5$ nub=yes end parm

read geon

unit 1

cyl inder $1110.63245 \quad 91.44 \quad 0.0$

cyl inder $0.10 .64135 \quad 91.44 \quad 0.0$

cylinder $710.64135 \quad 93.98 \quad-2.54$

cyl inder $210.70735 \quad 93.98 \quad-2.54$

$\begin{array}{lllll}\text { cuboid } 314 \mathrm{p0} .946 & 93.98 & -2.54\end{array}$

unit 2

array $13 * 0.0$

unit 3

$\begin{array}{lllllllll}\text { cuboid } & 5 & 1 & 17.2627 & 0.0 & 30.272 & 0.0 & 93.98 & -2.54\end{array}$

global unit 4

array $2-51.3187-15.1360$.

reflector $\quad 415 r 0.0 \quad 2.54$

$\begin{array}{llllll}\text { cuboid } \quad 512 p 82.0 & 2 p 15.136 & 103.02 & -20.38\end{array}$

$\begin{array}{llllll}\text { reflector } \quad 6 & 1 & 2 \mathrm{r} 0.0 & 2 \mathrm{r} 10.2 & 2 \mathrm{r} 0.0 & 1\end{array}$

reflector $\quad 512 r 30.5 \quad 2 r 20.3 \quad 6.1601$

end geometry

read array

ara $=1$ nux $=12$ nuy=16 nuz=1 $\mathrm{fill} f 1$ end $\mathrm{fill}$

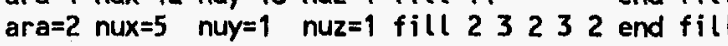

end array 
end data

end

\section{p392615}

\section{$=\operatorname{csas} 25$}

p392615

$\begin{array}{lllll}\text { 44group latticecell } & & & \\ \text { uo2 } & 1 & 0.949 & 293922354.319223895 .69\end{array}$

$\begin{array}{lllllll}\text { uo2 } & 1 & 0.949293922354 .319223895 .69 & \text { end } \\ \text { al } & 2 & 1.0 & 3 & \end{array}$

al 21.0

hexiglas 41.0

h20 $\quad 51.0$

pb. $\quad 6.9758$

arbmrubber $1.3216000601258,10016.52000011 .4$

160001.7801622 .1140000 .37

end comp

squarepitch 1.8921 .2649131 .414721 .28270

separated from lead reflecting walls by $0.66 \mathrm{~cm}$ of water

read parm run=yes gen $=405 \mathrm{npg}=600 \mathrm{nsk}=5$ nub=yes end parm

read geom

unit 1

cylinder 110.63245

cylinder 010.64135

cylinder 710.64135

cylinder 210.70735

cuboid $314 \mathrm{p0} .946$

cuboid

unit 2 array $13 * 0.0$

array 3

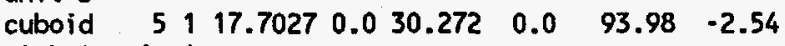

global unit 4

array $2-51.7587-15.136 \quad 0$

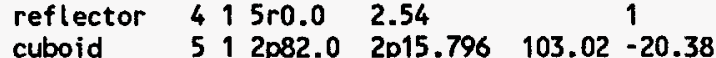

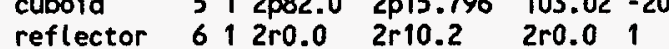

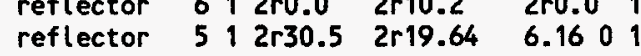

end geometry

\section{read array}

ara=1 nux=12 nuy=16 nuz=1 fill $f 1$ end fill

ara=2 nux=5 nuy=1 nuz=1 fill 232232 end fill

end array

end data

end
1292616

$=\operatorname{csas} 25$

p392616

44 group latticecel!

uo2 10.949293922354 .319223895 .69 end

at 21.0

h20 $\quad 31.0$

plexiglas 41.0

h20 51.0

pb $\quad 6.9758$

1.32160000601258 .10016 .52000011 .4 160001.7801622 .1140000 .37

end comp

squarepitch $1.8921 .2649131 .41472 \quad 1.28270$

作 separated from lead reflecting walls by $1.956 \mathrm{~cm}$ of water read parm run=yes gen $=405 \mathrm{npg}=600 \mathrm{nsk}=5$ nub=yes end parm

read geom

unit 1

cylinder 110.63245

cylinder 0

cylinder 0

cylinder 710.64135

$\begin{array}{lllll}\text { cylinder } & 2 & 1 & 0.70735 \\ \text { cuboid } & 3 & 1 & 4 \mathrm{p} 0.946\end{array}$

$91.44 \quad 0.0$

$\begin{array}{ll}91.44 & 0.0 \\ 93.98 & -2.54\end{array}$

$93.98-2.54$

array $13 * 0.0$

unit 3

$\begin{array}{llllllllll}\text { cuboid } & 5 & 1 & 16.9527 & 0.0 & 30.272 & 0.0 & 93.98 & -2.54\end{array}$

global unit 4

array $2-51.0087-15.1360$

reflector $\quad 415 \mathrm{ro} 0.0 \quad 2.54$

$\begin{array}{lllllll}\text { cuboid } \quad 5 & 1 & 2 p 82.0 & 2 p 17.092 & 103.02 & -20.38\end{array}$

$\begin{array}{lllllll}\text { reflector } & 6 & 1 & 2 \mathrm{r} 0.0 & 2 \mathrm{r} 10.2 & 2 \mathrm{r} 0.0 & 1\end{array}$

reflector $\quad 512 r 30.5 \quad 2 r 18.3446 .1601$

end geometry

read array

ara $=1$ nux $=12$ nuy=16 nuz=1 fill $f 1$ end $f i l l$ ara=2 nux=5 nuy=1 nuz=1 fill $23 \quad 3 \quad 2 \quad 3 \quad 2$ end fill end array

end data

end

03926sl1

$=\operatorname{csas} 25$

p3926s 11

44group latticecell

uo2

10.84293922352 .359223897 .65 


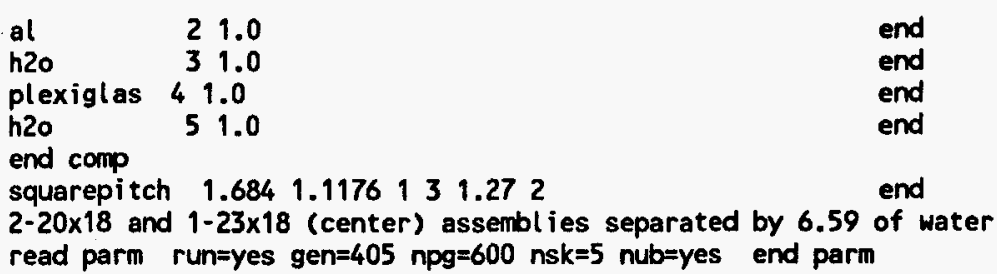

\section{read geon}

unit

cylinder 110.5588

cylinder 210.635

cuboid $314 \mathrm{p} 0.842$

$91.44 \quad 0.0$

unit 2

array $13 * 0.0$

unit 3

$\begin{array}{lllllllll}\text { cuboid } & 5 & 1 & 6.176 & 0.0 & 30.312 & 0.0 & 96.52 & -1.27\end{array}$

unit 4

array $23 * 0.0$

global unit 5

array $3 \quad-59.222-15.156 \quad 0$.

reflector $415 \mathrm{ro} \quad 2.54$

reflector $5,4 r 30.515 .215 .3$

$\stackrel{\omega}{\circ}$

end geometry

read array

ara $=1$ nux $=23$ nuy $=18$ nuz $=1$ fill $f 1$ end $f i l l$

ara $=2$ nux $=20$ nuy=18 nuz=1 fill $f 1$ end fill

ara=3 nux=5 nuy=1 nuz=1 fill 432234 end fill

end array

end data

end

\section{6s12}

$=\operatorname{csas} 25$

p3926s 12

44group latticecell

uo2 10.949293922354 .319223895 .69

al 21.0

h2o $\quad 31.0$

plexiglas 41.0

h2o $\quad 51.0$

arbmrubber 1.3216000061258 .10016 .52000011 .4 160001.7801622 .1140000 .36

end comp

squarepitch $1.892 \quad 1.2649131 .41472 \quad 1.28270$

3-12×16 assemblies separated by $12.79 \mathrm{~cm}$ of water

read parm run=yes gen $=405 \mathrm{npg}=600 \mathrm{nsk}=5$ nub=yes end parm read geom

unit 1

cylinder $110.63245 \quad 91.44 \quad 0.0$

cylinder $0110.64135 \quad 91.44 \quad 0.0$

cyl inder $610.64135 \quad 93.98 \quad-2.54$

cylinder $210.70735 \quad 93.98$-2.54

cuboid $314 \mathrm{p} .9460 \quad 93.98 \quad-2.54$

unit 2

array $13 * 0.0$

unit 3

$\begin{array}{lllllllll}\text { cuboid } 5 & 5 & 12.4927 & 0.0 & 30.272 & 0.0 & 93.98 & -2.54\end{array}$

global unit 5

array $2-46.5487-15.1360$

reflector $415 \mathrm{r0.0} \quad 2.541$

reflector $514 r 30.415 .215 .31$

end geometry

read array

ara $=1$ nux $=12$ nuy=16 nuz=1 fill $f 1$ end fill

ara $=2$ nux $=5$ nuy=1 nuz=1 fill 232232 end fill

end array

end data

end

2392641

$=\operatorname{csas} 25$

p3926uit

44group latticecell

uo2 $10.84293922352 .359223897 .65 \quad$ end

al 21.0

h20 31.0

plexiglas 41.0

h20 $51.0 \quad 6 \quad .9816329392235 .1999223899 .801$ end

uranium 6.9816329392235 .1999223899 .801 end

end comp

squarepitch $1.6841 .1176+31.272$

-20x18 and $1-23 \times 18$ (center) assemblies separated by $8.06 \mathrm{~cm}$ of water assemblies separated from depleted uranium reflecting walls by $0.0 \mathrm{~cm}$ of water

read parm run=yes gen $=405 n p g=600$ nsk $=5$ nub=yes end parm

read geom

unit 1

cyl inder 110.558

cylinder 210.635

cuboid

$91.44 \quad 0.0$

unit 2

$96.52-1.27$

array $13 * 0.0$

unit 3 


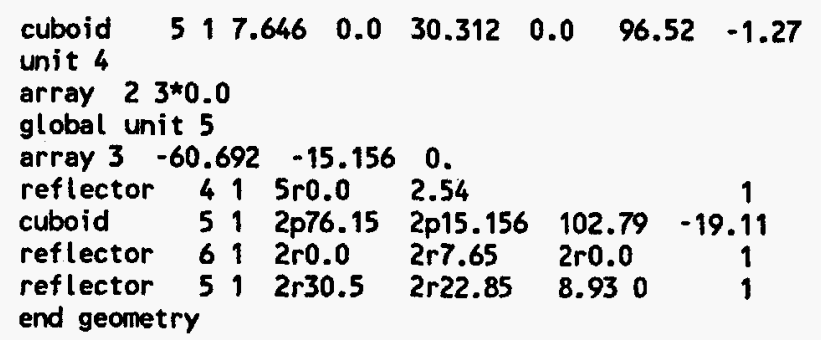

$\begin{array}{lllllll}\text { cuboid } & 5 & 1 & 2 p 76.15 & 2 p 16.477 & 102.79 & -19.11 \\ \text { reflector } & 6 & 1 & 2 r 0.0 & 2 r 7.65 & 2 r 0.0 & 1 \\ \text { reflector } 5 & 1 & 2 r 30.5 & 2 r 21.529 & 8.930 & 1 \\ \text { end geometry } & & & & & \end{array}$

\section{read array}

ara $=1$ nux $=23$ nuy=18 nuz=1 fill $f 1$ end $f i l l$

ara $=2$ nux $=20$ nuy $=18$ nuz=1 fill $f 1$ end fill

ara=3 nux=5 nuy=1 nuz=1 fill 433234 end fill

end array

end data

end

2392613

$=\operatorname{csas} 25$

p3926u3

44group latticecel

uo2 1 0.84293922352 .359223897 .65 end

al 1.0

h2o $\quad 31.0$

plexiglas 41.0

h20 51.0

end comp

6.9816329392235 .1999223899 .801

end

end

end

squarepitch 1.6841 .1176131 .272 end
$2-20 \times 18$ and $1-23 \times 18$ (center) assemblies separated by $9.19 \mathrm{~cm}$ of water assemblies separated from depleted uranium reflecting walls by 3.912 ' $\mathrm{cm}$ of water

read parm run=yes gen $=405$ npg $=600$ nsk $=5$ nub=yes end parm

read geom

unit

cylinder $110.5588 \quad 91.440 .0$

cylinder $210.635 \quad 96.52 \quad-1.27$

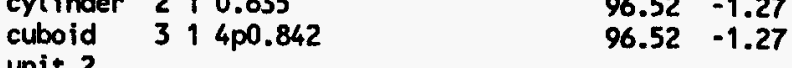

array $13 * 0.0$

unit 3

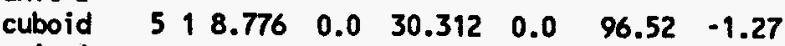

unit 4

array $23 * 0.0$

global unit 5

array $3-61.822-15.1560$.

reflector $415 \mathrm{ro.0} \quad 2.54$

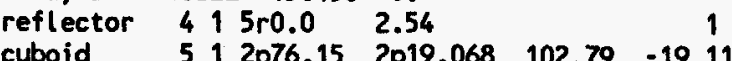

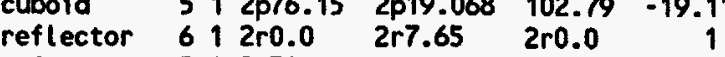

reflector $\quad 512 r 30.5 \quad 2 r 18.938 \quad 8.930 \quad 1$

end geometry 


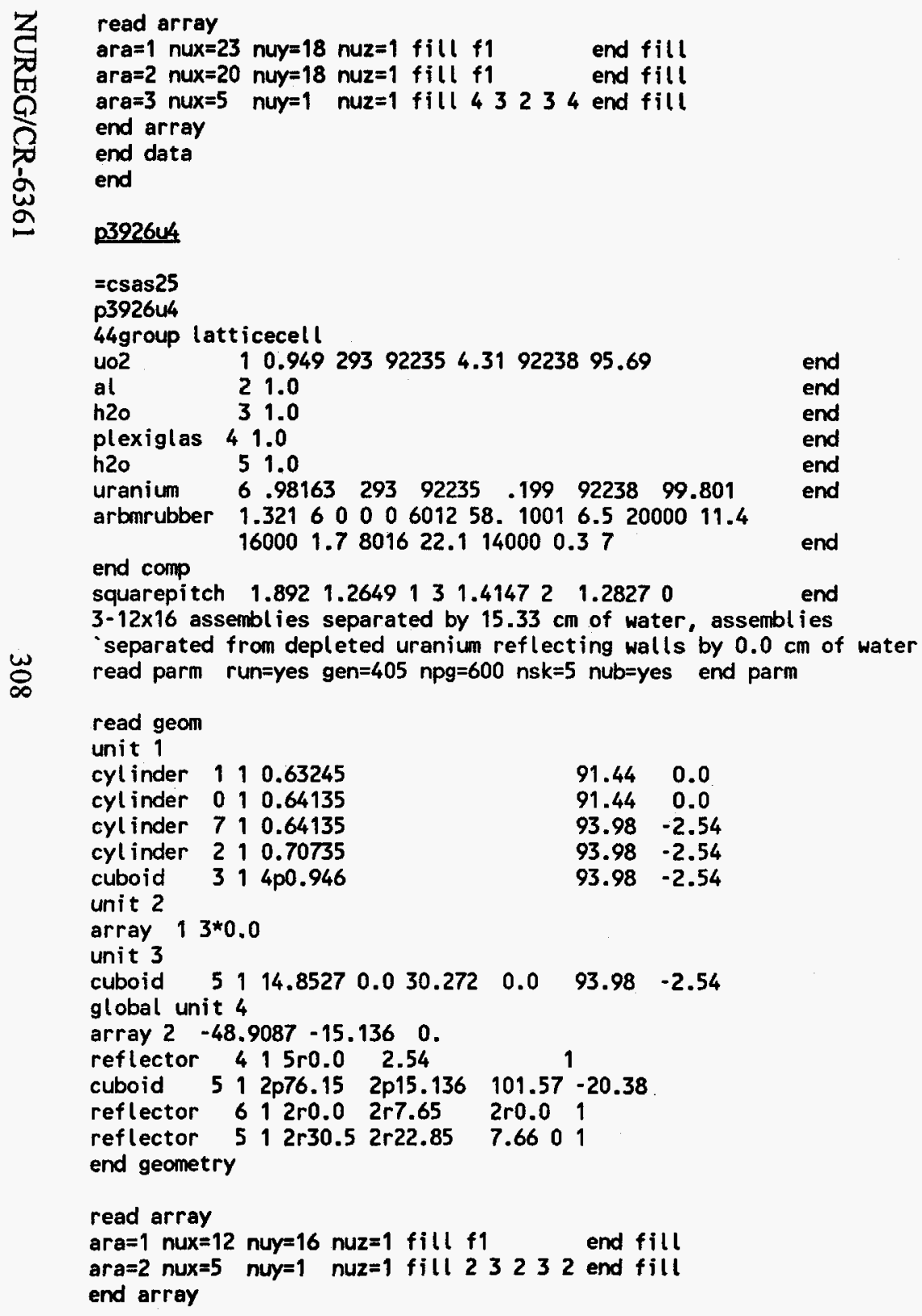

end data

end

12392645

$=\operatorname{csas} 25$

p3926u5

44 group latticecell

uo2 $\quad 10.949293922354 .319223895 .69$

al 1.902

at 21.0

plexiglas 41.0

h20 51.0

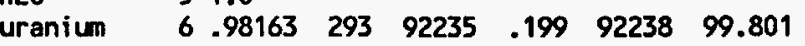

arbmrubber 1.32160000601258 .10016 .52000011 .4

160001.7801622 .1140000 .37

end comp

squarepitch $1.892 \quad 1.2649131 .414721 .28270$

$3-12 \times 16$ assemblies separated by $19.24 \mathrm{~cm}$ of water, assemblies

separated from depleted uranium reflecting walls' by $1.956 \mathrm{~cm}$ of water read parm run=yes gen $=405$ npg $=600$ nsk $=5$ nub=yes end parm

read geom

unit 1

cylinder 110.63245

cyl inder 0010.64135

cylinder $\begin{array}{llll}0 & 1 & 0.64135 \\ \text { cyl inder } & 7 & 1 & 0.64135\end{array}$

$\begin{array}{llll}\text { cyl inder } & 7 & 1 & 0.64135 \\ \text { cyl inder } & 2 & 1 & 0.70735\end{array}$

cuboid $314 \mathrm{p0} 0.946$

end

end

end

end

unit 2

array $13 * 0.0$

unit 3

$\begin{array}{lllllllll}\text { cuboid } 5 & 1 & 18.7627 & 0.0 & 30.272 & 0.0 & 93.98 & -2.54\end{array}$

global unit 4

array $2-52.8187-15.1360$.

reflector $\quad 4 \quad 15 r 0.0 \quad 2.54$

$\begin{array}{lllllll}\text { cuboid } \quad 5 & 1 & 2 p 76.15 & 2 p 17.092 & 101.57 & -20.38\end{array}$

reflector . $612 \mathrm{i} 0.0 \quad 2 r 7.65 \quad 2 \mathrm{r} 0.0$

reflector 512 r30.5 $2 r 20.894 \quad 7.6601$

end geometry

read array

ara $=1$ nux $=12$ nuy=16 nuz=1 fill $f 1$ end $f i l l$

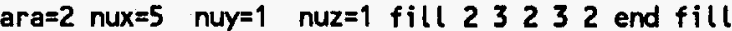

end array

end data

end

D392606

$=\operatorname{csas} 25$ 
p3926u6

44 group latticecell

uo2 10.949293922354 .319223895 .69 end

al 21.0

h20 31.0

h20

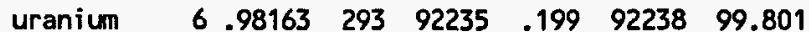

arbmrubber $1.32160000601258,10016.52000011 .4$ 160001.7801622 .1140000 .3

end comp

end

end

end

end

end

end

squarepitch $1.8921 .2649131 .41472 \quad 1.28270$

end

$3-12 \times 16$ assembl ies separated by $18.78 \mathrm{~cm}$ of water, assembl ies

separated from depleted uranium reflecting walls by $3.276 \mathrm{~cm}$ of water

read parm run=yes gen $=405 \mathrm{npg}=600 \mathrm{nsk}=5$ nub=yes end parm

read geom

unit 1

cylinder 110.63245

cylinder 0110.64135

cylinder $710.64135 \quad 93.98-2.54$

cylinder $210.70735 \quad 93.98-2.54$

$\begin{array}{lllll}\text { cuboid } 31400.946 & 93.98 & -2.54\end{array}$

unit 2

array $13 * 0.0$

unit 3

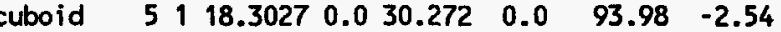

global unit 4

array $2-52.3587-15.136 \quad 0$

reflector

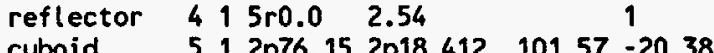

$\begin{array}{lllllll}\text { cuboid } & 5 & 1 & 2 p 76.15 & 2 p 18.412 & 101.57 & -20.38 \\ \text { reflector } & 6 & 1 & 2 \mathrm{r} 0.0 & 2 \mathrm{r} 7.65 & 2 \mathrm{r} 0.0 & 1\end{array}$

$\begin{array}{llllllll}\text { reflector } & 6 & 1 & 2 r 0.0 & 2 r 7.65 & 2 r 0.0 & 1 \\ \text { reflector } & 5 & 1 & 2 r 30.5 & 2 r 19.574 & 7.66 & 0 & 1\end{array}$

end geometry

read array

ara $=1$ nux $=12$ nuy=16 nuz=1 fill $f 1$ end fill

ara $=2$ nux=5 nuy=1 nuz=1 fill $23 \quad 3 \quad 3 \quad 2$ end fill

end array

end data

end

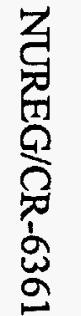

2426701

$=\operatorname{csas} 25$

p4267b1

44 group latticecell

no2 1 den=10.40 $1.029392234 \quad 022922354.30692236$ al $\quad 21.0$ $02292238 \quad 95.65$

boron 3 den $=.002151 .0$ h2o 3 1.0

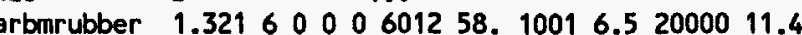
160001.7801622 .1140000 .34

arbmprop $\quad 0.9042010631261901351$

plexiglas 61.0

h20

71.0

end comp

squarepitch 1.8901 .2649131 .414721 .28270

Core of 923 fuel rods, 111.7 water height with $2150 \mathrm{ppm}$ boron

read parm run=yes gen $=405 \mathrm{npg}=600 \mathrm{nsk}=5$ nub=yes end parm

read geom

unit 1

cyl inder 410.63880

cylinder 210.70735

cuboid 51400.945

unit 2

cyl inder 110.63245

cyl inder 010.64135

cyl inder 210.7073

cuboid $314 \mathrm{p0} .945$

unit 3

cyl inder 110.63245

cyl inder 010.64135

cyl inder 210.70735

cuboid $514 \mathrm{p0} .945$

unit 4

cyl inder 110.63245

cyl inder 010.64135

cylinder 210.70735

cuboid 31400.945

unit 5

cyl inder 410.63880

cyl inder 210.70735

cuboid 314 p0.945

unit 6

$\begin{array}{ll}2.54 & 0.0 \\ 2.54 & 0.0\end{array}$

$67.31 \quad 0.0$

67.310 .0

$\begin{array}{ll}67.31 & 0.0 \\ 67.31 & 0.0\end{array}$

$\begin{array}{ll}1.27 & 0.0\end{array}$

1.270 .0

$1.27 \quad 0.0$

1.270 .0

$22.86 \quad 0.0$

$22.86 \quad 0.0$

$22.86 \quad 0.0$

$22.86 \quad 0.0$

$\begin{array}{ll}2.54 & 0.0 \\ 2.54 & 0.0\end{array}$

2.540 .0

array $13 * 0.0$

unit 7

cuboid $614 \mathrm{p0} .945$

cuboid $314 \mathrm{p0.945}$

cuboid $614 \mathrm{p0} .945$

cuboid $\quad 314 \mathrm{p0} .945$

$2.54 \quad 0.0$

69.850 .0

$\begin{array}{ll}71.12 \quad 0.0 \\ 96.52 & 0.0\end{array}$

$96.52 \quad 0.0$

global

unit 8

array 2 -22.68 $-37.80 \quad 0.0$

$\begin{array}{llllllllll}\text { cuboid } & 3 & 1 & 22.68 & -56.92 & 2037.8 & 111.7 & 0.0\end{array}$

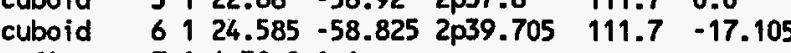

reflector 714 r3o $2 \mathrm{rO} 1$

end

end geometry end

end

end

end 


\section{$\$ 267 \mathrm{~b} 2$}

$=\operatorname{csas} 25$

p4267b2

44 group latticecell

uo2 1 den=10.40 $1.029392234 \quad 022922354.30692236$

0229223895.65

$\begin{array}{lll}\text { al } & 2 & 1.0 \\ \text { boron } & 3 & \text { den }=.00255 \\ \text { hed } & 3.0\end{array}$

$\begin{array}{lll}\text { boron } & 3 \text { den }=.00255 & 1.0 \\ \text { h2o } & 3 & 1.0\end{array}$

arbmrubber 1.3216000601258 .10016 .52000011 .4 $160001.7801622 .114000 \quad 0.34$

arbmprop 0.904201063126190135

plexiglas 61.0

h2o

$$
7 \text { i.o }
$$

end comp

squarepitch 1.8901 .2649131 .414721 .28270

$\omega \quad$ Core of 1237 fuel rods, 111.7 water height with $2550 \mathrm{ppm}$ boron

read parm run=yes gen $=405$ npg $=600$ nsk $=5$ nub=yes end parm

\section{read geom}

unit 1

cylinder 490.63880

cylinder 210.70735

cuboid 5 1 4 p0.945

unit 2

cylinder 110.63245

cylinder $\quad 010.64135$

cyl inder 210.70735

cuboid $314 \mathrm{p0} 0.945$

unit 3

cylinder 110.63245

cylinder 010.64135

cylinder 210.70735

cuboid $514 \mathrm{p0} .945$

unit 4

$2.54 \quad 0.0$

unit 4 cyler 1110.63245

cylinder 010.64135

cylinder 210.70735

cuboid $314 \mathrm{p0} 0.945$

unit 5

cyl inder 410.63880

cylinder 210.70735

$2.54 \quad 0.0$

2.540 .0

$67.31 \quad 0.0$

$67.31 \quad 0.0$

$67.31 \quad 0.0$

$67.31 \quad 0.0$

$\begin{array}{ll}1.27 & 0.0\end{array}$

$1.27 \quad 0.0$

$\begin{array}{ll}1.27 & 0.0 \\ 1.27 & 0.0\end{array}$

1.270 .0

$22.86 \quad 0.0$

$22.86 \quad 0.0$

$\begin{array}{ll}22.86 & 0.0 \\ 22.86 & 0.0\end{array}$

$\begin{array}{lllll}\text { cylinder } & 2 & 1 & 0.70735 \\ \text { cuboid } & 3 & 1 & 4 \mathrm{p} 0.945\end{array}$

unit 7

cuboid

cuboid $314 \mathrm{p0} .945$

cuboid $614 \mathrm{p0} .945$

cuboid $314 \mathrm{p0.945}$

$2.54 \quad 0.0$

$\begin{array}{ll}71.12 & 0.0\end{array}$

global

unit 8

array $2-29.295 \quad-37.80 \quad 0.0$

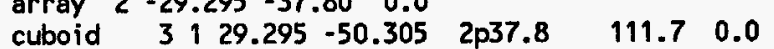

$\begin{array}{llllllll}\text { cuboid } & 6 & 1 & 31.2 & -52.21 & 2 \mathrm{p} 39.705 & 111.7 & -17.105\end{array}$

reflector 714 r30 2ro 1

end geometry

read array

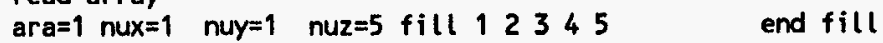
ara $=2$ nux $=31$ nuy $=40$ nuz $=1$ fill 1147 ro 730 r6 $2 q 31$ end fill end array

end data

end

\section{$04267 \mathrm{b3}$}

$=\operatorname{csas} 25$

p4267b3

44group latticecell

uo2 1 den=10.40 1.0 $29392234 \quad 022922354.30692236$

al 21.0 end

boron 3 den $=.001031 .0 \quad$ end

$\begin{array}{llll}\text { boron } & 3 & \\ \text { h2o den }=.00103 & 3.0 & \text { end } \\ \text { aromraber } & 1.0 & 1.0 & \text { end }\end{array}$

$\begin{array}{lllllllllll}\text { arbmrubber } & 1.321 & 6 & 0 & 0 & 0 & 6012 & 58.1001 & 6.5 & 20000 & 11.4 \\ 16000 & 1.7 & 8016 & 22.1 & 14000 & 0.3 & 4 & \end{array}$

arbmprop 0.9042010631261901351 end

plexiglas 61.0 end

end comp

squarepitch 1.7151 .2649131 .414721 .28270 end

Core of 737 fuel rods, 111.7 water height with $1030 \mathrm{ppm}$ boron

read parm run=yes gen $=405$ npg $=600$ nsk $=5$ nub=yes end parm

\section{read geom}

unit 1

cylinder 410.63880

cylinder 210.70735

cuboid $514 \mathrm{p0} .8575$

unit 2

cylinder 1110.63245

cyl inder 010.64135

cylinder 210.70735

$\begin{array}{ll}2.54 & 0.0 \\ 2.54 & 0.0 \\ 2.54 & 0.0 \\ & \\ 67.31 & 0.0 \\ 67.31 & 0.0 \\ 67.31 & 0.0\end{array}$




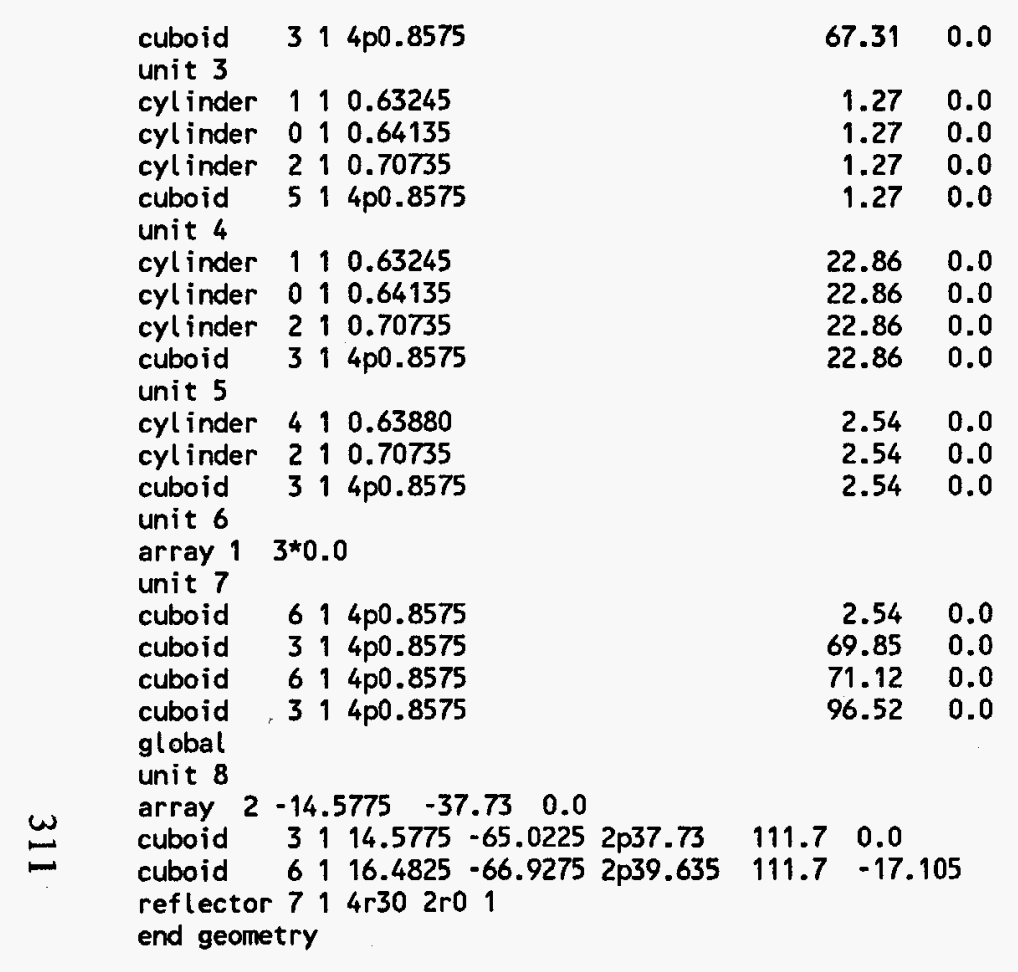

read array

ara $=1$ nux=1 nuy=1 nuz=5 fill $1 \begin{array}{lllllll} & 2 & 3 & 4 & 5\end{array}$ end fill ara $=2$ nux $=17$ nuy $=44$ nuz=1 fill 561 r6 $7 \quad 16 r 6 \quad 10 q 17$ end fill end array

end data

end

\section{p426704}

\section{$=\operatorname{csas} 25$}

p4267b4 uo2 1 den=10.40 1.029392234 .022922354 .30692236 end al boron

arbmrubber 1.32160000601258 .10016 .52000011 .4 3 den $=.001821 .0$ 3 den $=.001821 .0$ $\begin{array}{lllllllllll}1.321 & 6 & 0 & 0 & 0 & 6012 & 58.1001 & 6.5 \\ 16000 & 1.7 & 8016 & 22.1 & 14000 & 0.3 & 4\end{array}$ arbmprop $\quad 0.9042010631261901351$ plexiglas 61.0 h2o

71.0

end

end comp

squarepitch 1.7151 .2649131 .414721 .28270 read parm run=yes gen $=405 \mathrm{npg}=600$ nsk $=5$ nub=yes end parm

read geom

unit 1

cyl inder 410.63880

cylinder 210.70735

cylinder 21 0.707

cuboid $514 \mathrm{p0.8575}$

unit 2

cyl inder 110.63245

cyl inder 010.64135

cyl inder 2110.70735

cuboid $314 \mathrm{p0} 0.8575$

unit 3

cylinder 110.63245

cylinder $\begin{array}{lllll}1 & 1 & 0.63245 \\ \text { cylind } & 1 & 0.64135\end{array}$

cylinder $\begin{array}{llll}\text { cylinder } & 2 & 1 & 0.70735\end{array}$

cuboid $514 \mathrm{p0} .8575$

unit 4

cyl inder 1110.63245

cylinder 010.64135

cylinder 2110.70735

$\begin{array}{llll}\text { cylinder } & 2 & 1 & 0.70735 \\ \text { cuboid } & 3 & 1 & 4 \mathrm{p} 0.8575\end{array}$

cuboid

unit 5

cylinder 410.63880

cylinder 210.70735

cuboid 31400.8575

unit 6

array $13 * 0.0$

unit 7

cuboid 614 p0.8575

cuboid $314 \mathrm{p0} 0.8575$

cuboid $614 \mathrm{p0} 0.8575$

cuboid $314 \mathrm{p0} 0.8575$

2.540 .0

$2.54 \quad 0.0$

2.540 .0

67.310 .0

$67.31 \quad 0.0$

$67.31 \quad 0.0$

1.270 .0

$\begin{array}{ll}1.27 & 0.0\end{array}$

$\begin{array}{ll}1.27 & 0.0 \\ 1.27 & 0.0\end{array}$

$\begin{array}{ll}1.27 & 0.0\end{array}$

$22.86 \quad 0.0$

$22.86 \quad 0.0$

$\begin{array}{ll}22.86 & 0.0 \\ 22.86 & 0.0\end{array}$

$2.54 \quad 0.0$

$\begin{array}{ll}2.54 & 0.0 \\ 2.54 & 0.0\end{array}$

global

unit 8

$\begin{array}{lllll}\text { array } 2 & -18.0075 & -37.73 & 0.0\end{array}$

$\begin{array}{llllllll}\text { cuboid } & 3 & 1 & 18.0075 & -61.5925 & 2 p 37.73 & 111.7 & 0.0\end{array}$

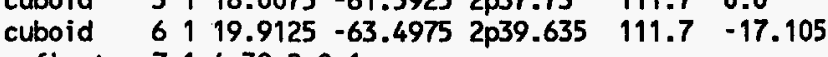
reflector $714 \mathrm{r} 30$ 2ro 1

end geometry

read array

ara=1 nux=1 nuy=1 nuz=5 fill 122345 end fill ara $=2$ nux=21 nuy=44 nuz=1 fill 777 r6 7 20r6 6 q21 end fill ara $=2$ nux $=21$

end array

end data

end 


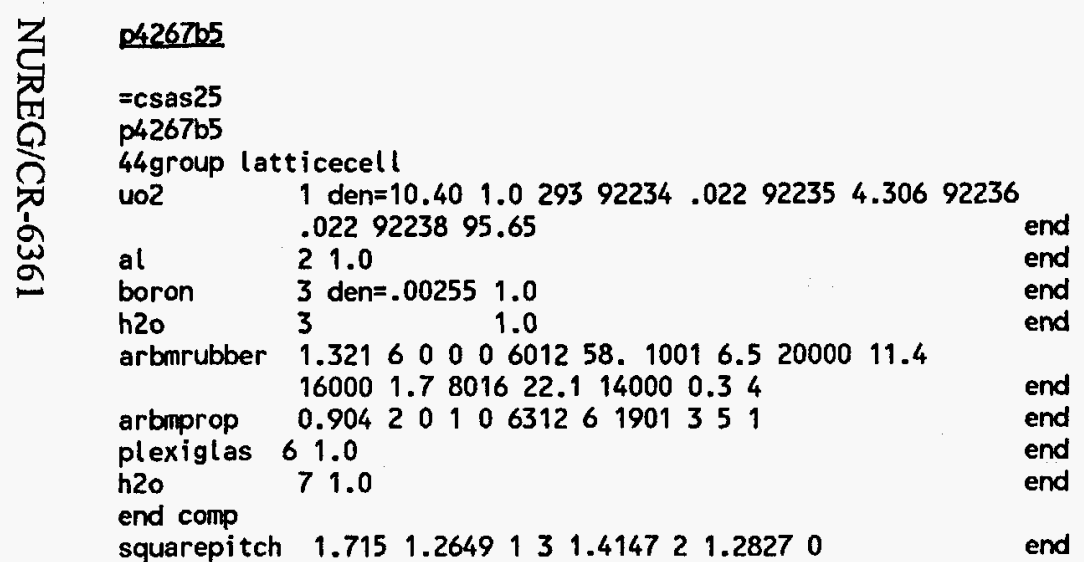

Core of 1192 fuel rods, 111.7 water height with $2550 \mathrm{ppm}$ boron read parm run=yes gen $=405$ npg $=600$ nsk $=5$ nub $=y e s$ end parm

read geom

unit 1

cyl inder 490.63880

cylinder 210.70735

$\omega$

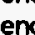

cuboid 51400.8575

unit 2 nd

cylinder 1110.63245

cylinder 0110.64135

cylinder 210.70735

cuboid $314 \mathrm{p0} .8575$

unit 3

cylinder 110.63245

cylinder 0010.64135

cylinder 210.70735

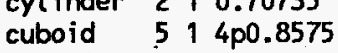

unit 4

cylinder 110.63245

cylinder 0010.64135

cylinder 210.70735

$\begin{array}{llll}\text { cylinder } & 2 & 1 & 0.70735 \\ \text { cuboid } & 3 & 1 & 4 \mathrm{p} 0.8575\end{array}$

cuboid
unit 5

cyl inder 410.63880

cylinder 210.70735

cuboid $314 \mathrm{p0} 0.8575$

unit 6

array $13 * 0.0$

unit?

cuboid $614 \mathrm{p0} .8575$

cuboid $314 \mathrm{p0} 0.8575$

cuboid $614 \mathrm{p0} .8575$

cuboid 314 po.8575

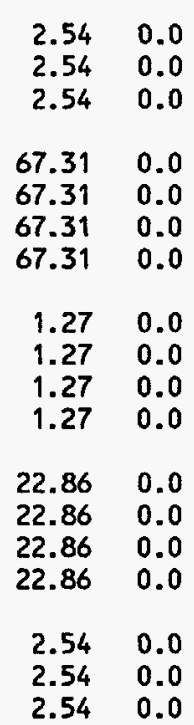

$2.54 \quad 0.0$

$69.85 \quad 0.0$

$\begin{array}{ll}71.12 & 0.0 \\ 96.52 & 0.0\end{array}$ global

unit 8

array $2-24.01-37.73 \quad 0.0$

$\begin{array}{lllllll}\text { array } & -24.01 & -37.73 & 0.0 & & & \end{array}$

$\begin{array}{llllll}\text { cuboid } 6125.915 & -57.495 & 2 \mathrm{p} 39.635 & 111.7 & -17.105\end{array}$

reflector 714 r3o 2ro 1

end geometry

read array

ara=1 nux=1 nuy=1 nuz=5 fill 123345 end fill ara $=2$ nux $=28$ nuy $=44$ nuz=1 fill 112 r6 $7 \quad 27 r 639 q 28$ end $\mathrm{fill}$

end array

end data

end

$04267 \mathrm{~s} 11$

$=\operatorname{csas} 25$

p4267s 11

44group latticecell

uo2 1 den=10.40 1.0 29392234.02292235 4.30692236

$\begin{array}{llll} & .0229223895 .65 & \text { end } \\ \text { at } & 21.0 & \text { end }\end{array}$

arbmrubber 1.32160000601258 .10016 .52000011 .4

arbmrubber 1.3216001 .7801622 .1140000 .34

arbmprop 0.9042010631261901351 end

plexiglas 61.0 end

end comp

squarepitch 1.8901 .2649131 .414721 .28270

Core of 357 fuel rods, $111.7 \mathrm{~cm}$ of water

read parm run=yes gen $=405 \mathrm{npg}=600 \mathrm{nsk}=5$ nub=yes end parm

read geom

unit

cylinder $4 \begin{array}{llll} & 0.63880 & 2.54 & 0.0\end{array}$

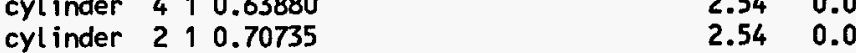

$\begin{array}{lllll}\text { cuboid } 514 \mathrm{p} 0.945 & 2.54 & 0.0\end{array}$

unit 2

$67.31 \quad 0.0$

cylinder 110.63245

cylinder 010.64135

cylinder 210.70735

cuboid $314 \mathrm{p0.945}$

unit 3

cylinder 1110.63245

cylinder 010.64135

cyl inder 210.70735

cuboid 5 1 4 p0.945

unit 4

cylinder 1110.63245

cylinder 0110.64135

$\begin{array}{ll}67.31 & 0.0 \\ 67.31 & 0.0\end{array}$

67.310 .0

$1.27 \quad 0.0$

$\begin{array}{ll}1.27 & 0.0\end{array}$

$\begin{array}{ll}1.27 & 0.0\end{array}$

$1.27 \quad 0.0$

$22.86 \quad 0.0$

$\begin{array}{ll}22.86 & 0.0 \\ 22.86 & 0.0\end{array}$ 


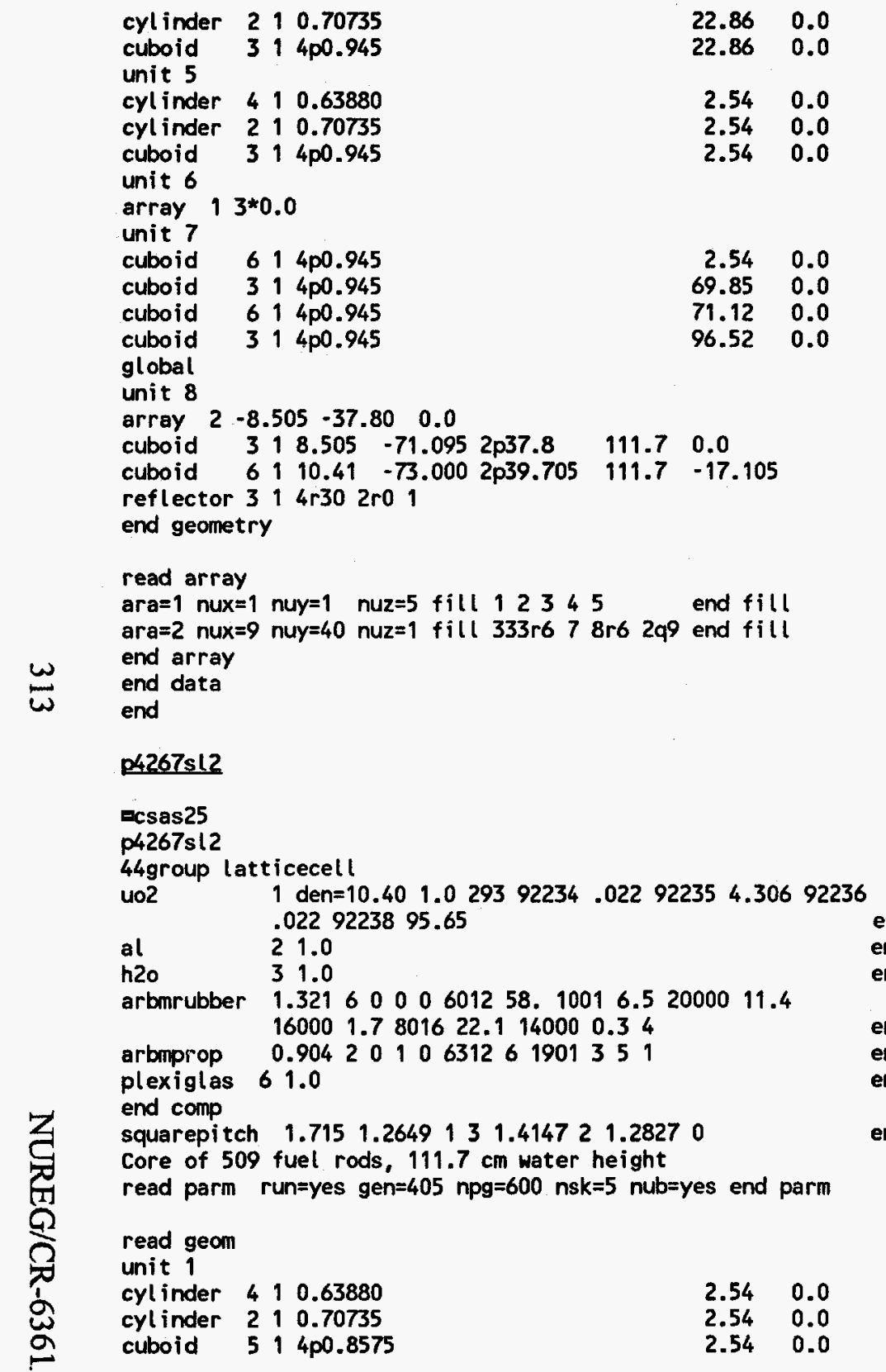

unit 2

cylinder 110.63245

$67.31 \quad 0.0$

cylinder 0110.64135

$67.31 \quad 0.0$

cylinder $210.70735 \quad 67.31 \quad 0.0$

cuboid $314 \mathrm{p0} .8575$

67.310 .0

cyl inder 110.63245

$\begin{array}{lll}1.27 & 0.0\end{array}$

cylinder 0010

$1.27 \quad 0.0$

cylinder 210.70735

cuboid $514 \mathrm{p} 0.8575$

$\begin{array}{ll}1.27 & 0.0\end{array}$

unit 4

$1.27 \quad 0.0$

cylinder 110.63245

$22.86 \quad 0.0$

cylinder 0110.64135

$22.86 \quad 0.0$

cylinder $210.70735 \quad 22.86 \quad 0.0$

cuboid 31400.8575

$22.86 \quad 0.0$

unit 5

cylinder $4 \begin{array}{lll}\mathbf{1} & 0.63880\end{array}$

0.860

cyl inder 210.70735

$\begin{array}{ll}2.54 & 0.0 \\ 2.54 & 0.0\end{array}$

cuboid $314 \mathrm{p0} .8575$

$2.54 \quad 0.0$

array $13 * 0.0$

unit 7

cuboid 61400.8575

$2.54 \quad 0.0$

cuboid

cuboid $614 \mathrm{p0.8575}$

$69.85 \quad 0.0$

cuboid $314 \mathrm{p0.8575}$

$\begin{array}{ll}71.12 & 0.0\end{array}$

global

$96.52 \quad 0.0$

unit 8

array $2-10.29-37.73 \quad 0.0$

$\begin{array}{llllllll}\text { cuboid } & 3 & 1 & 10.29 & -69.31 & 2 p 37.73 & 111.7 & 0.0\end{array}$

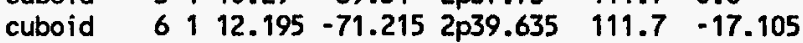

reflector $314 \mathrm{r} 302 \mathrm{rO} 1$

end geometry

read array

ara=1 nux=1 nuy=1 nuz=5 fill $123 \quad 345 \quad$ end fill ara $=2$ nux $=12$ nuy $=44$ nuz $=1$ fill $300 r 6711$ r6 $18 q 12$ end fill end array

end data

end

p62ft231

\section{$=c s a s 25$}

p62ft231

44 group latticecell

uo2 $\quad 10.9489293922340 .022922354 .306922360 .022$

9223895.65

al $\quad 21.0$ 
arbmboral $\quad 2.645101500035 .6360129 .951302754 .33$ 80160.07260000 .024

arbmrubber 1.3216000601258 .10016 .52000011 .4 $160001.7801622 .1 \quad 14000 \quad 0.35$

plexiglas 61.0

end comp

squrepitch 1.8911 .265131 .41521 .2830

and $1-12 \times 16$ plus $3 \times 17$ assemblies separated by $0.683-\mathrm{cm}-$ thick Boral plates and $3.824 \mathrm{~cm}$ of water

read parm run=yes gen $=405 \mathrm{npg}=600$ nsk $=5$ nub=yes end parm

read geometry

unit 1

cylinder 11.6325

cyl inder 01.6415

cylinder $5, .6415$

cylinder 21.7075

cuboid $314 \mathrm{p} .9455$

unit 2

array $23 * 0.0$

$3132.4220-11.8750$

cuboid

cuboid $2133.1250-11.875$

unit 3

$\omega$ array $13 * 0.0$

cuboid $3144.0220-0.2950$

cuboid $2144.0220-0.3965$

cuboid $4144.0220-0.8765$

cuboid $2144.0220-0.9780$

unit 4

array $23 * 0.0$

cuboid $3132.4220-11.8750$

cuboid $2132.5435-11.8750$

cuboid $4133.0235-11.8750$

cuboid $2133.1250-11.8750$

unit 5

array $33 * 0.0$

$\begin{array}{llllllllll}\text { cuboid } & 3 & 1 & 44.0220 & -0.2955 & 28.6600 & -15.657 & 96.00 & 0.0 \\ \text { cuboid } & 2 & 1 & 44.0220 & -0.3965 & 28.7615 & -15.657 & 96.00 & 0.0\end{array}$

$\begin{array}{lllllllll}\text { cuboid } 4 & 1 & 44.0220 & -0.8765 & 29.2415 & -15.657 & 96.00 & 0.0\end{array}$

$\begin{array}{lllllllll}\text { cuboid } 21 & 44.0220 & -0.9780 & 29.3430 & -15.657 & 96.00 & 0.0\end{array}$

unit 6

$4.0220-0.2950$ $44.0220-0.3965$ $44.0220-0.8765$

$44.0220-0.9780$

$93.98 \quad 2.54$

$93.98 \quad 2.54$

$96.00 \quad 0.0$

$96.00 \quad 0.0$

$96.00 \quad 0.0$

$44.0220-0.2950$

$4.0220-0.3965$

$\begin{array}{llll}44.0220 & -0.9780 \quad 96.00 \quad 0.0\end{array}$

$28.6600-15.6570 \quad 96.00 \quad 0.0$

$8.7615-15.6570 \quad 96.00 \quad 0.0$

$29.2415-15.6570 \quad 96.00 \quad 0.0$

$29.3430-15.6570$

cuboid

$313.71 \quad 0.0$

45.0

0.0

$96.00 \quad 0.0$

cuboid

unit 8

cuboid

$3145.0 \quad 0.0$

3.71

0.0

$96.00 \quad 0.0$

unit 9

$\begin{array}{llll}3 & 1 & 3.71 & 0.0\end{array}$

3.71

0.0

$96.00 \quad 0.0$

$96.00 \quad 0.0$

cuboid $314 \mathrm{p} .9455$

global unit 10 array $43 * 0.0$

reflector $615 \mathrm{ro} \quad 5.081$

reflector 314 r15 15.15 .881

end geom

read array

ara $=1$ nux $=17$ nuy=15 fill $16 r 1914 q 17$ end fill

ara $=2$ nux $=17$ nuy=15 fill $916 r 1 \quad 14 q 17$ end fill

ara $=3$ nux $=17$ nuy=15 fill 51r1 16r1 911 q17 end fill ara $=4$ nux $=3$ nuy $=3$ fill 465787263 end fill end array

end data

end

\section{p71f14f3}

$=\operatorname{csas} 25$

p71f14f3

44group latticecell

uo2 $\quad 10.9489293922340 .022922354 .306922360 .022$ 9223895.650 end

at 21.0

h20 31.0

arbmboral 2.645101500029 .2260128 .161302762 .54

end

80160.06260000 .02

end

arbmrubber 1.3216000601258 .10016 .52000011 .4

end

plexiglas 160001.7801622 .1140000 .35

plexiglas

61.0

end

squarepitch 1.8911 .265131 .41521 .2830

$2-15 \times 14$ and $1-15 \times 15$ and $1-12 \times 14$ plus $3 \times 15$ assembl ies separated by $-0.673-\mathrm{cm}$ thick Boral plates, 14 fuel rods, and $3.844 \mathrm{~cm}$ of water read parm run=yes gen $=405 \mathrm{npg}=600 \mathrm{nsk}=5$ nub=yes end parm

\section{read geometry}

unit 1

cylinder 11.6325

cylinder 011.6415

cyl inder 51.6415

cyl inder 21.7075

cuboid $314 \mathrm{p} .9455$

$93.98 \quad 2.54$

$96.00 \quad 0.0$

$96.00 \quad 0.0$

unit 2

array $13 * 0.0$

$\begin{array}{lllllll}\text { cuboid } 3128.4220 & -15.9050 & 44.2700 & -.0570 & 96.00 & 0.0\end{array}$

$\begin{array}{llllllll} & & \end{array}$

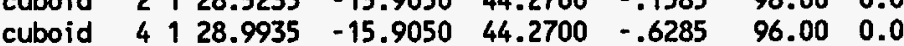

$\begin{array}{llllllll}\text { cuboid } 2 & 1 & 29.0950 & -15.9050 & 44.2700 & -.7300 & 96.00 & 0.0\end{array}$

unit 3

array $33 * 0.0$

cuboid $3144.2700 \quad-0.0570 \quad 44.2700 \quad-0.0570 \quad 96.00 \quad 0.0$

$\begin{array}{lllllllll}\text { cuboid } 2 & 1 & 44.2700 & -0.1585 & 44.2700 & -0.1585 & 96.00 & 0.0\end{array}$ 


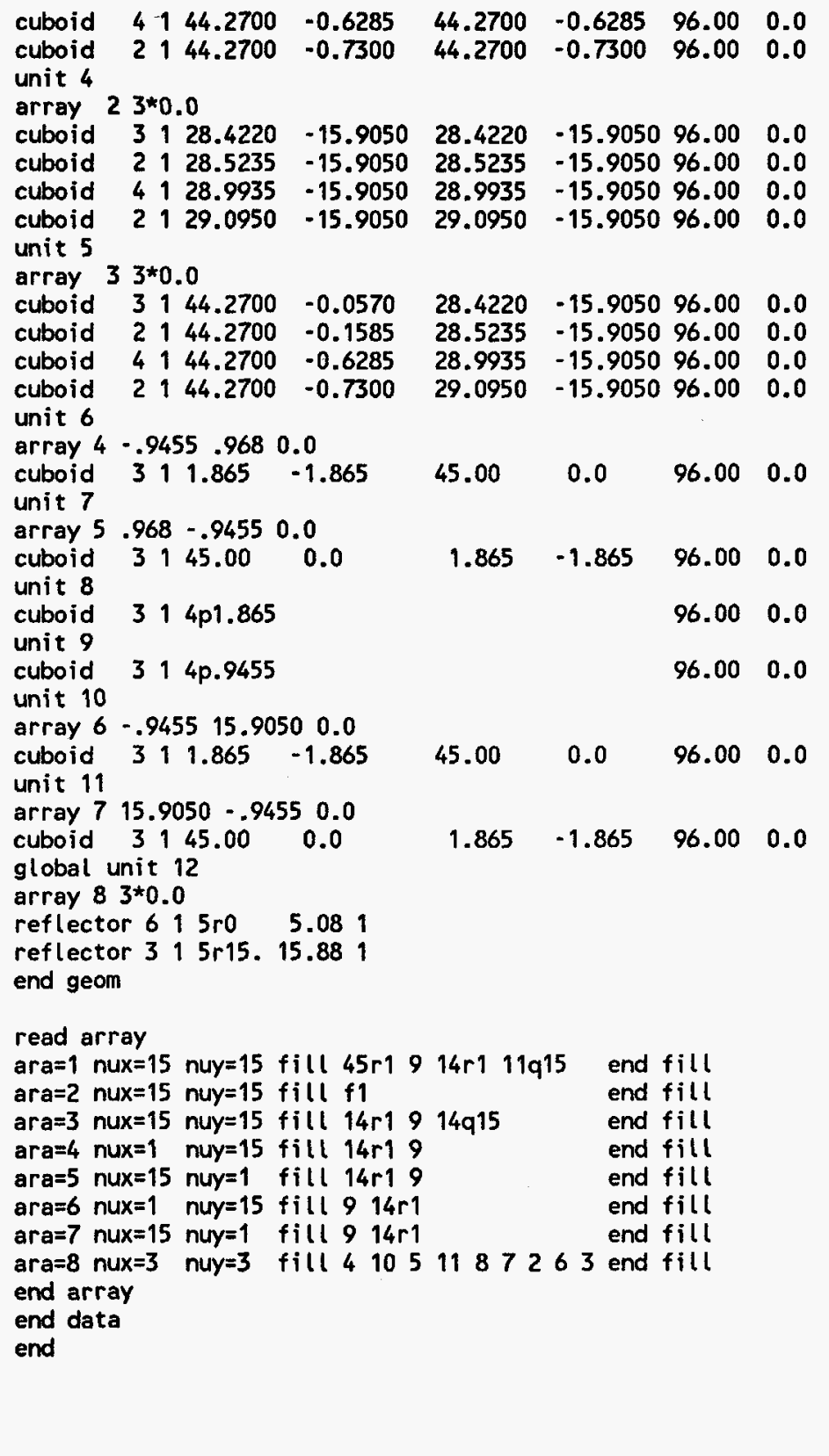

\section{p71f14\%3}

$=\operatorname{csas} 25$

p71f14v3

44 group latticecell

uo2 $\quad 10.9489293922340 .022922354 .306922360 .022$ 9223895.650

al 21.0 end

h20 $31.0 \quad$ end

$\begin{array}{llllllll}\text { arbmboral } & 2.645101500029 .2260128 .161302762 .54 \\ & 8016 & 0.0626000 & 0.024\end{array}$

arbmrubber 1.32160000601258 .10016 .52000011 .4

plexiglas $61.0 \quad$ end

squarepitch 1.8911 .265131 .41521 .2830

$2-15 \times 14$ and $1-15 \times 15$ and $1-8 \times 14$ plus $7 \times 15$ assembl ies separated by

$0.673-\mathrm{cm}$ thick Boral plates, $0.62-\mathrm{cm}$-thick aluminum plates, and 3.844 " $\mathrm{cm}$ of water

read parm run=yes gen $=405 \mathrm{npg}=600$ nsk $=5$ nub=yes end parm

\section{read geometry}

unit 1

cylinder 11 . 1.6325

cylinder 011.6415

cyl inder 51.6415

cylinder 21.7075

cuboid $314 \mathrm{p} .9455$

unit 2

array $13 * 0.0$

cuboid $3126.5310-17.7960$

cuboid $2126.6325-17.7960$

cuboid $4127.1025 \quad-17.7960$

cuboid $2127.2040 \quad-17.7960$

unit 3

array $23 * 0.0$

cuboid $3144.2700-0.0570$

$\begin{array}{llllll}\text { cuboid } & 2 & 1 & 44.2700 & -0.1585 \\ \text { cuboid } & 4 & 1 & 44.2700 & -0.6285\end{array}$

cuboid $2144.2700 \quad-0.7300$

unit 4

array $13 * 0.0$

cuboid $3126.5310-17.7960$

cuboid $3126.5310-17.7960$

$\begin{array}{lllll}\text { cuboid } & 2 & 1 & 26.6325 & -17.7960 \\ \text { cuboid } & 4 & 1 & 27.1025 & -17.7960\end{array}$

$\begin{array}{llllll}\text { cuboid } & 4 & 1 & 27.1025 & -17.7960 \\ \text { cuboid } & 2 & 1 & 27.2040 & -17.7960\end{array}$

unit 5

array $33 * 0.0$

$\begin{array}{lllllll}\text { cuboid } 3 & 144.2700 & -0.0570 & 28.4220 & -15.9050 & 96.00 & 0.0\end{array}$
$93.98 \quad 2.54$

$93.98 \quad 2.54$

$96.00 \quad 0.0$

$96.00 \quad 0.0$

$96.00 \quad 0.0$

$44.2700 \quad-.057096 .00 \quad 0.0$

$\begin{array}{llll}44.2700 & -.1585 & 96.00 & 0.0\end{array}$

$\begin{array}{llll}44.2700 & -.6285 & 96.00 & 0.0\end{array}$

$\begin{array}{llll}44.2700 & -.7300 & 96.00 & 0.0\end{array}$

$\begin{array}{llll}44.2700 & -.0570 & 96.00 & 0.0\end{array}$

$\begin{array}{llll}44.2700 & -.1585 & 96.00 & 0.0\end{array}$

$\begin{array}{lllll}44.2700 & -.6285 & 96.00 & 0.0\end{array}$

$\begin{array}{llll}44.2700 & -.7300 \quad 96.00 \quad 0.0\end{array}$

$\begin{array}{lllll}28.5235 & -15.9050 & 96.00 & 0.0\end{array}$

$\begin{array}{lllll}28.9935 & -15.9050 & 96.00 & 0.0\end{array}$

$\begin{array}{lllll}29.0950 & -15.9050 & 96.00 & 0.0\end{array}$ $\begin{array}{lllll}28.4220 & -15.9050 & 96.00 & 0.0\end{array}$ 


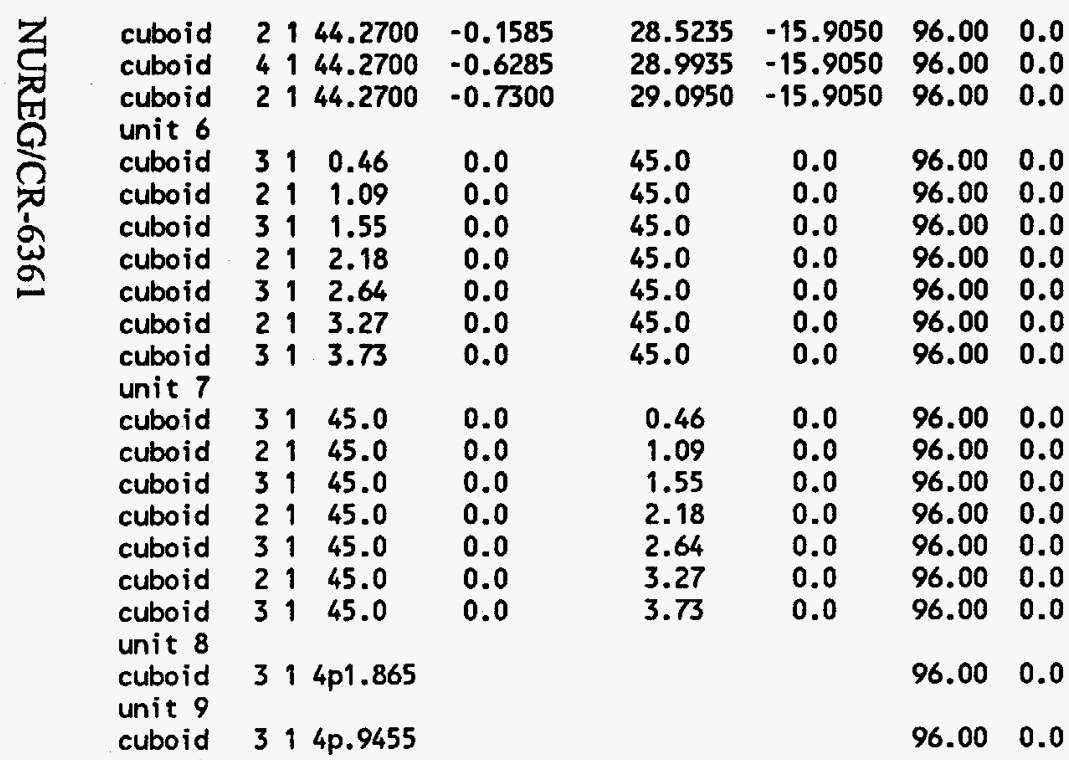

arbmrubber 1.3216000601258 .10016 .52000011 .4

plexiglas $\quad 61.0$

end comp 1.8911 .265131 .41521 .2830

$2-15 \times 15$ and $1-16 \times 15$ and $1-15 \times 15$ plus $1 \times 10$ assemblies separated by

$-0.673-\mathrm{cm}$ thick Boral plates, 14 aluminum rods, and $3.844 \mathrm{~cm}$ of water read parm run=yes gen $=405$ npg $=600$ nsk $=5$ nub=yes end parm

\section{read geometry}

unit 1

cylinder 11.6325

cyl inder 010.6415

cyl inder 51 . 6415

cyl inder 21.7075

cuboid $314 \mathrm{p} .9455$

unit 2

array $33 * 0.0$

cuboid

$93.98 \quad 2.54$

$93.98 \quad 2.54$

$96.00 \quad 0.0$

$96.00 \quad 0.0$

$96.00 \quad 0.0$

end geom

read array

ara=1 nux $=14$ nuy=15 fill $f 1$

end $f i l l$

ara $=4$ nux $=3$ nuy $=3$ fill 465787263 end fill

end array

end data

$=\operatorname{csas} 25$

p71f14v5

44 group latticecell

uo2 10.9489293922340 .022922354 .306922360 .022 9223895.650

$\begin{array}{lll}\text { al } & 2 & 1.0 \\ \text { h20 } & 3 & 1.0\end{array}$

arbmboral 2.6451101500029 .2260128 .161302762 .54 80160.06260000 .024

cuboid $3,128.4220-15.9050$

cuboid $2128.5235-15.9050$

cuboid $4128.9935-15.9050$

cuboid $2129.0950-15.9050$

unit 3

array $23 * 0.0$

cuboid $3144.2700=.0570$

cuboid $2144.2700-.1585$

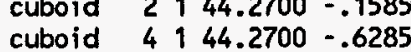

cuboid 4 cuboid $2144.2700-.6285$

cuboid

$44.2700-.1585$
$44.2700-.6285$

$44.2700-.7300$

unit 4

array $13 * 0.0$

cuboid $3128.4220-15.9050$

cuboid $2128.5235-15.9050$

cuboid $4128.9935-15.9050$

cuboid $2129.0950-15.9050$

unit 5

array $13 * 0.0$

cuboid $3144.2700-.0570$

cuboid $2144.2700-.1585$

cuboid $4144.2700-.6285$

cuboid $2144.2700-.7300$

unit 6

array $4-0.9455$ 2r0.0

$44.2700 \cdot .0570$ $44.2700-.1585$ $44.2700-.6285$ $44.2700-.7300$

$96.00 \quad 0.0$ $96.00 \quad 0.0$ $96.00 \quad 0.0$ $96.00 \quad 0.0$

$96.00 \quad 0.0$

$96.00 \quad 0.0$

$96.00 \quad 0.0$

$96.00 \quad 0.0$

$\begin{array}{lllll}28.4220 & -15.9050 & 96.00 & 0.0\end{array}$ $28.5235-15.9050 \quad 96.00 \quad 0.0$ $\begin{array}{lllll}28.9935 & -15.9050 & 96.00 & 0.0\end{array}$ $29.0950-15.9050 \quad 96.00 \quad 0.0$

$\begin{array}{lllll}28.4220 & -15.9050 & 96.00 & 0.0\end{array}$ $\begin{array}{llll}28.5235 & -15.9050 & 96.00 & 0.0\end{array}$

$28.9935 \quad-15.9050 \quad 96.00 \quad 0.0$

$29.0950-15.9050 \quad 96.00 \quad 0.0$

$\begin{array}{llll}44.2700 & -0.7300 & 96.00 & 0.0\end{array}$

unit 7

array $5 \quad 0.0 \quad-0.9455 \quad 0.0$

$\begin{array}{lll}\text { cuboid } 3144.27 & -0.73\end{array}$

unit 8

$\begin{array}{llll}1.865 & -1.865 \quad 96.00 \quad 0.0\end{array}$

$96.00 \quad 0.0$

$96.00 \quad 0.0$

unit 9

cuboid $314 p .9455$

unit 10 
array $6-0.9455 \quad 2 r 0.0$

cuboid $3,1.865 \cdot 1.865$

$\begin{array}{lllll}29.0950 & -15.9050 & 96.00 & 0.0\end{array}$

unit 11

$\begin{array}{llll}\text { array } 7 & 0.0 & -0.9455 & 0.0\end{array}$

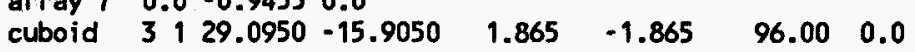

unit 12

cyl inder 21.635

$96.00 \quad 0.0$

cuboid $314 \mathrm{p} .9455$

global unit 13

array $83 * 0.0$

reflector $615 \mathrm{ro} \quad 5.081$

reflector 315 r 15.15 .881

end geom

\section{read array}

ara $=1$ nux $=15$ nuy $=15$ fill $f 1$

ara=2 nux=15 nuy=16 fill fi

ara $=3$ nux $=15$ nuy $=16$ fill $225 r 1 \quad 5 r 9 \quad 10 r 1$

end fill

end fill

end fill

end fill

ara $=4$ nux $=1$ nuy $=16$ fill $14 r 12$ 2ro

ara $=5$ nux $=15$ nuy $=1$ fill $14 r 129$

ara $=6$ nux $=1$ nuy $=15$ fill 914 r 12

end fill

end fill

ara $=7$ nux $=15$ nuy=1 fill $914 r 12$

end fill

ara $=8$ nux $=3$ nuy=3 fill 41051187263 end fill

end array

data

end

\section{p71f214r}

$=\operatorname{csas} 25$

p71f214r

44group latticecell

uo2 10.9489293922340 .022922354 .306922360 .022 9223895.650

$\begin{array}{lll}\text { al } & 2 & 1.0 \\ \text { h20 } & 3 & 1.0\end{array}$

arbmboral $\quad 2.645901500029 .2260128 .161302762 .54$

80160.06260000 .024

arbmrubber 1.32160000601258 .10016 .52000011 .4 $160001.7801622 .114000 \quad 0.35$

\section{plexiglas}

$$
61.0
$$

end comp

squarepitch 1.8911 .265131 .41521 .2830

$3-15 \times 16$ and $1-8 \times 15$ and plus $7 \times 16$ assemblies separated by $0.673-\mathrm{cm}$

thick Boral plates and $3.844 \mathrm{~cm}$ of water

read parm run=yes gen $=405 \mathrm{npg}=600 \mathrm{nsk}=5$ nub=yes end parm

read geometry

unit 1 end

end

cylinder 11.6325

cylinder 011.6415

cylinder 51.6415

cyl inder 21.7075

cuboid $314 p .9455$

unit 2

array $13 * 0.0$

$\begin{array}{lllll}\text { cuboid } 3 & 1 & 30.313 & -14.0140\end{array}$

cuboid $2130.4145-14.0140$

cuboid $4130.8845-14.0140$

cuboid $2130.9860-14.0140$

unit 3

array $13 * 0.0$

cuboid $3144.270-0.0570$

cuboid $2144.270-0.1585$

cuboid $4144.270 \quad-0.6285$

$\begin{array}{lll}\text { cuboid } 2144.270 & -0.7300\end{array}$

unit 4

array $13 * 0.0$

cuboid $3130.3130-14.0140$

cuboid $2130.4145-14.0140$

cuboid $4130.8845-14.0140$

cuboid $2130.9860-14.0140$

unit 5

array $23 * 0.0$

cuboid $3144.270 \quad-0.0570$

cuboid $2144.270-0.1585$

cuboid $4144.270-0.6285$

$\begin{array}{llll}\text { cuboid } 2144.270 & -0.7300\end{array}$

unit 6

cuboid

cuboid
unit 7

$312 p 1.865$

cuboid

unit 8

cuboid

0.0

$314 p 1.865$

cuboid 3140.9455

global unit 10

array $3 * 0.0$

reflector $695 \mathrm{r} 0, \quad 5.081$

reflector $315 r 15.15 .881$

end geom

read array

ara $=1$ nux $=16$ nuy $=15$ fill $f 1$ end fill

$93.98 \quad 2.54$

$93.98 \quad 2.54$

$96.00 \quad 0.0$

$96.00 \quad 0.0$

$96.00 \quad 0.0$

$44.270-.0570$

$96.00 \quad 0.0$

$96.00 \quad 0.0$

$\begin{array}{llll}44.270 & -.6285 & 96.00 \quad 0.0\end{array}$

$44.270 \quad-.7300 \quad 96.00 \quad 0.0$

$\begin{array}{lllll}44.270 & -0.0570 & 96.00 & 0.0\end{array}$

$\begin{array}{llll}44.270 & -0.1585 & 96.00 & 0.0\end{array}$

$\begin{array}{llll}44.270 & -0.6285 & 96.00 & 0.0\end{array}$

$\begin{array}{llll}44.270 & -0.7300 \quad 96.00 & 0.0\end{array}$

$\begin{array}{lllll}28.4220 & -15.9050 & 96.00 & 0.0\end{array}$

$28.5235-15.9050 \quad 96.00 \quad 0.0$

$28.9935-15.9050 \quad 96.00 \quad 0.0$

$29.0950 \quad-15.9050 \quad 96.00 \quad 0.0$

$28.4220-15.9050 \quad 96.00 \quad 0.0$

$28.5235-15.9050 \quad 96.00 \quad 0.0$ $28.9935 \quad-15.9050 \quad 96.00 \quad 0.0$ $29.0950-15.9050 \quad 96.00 \quad 0.0$

$\begin{array}{llll}45.0 & 0.0 & 96.00 & 0.0\end{array}$

$2 p 1.865$

$96.00 \quad 0.0$

$96.00 \quad 0.0$

$96.00 \quad 0.0$

ara=2 nux=16 nuy=15 fill $15 r 197916 \quad 112 r 1$ end fill ara $=3$ nux $=3$ nuy $=3$ fill 465787263 end fill end array

end data

end 


\section{pat8011}

$=\operatorname{csas} 25$

pat8011

44group latticecell

uo2 1 den=10.38 1.0 293922354.7429223895 .258 end

arbmag5 2.7040011302798 .85120000 .47140000 .4326000 0.22
31.0

end

$\begin{array}{lll}\text { h2o } & 3 & 1.0 \\ \mathrm{pb} & 4 \text { den }=11.34\end{array}$

arbmss $\quad \begin{aligned} & 7.90 \quad 30 \\ & \text { arbmboral }\end{aligned}$ arbmboral $2.618931005000 \quad 22.20 \quad 13027 \quad 71.646012 \quad 6.176$ end uo2 7 den=10.38 1.0293922354 .7429223895 .258 end 8 den $=2.651$ end

al $\quad 91.0$

end comp

squarepitch $1.6 \quad 0.79130 .9420 .820$ end

more data res $=7$ cyl inder 0.395 dan $(7)=0.991436$ end more

$4-18 \times 18$ assemblies separated by $0.65-\mathrm{cm}$-thick Boral plates and $3.6 \mathrm{~cm}$ I of water, plate-to-assembly distance of $0.8 \mathrm{~cm}$, assembl ies separated I from lead reflecting walls by $1.68 \mathrm{~cm}$ of water, $53.98 \mathrm{~cm}$ water height read parm run=yes gen $=405 \mathrm{npg}=600 \mathrm{nsk}=5$ nub=yes end parm

\section{read geom}

unit 1

cuboid $014 \mathrm{p0} 800$

cylinder 1110.395

cylinder $0 \begin{array}{llll}1 & 0.410\end{array}$

cylinder 210.470

cuboid $314 \mathrm{p0.800}$

unit 3

cyl inder 910.470

cyl inder 310.500

cylinder 3 id 5 i $4 \mathrm{p} 0.800$

unit 4

cylinder 910.470

cuboid $314 \mathrm{p0} .800$

cuboid 51400.800

unit 5

10

array

unit 6

cylinder 510.500

cuboid $314 \mathrm{p0} .800$

cuboid $5+4 \mathrm{p} 0.800$

unit 7

array $23 * 0.0$

reflector $814 \mathrm{r} 0.112 \mathrm{ro} 1$

$1.95 \quad 0.4$

$1.95 \quad 0.0$

56.180 .4

$\begin{array}{lll}56.18 & 0.4\end{array}$

56.180 .0 reflector $614 \mathrm{ro} .432 \mathrm{rO}$

reflector $814 \mathrm{ro} .112 \mathrm{ro}$

unit 8

cuboid $312.0 \quad 0.030 .1 \quad 0.056 .180 .4$

cuboid $512.0 \quad 0.030 .1 \quad 0.056 .180 .0$

unit 9

array $33 * 0.0$

unit 10

cuboid 3162.20 .02 .00 .056 .180 .4

cuboid 5162.20 .02 .00 .056 .180 .0

unit 11

array $43 * 0.0$

reflector $314 \mathrm{ro} 0.232 \mathrm{ro} 0$

reflector $414 \mathrm{r} 10.02 \mathrm{r} 0.0$

reflector $314 r 18.72 r 0.0$

reflector $515 r 0.0 \quad 0.8$

reflector 315 ro.0 20.0

unit 12

cyl inder $510.500 \quad 36.020 .0$

cuboid $014 \mathrm{p0.800} \quad 36.020 .0$

unit 13

array $53 * 0.0$

reflector $814 \mathrm{ro} .112 \mathrm{ro}$

reflector $614 \mathrm{ro} 432 \mathrm{2r}$

reflector $6114 \mathrm{ro} 432 \mathrm{2ro}$

unit 14

cuboid $012.0 \quad 0.030 .1 \quad 0.0 \quad 36.02 \quad 0.0$

unit 15

array $63 * 0.0$

unit 16

cuboid 0162.20 .02 .00 .036 .020 .0

unit 17

array $73 * 0.0$

reflector 0 \$ 4 ro.23 $2 \mathrm{r} 0.0$

reflector $414 \mathrm{r} 10.02 \mathrm{2} 0.0$

reflector $014 \mathrm{r} 18.72 \mathrm{r0} 0.0$

global unit 18

array $83 \star 0.0$

end geom

read array

ara $=1$ nux=1 nuy=1 nuz=3 fill $4 \begin{array}{llll} & 3\end{array}$

end

ara=2 nux=18 nuy=18 nuz=1 fill $6 \quad 16 r 5 \quad 6 \quad 18 r 5 \quad 15 q 18 \quad 6 \quad 16 r 5 \quad 6 \quad$ end

fill

ara $=3$ nux $=3$ nuy=1 nuz=1 fill 787

end

fill

ara $=4$ nux=1 nuy=3 nuz=1 fill 9109

end

ara=5 nux=18 nuy=18 nuz=1 fill 12 16ri 12 18r1 $15 q 1812 \quad 16 r 1 \quad 12$ end fill 
ara=6 nux=3 nuy=1 nuz=1 fill $13 \quad 14 \quad 13$ fill

ara $a=7$ nux=1 nuy=3 nuz=1 fill $15 \quad 16 \quad 15$

fill

ara $=8$ nux=1 nuy=1 nuz=2 fill $11 \quad 17$

fill

end array

end data

end

pat8012

$=\operatorname{csas} 25$

pat80l2

44 group latticecell

uo2 1 den=10.38 $1.0293922354 .7429223895 .258 \quad$ end

arbmag5 $2.7040011302798 .8512000 \quad 0.47 \quad 14000 \quad 0.43 \quad 26000$

$\begin{array}{llllllllllllllll}\text { arbmags } & 2.70 & 4 & 0 & 0 & 1 & 13027 & 98.85 & 12000 & 0.47 & 14000 & 0.43 & 26000\end{array}$

h20

$\mathrm{pb} \quad 4$ den=11.34

end

$\begin{array}{lllllllll}7.90 & 3 & 0 & 0 & 1 & 26000 & 72.002400018 .0028000 & 10.05 & 5 \text { end }\end{array}$ arbmboral $2.618931005000 \quad 22.201302771 .646012 \quad 6.176$ end uo2 7 den=10.38 $1.0293922354 .7429223895 .258 \quad$ end al 8 den $=2.65$

end comp

91.0

squarepitch $1.6 \quad 0.79130 .9420 .820$ end

more data res $=7$ cyl inder 0.395 dan $(7)=0.991436$ end more

4-18x18 assemblies separated by $0.65-\mathrm{cm}$-thick Boral plates and $3.6 \mathrm{~cm}$ 'of water, plate-to-assembly distance of $0.8 \mathrm{~cm}$, assemblies separated Ifrom lead reflecting walls by $3.95 \mathrm{~cm}$ of water, $63.85 \mathrm{~cm}$ water height read parm run=yes gen $=405 \mathrm{npg}=600 \mathrm{nsk}=5$ nub=yes end parm read geom

unit 1

cyl inder 710.395

cyl inder 0110.410

cylinder 210.470

cuboid $014 \mathrm{p0} .800$

unit 2

cyl inder 110.395

cylinder 010.410

cylinder 210.470

cuboid $314 \mathrm{p0} .800$

unit 3

cyl inder 910.470

cylinder 310.500

cuboid 51400.800

unit 4

cyl inder 910.470

cuboíd $314 \mathrm{p0} .800$

cuboid $514 \mathrm{p0} .800$

26.150 .0

$26.15 \quad 0.0$

26.150 .0

26.150 .0

63.850 .0

63.850 .0

63.850 .0

$63.85 \quad 0.0$

$0.25 \quad 0.0$

$0.25 \quad 0.0$

$\begin{array}{ll}1.95 & 0.4\end{array}$

1.950 .4

$1.95 \quad 0.0$ unit 5

array $13 * 0.0$

unit 6

cylinder $510.500 \quad 66.050 .4$

cuboid $314 \mathrm{p0} .800 \quad 66.050 .4$

cuboid 514 p0.800 66.050 .0

unit 7

array $23 * 0.0$

reflector 814 ro.11 2ro 1

reflector $614 \mathrm{r} 0.432 \mathrm{r} 01$

reflector $814 \mathrm{ro} .112 \mathrm{ro} 1$

unit 8

cuboid $\quad 312.00 .030 .1 \quad 0.066 .050 .4$

cuboid $512.00 .030 .1 \quad 0.066 .05 \quad 0.0$

unit 9

array $33 * 0.0$

unit 10

cuboid $3162.2 \quad 0.02 .0 \quad 0.066 .05 \quad 0.4$

cuboid $5162.2 \quad 0.02 .0 \quad 0.066 .05 \quad 0.0$

unit 11

array $43 * 0.0$

reflector $314 \mathrm{r} 2.502 \mathrm{ro} .0$

reflector $414 r 10.02 r 0.0$

reflector $314 r 18.72 r 0.0$

reflector $515 \mathrm{ro.0} \quad 0.8$

reflector $315 r 0.0 \quad 20.0$

unit 12

cyl inder $510.500 \quad 26.150 .0$

cuboid $014 \mathrm{p0} 0.800 \quad 26.150 .0$

unit 13

array $53 * 0.0$

reflector 814 ro.11 2ro 1

reflector $614 \mathrm{r} 0.432 \mathrm{r} 01$

reflector $814 \mathrm{ro} 0.112 \mathrm{r} 01$

unit 14

$\begin{array}{llllllll}\text { cuboid } \quad 0 & 1 & 2.0 & 0.0 & 30.1 & 0.0 & 26.15 & 0.0\end{array}$

unit 15

array $63 * 0.0$

unit 16

cuboid $0162.20 .02 .0 \quad 0.026 .15 \quad 0.0$

unit 17

array $73 * 0.0$

reflector $014 r 2.502 r 0.0$

reflector $414 \mathrm{r} 10.0 \mathrm{2r} 0.0$

reflector $014 r 18.72 r 0.0$

global unit 18

array $83 * 0.0$

end geom

read array

ara=1 nux=1 nuy=1 nuz=3 fill 432 
fill

ara $=2$ nux $=18$ nuy=18 nuz=1 fill $6 \quad 16 r 5 \quad 6 \quad 18 r 5 \quad 15 q 18 \quad 6 \quad 16 r 5 \quad 6$ and

fill

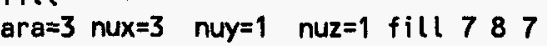

end

ara $=4$ nux=1 nuy=3 nuz=1 fill 9 10 9

end

ara=5 nux=18 nuy=18 nuz=1 fill $12 \quad 16 r 1 \quad 12 \quad 18 r 1 \quad 15 q 18 \quad 12 \quad 16 r 1 \quad 12$ end fill

ara=6 nux=3 nuy=1 nuz=1 fill 131413 end

fill

ara $a=7$ nux=1 nuy=3 nuz=1 fill $15 \quad 16 \quad 15$

end

fill

ara $=8$ nux $=1$ nuy=1 nuz $=2$ fill $11 \quad 17$

end

fill

end array

end data

end

pat80ss1

$=\operatorname{csas} 25$

pat80ss1

44 group latticecell

402 1 den=10.381.0293922354.7429223895.258

$\begin{array}{lllllllll}\text { arbmag5 } 2.7040 & 011302798.85 & 12000 & 0.47 & 14000 & 0.4326000\end{array}$ 0.2221293

n20 31.0

arbmst $\quad \begin{array}{lllllllllllllll} & 7.80 & 3 & 0 & 0 & 1 & 26000 & 99.66 & 6012 & 0.14 & 14000.20 & 4\end{array}$ arbmss $\quad 7.90 \quad 300012600072.002400018 .00 \quad 28000 \quad 10.05$

arbmboral $2.618931005000 \quad 22.20 \quad 13027 \quad 71.646012 \quad 6.176$

uo2 7 den=10.38 1.0293922354 .7429223895 .258

at 8 den $=2.651$

al 91.0

end comp

squarepitch 1.60 .79130 .9420 .820

more data res $=7$ cyl inder 0.395 dan(7) $=0.991436$ end more

4-18x18 assembl ies separated by $0.65-\mathrm{cm}$-thick Boral plates and $3.6 \mathrm{~cm}$ 'of water, plate-to-assembly distance of $0.8 \mathrm{~cm}$, assemblies separated I from stainless steel reflecting walls by $1.68 \mathrm{~cm}$ of water, $51.55 \mathrm{~cm}$ iwater height

read parm run=yes gen $=405 \mathrm{npg}=600$ nsk $=5$ nub=yes end parm

\section{read geom}

unit 1

cyl inder 710.395

cylinder 011

cylinder 2

inder 210.470

cuboid $014 \mathrm{p0.800}$

38.450 .0

38.450 .0

38.450 .0

38.450 .0

51.550 .0 cyl inder 010.410

\begin{tabular}{ll}
51.550 .0 \\
\hline
\end{tabular}

cuboid 314 p0.800 $\quad 51.550 .0$

unit 3

cylinder $910.470 \quad 0.250 .0$

cyl inder $310.500 \quad 0.25 \quad 0.0$

$\begin{array}{llll}\text { cuboid } 514 \mathrm{p0} .800 & 0.25 & 0.0\end{array}$

unit 4

cylinder 910.470

cuboid $514 \mathrm{p0.800} \quad 1.95 \quad 0.0$

unit 5

unit 6

cyl inder $510.500 \quad 53.75 \quad 0.4$

cuboid $314 \mathrm{p0} .800 \quad 53.750 .4$

cuboid $514 \mathrm{p0.800} \quad 53.750 .0$

unit 7

array $23 * 0.0$

reflector 814 ro.112ro

reflector $614 \mathrm{ro} 0.432 \mathrm{ro}$

reflector $814 \mathrm{ro} .112 \mathrm{rO}$

unit 8

cuboid 312.00 .030 .10 .053 .750 .4

cuboid $5,2.00 .030 .10 .053 .750 .0$

unit 9

array $3 * 0.0$

unit 10

cuboid $3162.2 \quad 0.02 .0 \quad 0.053 .75 \quad 0.4$

cuboid $5162.20 .02 .0 \quad 0.053 .750 .0$

unit 11

array $43 * 0.0$

reflector $314 \mathrm{ro} .232 \mathrm{r0} 0$

reflector $414 \mathrm{r} 15.02 \mathrm{r0.0}$

reflector 314 r 13.7 2ro.0

reflector 51 5ro.0 0.8

reflector $315 r 0: 0 \quad 20.0$

unit 12

$\begin{array}{llllll}\text { cyl inder } 5 & 1 & 0.500 & 38.45 & 0.0 \\ \text { cuboid } & 1 & 400.800 & 38.45 & 0.0\end{array}$

cuboid

array $53 * 0.0$

reflector 814 ro.11 2ro 1

reflector $614 \mathrm{ro} .432 \mathrm{rO} 1$

reflector $814 \mathrm{ro} .112 \mathrm{ro} 1$

$\begin{array}{lllllllllll}\text { unit } 14 & & & & & \\ \text { cuboid } & 0 & 1 & 2.0 & 0.0 & 30.1 & 0.0 & 38.45 & 0.0\end{array}$

unit 15

array $63 * 0.0$

unit 16

cuboid $0162.2 \quad 0.02 .0 \quad 0.038 .450 .0$ 
unit 17

array $73 * 0.0$

reflector 014 ro.23 2r0.0 1

reflector $414 \mathrm{r} 15.02 \mathrm{2r0.0} \quad 1$

reflector $014 r 13.7 \quad 2 r 0.0$

globa! unit 18

array $83 * 0.0$

end geom

read array

ara=1 nux=1 nuy=1 nuz=3 fill 432

end

ara=2 nux=18 nuy=18 nuz=1 fill $6 \quad 16 r 5 \quad 6 \quad 18 r 515 q 18 \quad 6 \quad 16 r 56$ end

fill

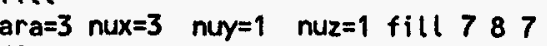

fill

ara $=4$ nux=1 nuy=3 nuz=1 fill 9109

fill

ara=5 nux=18 nuy=18 nuz=1 fill $12 \quad 16 r 1 \quad 12 \quad 18 r 1 \quad 15 q 18 \quad 12 \quad 16 r 1 \quad 12$ end

fill

ara $=6$ nux $=3$ nuy=1 nuz=1 fill $13.1413 \quad$ end

fill

ara=7 nux=1 nuy=3 nuz=1 fill 151615 end

fill

N fill

nux $=1$ nuy $=1$ nuz=2 fill $11 \quad 17$

end

end array

end data

end

pat80ss?

$=\operatorname{csas} 25$

pat80ss2

44group latticecel

uo2 1 den=10.38 1.0293922354 .7429223895 .258

end

$\begin{array}{lllllllllll}\text { arbmag5 } 2.7040 & 0 & 1 & 1302798.85 & 12000 & 0.47 & 14000 & 0.43 & 26000\end{array}$ 0.2221293

h2o $\quad 31.0$

end

$\begin{array}{lllllllllllll}\text { arbmst } \quad 7.80 & 3 & 0 & 0 & 1 & 26000 & 99.66 & 6012 & 0.14 & 14000 & 20 & 4 & \text { end }\end{array}$

arbmss $\quad 7.90 \quad 3 \quad 0 \quad 0 \quad 12600072.002400018 .0028000 \quad 10.05$ end

arbmboral $2.618931005000 \quad 22.201302771 .646012 \quad 6.176$ end

402 7 den=10.381.029392235 4.7429223895 .258 end

al 8 den $=2.651$

at

end comp

1.0

end

squarepitch $1.6 \quad 0.79130 .9420 .820$

end

data res $=7$ cylinder 0.395 dan(7) $=0.991436$ end more

$4-18 \times 18$ assemblies separated by $0.65-\mathrm{cm}$-thick Boral plates and $3.6 \mathrm{~cm}$

'of water, plate-to-assembly distance of $0.8 \mathrm{~cm}$, assembl ies separated 'from stainless steel reflecting walls by $3.95 \mathrm{~cm}$ of water, $61.84 \mathrm{~cm}$ 'water height

read parm run=yes gen=405 npg=600 nsk=5 nub=yes end parm

\section{read geom}

unit

cyl inder 710.395

cyl inder 010.410

cylinder 210.470

cuboid $0,4 \mathrm{p} 0.800$

28.160 .0

28.160 .0

28.160 .0

unit 2

cylinder 190.395

cylinder 0 1 0.410

28.160 .0

61.840 .0

61.840 .0

61.840 .0

61.840 .0

cuboid 31400.800

unit 3

cylinder $910.470 \quad 0.250 .0$

cylinder $310.500 \quad 0.250 .0$

cuboid $514 \mathrm{p0} .800 \quad 0.250 .0$

$\begin{array}{lll}0.25 & 0.0\end{array}$

cylinder 910.470

$1.95 \quad 0.4$

cuboid $314 \mathrm{p0} .800 \quad 1.95 \quad 0.4$

cuboid $514 \mathrm{p0.800} \quad 1.95 \quad 0.0$

unit 5

array $13 * 0.0$

unit 6

cylinder $510.500 \quad 64.040 .4$

cuboid $31400.800 \quad 64.040 .4$

cuboid 514 p0.800 64.040 .0

unit 7

array $23 * 0.0$

reflector $814 \mathrm{ro} .11$ 2ro 1

reflector $614 \mathrm{r} 0.432 \mathrm{ro}$

reflector $814 \mathrm{ro} .112 \mathrm{ro}$

unit 8

cuboid 312.00 .030 .10 .064 .040 .4

cuboid $512.0 \quad 0.030 .1 \quad 0.064 .04 \quad 0.0$

cuboid

unit 9

array $3 * 0.0$

unit 10

cuboid 3162.20 .02 .00 .064 .040 .4

cuboid $5162.2 \quad 0.02 .00 .064 .040 .0$

unit 11

array $43 * 0.0$

reflector $314 \mathrm{r} 2.502 \mathrm{r0.0}$

reflector $414 r 15.02 r 0.0$

reflector $314 \mathrm{r} 13.72 \mathrm{r0.0}$

reflector $515 \mathrm{ro.0} \quad 0.8$

reflector $315 \mathrm{r0} .0 \quad 20.0$

unit 12

cýl inder 510.500

28.160 .0 
cuboid $014 \mathrm{p} 0.800 \quad 28.160 .0$

unit 13

array $53 * 0.0$

reflector 814 ro.11 2ro 1

reflector $614 \mathrm{ro} 0.432 \mathrm{ro}$ ?

reflector $814 \mathrm{ro} .112 \mathrm{ro} 1$

unit 14

cuboid $012.0 \quad 0.030 .1 \quad 0.028 .160 .0$

unit 15

array $63 * 0.0$

unit 16

cuboid 0162.20 .02 .00 .028 .160 .0

unit 17

array $73 * 0.0$

reflector $014 \mathrm{r} 2.50 \quad 2 \mathrm{r} 0.0$

reflector $414 \mathrm{rr} 15.0$ 2r0.0

reflector $014 \mathrm{r} 13.72 \mathrm{r} 0.0$

global unit 18

array $8 * 0.0$

end geom

read array

ara=1 nux=1 nuy=1 nuz=3 fill $4 \begin{array}{llll}4 & 3\end{array}$

fill

$\underset{N}{\omega}$

a $a=2$ nux $=18$ nuy=18 nuz=1 fill $6 \quad 16 r 5 \quad 6 \quad 18 r 5 \quad 15 q 18 \quad 6 \quad 16 r 56$

ara $=3$ nux $=3$ nuy $=1$ nuz=1 fill $7 \quad 87$

fill

ara $=4$ nux=1 nuy $=3$ nuz=1 fill 9109

fill

ara=5 nux=18 nuy=18 nuz=1 fill $12 \quad 16 r 1 \quad 12 \quad 18 r 1 \quad 15 q 18 \quad 12 \quad 16 r 1 \quad 12$ end fill

ara=6 nux=3 nuy $=1$ nuz=1 fill $13 \quad 14 \quad 13$

fill

ara $=7$ nux=1 nuy $=3$ nuz=1 fill $15 \quad 16 \quad 15$

fill

ara=8 nux=1 nuy=1 nuz=2 fill $11 \quad 17$

fill

end array

end data

end

\section{$33269 a$}

acsas 25

$43269 a$

44group Latticecel

no2 $\quad 10.93029392235 \quad 5.709223894 .30$

ss304 21.0

h20 31.0

al 41.0 uo2 $\quad 5 \quad 0.930293922355 .709223894 .30$

arbmagincd 9.9130014700079 .604900015 .17480005 .086 end end comp

squarepitch 1.42240 .9068130 .993120 .91690

more date dan(5) $=927954$ res=5 cyl .4534 end more

Core of 453 fuel rods, 6-1.024-cm-diam Ag-In-Cd rods, $88.27 \mathrm{~cm}$ water

'height

read parm run=yes gen $=405 \mathrm{npg}=600 \mathrm{nsk}=5$ nub=yes end parm

read geom

unit 1

cylinder 111.4534

cylinder 011.4585

cyl inder 21.4966

cuboid $314 \mathrm{p} .7112$

unit 2

cylinder 11 1 .4534

cylinder 019.4585

cylinder 2,4966

cuboid $414 p .7112$

unit 3

cyl inder 11.4534

cyl inder 001 . 14585

cylinder 21 . 4966

cuboid $314 \mathrm{p} .7112$

unit 4

cylinder 51.4534

cylinder 001.4585

cylinder 21.4966

cuboid $014 \mathrm{p} .7112$

unit 5

cylinder $61 \quad .5118$

cuboid $314 \mathrm{p} .7112$

unit 6

cylinder 61

cuboid 414 p.7112

unit 7

cylinder $61 \quad .5118$

cuboid $314 \mathrm{p} .7112$

unit 8

cylinder $61 \quad .5118 \quad 93.97988 .27$

cuboid 014 p.7112 93.97988 .27

unit 9

array $13 * 0.0$

reflector $312 r 11.27762 r 18.38962$ 2ro

reflector $415 \mathrm{ro} 3.175$

reflector 312 2r3.7224 3r0 6.35

unit 10

array $23 * 0.0$

reflector $4 \quad 12$ 2r11.2776 2r18.3896 2ro 1 
array $43 * 0.0$

reflector 012 2r15. 2r18.3896 2r0 I

reflector 414 ro 1.270

global

unit 13

array $53 * 0.0$

end geom

\section{read array}

ara $=1$ nux $=27$ nuy=17 nuz=1 fill

$113 r 15$ 7r1 5 7r1 5 199r1 5 7r1 5 7r1 $5 \quad 113 r 1$ end fill ara=2 nux=27 nuy=17 nuz=1 fill

$113 r 267 r 267 r 26$ 199r2 6 7r2 6 7r2 6113 r2 end fill ara $=3$ nux=27 nuy=17 nuz=1 fill

$113 r 377 r 377 r 37199 r 377 r 377 r 37113 r 3$ end fill ara $=4$ nux $=27$ nuy $=17$ nuz=1 fill

$113 r 48$ 7r4 $87 r 48199 r 487 r 487 r 48113 r 4$ end fill

ara $=5$ nux $=1$ nuy=1 nuz=4
fill $91011 \quad 12 \quad$ end fill

$\omega$ end array

fill

$\omega$

\section{end}

\section{$43269 \mathrm{~b} 1$}

$=\operatorname{csas} 2 x$

H3269b1

44 group

no2

latticecell

ss304 20.950

3

h20

al 5

h20 6

$\begin{array}{llllll}\text { uo2 } & 7 & 0.95029392235 & 3.709223896 .30\end{array}$

553048

90.950293922353 .709223896 .30

end comp

squarepitch $1.10490 .754 \quad 1 \quad 3 \quad 0.855520 .77880$

more data dan $(7)=.301909$ res $=7$ cyl .3772

$\operatorname{dan}(9)=.924574$ res $=10 \mathrm{cyl} .3772$ end more

Core of 2221 fuel rods, 12-0.838-cm-diam Ag-In-Cd rods, $55.41 \mathrm{~cm}$ water

'height

read parm run=yes gen $=405$ npg $=600$ nsk $=5$ nub=yes end parm

end
end
end
end
end
end
end
end
end
end
water

cylinder 710.3772

cyl inder 010.3894

cyl inder 810.4300

cuboid $614 \mathrm{p} .5525$

unit 2

cuboid 500 \& $4 p .5525$

unit 3

cylinder 410.4191

cuboid $614 \mathrm{p} .5525$

unit 4

cuboid

$614 p .5525$

unit 5

cylinder 710.3772

cylinder 010.3894

cylinder 810.4300

cuboid $514 \mathrm{p} .5525$

unit 6

cuboid $500+4 \mathrm{p} .5525$

unit 7

cylinder 490.4191

cuboid $514 \mathrm{p} .5525$

unit 8

cuboid 514 p.5525

unit 9

cylinder $\begin{array}{lll}7 & 1 & 0.3772\end{array}$

cylinder $\quad 0 \quad 1 \quad 0.3894$

cylinder 810.4300

cuboid 614 p.5525

unit 10

cuboid 500 I $4 \mathrm{p} .5525$

unit 11

cylinder $4 \quad 10.4191$

cuboid $614 \mathrm{p} .5525$

unit 12

14 p.5525

cuboid

614 p. 5525

unit 13

cylinder 910.377

cylinder $\quad 0 \quad 1 \quad 0.389$

cylinder 810.430

cuboid $014 \mathrm{p} .5525$

unit 14

40.5525

cuboid $50014 \mathrm{p} .5525$

unit 15

cyl inder $\quad 4 \quad 10.4191$

cuboid $014 \mathrm{p} .5525$

unit 16

$014 \mathrm{p} .5525$

cuboid

array $13 * 0.0$

$\begin{array}{ll}35.88 & 0.00 \\ 35.88 & 0.00 \\ 35.88 & 0.00 \\ 35.88 & 0.00 \\ 35.88 & 0.00 \\ 35.88 & 0.00 \\ 35.88 & 0.00 \\ 35.88 & 0.00 \\ 1.27 & 0.00 \\ 1.27 & 0.00 \\ 1.27 & 0.00 \\ 1.27 & 0.00 \\ 1.27 & 0.00 \\ 1.27 & 0.00 \\ 1.27 & 0.00 \\ 1.27 & 0.00 \\ 18.26 & 0.00 \\ 18.26 & 0.00 \\ 18.26 & 0.00 \\ 18.26 & 0.00 \\ 18.26 & 0.00 \\ 18.26 & 0.00 \\ 18.26 & 0.00 \\ 18.26 & 0.00 \\ 121.92 & 55.41 \\ 121.92 & 55.41 \\ 121.92 & 55.41 \\ 121.92 & 55.41 \\ 121.92 & 55.41 \\ 121.92 & 55.41 \\ 121.92 & 55.41 \\ 121.92 & 55.41\end{array}$




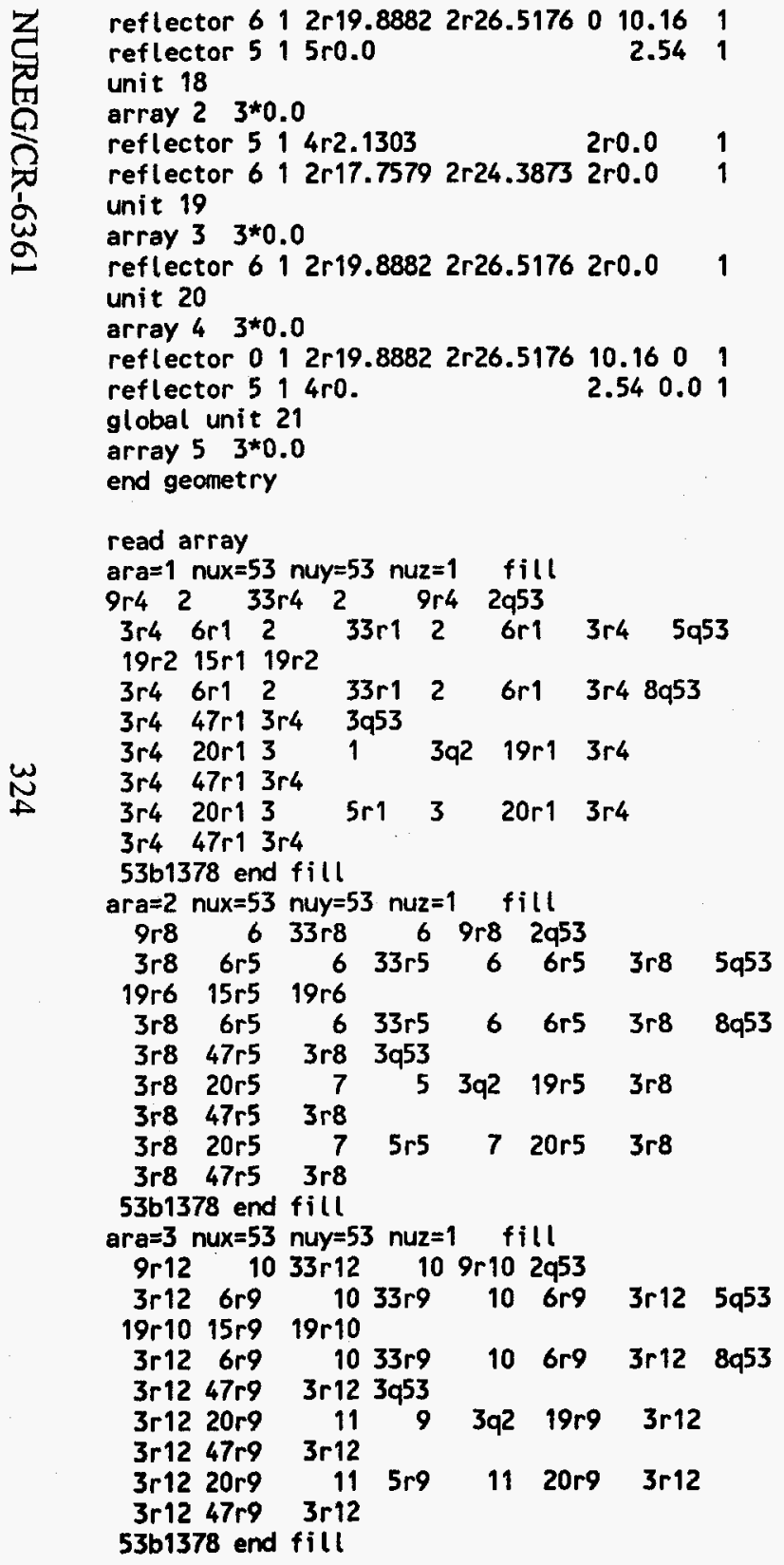

$53 b 1378$ end $f i l l$

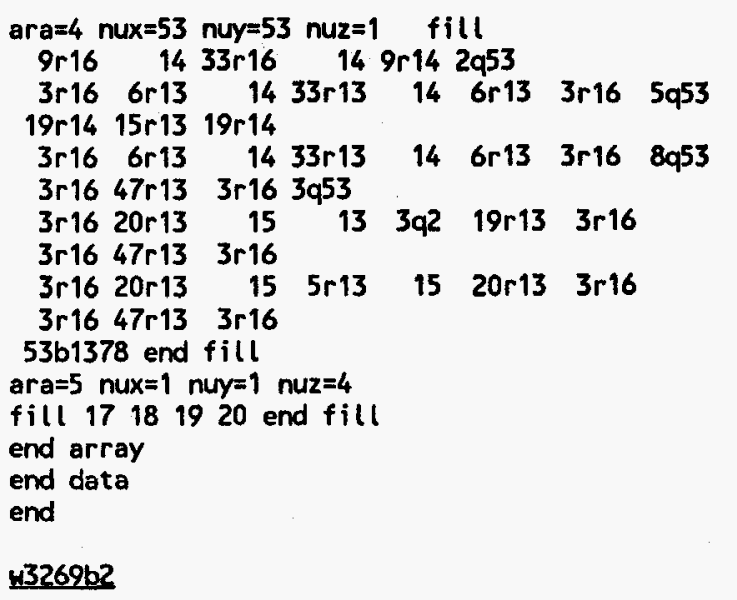

-csas $2 x$

$43269 b 2$

44 group latticecell

uo2 10.950293922353 .709223896 .30 end

$\begin{array}{lll}s s 304 & 2 & 1.0 \\ \text { h20 } & 3 & 1.0\end{array}$

end

arbmagincd 9.9130014700079 .604900015 .17480005 .084 end

$\begin{array}{lllll}\text { al } & 5 & 1.0 & \text { end } \\ \text { h20 } & 6 & 1.0 & \text { end }\end{array}$

uo2 $\quad 7 \quad 0.950293922353 .709223896 .30$ end

ss304 $81.0 \quad$ end

$\begin{array}{llllll}\text { uo2 } & 9 & 0.950293922353 .709223896 .30 & \text { end }\end{array}$

end comp

squarepitch $1.10490 .754 \quad 130.855520 .77880 \quad$ end

more data dan $(7)=.301909$ res $=7$ cyl .3772

dan(9) $=.924574$ res=10 cyl .3772 end more

Core of 2209 fuel rods, $24-0.838-\mathrm{cm}-\mathrm{diam} \mathrm{Ag}-\mathrm{In}$-Cd rods, $64.56 \mathrm{~cm}$ water

'height

read parm run=yes gen=405 npg=600 nsk=5 nub=yes end parm

read geom

unit 1

cyl inder 710.3772

cylinder $0 \begin{array}{llll}0 & 0.3894 & 35.88 & 0.00\end{array}$

cylinder $810.4300 \quad 35.88 \quad 0.00$

$\begin{array}{lllll}\text { cuboid } 614 p .5525 & 35.88 & 0.00\end{array}$

$\begin{array}{lllll}\text { unit } 2 & & & & \\ \text { cuboid } & 500 & 14 \mathrm{p} .5525 & 35.88 & 0.00\end{array}$

$\begin{array}{lllll}\text { cuboid } 50014 p .5525 & 35.88 & 0.00\end{array}$

cylinder 4 1 $0.4191 \quad 35.88 \quad 0.00$

$\begin{array}{lllll}\text { cuboid } 614 \mathrm{p} .5525 & 35.88 & 0.00\end{array}$ 


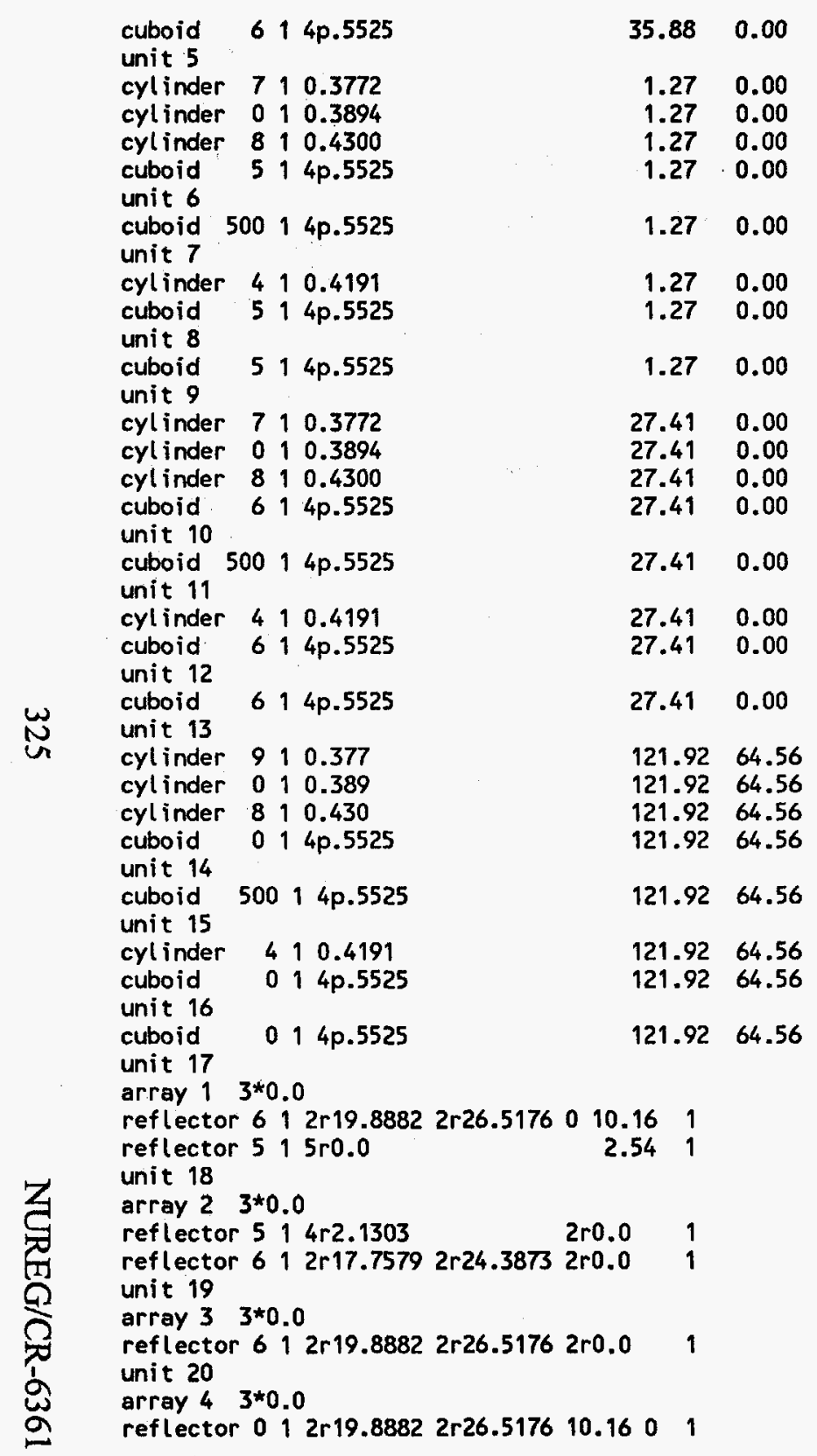

reflector $514 \mathrm{ro}$

global unit 21

end geometry

read array

ara $=1$ nux $=53$ nuy=53 nuz=1 fill

$9 r_{4} \quad 233 r 429 r_{4} 2 \mathrm{q} 53$

$3 r 4$ 6r1 2 33r1 2 6r1 3r4 $5 q 53$

$19 r 215 r 119 r 2$

$3 r 46 r 1 \quad 2 \quad 33 r 1 \quad 26 r 13 r 48953$

$3 r 4$ 47r1 3r4 3q53

$3 r 420 r 1 \quad 3 \quad 1 \quad 3 q 219 r 13 r 4$

$3 r 4 \quad 47 r 1 \quad 3 r 4$

$3 r 420 r 1 \quad 3 \quad 5 r 1 \quad 320 r 13 r 4$

$3 r 447 r 13 r 453 b 424$

$\begin{array}{lllllllllllllllll}3 r 4 & 6 r 1 & 2 & 1 & 3 & 1 & 3 q 2 & 24 r 1 & 2 & 6 r 1 & 3 r 4\end{array}$

$3 r 4$ 6ri $233 r 12$ 2 $6 r 13 r 4$

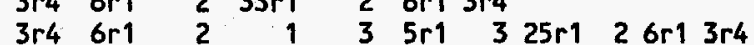

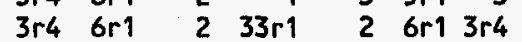

$3 r 46 r 1 \quad 2 \quad 1 \quad 35 r 1325 r 126 r 13 r 4$

$3 r 4$ 6r1 $233 r 1 \quad 26 r 13 r 4$

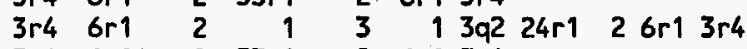

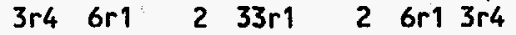

$19 r 215 r 119 r 2$

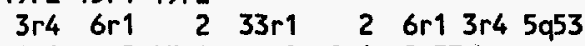

$9 r 4233 r 4 \quad 2 \quad 9 r 4 \quad 2 q 53$

end fill

ara $=2$ nux $=53$ nuy=53 nuz $=1$ fill

$\begin{array}{llllll}9 r 8 & 6 & 33 r 8 & 6 & 9 r 8 & 2 q 53\end{array}$

$\begin{array}{lllllllll}3 r 8 & 6 r 5 & 6 & 33 r 5 & 6 & 6 r 5 & 3 r 8 & 5 q 53\end{array}$

$19 r 615 r 519 r 6$

$\begin{array}{lllllllll}3 r 8 & 6 r 5 & 6 & 33 r 5 & 6 & 6 r 5 & 3 r 8 & 8 q 53\end{array}$

$3 r 8 \quad 47 r 5 \quad 3 r 8 \quad 3 a 53$

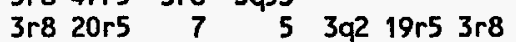

$3 r 8 \quad 47 r 5 \quad 3 r 8$

$\begin{array}{llr}3 r 8 & 20 r 5 & 7 \\ 3 r 8 & 47 r 5 & 3 r 8\end{array}$

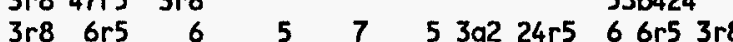

$\begin{array}{lllllll}3 r 8 & 6 r 5 & 6 & 33 r 5 & 6 & 6 r 5 & 3 r 8\end{array}$

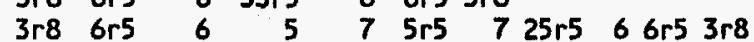

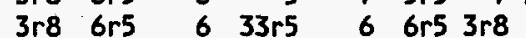

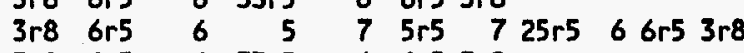

$\begin{array}{lllllll}3 r 8 & 6 r 5 & 6 & 33 r 5 & 6 & 6 r 5 & 3 r 8\end{array}$

$\begin{array}{lllllllllll}3 r 8 & 6 r 5 & 6 & 5 & 7 & 5 & 3 q 2 & 24 r 5 & 6 & 6 r 5 & 3 r 8\end{array}$

$\begin{array}{lllllll}3 r 8 & 6 r 5 & 6 & 33 r 5 & 6 & 6 r 5 & 3 r 8\end{array}$

$19 r 615 r 519 r 6$

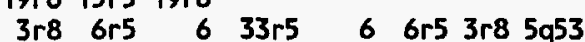

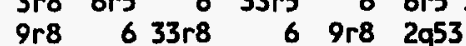

end $f i l l$

ara $=3$ nux $=53$ nuy $=53$ nuz $=1$ fill 


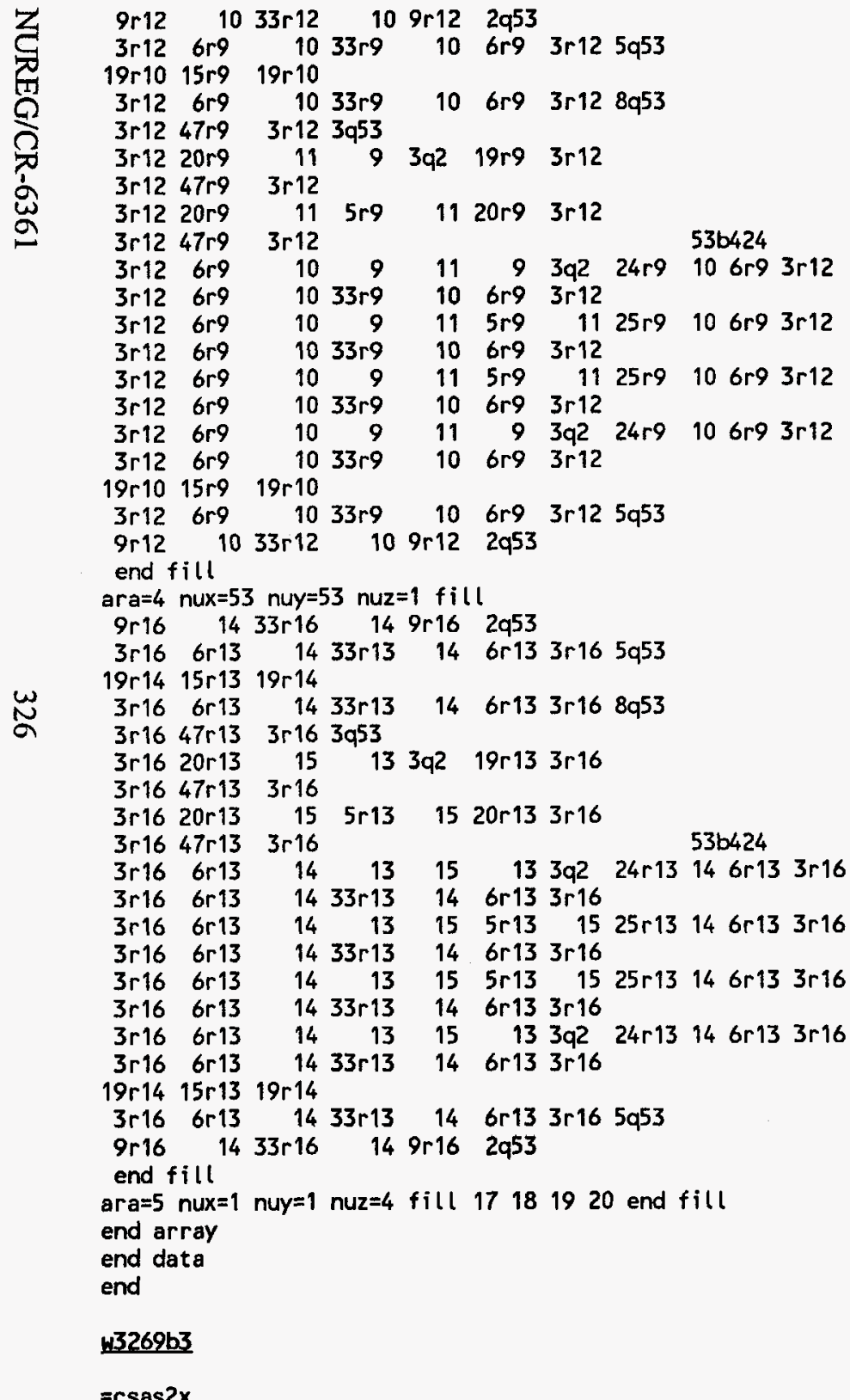

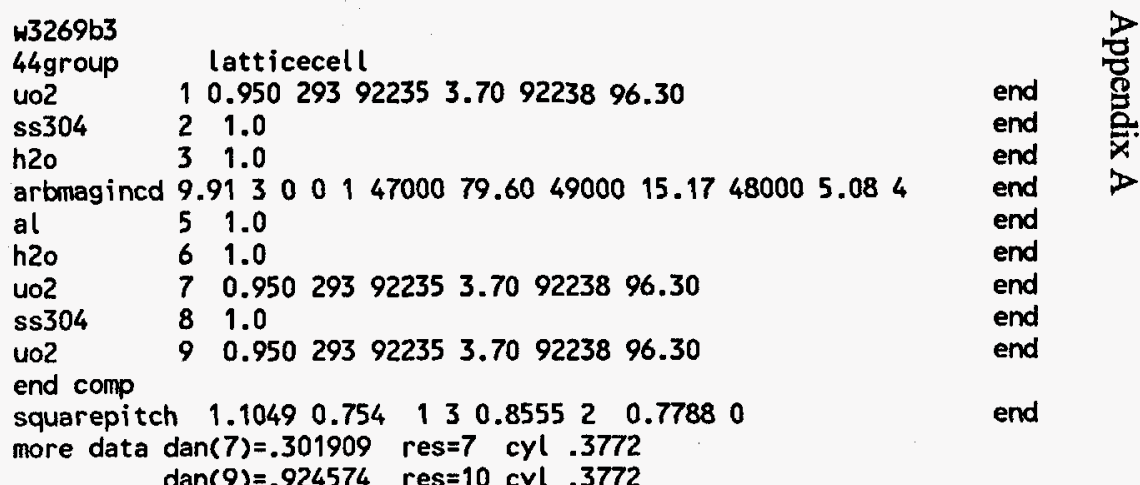

end more

Core of 2185 fuel rods, $48-0.838-\mathrm{cm}$-diam Ag-In-Cd rods, $57.00 \mathrm{~cm}$ water height

read parm run=yes gen $=405 \mathrm{npg}=600$ nsk $=5$ nub=yes end parm

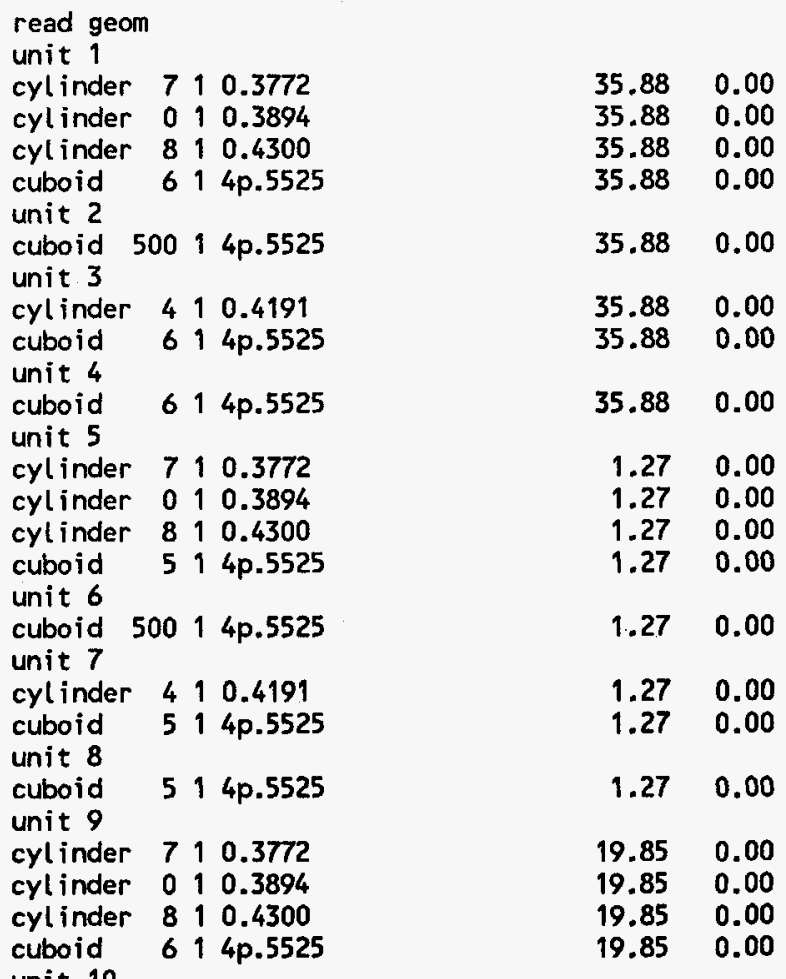




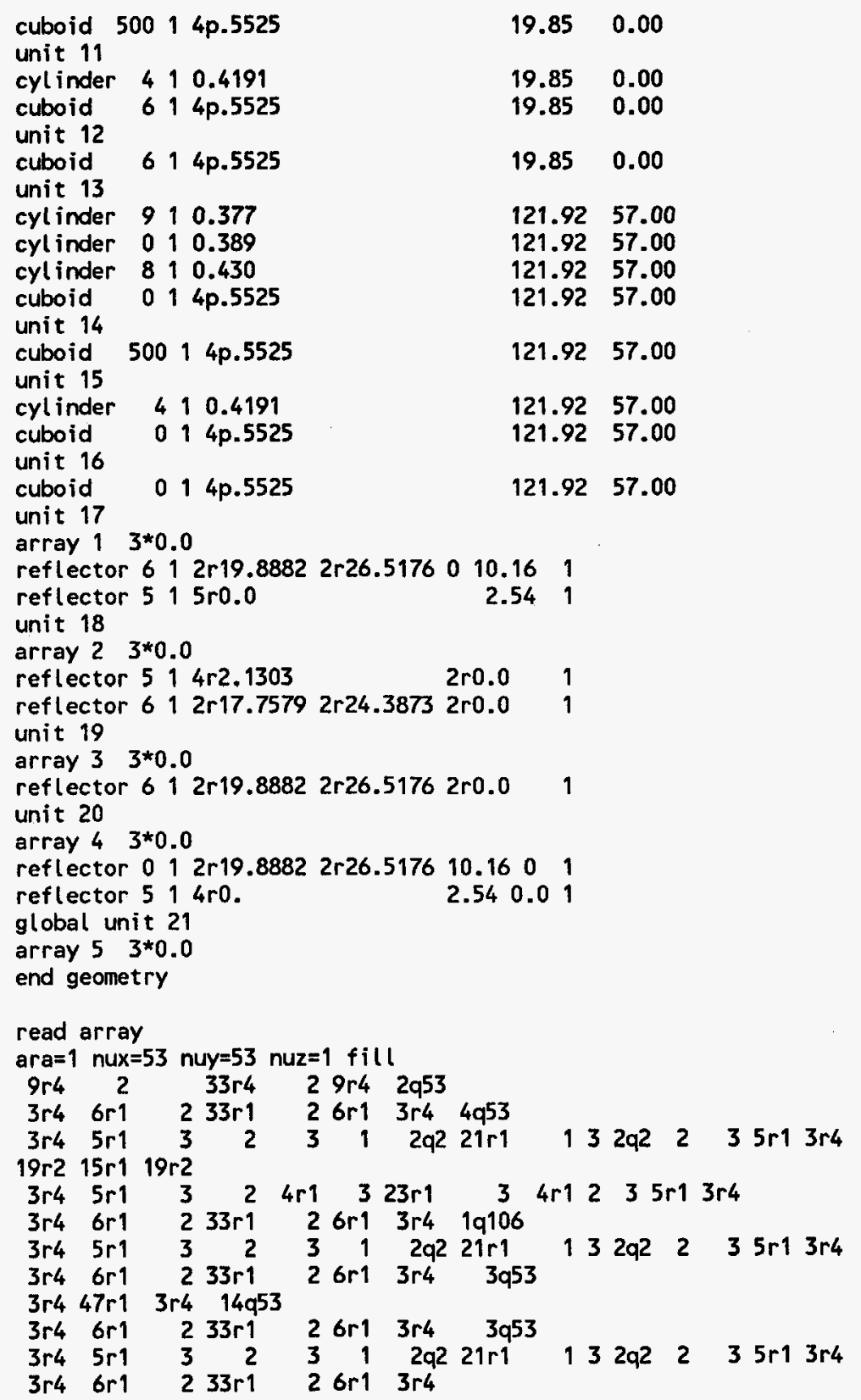

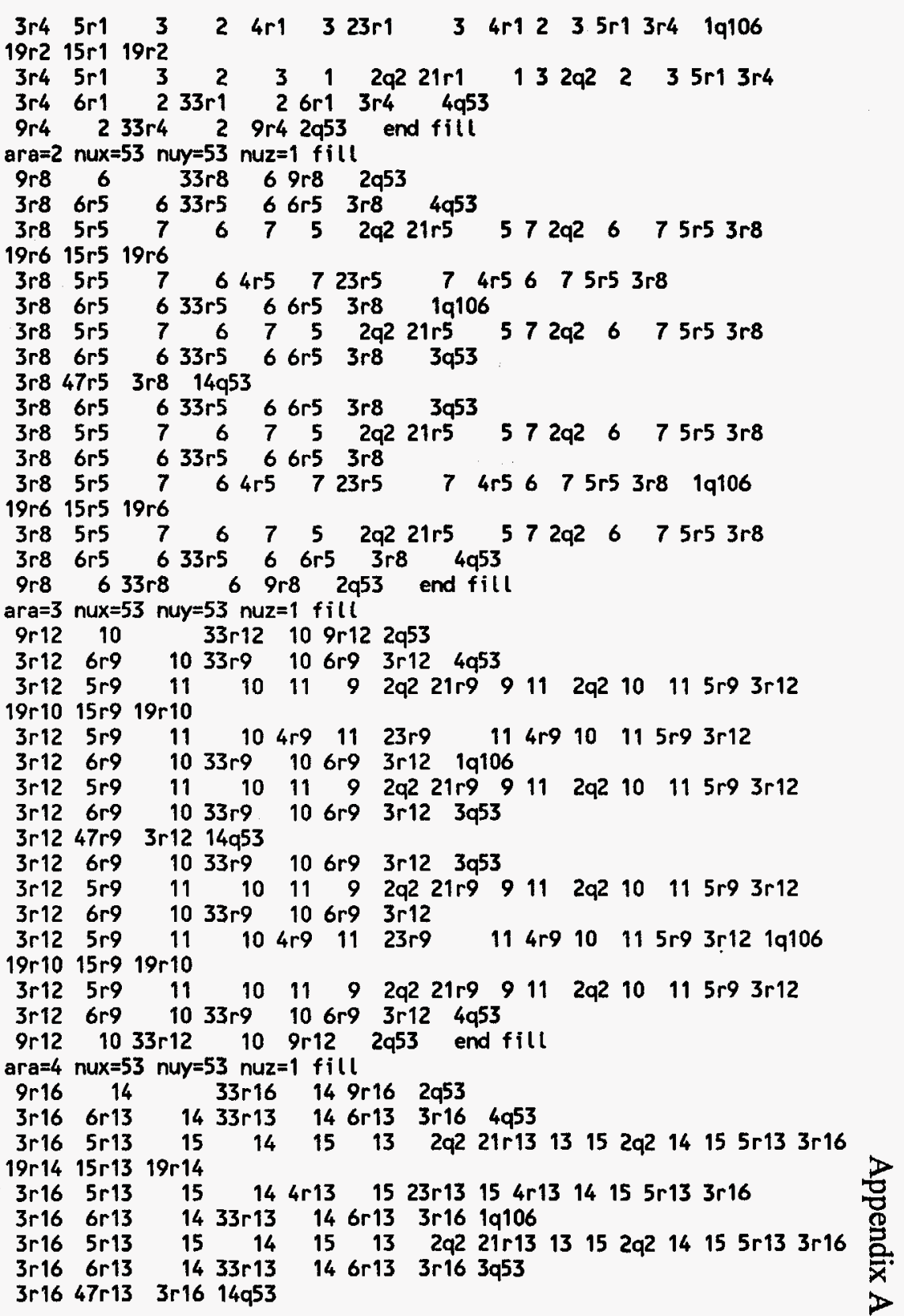




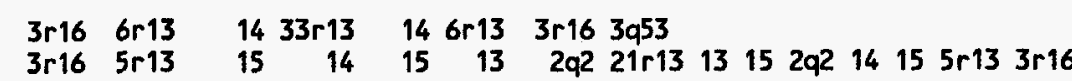
$3 r 16$ 6r13

$3 r 16 \quad 5 r 13$

$19 r 14+15 r 1319 r 14$

$33 r 13 \quad 146 r 13 \quad 3 r 16$

$19 r 14$ 15r13 19r14 $\begin{array}{llllllll}3 r 16 & 6 r 13 & 14 & 33 r 13 & 14 & 6 r 13 & 3 r 16 & 4953\end{array}$

9r16 $1433 r 16 \quad 149 r 162 q 53$ end fill

ara $=5$ nux $=1$ nuy $=1$ nuz $=4$

fill 17181920

end $f i l l$

end array

end data

end

W3269c

$=\operatorname{csas} 25$

$3269 \mathrm{c}$

44 group

4o2

latticecell

arbmzirz 6.5040014000098 .20260000 .21500001 .424000 0.102

h2o 31.0

arbmagincd 9.9130014700079 .604900015 .17480005 .084 end

al $\quad 51.0$

$\underset{\infty}{\infty}$

Uo2 end comp

squarepitch $1.524 \quad 1.016 \quad 131.189 \quad 2 \quad 1.0297 \quad 0$

more data res $=6 \mathrm{cyl} .508$ dan(6) $=.948580$ end more

'height

read parm run=yes gen $=405$ npg $=600$ nsk $=5$ nub=yes end parm

read geom

unit 1

cyl inder 110.5080

cyl inder 0110.5149

cylinder 2110.5947

cuboid $314 p .762$

unit

cyl inder 110.5080

cyl inder 0110.5149

cyl inder 210.5947

cuboid 514 p.762

unit 3

cyl inder 110.5080

cylinder 0110.5149

cyl inder 210.5947

cuboid 3 i 4 p.762

unit 4

14 p.762

cylinder 610.5080 $\begin{array}{ll}16.19 & 0.00 \\ 16.19 & 0.00\end{array}$

$16.19 \quad 0.00$

$32.17 \quad 0.00$ cylinder 210.5947

cuboid $014 \mathrm{p} .762$

unit 5

cylinder 410.5118

cuboid 3140.762

unit 6

cylinder 410.5118

cuboid 5 i 4 p.762

unit 7

cylinder 410.5118

cuboid $314 p .762$

unit 8

cylinder 410.5118

cuboid $014 \mathrm{p} .762$

unit 9

array $13 * 0.0$

reflector 314 r6.858 2 ro 1

$\begin{array}{lll}\text { reflector } 515 r 0 & 1.271\end{array}$

reflector $314 \mathrm{r8.142} 0.481$

reflector $515 \mathrm{ro} \quad 5.081$

unit 10

array $23 * 0.0$

reflector $514 r 6.858 \quad 2 r 01$

reflector $314 r 8.142$ 2ro

unit 11

array $33 * 0.0$

reflector $314 r 15.0 \quad 2 r 01$

unit 12

array $43 * 0.0$

reflector 014 r $15.0 \quad 2$ ro 1

global

unit 13

array $53 * 0.0$

end geom

read array

ara $=1$ nux $=31$ nuy $=31$ nuz $=1$ fill

$31 r 19 q 31$ 10r1 5 2r1 3q3 9r1 31r1 12r1 5 4r1 5 13r1 10r1 5 8r1 5

$11 r 1$

$31 r 1 \quad 1 b 464 \quad 32 r 1$ end fill

ara $=2$ nux $=31$ nuy $=31$ nuz $=1$ fill

$31 r 29 q 31$ 10r2 6 2r2 3q3 9r2 $31 r 2 \quad 12 r 264 r 2613 r 2 \quad 10 r 268 r 26$

$11 r^{2}$

$31 r 2 \quad 1 b 464 \quad 32 r 2$ end $f i l l$

ara $=3$ nux $=31$ nuy $=31$ nuz=1 fill

$\begin{array}{lllllllllllllllll}31 r 3 & 9 q 31 & 10 r 3 & 72 r 3 & 3 q 3 & 9 r 3 & 31 r 3 & 12 r 3 & 7 & 4 r 3 & 7 & 13 r 3 & 10 r 3 & 7 & 8 r 37\end{array}$

$11 r 3$

$31 \mathrm{r} 31 \mathrm{~b} 464 \quad 32 \mathrm{r} 3$ end fill

ara $=4$ nux $=31$ nuy=31 nuz=1 fill

$31 r 49 q 31$ 10r4 \& $2 r 43 q 39 r 4 \quad 31 r 4 \quad 12 r 48$ sr4 8 13r4 $10 r 48$ 8r4 8

$\begin{array}{ll}32.17 & 0.00 \\ 47.17 & 0.00 \\ 47.17 & 0.00 \\ 72.93 & -2.07 \\ 72.93 & -2.07 \\ & \\ 0.63 & 0.00 \\ 0.63 & 0.00 \\ & \\ 16.19 & 0.00 \\ 16.19 & 0.00 \\ 32.17 & 0.00 \\ 47.17 & 0.00\end{array}$


$11 \mathrm{r} 4$

$31{ }^{4} \quad 16464 \quad 32 r 4$ end fill

ara $=5$ nux $=1$ nuy $=1$ nuz $=4$

fill $91011 \quad 12$ end fill

end array

end data

end

\section{9s11}

$=\operatorname{csas} 25$

W3269s 11

44 group

uo2

latticecel

end

0.102

heo $\quad 31.0$

uo2 $\quad 50.950293922352 .729223897 .28$

end comp

squarepitch $1.524 \quad 1.016 \quad 1 \quad 3 \quad 1.189 \quad 2 \quad 1.030 \quad 0$

more data res $=5 \mathrm{cyl} .508$ dan(5) $=.948684$ end more

Core of $31 \times 31$ fuel rods, $38.58 \mathrm{~cm}$ water height

read parm run=yes gen $=405$ npg $=600$ nsk $=5$ nub=yes end parm

N read geom

cylinder 110.508

cylinder 010.515

cylinder 210.5945

cuboid $314 \mathrm{p} .762$

unit 2

cylinder 5110.508

cyl inder $\quad 0 \quad 1 \quad 0.515$

cyl inder 210.5945

cuboid $014 \mathrm{p} .762$

$\begin{array}{ll}38.58 & 0.00\end{array}$

$\begin{array}{ll}38.58 & 0.00\end{array}$

$\begin{array}{ll}38.58 & -2.07\end{array}$

$38.58-2.07$

$121.92 \quad 38.58$

$121.92 \quad 38.58$

$121.92 \quad 38.58$

unit 3

array $13 * 0.0$

unit 4

array $23 * 0.0$

global unit 5

array $3 \quad 3 * 0.0$

reflector $\quad 314 r 13.722 r 0 \quad 1$

reflector $415 \mathrm{ro.0} \quad 1.27$

reflector 314 r15 00.48

reflector $415 \mathrm{r} 0.0 \quad 5.081$

end geometry

read array

ara=1 nux $=31$ nuy=31 nuz=1 fill $31 r 130 \mathrm{q} 31$ end fill

ara $=2$ nux $=31$ nuy $=31$ nuz=1 fill 31 r2 $30 q^{31}$ end fill ara=3 nux=1 nuy=1 nuz=2 fill 34 end fill

end array

end data

end

\section{9s12}

$=\operatorname{csas} 25$

w3269s12

44group

10.930293922355 .709223894 .30

h20 31.0

41.0

uo2 $\quad 5 \quad 0.93029392235 \quad 5.709223894 .30$

end comp

squarepitch 1.42240 .9068130 .993120 .91690

more data dan $(5)=.927954$ res $=5 \mathrm{cyl} .4534$ end more

end

end

end

end

Core of $27 \times 17$ fuel rods, $52.97 \mathrm{~cm}$ water height

read parm run=yes gen $=405$ npg=600 nsk $=5$ nub=yes end parm

end

read geon

unit 1

cylinder 11.4534

cylinder 010

cylinder 21.4966

cuboid $314 \mathrm{p} .7112$

$45.72 \quad 0.0$

45.720 .0

$45.72-1.27$

cuboid

array $13 * 0.0$

reflector $314 r 15.02 \mathrm{ro}$.

reflector $415 \mathrm{r} 0.03 .175$

reflector $315 \mathrm{r0.0} 6.350$

reflector $415 r 0.05 .080$

unit 3

$\begin{array}{lll}0.653 & 0.0\end{array}$

cylinder $01.4585 \quad 0.6530 .0$

$\begin{array}{llll}\text { cylinder } 2 & 1.4966 & 0.653 & 0.0\end{array}$

$\begin{array}{llll}\text { cuboid } 414 \mathrm{p} .7112 & 0.653 & 0.0\end{array}$

unit 4

array $23 * 0.0$

reflector 414 r15.0 2ro.

unit 5

$\begin{array}{llll}\text { cylinder } 11.4534 & 6.597 & 0.0\end{array}$

$\begin{array}{llll}\text { cuboid } 3 & 14 \mathrm{p} .7112 & 6.597 & 0.0\end{array}$

unit 6

array $33 * 0.0$

reflector 314 r15.0 2 ro.

unit 7

cyl inder $5 \quad 1 \quad .4534$

cyl inder $0 \quad 1.4585$

cyl inder 21.4966

$39.994 \quad 0.0$

$39.994 \quad 0.0$

$41.009 \quad 0.0$ 
end geom

\section{read array}

ara=1 nux=27 nuy=17 nuz=1 fill 459r1 end fill ara $=2$ nux $=27$ nuy=17 nuz=1 fill $459 \mathrm{r} 3$ end fill ara=3 nux=27 nuy=17 nuz=1 fill $459 r 5$ end fill ara $=4$ nux $=27$ nuy=17 nuz=1 fill $459 r 7$ end fill ara $=5$ nux $=1$ nuy $=1$ nuz $=4$ fill 2468 end fill

end array

end data

end

w3269w1

\section{$=\operatorname{csas} 25$}

w3269w1

44group latticecell

$$
\text { 5 } 0.950293922352 .729223897 .28
$$

squarepitch $1.524 \quad 1.016 \quad 1 \quad 3 \quad 1.189 \quad 2 \quad 1.030 \quad 0$

more data res $=5 \mathrm{cyl} .508$ dan $(5)=.948684$ end more

Core of 945 fuel rods, 16 water holes, $37.21 \mathrm{~cm}$ water height read parm run=yes gen $=405 \mathrm{npg}=600 \mathrm{nsk}=5$ nub=yes end parm

\section{read geom}

unit 1

cylinder 110.508

cylinder 010.515

cyl inder 2110.5945

$\begin{array}{llll}\text { cylinder } & 2 & 1 & 0.5945 \\ \text { cuboid } & 3 & 1 & 4 \mathrm{p} .762\end{array}$

unit 2

cyl inder 510.508

cylinder 0110.515

cylinder 210.5945

cuboid $0014 \mathrm{p} .762$

cubit 3

4 p.762

cuboid

$314 p .762$

$\begin{array}{rr}37.21 & 0.00 \\ 37.21 & 0.00 \\ 37.21 & -2.07 \\ 37.21 & -2.07 \\ & \\ 121.92 & 37.21 \\ 121.92 & 37.21 \\ 121.92 & 37.21 \\ 121.92 & 37.21 \\ & \\ 37.21 & -2.07\end{array}$

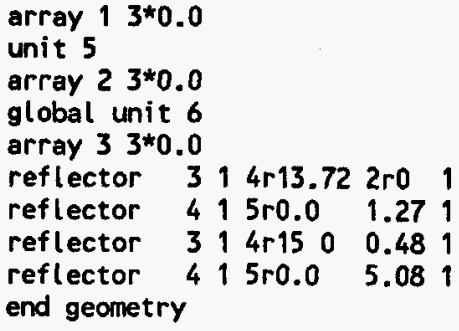

\section{9w2}

$=$ csas 25

W3269w2

44group latticecell

uo2 $10.930293922355 .709223894 .30 \quad$ end

ss304 21.0

h2o 31.0

al $\quad 41.0$

squarepitch 1.42240 .9068130 .993120 .91690 end more data $\operatorname{dan}(5)=.927954$ res $=5 \mathrm{cyl} .4534$ end more

Core of $27 \times 17$ positions: 453 fuel rods and 6 water holes, $37.21 \mathrm{~cm}$ water height

read parm run=yes gen $=405$ npg=600 nsk $=5$ nub=yes end parm

read geom

unit 1 
cylinder 11.4534

cylinder 011.4585

cylinder 21.4966

cuboid $314 p .7112$

unit 2

cylinder 11.4534

cyl inder 010.4585

cylinder 21.4966

cuboid $414 p .7112$

unit 3

cyl inder 11.4534

cylinder 01.4585

cylinder 21.4966

cuboid $314 \mathrm{p} .7112$

unit 4

cylinder 51.4534

cyl inder 01.4585

cylinder

cylinder 21.4966

cuboid 014 p.7112

unit 5

cuboid $314 p .7112$

unit 6

cuboid $414 \mathrm{p} .7112$

$45.72 \quad 0.0$

$\begin{array}{ll}45.72 & 0.0\end{array}$

$\begin{array}{ll}45.72 & -1.27\end{array}$

$\begin{array}{ll}45.72 & -1.27\end{array}$

0.6350 .0

0.6350 .0

0.6350 .0

0.6350 .0

$\begin{array}{ll}5.575 & 0.0\end{array}$

$\begin{array}{lll}5.575 & 0.0\end{array}$

5.5750 .0

5.5750 .0

92.96451 .93

92.96451 .93

93.97951 .93

93.97951 .93

$45.72-1.27$

unit 7

0.6350 .0

$\begin{array}{lll}5.575 & 0.0\end{array}$

cuboid $314 \mathrm{p} .7112$

unit

$14 p .7112$

93.97951 .93

unit 9

array $13 * 0.0$

reflector $312 r 11.27762 r 18.38962 r 0$ reflector 415 ro 3.175

reflector 312 2r3.7224 3r0 6.35

reflector 415 r0 5.08

unit 10

array $23 * 0.0$

reflector $4 \quad 12 r 11.2776$ 2r18.3896 2ro 1 reflector 312 1 3.7224

unit 11

reflector 312 2r15. 2r18.3896 2r0 1

unit 12

array $4 * 0.0$

reflector 012 r15. $2 r 18.38962 r 0$

reflector 414 ro 1.270

global

unit 13

array $53^{\star} 0.0$

end geom

read array

ara $=1$ nux $=27$ nuy=17 nuz=1 fill
$113 r 157 r 157 r 15$ 199r1 5 7r1 5 7r1 5 113r1 end fill ara $=2$ nux $=27$ nuy $=17$ nuz=1 fill

113r2 6 7r2 $67 r_{2} 6$ 199r2 $67 r^{2} 67 r_{2} 6113 r 2$ end fill ara $=3$ nux $=27$ nuy=17 nuz=1 fill

$113 r 377 r 377 r 37199 r 377 r 377 r 37113 r 3$ end fill ara $=4$ nux $=27$ nuy $=17$ nuz $=1$ fill

$113 r 487 r 487 r 48199 r 487 r 487 r 48113 r 4$ end fill ara=5 nux=1 nuy=1 nuz=4 fill $91011 \quad 12$ end fill

end array

end data

end

$43385 \$ 11$

\#csas25

W3385s 11

44 group

uo2

ss304

h20

al

uo2 5 den=10.1928 $1.0291 .0 \quad 92235 \quad 5.7429223894 .258$

$6 \quad .85293$

end comp

squarepitch $1.4224 .906813 \quad .99312 \quad .91690$

more data res $=5 \mathrm{cyl} .4534$ dan(5) $=.922095$ end more

Core of $19 \times 19$ fuel rods, $83.71 \mathrm{~cm}$ water height

read parm gen $=405 \mathrm{npg}=600$ nsk $=5$ nub=yes run=yes end parm

read geom

unit 1

cylinder

$\begin{array}{lllll}21.4966 & .6350\end{array}$

cyl inder 31.5042 .6350

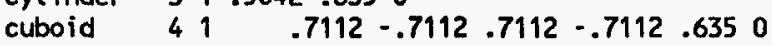

unit 2

cylinder 11.453447 .6251 .905

cylinder $\quad 0 \quad 1.458547 .6251 .905$

cylinder $\quad 21.4966 \quad 47.625 .635$

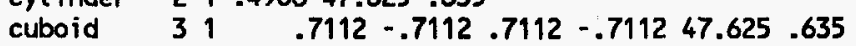

unit 3

cyt inder

cyl inder

cylinder

cyl inder

cuboid

unit 4

cyl inder

cyl inder

cyl inder

11.453448 .2647 .625

$01.458548 .26 \quad 47.625$

$21.496648 .26 \quad 47.625$

$31.504248 .26 \quad 47.625$

61

$.7112 \cdot .7112 \quad .7112 \cdot .711248 .26 \quad 47.625$

$\begin{array}{lllll}1 & 1 & .4534 & 83.955 & 48.26\end{array}$

$0 \begin{aligned} & 1 \\ & 0.458583 .95548 .26\end{aligned}$

21.496683 .95548 .26

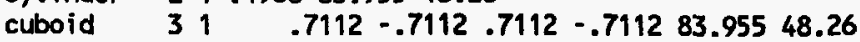

end end end end end end 
unit 5

cyl inder $\quad 5 \quad 1 \quad 4534 \quad 94.86983 .955$

cyl inder $\quad 01.458594 .86983 .955$

cylinder 21.496694 .86983 .955

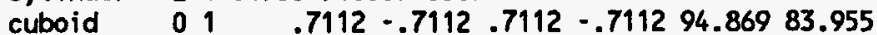

unit 6

cyl inder 21.496695 .88594 .869

cuboid $\quad 010 \quad .7112-.7112 .7112-.711295 .88594 .869$

unit 7

cylinder $\quad 21.496697 .15595 .885$

cylinder 01.504297 .15595 .885

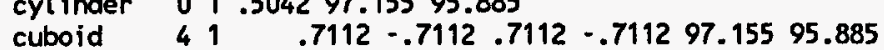

unit 8

array

1 13030303000

reflector 4100000002.54

reflector 3110000006.35

reflector 41000005.081

unit 9

array

20000

reflector 013030303000

end geom

\section{read array}

ara=1 nux=19 nuy=19 nuz=4 fill $361 r 1361 \mathrm{r2} \quad 361 \mathrm{r} 3 \quad 361 \mathrm{r} 4$ end $\mathrm{fill}$ ara $=2$ nux $=19$ nuy=19 nuz=3 fill $361 \times 5361 r 6361 r 7$ end fill ara $=2$ nux $=19$ nuy=19 nuz=3 fill $361 r 5361$ r6 $361 \mathrm{r} 7$ end fill end array

end data

end

\section{$3385 \mathrm{~s} 12$}

\section{\#csas 25}

w3385s 12

44group

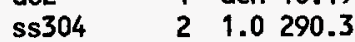

$\begin{array}{llll}\text { h20 } & 3 & 1.0 & 290.3\end{array}$

al $\quad 4 \quad 1.0290 .3$

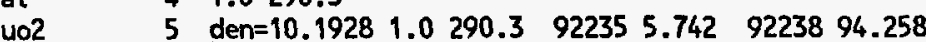

al $\quad 6 \quad .94293$

h20 $\quad 6 \quad .06293$

end comp

squarepitch $2.01168 .906813 \quad .99312 .91690$

more data res $=5$ cyl .4534 dan $(5)=.945902$ end more

Core of $13 \times 14$ fuel rods, $90.60 \mathrm{~cm}$ water height

read parm gen $=405 \mathrm{npg}=600 \mathrm{nsk}=5$ nub=yes run=yes end parm read geom

unit 1

cylinder 21.4966 .6350

cylinder 31.5042 .6350

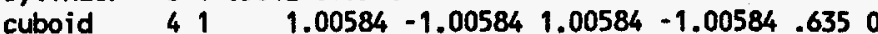

unit

cylinder

cyl inde

1.453447 .6251 .905

$\begin{array}{llllll}\text { cyl inder } & 0 & 1 & .4585 & 47.625 & 1.905 \\ \text { cyl inder } & 2 & .4966 & 47.625 & .635\end{array}$

unit 3

cylinder

cylinder $01 . .458548 .2647 .625$

31.504248 .2647 .625

cuboid $61 \quad 1.00584-1.005841 .00584-1.0058448 .2647 .625$

cylinder 11.453490 .86548 .26

cylinder 09.458590 .86548 .26

cylinder

cuboid $31.1 .00584-1.005841 .00584-1.0058490 .86548 .26$

cubit

unit s

cylinder 01.458594 .86990 .865

cylinder $\quad 21.496694 .86990 .865$

cuboid

cyl inder

cuboid

unit 7

cylinder

cyl inder

cuboid

unit 8

unit 8

$\begin{array}{lllllllllll}\text { reflector } & 3 & 1 & 30 & 30 & 30 & 30 & 0 & 0 & 1\end{array}$

reflector $4 \begin{array}{lllllllll}4 & 0 & 0 & 0 & 0 & 0 & 2.54 & 1\end{array}$

reflector 31100000006.35

reflector 410000005.08

unit 9

2000

reflector 013030303000

end geom

read array

ara $=1$ nux=13 nuy=14 nuz=4 fill 182r1 $182 r 2 \quad 182 r 3 \quad 182 r 4$ end fill ara $=2$ nux $=13$ nuy=14 nuz $=3$ fill $182 r 5 \quad 182 r 6 \quad 182 r 7$ end fill

ara=3 $\mathrm{gbl}=3$ nux=1 nuy=1 nuz=2 fill 89 and fill

end array

end data

end 


\section{vdr14pl2}

\section{\#csas25}

ydr14pl2

infhommedium

$101.1910-4$

$100.4152 \mathrm{e}-3$

h $104.5472 \mathrm{e}-2$

$\begin{array}{lllll}c & 1 & 0 & 2.1861 \mathrm{e}-2 \\ \mathrm{f} & 1 & 0 & 2.2109 \mathrm{e}-2\end{array}$

para(h20) 21.0

plexiglas 30.918

al 30.062

end comp

$2.00 \mathrm{Wt} \%{ }^{235} \mathrm{UUF}_{4}$-paraffin parallelpiped, paraffin reflected

read param run=yes gen $=405 \mathrm{npg}=600 \mathrm{nsk}=5$ nub=yes $f d n=y e s$ end param

read geom

unit

cuboid $114 \mathrm{p} 26.8352 \mathrm{p} 27.145$

reflector $215 * 15.20 .01$

unit 2

cuboid

$314 p 42.03515 .2 \quad 0.0$

end geom

w nux=1 nuy=1 nuz=2 fill $2 \quad 1$ end fill

end array

end

ydr14pl3

\#csas25

ydr14p13

44group infhommedium

u-235 $102.3494 \mathrm{e}-4$

$\begin{array}{llll}\mathrm{u}-235 & 1 & 0 & 2.3494 \mathrm{e}-4 \\ \mathrm{u}-238 & 1 & 0 & 7.4999 \mathrm{e}-3\end{array}$

h $\quad 103.1341 \mathrm{e}-2$

c $\quad 101.5067 \mathrm{e}-2$

para(h20) 21.0

plexiglas 30.918

al 30.062

$3.00 \mathrm{wt} \%{ }^{235} \mathrm{UUF}_{4}$-paraffin parallelpiped, paraffin reflected read param gen $=405 \mathrm{npg}=600 \mathrm{nsk}=5$ run=yes nub=yes end param

read geom

unit 1

cuboid $\quad 112 p 21.7352 p 21.7352 p 43.1950$ reflector $215 * 15.20 .01$

unit 2

cuboid $\quad 312 p 36.9352 p 36.93515 .20$.

end geom

gbl $=1$ ara $a=1$ nux $=1$ nuy=1 nuz=2 fill $2 \quad 1$ end fill

end array

end data

end

ydr14un?

$=\operatorname{csas} 25$

ydr14un2

44group inthormedium

$u-2351001.3303 \mathrm{e}-4$ end

$u-238 \quad 1 \quad 0 \quad 6.4370 \mathrm{e}-3$ end

$h \quad 103.9097 \mathrm{e}-2$ end

c $\quad 1001.8797 \mathrm{e}-2$ end

$f \quad 102.6280 \mathrm{e}-2$ end

$2.00 \mathrm{Wt} \%{ }^{235} \mathrm{U} U \mathrm{~F}_{4}$-paraffin parallelpiped, unreflected

read param gen $=405$ npg $=600$ nsk $=5$ nub=yes fdn=yes run=yes end param

read geom

cuboid $1128.11-28.1128 .11-28.1161 .235 \quad-61.235$

end geom

end data

end

ydr14un3

$=\operatorname{csas} 25$

ydr14un3 3 infhoup inmedium

$\begin{array}{lllll}\text { 44group } & \text { infhomedium } \\ 4-235 & 1 & 0 & 2.3494 \mathrm{e}-4 & \text { end }\end{array}$

u-238 $1107.4999 \mathrm{e}-3$

h $\quad 103.1341 \mathrm{e}-2$

c $\quad 101.5067 \mathrm{e}-2$

$f \quad 103.0939 \mathrm{e}-2$

end

end comp

$3.00 \mathrm{Wt} \%{ }^{235} \mathrm{UUF}_{4}$-paraffin parallelpiped, unreflected

read param gen $=405$ npg $=600$ nsk $=5$ nub $=y e s$ fdn=yes run $=y e s$ end param

read geom

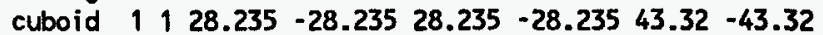

end geom

end data

end 



\section{APPENDIX B}

\section{Comparison of 44- and 238-Group Results}

To address the effectiveness of the broad-group structure in the 44GROUPNDF5 library, the benchmark calculations were repeated using SCALE-4.3 and the 238GROUPNDF5 library. The calculated $k_{\text {eff }}$ values obtained with the two libraries are compared in Table B.1. These results indicate that, on average, the fine-group library underpredicts the critical condition by $0.35 \%$ while the broad-group library predicts the critical condition almost exactly. Note, however, that the additional group structure in the 238-group library enables it to more accurately predict most of the homogeneous uranium critical experiments. 
Appendix B

Table B.1 Comparison of 44- and 238-group results

\begin{tabular}{|c|c|c|c|c|c|c|c|c|c|}
\hline $\begin{array}{l}\text { Case } \\
\text { No. }\end{array}$ & $\begin{array}{c}\text { Case } \\
\text { designation }\end{array}$ & $\begin{array}{c}\text { 44-Group } \\
\mathrm{k}_{\text {eff }}\end{array}$ & $\begin{array}{c}238 \text {-Group } \\
k_{\text {eff }}\end{array}$ & $\begin{array}{c}\Delta \mathrm{k}_{\text {eff }} \\
(\%)\end{array}$ & $\begin{array}{c}\text { Case } \\
\text { No. }\end{array}$ & $\begin{array}{c}\text { Case } \\
\text { designation }\end{array}$ & $\begin{array}{c}\text { 44-Group } \\
\mathrm{k}_{\text {eff }}\end{array}$ & $\begin{array}{c}238-G r o u p \\
k_{\text {eff }}\end{array}$ & $\begin{array}{r}\Delta \mathrm{k}_{\text {eff }} \\
(\%)\end{array}$ \\
\hline 1 & ANS33AL1 & 1.0027 & 1.0036 & -0.09 & 48 & EPRU65 & 0.9977 & 0.9900 & 0.77 \\
\hline 2 & ANS33AL2 & 1.0129 & 1.0092 & 0.37 & 49 & EPRU65B & 0.9985 & 0.9965 & 0.20 \\
\hline 3 & ANS33AL3 & 1.0035 & 0.9991 & 0.44 & 50 & EPRU75 & 0.9974 & 0.9941 & 0.33 \\
\hline 4 & ANS33EB1 & 0.9979 & 0.9906 & 0.73 & 51 & EPRU75B & 1.0023 & 1.0004 & 0.19 \\
\hline 5 & ANS33EB2 & 1.0096 & 1.0073 & 0.23 & 52 & EPRU87 & 0.9986 & 0.9974 & 0.12 \\
\hline 6 & ANS33EP1 & 0.9964 & 0.9941 & 0.23 & 53 & EPRU87B & 0.9985 & 0.9968 & 0.17 \\
\hline 7 & ANS33EP2 & 1.0020 & 0.9984 & 0.36 & 54 & NSE71H1 & 0.9982 & 0.9939 & 0.43 \\
\hline 8 & ANS33SLG & 0.9964 & 0.9960 & 0.04 & 55 & NSE71H2 & 1.0009 & 0.9973 & 0.36 \\
\hline 9 & ANS33STY & 0.9909 & 0.9876 & 0.33 & 56 & NSE71H3 & 1.0036 & 0.9979 & 0.57 \\
\hline 10 & $\mathrm{~B} 1645 \mathrm{SO} 1$ & 0.9976 & 0.9947 & 0.29 & 57 & NSE71SQ & 1.0014 & 0.9959 & 0.55 \\
\hline 11 & $\mathrm{~B} 1645 \mathrm{SO} 2$ & 1.0007 & 0.9983 & 0.24 & 58 & NSE71W1 & 0.9993 & 0.9919 & 0.74 \\
\hline 12 & BW1231B1 & 0.9969 & 0.9936 & 0.33 & 59 & NSE71W2 & 0.9976 & 0.9957 & 0.19 \\
\hline 13 & BW1231B2 & 0.9985 & 0.9947 & 0.38 & 60 & P2438AL & 0.9951 & 0.9943 & 0.08 \\
\hline 14 & BW1273M & 0.9947 & 0.9943 & 0.04 & 61 & P2438BA & 0.9972 & 0.9958 & 0.14 \\
\hline 15 & BW1484A1 & 0.9965 & 0.9948 & 0.17 & 62 & P2438CU & 0.9957 & 0.9958 & -0.01 \\
\hline 16 & BW1484A2 & 0.9954 & 0.9888 & 0.66 & 63 & P2438SLG & 0.9997 & 0.9954 & 0.43 \\
\hline 17 & BW1484B1 & 0.9973 & 0.9964 & 0.09 & 64 & P2438SS & 0.9978 & 0.9987 & -0.09 \\
\hline 18 & BW1484B2 & 0.9973 & 0.9940 & 0.33 & 65 & P2438ZR & 0.9956 & 0.9937 & 0.19 \\
\hline 19 & BW1484B3 & 0.9992 & 0.9955 & 0.37 & 66 & P2615AL & 1.0004 & 0.9940 & 0.64 \\
\hline 20 & $\mathrm{BW} 1484 \mathrm{Cl}$ & 0.9937 & 0.9914 & 0.23 & 67 & P2615BA & 0.9970 & 0.9961 & 0.09 \\
\hline 21 & BW1484C2 & 0.9936 & 0.9937 & -0.01 & 68 & $\mathrm{P} 2615 \mathrm{CD} 1$ & 0.9985 & 0.9986 & -0.01 \\
\hline 22 & BW1484S1 & 0.9981 & 0.9947 & 0.34 & 69 & P2615CD2 & 0.9990 & 0.9930 & 0.60 \\
\hline 23 & BW1484S2 & 0.9989 & 0.9980 & 0.09 & 70 & P2615CU & 1.0003 & 0.9968 & 0.35 \\
\hline 24 & BW1484SL & 0.9966 & 0.9911 & 0.55 & 71 & P2615SS & 0.9998 & 0.9969 & 0.29 \\
\hline 25 & BW1645S1 & 1.0010 & 0.9906 & 1.04 & 72 & P2615ZR & 0.9980 & 0.9940 & 0.40 \\
\hline 26 & BW1645S2 & 1.0035 & 0.9940 & 0.95 & 73 & P2827Ll & 1.0023 & 1.0006 & 0.17 \\
\hline 27 & BW1645T1 & 1.0062 & 0.9951 & 1.11 & 74 & P2827L2 & 0.9997 & 0.9964 & 0.33 \\
\hline 28 & BW1645T2 & 1.0068 & 0.9965 & 1.03 & 75 & P2827L3 & 1.0081 & 1.0088 & -0.07 \\
\hline 29 & BW1645T3 & 1.0007 & 0.9924 & 0.83 & 76 & $\mathrm{P} 2827 \mathrm{~L} 4$ & 1.0073 & 1.0044 & 0.29 \\
\hline 30 & BW1645T4 & 0.9986 & 0.9888 & 0.98 & 77 & P2827SLG & 0.9948 & 0.9939 & 0.09 \\
\hline 31 & BW1810A & 0.9971 & 0.9945 & 0.26 & 78 & P2827U1 & 1.0004 & 0.9955 & 0.49 \\
\hline 32 & BW1810B & 0.9980 & 0.9969 & 0.11 & 79 & P2827U2 & 0.9998 & 0.9979 & 0.19 \\
\hline 33 & BW1810C & 1.0005 & 0.9984 & 0.21 & 80 & P2827U3 & 1.0018 & 0.9999 & 0.19 \\
\hline 34 & BW1810D & 0.9999 & 0.9962 & 0.37 & 81 & P2827U4 & 1.0042 & 1.0011 & 0.31 \\
\hline 35 & BW1810E & 0.9976 & 0.9940 & 0.36 & 82 & P3314AL & 0.9973 & 0.9944 & 0.29 \\
\hline 36 & BW1810F & 1.0032 & 0.9983 & 0.49 & 83 & P3314BA & 1.0002 & 1.0075 & -0.73 \\
\hline 37 & BW1810G & 0.9971 & 0.9949 & 0.22 & 84 & P3314BC & 1.0008 & 1.0072 & -0.64 \\
\hline 38 & BW1810H & 0.9973 & 0.9939 & 0.34 & 85 & P3314BF1 & 1.0022 & 1.0055 & -0.33 \\
\hline 39 & BW1810I & 1.0022 & 0.9953 & 0.69 & 86 & P3314BF2 & 0.9981 & 0.9985 & -0.04 \\
\hline 40 & BW1810J & 0.9980 & 0.9970 & 0.10 & 87 & P3314BS1 & 0.9970 & 0.9965 & 0.05 \\
\hline 41 & CR1071AS & 1.0207 & 1.0125 & 0.82 & 88 & P3314BS2 & 0.9959 & 0.9926 & 0.33 \\
\hline 42 & CR1653AS & 1.0169 & 1.0097 & 0.72 & 89 & P3314BS3 & 0.9972 & 0.9936 & 0.36 \\
\hline 43 & CR2500S & 1.0194 & 1.0129 & 0.65 & 90 & P3314BS4 & 1.0005 & 0.9941 & 0.64 \\
\hline 44 & DSN399-1 & 1.0056 & 0.9973 & 0.83 & 91 & $\mathrm{P} 3314 \mathrm{CD} 1$ & 1.0001 & 0.9939 & 0.62 \\
\hline 45 & DSN399-2 & 1.0010 & 0.9948 & 0.62 & 92 & P3314CD2 & 0.9929 & 0.9910 & 0.19 \\
\hline 46 & DSN399-3 & 1.0024 & 0.9977 & 0.47 & 93 & P3314CU1 & 0.9941 & 0.9877 & 0.64 \\
\hline 47 & DSN399-4 & 0.9965 & 0.9910 & 0.55 & 94 & P3314CU2 & 1.0005 & 0.9953 & 0.52 \\
\hline
\end{tabular}


Appendix B

Table B.1 (continued)

\begin{tabular}{|c|c|c|c|c|c|c|c|c|c|}
\hline $\begin{array}{l}\text { Case } \\
\text { No. }\end{array}$ & $\begin{array}{c}\text { Case } \\
\text { designation }\end{array}$ & $\begin{array}{c}\text { 44-Group } \\
\mathrm{k}_{\mathrm{eff}}\end{array}$ & $\begin{array}{c}238-\text { Group } \\
\mathrm{k}_{\text {eff }}\end{array}$ & $\begin{array}{l}\Delta \mathrm{k}_{\text {eff }} \\
(\%)\end{array}$ & $\begin{array}{l}\text { Case } \\
\text { No. }\end{array}$ & $\begin{array}{c}\text { Case } \\
\text { designation }\end{array}$ & $\begin{array}{c}\text { 44-Group } \\
k_{\text {eff }}\end{array}$ & $\begin{array}{c}\text { 238-Group } \\
\mathrm{k}_{\text {eff }}\end{array}$ & $\begin{array}{r}\Delta \mathrm{k}_{\text {eff }} \\
(\%)\end{array}$ \\
\hline$\overline{95}$ & P3314CU3 & 0.9972 & 0.9989 & -0.17 & 141 & P3926SL1 & 0.9942 & 0.9926 & 0.16 \\
\hline 96 & P3314CU4 & 0.9992 & 0.9950 & 0.42 & 142 & P3926SL2 & 0.9990 & 0.9928 & 0.62 \\
\hline 97 & P3314CUS & 0.9938 & 0.9926 & 0.12 & 143 & P3926U1 & 0.9979 & 0.9939 & 0.40 \\
\hline 98 & P3314CU6 & 0.9958 & 0.9910 & 0.48 & 144 & P3926U2 & 0.9980 & 0.9931 & 0.49 \\
\hline 99 & P3314SLG & 0.9982 & 0.9942 & 0.40 & 145 & P3926U3 & 1.0001 & 0.9959 & 0.42 \\
\hline 100 & P3314SS1 & 0.9969 & 0.9946 & 0.23 & 146 & P3926U4 & 1.0026 & 0.9974 & 0.52 \\
\hline 101 & P3314SS2 & 1.0011 & 0.9960 & 0.51 & 147 & P3926U5 & 0.9999 & 0.9998 & 0.01 \\
\hline 102 & P3314SS3 & 0.9990 & 0.9926 & 0.64 & 148 & P3926U6 & 1.0024 & 0.9970 & 0.54 \\
\hline 103 & P3314SS4 & 0.9957 & 0.9957 & 0.00 & 149 & P4267B1 & 0.9969 & 0.9981 & -0.12 \\
\hline 104 & P3314SS5 & 0.9944 & 0.9875 & 0.69 & 150 & P4267B2 & 1.0035 & 1.0010 & 0.25 \\
\hline 105 & P3314SS6 & 1.0003 & 0.9935 & 0.68 & 151 & P4267B3 & 1.0025 & 1.0053 & -0.28 \\
\hline 106 & P3314W1 & 1.0015 & 0.9992 & 0.23 & 152 & P4267B4 & 0.9993 & 0.9977 & 0.16 \\
\hline 107 & P3314W2 & 0.9958 & 0.9927 & 0.31 & 153 & P4267B5 & 1.0031 & 1.0007 & 0.24 \\
\hline 108 & P3314ZR & 0.9978 & 0.9957 & 0.21 & 154 & P4267SL1 & 0.9974 & 0.9940 & 0.34 \\
\hline 109 & P3602BB & 0.9988 & 0.9947 & 0.41 & 155 & P4267SL2 & 0.9993 & 0.9958 & 0.35 \\
\hline 110 & P3602BS1 & 1.0000 & 0.9928 & 0.72 & 156 & P49-194 & 1.0081 & 0.9981 & 1.00 \\
\hline 111 & P3602BS2 & 1.0001 & 0.9977 & 0.24 & 157 & P62FT231 & 1.0005 & 0.9958 & 0.47 \\
\hline 112 & P3602CD1 & 0.9988 & 0.9943 & 0.45 & 158 & P71F14F3 & 1.0029 & 0.9944 & 0.85 \\
\hline 113 & $\mathrm{P} 3602 \mathrm{CD} 2$ & 1.0002 & 0.9931 & 0.71 & 159 & P71F14V3 & 0.9973 & 0.9929 & 0.44 \\
\hline 114 & P3602CU1 & 0.9964 & 0.9945 & 0.19 & 160 & P71F14V5 & 0.9977 & 0.9959 & 0.18 \\
\hline 115 & P3602CU2 & 0.9980 & 0.9926 & 0.54 & 161 & P71F214R & 1.0010 & 0.9949 & 0.61 \\
\hline 116 & P3602CU3 & 1.0039 & 0.9938 & 1.010 & 162 & PAT80L1 & 1.0031 & 0.9987 & 0.44 \\
\hline 117 & P3602CU4 & 1.0040 & 0.9944 & 0.96 & 163 & PAT80L2 & 0.9920 & 0.9954 & -0.34 \\
\hline 118 & P3602N11 & 1.0003 & 0.9918 & 0.85 & 164 & PAT80SS1 & 1.0004 & 1.0003 & 0.01 \\
\hline 119 & $\mathrm{P} 3602 \mathrm{~N} 12$ & 0.9963 & 0.9943 & 0.20 & 165 & PAT80SS2 & 0.9926 & 0.9946 & -0.20 \\
\hline 120 & $\mathrm{P} 3602 \mathrm{~N} 13$ & 0.9957 & 0.9939 & 0.18 & 166 & W3269A & 0.9934 & 0.9920 & 0.14 \\
\hline 121 & P3602N14 & 0.9984 & 0.9951 & 0.33 & 167 & W3269B1 & 0.9963 & 0.9900 & 0.63 \\
\hline 122 & P3602N21 & 0.9995 & 0.9972 & 0.23 & 168 & W3269B2 & 0.9964 & 0.9898 & 0.66 \\
\hline 123 & $\mathrm{P} 3602 \mathrm{~N} 22$ & 0.9967 & 0.9972 & -0.05 & 169 & W3269B3 & 0.9948 & 0.9878 & 0.70 \\
\hline 124 & P3602N31 & 1.0010 & 0.9966 & 0.44 & 170 & W3269C & 0.9986 & 0.9946 & 0.40 \\
\hline 125 & P3602N32 & 1.0015 & 0.9967 & 0.48 & 171 & W3269SL1 & 0.9952 & 0.9919 & 0.33 \\
\hline 126 & P3602N33 & 1.0045 & 0.9976 & 0.69 & 172 & W3269SL2 & 1.0040 & 0.9947 & 0.93 \\
\hline 127 & P3602N34 & 1.0027 & 0.9961 & 0.66 & 173 & W3269W1 & 0.9957 & 0.9938 & 0.19 \\
\hline 128 & P3602N35 & 1.0036 & 0.9960 & 0.76 & 174 & W3269W2 & 1.0009 & 0.9995 & 0.14 \\
\hline 129 & P3602N36 & 1.0009 & 0.9964 & 0.45 & 175 & W3385SL1 & 0.9964 & 0.9918 & 0.46 \\
\hline 130 & P3602N41 & 1.0002 & 0.9948 & 0.54 & 176 & W3385SL2 & 1.0005 & 0.9995 & 0.10 \\
\hline 131 & $\mathrm{P} 3602 \mathrm{~N} 42$ & 1.0019 & 0.9995 & 0.24 & 177 & YDR14PL2 & 1.0011 & 1.0011 & 0.00 \\
\hline 132 & P3602N43 & 0.9994 & 0.9967 & 0.27 & 178 & YDR14PL3 & 1.0115 & 1.0089 & 0.26 \\
\hline 133 & P3602SS1 & 0.9978 & 0.9904 & 0.74 & 179 & YDR14UN2 & 1.0047 & 1.0024 & 0.23 \\
\hline 134 & P3602SS2 & 1.0012 & 0.9962 & 0.50 & 180 & YDR14UN3 & $3 \quad 1.0163$ & 1.0128 & 0.35 \\
\hline 135 & P3926L1 & 0.9996 & 0.9966 & 0.30 & & & & & \\
\hline 136 & P3926L2 & 1.0028 & 0.9994 & 0.34 & & MEAN & 0.9999 & 0.9964 & \\
\hline 137 & P3926L3 & 1.0017 & 0.9959 & 0.58 & & MEDIAN & 0.9993 & 0.9957 & \\
\hline 138 & P3926L4 & 1.0055 & 1.0114 & -0.59 & & STD. DEV. & 0.0045 & 0.0064 & \\
\hline 139 & P3926L5 & 1.0070 & 1.0032 & 0.38 & & MIN. & 0.9909 & 0.9875 & \\
\hline 140 & P3926L6 & 1.0026 & 0.9994 & 0.32 & & MAX. & 1.0207 & 1.0129 & \\
\hline
\end{tabular}





\section{APPENDIX C \\ USER'S MANUAL FOR USLSTATS V1.0}

\section{C.1 Introduction}

The ANS/ANSI-8.1 Standard ${ }^{39}$ recommends that calculational methods used in determining criticality safety limits for applications outside reactors be validated by comparison with appropriate critical experiments. An upper safety limit (USL) provides a high degree of confidence that a given system is subcritical if a criticality calculation based on the system yields a multiplication factor $\left(k_{\text {eff }}\right)$ below the USL. Section 4 describes two different methods for the determination of a USL for criticality based on any set of calculations based on criticality experiments.

The USLSTATS computer program uses these two methods [i.e., (1) confidence band with administrative margin and (2) single-sided uniform-width closed-interval] to calculate and print USL correlations based on a set of user-supplied $k_{\text {eff }}$ values and corresponding values of a single associated parameter $X$ [e.g., lattice pitch, fuel enrichment, average energy group causing fission (AEG), etc.], for a set of criticality benchmark calculations. In addition, other statistical parameters computed as a part of the USL calculation are included in the output. The user is referred to Sect. 4 for the definition of these factors and for additional details relative to the theory and application of upper safety limit calculations.

\section{C.2 Background}

The USLSTATS program is derived from the V_STATS program,,$^{45}$ originally developed to calculate and plot the USL based on a single-sided, uniform-width, closed-interval approach ${ }^{40,41}$ (method 2). Because USL method 1 is essentially a simplification of method 2, the calculation of USLs via the results of a method 2 calculation are straightforward. In creating USLSTATS, the input was simplified somewhat, and the output was completely redesigned, in order to print only the information required for USL calculations using the nomenclature given in Sect. 4.

\section{C.3 Input Description}

The input required by the USLSTATS program is very minimal. The input consists of three sections, all read in a free-format fashion. The first line contains a title, up to 80 characters. The second line contains problemspecification parameters: $P,(1-\gamma), \alpha, x_{\min }, x_{\max }, \sigma_{\text {sample }}$, and $\Delta k_{m}$, where

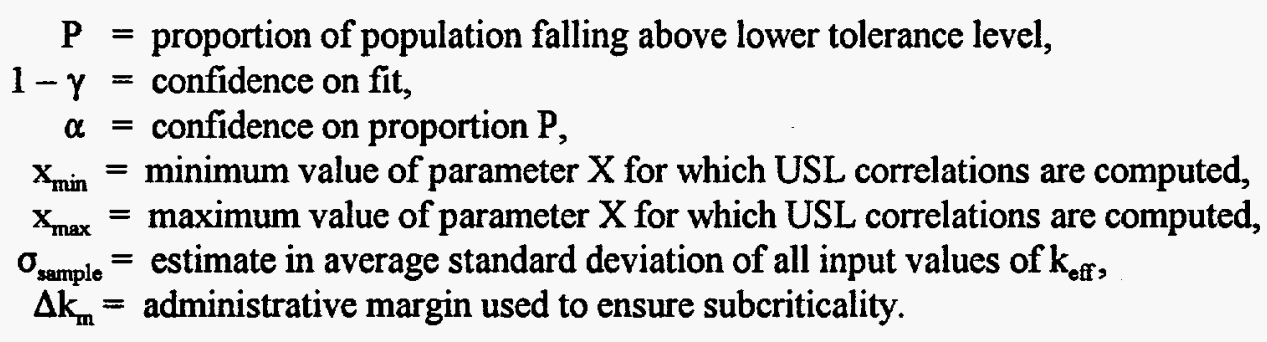

The final section of input read is the set of values (parameter $X, \mathrm{k}_{\mathrm{eff}}$, and, if $\sigma_{\text {sample }}<0.0$, the value of $\sigma$ corresponding to $\mathrm{k}_{\mathrm{eff}}$ ) for each of up to 1000 benchmark critical experiments. A minimum of 25 experiments is recommended to have a sufficient number of data points to determine the statistical normality of the data. USLSTATS reads $\mathrm{X}, \mathrm{k}_{\text {eff }}$ 
pairs (or X, $\mathrm{k}_{\mathrm{eff}}, \sigma$ triplets) until the end-of-file marker is reached. Again, $\mathrm{X}$ is the value of an independent parameter for which a trend in $\mathrm{k}_{\text {eff }}$ is known or suspected to exist.

The value of $P$ is typically selected as 0.995 . This statistical parameter is used only in the computation of USL by method 2, and represents the proportion of the population of criticality experiments that will lie above the line representing one tolerance level below the linear regression fit to $\mathrm{k}_{\text {eff }}$. The value $1-\gamma$, used in the Student-t calculation of USL methods 1 and 2, is typically assigned a value of 0.95 and represents the desired confidence level to be assigned the linear regression fit of $k_{\mathrm{eff}}$ values. (It is also used in a $\chi^{2}$ test for normality of input data. USL methods 1 and 2 are valid only for normally distributed data.) The third statistical parameter, $\alpha$, represents the confidence on the proportion $P$, and is also typically assigned a value of 0.95 .

The parameters $\mathrm{x}_{\min }$ and $\mathrm{x}_{\max }$ represent the lower and upper limits, respectively, for parameter $\mathrm{X}$ in the USL correlations of methods 1 and 2 . These limit the range in which the USL correlation is valid, but a smaller range will raise the USL. Typically, values of 0.0 are entered for both $x_{\min }$ and $x_{\max }$; this instructs the code to use the minimum and maximum values of $\mathrm{X}$ found in the user-supplied data.

The parameter $\sigma_{\text {sample }}$ is the average standard deviation estimated for the supplied values of $k_{\text {eff }}$. For the results of a set of deterministic calculations, an average standard deviation of 0.0 may be specified; a positive value may be supplied to account for modeling and numerical uncertainties associated with the set of values. However, if an estimate of the standard deviation associated with each value of $k_{\text {eff }}$ is available (such as is computed for Monte Carlo criticality calculations), USLSTATS may be instructed to read this value for each value of $\mathrm{k}_{\mathrm{eff}}$ by supplying any negative value for $\sigma_{\text {sample }}$.

The final parameter supplied in line 2 of the input file is the administrative margin that is to be included in calculation of the USL by method 1. Based on general experience, a value of 0.05 is most often used in criticality calculations as the value for the administrative subcritical margin. A statistically based margin is computed and used in the method 2 approach, and is supplied in output as the "minimum margin of subcriticality." Typically, this statistical margin is found to be less than the arbitrary $5 \%$ margin; for this reason, the method 2 USL is generally less restrictive.

A sample input case is given on the following page. In this case, entitled "34.fresh fuel LWR-type criticals," typical values for $P, 1-\gamma$, and $\alpha$ of $0.995,0.95$, and 0.95 , respectively, are given. A value of 0.0 is assigned to both $x_{\min }$ and $\mathrm{x}_{\max }$, indicating that the minimum and maximum limits of the USL correlations are to be set as the minimum and maximum values of $X$ from the list supplied by the user. A value of -1.0 is given for $\sigma_{\text {sample }}$, indicating that individual uncertainties are to be read for each value of $k_{e f f}$. Finally, 34 sets of $X, k_{e f f}$, and $\sigma$ are supplied. (In this case, parameter X is the AEG computed by KENO V.a based on the SCALE 27-group library group structure.) 
Sample input for a USLSTATS calculation

34.fresh fuel LWR-type criticals

$0.9950 .95 \quad 0.950 .0 \quad 0.0-1.00 .05$

$\begin{array}{lll}2.26322 E+01 & .9912 \quad .0010\end{array}$

$\begin{array}{lll}2.16825 E+01 & .9953 & .0011\end{array}$

$2.17734 E+01 \quad .9919 \quad .0010$

$2.18186 E+01 \quad .9949 \quad .0010$

$\begin{array}{lll}2.22143 E+01 & .9904 \quad .0014\end{array}$

$\begin{array}{lll}2.37713 E+01 & .9946 \quad .0013\end{array}$

$2.06628 E+01 \quad .9955 \quad .0014$

$2.10962 E+01 \quad .9956 \quad .0013$

$\begin{array}{lll}2.20739 E+01 & 1.0071 & .0012\end{array}$

$2.26189 E+01 \quad 1.0037 \cdot .0010$

$\begin{array}{lll}2.26746 E+01 & 1.0072 & .0009\end{array}$

$\begin{array}{lll}2.30862 E+01 & 1.0077 & .0015\end{array}$

$\begin{array}{lll}2.16605 E+01 & .9971 & .0016\end{array}$

$2.16390 E+01 \quad .9924 \quad .0011$

$\begin{array}{lll}2.35796 E+01 & .9912 & .0014\end{array}$

$2.35464 E+01 \quad .9927 \quad .0010$

$\begin{array}{lll}2.35761 E+01 & .9924 & .0013\end{array}$

$2.35623 E+01 \quad .9921 \quad .0014$

$2.19304 E+01 \quad .9970 \quad .0016$

$2.19636 E+01 \quad 1.0000 \quad .0015$

$\begin{array}{lll}2.13056 E+01 & .9994 & .0014\end{array}$

$2.20520 E+01 \quad .9952 \quad .0011$

$2.09066 \mathrm{E}+01 \quad .9991 \quad .0012$

$\begin{array}{lll}2.11516 E+01 & .9940 \quad .0011\end{array}$

$\begin{array}{lll}1.99756 E+01 & .9904 & .0013\end{array}$

$\begin{array}{lll}1.82077 \mathrm{E}+01 \quad .9869 & .0012\end{array}$

$\begin{array}{llll}2.34646 E+01 & 1.0057 & .0022\end{array}$

$\begin{array}{lll}2.07944 E+01 & 1.0027 & .0015\end{array}$

$\begin{array}{llll}2.25639 E+01 & 1.0031 & .0017\end{array}$

$\begin{array}{lll}2.28514 E+01 & 1.0040 & .0018\end{array}$

$\begin{array}{lll}2.03398 E+01 & 1.0015 \quad .0012\end{array}$

$\begin{array}{llll}2.10385 E+01 & 1.0014 & .0018\end{array}$

$\begin{array}{lll}2.18995 \mathrm{E}+01 & .9861 \quad .0016\end{array}$

$\begin{array}{llll}2.34236 E+01 & .9972 & .0011\end{array}$ 
Appendix C

\title{
C.4 Output Description
}

The output generated by USLSTATS (corresponding to the sample input supplied earlier) is given at the end of this appendix. The first section lists the filename corresponding to the input file and supplies all input in a descriptive format. The value of $\chi$ from the $\chi^{2}$ test is printed and compared to the value required for normal data. A message is printed indicating whether or not the supplied data is normal. Note that if fewer than 25 data points are supplied, a warning message is also printed indicating that too few points are available for an accurate assessment of normality. The final section of the output supplies the two USLs, together with important input and intermediate parameters used in the calculation of the USL correlations.

\section{C.5 How to Run USLSTATS}

The USLSTATS program has an interactive interface to allow the user to specify the location of an input file and to select the name of the output file. Below is an example of typical USLSTATS usage. Input and output file names are limited to 20 characters. Note that text supplied by the user is shown in boldface in the following example.

\author{
\$ uslstats \\ Enter filename containing input data. \\ (Enter "test" to generate test case input.) \\ list34_uslstats.in
}

Enter output filename (will overwrite existing file):

\section{list34_uslstats.out}

$\$$

Note that USLSTATS will create a sample input file and will then run using the information in the sample file if the word "test" is given as the name for the input file. This is illustrated in the sample interactive session below.

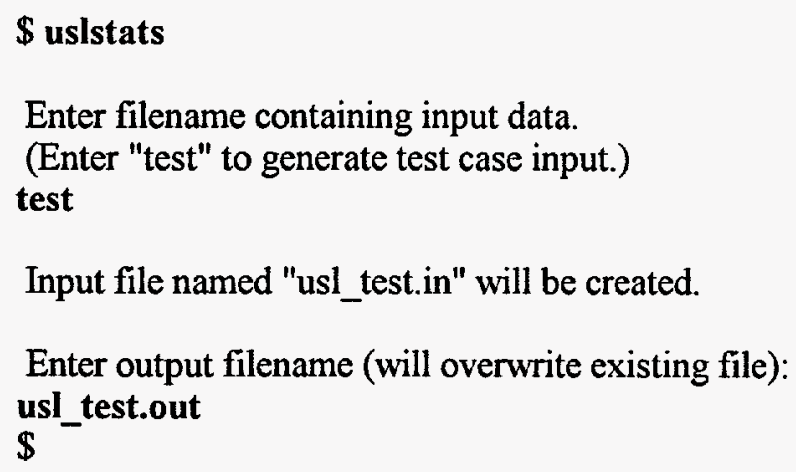

USLSTATS does not require significant computing space or time. It will typically execute in less than 1 second once the interactive input is completed.

\section{C.6 How to Obtain USLSTATS}

The USLSTATS program may be downloaded from the World Wide Web (WWW) by accessing the SCALE home page. A pointer for the SCALE home page may be found at http://www.cad.ornl.gov 
Sample output from a USLSTATS calculation

uslstats: a utility to calculate upper subcritical

limits for criticality safety applications

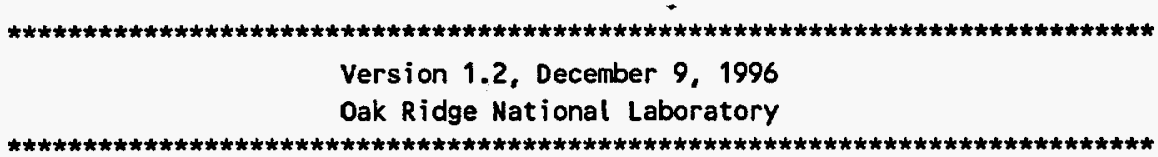

Input to statistical treatment from file:usl_test.in

Title: 34 Fresh-fuel U02 criticals

$\begin{array}{ll}\text { Proportion of the population } & =.999 \\ \text { Confidence of fit } & =.950 \\ \text { Confidence on proportion } & =.950 \\ \text { Number of observations } & =29 \\ \text { Minimum value of closed band } & =.00 \\ \text { Maximum value of closed band }= & .00 \\ \text { Administrative margin } & =\end{array}$

$\begin{array}{lll}\begin{array}{l}\text { independent } \\ \text { variable }-x\end{array} & \begin{array}{l}\text { dependent } \\ \text { variable }-y\end{array} & \begin{array}{c}\text { deviation } \\ \text { in } Y\end{array} \\ 1.48200 E+01 & 9.96470 E-01 & 3.37000 E-03 \\ 1.48100 E+01 & 9.97760 E-01 & 3.26000 E-03 \\ 1.48300 E+01 & 1.00764 E+00 & 3.11000 E-03 \\ 1.44400 E+01 & 9.95870 E-01 & 3.65000 E-03 \\ 1.42800 E+01 & 9.97440 E-01 & 3.27000 E-03 \\ 1.48400 E+01 & 1.00337 E+00 & 3.95000 E-03 \\ 1.47300 E+01 & 9.96090 E-01 & 3.95000 E-03 \\ 1.50800 E+01 & 1.00108 E+00 & 3.78000 E-03 \\ 1.52000 E+01 & 9.97370 E-01 & 3.25000 E-03 \\ 1.53100 E+01 & 9.84080 E-01 & 3.42000 E-03 \\ 1.52600 E+01 & 9.88710 E-01 & 3.61000 E-03 \\ 1.55000 E+01 & 9.95270 E-01 & 2.92000 E-03 \\ 1.54900 E+01 & 9.88040 E-01 & 2.73000 E-03 \\ 1.43600 E+01 & 1.01363 E+00 & 4.01000 E-03 \\ 1.43600 E+01 & 1.01660 E+00 & 4.45000 E-03 \\ 1.43600 E+01 & 1.00874 E+00 & 4.85000 E-03 \\ 1.43800 E+01 & 1.01190 E+00 & 4.79000 E-03 \\ 1.43500 E+01 & 1.00980 E+00 & 4.98000 E-03 \\ 1.41000 E+01 & 1.00565 E+00 & 3.97000 E-03 \\ 1.41200 E+01 & 1.01929 E+00 & 4.07000 E-03 \\ 1.41000 E+01 & 1.00860 E+00 & 4.11000 E-03 \\ 1.50400 E+01 & 9.94870 E-01 & 4.62000 E-03 \\ 1.49000 E+01 & 9.92570 E-01 & 3.82000 E-03 \\ 1.49000 E+01 & 1.00132 E+00 & 4.50000 E-03 \\ 1.49000 E+01 & 9.91540 E-01 & 4.20000 E-03 \\ 1.54300 E+01 & 1.00028 E+00 & 3.74000 E-03 \\ 1.54400 E+01 & 9.95650 E-01 & 4.13000 E-03 \\ 1.54300 E+01 & 9.85740 E-01 & 4.15000 E-03 \\ 1.54300 E+01 & 9.87330 E-01 & 4.16000 E-03\end{array}$




\section{Appendix C}

WARNING *** the test for normal may be unreliable due to insufficient data.

chi $=4.2759$ (upper bound $=9.49$ ). The data tests normal.

Output from statistical treatment

34 Fresh-fuel U02 criticals

Number of data points $(n)$

Linear regression, $k(X)$

Confidence on fit (1-gamma) [input]

Confidence on proportion (alpha) [input]

Proportion of population falling above

lower tolerance interval (rho) [input]

Minimum value of $x$

Maximum value of $x$

Average value of $x$

Average value of $k$

Minimum value of $k$

Variance of $f i t, s(k, X)-2$

Within variance, $s(w)^{` 2}$

Pooled variance, $s(p)-2$

Pooled std. deviation, $s(p)$

c(alpha, rho)* $s(p)$

student-t a (n-2,1-gamma)

Confidence band width, $W$

Minimum margin of subcriticality, $C^{*} s(p)-H$
29

$1.2267+(-1.5296 E-02) * x$

$95.0 \%$

$95.0 \%$

$99.9 \%$

14.1000

15.5000

14.83414

.99975

.98408

$3.8263 E-05$

$1.5455 E-05$

5.3718E-05

7.3293E-03

$3.6627 \mathrm{E}-02$

$1.70300 E+00$

$1.3223 E-02$

2.3404E-02

Upper subcritical limits: $(14.10000<x<=15.50000)$

$\star \star * * * * \star * * * * * * * * * * * * * * * * * *$

USL Method 1 (Confidence Band with Administrative Margin)

$1.1634+(-1.5296 E-02) * x(x>14.818)$ .9368

$(X<=14.818)$

USL Method 2 (single-sided Uniform

Width Closed Interval Approach) $1.1900+(-1.5296 E-02) * X(X>14.818)$ .9634

$(X<14.818)$ 
(See instructions on the reverse)

2. TITLE AND SUBTITLE

Criticality Benchmark Guide for Light-Water-Reactor Fuel in

Transportation and Storage Packages

8. PERFORMING ORGANIZATION - NAME AND ADDRESS IIf NRC, provide Division, Office or Region, U.S. Nuclear Regulatory Commission, and mailing address; if contractor, provide

make Ridge National Laboratory

Oak Ridge, TN 37831-6370

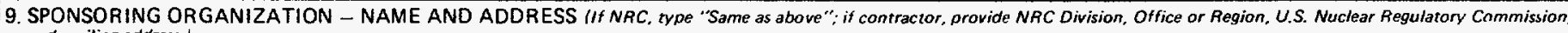
and mailing address.)

Spent Fuel Project Office

Office of Nuclear Material Safety and Safeguards

U.S. Nuclear Regulatory Commission

Washington, DC 20555-0001

10. SUPPLEMENTARY NOTES

11. ABSTRACT (200 words or less)

This report is designed as a guide for performing criticality benchmark calculations for light-water-reactor (LWR) fuel applications. The guide provides documentation of 180 criticality experiments with geometries, materials, and neutron interaction characteristics representative of transportation packages containing LWR fuel or uranium oxide pellets or powder. These experiments should benefit the U.S. Nuclear Regulatory Commission (NRC) staff and licensees in validation of computational methods used in LWR fuel storage and transportation concerns. The experiments are classified by key parameters such as enrichment, water/fuel volume, hydrogen-to-fissile ratio $(\mathrm{H}, \mathrm{X})$, and lattice pitch. Groups of experiments with common features such as separator plates, shielding walls, and soluble boron are also identified. In addition, a sample validation using these experiments and a statistical analysis of the results are provided. Recommendations for selecting suitable experiments and determination of calculational bias and uncertainty are presented as part of this benchmark guide. 